\title{
INCREASED USE OF FLY ASH IN HYDRAULIC CEMENT CONCRETE (HCC) FOR PAVEMENT LAYERS AND TRANSPORTATION STRUCTURES
}
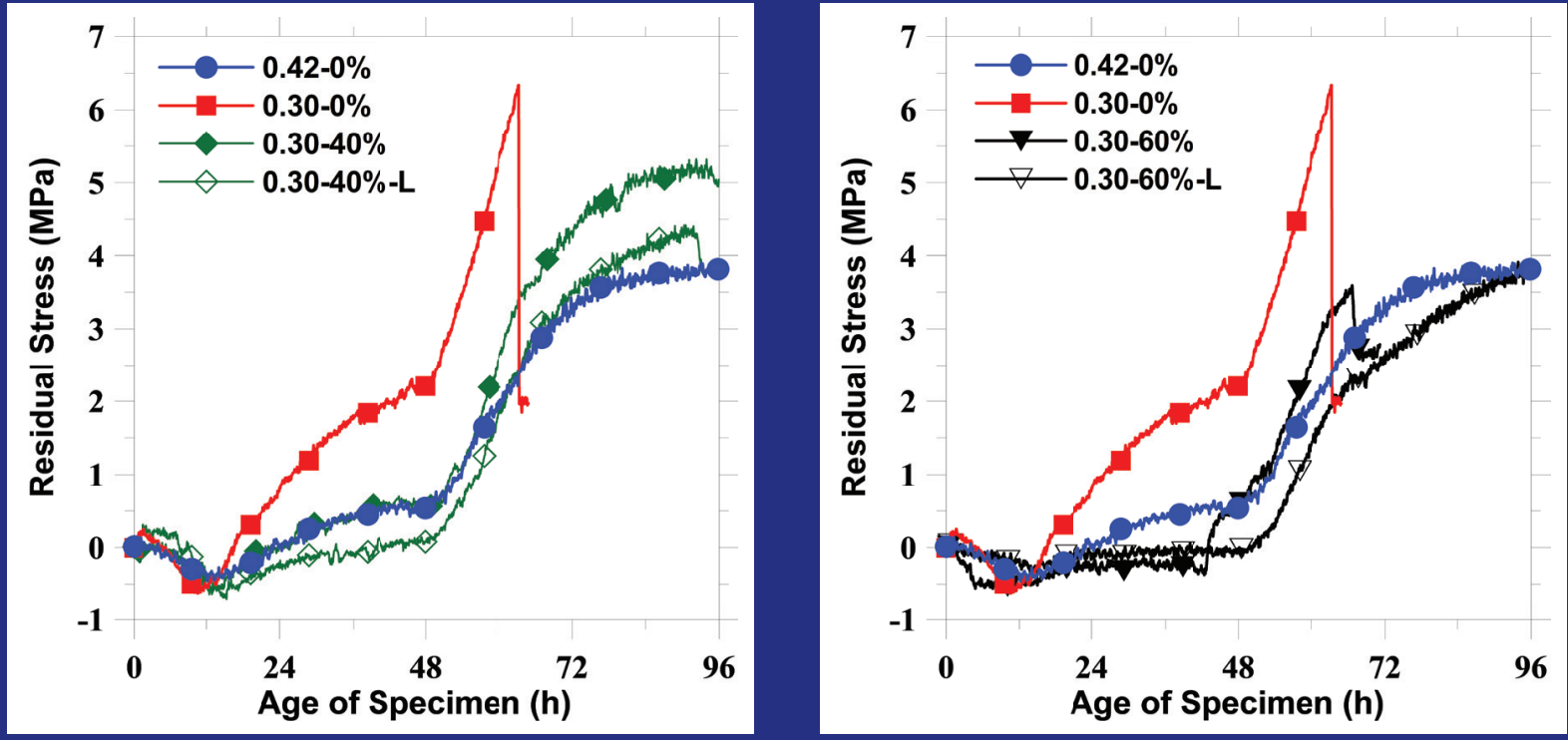

Igor De la Varga, Dale P. Bentz, W. Jason Weiss, Javier Castro, Timothy J. Barrett, Robert P. Spragg, Carmelo Di Bella, Karthik H. Obla, Haejin Kim, Anton K. Schindler, Kevin P. Keith, Taijiro Sato

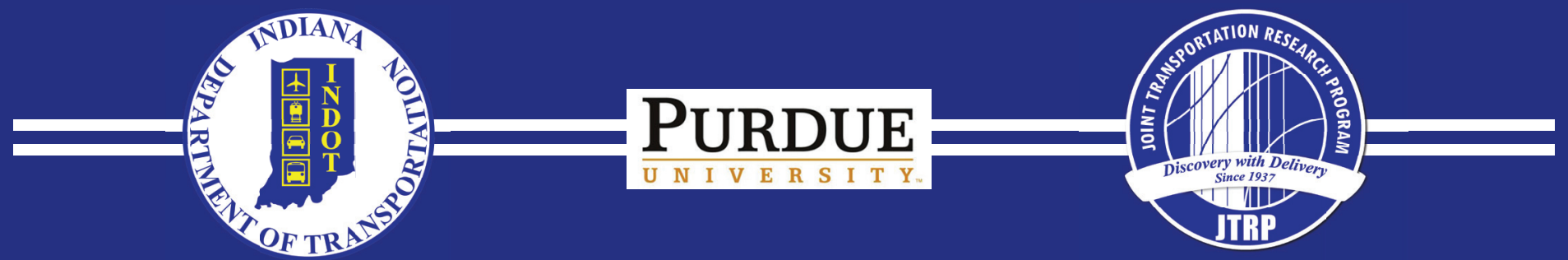




\section{INCREASED USE OF FLY ASH IN HYDRAULIC CEMENT CONCRETE (HCC) FOR PAVEMENT LAYERS AND TRANSPORTATION STRUCTURES}

Project BAA No. DTFH61-08-R-00034

Prime Award No. DTFGH61-09-H-00005

Subaward No. 4108-30885

Igor De la Varga

Dale P. Bentz

W. Jason Weiss

Javier Castro

Timothy J. Barrett

Robert P. Spragg

Carmelo Di Bella

Karthik H. Obla

Haejin Kim

Anton K. Schindler

Kevin P. Keith

Taijiro Sato 


\section{CONTENTS}

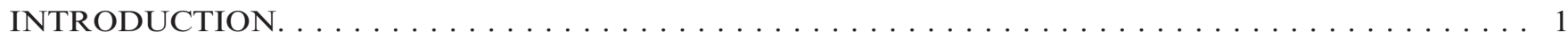

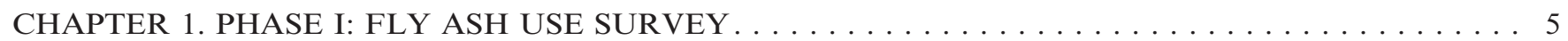

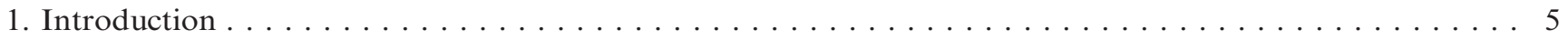

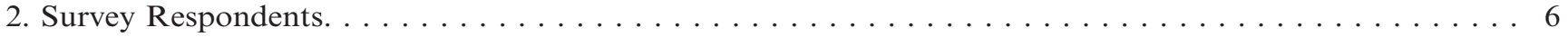

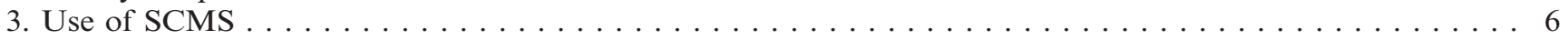

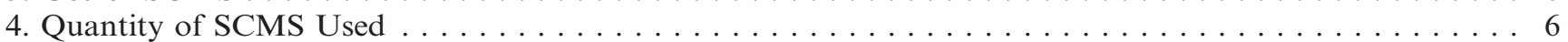

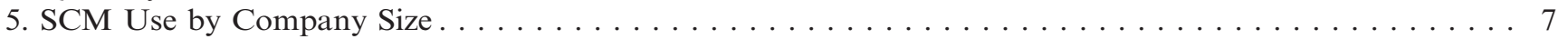

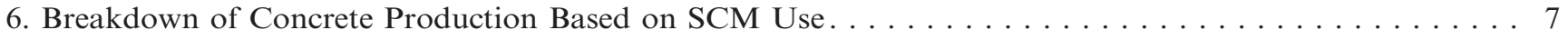

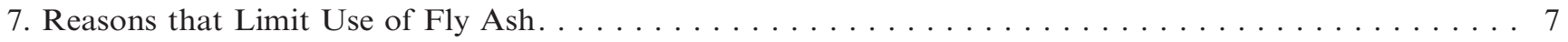

8. Opportunities for Increasing the Use of Fly Ash $\ldots \ldots \ldots \ldots \ldots \ldots \ldots \ldots \ldots \ldots \ldots \ldots$

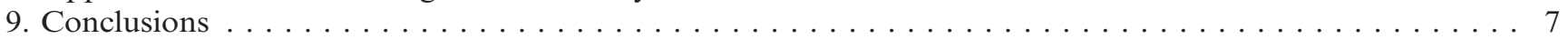

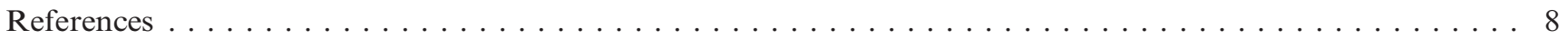

APPENDIX A.1 Supplementary Cementitious Materials (SCM) Use Survey . . . . . . . . . . . . 9

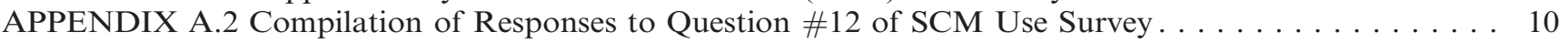

CHAPTER 2. PHASE II: DEVELOPMENT OF AN ACTIVATION ENERGY DATABASE AND

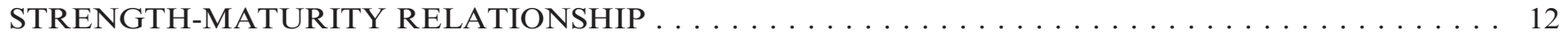

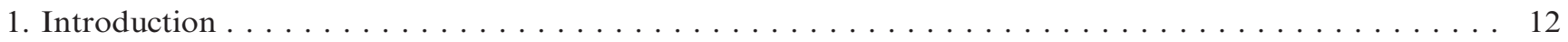

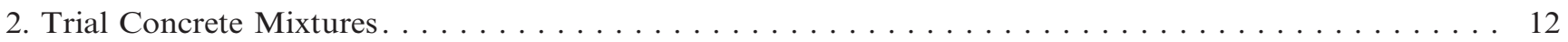

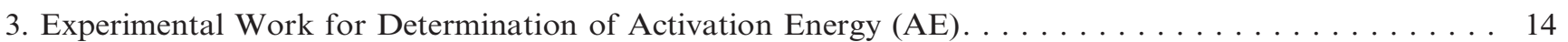

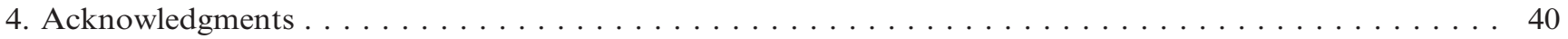

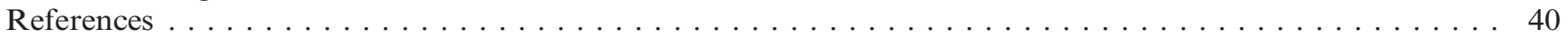

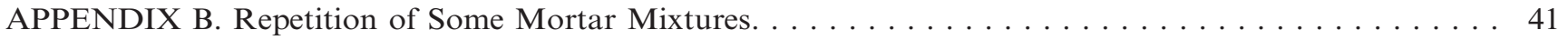

APPENDIX C. Compressive Strength Test Results of Mortar Mixtures . . . . . . . . . . . . . . . 44

CHAPTER 3. PHASE II, TASK 3: SETTING AND TEMPERATURE DEVELOPMENT $\ldots \ldots \ldots \ldots$

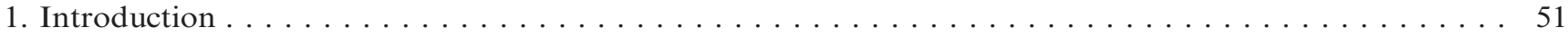

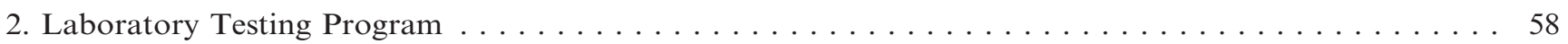

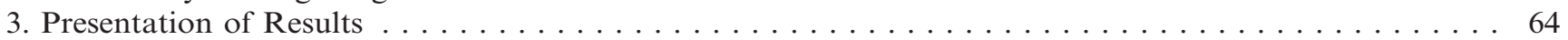

4. Development of HVFA Concrete Hydration Model . . . . . . . . . . . . . . . . . . . . . . 77

5. Evaluation of the Modeled In-Place Performance of HVFA Concrete $\ldots \ldots \ldots \ldots \ldots \ldots \ldots \ldots$

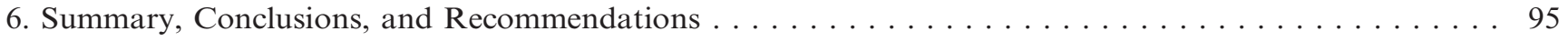

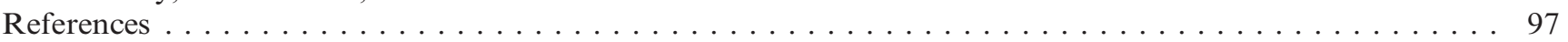

PHASE II, TASKS 4 AND 5: INTERNAL CURING AND EARLY AGE CRACKING-INTRODUCTION. . . 99

CHAPTER 4. PHASE II, TASKS 4 AND 5: APPLICATION OF INTERNAL CURING FOR MIXTURES CONTAINING HIGH VOLUMES OF FLY ASH $\ldots \ldots \ldots \ldots \ldots \ldots \ldots \ldots \ldots \ldots \ldots \ldots \ldots \ldots$

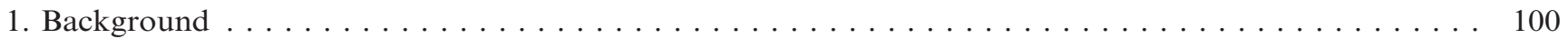

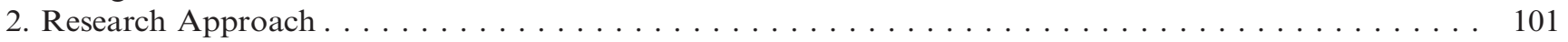

3. Proportioning Mixtures for Internal Curing $\ldots \ldots \ldots \ldots \ldots \ldots \ldots \ldots \ldots \ldots \ldots \ldots \ldots \ldots \ldots$

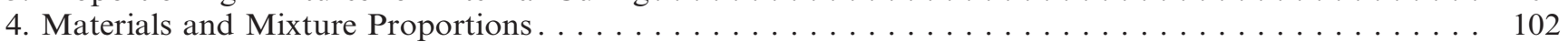

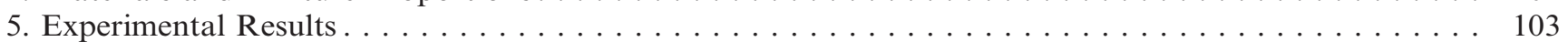

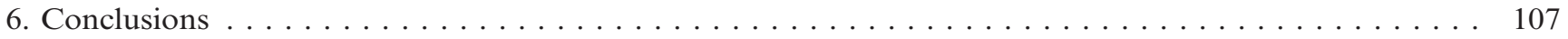

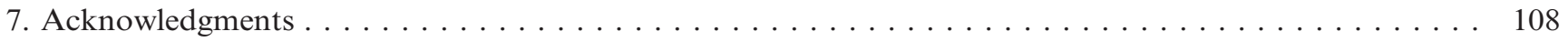

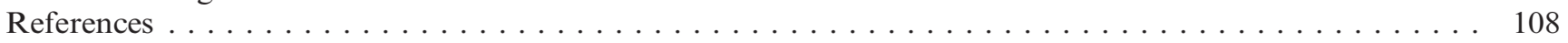

CHAPTER 5. PHASE II, TASKS 4 AND 5: INFLUENCE OF INTERNAL CURING ON THE ISOTHERMAL AND SEMI-ADIABATIC CRACKING BEHAVIOR OF HIGH VOLUME FLY ASH MIXTURES . . . 110

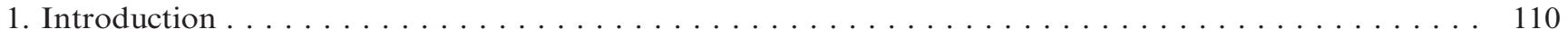

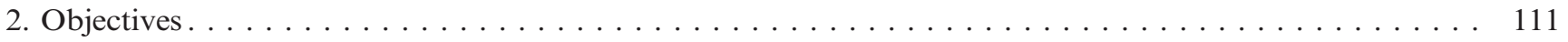

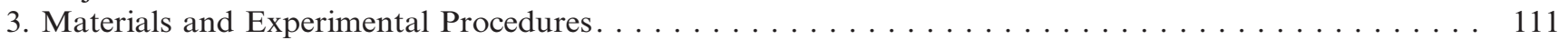

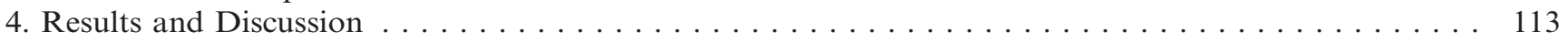

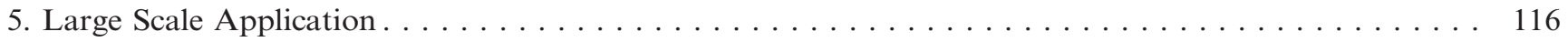

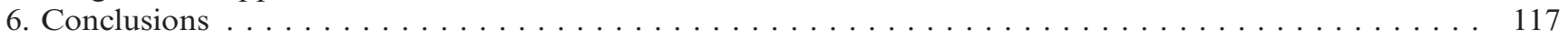

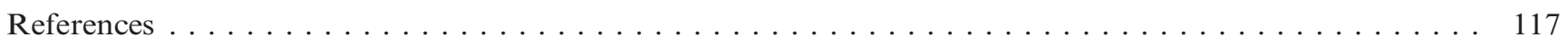


CHAPTER 6. PHASE II, TASKS 4 AND 5: FLUID TRANSPORT IN HIGH-VOLUME FLY ASH

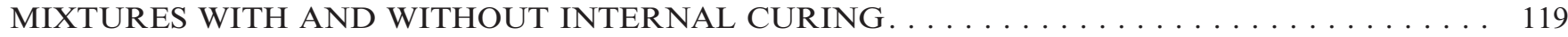

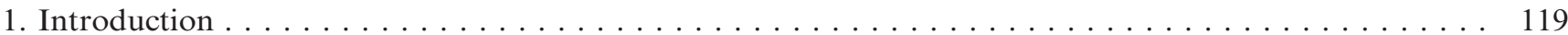

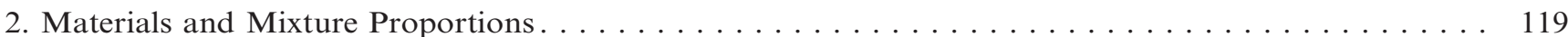

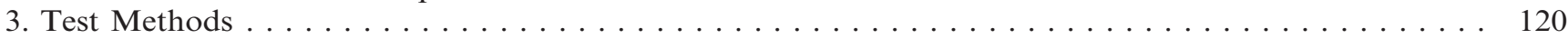

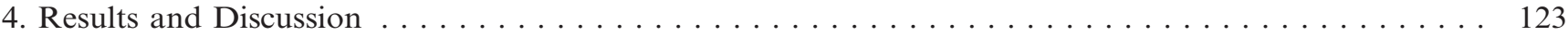

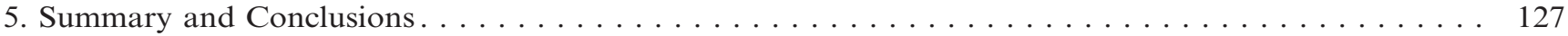

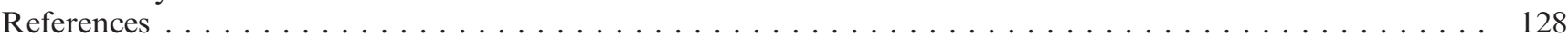

CHAPTER 7. PHASE II, TASKS 4 AND 5: ADDITION OF A TERNARY COMPONENT TO HIGH

VOLUME FLY ASH MIXTURES TO REGULATE SETTING . . . . . . . . . . . . . . . . . . . . 130

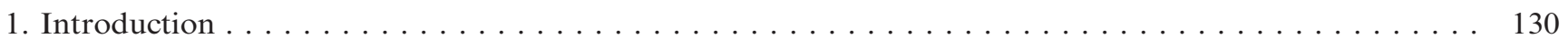

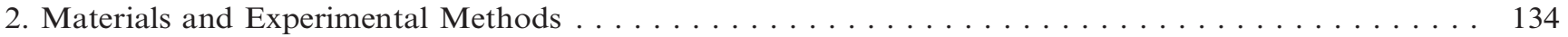

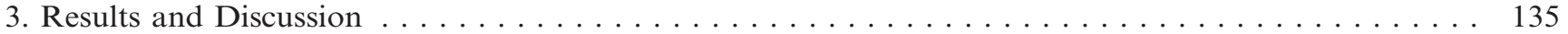

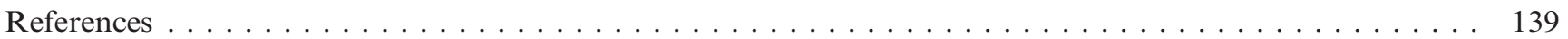

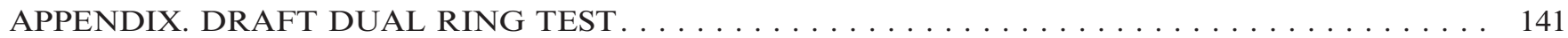




\section{LIST OF TABLES}

Note: Tables are numbered by section in all chapters

Table

Chapter 1

Table 1.1 Fly Ash and Slag Cement Use in Ready Mixed Concrete (RMC)

Table 2.1 Percentile Breakdown, Average, Minimum, and Maximum Amounts of Concrete Production, Fly Ash and SCM Use

Table 5.1 Average Portland Cement, Fly Ash, SCM Usage Based on Company Production Levels

Table 7.1 Analysis of Company Responses of Causes Preventing Increased Use of Fly Ash

Chapter 2

Table 2.1 Chemical and Physical Characteristics of Portland Cements and Fly Ashes

Table 2.2 Summary of Concrete Trial Mixtures

Table 3.1 Characteristics of Mortar Mixtures for Activation Energy Measurement

Table 3.2 Summary of Mortar Test Result @ Control-73 ${ }^{\circ} \mathrm{F}$ (Low Alkali Cement)

Table 3.3 Summary of Mortar Test Result @ Hot-100 ${ }^{\circ} \mathrm{F}$ (Low Alkali Cement)

Table 3.4 Summary of Mortar Test Result @ Cold- $45^{\circ} \mathrm{F}$ (Low Alkali Cement)

Table 3.5 Summary of Mortar Test Result @ Control-73 F (High Alkali Cement)

Table 3.6 Summary of Mortar Test Result @ Hot- $100^{\circ} \mathrm{F}$ (High Alkali Cement)

Table 3.7 Summary of Mortar Test Result @ Cold- $45^{\circ} \mathrm{F}$ (High Alkali Cement)

Table 3.8 Summary of Mortar Strength Result @ Control-73F (Low Alkali Cement)

Table 3.9 Summary of Mortar Strength Result @ Hot-100 F (Low Alkali Cement)

Table 3.10 Summary of Mortar Strength Result @ Cold-45F (Low Alkali Cement)

Table 3.11 Summary of Mortar Strength Result @ Control-73 ${ }^{\circ}$ F (High Alkali Cement)

Table 3.12 Summary of Mortar Strength Result @ Hot-100F (High Alkali Cement)

Table 3.13 Summary of Mortar Strength Result @ Cold-45F (High Alkali Cement)

Table 3.14 Strength vs. Age Curve Fit Parameters for Low Alkali Cement Mixtures Based on ASTM C1074

Table 3.15 Activation Energies Determined for Low Alkali Cement Mixtures Based on ASTM C1074

Table 3.16 Strength vs. Age Curve Fit Parameters for Low Alkali Cement Mixtures Based on Modified ASTM C1074 Approach

Table 3.17 Activation Energies for Low Alkali Cement Mixtures Based on Modified ASTM C1074 Approach

Table 3.18 Strength vs. Age Curve Fit Parameters for High Alkali Cement Mixtures Based on ASTM C1074

Table 3.19 Activation Energies Determined for High Alkali Cement Mixtures Based on ASTM C1074

Table B.1 Mortar Mixtures to Be Repeated 
Table 2.5 Fly Ash Properties

Table 3.1 Summary of Initial and Final Set Times for All $73^{\circ} \mathrm{F}$ Mixtures

Table 3.2 LA Cement Mixtures Activation Energy Summary

Table 3.3 HA Cement Mixtures Activation Energy Summary

Table 4.1 Best-Fit Hydration Parameters from SAC Testing

Table 4.2 Best-Fit Hydration Parameters from SAC Testing from Schindler and Folliard (2005)

Table 5.1 ConcreteWorks Hydration Parameter Inputs

Table 5.2 ConcreteWorks Placement Conditions for Column Elements

Table 5.3 ConcreteWorks Placement Conditions for Flatwork Elements

Table 5.4 Equivalent Age at Initial Set for Mixtures Considered

Table 5.5 LA Cement Mixtures ASTM C 1074 Activation Energy Values

Chapter 4

Table 4.1 Chemical Composition of the Cement and Fly Ash Used in This Study

Table 4.2 List of Mortar Mixtures Prepared in the Study

Table 3.3 Measured Heat Capacities of the Raw Materials

Table 3.4 Measured Thermal Properties of the Five Mortar Mixtures 


\section{LIST OF FIGURES}

Note: Figures are numbered by section in all chapters

Figure

\section{Chapter 1}

Figure 4.1 Percentile distribution of SCM used by companies

Chapter 2

Figure 3.1 Compressive strength test results of the low alkali cement control mortar mixture vs. (a) $35 \%$ fly ash; (b) $50 \%$ fly ash. All mixtures cured at Control- $73^{\circ} \mathrm{F}$

Figure 3.2 Compressive strength test results of the low alkali cement control mortar mixture vs. (a) 35\% fly ash; (b) 50\% fly ash. All mixtures cured at $\mathrm{Hot}-100^{\circ} \mathrm{F}$

Figure 3.3 Compressive strength test results of the low alkali cement control mortar mixture vs. (a) 35\% fly ash; (b) $50 \%$ fly ash. All mixtures cured at Cold $-45^{\circ} \mathrm{F}$

Figure 3.4 Compressive strength test results of the high alkali cement control mortar mixture vs. (a) $35 \%$ fly ash; (b) $50 \%$ fly ash. All mixtures cured at Control- $73^{\circ} \mathrm{F}$

Figure 3.5 Compressive strength test results of the high alkali cement control mortar mixture vs. (a) $35 \%$ fly ash; (b) $50 \%$ fly ash. All mixtures cured at $\mathrm{Hot}-100^{\circ} \mathrm{F}$

Figure 3.6 Compressive strength test results of the high alkali cement control mortar mixture vs. (a) $35 \%$ fly ash; (b) $50 \%$ fly ash. All mixtures cured at Cold $-45^{\circ} \mathrm{F}$

Figure 3.7 Strength-age plot: (a) plot to compute activation energy based on ASTM C1074 (b) and strength-equivalent age plot (c) for low alkali portland cement $\mathrm{B}$ mixture

Figure 3.8 Strength-equivalent age plot for Low Alkali Cement B Mixtures based on ASTM C1074: (a) 0.39FAA35, (b) 0.39FAB35, (c) 0.39FAC35, (d) 0.39FAA50, (e) 0.39FAB50, and (f) 0.39FAC50

Figure 3.9 Strength-age plot: (a) plot to compute activation energy based on modified ASTM C1074 (b) and strength-equivalent age plot (c) for low alkali portland cement B mixture

Figure 3.10 Strength-equivalent age plot for low alkali Cement B mixtures based on modified ASTM C1074: (a) 0.39FAA35, (b) 0.39FAB35, (c) 0.39FAC35, (d) 0.39FAA50, (e) 0.39FAB50, and (f) 0.39FAC50

Figure 3.11 Strength-age plot: (a) plot to compute activation energy based on ASTM C1074 (b) and strength-equivalent age plot (c) for high alkali portland cement mixture

Figure 3.12 Strength-equivalent age plot for high alkali cement mixtures based on ASTM C1074: (a) 0.39FAA35, (b) 0.39FAB35, (c) $0.39 \mathrm{FAC} 35$, (d) 0.39FAA50, (e) 0.39FAB50, and (f) 0.39FAC50

Figure 3.13 Strength-age plot: (a) plot to compute activation energy based on modified ASTM C1074 (b) and strength-equivalent age plot (c) for high alkali portland cement mixture

Figure 3.14 Strength-equivalent age plot for high alkali cement mixtures based on modified ASTM C1074: (a) 0.39FAA35, (b) 0.39FAB35, (c) $0.39 \mathrm{FAC} 35$, (d) 0.39FAA50, (e) 0.39FAB50, and (f) 0.39FAC50

Figure 3.15 Compressive strength vs. equivalent age plots for concrete mixtures: (a) 0.34FAA50LAC, (b) 0.34FAC50LAC, (c) $0.34 \mathrm{FAA} 50 \mathrm{HAC}$, and (d) 0.34FAC50HAC

Figure B.1 Compressive strength test result of repeated mortar mixture

Figure B.2 Curing temperature history comparisons of repeat mortar and original mortar mixtures

Figure C.1 Strength-age plot with low and high alkali cement at (a) Hot, (b) Control, and (c) Cold temperature; (d) strength-age plot with low alkali cement; (e) strength-age plot with high alkali cement for $0.43 \mathrm{PC}$ mixture

Figure C.2 Strength-age plot with low and high alkali cement at (a) Hot, (b) Control, and (c) Cold temperature; (d) strength-age plot with low alkali cement; (e) strength-age plot with high alkali cement for 0.39FAA35 mixture

Figure C.3 Strength-age plot with low and high alkali cement at (a) Hot, (b) Control, and (c) Cold temperature; (d) strength-age plot with low alkali cement; (e) strength-age plot with high alkali cement for $0.39 \mathrm{FAB} 35$ mixture

Figure C.4 Strength-age plot with low and high alkali cement at (a) Hot, (b) Control, and (c) Cold temperature; (d) strength-age plot with low alkali cement; (e) strength-age plot with high alkali cement for 0.39 FAC 35 mixture 
Figure C.5 Strength-age plot with low and high alkali cement at (a) Hot, (b) Control, and (c) Cold temperature; (d) strength-age plot with low alkali cement; (e) strength-age plot with high alkali cement for 0.34FAA50 mixture

Figure C.6 Strength-age plot with low and high alkali cement at (a) Hot, (b) Control, and (c) Cold temperature; (d) strength-age plot with low alkali cement; (e) strength-age plot with high alkali cement for 0.34FAB50 mixture

Figure C.7 Strength-age plot with low and high alkali cement at (a) Hot, (b) Control, and (c) Cold temperature; (d) strength-age plot with low alkali cement; (e) strength-age plot with high alkali cement for 0.34FAC50 mixture

Chapter 3

Figure 1.1 Effect of curing temperature on degree of hydration

Figure 1.2 Sample isothermal calorimetry results

Figure 1.3 Sample semi-adiabatic calorimetry results

Figure 1.4 Influence of fly ash $\mathrm{CaO}$ content on heat of hydration

Figure 1.5 Comparison of compressive strength versus real concrete age and equivalent age for samples cured at different temperatures

Figure 2.1 Nomenclature used for control and fly ash concrete mixtures

Figure 3.1 Effect of fly ash dosage on set times for (a) LA-1\%, (b) LA-15\%, (c) LA-24\%, and (d) LA-28\% mixtures $\left(73^{\circ} \mathrm{F}\right)$

Figure 3.2 Effect of fly ash dosage on set times for (a) HA-1\%, (b) HA-15\%, (c) HA-24\%, and (d) HA-28\% mixtures (73 ${ }^{\circ} \mathrm{F}$ )

Figure $3.373^{\circ} \mathrm{F}$ Setting test results for (a) LA-35FA, (b) LA-50FA, (c) HA-35FA, and (d) HA-50FA mixtures

Figure 3.4 Effect of temperature on HVFA concrete set times for (a) LA-35FA-15\%, (b) LA-50FA-28\%, (c) HA-50FA-1\%, and (d) HA$35 \mathrm{FA}-24 \%$

Figure 3.5 Sample mortar temperature history (LA-35FA-28\%)

Figure 3.6 Penetration resistance versus equivalent age results for (a) LA-35FA-15\% and (b) HA-50FA-1\% concrete mixtures

Figure 3.7 Rate of heat evolution for (a) LA-35FA and (b) LA-50FA mixtures

Figure 3.9 Rate of heat evolution for (a) LA-35FA-15\%, (b) LA-50FA-28\%, (c) HA-50FA-1\%, and (d) HA-35FA-24\%

Figure 3.10 Arrhenius rate plots for (a) LA-35FA-15\%, (b) LA-50FA-28\%, (c) HA-50FA-1\%, and (d) HA-35FA-24\%

Figure 3.11 Heat generation per volume of concrete for a) LA-1\%, b) LA-15\%, c) LA- $24 \%$ and d) LA- $28 \%$ concrete mixtures $\left(1 \mathrm{MJ} / \mathrm{m}^{3}=\right.$ $\left.0.725 \mathrm{kBTU} / \mathrm{yd}^{3}\right)$

Figure 3.12 Heat generation per volume of concrete for (a) HA-1\%, (b) HA-15\%, (c) HA-24\%, and (d) HA-28\% concrete mixtures (1 MJ/m ${ }^{3}$ $\left.=0.725 \mathrm{kBTU} / \mathrm{yd}^{3}\right)$

Figure 3.13 Effect of fly ash $\mathrm{CaO}$ content on heat generation for (a) HA-35FA and (b) HA-50FA concrete mixtures

Figure 3.14 Compressive strength testing results for (a) LA-35FA-Class F, (b) LA-35FA-Class C, (c) LA-50FA-Class F, and (d) LA-50FAClass C mixtures

Figure 3.15 Compressive strength testing results for (a) HA-35FA-Class F, (b) HA-35FA-Class C, (c) HA-50FA-Class F, and (d) HA-50FAClass C mixtures

Figure 3.16 Effect of fly ash $\mathrm{CaO}$ content on compressive strength development of (a) HA-35FA and (b) HA-50FA mixtures

Figure 4.1 Effect of changes to: (a) hydration time parameter, (b) hydration shape parameter, and (c) ultimate degree of hydration on the overall hydration development; (d) example of hydration equation fitted to SAC testing results

Figure 4.2 Fly ash heat contribution versus fly ash $\mathrm{CaO}$ content

Figure 4.3 Plot of measured versus predicted degree of hydration for final model

Figure 5.1 In-place temperature profiles for $6 \times 6 \mathrm{ft}$ columns placed at $70^{\circ} \mathrm{F}$ for the following mixtures: (a) $35 \mathrm{FA}-1 \%$, (b) $50 \mathrm{FA}-1 \%$, (c) $35 \mathrm{FA}-$ $24 \%$, and (d) $50 \mathrm{FA}-24 \%$

Figure 5.2 In-place temperature profiles for HVFA concrete proportioned with 50\% fly ash dosage placed in $6 \times 6 \mathrm{ft}$ columns at $70^{\circ} \mathrm{F}$

Figure 5.3 In-place temperature profiles for HVFA concrete proportioned with 50\% fly ash dosage placed in $6 \times 6 \mathrm{ft}$ columns at (a) $40^{\circ} \mathrm{F}$ and (b) $95^{\circ} \mathrm{F}$ 
Figure 5.4 In-place temperature profiles for HVFA concrete proportioned with 50\% fly ash dosage placed in: (a) $10 \times 10 \mathrm{ft}$ column and (b) 8 in. bridge deck elements at $70^{\circ} \mathrm{F}$

Figure 5.5 Maximum in-place concrete temperature versus concrete placement temperature for $3 \times 3 \mathrm{ft}$ columns 88

Figure 5.6 Maximum in-place concrete temperature versus concrete placement temperature for 6 x $6 \mathrm{ft}$ columns 89

Figure 5.7 Maximum in-place concrete temperature versus concrete placement temperature for $10 \times 10 \mathrm{ft}$ columns 89

Figure 5.8 Maximum in-place concrete temperature versus concrete placement temperature for 8 in. bridge deck element 90

Figure 5.9 Maximum in-place concrete temperature versus concrete placement temperature for 14 in. pavement element 91

Figure 5.10 Time to reach initial for the (a) $35 \%$ fly ash dosage and (b) $50 \%$ fly ash dosage concrete mixtures when placed in the column or bridge deck elements

Figure 5.11 Time to reach initial for the (a) 35\% fly ash dosage and (b) 50\% fly ash dosage concrete mixtures when placed in the pavement element

Figure 5.12 Time to reach $70 \%$ of the 28 -day strength for (a) $35 \%$ fly ash dosage and (b) $50 \%$ fly ash dosage concrete mixtures when placed in the $6 \times 6 \mathrm{ft}$ column

Figure 5.13 Time to reach $70 \%$ of the 28 -day strength for a) $35 \%$ fly ash dosage and b) $50 \%$ fly ash dosage concrete mixtures when placed in the $10 \times 10 \mathrm{ft}$ column

Figure 5.14 Time to reach 70\% of the 28-day strength for (a) $35 \%$ fly ash dosage and (b) $50 \%$ fly ash dosage concrete mixtures when placed in the bridge deck element

Figure 5.15 Time to reach 70\% of the 28-day strength for a) 35\% fly ash dosage and b) $50 \%$ fly ash dosage concrete mixtures when placed in the pavement element

Chapter 4

Figure 1.1 Conceptual illustration of the differences between external and internal curing

Figure 4.1 Particle Size Distributions (PSD) of cement and fly ash, as measured by laser diffraction

Figure 5.1 Effect of decreasing $w / \mathrm{cm}$ on the compressive strength. (Error bars represent the standard deviation from the average of three samples.)

Figure 5.2 Effect of adding high volumes of fly ash on the compressive strength. (Error bars represent the standard deviation from the average of three samples.)

Figure 5.3 Effect of including IC in HVFA mixtures on the compressive strength. (Error bars represent the standard deviation from the average of three samples.)

Figure 5.4 Effect of including IC in HVFA mixtures on the elastic modulus. (Error bars represent the standard deviation from the average of two samples.)

Figure 5.5 Autogenous shrinkage as a function of time. (Error bars represent the standard deviation from the average of three samples.)

Figure 5.6 Measured internal relative humidity of an aging mortar. (Error bars represent the standard deviation from the average of two samples.)

Figure 5.7 Chemical shrinkage in mortar samples. (Error bars represent plus and minus one standard deviation from the average of two samples.)

Figure 5.8 Stress developed in ring specimens as a function of time. (Error bars represent the deviation from the average of two samples.) 106

Figure 5.9 Temperature rise as a function of time

Figure 5.10 Cumulative heat released (Isothermal) curves vs. time on a per milliliter of water basis

Figure 5.11 Measured compressive strengths vs. measured cumulative heat on a per milliliter initial water basis for mortars at the ages of 1, 3, 7 , and $14 \mathrm{~d}$. Solid line shows the best fit while the two dashed lines indicate $\pm 10 \%$ from the best fit

Figure 5.12 Effect of changing IC replacement level

Chapter 5

Figure 4.1 2-day residual stress comparison

Figure 4.2 4-day residual stress comparison. Three of the mixes did not crack at any time: $0.42-0 \%, 0.30-40 \%-\mathrm{L}$, and $0.30-60 \%-\mathrm{L}$ 
Figure 4.4 Remaining stress capacity comparison at $2 \mathrm{~d}, 4 \mathrm{~d}$, and $7 \mathrm{~d}$ of age

Figure 4.5 Semi-adiabatic temperature profiles

Figure 4.6 Semi-adiabatic residual stress development

Figure 4.7 Concrete beam preparation

Figure 4.8 Basic configuration for measuring thermal properties of mortar samples using the Hot Disk Thermal Constants Analyzer

Figure 3.1 Rapid Chloride Migration (RCM) experimental set-up

Figure 3.2 A sample from the Rapid Chloride Migration (RCM) test that was split and sprayed with Silver $\mathrm{Nitrate}\left(\mathrm{AgNO}_{3}\right)$

Figure 3.3 Rapid Chloride Penetration Test (RCPT) cells

Figure 3.4 Wenner probe surface testing device

Figure 4.1 Chloride penetration profiles at different curing/ponding time periods. (Error bars represent the standard deviation from the average of three samples.)

Figure 4.2 Formation factor as a function of time of mortars samples stored in lime water up to 365 d. (Error bars represent the standard deviation from the average of three samples.)

Figure 4.3 Absorbed water of sealed-cured mortars samples (a) as a function of fly ash content curing during $28 \mathrm{~d}$, (b) as a function of fly ash content curing during $90 \mathrm{~d}$, (c) including IC and curing $28 \mathrm{~d}$, and (d) including IC and curing $90 \mathrm{~d}$. (Error bars represent the standard deviation from the average of three samples.)

Figure 4.4 (a) and (b) Initial sorptivity of the sealed-cured mortars samples; (c) and (d) Secondary sorptivity of the sealed-cured mortars samples

\section{Chapter 7}

Figure 1.1 Isothermal calorimetry for $w / \mathrm{cm}=0.36$ pastes, indicating about $3 \mathrm{~h}$ of retardation due to the Class $\mathrm{C}$ fly ash

Figure 1.2 Isothermal calorimetry for $w / \mathrm{cm}=0.3$ pastes prepared with various additions of gypsum to a $50 \% \mathrm{Class} \mathrm{C}$ fly ash blended cement

Figure 1.3 Isothermal calorimetry curves for $w / \mathrm{cm}=0.36$ pastes prepared with the Type I/II cement and the high calcium Class C fly ash 131

Figure 1.4 Isothermal calorimetry heat flow (a) and cumulative heat release ( $\mathrm{b}$ and c) curves for $\mathrm{w} / \mathrm{cm}=0.36$ blended cement pastes prepared with two levels of addition of a rapid set cement. RSC = rapid set cement

Figure 1.5 Isothermal calorimetry heat flow (a) and cumulative heat release ( $\mathrm{b}$ and c) curves for $w / \mathrm{cm}=0.36$ blended cement pastes prepared with various additions of aluminate. $\mathrm{CAC}=$ calcium aluminate cement

Figure 2.1 Measured particle size distributions for the cement and fly ash investigated in the study. Results are the average of six individual measurements and error bars (one standard deviation) would fall within the size of the symbols

Figure 2.2 Particle size distributions for the limestone and silica powders investigated in the study

Figure 3.1 Measured Vicat needle penetrations vs. time for the nine cement pastes

Figure 3.2 Isothermal calorimetry curves vs. time for the nine cement pastes. For each curve, the short vertical line indicates the initial setting time

Figure 3.3 Cumulative heat release curves vs. time for the nine cement pastes on a per $\mathrm{ml}$ of water basis

Figure 3.4 Measured compressive strengths for mortar cubes at four ages

Figure 3.5 Isothermal calorimetry results for mortars with various volumetric substitutions of silica powder for cement

Figure 3.7 Measured compressive strengths vs. measured cumulative heat release on either a per ml paste basis (top) or a per ml initial water basis (bottom) for mortars at ages of $1 \mathrm{~d}$ and $7 \mathrm{~d}$ 


\section{INTRODUCTION}

High volume fly ash (HVFA) concrete mixtures can have the benefit of reduced cost, reduced energy content, enhanced environmental sustainability, and improved long-term performance. While many producers and transportation agencies desire to increase the use of fly ash in the transportation infrastructure, several barriers exist to adopting these mixtures in practice. These barriers include:

- specifications which prohibit the use of high volumes of fly ash based on historical performance observations,

- a lack of contractor/agency familiarity with prediction of setting and strength development for construction sequencing,

- concerns about potential material incompatibilities which can dramatically alter the performance of admixtures or inhibit the chemical reactions that lead to setting and strength development,

- questions about the practical ability of providing a sufficient length of curing to ensure durability due to the delayed reactions,

- questions about long-term durability especially with respect to scaling and freeze thaw performance that can lead to volume or time of placement limits, and

- a lack of understanding of all the benefits associated with using HFVA mixtures.

The conventional approach to using fly ash has relied on the establishment of strict limits on the maximum amount of fly ash that can be used or limits on the times of year that fly ash can be used in construction. Further, conventional applications have set limits on the composition of the fly ash to enable the fly ash-cement system to be treated the same way conventional portland cement has been treated in concrete. This project moved in a bold new direction by proposing innovative solutions to approach the use of fly ash in mixture proportioning, new design methodologies to predict strength, and new strategies to overcome the hurdles associated with HVFA use.

\section{REPORT ORGANIZATION}

The project was divided into two phases. Phase I was performed by the National Ready Mix Concrete Association (NRMCA) and is summarized in Chapter 1. This portion of the work consisted of an industry survey. The American Coal Ash Association (ACAA) conducted a survey in 2004 that concluded that of the 70 million tons of fly ash produced annually; only $40 \%$ was beneficially utilized. This means that a large portion of this fly ash $(60 \%)$ was land-filled. While concrete is the largest single market for fly ash, it offers the potential for even greater fly ash utilization. A large body of research exists on the development of HVFA concrete mixtures. Despite that, applications of HVFA concrete are still somewhat limited. Surveys suggest that the average fly ash content in all ready-mixed concrete is still only about $10 \%$ (of total cementitious content). Even though some producers may be using fly ash contents as high as $30 \%$ in summer months, this may change dramatically in the late fall, winter, and early spring as temperature drops.

Phase II of this project was the research and innovation phase. This phase is divided into six chapters and an appendix, as described below.

\section{Activation Energy}

The premise of this chapter is that even though laboratory or field cured HVFA concrete cylinders may show low early-age strengths, the strength that develops in the structure is usually higher. Pavements and structures retain heat from the hydration reactions, resulting in in-place concrete temperatures that enable faster rates of strength gain than those experienced by standard cylinders. In summary, HVFA concrete mixtures may be unfairly penalized by strengths measured using standard cylinder tests at constant curing temperature.

The challenge in accurately predicting the concrete strength in a structure may be answered if the maturity method can be easily extended to HVFA mixtures (Carino, 1991). In-place strength can be estimated by monitoring the concrete's temperature history, calculating the accumulated maturity, and obtaining an estimated strength from the pre-calibrated strengthmaturity relationship. While maturity concepts are well accepted and utilized in practice for portland cement concretes, they are not so established for HVFA concrete mixtures containing chemical admixtures. The Arrhenius maturity function requires the use of a binder-specific activation energy. While this can be reasonably estimated for many OPC materials, obtaining an estimate for an HVFA binder is more difficult. A methodology is needed to establish specific fundamental relationships that will have broad application for HVFA materials.

\section{Setting and Temperature Development}

Increases in the volume fraction of fly ash generally reduce the rate of overall hydration, which in turn leads to a decreased rate of strength development in structures. Since the hydration process is highly dependent on temperature, in-place setting times, timing of finishing operations, and rates of strength development are significantly impacted by the size of the structure built and ambient conditions experienced during placement. These effects associated with the use of HVFA concrete can be very difficult to manage during construction. Limited guidance is currently available to the concrete industry that may want to consider using HVFA concretes.

Currently, the majority of contractors have limited experience with HVFA mixtures which places these mixtures at a disadvantage in an environment where contractor incentives are tied to rapid construction schedules using processes they know well. As a result, many contractors do not pursue the benefits of HVFA since they are concerned about many of the side effects 
that can occur. For example, delayed initial setting times prolong the time that the concrete finishing crew has to wait at the job site before they can do the final finishing of the concrete slab. This "idle waiting time" substantially increases concrete construction costs of primarily the slab type elements that need to be finished. Delayed initial setting times also increases the chances of plastic shrinkage cracking. The setting times of high volume Class $\mathrm{C}$ fly ash concrete can be delayed as compared to either plain portland cement or Class F fly ash concretes (Wade, Nixon, Schindler, \& Barnes, 2004). Accelerating chemical admixtures have been used to reduce these setting times. However, the use of non-chloride based accelerators is not as effective. Research is needed to quantify the effect of fly ash dosage, type, and curing temperature on setting times of HVFA concretes.

It should be noted that recently several user-friendly, computer programs have been developed to help characterize the temperature development and behavior of various concrete structures exposed to in-place conditions. Prof. Schindler has developed models for some of the most commonly used programs in the United States, which include HIPERPAV (Schindler, 2004) and ConcreteWorks (Riding, Poole, Schindler, Juenger, \& Folliard, 2007). Research at Purdue using FEM MASSE (Raoufi, Radlinska, Nantung, \& Weiss, 2008) has demonstrated how environmental conditions can influence the timing of saw-cutting. While these programs are powerful, the limited database of input parameters restricts their application to HVFA concretes.

Hydration is an exothermic process, and the total amount of heat generated may affect the in-place performance of some structures. The total heat released during hydration is a function of the composition of cementitious materials, amount of cementitious materials, and the $w / \mathrm{cm}$ of the mixture. Semi-adiabatic calorimetry provides means to quantify how the heat of hydration development of a mixture progresses (Schindler $\&$ Folliard, 2005). The ratio of the heat developed to the maximum heat available in the system, is a practical method to quantify the degree of hydration development (Raoufi et al., 2008).

Research is required to quantify the hydration behavior and temperature dependence of HVFA concretes that contain fly ash with different chemical compositions. This can reduce some of the concerns associated with strength gain and setting. Further, this will enable the beneficial effect of HVFA concretes to be quantified as it reduces the total temperature rise in concrete members, which may help to control durability issues related to thermal cracking (particularly for mass concrete applications) and delayed-ettringite formation.

\section{Design of HVFA with IC}

It is known that HVFA have great potential to improve the performance of concrete. For example, the use of fly ash can reduce the propensity of a mixture to exhibit Alkali-Silica Reactions (ASR). Further, it is known that HVFA mixtures hydrate more slowly, thereby reducing the propensity for early age cracking caused by rapid temperature development or restrained shrinkage. However, the slower hydration will also cause a slower strength development and the difficulty of obtaining proper curing in HVFA systems, as they are more susceptible to the quality and longevity of applied curing conditions than conventional concrete systems (Bentz, 2002). For example, many state DOT's currently advocate/mandate increasing their standard "wet" curing time from $7 \mathrm{~d}$ to $14 \mathrm{~d}$ when the concrete mixtures contain fly ash. For HVFA mixtures, the fly ash will continue to react and participate in microstructural refinement and strength development processes for well beyond $14 \mathrm{~d}$, provided that sufficient water is present to support these reactions. It may not be practical to mandate these long periods of external curing. To overcome this limitation, it may be possible to use emerging technology where the concrete can in effect 'cure itself.'

The recent development of internal curing (IC) technology using pre-wetted lightweight aggregates (Bentz, Lura, \& Roberts, 2005; Kovler \& Jensen, 2007) is particularly applicable to high volume fly ash mixtures, as has already been demonstrated in practice in Texas (Mehta, 2004). The use of pre-wetted lightweight aggregates (fine, coarse, or a mixture of both) will provide a set of internal reservoirs of additional curing water that are critical to achieving the full hydration potential of high volume fly ash blended cements.

Specifically, as the cement and fly ash react with water to hydrate, the volume occupied by these products is significantly less than that of the starting materials (Bentz, 2007). If a source of additional water is not available, this chemical shrinkage will result in the creation of a set of empty pores and a corresponding reduction in internal relative humidity as the cement paste self-desiccates, causing shrinkage and reducing hydration rates. Obviously, it is desirable to avoid this scenario, but with conventional external curing, the additional curing water applied to the top concrete surface is only able to penetrate a limited depth (perhaps only a few millimeters) into the concrete. In this case, the interior of the concrete undergoes selfdesiccation and cracking may ensue. With internal curing, the water reservoirs are distributed uniformly throughout the three-dimensional concrete microstructure and the blended cement/fly ash paste remains saturated as the larger pores in the lightweight aggregates sacrificially empty instead (Henkensiefken, Castro, Bentz, Nantung, \& Weiss, 2009). This mitigates the early-age autogenous shrinkage and also provides adequate water for the longer term reaction of the fly ash (Bentz, 2007).

This chapter presents an innovative method to design HVFA concrete mixtures with IC. It will be shown that IC not only improves curing conditions in HVFA mixtures, but also provides benefits in terms of increased mechanical properties, increased hydration, and reduced early age shrinkage. 


\section{Cracking in HVFA with IC}

The problem of early age cracking has garnered much attention over the last two decades in the concrete industry (Bentur, 2003). As the paste content and concrete strength have increased, many have observed cracking, causing the concrete community to examine the factors responsible for these premature failures. Premature cracking can frequently be traced to three driving forces, namely: thermal shrinkage, drying shrinkage or autogenous shrinkage. Currently, researchers can use tools like the standard ring test (ASTM C 1581 or AASHTO PP-34) to assess drying and autogenous (hygral) shrinkage under isothermal conditions. However, relatively little work has been done to assess the combined effects of temperature with hygral shrinkage. It is known that the cracking potential is dependent on the shrinkage, the coefficient of thermal expansion, the elastic modulus development, creep/relaxation and the fracture properties of the mixture (Shah, Weiss, \& Yang, 1998). HVFA mixtures may show specific benefits due to lower heat of hydration and slower development of elastic modulus and creep which would enable stresses to be "relaxed" out of the system. The benefits of HVFA with respect to reduced cracking potential are not fully realized or appreciated by the concrete community. As such, research is needed to demonstrate and quantify these benefits.

The majority of work that has been performed uses linear passive resistance cracking frames or active resistance frames. While the linear frames are easy to interpret, difficulties associated with end restraint and the costs of the active resistance frames can make these difficult to be used in quality control or materials screening applications. Recently, new tests have been developed to use the dual ring specimen geometry to assess residual tensile stress and compressive stress development. While the dual ring was initially used for paste at isothermal conditions, a recent project with INDOT enabled the Purdue-NIST team to develop a dual ring setup that can be used for concrete under a variety of temperature conditions (isothermal, adiabatic, or specified) and which enables tensile and compressive residual stress and cracking potential to be assessed (Schlitter, Bentz, \& Weiss, 2010;Schlitter, Senter, Bentz, Nantung, \& Weiss, 2010).

This chapter quantifies the early-age tensile stress development due to both autogenous and thermal shrinkage using the dual ring test. The results show the lower potential for cracking of HVFA mixtures including IC.

\section{Fluid Transport in HVFA with IC}

Salt infiltration has always been a problem in concrete structures (especially bridge decks). Likewise, other ions and species (e.g., sulfates, $\mathrm{CO}_{2}$ ), can enter the concrete and cause general degradation, thus reducing the service life of the material. This has catalyzed efforts in the concrete community into producing less permeable concretes by reducing the $w / \mathrm{cm}$.
It is also known that fly ash in concrete will further refine the microstructure due mainly to a pozzolanic effect. This should be even more evident in HVFA mixtures. However, this pore refinement along with the low $w / \mathrm{cm}$ used in this type of concrete mixtures won't allow the material to properly cure if conventional (external) curing techniques are deployed. This chapter will show how IC might be beneficial in further reducing the permeability of the concrete at the same time that it ensures the provision of curing water to the material.

\section{Ternary Component in HVFA}

Two of the common issues associated with HVFA concrete mixtures are delays in setting times and reductions in early-age strengths, both due to the lower reactivity of the fly ash relative to the cement that it is replacing. Industry has developed protocols/practices for partially mitigating these issues including reducing the $w / \mathrm{cm}$ of the HVFA concrete, switching from an ASTM C150 Type I to a more reactive Type III cement, and utilizing chemical accelerators. However, the mitigation is often only partially successful, even when large dosages of expensive chemical admixtures are employed.

This chapter details efforts to investigate an alternate route to reducing setting times and enhancing early-age strengths in HVFA concretes by adding a ternary component to the binder, namely a fine limestone (calcium carbonate) powder. The limestone surfaces provide nucleation sites for the precipitation of cement hydration and fly ash reaction products, while the limestone itself contributes calcium and carbonate ions that lead to the production of carboaluminate phases in preference to sulfoaluminate phases (Ramachandran \& Zhang, 1986). Because the performance of the limestone powder is regulated by its exposed surface area, the particle size of the added powder is the critical parameter controlling early-age performance in these ternary blends.

\section{Draft Dual Ring Test}

The team drafted a document for AASTHO describing the dual ring test. It is attached as an appendix to the report.

\section{REFERENCES}

Bentz, D. P. (2002). Influence of curing conditions on water loss and hydration in cement pastes with and without fly ash substitution (NISTIR 6886). Washington, DC: U.S. Department of Commerce. Retrieved from http://ws680.nist.gov/ publication/get_pdf.cfm?pub_id $=860431$

Bentz, D. P. (2007). Internal curing of high-performance blended cement mortars. ACI Materials Journal, 104(3), 408414. http://dx.doi.org/10.14359/18831

Bentz, D. P., Lura, P., \& Roberts, J. W. (2005). Mixture proportioning for internal curing. Concrete International, 27(2), 35-40. 
Bentur, A. (Ed.). (2003). Early age cracking in cementitious systems-Report of RILEM Technical Committee 181EAS-Early age shrinkage induced stresses and cracking in cementitious systems (RILEM Report 25). Paris, France: RILEM Publications SARL.

Carino, N. (1991). The maturity method. In V. M. Malhotra \& N. J. Carino (Eds.), CRC Handbook on Nondestructive Testing of Concrete (pp. 101-146). Boca Raton, FL: CRC Press.

Henkensiefken, R., Castro, J., Bentz, D., Nantung, T., \& Weiss, J. (2009). Water absorption in internally cured mortar made with water-filled lightweight aggregate. Cement and Concrete Research, 39(10), 883-892. http://dx.doi.org/ 10.1016/j.cemconres.2009.06.009

Kovler, K., \& Jensen, O. M. (Eds.). (2007). Internal curing of concrete-State of the art report of RILEM Technical Committee 196-ICC (RILEM Report 41). Paris, France: RILEM Publications SARL.

Mehta, P. K. (2004). High-performance, high-volume fly ash concrete for sustainable development. In Proceedings of the International Workshop on Sustainable Development and Concrete Technology (pp. 3-14). Ames, IA: Iowa State University, Center for Transportation Research and Education.

Ramachandran, V. S., \& Zhang, C. (1986). Influence of $\mathrm{CaCO} 3$ on hydration and microstructural characteristics of Tricalcium Silicate. Il Cemento, 83, 129-152.

Raoufi, K., Radlinska, A., Nantung, T., \& Weiss, W. J. (2008). Practical considerations to determine the time and depth of saw-cuts in concrete pavements. Transportation Research Record, 2081, 110-117. http://dx.doi.org/10.3141/2081-12
Riding, K. A., Poole, J. L., Schindler, A. K., Juenger, M. G., \& Folliard, K. J. (2007). Temperature boundary condition models for concrete bridge members. ACI Materials Journal, 104(4), 379-387. http://dx.doi.org/10.14359/18827

Schindler, A. K. (2004). Prediction of concrete setting. In J. Weiss, K. Kovler, J. Marchand, \& S. Mindess (Eds.), International RILEM Symposium on Concrete Science and Engineering: A Tribute to Arnon Bentur. Paris, France: RILEM Publications SARL.

Schindler, A. K., \& Folliard, K. J. (2005). Heat of hydration models for cementitious materials. ACI Materials Journal, 102(1), 24-33. http://dx.doi.org/10.14359/14246

Schlitter, J. L., Bentz, D. P., \& Weiss, J. (2010). Quantifying residual stress development and reserve strength in restrained internally cured concrete. ACI Materials Journal, 110(1), 3-12. http://dx.doi.org/10.14359/51684361

Schlitter, J. L., Senter, A. H., Bentz, D. P., Nantung, T., \& Weiss, W. J. (2010). A dual concentric ring test for evaluating residual stress development due to restrained volume change. Journal of ASTM International, 7(9), 1-13. https:// doi.org/10.1520/JAI103118

Shah, S. P., Weiss, W. J., \& Yang, W. (1998). Shrinkage cracking - Can it be prevented? Concrete International, 20(4), 51-55.

Wade, S. A., Nixon, J. M., Schindler, A. K., \& Barnes, R. W. (2004). Setting behavior of concrete cured at fluctuating temperatures. In Transportation Research Board annual meeting compendium of papers [CD-ROM]. Washington, DC: Transportation Research Board. 


\section{CHAPTER 1. PHASE I: FLY ASH USE SURVEY}

\section{Karthik H. Obla, Haejin Kim}

\section{INTRODUCTION}

The purpose of this phase is to evaluate the past, current and future trends of use of fly ash in concrete and restrictions to its use. The American Coal Ash Association (ACAA, 2013) conducts an annual survey of fly ash production and use. Typically, on an annual basis out of the 70 million tons of fly ash generated, about $40 \%$ is beneficially utilized. Out of the fly ash that is beneficially used about $50 \%$ is used in cement and concrete applications. Table 1.1 has been developed based on fly ash use as reported by ACAA, slag cement use as reported by the Slag Cement Association (n.d.) and cement use as reported by the U.S. Geological Survey (n.d.). Ready mixed concrete production is estimated from cement shipments reported by USGS. Several other assumptions as stated in Table 1.1 are made to derive the fly ash volume used in ready mixed concrete. Table 1.1 shows that even though concrete volume had decreased, fly ash and fly ash+slag cement use had steadily increased and stood at $12 \%$ and $16 \%$ respectively of the total cementitious content in 2008 . These percentages should not be confused to percent of cementitious materials used in typical concrete mixtures.

Assumptions: $75 \%$ of all cement (including blended cement) is used in $\mathrm{RMC}$-blended cement is $2.5 \%$ of total cement consumption (USGS) and assumed to contain $30 \%$ SCM on average. Fly ash use is derived from ACAA survey. Ready mixed concrete is assumed to consume $88 \%$ of the value reported under Concrete/ Concrete Products/Grout of the ACAA survey and $10 \%$ of the value reported under Blended Cement/ Raw Feed for Clinker of the ACAA survey. Slag use is determined from the Slag Cement Association use reports.

Even though ready mixed concrete forms the single largest market for fly ash it can still offer the largest potential for increased fly ash utilization. There is a large body of research and literature (Naik \& Singh, 1991; Obla, Hill, \& Martin, 2003; Sivasundaram, Carette, \& Malhotra, 1989) on the development and use of High-Volume Fly Ash (HVFA) concrete but actual use still is much lower. In order to clarify some of the assumptions and corroborate the findings made in Table 1.1 a survey of ready mixed concrete producers was conducted to determine:

1. Average amounts of cement, fly ash, slag cement, and silica fume used in a cubic yard of ready mixed concrete. This will serve as a benchmark for future comparisons.

2. Differences between the percent of supplementary cementitious materials (SCM) use between companies.

3. Primary reasons for not using more fly ash. This will help devise programs for enhancing fly ash use in ready mixed concrete.

The survey was based on excel and is reproduced in Appendix A.1 to this chapter. The survey findings are presented in the following sections.

TABLE 1.1

Fly Ash and Slag Cement Use in Ready Mixed Concrete (RMC)

\begin{tabular}{|c|c|c|c|c|c|c|c|c|c|}
\hline Year & $\begin{array}{l}\text { RMC Volume } \\
\left(\text { Million } \mathrm{yd}^{3}\right)\end{array}$ & $\begin{array}{c}\text { PC (Million } \\
\text { tons) }\end{array}$ & $\begin{array}{l}\text { FA (Million } \\
\text { tons) }\end{array}$ & FA (\%) & $\begin{array}{l}\text { SL (Million } \\
\text { tons) }\end{array}$ & $\mathbf{F A}+\mathbf{S L}(\%)$ & PC $\left(l b / y d^{3}\right)$ & FA $\left(l b / y d^{3}\right)$ & SL $\left(l b / y d^{3}\right)$ \\
\hline 2001 & 406.1 & 91.4 & 11.0 & 10.5 & 2.6 & 13.1 & 450 & 54 & 13 \\
\hline 2003 & 404.3 & 91.0 & 11.1 & 10.6 & 3.3 & 13.8 & 450 & 55 & 17 \\
\hline 2005 & 458.3 & 103.1 & 13.5 & 11.3 & 3.9 & 14.5 & 450 & 59 & 17 \\
\hline 2006 & 456.8 & 102.8 & 13.7 & 11.4 & 4.0 & 14.8 & 450 & 60 & 18 \\
\hline 2007 & 414.6 & 93.3 & 12.4 & 11.4 & 3.7 & 14.8 & 450 & 60 & 18 \\
\hline 2008 & 351.7 & 79.1 & 11.4 & 12.2 & 3.3 & 15.8 & 450 & 65 & 19 \\
\hline
\end{tabular}

$\mathrm{PC}=$ portland cement $\mathrm{FA}=$ fly ash $; \mathrm{SL}=$ slag cement. 


\section{SURVEY RESPONDENTS}

A total of 57 companies/divisions responded constituting total ready mixed concrete production in 2010-2011 (12 months) of 35 million $\mathrm{yd}^{3}$ which was $13 \%$ of total production for that period. The breakdown of survey respondents is: $53 \%$ of the companies produced less than $250,000 \mathrm{yd}^{3}, 32 \%$ between 250,000 and 1 million $\mathrm{yd}^{3}$, and $16 \%$ of the companies produced more than 1 million $\mathrm{yd}^{3}$. The average reported production per respondent was $610,000 \mathrm{yd}^{3}$. Table 2.1 shows the minimum, maximum and percentile breakdown of production amounts. The respondents cover a broad range of production.

\section{USE OF SCMS}

About $98 \%$ of the companies had used fly ash in some of their concrete over that time period. It does not mean that fly ash was used in all the concrete produced. Similarly, $61 \%$ of the companies had used slag cement and $39 \%$ of the companies used silica fume. $9 \%$ of the companies used blended cement. $61 \%$ of the companies reported using blended cement and/or more than one SCM but not necessarily in the same concrete mixture.

\section{QUANTITY OF SCMS USED}

The average reported portland cement consumption was $457 \mathrm{lb}$ per $\mathrm{yd}^{3}$ produced; blended cement was 2.7 $\mathrm{lb} / \mathrm{yd}^{3}$; fly ash was $83 \mathrm{lb} / \mathrm{yd}^{3}$; slag cement was $18 \mathrm{lb} / \mathrm{yd}^{3}$; silica fume was $0.2 \mathrm{lb} / \mathrm{yd}^{3}$. These numbers do not reflect typical concrete mixture proportions being used. Rather for each specific material (for example slag cement) they are arrived at by dividing the total material consumed annually by all respondents by the total annual concrete produced by all respondents. In the discussions below the fly ash and total SCM was calculated after assuming that the blended cement had on average $70 \%$ portland cement, $10 \%$ fly ash, and $30 \%$ total SCM (this includes the $10 \%$ fly ash). Table 2.1 shows the minimum, maximum and percentile breakdown of portland cement, fly ash and total SCM used. Figure 4.1 shows the cumulative distribution function of the total SCM use. When the 20th and 80th percentile numbers are compared it is clear that there is a $2-3$ times increase in the amount of fly ash and total SCM used between companies.

TABLE 2.1

Percentile Breakdown, Average, Minimum, and Maximum Amounts of Concrete Production, Fly Ash and SCM Use

\begin{tabular}{|c|c|c|c|c|c|c|}
\hline Survey Participants & $20 \%$ ile & $\mathbf{5 0} \%$ ile & $\mathbf{8 0} \%$ ile & Average & Min & Max \\
\hline Annual concrete volume, $\mathrm{yd}^{3}$ & 58,547 & 197,535 & 733,356 & 610,400 & 13,000 & $5,216,519$ \\
\hline Annual fly ash used, lb/yd ${ }^{3}$ & 39 & 75 & 100 & 83 & 0 & 170 \\
\hline Annual SCM used, lb/yd ${ }^{3}$ & 62 & 94 & 144 & 102 & 28 & 198 \\
\hline Annual fly ash used, \% & 7 & 13 & 18 & 15 & 0 & 27 \\
\hline Annual SCM used, \% & 12 & 17 & 24 & 18 & 3 & 33 \\
\hline
\end{tabular}

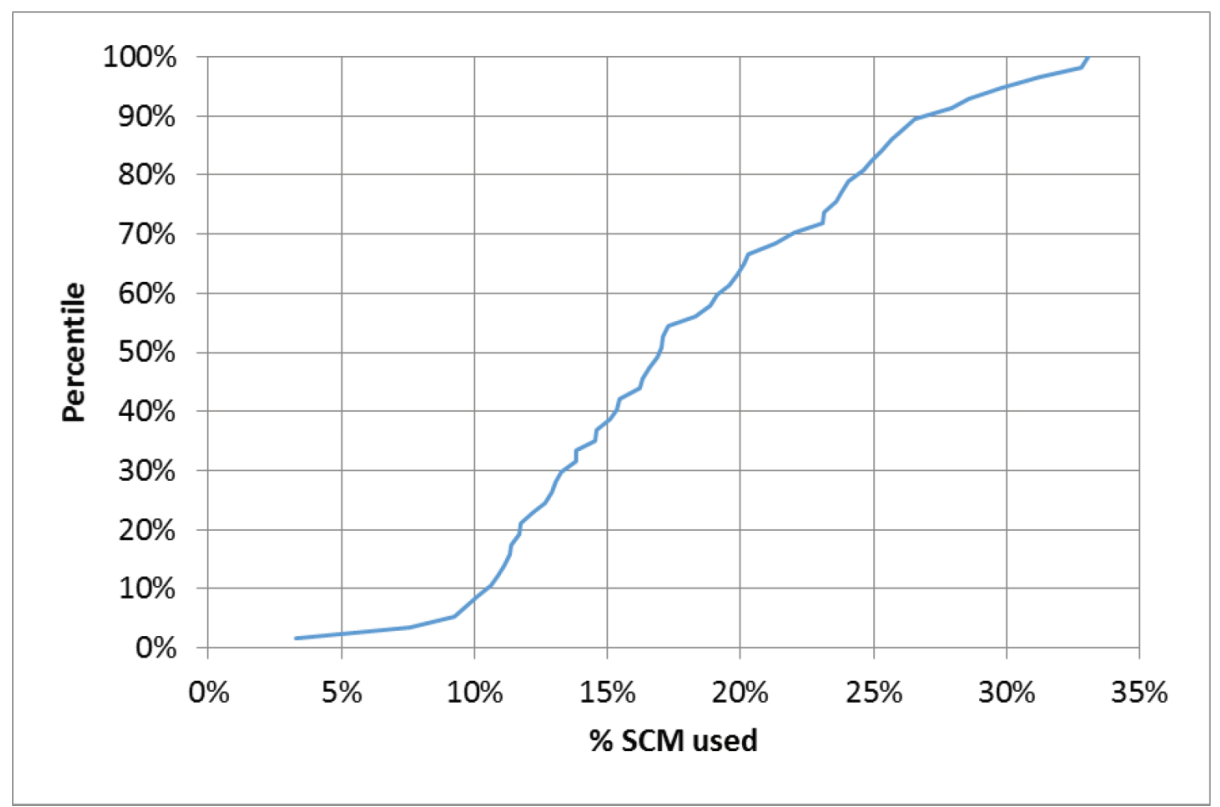

Figure 4.1 Percentile distribution of SCM used by companies. 
TABLE 5.1

Average Portland Cement, Fly Ash, SCM Usage Based on Company Production Levels

\begin{tabular}{|c|c|c|c|c|}
\hline & $<250,000 \mathbf{y d}^{3}$ & 250 to 1 Million $\mathrm{yd}^{3}$ & $>1$ Million $\mathrm{yd}^{3}$ & All \\
\hline Annual portland cement used, $\mathrm{lb} / \mathrm{yd}^{3}$ & 451 & 466 & 454 & 457 \\
\hline Annual fly ash used, $l b / y d^{3}$ & 62 & 86 & 85 & 83 \\
\hline Annual SCM used, $\mathrm{lb} / \mathrm{yd}^{3}$ & 106 & 99 & 104 & 102 \\
\hline
\end{tabular}

\section{SCM USE BY COMPANY SIZE}

No trends can be observed in average portland cement, and/or SCM usage when companies are differentiated based on their production levels. Table 5.1 shows the average portland cement, fly ash, and total SCM consumption for companies producing less than $250,000 \mathrm{yd}^{3}$, between 250,000 and 1 million $\mathrm{yd}^{3}$, and over 1 million $\mathrm{yd}^{3}$. The averages for "all" production are also provided. The average portland cement content was consistently around $455 \mathrm{lb} / \mathrm{yd}^{3}$ and average total SCM content was consistently around $102 \mathrm{lb} / \mathrm{yd}^{3}$. Smaller producers used more slag cement as compared to fly ash but this may be a result of the regional availability and companies responding to the survey.

\section{BREAKDOWN OF CONCRETE PRODUCTION BASED ON SCM USE}

Producers were asked to state how much of their production was with straight portland cement etc. It is not clear how many companies were tracking these numbers; therefore, there is some uncertainty associated with responses to this question. Data analysis reveals that $34 \%$ of all ready mixed concrete produced was with straight portland cement; $2.2 \%$ with blended cement only, $56 \%$ with fly ash as the only SCM, 5.1\% with slag cement as the only SCM, $0.1 \%$ was with silica fume as the only SCM, and $2.8 \%$ was with more than one SCM (ternary mixtures).

\section{REASONS THAT LIMIT USE OF FLY ASH}

Producers were asked to rank the reasons for not substantially increasing the use of fly ash with " 1 " being extremely important and "6" being least important. Table 7.1 illustrates the average ratings. Restrictive specifications and concrete performance issues ranked as the most important reasons (average rating of 2.5), followed by customer objection (2.9), variable fly ash properties (3.9) and finally fly ash availability and use of other SCMs (4.6). Lack of fly ash availability may be a localized concern in some areas. Use of other SCMs is not considered as an important reason. Use of other SCMs like slag cement also helps improve concrete performance and makes concrete more sustainable. In a slightly different way of analyzing these data, producer rankings 1 , and 2 were compiled together as "strongly agree", 3-4 were termed "agree" and 5-6 were termed "neutral." Table 7.1 illustrates that by this approach the rankings are the same as before with restrictive specifications and performance issues ranked as the most important reasons for limiting use. There was no correlation between how producers responded to this question and their production numbers, and/or extent of SCM use.

\section{OPPORTUNITIES FOR INCREASING THE USE OF FLY ASH}

Producers were also asked to list the various opportunities that exist to substantially increase the use of fly ash. Individual comments from the survey are captured in Appendix A.2 to this chapter. A vast majority of them listed education of specifiers and owners on the benefits of use of SCMs as the most important reason; some of them listed cost and performance issues such as setting time and early age strength; a few listed lack of availability but that might again be a localized phenomenon.

\section{CONCLUSIONS}

If the overall average SCM use increases to the currently reported 80th percentile level in Table 1.2 that would lead to an increase in SCM use from $102 \mathrm{lb}$ to $144 \mathrm{lb}$ per cubic yard produced, i.e., an increase of $42 \mathrm{lb}$. If all of this increase is attributed to increase in fly ash use and if the annual ready mixed concrete production were to recover to the pre 2008 recession

TABLE 7.1

Analysis of Company Responses of Causes Preventing Increased Use of Fly Ash

\begin{tabular}{|c|c|c|c|c|}
\hline Causes & Avg. Rating & Strongly Agree & Agree & Neutral \\
\hline Fly ash supply_not available or inconsistent & 4.6 & $14 \%$ & $18 \%$ & $68 \%$ \\
\hline Fly ash properties are variable & 3.9 & $18 \%$ & $38 \%$ & $45 \%$ \\
\hline Use other SCMs (slag, etc.) & 4.6 & $16 \%$ & $20 \%$ & $64 \%$ \\
\hline Concrete performance issues - setting time, strength gain, etc. & 2.5 & $52 \%$ & $36 \%$ & $13 \%$ \\
\hline Specifications restrict use & 2.5 & $57 \%$ & $29 \%$ & $14 \%$ \\
\hline Customers object to use & 2.9 & $38 \%$ & $48 \%$ & $14 \%$ \\
\hline
\end{tabular}


level of 460 million $\mathrm{yd}^{3}$ that would increase the beneficial use of fly ash by an additional 14 million tons. This represents an increase of fly ash utilization from $40 \%$, currently, to $61 \%$. For all ready mixed concrete produced SCM use will then constitute $26 \%$ of the total cementitious content as opposed to the current levels of $18 \%$. In order to accomplish this, it is suggested that a series of seminars with the target audience of specifying engineers, contractors and concrete producers be conducted with the following goals:

1. Replace specification restrictions on SCM dosages with concrete performance requirements.

2. Share laboratory/field data showing acceptable concrete performance with higher amounts of SCMs.

3. Share best practices for successfully incorporating higher amounts of SCMs and achieving concrete performance requirements.

It may be useful to include presentations by concrete producers who are already at the $80 \%$ percentile in terms of use of SCMs.

\section{REFERENCES}

ACAA. (2013). 2013 Coal Combustion Product (CCP) Production \& Use Survey Report. Farmington Hills, MI: American Coal Ash Association. Retrieved from https:// www.acaa-usa.org/Publications/Production-Use-Reports

Naik, T. R., \& Singh, S. S. (1991). Superplasticized structural concrete containing high volumes of Class C fly ash. $A S C E$ Journal of Energy Engineering, 117(2), 87-95. http://dx.doi. org/10.1061/(ASCE)0733-9402(1991)117:2(87)

Obla, K., Hill, R., \& Martin, R. (2003). HVFA concrete-An industry perspective. ACI Concrete International, 25(8), 49-54.

Sivasundaram, V., Carette, G. G., \& Malhotra, V. M. (1989). Properties of concrete incorporating low quantity of cement and high volumes of low-calcium fly ash. In V. M. Malhotra (Ed.), ACI SP-114: Fly ash, silica fume, slag, and natural pozzolans in concrete-Proceedings of the Third International Conference (pp. 45-71). Farmington Hills, MI: American Concrete Institute. http://dx.doi.org/10.14359/1847

Slag Cement Association. (n.d.). U.S. Slag Cement Shipments. Retrieved from http://www.slagcement.org/News/Shipments.html

U.S. Geological Survey. (n.d.). Cement Statistics and Information. Retrieved from http://minerals.usgs.gov/minerals/ pubs/commodity/cement/ 
As part of a funded research project on increased fly ash utilization in hydraulic cement concrete, NRMCA is tasked with conducting a survey to better understand SCM use. SCM can consist of fly ash meeting ASTMC618/AASHTO M295, slag cement meeting C989, and silica fume meeting C1240. Report portland cement conforming to ASTM C150/AASHTO M85; blended cement conforming to ASTM C595/AASHTO M240 or ASTM C1157. This survey should be completed by the concrete producer for calendar year 2011 (use 2010 data if 2011 is not available). This survey only pertains to SCM used in all concrete supplied by a ready mixed concrete producer. Do not include SCM use for nonconcrete applications. You are requested to answer as many questions as possible without guessing. The data you report will be kept confidential and only the overall nationwide survey results will be shared with all survey participants and other researchers.

\begin{tabular}{|c|l|l|}
\hline 1 & Name & \multicolumn{1}{|l|}{} \\
\hline 2 & Company/Division Name & \\
\hline 3 & Email id & \\
\hline 4 & Annual Concrete Volume (cyds) & \\
\hline 5 & Annual portland cement used (tons) & \\
\hline 6 & Annual blended cement used (tons) & \\
\hline 7 & Annual fly ash used (tons) & \\
\hline 8 & Annual slag cement used (tons) & \\
\hline 9 & Annual silica fume used (tons) & \\
\hline 10 & Breakdown percentage of concrete produced - All of it should add up to 100 & \\
\hline 10.1 & Percent with portland cement only, \% & \\
\hline 10.2 & Percent with blended cement only, \% & \\
\hline 10.3 & Percent with fly ash, \% & \\
\hline 10.4 & Percent with slag cement, \% & \\
\hline 10.5 & Percent with silica fume, \% & \\
\hline 10.6 & Percent with more than one SCM (ternary), \% & \\
\hline
\end{tabular}

The following questions pertain only to fly ash use

\begin{tabular}{|c|l|l|}
\hline 11 & \multicolumn{2}{|l|}{$\begin{array}{l}\text { In your view what are the reasons why you don't substantially increase the use of fly ash? Rank them } \\
\text { from } \mathbf{1} \text { to } \mathbf{6} \text { in the order of importance with 1=most important }\end{array}$} \\
\hline 11.1 & Fly ash supply - not available or inconsistent & \\
\hline 11.2 & Fly ash properties are variable & \\
\hline 11.3 & Use other SCMs (slag, etc.) & \\
\hline 11.4 & Concrete performance issues - setting time, strength gain, etc. & \\
\hline 11.5 & Specifications restrict use & \\
\hline 11.6 & Customers object to use \\
\hline 12 & What opportunities exist to substantially increase use of fly ash? List one or more items below. \\
\hline & \multicolumn{2}{|l}{} \\
\hline 13 & Comment - state any assumptions or details about reported data. \\
\hline & \multicolumn{2}{l}{} \\
\hline
\end{tabular}




\begin{tabular}{|c|c|}
\hline ID & What opportunities exist to substantially increase use of fly ash? List one or more items. \\
\hline 1 & $\begin{array}{l}\text { It would be used more if it is not perceived as a hazardous material by the EPA and the classification issue gets } \\
\text { resolved. It would be used more if it is required, instead of being listed as an option. }\end{array}$ \\
\hline 2 & Teach engineers and architects the benefits of using SCM's as so they will allow it on more projects. \\
\hline 4 & LEED Projects, Flowable fill Mix Designs, \\
\hline 5 & Commercial Specs \\
\hline 7 & $\begin{array}{l}\text { 1) Improved specifications that don't restrict the } \% \text { of fly ash allowed (i.e. } 15 \% \max \text { ) 2) Continued availability of } \\
\text { beneficiated ash that allows for more consistent concrete performance }\end{array}$ \\
\hline 8 & $\begin{array}{l}\text { educate engineers municipal and private, fly ash was not permitted in Wind power turbine foundations so ..much } \\
\text { for green energy.... }\end{array}$ \\
\hline 9 & $\begin{array}{l}\text { green movement and specifications. } \\
\text { we will always want to use as much ash as possible except where cold weather limits us. (durability, set time, } \\
\text { strength gain) }\end{array}$ \\
\hline 10 & Cell fill (High Replacement) / Specified green mixes \\
\hline 12 & $\begin{array}{l}\text { Unfortunately, the use of Fly Ash in the North West could see a decrease. Both of our local plants are currently shut } \\
\text { down due to natural gas prices and they are both scheduled to be shut down by } 2019 \text { if not sooner. }\end{array}$ \\
\hline 13 & Specification limitation to $15 \%$ common....Wider acceptance to ACI limitations would help. \\
\hline 16 & Acceptance of high volume fly ash mixes. Emerging technology creating modified fly ash products. \\
\hline 17.1 & We use the maximum quantities as allowed by spec writers. \\
\hline 17.2 & None currently. Supply is very limited. \\
\hline 20 & More LEED projects and increased use in residential jobs in "Green" type mixes. \\
\hline 22 & Educate specifiers and owners \\
\hline 23.1 & $\begin{array}{l}\text { Many opportunities exist, we just need to push them as an industry. LEED may help us achieve this as straight } \\
\text { portland cement is starting to be looked at as non-environmentally friendly. }\end{array}$ \\
\hline 23.2 & LEED Project Requirements. Sustainability Initiatives. Durability \\
\hline 24 & $\begin{array}{l}\text { set times need to be decreased, design and engineering fields need to be educated on the benefits of higher } \% \text { cement } \\
\text { replacement }\end{array}$ \\
\hline 25 & The green building initiative, Usage of fly ash in warmer temp for slab on grade applications. Slower set time \\
\hline 27 & $\begin{array}{l}\text { LEED complaint construction and other "Green" Building systems. } \\
2011 \text { saw a change in sources of fly ash and shortages. Generally \% fly ash was reduced for a significant portion of } \\
\text { the year but not removed from the mix designs completely. }\end{array}$ \\
\hline 28 & $\begin{array}{l}\text { If the specifiers will allow a higher percent of fly ash replacement. We would actively develop usable mixes with } \\
\text { higher fly ash content }\end{array}$ \\
\hline 29 & New government specifications are calling for higher volumes of SCM in the $30-40 \%$ range on most projects. \\
\hline 30 & 1. Residential Concrete. 2. Commercial Floors. \\
\hline 32 & An increase in specified acceptance age. For example 56 days in lieu of 28 days. \\
\hline 33 & Floors, walls, footings \\
\hline 34 & $\begin{array}{l}\text { Increased density to mitigate salt intrusion form marine environment, need to educate the end user of the benefits } \\
\text { and of the need to increase the ultimate strength test date beyond } 28 \text { days. } \\
\text { Normally designers and contractors are VERY reluctant to permit higher than "normal" ratios of FA to Cem. I think } \\
\text { based on old stereotypical thought processes. We have had difficulty in pushing the higher ratio mixes. }\end{array}$ \\
\hline 35 & $\begin{array}{l}\text { The specs for green uses have increased. } \\
\text { We do raise the levels of Fly Ash in our concrete in the warmer summer months up to } 25 \% \text { of cementitous content } \\
\text { in our standard mixes and in specialty mixes for state highway work and other mixes. But generally it's no more } \\
\text { than } 15 \% \text {. }\end{array}$ \\
\hline 36 & $\begin{array}{l}\text { Educate contractors, specifiers and owners (including governmental agencies) on benefits of fly ash, dispel incorrect } \\
\text { performance assumptions, and get specifications updated to allow for the use of more fly ash. }\end{array}$ \\
\hline 38 & Set time with ash to be equal with all cement mixes \\
\hline 40 & More focus on sustainability \& green building. Designers yielding to the pressure of LEEDs-type projects. \\
\hline
\end{tabular}




\begin{tabular}{|c|c|}
\hline ID & What opportunities exist to substantially increase use of fly ash? List one or more items. \\
\hline 41 & infrastructure projects; flowable fill, CLSM, grouts .... \\
\hline 43 & $\begin{array}{l}\text { (1) Relax specification restrictions (2) Where feasible, have specifiers increase the strength acceptance age to } 56 \text { or } \\
90 \text { days for mixes with higher percentages of fly ash }\end{array}$ \\
\hline 44 & Talk to more architects and engineers about the usage. Restrictive specs are the biggest reason to not include SCM. \\
\hline 45 & $\begin{array}{l}\text { Projects with severe ASR problems can utilize a higher quantity of some types of Fly Ash. As well as more backfill } \\
\text { opportunities. }\end{array}$ \\
\hline 49 & Reduction of concrete early strength, Reduction in Cost \\
\hline 50 & $\begin{array}{l}\text { Until there is a savings to use Fly Ash, it is going to be difficult to increase usage. Furthermore, supply has been } \\
\text { getting tighter, and tighter, which has proven to be problematic when Fly Ash is specified in a project--we have } \\
\text { actually had instances where supply has forced projects to be delayed until Fly Ash was available. Another issue, } \\
\text { recently, has been with Fly Ash being on the news as part of the group of by-products the Federal EPA wants to } \\
\text { classify as hazardous waste, customers are afraid to have Fly Ash mixes, particularly in residential applications, out } \\
\text { of fear they will face future liability as a result. }\end{array}$ \\
\hline 51 & Spec change is by far biggest for us. Education of specifiers and promotion of LEED? \\
\hline 52 & $\begin{array}{l}\text { New high ranges designed to increase the strength efficiency of fly ash. Air entraining agents that provide a stable } \\
\text { air content }\end{array}$ \\
\hline 54 & Supply and consistent quality \\
\hline 55 & $\begin{array}{l}\text { The opportunities are changing gradually. The biggest problem we see is that a lot of the engineers in our area are } \\
\text { still "old school" in the fact that they believe that straight bag mixes are the only way to make quality concrete. }\end{array}$ \\
\hline 56 & $\begin{array}{l}\text { LEED pushes for more fly ash but the Northwest availability is totally cut off between March to June due to } \\
\text { hydroelectric power being so much cheaper than coal burned power that coal burning power plants totally shutdown. }\end{array}$ \\
\hline
\end{tabular}


CHAPTER 2. PHASE II: DEVELOPMENT OF AN ACTIVATION ENERGY DATABASE AND STRENGTH-MATURITY RELATIONSHIP

\section{Karthik H. Obla, Haejin Kim}

\section{INTRODUCTION}

One of the primary reasons provided for not using HVFA concrete is its slower setting characteristics and rate of strength development, especially at early ages. This can be addressed to some extent through the effective use of chemical admixtures and proportions and characteristics of other ingredients when developing and producing concrete mixtures. In an earlier research study (Obla, Upadhyaya, Goulias, Schindler, \& Carino, 2008) it was clearly shown that even though laboratory or field cured measured strengths at early ages of HVFA concrete mixtures are low the actual strengths in the structure is higher. This is because the greater mass of concrete in most structures entraps more heat generated by the hydration reactions of cementitious materials. Higher in-place concrete temperatures allow for faster rate of strength gain in the structure than indicated by strength of standard-cured or field-cured cylinders.

Maturity techniques have been developed and used to predict the concrete strength in the structure. The strength of in-place concrete in the structure is estimated by monitoring its temperature history over time, calculating the accumulated maturity, and by obtaining an estimated strength from the pre-determined strength-maturity relationship that is unique to that set of materials and the mixture. Maturity concepts are well established for concrete mixtures containing only portland cement. Not much work has been done with HVFA concrete mixtures containing chemical admixtures. The Arrhenius and Nurse-Saul maturity functions are commonly used to calculate the maturity index. The Arrhenius maturity function is considered to be more accurate and it requires the use of a mixture-specific activation energy to yield accurate results. Activation energy parameters for HVFA have not been established to any degree of accuracy. This phase of the project therefore focuses on developing an activation energy database comprising of typical fly ashes and portland cements used commercially. The activation energy quantifies the temperature sensitivity of the concrete mixture. The activation energy of each of the concrete mixture is established using the procedure outlined in ASTM C1074-11 (ASTM, 1993b).

\section{TRIAL CONCRETE MIXTURES}

The physical and chemical properties of the three fly ashes and the two cements used in this study are provided in Table 2.1. An ASTM C494 (ASTM, 1993b) Type $\mathrm{F}$ polycarboxylate based super plasticizer and a Type $\mathrm{C}$ accelerating admixture were used.

Before starting on the activation energy testing several trial mixtures were prepared to finalize the most optimal HVFA concrete mixture combination that will yield adequate early age strengths and workability.

Table 2.2 shows the concrete mixture proportions and test results:

- Mixture 1 is the control mixture with low alkali Type I cement (Cement A) and no fly ash.

- Mixture 2 is the HVFA concrete mixture containing 50\% fly ash (fly ash FAA) designed to attain higher early age strength by using a low $w / \mathrm{cm}$ (low mixing water content and high cementitious content). Mixture 2 attained about 3000 psi at 2 days and 3800 psi at 4 days. This rate of strength gain is adequate for most applications. Mixture 2 could be used for vertical elements but it was determined that the consistency was very sticky. This was likely due to the high paste volume and the use of a low water content and high HRWRA. Mortar mixtures were evaluated by varying proportions to improve the workability. To improve this in Mixture 3 cement replacement with fly ash was done on a volume basis while maintaining the total paste volume equal to that of the control concrete Mixture 1. This led to the development of HVFA Mixture 3.

- Mixture 3 is a HVFA mixture. The fly ash was at $50 \%$ volume of total cementitious (approximately $40 \%$ by weight). Mixture 3 had a lower mixing water content and HRWR admixture dosage level than Mixture 2 and similar workability. Comparing the strength at early age of 2 and 4 days of this mixture to Mixture 2 it is clear that the fly ash does not appear to be contributing much to strength gain. At a $45^{\circ} \mathrm{F}$ curing temperature (by placing the cylinders in a refrigerator maintained at $45^{\circ} \mathrm{F}$ ) the 2-day compressive strengths were 1700 psi.

- Mixture 4 is a modification of Mixture 3 by using $30 \mathrm{oz} /$ cwt of a Type $\mathrm{C}$ non-chloride accelerating admixture. This caused an increase in the 2 and 4 day strengths for concrete cured at $73^{\circ} \mathrm{F}$ and at the $45^{\circ} \mathrm{F}$ curing temperature, compared to Mixture 3.

- In Mixture 5 a Type III cement from a different source replaced the Type I cement used in Mixture 4. Using the Type III cement yielded significantly higher compressive strengths for specimens cured at $73^{\circ} \mathrm{F}$ and at $45^{\circ} \mathrm{F}$ as indicated in Table 2.2. The measured strengths at early age of Mixture 5 are even higher than the control Mixture 1. The use of a Type III cement and higher dosage of a Type $\mathrm{C}$ accelerating admixture will increase materials cost and may only be necessary in fast-track construction applications. 
TABLE 2.1

Chemical and Physical Characteristics of Portland Cements and Fly Ashes

\begin{tabular}{|c|c|c|c|c|c|c|}
\hline Item & Cement A & Cement B & Cement $\mathrm{C}$ & FA-A & FA-B & FA-C \\
\hline NRMCA Sample ID & 143 & 078 & 067 & FAA & FAB & FAC \\
\hline Silicon oxide $\left(\mathrm{SiO}_{2}\right), \%$ & 20.59 & 19.49 & 19.34 & 60.5 & 53.38 & 38.71 \\
\hline Aluminum oxide $\left(\mathrm{Al}_{2} \mathrm{O}_{3}\right), \%$ & 4.76 & 4.93 & 5.95 & 29.1 & 19.05 & 19.15 \\
\hline Iron oxide $\left(\mathrm{Fe}_{2} \mathrm{O}_{3}\right), \%$ & 1.96 & 3.74 & 1.94 & 2.9 & 4.8 & 6.49 \\
\hline Sum of $\mathrm{SiO}_{2}, \mathrm{Al}_{2} \mathrm{O}_{3}, \mathrm{Fe}_{2} \mathrm{O}_{3}, \%$ & & & & 92.5 & 77.23 & 64.35 \\
\hline Calcium oxide $(\mathrm{CaO}), \%$ & 63.77 & 64.24 & 62.3 & 0.7 & 15.09 & 23.51 \\
\hline Magnesium oxide $(\mathrm{MgO}), \%$ & 2.68 & 1.04 & 2.93 & - & 3.09 & 5.29 \\
\hline Sulfur trioxide $\left(\mathrm{SO}_{3}\right), \%$ & 3 & 3.18 & 3.89 & 0 & 0.63 & 1.36 \\
\hline Sodium Oxide $\left(\mathrm{Na}_{2} \mathrm{O}\right), \%$ & 0.13 & 0.19 & 0.24 & 0.12 & 0.65 & 1.64 \\
\hline Potassium Oxide $\left(\mathrm{K}_{2} \mathrm{O}\right), \%$ & 0.25 & 0.36 & 1.06 & 0.64 & 1.03 & 0.58 \\
\hline Loss of Ignition, \% & 2.61 & 2.34 & 1.46 & 1.3 & 0.28 & 0.3 \\
\hline Fineness $45 \mu \mathrm{m}$ sieve, $\%$ retained & - & - & - & 27.2 & 27.23 & 12.3 \\
\hline Blaine (Specific Surface) $\mathrm{m}^{2 /} \mathrm{kg}$ & 379 & 388 & 369 & - & - & - \\
\hline Relative Density & 3.15 & 3.15 & 3.15 & 2.14 & 2.47 & 2.63 \\
\hline $\begin{array}{l}\text { Strength Activity Index with Portland Cement at } 7 \\
\text { days, \% Control }\end{array}$ & - & - & - & 80.4 & 84.6 & 84 \\
\hline $\begin{array}{l}\text { Strength Activity Index with Portland Cement at } \\
28 \text { days, \% Control }\end{array}$ & - & - & - & 88.5 & 102.7 & - \\
\hline Water Requirement, \% Control & - & - & - & 100.4 & 93.8 & 93 \\
\hline Autoclave Expansion \% & - & - & - & -0.06 & -0.01 & 0.03 \\
\hline Total Alkali (as $\mathrm{Na}_{2} \mathrm{O}$ eq), \% & 0.29 & 0.42 & 0.94 & 0.54 & 1.33 & 2.02 \\
\hline Available Alkali (as $\mathrm{Na}_{2} \mathrm{O}$ ), \% & - & - & - & 0.50 & 0.61 & - \\
\hline Tricalcium Silicate $\left(\mathrm{C}_{3} \mathrm{~S}\right), \%$ & 60 & 66 & 53 & - & - & - \\
\hline Dicalcium silicate $\left(\mathrm{C}_{2} \mathrm{~S}\right), \%$ & 14 & 6 & 16 & - & - & - \\
\hline Tricalcium Aluminate $\left(\mathrm{C}_{3} \mathrm{~A}\right), \%$ & 9 & 7 & 12 & - & - & - \\
\hline Tetracalcium Aluminoferrite $\left(\mathrm{C}_{4} \mathrm{AF}\right), \%$ & 6 & 11 & 6 & - & - & - \\
\hline
\end{tabular}

FAA = Sample Source: Brandon shore, MD; FAB = Sample Source: Big Brown, TX (from Boral); FAC = Sample Source: Muskogee (from Lafarge through Purdue).

Cement A, B, and C were supplied by W.R. Grace.

TABLE 2.2

Summary of Concrete Trial Mixtures

\begin{tabular}{|c|c|c|c|c|c|}
\hline Calculated Batch Quantities & Mix1 & Mix2 & Mix3 & Mix4 & Mix 5 \\
\hline Cement A, lb/yd ${ }^{3}$ & 619 & 380 & 389 & 387 & \\
\hline Type III cement, lb/yd ${ }^{3}$ & & & & & 388 \\
\hline Fly ash FAA, lb/yd & & 380 & 265 & 263 & 264 \\
\hline Fly ash, \% & 0 & 50 & 41 & 40 & 40 \\
\hline Coarse Agg. (No.57), lb/yd ${ }^{3}$ & 2056 & 2089 & 2089 & 2075 & 2080 \\
\hline Fine Aggregate, $\mathrm{lb} / \mathrm{yd}^{3}$ & 1243 & 1100 & 1260 & 1278 & 1281 \\
\hline Mixing Water, $\mathrm{lb} / \mathrm{yd}^{3}$ & 265 & 233 & 223 & 221 & 222 \\
\hline$w / \mathrm{cm}$ & 0.43 & 0.31 & 0.34 & 0.34 & 0.34 \\
\hline ASTM C494 Type F, oz/cwt & 4.0 & 11.5 & 9.2 & 7.9 & 8.0 \\
\hline ASTM C494 Type C, oz/cwt & - & - & - & 30.0 & 30.0 \\
\hline \multicolumn{6}{|l|}{ Fresh Concrete Properties } \\
\hline ASTM C143, Slump, in. & 8.00 & 9.00 & 7.50 & 7.75 & 6.75 \\
\hline ASTM C231, Air, \% & 2.3 & 0.5 & 1.2 & 1.2 & 1.6 \\
\hline ASTM C138, Density, lb/ft ${ }^{3}$ & 155.7 & 155.7 & 157.3 & 156.9 & 157.3 \\
\hline ASTM C1064, Temperature, ${ }^{\circ} \mathrm{F}$ & 68 & 68 & 71 & 71 & 71 \\
\hline \multicolumn{6}{|l|}{ Hardened Concrete Properties } \\
\hline \multicolumn{6}{|l|}{ Compressive Strength, psi } \\
\hline 2 days & 4,631 & 2,965 & 3,025 & 3,895 & 6,029 \\
\hline $4 \mathrm{~d}$ & 6,467 & 3,767 & - & - & - \\
\hline $6 \mathrm{~d}$ & - & - & 4,617 & 5,424 & 7,274 \\
\hline $2 \mathrm{~d}$ at $45 \mathrm{~F}$ & - & - & 1,685 & 2,132 & 3,281 \\
\hline $6 \mathrm{~d}$ at $45 \mathrm{~F}$ & - & - & 3,945 & 4,567 & 6,285 \\
\hline
\end{tabular}

Type F polycarboxylate based super plasticizer (Advacast 575), Type C accelerating admixture (Darset HES) provided by W.R. Grace. 


\section{EXPERIMENTAL WORK FOR DETERMINATION OF ACTIVATION ENERGY (AE)}

In this portion of the study, for determining the activation energy of HVFA mixtures, mortar mixtures were used by proportioning the mortar to have a fine aggregate-to-cement ratio equal to the coarse aggregate-to-cement ratio of the concrete. This is as recommended in ASTM C1074. The mortar mixture with 50\% fly ash is equivalent to concrete Mixture 4 (Table 2.2) since it yielded acceptable early age strengths.

\subsection{Variables}

Table 3.1 summarizes the variables for the 14 mortar mixtures used in the determination of the activation energy. The variables were as follows:

- Two portland cements: Cement B (low alkali) and Cement C (high alkali);

- Three fly ashes: Fly ash A (Class F, low calcium); Fly ash B (Class F, intermediate calcium), and Fly ash C (Class $\mathrm{C}$, high calcium);

- Fly ash quantity: $35 \%$ and $50 \%$ of cementitious materials by volume.

The $w / \mathrm{cm}$ of the mortar mixtures was varied: the $w / \mathrm{cm}$ of the control mixture was 0.43 ; and 0.39 and 0.34 for the $35 \%$ and $50 \%$ fly ash mixtures, respectively.

\subsection{Procedures}

Mortar mixtures were mixed and the cubes were molded in accordance with ASTM C109 (ASTM, 1993a). Mortar cubes were conditioned in limesaturated water baths maintained at three different temperatures: $45^{\circ} \mathrm{F}, 73^{\circ} \mathrm{F}, 100^{\circ} \mathrm{F}$. All ingredient materials for mortar mixtures were conditioned at the respective temperatures for 24 hours before mixing. For the $73^{\circ} \mathrm{F}$ condition, the standard curing room was used; for the higher temperature, the specimens were stored in a room maintained at $100^{\circ} \mathrm{F}$; and for the $45^{\circ} \mathrm{F}$ condition, the specimens were stored in a refrigerator maintained at that temperature.

TABLE 3.1

Characteristics of Mortar Mixtures for Activation Energy Measurement

\begin{tabular}{|c|c|c|c|c|c|}
\hline \multirow{2}{*}{$\begin{array}{l}\text { Portland } \\
\text { Cement }\end{array}$} & \multirow[b]{2}{*}{$w / c m$} & \multirow{2}{*}{$\begin{array}{l}\text { Fly Ash } \\
\text { Dosage }\end{array}$} & \multicolumn{3}{|c|}{ Fly Ash Source } \\
\hline & & & FAA & FAB & FAC \\
\hline \multirow{3}{*}{$\begin{array}{c}\text { Cement B } \\
\text { (low } \\
\text { alkali) }\end{array}$} & 0.43 & $0 \%$ & \multirow{3}{*}{$\begin{array}{l}\square \\
\square\end{array}$} & \multirow{3}{*}{$\begin{array}{l}\square \\
\square\end{array}$} & \multirow{3}{*}{$\begin{array}{l}\square \\
\square\end{array}$} \\
\hline & 0.39 & $35 \%$ & & & \\
\hline & 0.34 & $50 \%$ & & & \\
\hline \multirow{3}{*}{$\begin{array}{l}\text { Cement C } \\
\text { (high } \\
\text { alkali) }\end{array}$} & 0.43 & $0 \%$ & \multirow{3}{*}{$\begin{array}{l}\square \\
\square\end{array}$} & \multirow{3}{*}{$\begin{array}{l}\square \\
\square\end{array}$} & \multirow{3}{*}{$\begin{array}{l}\square \\
\square\end{array}$} \\
\hline & 0.39 & $35 \%$ & & & \\
\hline & 0.34 & $50 \%$ & & & \\
\hline
\end{tabular}

Target flow $=105-115$ per C109 attained with a dosage of Type F admixture.
A total of 18 cubes were made for each batch-two 2 in. cubes at each of 7 test ages, 1 cube with embedded temperature sensor, and 3 extra cubes. A temperature sensor placed in the water bath as a back-up record of curing temperature. Compressive strength was measured at equivalent ages $\left(73^{\circ} \mathrm{F}\right)$ of early age (less than 1 day), 1, 2, 4, 8, 16, 32 days. This should not be confused with chronological test ages. Equivalent age represents the age at a reference curing temperature $\left(73^{\circ} \mathrm{F}\right)$ that results in the same maturity as under the actual curing temperature. To estimate the chronological test age for the temperature conditions of $45^{\circ} \mathrm{F}$ and $100^{\circ} \mathrm{F}$, an initial approximation of activation energy based on past experience was used. The early age (less than 1 day) was chosen by estimating when a compressive strength in the range of 600-1000 psi would be achieved. Typically, this strength level is attained at an equivalent age of 12 hours and was used as a starting point.

\subsection{Results and Discussion}

Tables 3.2, 3.3, and 3.4 summarize the mortar mixture proportions and test results of fresh mortar mixtures with the low alkali cement at curing temperatures $73^{\circ} \mathrm{F}, 100^{\circ} \mathrm{F}$ and $45^{\circ} \mathrm{F}$, respectively. Tables $3.5,3.6$, and 3.7 summarize the mortar compressive strength results of corresponding mixtures. Figures 3.5, 3.6, and 3.7 illustrate the compressive strength test results of low alkali cement mixtures conditioned in $73^{\circ} \mathrm{F}, 100^{\circ} \mathrm{F}$, and $45^{\circ} \mathrm{F}$ respectively. In the following discussion, the fly ash quantity in the mixtures is on the basis of volume of cementitious materials.

From Figure $3.1\left(73^{\circ} \mathrm{F}\right)$ the following observations are made:

1. The control mixture, Mix 1, had the fastest rate of strength gain at early age as expected and the rate slowed considerably by 16 days.

2. For the fly ash mixtures with $35 \%$ fly ash, the faster rate of strength gain at early ages was evident with the FAA fly ash. The strength of this mixture exceeded that of the control mixture by 18 days. The strength of the mixtures with fly ashes FAB and FAC exceeded that of the control mixture after 80 days. Later age strength for the fly ash mixtures is ranked from highest to lowest with fly ashes FAA, FAB and FAC.

3. For the mixtures with $50 \%$ fly ash, there was little difference between the mixtures at early ages. These mixtures had a slower rate of strength gain compared to the control mixture and exceeded it by 28 days. Ranking later age strength followed the same trend for highest to lowest: FAA, FAB and FAC.

4. Two of the mixtures (0.43PC and 0.34FAA50) had to be repeated because the early age strength measured exceeded the target range of $600-1000$ psi. The repeat mixtures (0.43PC-R and 0.34FAA50-R) show compressive strength test results very close to the original mixtures thus confirming good repeatability.

Figure 3.2 illustrates strength curves for mortars maintained at $100^{\circ} \mathrm{F}$. The strength of $35 \%$ and $50 \%$ fly ash mixtures exceeded that of the control mixture 
TABLE 3.2

Summary of Mortar Test Result @ Control-73 ${ }^{\circ}$ (Low Alkali Cement)

\begin{tabular}{|c|c|c|c|c|c|c|c|c|c|}
\hline Batch Quantities & Mix1 & Mix2 & Mix3 & Mix4 & Mix5 & Mix6 & Mix7 & Mix1R & Mix5R \\
\hline & $0.43 \mathrm{PC}$ & 0.39FAA35 & 0.39FAB35 & 0.39FAC35 & 0.34 FAA50 & $0.34 F A B 50$ & 0.34FAC50 & 0.43PC-R & 0.34FAA50-R \\
\hline Cement $\mathrm{B}, \mathrm{lb} / \mathrm{yd}^{3}$ & 620 & 454 & 444 & 439 & 384 & 373 & 368 & 620 & 384 \\
\hline Fly ash, $1 \mathrm{~b} / \mathrm{yd}^{3}$ & 0 & 166 & 187 & 198 & 261 & 292 & 307 & 0 & 261 \\
\hline Fly ash, $\%$ by Mass & 0 & 26.8 & 29.6 & 31.1 & 40.5 & 43.9 & 45.5 & 0 & 40.5 \\
\hline Fly ash, \% by Vol. & 0 & 35 & 35 & 35 & 50 & 50 & 50 & 0 & 50 \\
\hline Fine Aggregate, $\mathrm{lb} / \mathrm{yd}^{3}$ & 2060 & 2060 & 2060 & 2060 & 2060 & 2060 & 2060 & 2060 & 2060 \\
\hline Mixing Water, $1 \mathrm{~b} / \mathrm{yd}^{3}$ & 266 & 242 & 246 & 248 & 219 & 226 & 229 & 266 & 219 \\
\hline$w / \mathrm{cm}$ & 0.43 & 0.39 & 0.39 & 0.39 & 0.34 & 0.34 & 0.34 & 0.43 & 0.34 \\
\hline ASTM C494 Type F, oz/cwt & 4.0 & 5.8 & 4.9 & 2.7 & 18.0 & 4.0 & 2.6 & 4.0 & 18.0 \\
\hline ASTM C494 Type C, oz/cwt & - & - & - & - & 30.0 & 30.0 & 30.0 & - & 30.0 \\
\hline \multicolumn{10}{|l|}{ Fresh Mortar Properties } \\
\hline ASTM C1437, Flow, \% & 114 & 105 & 112 & 106 & 112 & 106 & 112 & 107 & 107 \\
\hline ASTM C185, Density, lb/ft ${ }^{3}$ & 138.2 & 137.1 & 134.6 & 136.3 & 137.2 & 133.5 & 138.1 & 138 & 139.5 \\
\hline ASTM C185, Air, \% & 7.7 & 8.6 & 10.8 & 7.3 & 6.4 & 9.8 & 7.1 & 6.1 & 4.9 \\
\hline
\end{tabular}

Note: FAA, FAB, FAC are different fly ash sources.

TABLE 3.3

Summary of Mortar Test Result @ Hot-100 $^{\circ}$ F (Low Alkali Cement)

\begin{tabular}{|c|c|c|c|c|c|c|c|}
\hline Batch Quantities & Mix8 & Mix9 & Mix10 & Mix11 & Mix12 & Mix13 & Mix14 \\
\hline & $0.43 P C$ & 0.39FAA35 & 0.39FAB35 & 0.39FAC35 & 0.34FAA50 & 0.34FAB50 & 0.34 FAC50 \\
\hline Cement $\mathrm{B}, \mathrm{lb} / \mathrm{yd}^{3}$ & 620 & 454 & 444 & 439 & 384 & 373 & 368 \\
\hline Fly ash, $1 \mathrm{~b} / \mathrm{yd}^{3}$ & 0 & 166 & 187 & 198 & 261 & 292 & 307 \\
\hline Fly ash, $\%$ by Mass & 0 & 26.8 & 29.6 & 31.1 & 40.5 & 43.9 & 45.5 \\
\hline Fly ash, \% by Vol. & 0 & 35 & 35 & 35 & 50 & 50 & 50 \\
\hline Fine Aggregate, $1 \mathrm{~b} / \mathrm{yd}^{3}$ & 2060 & 2060 & 2060 & 2060 & 2060 & 2060 & 2060 \\
\hline Mixing Water, $\mathrm{lb} / \mathrm{yd}^{3}$ & 266 & 242 & 246 & 248 & 219 & 226 & 229 \\
\hline$w / \mathrm{cm}$ & 0.43 & 0.39 & 0.39 & 0.39 & 0.34 & 0.34 & 0.34 \\
\hline ASTM C494 Type F, oz/cwt & 4.0 & 9.6 & 4.0 & 2.7 & 19.8 & 4.0 & 2.6 \\
\hline ASTM C494 Type C, oz/cwt & - & - & - & - & 30.0 & 30.0 & 30.0 \\
\hline \multicolumn{8}{|l|}{ Fresh Mortar Properties } \\
\hline ASTM C1437, Flow, \% & 108 & 107 & 109 & 114 & 106 & 105 & 115 \\
\hline ASTM C185, Density, lb/ft ${ }^{3}$ & 137.2 & 136.7 & 134.9 & 137.9 & 139 & 134.5 & 139.2 \\
\hline ASTM C185, Air, \% & 6.7 & 8.9 & 10.6 & 6.2 & 5.2 & 9.1 & 6.3 \\
\hline
\end{tabular}

Note: FAA, FAB, FAC are different fly ash sources.

within 4-6 days. The mixture with the FAA fly ash had higher later age strength than the mixtures with the FAB and FAC fly ashes. The higher curing temperature caused an accelerated rate of strength development for the fly ash mixtures compared to the control mixture. Later age ( $>90$ days) data shows the fly ash mixtures had a mortar strength almost 3000 to 4000 psi higher than the control mixture. Ultimate strengths of the fly ash mixtures appear to be less affected by higher temperature compared to the control mixture without fly ash.

Figure 3.3 illustrates strength curves for mortars maintained at $45^{\circ} \mathrm{F}$. The strength of the $35 \%$ FAA and FAC mixtures exceeded the control mixture after 1 year. The strength of the $50 \%$ FAC and FAA mixture exceeded that of the control mixture after 200 days and
1 year respectively. Both the FAB mixtures were lagging behind the control even after 1 year. The lower curing temperature resulted in a slower rate of strength development for the fly ash mixtures as compared to the control mixture.

Tables 3.8, 3.9, and 3.10 summarize the mortar mixture proportions and test results of fresh mortar mixtures with the high alkali cement at curing temperatures $73^{\circ} \mathrm{F}, 100^{\circ} \mathrm{F}$ and $45^{\circ} \mathrm{F}$, respectively. Tables 3.11 , 3.12 , and 3.13 summarize the mortar compressive strength results of corresponding mixtures. Figures 3.4, 3.5, and 3.6 illustrate the compressive strength test results of high alkali cement mixtures conditioned in $73^{\circ} \mathrm{F}, 100^{\circ} \mathrm{F}$, and $45^{\circ} \mathrm{F}$ respectively. In the following discussion, the fly ash quantity in the mixtures is on the basis of volume of cementitious materials. 
TABLE 3.4

Summary of Mortar Test Result @ Cold-45 ${ }^{\circ}$ (Low Alkali Cement)

\begin{tabular}{|c|c|c|c|c|c|c|c|}
\hline Batch Quantities & Mix15 & Mix16 & Mix17 & Mix18 & Mix19 & Mix20 & Mix21 \\
\hline & 0.43PC & 0.39FAA35 & 0.39FAB35 & 0.39FAC35 & 0.34FAA50 & $0.34 F A B 50$ & 0.34 FAC50 \\
\hline Cement $\mathrm{B}, \mathrm{lb} / \mathrm{yd}^{3}$ & 620 & 454 & 444 & 439 & 384 & 373 & 368 \\
\hline Fly ash, $1 \mathrm{~b} / \mathrm{yd}^{3}$ & 0 & 166 & 187 & 198 & 261 & 292 & 307 \\
\hline Fly ash, $\%$ by Mass & 0.0 & 26.8 & 29.6 & 31.1 & 40.5 & 43.9 & 45.5 \\
\hline Fly ash, \% by Vol. & & 35 & 35 & 35 & 50 & 50 & 50 \\
\hline Fine Aggregate, $1 \mathrm{~b} / \mathrm{yd}^{3}$ & 2060 & 2060 & 2060 & 2060 & 2060 & 2060 & 2060 \\
\hline Mixing Water, $1 \mathrm{~b} / \mathrm{yd}^{3}$ & 266 & 242 & 246 & 248 & 219 & 226 & 229 \\
\hline$w / \mathrm{cm}$ & 0.43 & 0.39 & 0.39 & 0.39 & 0.34 & 0.34 & 0.34 \\
\hline ASTM C494 Type F, oz/cwt & 4.7 & 11.2 & 2.8 & 1.8 & 4.0 & 4.4 & 2.2 \\
\hline ASTM C494 Type C, oz/cwt & - & - & - & - & 30.0 & 30.0 & 30.0 \\
\hline \multicolumn{8}{|l|}{ Fresh Mortar Properties } \\
\hline ASTM C1437, Flow, \% & 114 & 115 & 109 & 120 & 110 & 113 & 114 \\
\hline ASTM C185, Density, $1 \mathrm{~b} / \mathrm{ft}^{3}$ & 136.7 & 137.1 & 136.2 & 137.1 & 138.9 & 134.1 & 137.2 \\
\hline ASTM C185, Air, \% & 7.0 & 8.6 & 9.7 & 6.7 & 5.2 & 9.4 & 7.7 \\
\hline
\end{tabular}

Note: FAA, FAB, FAC are different fly ash sources.

TABLE 3.5

Summary of Mortar Test Result @ Control-73 ${ }^{\circ}$ (High Alkali Cement)

\begin{tabular}{|c|c|c|c|c|c|c|c|c|}
\hline Calculated Batch Quantities & Mix22 & Mix22R & Mix23 & Mix24 & Mix25 & Mix26 & Mix27 & Mix28 \\
\hline & $0.43 \mathrm{PC}$ & $0.43 \mathrm{PC}$ & 0.39FAA35 & 0.39FAB35 & 0.39FAC35 & 0.34FAA50 & $0.34 F A B 50$ & $0.34 F A C 50$ \\
\hline Cement $\mathrm{C}, \mathrm{lb} / \mathrm{yd}^{3}$ & 620 & 620 & 454 & 444 & 439 & 384 & 373 & 368 \\
\hline Fly ash, $1 \mathrm{~b} / \mathrm{yd}^{3}$ & 0 & 0 & 166 & 187 & 198 & 261 & 292 & 307 \\
\hline Fly ash, $\%$ by Mass & 0 & 0 & 27 & 30 & 31 & 40 & 44 & 45 \\
\hline Fly ash, $\%$ by Vol. & & & 35 & 35 & 35 & 50 & 50 & 50 \\
\hline Fine Aggregate, $1 \mathrm{~b} / \mathrm{yd}^{3}$ & 1246 & 1246 & 1244 & 1245 & 1245 & 1245 & 1245 & 1245 \\
\hline Mixing Water, $\mathrm{lb} / \mathrm{yd}^{3}$ & 266 & 266 & 242 & 246 & 248 & 219 & 226 & 229 \\
\hline$w / \mathrm{cm}$ & 0.43 & 0.43 & 0.39 & 0.39 & 0.39 & 0.34 & 0.34 & 0.34 \\
\hline ASTM C494 Type F, oz/cwt & 11.3 & 11.3 & 15.0 & 6.0 & 3.6 & 31.5 & 5.2 & 3.5 \\
\hline ASTM C494 Type C, oz/cwt & - & - & - & - & - & 30.0 & 30.0 & 30.0 \\
\hline \multicolumn{9}{|l|}{ Fresh Mortar Properties } \\
\hline Temperature, ${ }^{\circ} \mathrm{F}$ & 81 & 80 & 78 & 78 & 78 & 78 & 76 & 76 \\
\hline ASTM C1437, Flow, \% & 106 & 107 & 107 & 124 & 122 & 106 & 107 & 105 \\
\hline ASTM C185, Density, $1 \mathrm{~b} / \mathrm{ft}^{3}$ & 140.9 & 138.9 & 137.9 & 137.1 & 138.2 & 139.8 & 136 & 140.4 \\
\hline ASTM C 185 , Air, \% & 4.9 & 5.0 & 5.0 & 6.1 & 5.7 & 3.8 & 7.8 & 5.2 \\
\hline
\end{tabular}

Note: FAA, FAB, FAC are different fly ash sources.

From Figure $3.4\left(73^{\circ} \mathrm{F}\right)$ the following observations are made:

1. The control mixture had the fastest rate of strength gain at early age as expected and the rate slowed considerably by 8 days.

2. For the fly ash mixtures with $35 \%$ fly ash, the faster rate of strength gain at early ages was evident with the FAA fly ash. The strength of this mixture exceeded that of the control mixture by 33 days. The strength of the mixtures with fly ashes FAB and FAC exceeded that of the control mixture after 45 days. Later age strength for the fly ash mixtures is ranked from highest to lowest with fly ashes FAA, FAB and FAC.

3. For the $50 \%$ fly ash dosage the FAA and FAB fly ash mixtures showed a faster strength gain and had exceeded the control mixture by 25 days. The FAC mixture had exceeded the control by about 90 days. Later age data shows the FAA, FAB mixtures with a better strength gain as compared to FAC mixture.

4. One mixture $(0.43 \mathrm{PC})$ had to be repeated because the early age strength measured exceeded the target range of 600-1000 psi. The repeat mixtures (0.43PC-R) shows compressive strength test results very close to the original mixtures thus confirming good repeatability.

Figure 3.5 illustrates strength curves for mortars maintained at $100^{\circ} \mathrm{F}$. The strength of $35 \%$ FAA and FAC mixtures had exceeded the control mixture by 11 days. The $35 \%$ FAB mixture also exceeded the control mixture by 16 days. Later age strength for the fly ash mixtures is ranked from highest to lowest with fly ashes FAA, FAC and FAB. All of the $50 \%$ fly ash mixtures exceeded the control mixture by 7 days and by 90 days had over 2000 psi higher strength as compared to the control mixture. 
TABLE 3.6

Summary of Mortar Test Result @ Hot-100 F (High Alkali Cement)

\begin{tabular}{|c|c|c|c|c|c|c|c|}
\hline Calculated Batch Quantities & Mix29 & Mix30 & Mix31 & Mix32 & Mix33 & Mix34 & Mix35 \\
\hline & $0.43 \mathrm{PC}$ & 0.39FAA35 & $0.39 F A B 35$ & 0.39FAC35 & 0.34FAA50 & 0.34FAB50 & 0.34 FAC50 \\
\hline Cement $\mathrm{C}, \mathrm{lb} / \mathrm{yd}^{3}$ & 620 & 454 & 444 & 439 & 384 & 373 & 368 \\
\hline Fly ash, $1 \mathrm{~b} / \mathrm{yd}^{3}$ & & 166 & 187 & 198 & 261 & 292 & 307 \\
\hline Fly ash, $\%$ by Mass & 0.0 & 26.8 & 29.6 & 31.1 & 40.5 & 43.9 & 45.5 \\
\hline Fly ash, \% by Vol. & & 35 & 35 & 35 & 50 & 50 & 50 \\
\hline Fine Aggregate, $1 \mathrm{~b} / \mathrm{yd}^{3}$ & 1246 & 1245 & 1245 & 1245 & 1245 & 1245 & 1245 \\
\hline Mixing Water, $\mathrm{lb} / \mathrm{yd}^{3}$ & 266 & 242 & 246 & 248 & 219 & 226 & 229 \\
\hline$w / \mathrm{cm}$ & 0.43 & 0.39 & 0.39 & 0.39 & 0.34 & 0.34 & 0.34 \\
\hline ASTM C494 Type F, oz/cwt & 4.0 & 16.1 & 4.0 & 2.8 & 45.3 & 4.0 & 6.0 \\
\hline ASTM C494 Type C, oz/cwt & - & - & - & - & 30.0 & 30.0 & 30.0 \\
\hline \multicolumn{8}{|l|}{ Fresh Mortar Properties } \\
\hline Temperature, ${ }^{\circ} \mathrm{F}$ & 98 & 97 & 94 & 97 & 87 & 95 & 93 \\
\hline ASTM C1437, Flow, \% & 107 & 107 & 119 & 114 & 105 & 106 & 117 \\
\hline ASTM C185, Density, $1 \mathrm{~b} / \mathrm{ft}^{3}$ & 139.4 & 139.1 & 137.8 & 140 & 139.1 & 139.4 & 140.4 \\
\hline ASTM C185, Air, \% & 4.9 & 4.1 & 5.7 & 4.4 & 4.3 & 5.4 & 5.2 \\
\hline
\end{tabular}

Note: FAA, FAB, FAC are different fly ash sources.

TABLE 3.7

Summary of Mortar Test Result @ Cold-45 F (High Alkali Cement)

\begin{tabular}{|c|c|c|c|c|c|c|c|}
\hline Calculated Batch Quantities & Mix36 & Mix37 & Mix38 & Mix39 & Mix40 & Mix41 & Mix42 \\
\hline & $0.43 \mathrm{PC}$ & 0.39FAA35 & 0.39FAB35 & 0.39FAC35 & 0.34 FAA50 & 0.34FAB50 & $0.34 F A C 50$ \\
\hline Cement $\mathrm{C}, \mathrm{lb} / \mathrm{yd}^{3}$ & 620 & 454 & 444 & 439 & 384 & 373 & 368 \\
\hline Fly ash, $1 b / y^{3}$ & & 166 & 187 & 198 & 261 & 292 & 307 \\
\hline Fly ash, $\%$ by Mass & 0.0 & 26.8 & 29.6 & 31.1 & 40.5 & 43.9 & 45.5 \\
\hline Fly ash, \% by Vol. & & 35 & 35 & 35 & 50 & 50 & 50 \\
\hline Fine Aggregate, $1 \mathrm{~b} / \mathrm{yd}^{3}$ & 1246 & 1245 & 1245 & 1245 & 1245 & 1245 & 1245 \\
\hline Mixing Water, $\mathrm{lb} / \mathrm{yd}^{3}$ & 266 & 242 & 246 & 248 & 219 & 226 & 229 \\
\hline$w / \mathrm{cm}$ & 0.43 & 0.39 & 0.39 & 0.39 & 0.34 & 0.34 & 0.34 \\
\hline ASTM C494 Type F, oz/cwt & 6.7 & 11.5 & 8.3 & 1.9 & 28.8 & 4.4 & 2.2 \\
\hline ASTM C494 Type C, oz/cwt & - & - & - & - & 30.0 & 30.0 & 30.0 \\
\hline \multicolumn{8}{|l|}{ Fresh Mortar Properties } \\
\hline Temperature, ${ }^{\circ} \mathrm{F}$ & 67 & 63 & 66 & 64 & 72 & 67 & 64 \\
\hline ASTM C1437, Flow, \% & 106 & 105 & 105 & 122 & 108 & 112 & 114 \\
\hline ASTM C185, Density, lb/ft ${ }^{3}$ & 137.8 & 138 & 133.9 & 135.7 & 137.5 & 132.7 & 136.6 \\
\hline ASTM C185, Air, \% & 5.9 & 7.5 & 8.3 & 7.4 & 5.8 & 10.0 & 7.8 \\
\hline
\end{tabular}

Note: FAA, FAB, FAC are different fly ash sources.

Figure 3.6 illustrates strength curves for mortars maintained at $45^{\circ} \mathrm{F}$. Even after 1 year all of the fly ash mixtures were lagging behind the control mixture.

In some of the mortar mixtures the dosage of the Type F HRWRA used considerably exceeded the manufacturer's recommended dosage. This caused some retardation. These mixtures were repeated. It was decided, however, that the strengths measured were appropriate to use in the analysis. More details of the repeated mixtures are discussed in Appendix B to this chapter.

Charts in Appendix $\mathrm{C}$ to this chapter illustrate different comparisons of the mortar test results. The following conclusions can be drawn from those plots:

1. A cross-over effect is observed in these charts, where the later age strengths of the specimens cured at cooler temperatures are higher than that of specimens cured at warmer temperatures. This effect is observed for the control $0.43 \mathrm{PC}$, and to a lesser extent for the 0.39FAA35 and 0.39 FAB35 mixtures. It was not observed for the rest of the fly ash mixtures even when testing was extended to over 1 year.

2. For the control $0.43 \mathrm{PC}$ mixture the strengths of the specimens cured at $43^{\circ} \mathrm{F}$ exceeded that of specimens cured at $73^{\circ} \mathrm{F}$ after 80 days. For all the fly ash mixtures even after 400 days of curing at $43^{\circ} \mathrm{F}$ the strengths were similar to the 28 day strengths of specimens cured at $73^{\circ} \mathrm{F}$.

3. Alkali content of fly ash does not seem to influence the rate of strength development of fly ash mixtures. The high and low alkali cement mixtures for the control as well as the fly ash mixtures showed similar strengths at different curing temperatures except for the 0.39FAA35 and 0.39FAB35 mixtures cured at cooler temperatures, in which case the high alkali cement mixtures showed higher strengths. 


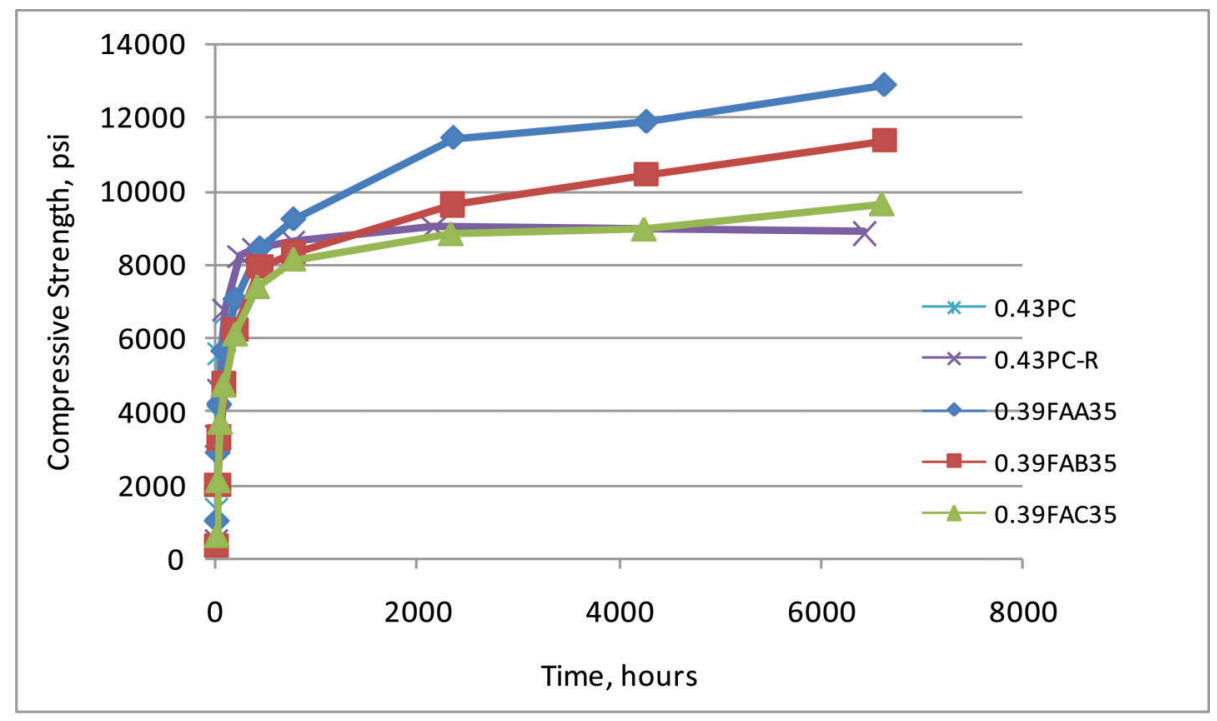

(a)

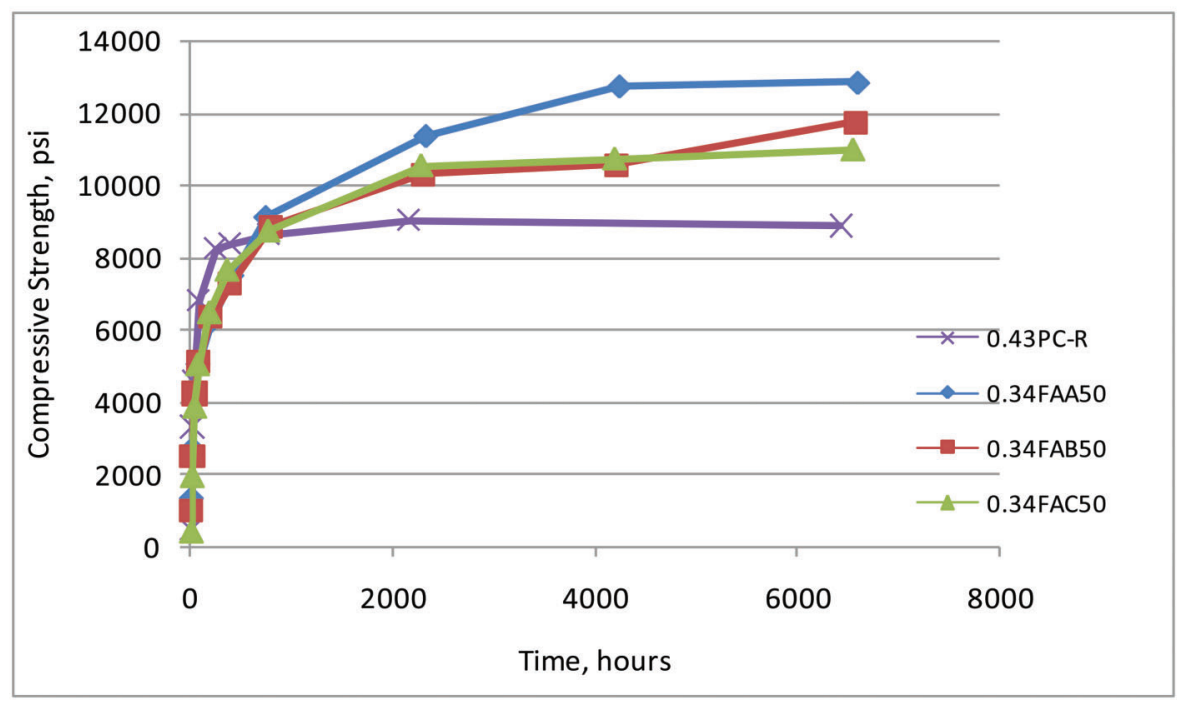

(b)

Figure 3.1 Compressive strength test results of the low alkali cement control mortar mixture vs. (a) $35 \%$ fly ash; (b) $50 \%$ fly ash. All mixtures cured at Control- $73^{\circ} \mathrm{F}$.

\subsection{Determination of Activation Energy}

The Activation Energy (AE) was determined for all 14 mixtures: 7 with low alkali cement and 7 with high alkali cement. For each mixture after the completion of the mortar cube testing the compressive strength and equivalent age data at each of the three temperatures were fitted with a hyperbolic function in accordance with ASTM C1074. The hyperbolic function is:

$$
S=S_{u} \frac{k\left(t-t_{0}\right)}{1+k\left(t-t_{0}\right)}
$$

Where:

$S=$ average cube compressive strength at age t,

$t=$ test age,
$S_{u}=$ limiting strength,

$t_{0}=$ age when strength development is assumed to begin, and

$k=$ rate constant

The solver process of Microsoft Excel software was used to calculate the best-fit values of $S_{u}, t_{0}$, and $k$ through a regression analysis. For each mixture the rate constants were plotted against temperature and fitted with the Arrhenius equation, from which the $\mathrm{AE}$ was estimated. Using the calculated AE values for each mixture the strength-to-equivalent age for the 3 temperatures were plotted.

This is depicted in Figures 3.7 and 3.8 for low alkali cements and Figures 3.11 and 3.12 for high alkali cements. Figures 3.7 and 3.11 are for the low alkali 


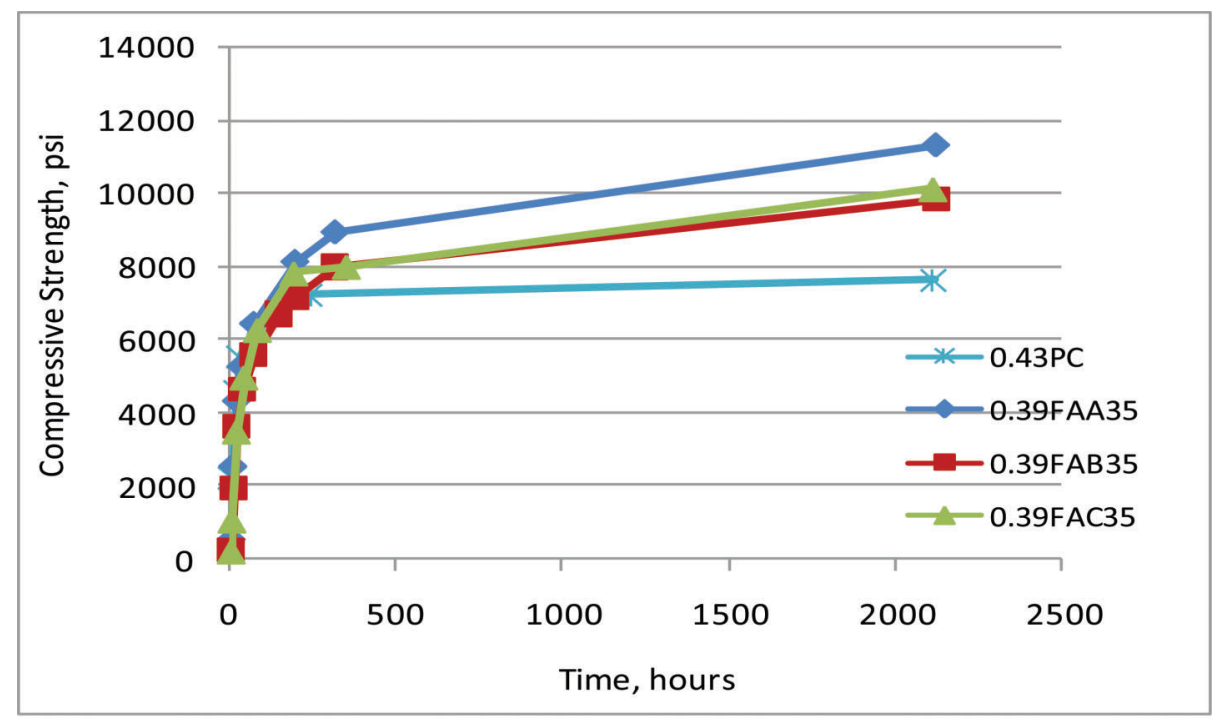

(a)

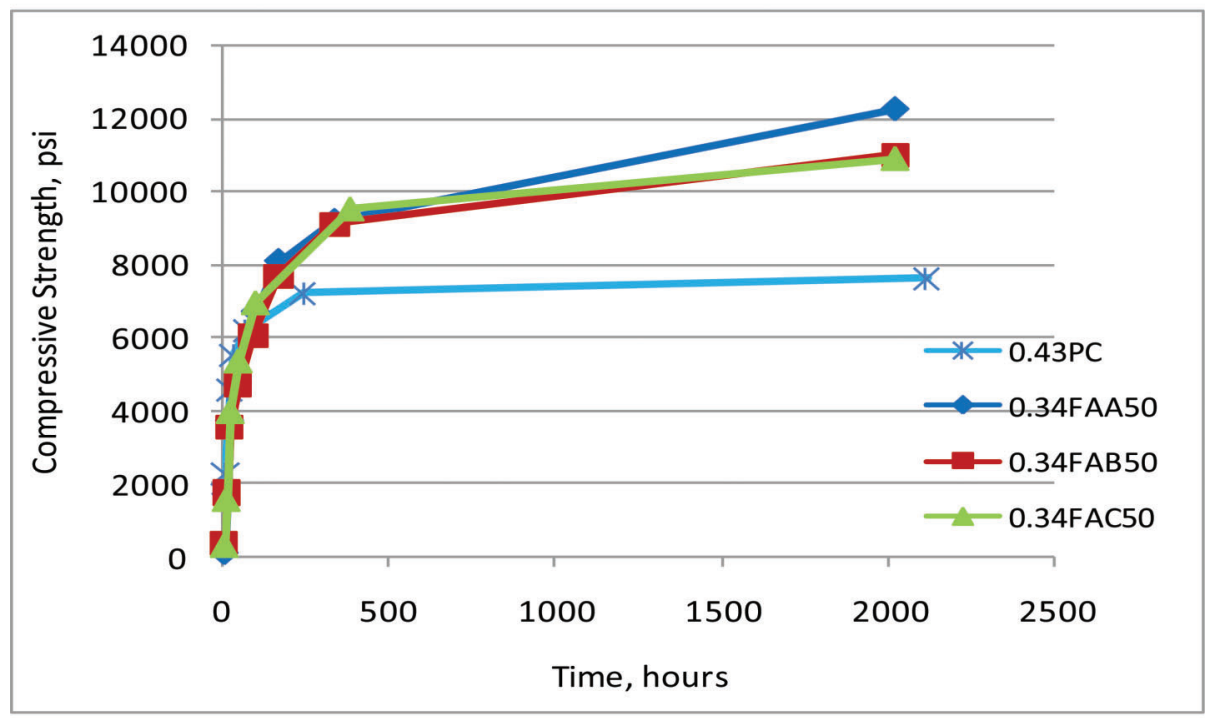

(b)

Figure 3.2 Compressive strength test results of the low alkali cement control mortar mixture vs. (a) $35 \%$ fly ash; (b) $50 \%$ fly ash. All mixtures cured at $\mathrm{Hot}-100^{\circ} \mathrm{F}$.

and high alkali cement control mixtures, respectively. Each of these figures has 3 plots depicted as a, b, c. In Figure 3.7, plot 8a shows the strength vs. age results (for age up to 20 days) for all 3 curing temperatures and the hyperbolic curve fit. Plot $8 \mathrm{~b}$ shows the logarithm of rate constant vs. inverse of curing temperature in degree Kelvin. The fitted line is the Arrhenius curve fit. The slope of this line is the activation energy. Plot $8 \mathrm{c}$ shows the strength vs. equivalent age results (for equivalent age up to 20 days) for all 3 curing temperatures. For the fly ash mixtures in the interest of space only the strengthequivalent age plots are shown in Figures 3.8, and 3.12 for the low alkali and high alkali cement mixtures respectively.
Tables 3.14 and 3.18 summarize the hyperbolic curve fit data for the low alkali and high alkali mixtures respectively. Generally, one would expect $t_{0}$ to be similar to the final setting time but Tables 3.14 and 3.18 show some zero values. For these mixtures the curve fit had suggested a negative $t_{0}$ value and since that is not physically possible a zero value is assumed. $S_{u}$ values also are slightly lower than the actual later age strengths measured in some cases. However, this also is acceptable as long as the hyperbolic curve fits are good for the first 7 days and the overall coefficient of determinations $\left(\mathrm{R}^{2}\right)$ values are good. It can be seen that the hyperbolic function gives good correlations with $\mathrm{R}^{2}$ values between 0.94 and 1.00 for all 42 cases (14 mixtures x 3 curing temperatures). For comparison, curve 


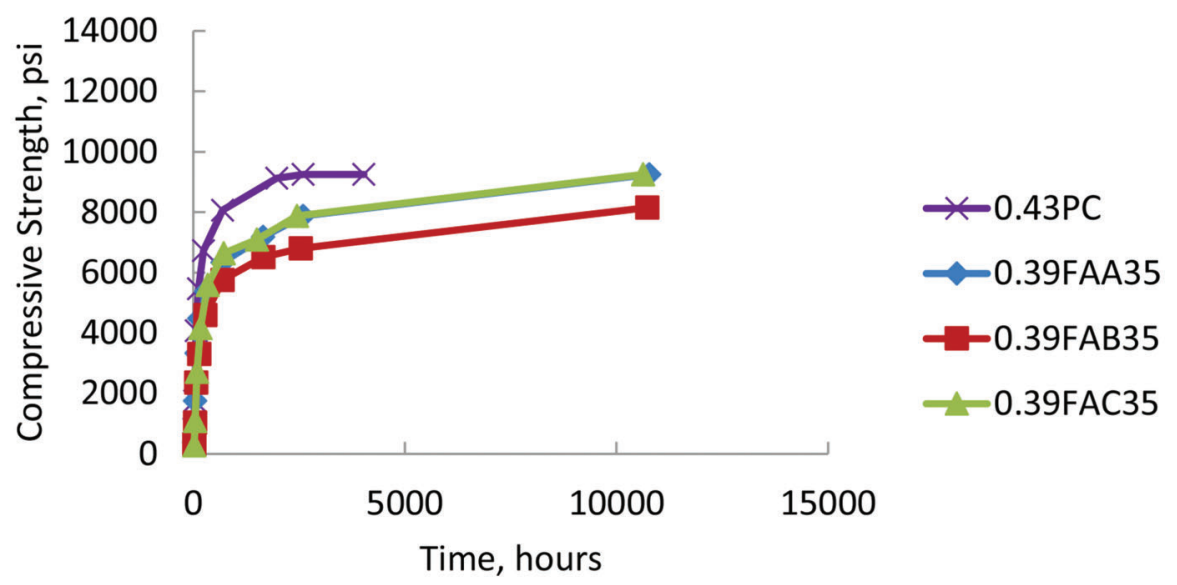

(a)

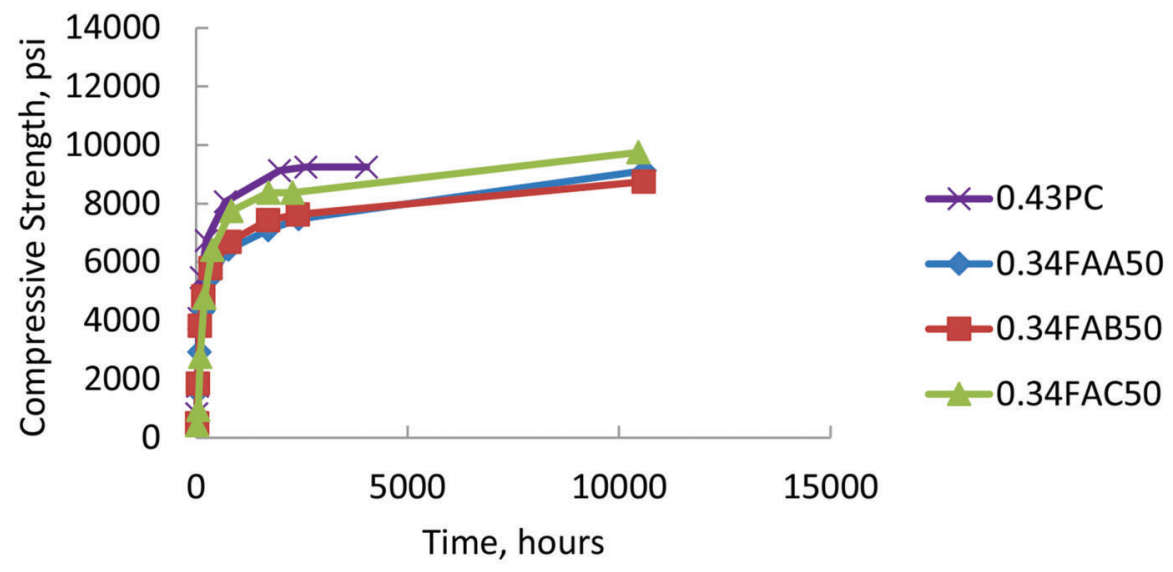

(b)

Figure 3.3 Compressive strength test results of the low alkali cement control mortar mixture vs. (a) $35 \%$ fly ash; (b) $50 \%$ fly ash. All mixtures cured at Cold $-45^{\circ} \mathrm{F}$.

fits of the strength age data for each case was also done using the logarithmic function and the $\mathrm{R}^{2}$ values are listed in the last column of Tables 3.14 and 3.18. It can be seen that for the control Mixture (0.43PC) and for the mixtures containing Fly ash FAC (Class C fly ash) the hyperbolic function gave better $\mathrm{R}^{2}$ values. For the mixtures containing Fly ash FAA and FAB (Class F fly ashes) the logarithmic function gave better $\mathrm{R}^{2}$ values for low alkali cement mixtures. Since in our case the hyperbolic function gave overall good curve fits for all mixtures $\left(\mathrm{R}^{2}\right.$ values of 0.94 to 1.00$)$ it was decided to use the hyperbolic function for all mixtures.

Tables 3.15 and 3.19 summarize the AE values determined from the curve fits for the logarithm of rate constant (k) vs. inverse of temperature plots. The $\mathrm{R}^{2}$ values ranging 0.94-1.00 indicate a good fit. Using these $\mathrm{AE}$ values the strength vs. equivalent age plots for the 3 curing temperatures were illustrated in Figures $3.7 \mathrm{c}, 3.8,3.11 \mathrm{c}$ and 3.12 . Ideally the strength vs. equivalent age plots for all 3 curing temperatures should plot on a straight line. Some variation may be acceptable.
Unfortunately, the variation is too high. For the control mixtures (Figures 3.8c and 3.12c) the strength vs. equivalent age for the hot temperature was much lower whereas for the fly ash mixtures (Figures 3.8 and 3.12) the strength vs. equivalent age for the cold temperature was much lower. Different methods were tried to reduce the variation in the strength vs. equivalent age plots for the 3 curing temperatures. Eventually, the following approach was used for each mixture:

1. The measured 28-day strength at the control curing temperature $\left(73^{\circ} \mathrm{F}\right)$ was set as the value of the ultimate strength, $S_{u}$ for all 3 curing temperatures.

2. $70 \%$ of this $S_{u}$ value was calculated. For all 3 curing temperatures only test results up to this strength level were included in the analysis.

3. $t_{0}, k$, and $\mathrm{AE}$ values were determined using the best fit with the hyperbolic function discussed earlier. The log of rate constant $(\mathrm{k})$ was plotted against inverse of temperature and this slope of this fitted line is reported as the estimate of the activation energy. 

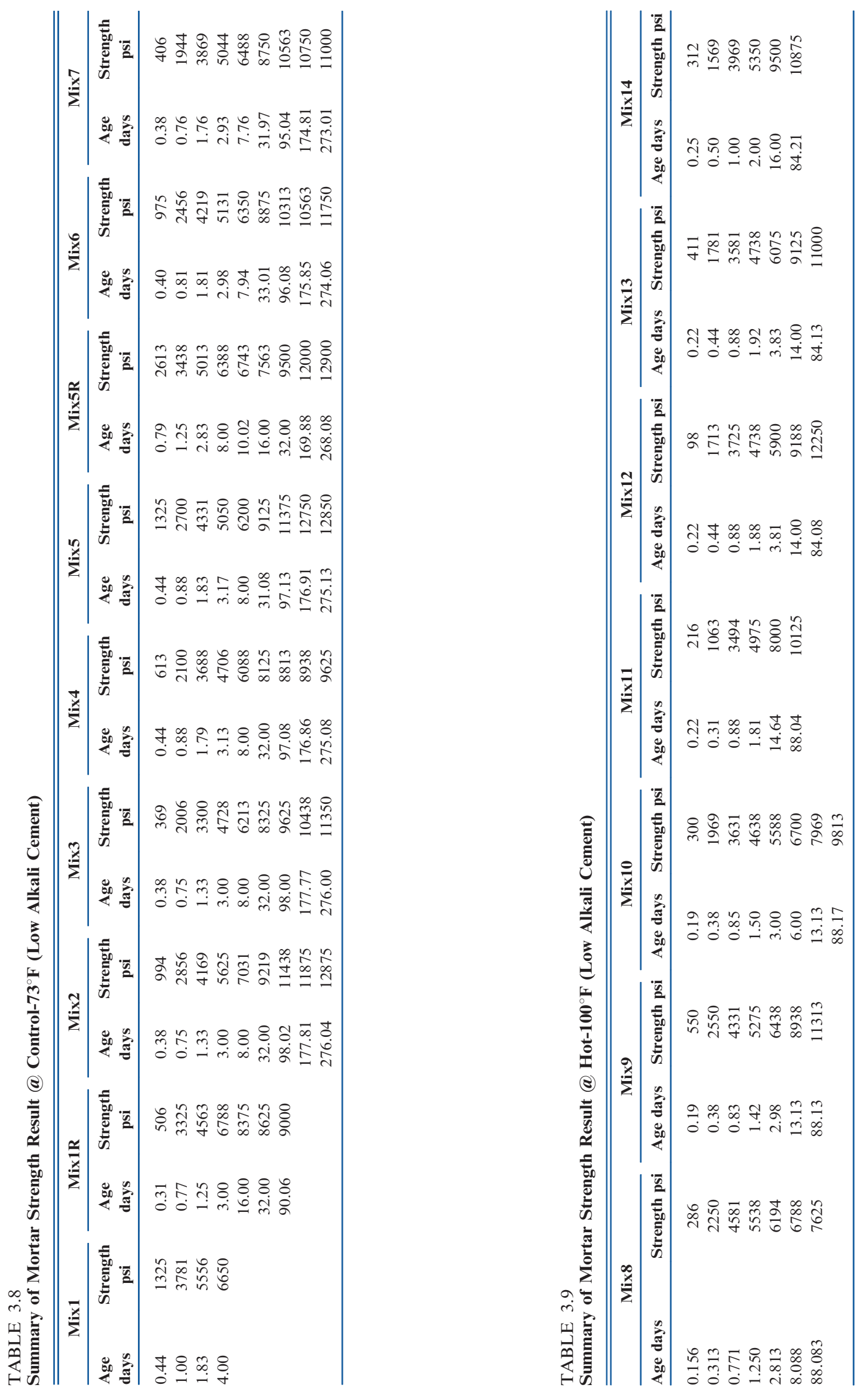

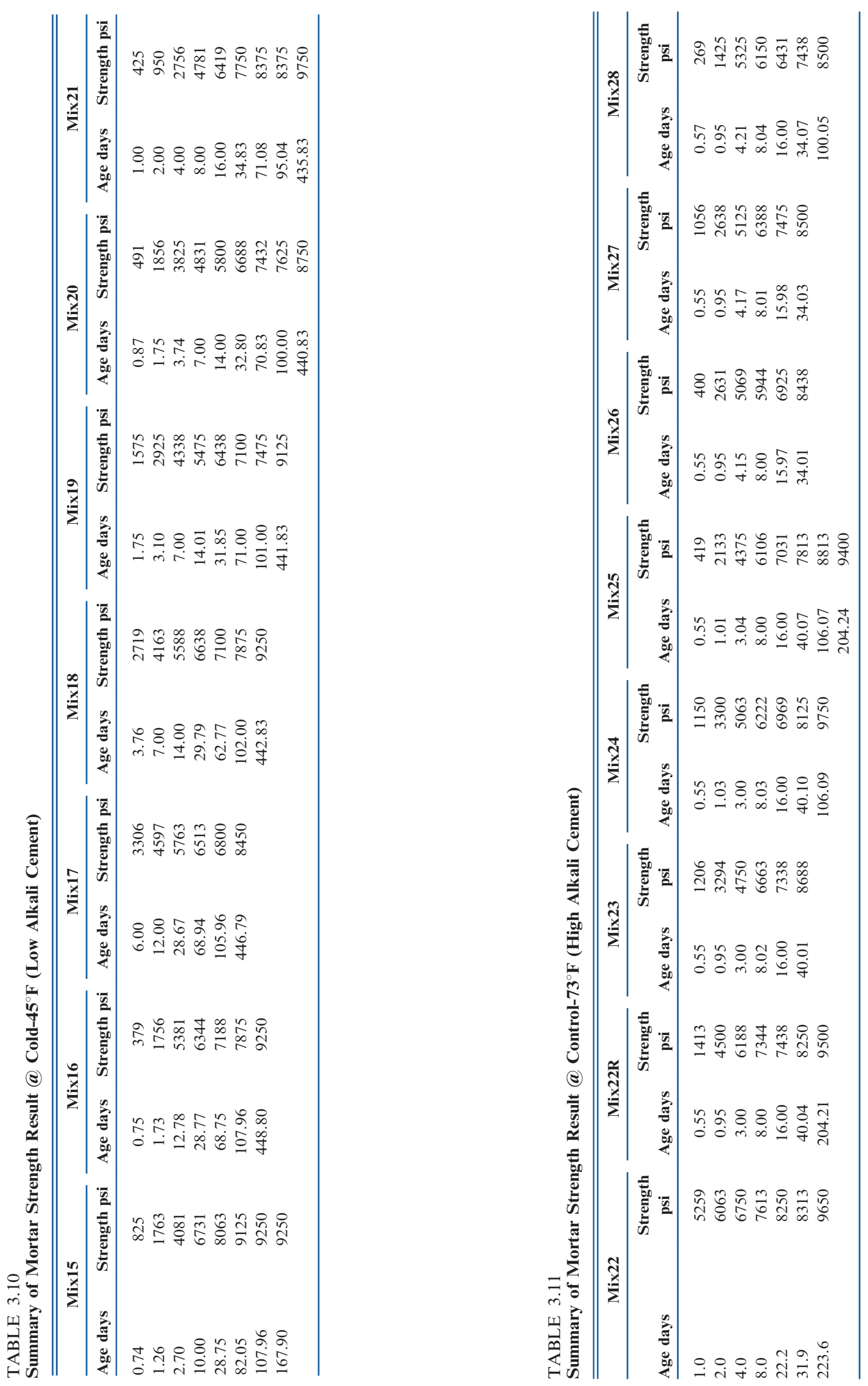
TABLE 3.12

Summary of Mortar Strength Result @ Hot-100 ${ }^{\circ}$ F (High Alkali Cement)

\begin{tabular}{|c|c|c|c|c|c|c|c|c|c|c|c|c|c|}
\hline \multicolumn{2}{|c|}{ Mix29 } & \multicolumn{2}{|c|}{ Mix30 } & \multicolumn{2}{|c|}{ Mix31 } & \multicolumn{2}{|c|}{ Mix32 } & \multicolumn{2}{|c|}{ Mix33 } & \multicolumn{2}{|c|}{ Mix34 } & \multicolumn{2}{|c|}{ Mix35 } \\
\hline $\begin{array}{l}\text { Age } \\
\text { days }\end{array}$ & $\begin{array}{c}\text { Strength } \\
\text { psi }\end{array}$ & $\begin{array}{l}\text { Age } \\
\text { days }\end{array}$ & $\begin{array}{c}\text { Strength } \\
\text { psi }\end{array}$ & $\begin{array}{l}\text { Age } \\
\text { days }\end{array}$ & $\begin{array}{l}\text { Strength } \\
\text { psi }\end{array}$ & $\begin{array}{l}\text { Age } \\
\text { days }\end{array}$ & $\begin{array}{c}\text { Strength } \\
\text { psi }\end{array}$ & $\begin{array}{l}\text { Age } \\
\text { days }\end{array}$ & $\begin{array}{c}\text { Strength } \\
\text { psi }\end{array}$ & $\begin{array}{l}\text { Age } \\
\text { days }\end{array}$ & $\begin{array}{c}\text { Strength } \\
\text { psi }\end{array}$ & $\begin{array}{l}\text { Age } \\
\text { days }\end{array}$ & $\begin{array}{c}\text { Strength } \\
\text { psi }\end{array}$ \\
\hline 0.33 & 1335 & 0.38 & 1556 & 0.30 & 1156 & 1.08 & 2968 & 0.96 & 3006 & 0.50 & 2106 & 0.31 & 474 \\
\hline 0.75 & 5088 & 0.93 & 3863 & 0.98 & 3781 & 1.25 & 4350 & 1.25 & 3588 & 1.00 & 3794 & 0.50 & 1631 \\
\hline 3.00 & 6444 & 3.00 & 5306 & 2.00 & 4575 & 2.27 & 5050 & 2.29 & 4719 & 2.00 & 4950 & 1.25 & 4281 \\
\hline 5.96 & 6906 & 5.99 & 6100 & 4.13 & 5313 & 5.02 & 6231 & 5.14 & 6044 & 4.98 & 6706 & 2.26 & 6038 \\
\hline 12.22 & 7156 & 13.18 & 8125 & 8.13 & 6413 & 11.26 & 7360 & 10.06 & 8813 & 8.01 & 8000 & 5.08 & 7500 \\
\hline 93.02 & 8375 & & & 27.06 & 8250 & 20.19 & 8375 & 20.04 & 9750 & 15.96 & 9250 & 10.03 & 8688 \\
\hline & & & & 93.11 & 8875 & 92.10 & 9563 & & & 92.08 & 10750 & 72.09 & 10188 \\
\hline
\end{tabular}

TABLE 3.13

Summary of Mortar Strength Result @ Cold-45 ${ }^{\circ}$ F (High Alkali Cement)

\begin{tabular}{|c|c|c|c|c|c|c|c|c|c|c|c|c|c|}
\hline \multicolumn{2}{|c|}{ Mix36 } & \multicolumn{2}{|c|}{ Mix37 } & \multicolumn{2}{|c|}{ Mix38 } & \multicolumn{2}{|c|}{ Mix39 } & \multicolumn{2}{|c|}{ Mix40 } & \multicolumn{2}{|c|}{ Mix41 } & \multicolumn{2}{|c|}{ Mix42 } \\
\hline $\begin{array}{l}\text { Age } \\
\text { days }\end{array}$ & $\begin{array}{c}\text { Strength } \\
\text { psi }\end{array}$ & $\begin{array}{l}\text { Age } \\
\text { days }\end{array}$ & $\begin{array}{c}\text { Strength } \\
\text { psi }\end{array}$ & $\begin{array}{l}\text { Age } \\
\text { days }\end{array}$ & $\begin{array}{c}\text { Strength } \\
\text { psi }\end{array}$ & $\begin{array}{l}\text { Age } \\
\text { days }\end{array}$ & $\begin{array}{c}\text { Strength } \\
\text { psi }\end{array}$ & $\begin{array}{l}\text { Age } \\
\text { days }\end{array}$ & $\begin{array}{c}\text { Strength } \\
\text { psi }\end{array}$ & $\begin{array}{l}\text { Age } \\
\text { days }\end{array}$ & $\begin{array}{c}\text { Strength } \\
\text { psi }\end{array}$ & $\begin{array}{l}\text { Age } \\
\text { days }\end{array}$ & $\begin{array}{c}\text { Strength } \\
\text { psi }\end{array}$ \\
\hline 1.73 & 2069 & 1.77 & 1513 & 2.00 & 1575 & 2.19 & 1169 & 1.75 & 869 & 1.85 & 1363 & 2.01 & 875 \\
\hline 2.99 & 4281 & 3.06 & 3275 & 4.00 & 3225 & 5.02 & 2950 & 3.02 & 2038 & 3.74 & 3238 & 3.99 & 2713 \\
\hline 5.00 & 6038 & 6.90 & 5000 & 8.00 & 4856 & 8.90 & 4025 & 7.01 & 3944 & 7.50 & 4944 & 7.99 & 4725 \\
\hline 11.06 & 7390 & 13.00 & 6363 & 17.06 & 5738 & 17.06 & 4674 & 13.98 & 4669 & 15.06 & 5319 & 16.03 & 5194 \\
\hline 28.79 & 8000 & 29.84 & 6738 & 33.06 & 6438 & 62.15 & 6063 & 31.91 & 5144 & 32.18 & 6050 & 33.81 & 6288 \\
\hline 82.05 & 9375 & 68.79 & 7188 & 68.94 & 7313 & & & 397.80 & 7500 & 71.04 & 6181 & 71.01 & 6506 \\
\hline 390.80 & 10375 & 398.80 & 8125 & 396.80 & 8000 & & & & & 392.80 & 8250 & 391.80 & 8000 \\
\hline
\end{tabular}

The above process is depicted in Figures 3.9 and 3.10 for low alkali cements and Figures 3.13 and 3.14 for high alkali cements. Figures 3.9 and 3.13 are for the low alkali and high alkali cement control mixtures, respectively. Each of these figures has 3 plots depicted as a, b, c. In Figure 3.9, Plot 10a illustrates the strength vs. age results for all 3 curing temperatures and the hyperbolic curve fit to the data. Only strength test results up to $70 \%$ of the $S_{u}$ value were used in the analysis. Plot 10b plots the logarithm of rate constant (k) vs. inverse of curing temperature in degree Kelvin and the line fit. The slope of this line is used to calculate the activation energy. Plot 10c shows the strength vs. equivalent age results (for equivalent age up to 7 days) for all 3 curing temperatures. For the fly ash mixtures in the interest of space only the strength-to-equivalent age plots are illustrated in Figures 3.10 and 3.14 for the low alkali and high alkali cement mixtures respectively.

Using the modified analysis, Tables 3.16 and 3.20 summarize the hyperbolic curve fit data for the low alkali and high alkali mixtures, respectively. The values of $t_{0}$ determined from this analysis are more reasonable than those in Table 3.14 with the prior analysis. The values of $S_{u}$ are lower than the actual measured later age strengths. However, this was considered to be acceptable as long as the hyperbolic curve fits are good for the first 7 days and the overall coefficient of determinations $\left(\mathrm{R}^{2}\right)$ values are good. It can be seen that the hyperbolic function indicates a good fit with $\mathrm{R}^{2}$ values between 0.91 and 1.00 for all 42 cases.
Tables 3.17 and 3.21 summarize the activation energy values determined from the curve fits from the plots of the logarithm of rate constant (k) vs. inverse of temperature. Using these derived values of activation energy, the strength vs. equivalent age plots for the 3 curing temperatures were drawn (Figures 3.9c, 3.10, and Figures $3.13 \mathrm{c}$ and 3.14). It can be seen that with this modified analysis the curves for 3 different curing temperatures converge together as should be expected.

\subsubsection{Significance of the Activation Energy Values Calculated}

For any concrete mixture the rate of strength development increases as concrete temperature increases. The activation energy values provide a relative indicator of the rate of hydration and strength gain characteristics of cementitious systems dependent on temperature. A higher value of the activation energy has a lower rate of strength gain at lower temperature and this rate increases as temperature rises. Lower values of activation energy indicate that the cementitious materials are less temperature sensitive. This can be observed in age conversion factors calculated from the $\mathrm{AE}$ of the various mixes listed in Tables 3.17 and 3.21. Age conversion factors of a mixture at a specific temperature can be seen as the ratio of strength of that mixture at that temperature to the strength at a reference (datum) temperature, which is commonly $73^{\circ} \mathrm{F}$.

So for the low alkali 0.43PC mixture (Table 3.17) at $41^{\circ} \mathrm{F}$ the strength is only $40 \%$ of the strength at $73^{\circ} \mathrm{F}$ 


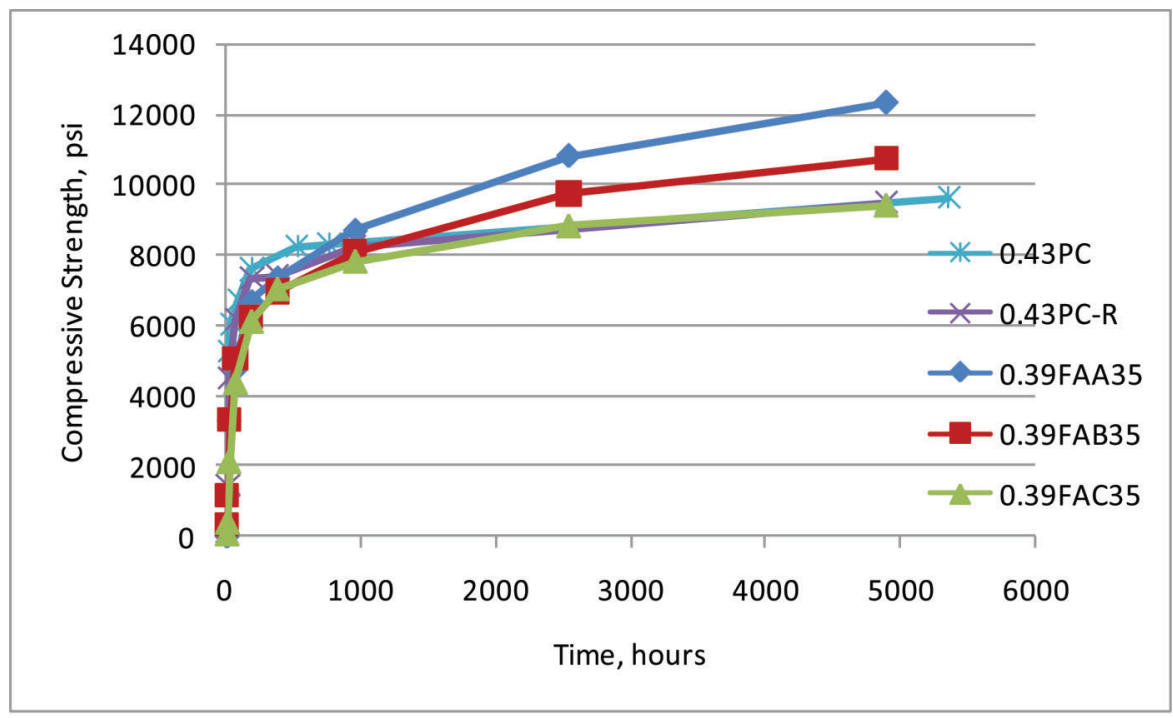

(a)

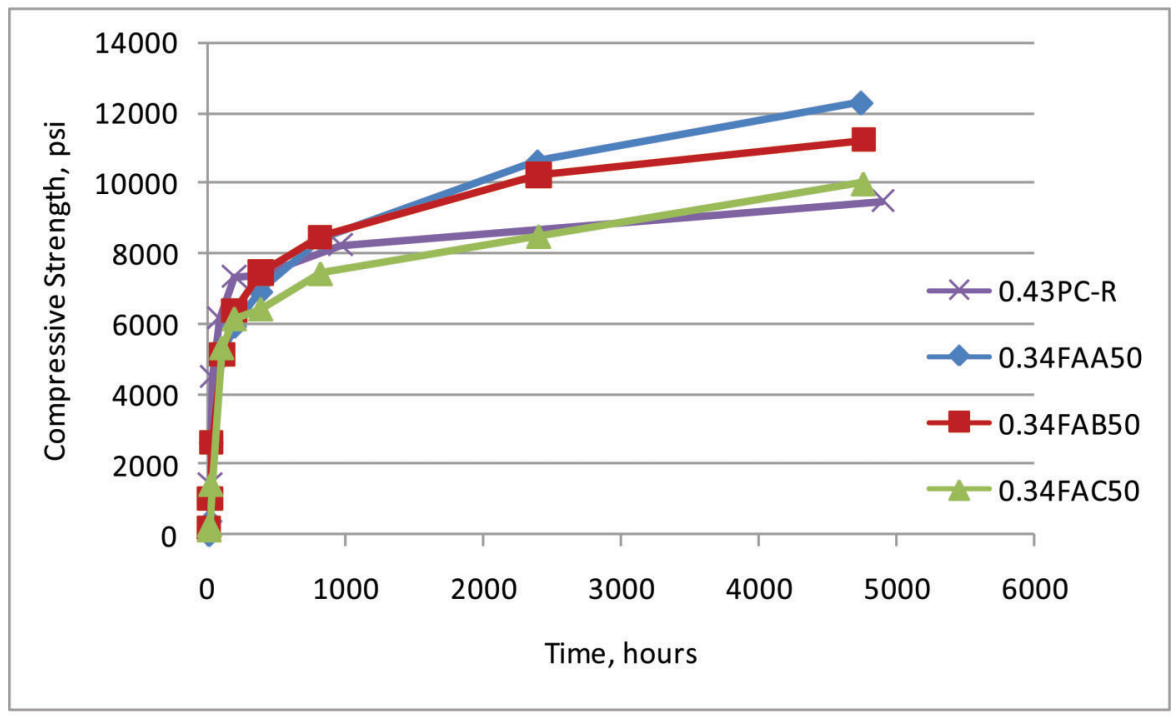

(b)

Figure 3.4 Compressive strength test results of the high alkali cement control mortar mixture vs. (a) $35 \%$ fly ash; (b) $50 \%$ fly ash. All mixtures cured at Control- $73^{\circ} \mathrm{F}$.

which means that in order to attain the 2-day strength at $73^{\circ} \mathrm{F}$ one would have to cure the specimen at $41^{\circ} \mathrm{F}$ for 5 days $(2 / 0.40)$. The low alkali $0.43 \mathrm{PC}$ mix had an AE of $34,981 \mathrm{~J} / \mathrm{mol}$. The fly ash mixtures made with low alkali cement have up to $50 \%$ higher $\mathrm{AE}$ values. For the $0.39 \mathrm{FAA} 35$ mixture at $41^{\circ} \mathrm{F}$ the strength is only $24 \%$ of the strength at $73^{\circ} \mathrm{F}$ which means that in order to attain the 2-day strength at $73^{\circ} \mathrm{F}$ one would have to cure the specimen at $41^{\circ} \mathrm{F}$ for 8.3 days. The AE values of mixtures made with the high alkali cement in general were lower for the 0.39FAA35 and 0.39FAB35 mixtures; for the rest of the mixtures it was similar. The AE values were up to $25 \%$ higher for the $50 \%$ low alkali cement fly ash mixtures and 50\% higher for the 50\% high alkali cement fly ash mixtures when compared to their respective control mixtures. No particular trends between $\mathrm{AE}$ values and cement or fly ash properties could be discerned. The age conversion factor is used to convert the temperature history of the concrete in the field into an equivalent age at $73^{\circ} \mathrm{F}$ and based on a predetermined strength-equivalent age relationship the strength is estimated.

\subsection{Development of Concrete Strength-Maturity Relationship}

Concrete testing was conducted on the 50\% fly ash (by volume) mixtures with low and high $\mathrm{CaO}$ fly ash in conjunction with low and high alkali cements (total of 4 concrete mixtures). Table 3.22 shows the mixture 


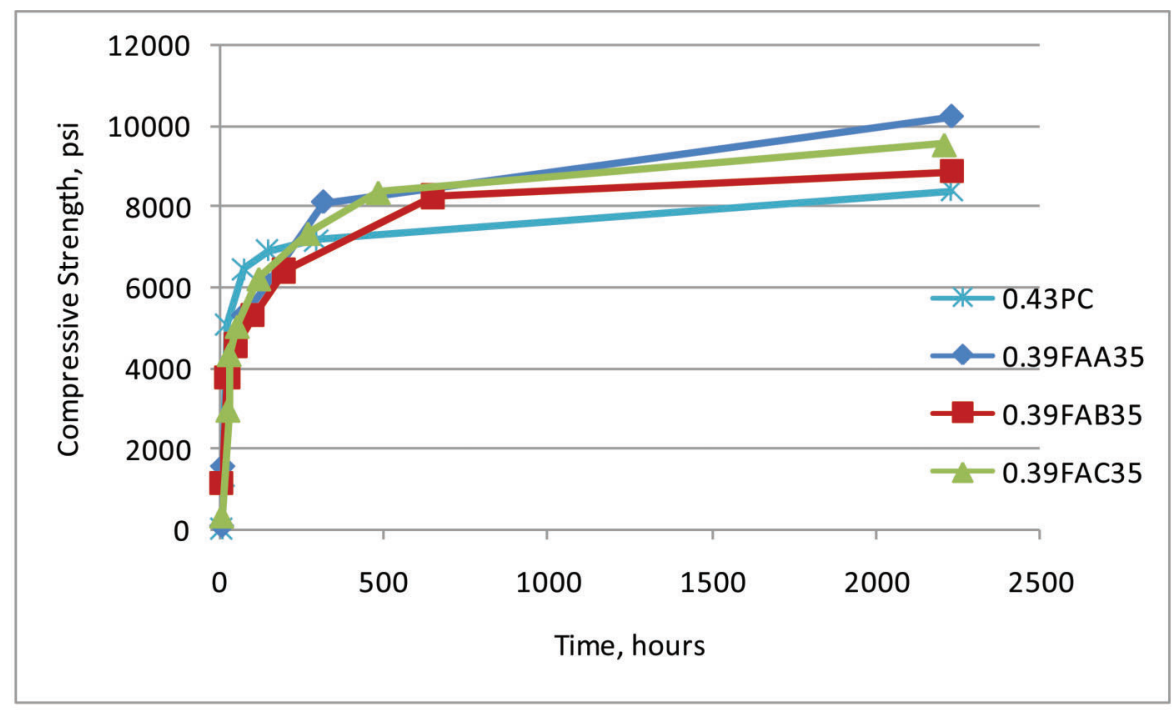

(a)

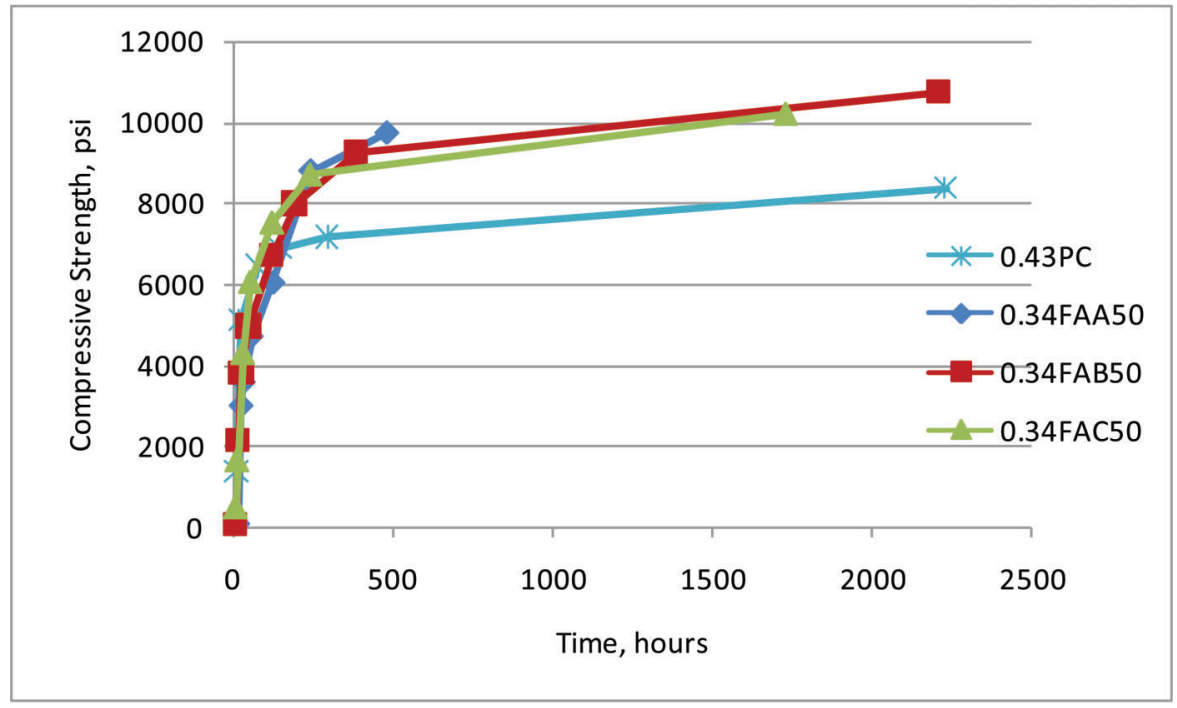

(b)

Figure 3.5 Compressive strength test results of the high alkali cement control mortar mixture vs. (a) $35 \%$ fly ash; (b) $50 \%$ fly ash. All mixtures cured at $\mathrm{Hot}-100^{\circ} \mathrm{F}$.

proportions and test results. Mixtures 1 and 2 used low alkali cement (Cement B) and were the concrete equivalents of mortar Mixtures 0.34FAA50 and 0.34 FAC50 respectively in Table 3.2. Therefore, they will be referred to as 0.34FAA50LAC, and 0.34FAC50LAC respectively. Mixtures 3 and 4 used high alkali cement (Cement C) and were the concrete equivalents of mortar mixtures $0.34 \mathrm{FAA} 50$ and $0.34 \mathrm{FAC} 50$ respectively in Table 3.8. Therefore, they will be referred to as $0.34 \mathrm{FAA} 50 \mathrm{HAC}$, and 0.34FAC50HAC respectively. Concrete slump (ASTM C143), temperature (C1064), density (C138), air content (C231), and compressive strength (C39) was measured. The $w / \mathrm{cm}$ was 0.34 for all the mixtures and a target concrete slump of 4 to $8 \mathrm{in}$. was attained through the use of a HRWR. All concrete mixtures were non air entrained. A $30 \mathrm{oz} / \mathrm{cwt}$ of a nonchloride Type $\mathrm{C}$ accelerating admixture was also used. The water content from the admixture was subtracted from the mixing water. Two concrete cylinders (4 in. x 8 in.) were tested in compression at equivalent ages of 1 , $2,4,7,14,28$ days and temperature was monitored in one additional cylinder.

As noted in the mortar mixtures the concrete mixtures containing fly ash FAA required a higher Type $\mathrm{F}$ admixture dosage as compared to the concrete mixtures containing fly ash FAC. But the admixture dosages were not excessive $(<10 \mathrm{oz} / \mathrm{cwt}$. as compared to 20 to $32 \mathrm{oz} / \mathrm{cwt}$ for corresponding mortar mixtures).

The two concrete mixtures with high alkali cement had higher strengths compared to the two mixtures with 


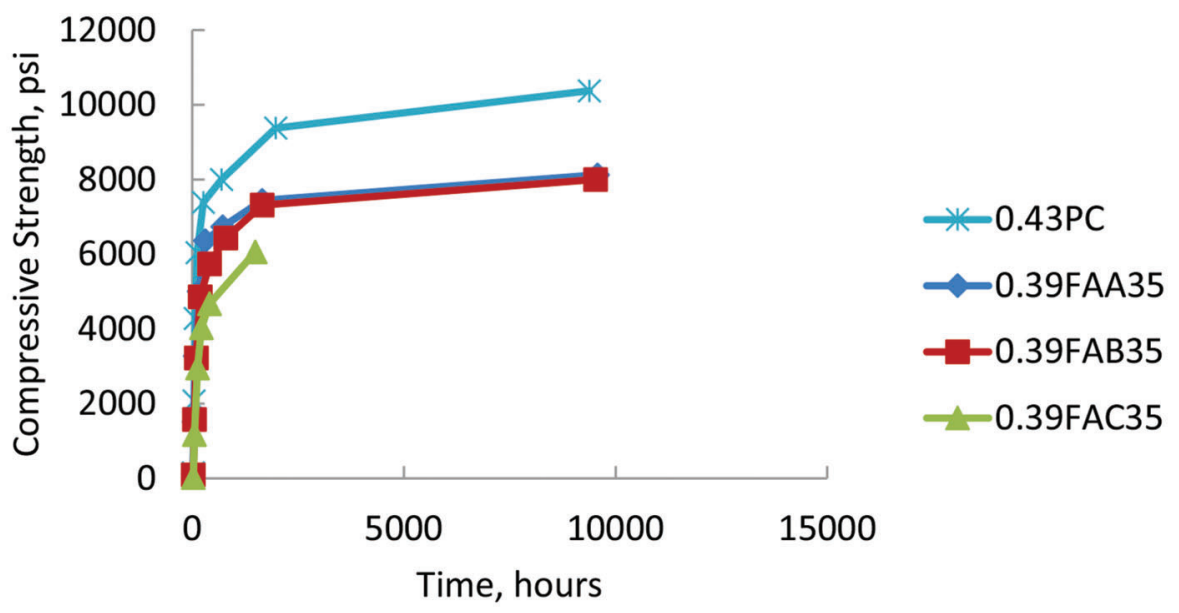

(a)

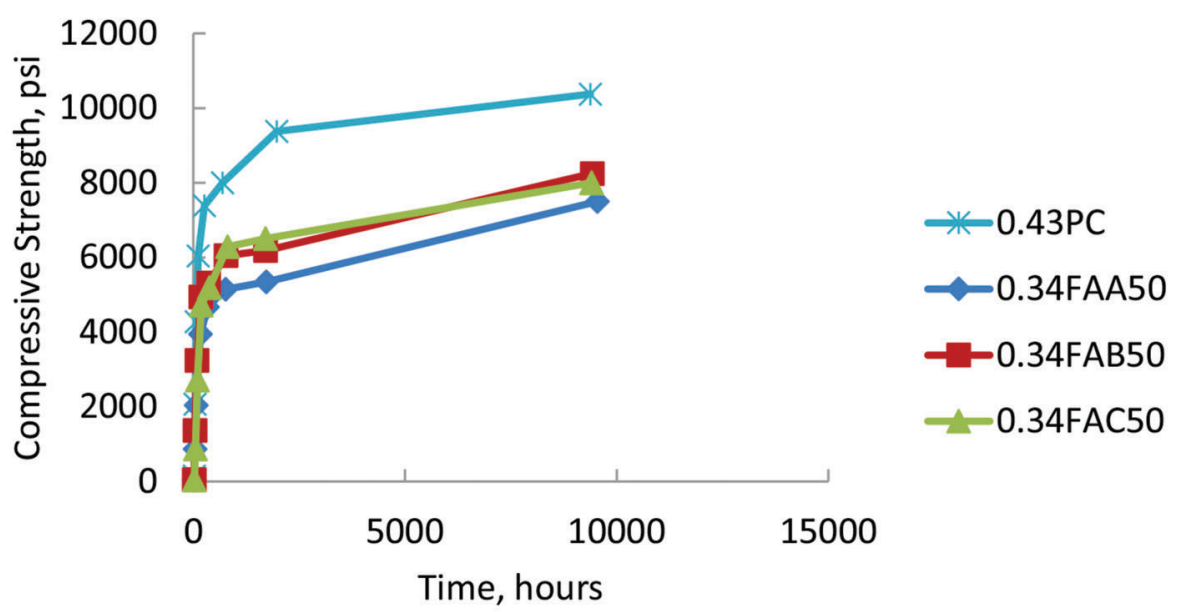

(b)

Figure 3.6 Compressive strength test results of the high alkali cement control mortar mixture vs. (a) $35 \%$ fly ash; (b) $50 \%$ fly ash. All mixtures cured at Cold $-45^{\circ} \mathrm{F}$.

the low alkali cement at all ages except at 1 day. The 1-day strength of the 0.34FAC50HAC mixture was 161 psi indicating severe retardation of that mixture. For both cement types the concrete mixtures containing the high $\mathrm{CaO}$ fly ash showed higher strengths at all ages except at 1 day. Figure 3.15a-d illustrates the strength vs. equivalent age at $73^{\circ} \mathrm{F}$ for the four concrete mixtures with the hyperbolic curve fit (indicated as best-fit within the figure). The equivalent age was calculated by the Arrhenius equation with the selected AE value obtained from the mortar study. The $\mathrm{R}^{2}$ values were higher than 0.95 . The best fit equations for the 4 mixtures are provided below:

0.34 FAA50LAC: $S=6774 \times \frac{0.023(t-0.0)}{1+0.023(t-0.0)}$
0.34FAC50LAC: $S=9608 \times \frac{0.015(t-1.5)}{1+0.015(t-1.5)}$

0.34 FAA 50 HAC $: S=9125 \times \frac{0.020(t-0.0)}{1+0.020(t-0.0)}$

0.34 FAC50HAC: $S=10423 \times \frac{0.018(t-22.2)}{1+0.018(t-22.2)}$

Where $\mathrm{t}=$ equivalent age at $73.4^{\circ} \mathrm{F}\left(23^{\circ} \mathrm{C}\right)$.

The above best fit equations can be used predicting in-place compressive strengths using maturity if the corresponding mixtures are used. A temperature sensor is recorded inside the structure. The time, and temperature data recorded is converted to equivalent at $73.4^{\circ} \mathrm{F}$ using the AE for that mixture. Using the above concrete Strength-Maturity equation the strength is predicted. 


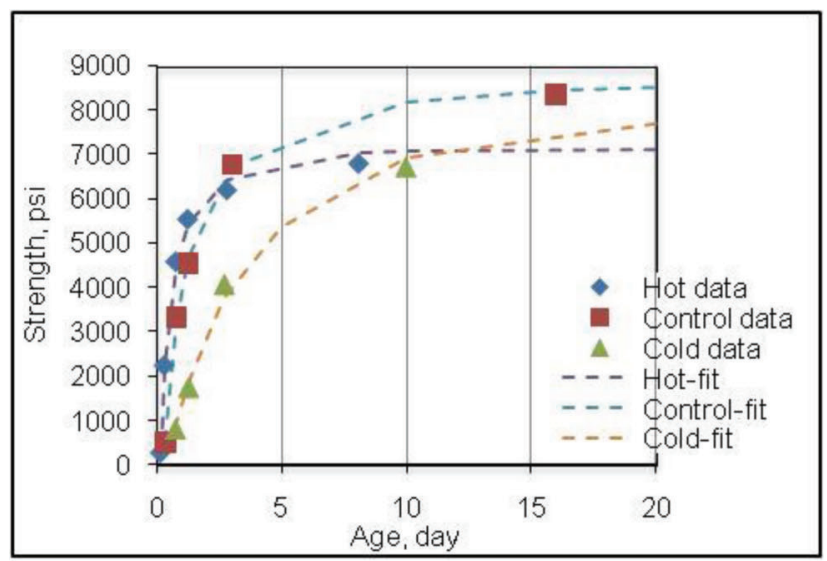

(a)

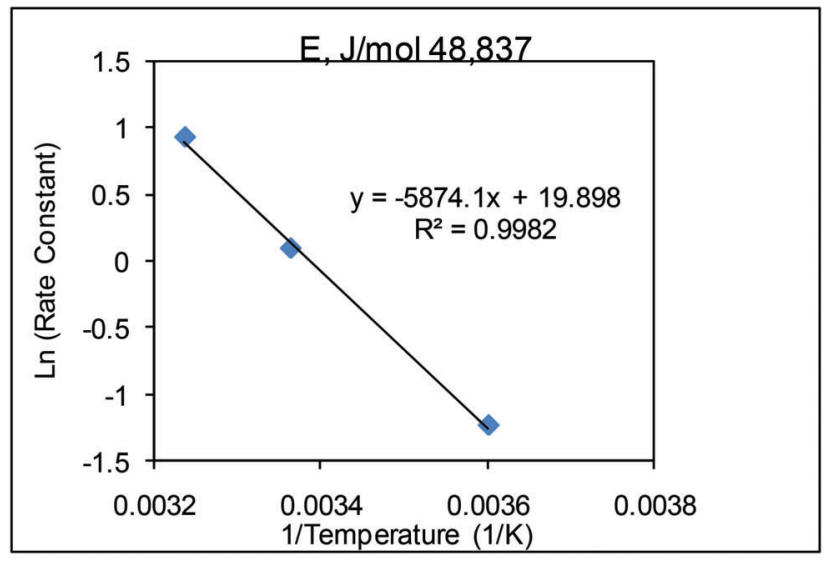

(b)

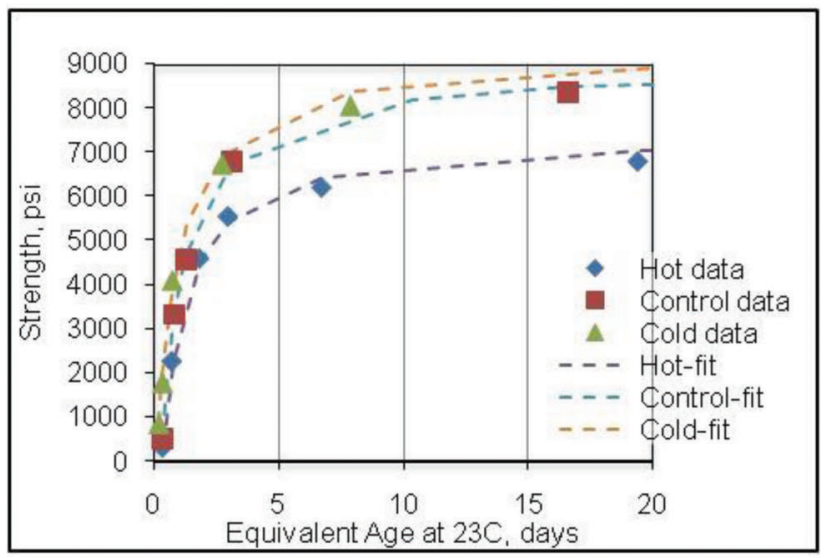

(c)

Figure 3.7 Strength-age plot: (a) plot to compute activation energy based on ASTM C1074 (b) and strength-equivalent age plot (c) for low alkali portland cement B mixture. 


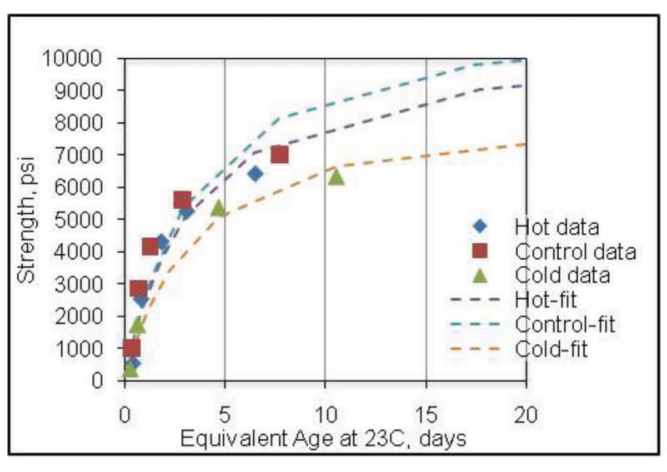

(a)

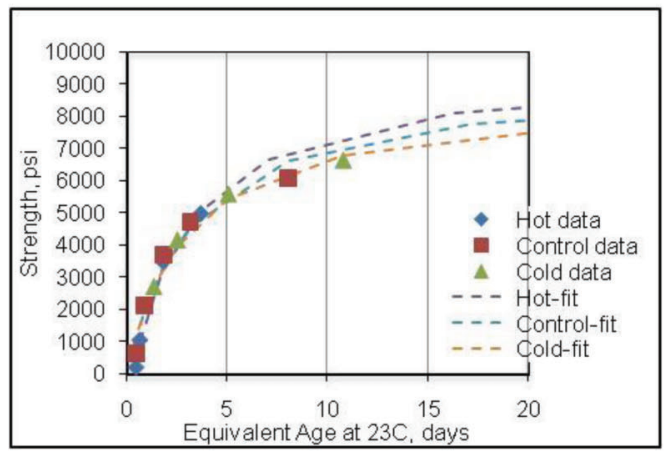

(c)

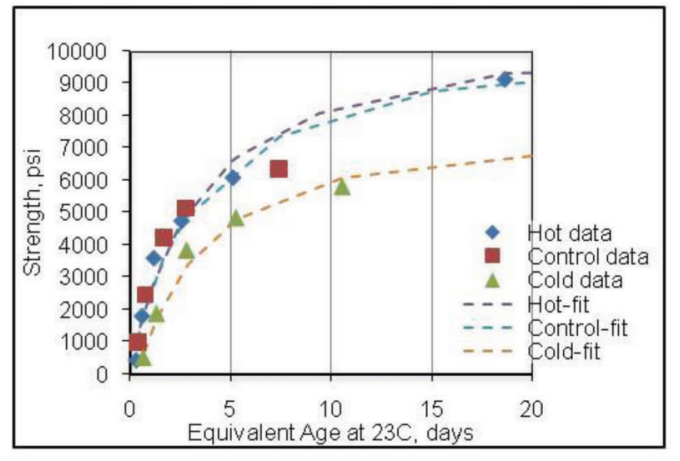

(e)

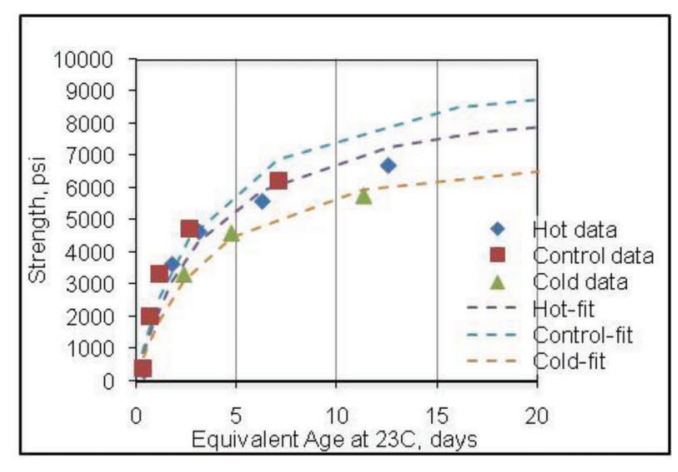

(b)

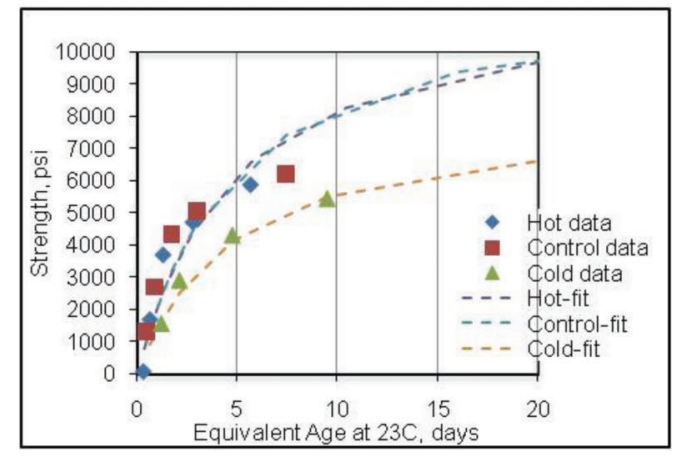

(d)

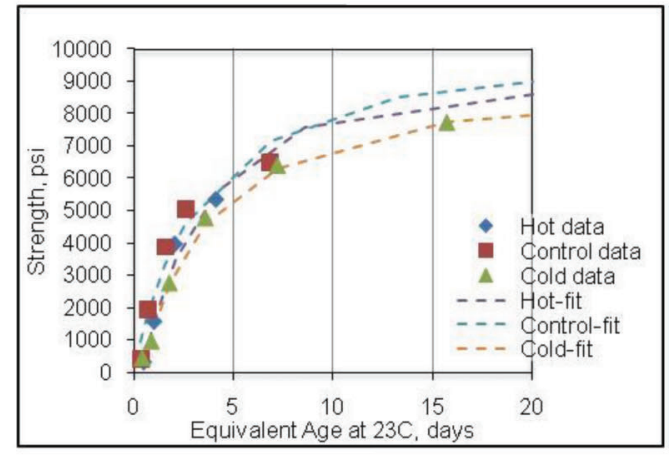

(f)

Figure 3.8 Strength-equivalent age plot for Low Alkali Cement B Mixtures based on ASTM C1074: (a) 0.39FAA35, (b) 0.39FAB35, (c) 0.39FAC35, (d) 0.39FAA50, (e) 0.39FAB50, and (f) 0.39FAC50. 


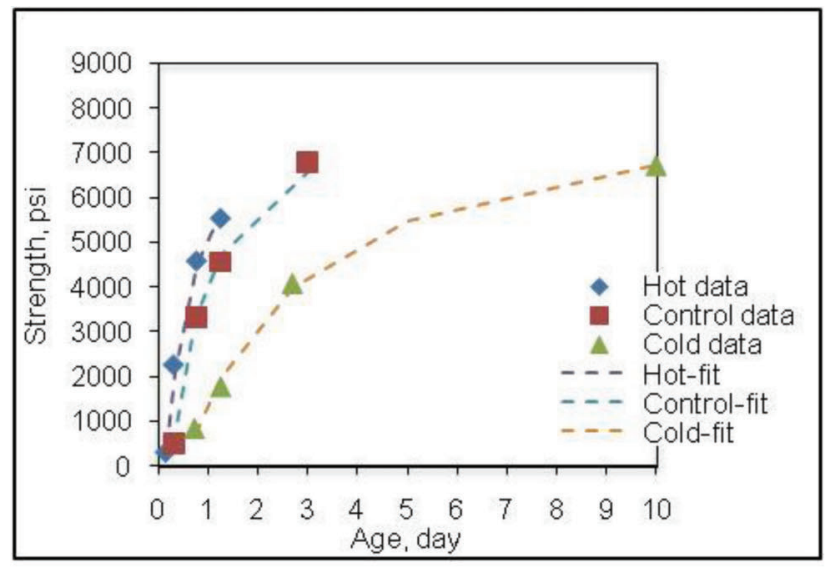

(a)

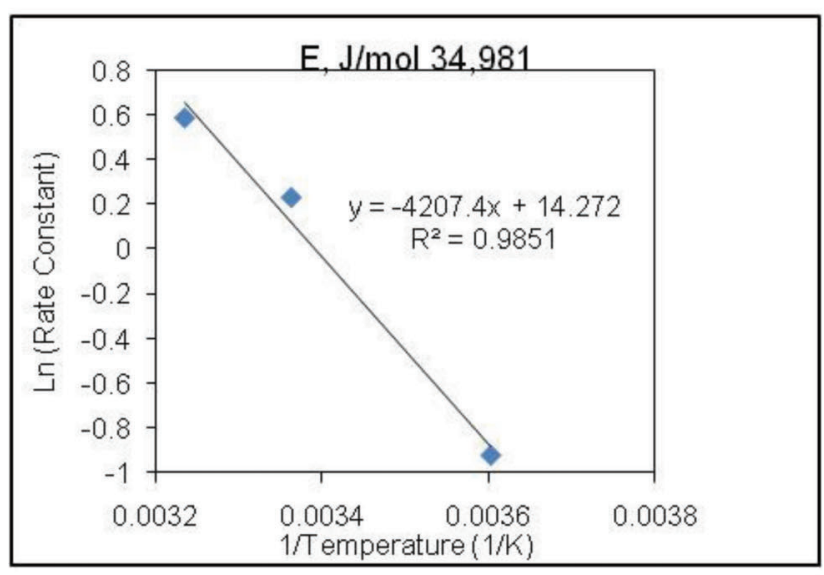

(b)

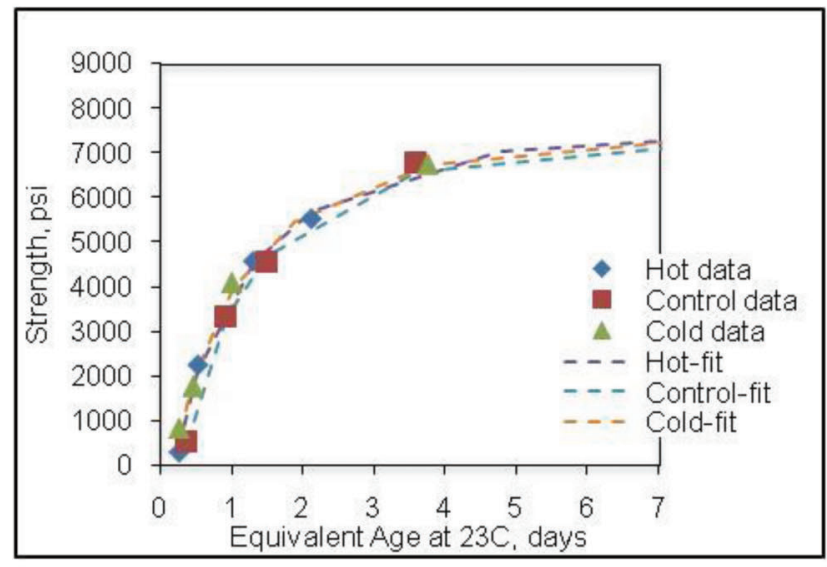

(c)

Figure 3.9 Strength-age plot: (a) plot to compute activation energy based on modified ASTM C1074 (b) and strength-equivalent age plot (c) for low alkali portland cement B mixture. 


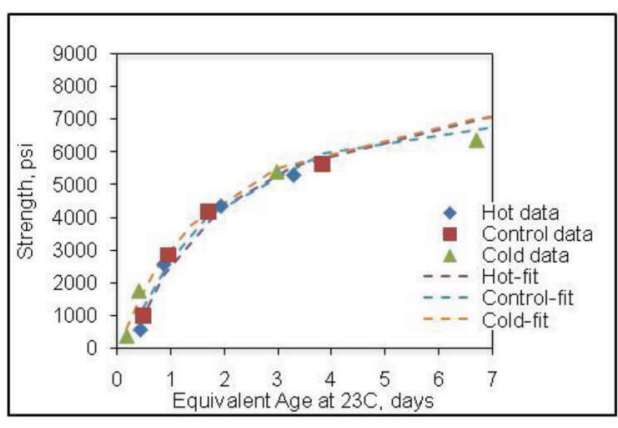

(a)

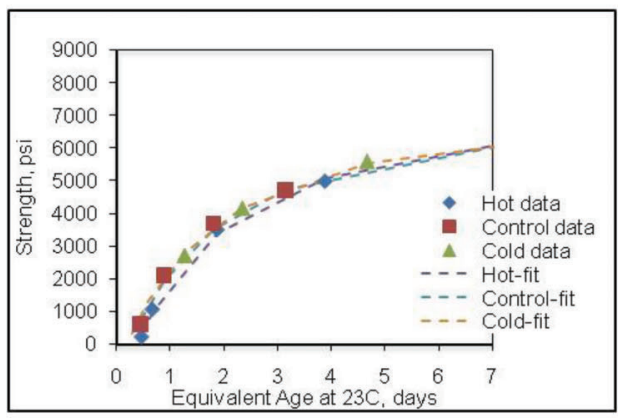

(c)

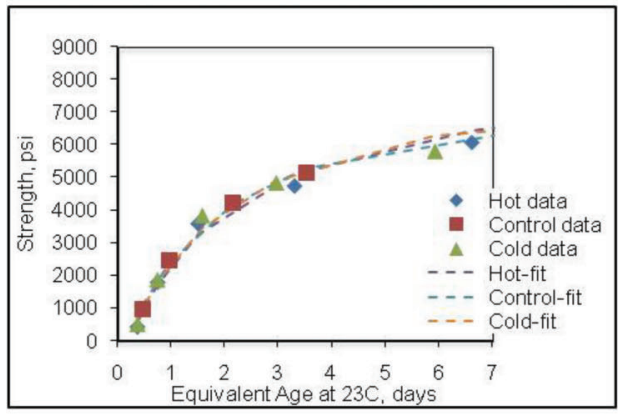

(e)

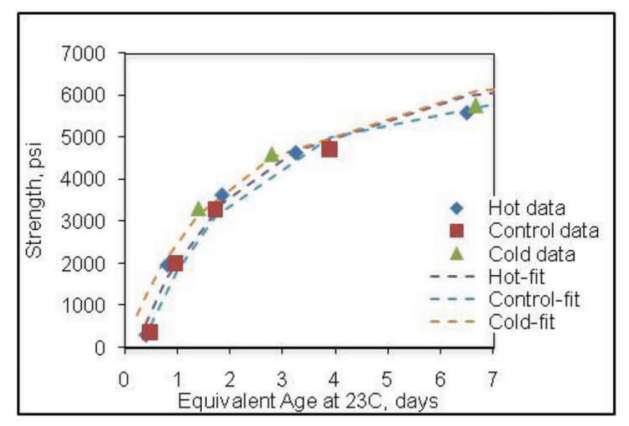

(b)

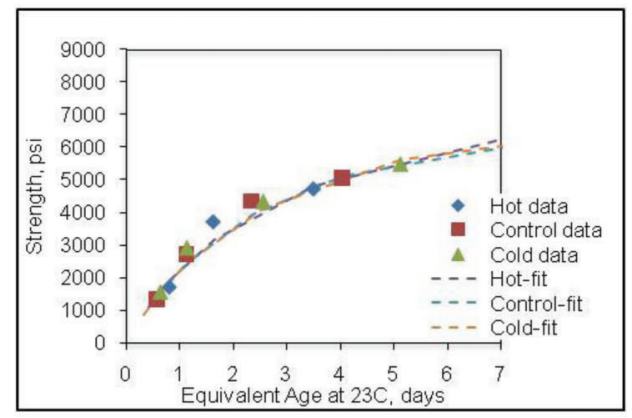

(d)

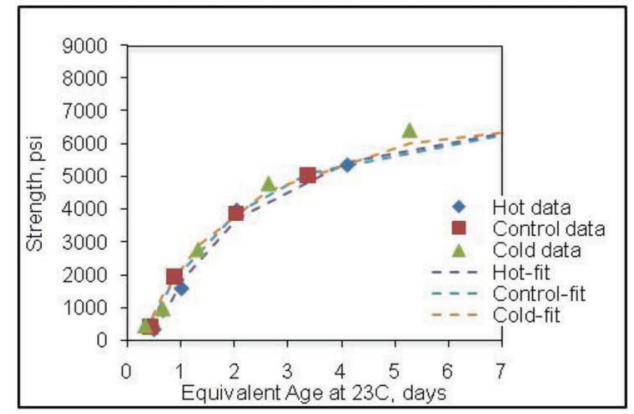

(f)

Figure 3.10 Strength-equivalent age plot for low alkali Cement B mixtures based on modified ASTM C1074: (a) 0.39FAA35, (b) 0.39FAB35, (c) 0.39FAC35, (d) 0.39FAA50, (e) 0.39FAB50, and (f) 0.39FAC50. 


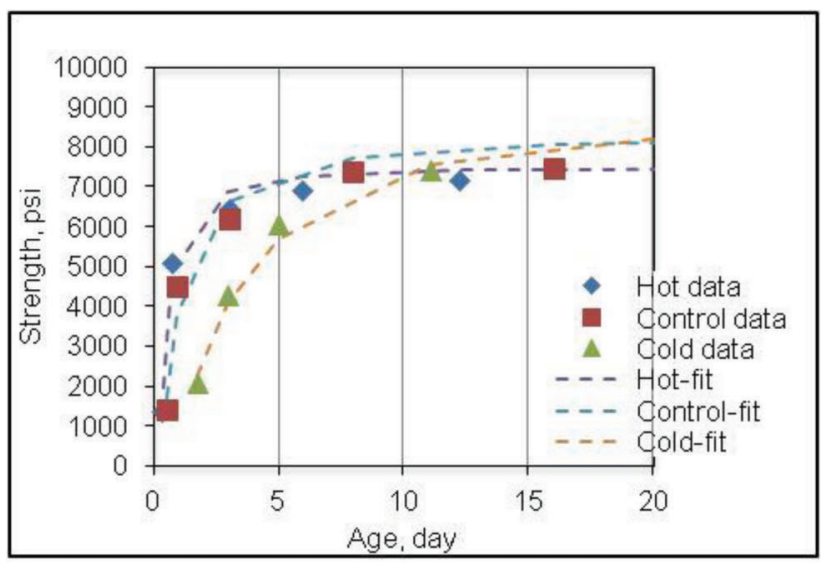

(a)

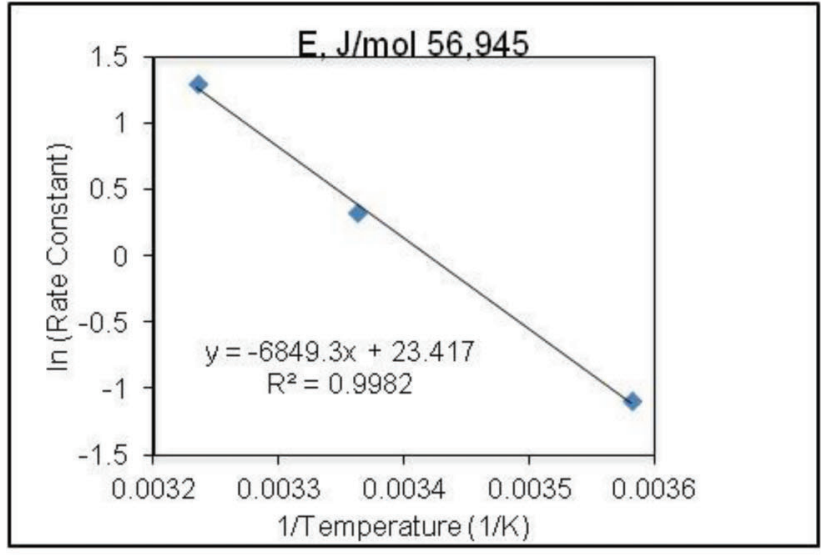

(b)

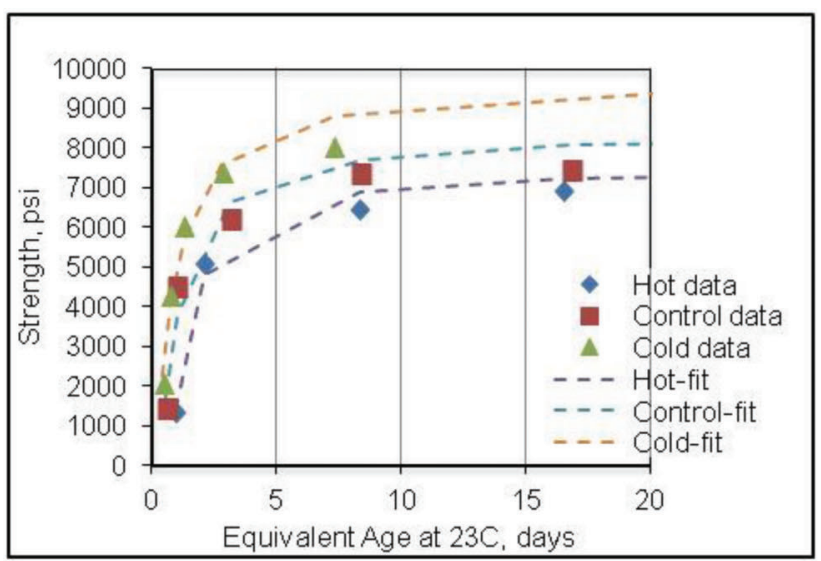

(c)

Figure 3.11 Strength-age plot: (a) plot to compute activation energy based on ASTM C1074 (b) and strength-equivalent age plot (c) for high alkali portland cement mixture. 


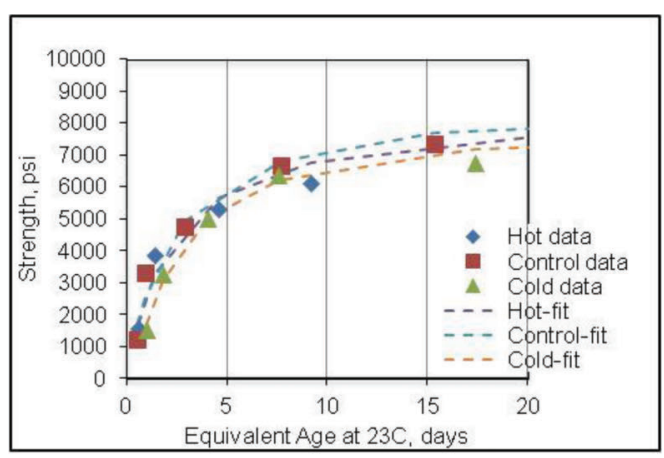

(a)

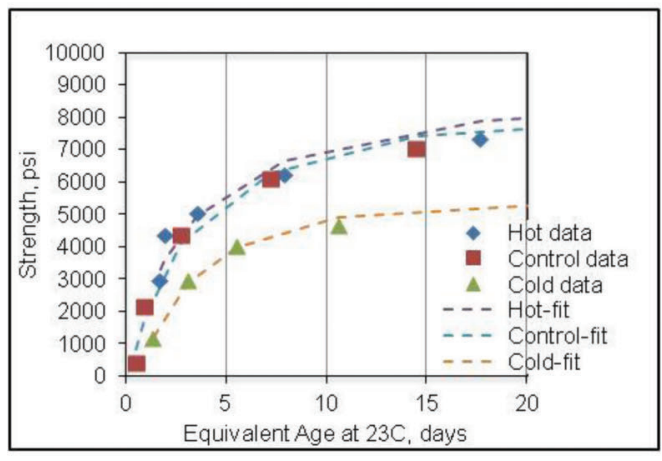

(c)

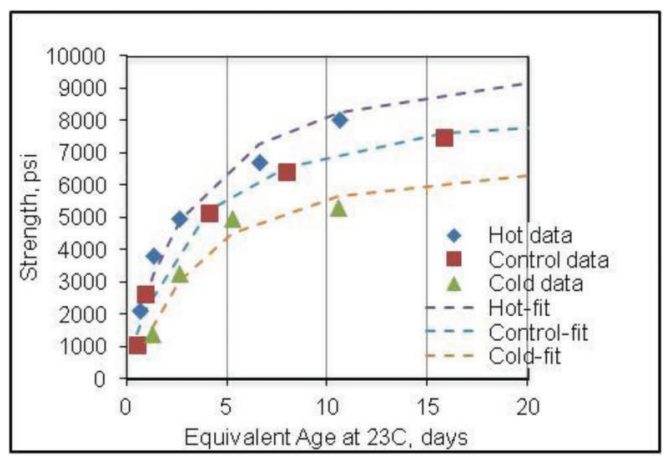

(e)

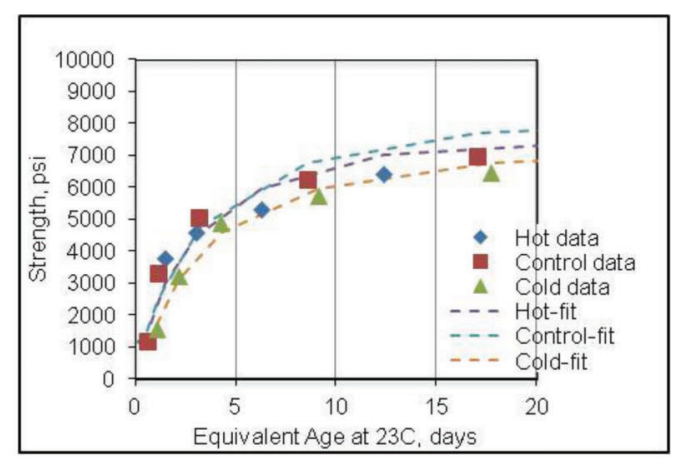

(b)

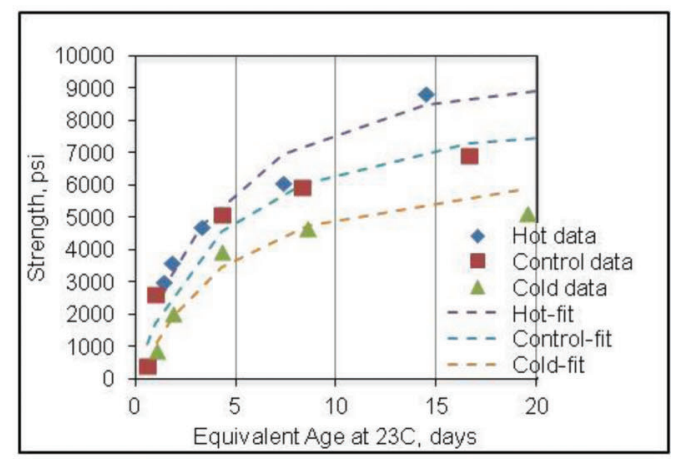

(d)

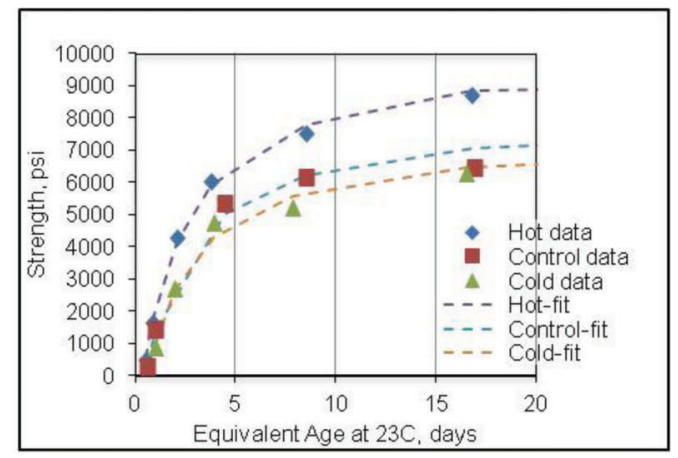

(f)

Figure 3.12 Strength-equivalent age plot for high alkali cement mixtures based on ASTM C1074: (a) 0.39FAA35, (b) 0.39FAB35, (c) 0.39FAC35, (d) 0.39FAA50, (e) 0.39FAB50, and (f) 0.39FAC50. 


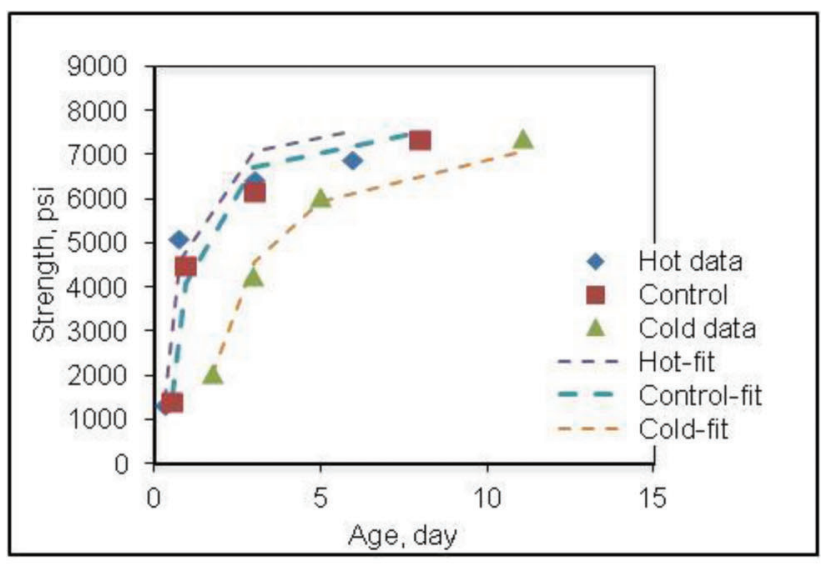

(a)

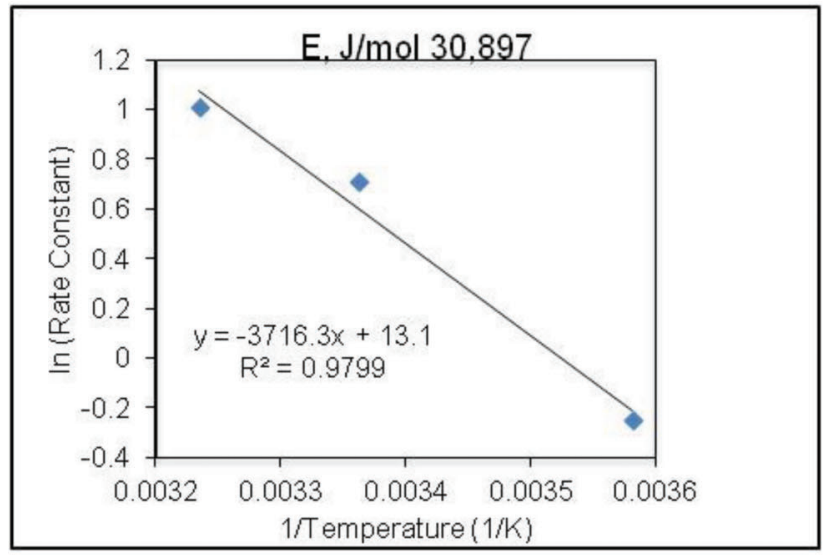

(b)

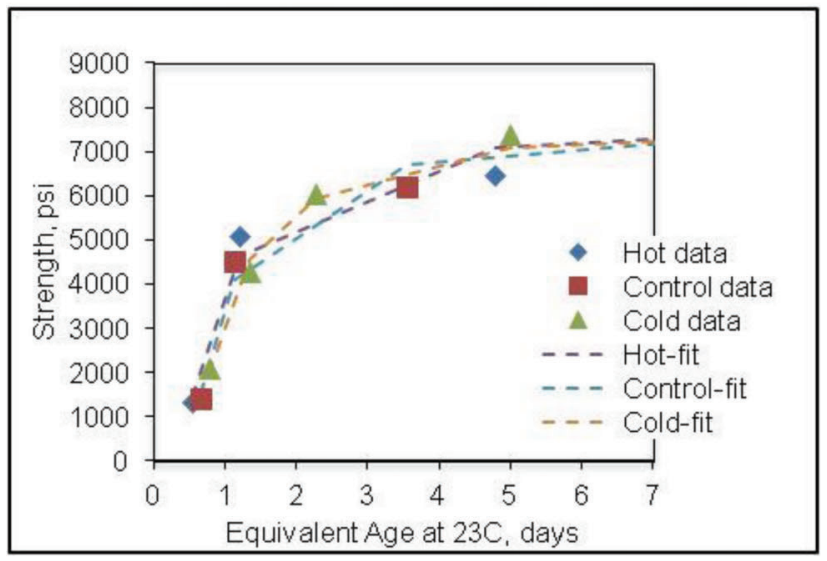

(c)

Figure 3.13 Strength-age plot: (a) plot to compute activation energy based on modified ASTM C1074 (b) and strength-equivalent age plot (c) for high alkali portland cement mixture. 


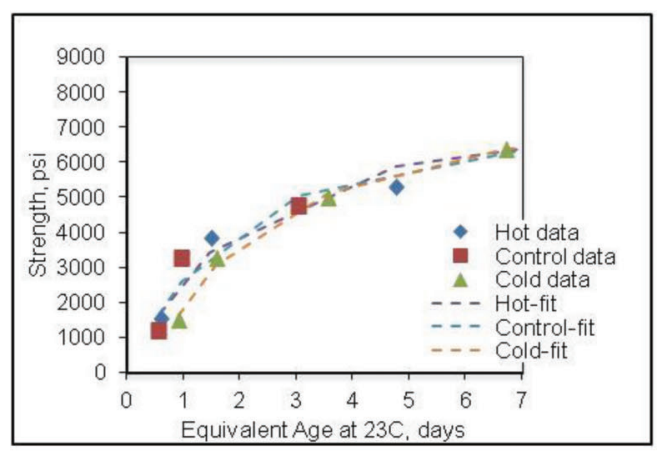

(a)

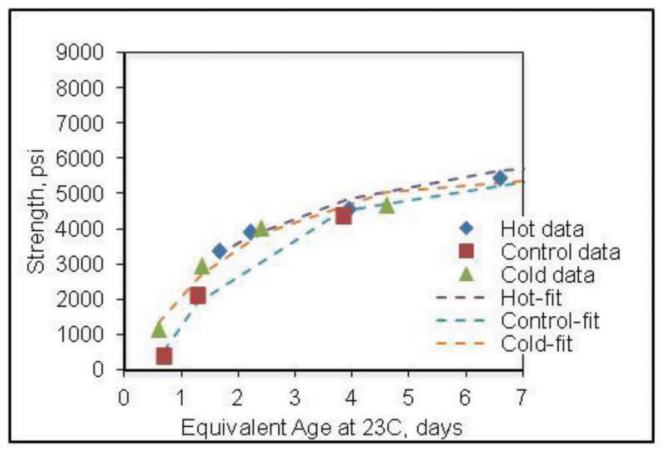

(c)

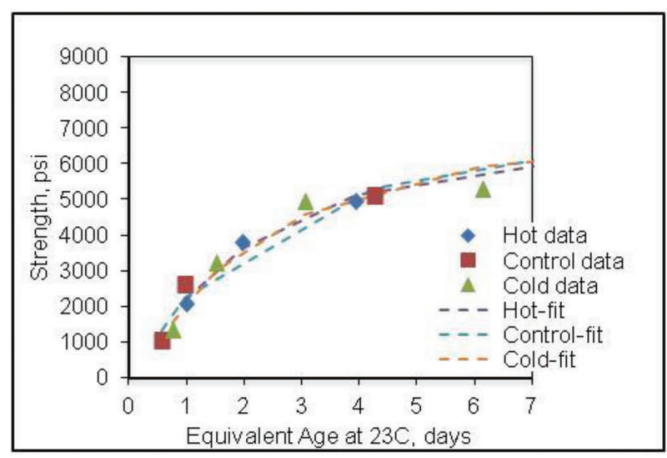

(e)

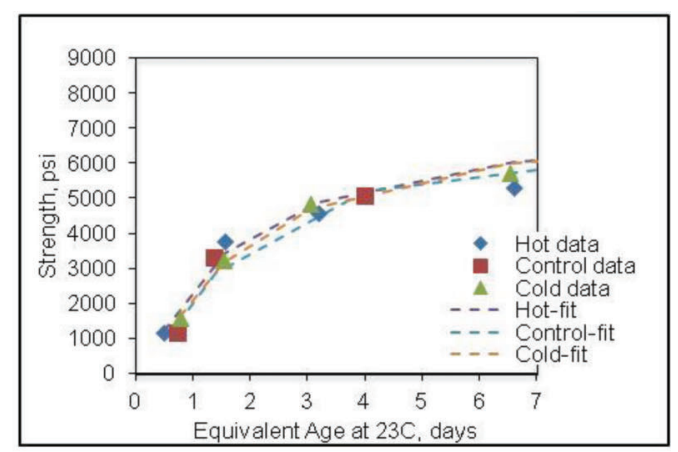

(b)

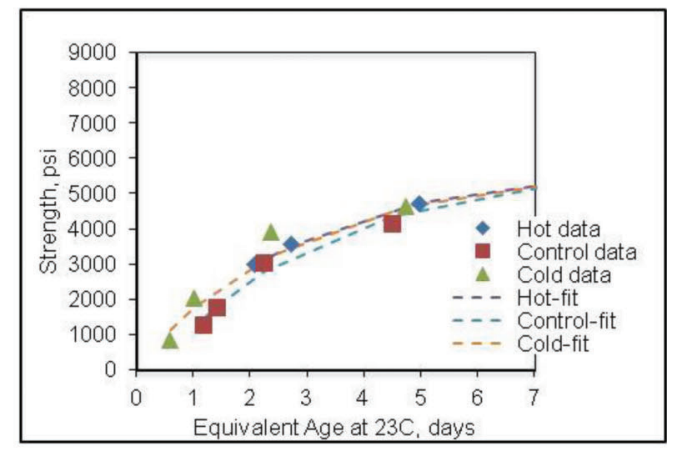

(d)

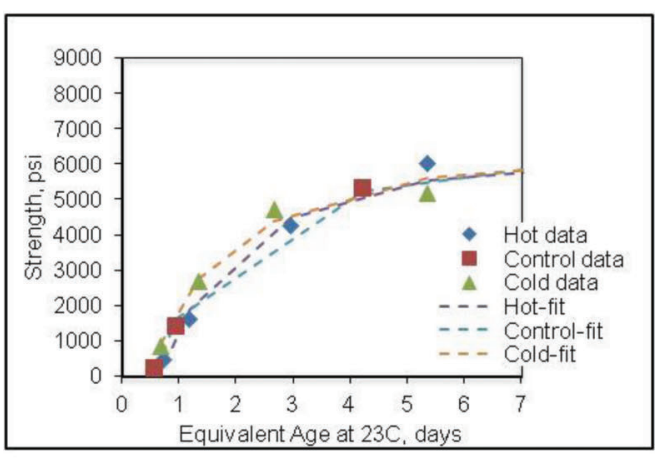

(f)

Figure 3.14 Strength-equivalent age plot for high alkali cement mixtures based on modified ASTM C1074: (a) 0.39FAA35, (b) 0.39FAB35, (c) 0.39FAC35, (d) 0.39FAA50, (e) 0.39FAB50, and (f) 0.39FAC50. 
TABLE 3.14

Strength vs. Age Curve Fit Parameters for Low Alkali Cement Mixtures Based on ASTM C1074

\begin{tabular}{|c|c|c|c|c|c|c|}
\hline Mixture ID & Curing Condition & $\mathbf{t}_{0}(\mathbf{h r})$ & $\mathrm{S}_{\mathbf{u}}(\mathrm{psi})$ & $\mathbf{k}\left(\mathbf{h r}^{-1}\right)$ & $\mathbf{R}^{2}$-Hyperbolic ${ }^{*}$ & $\mathbf{R}^{2}-\log \mathrm{Age}^{+}$ \\
\hline \multirow[t]{3}{*}{$0.43 \mathrm{PC}$} & Hot-97F & 3.4 & 7,361 & 2.533 & 1.00 & 0.79 \\
\hline & Control-76F & 6.2 & 8,953 & 1.097 & 1.00 & 0.89 \\
\hline & Cold-40F & 9.5 & 9,390 & 0.290 & 1.00 & 0.96 \\
\hline \multirow[t]{3}{*}{0.39 FAA35 } & Hot-98F & 0.0 & 10,781 & 0.636 & 0.97 & 0.98 \\
\hline & Control-76F & 0.0 & 11,797 & 0.282 & 0.96 & 0.99 \\
\hline & Cold-41F & 0.0 & 8,775 & 0.107 & 0.98 & 0.98 \\
\hline \multirow[t]{3}{*}{0.39 FAB35 } & Hot-97F & 0.1 & 9,391 & 0.569 & 0.97 & 0.98 \\
\hline & Control-75F & 0.0 & 10,440 & 0.242 & 0.97 & 0.99 \\
\hline & Cold-41F & 0.0 & 7,872 & 0.107 & 0.94 & 0.98 \\
\hline \multirow[t]{3}{*}{$0.39 \mathrm{FAC} 35$} & Hot-97F & 3.5 & 9,667 & 0.659 & 0.98 & 0.98 \\
\hline & Control-75F & 1.9 & 9,172 & 0.327 & 0.99 & 0.96 \\
\hline & Cold-41F & 0.0 & 8,750 & 0.117 & 0.97 & 0.96 \\
\hline \multirow[t]{3}{*}{$0.34 \mathrm{FAA} 50$} & Hot-98F & 0.0 & 12,115 & 0.310 & 0.97 & 0.99 \\
\hline & Control-76F & 0.0 & 12,258 & 0.194 & 0.95 & 1.00 \\
\hline & Cold-45F & 0.0 & 8,342 & 0.141 & 0.97 & 0.98 \\
\hline \multirow[t]{3}{*}{$0.34 \mathrm{FAB} 50$} & Hot-97F & 0.0 & 11,038 & 0.388 & 0.99 & 0.99 \\
\hline & Control-75F & 0.0 & 10,785 & 0.273 & 0.97 & 0.99 \\
\hline & Cold-45F & 11.0 & 8,121 & 0.220 & 0.98 & 0.95 \\
\hline \multirow[t]{3}{*}{$0.34 \mathrm{FAC} 50$} & Hot-98F & 4.6 & 10,826 & 0.592 & 1.00 & 0.97 \\
\hline & Control-75F & 0.0 & 10,759 & 0.254 & 0.98 & 0.98 \\
\hline & Cold-43F & 19.6 & 9,496 & 0.130 & 0.99 & 0.95 \\
\hline
\end{tabular}

*Coefficient of Determination for Hyperbolic strength age function.

${ }^{+}$Coefficient of Determination for Strength log-age function.

TABLE 3.15

Activation Energies Determined for Low Alkali Cement Mixtures Based on ASTM C1074

\begin{tabular}{|c|c|c|c|c|c|c|c|}
\hline \multirow[b]{2}{*}{ Mixture ID } & \multirow[b]{2}{*}{$\mathbf{R}^{2}$} & \multirow[b]{2}{*}{ Activation Energy (J/mol) } & \multicolumn{5}{|c|}{ Age Conversion Factor } \\
\hline & & & $41^{\circ} \mathrm{F}$ & $59^{\circ} \mathbf{F}$ & $73^{\circ} \mathrm{F}$ & $95^{\circ} \mathrm{C}$ & $113^{\circ} \mathrm{C}$ \\
\hline $0.43 \mathrm{PC}$ & 1.00 & 48,837 & 0.28 & 0.58 & 1.00 & 2.17 & 3.95 \\
\hline 0.39FAA35 & 0.99 & 40,273 & 0.35 & 0.63 & 1.00 & 1.89 & 3.10 \\
\hline $0.39 \mathrm{FAB} 35$ & 0.98 & 37,421 & 0.37 & 0.66 & 1.00 & 1.81 & 2.86 \\
\hline $0.39 \mathrm{FAC} 35$ & 1.00 & 39,760 & 0.35 & 0.64 & 1.00 & 1.88 & 3.06 \\
\hline $0.34 \mathrm{FAA} 50$ & 0.95 & 18,847 & 0.61 & 0.81 & 1.00 & 1.35 & 1.70 \\
\hline $0.34 \mathrm{FAB} 50$ & 0.94 & 13,685 & 0.70 & 0.86 & 1.00 & 1.24 & 1.47 \\
\hline $0.34 \mathrm{FAC} 50$ & 0.96 & 34,835 & 0.40 & 0.67 & 1.00 & 1.74 & 2.66 \\
\hline
\end{tabular}


TABLE 3.16

Strength vs. Age Curve Fit Parameters for Low Alkali Cement Mixtures Based on Modified ASTM C1074 Approach

\begin{tabular}{|c|c|c|c|c|c|c|}
\hline Mixture ID & Curing Condition & $\mathbf{t}_{0}(\mathbf{h r})$ & $\mathbf{S}_{\mathbf{u}}(\mathbf{p s i})$ & $k\left(h r^{-1}\right)$ & $\mathbf{R}^{2}$-Hyperbolic ${ }^{*}$ & $\mathbf{R}^{2}-\log \mathrm{Age}^{+}$ \\
\hline \multirow{3}{*}{$0.43 \mathrm{PC}$} & Hot-97F & 3.2 & 8,500 & 1.802 & 1.00 & 1.00 \\
\hline & Control-76F & 6.4 & 8,500 & 1.263 & 1.00 & 1.00 \\
\hline & Cold-40F & 12.2 & 8,500 & 0.399 & 1.00 & 0.99 \\
\hline \multirow[t]{3}{*}{0.39 FAA 35} & Hot-98F & 2.8 & 9,000 & 1.241 & 0.98 & 0.99 \\
\hline & Control-76F & 3.7 & 9,000 & 0.681 & 0.98 & 0.99 \\
\hline & Cold-41F & 4.3 & 9,000 & 0.124 & 0.99 & 1.00 \\
\hline \multirow[t]{3}{*}{0.39 FAB35 } & Hot-97F & 2.7 & 8,100 & 0.980 & 0.98 & 0.99 \\
\hline & Control-75F & 6.2 & 8,100 & 0.586 & 0.98 & 0.99 \\
\hline & Cold-41F & 0.0 & 8,100 & 0.105 & 0.96 & 0.99 \\
\hline \multirow[t]{3}{*}{$0.39 \mathrm{FAC} 35$} & Hot-97F & 4.4 & 8,000 & 1.073 & 1.00 & 1.00 \\
\hline & Control-75F & 5.6 & 8,000 & 0.504 & 0.99 & 0.99 \\
\hline & Cold-41F & 15.1 & 8,000 & 0.167 & 1.00 & 0.99 \\
\hline \multirow[t]{3}{*}{0.34 FAA 50} & Hot-98F & 0.0 & 8,900 & 0.628 & 0.98 & 0.97 \\
\hline & Control-76F & 0.0 & 8,900 & 0.430 & 0.99 & 0.98 \\
\hline & Cold-45F & 0.0 & 8,900 & 0.123 & 0.97 & 0.99 \\
\hline \multirow[t]{3}{*}{$0.34 \mathrm{FAB} 50$} & Hot-97F & 3.3 & 8,500 & 0.835 & 0.99 & 0.99 \\
\hline & Control-75F & 3.7 & 8,500 & 0.575 & 1.00 & 1.00 \\
\hline & Cold-45F & 10.9 & 8,500 & 0.205 & 0.99 & 0.99 \\
\hline \multirow[t]{3}{*}{$0.34 \mathrm{FAC} 50$} & Hot-98F & 5.4 & 8,500 & 1.000 & 1.00 & 0.98 \\
\hline & Control-75F & 6.6 & 8,500 & 0.563 & 1.00 & 1.00 \\
\hline & Cold-43F & 20.9 & 8,500 & 0.161 & 1.00 & 0.95 \\
\hline
\end{tabular}

*Coefficient of Determination for Hyperbolic strength age function.

${ }^{+}$Coefficient of Determination for Strength log-age function.

TABLE 3.17

Activation Energies for Low Alkali Cement Mixtures Based on Modified ASTM C1074 Approach

\begin{tabular}{|c|c|c|c|c|c|c|c|}
\hline \multirow[b]{2}{*}{ Mixture ID } & \multirow[b]{2}{*}{$\mathbf{R}^{2}$} & \multirow[b]{2}{*}{ Activation Energy (J/mol) } & \multicolumn{5}{|c|}{ Age Conversion Factor } \\
\hline & & & $41^{\circ} \mathrm{F}$ & $59^{\circ} \mathbf{F}$ & $73^{\circ} \mathbf{F}$ & $95^{\circ} \mathrm{C}$ & $113^{\circ} \mathrm{C}$ \\
\hline $0.43 \mathrm{PC}$ & 1.00 & 34,981 & 0.40 & 0.67 & 1.00 & 1.74 & 2.67 \\
\hline 0.39 FAA 35 & 0.99 & 53,717 & 0.24 & 0.55 & 1.00 & 2.34 & 4.53 \\
\hline 0.39 FAB 35 & 0.98 & 52,384 & 0.25 & 0.55 & 1.00 & 2.29 & 4.36 \\
\hline $0.39 \mathrm{FAC} 35$ & 1.00 & 42,890 & 0.32 & 0.62 & 1.00 & 1.97 & 3.34 \\
\hline $0.34 \mathrm{FAA} 50$ & 0.95 & 40,886 & 0.34 & 0.63 & 1.00 & 1.91 & 3.16 \\
\hline $0.34 \mathrm{FAB} 50$ & 0.94 & 35,328 & 0.39 & 0.67 & 1.00 & 1.75 & 2.70 \\
\hline $0.34 \mathrm{FAC} 50$ & 0.96 & 43,592 & 0.32 & 0.61 & 1.00 & 1.99 & 3.41 \\
\hline
\end{tabular}


TABLE 3.18

Strength vs. Age Curve Fit Parameters for High Alkali Cement Mixtures Based on ASTM C1074

\begin{tabular}{|c|c|c|c|c|c|c|}
\hline Mixture ID & Curing Condition & $\mathbf{t}_{0}(\mathrm{hr})$ & $\mathrm{S}_{\mathrm{u}}(\mathrm{psi})$ & $\mathbf{k}\left(\mathbf{h r}^{-1}\right)$ & $\mathbf{R}^{2}$-Hyperbolic ${ }^{*}$ & $\mathbf{R}^{2}-\log \mathrm{Age}^{+}$ \\
\hline \multirow[t]{3}{*}{$0.43 \mathrm{PC}$} & Hot-97F & 6.6 & 7,583 & 3.655 & 0.96 & 0.79 \\
\hline & Control-76F & 8.8 & 8,445 & 1.388 & 0.94 & 0.86 \\
\hline & Cold-43F & 20.2 & 9,964 & 0.336 & 0.97 & 0.89 \\
\hline \multirow[t]{3}{*}{0.39 FAA 35} & Hot-96F & 0.0 & 8,386 & 0.681 & 0.95 & 0.98 \\
\hline & Control-76F & 0.0 & 8,791 & 0.426 & 0.97 & 0.98 \\
\hline & Cold-43F & 18.4 & 8,125 & 0.259 & 0.98 & 0.85 \\
\hline \multirow[t]{3}{*}{$0.39 \mathrm{FAB} 35$} & Hot-97F & 0.0 & 8,620 & 0.537 & 0.96 & 0.97 \\
\hline & Control-75F & 0.0 & 8,973 & 0.376 & 0.95 & 0.98 \\
\hline & Cold-43F & 13.2 & 7,858 & 0.189 & 0.99 & 0.90 \\
\hline \multirow[t]{3}{*}{$0.39 \mathrm{FAC} 35$} & Hot-97F & 0.0 & 9,238 & 0.507 & 0.96 & 0.96 \\
\hline & Control-75F & 5.0 & 8,991 & 0.292 & 0.98 & 0.96 \\
\hline & Cold-44F & 24.8 & 6,443 & 0.200 & 0.99 & 0.96 \\
\hline \multirow[t]{3}{*}{$0.34 \mathrm{FAA} 50$} & Hot-98F & 0.0 & 11,053 & 0.329 & 0.97 & 0.98 \\
\hline & Control-76F & 0.0 & 9,177 & 0.238 & 0.96 & 0.98 \\
\hline & Cold-44F & 11.0 & 7,216 & 0.140 & 0.96 & 0.94 \\
\hline \multirow[t]{3}{*}{$0.34 \mathrm{FAB} 50$} & Hot-97F & 0.0 & 10,621 & 0.434 & 0.98 & 0.97 \\
\hline & Control-75F & 0.0 & 9,056 & 0.324 & 0.99 & 0.99 \\
\hline & Cold-44F & 16.4 & 7,353 & 0.228 & 0.93 & 0.91 \\
\hline \multirow[t]{3}{*}{$0.34 \mathrm{FAC} 50$} & Hot-98F & 5.5 & 10,167 & 0.677 & 1.00 & 0.93 \\
\hline & Control-75F & 11.3 & 8,131 & 0.427 & 0.98 & 0.94 \\
\hline & Cold-43F & 29.8 & 7,480 & 0.197 & 0.97 & 0.91 \\
\hline
\end{tabular}

*Coefficient of Determination for Hyperbolic strength age function.

${ }^{+}$Coefficient of Determination for Strength log-age function.

TABLE 3.19

Activation Energies Determined for High Alkali Cement Mixtures Based on ASTM C1074

\begin{tabular}{|c|c|c|c|c|c|c|c|}
\hline \multirow[b]{2}{*}{ Mixture ID } & \multirow[b]{2}{*}{$\mathbf{R}^{2}$} & \multirow[b]{2}{*}{ Activation Energy (J/mol) } & \multicolumn{5}{|c|}{ Age Conversion Factor } \\
\hline & & & $41^{\circ} \mathrm{F}$ & $59^{\circ} \mathbf{F}$ & $73^{\circ} \mathrm{F}$ & $95^{\circ} \mathrm{C}$ & $113^{\circ} \mathrm{C}$ \\
\hline $0.43 \mathrm{PC}$ & 1.00 & 56,945 & 0.22 & 0.53 & 1.00 & 2.46 & 4.96 \\
\hline 0.39FAA35 & 0.98 & 23,213 & 0.54 & 0.77 & 1.00 & 1.44 & 1.92 \\
\hline $0.39 \mathrm{FAB} 35$ & 1.00 & 25,167 & 0.52 & 0.75 & 1.00 & 1.49 & 2.03 \\
\hline $0.39 \mathrm{FAC} 35$ & 0.95 & 21,991 & 0.56 & 0.78 & 1.00 & 1.42 & 1.86 \\
\hline $0.34 \mathrm{FAA} 50$ & 1.00 & 20,457 & 0.58 & 0.79 & 1.00 & 1.38 & 1.78 \\
\hline $0.34 \mathrm{FAB} 50$ & 1.00 & 15,294 & 0.67 & 0.84 & 1.00 & 1.27 & 1.54 \\
\hline $0.34 \mathrm{FAC} 50$ & 1.00 & 29,127 & 0.46 & 0.72 & 1.00 & 1.59 & 2.27 \\
\hline
\end{tabular}


TABLE 3.20

Strength vs. Age Curve Fit Parameters for High Alkali Cement Mixtures Based on Modified ASTM C1074 Approach

\begin{tabular}{|c|c|c|c|c|c|c|}
\hline Mixture ID & Curing Condition & $\mathbf{t}_{\mathbf{0}}(\mathrm{hr})$ & $\mathbf{S}_{\mathbf{u}}(\mathbf{p s i})$ & $k\left(h r^{-1}\right)$ & $\mathbf{R}^{2}$-Hyperbolic ${ }^{*}$ & $\mathbf{R}^{2}-\log \operatorname{age}^{+}$ \\
\hline \multirow[t]{3}{*}{$0.43 \mathrm{PC}$} & Hot-97F & 6.1 & 8,000 & 2.746 & 0.96 & 0.84 \\
\hline & Control-76F & 10.4 & 8,000 & 2.034 & 0.98 & 0.88 \\
\hline & Cold-43F & 31.3 & 8,000 & 0.780 & 1.00 & 0.96 \\
\hline \multirow[t]{3}{*}{0.39 FAA 35} & Hot-96F & 0.1 & 8,400 & 0.800 & 0.91 & 0.96 \\
\hline & Control-76F & 1.8 & 8,400 & 0.510 & 0.97 & 0.96 \\
\hline & Cold-43F & 20.6 & 8,400 & 0.260 & 1.00 & 0.99 \\
\hline \multirow[t]{3}{*}{0.39 FAB35 } & Hot-97F & 0.1 & 7,700 & 0.900 & 0.92 & 0.96 \\
\hline & Control-75F & 6.2 & 7,700 & 0.752 & 0.97 & 0.95 \\
\hline & Cold-43F & 18.2 & 7,700 & 0.215 & 0.99 & 0.98 \\
\hline \multirow[t]{3}{*}{$0.39 \mathrm{FAC} 35$} & Hot-97F & 0.0 & 7,500 & 1.007 & 0.94 & 0.99 \\
\hline & Control-75F & 10.1 & 7,500 & 0.578 & 1.00 & 0.99 \\
\hline & Cold-44F & 10.9 & 7,500 & 0.124 & 0.96 & 0.98 \\
\hline \multirow[t]{3}{*}{$0.34 \mathrm{FAA} 50$} & Hot-98F & 0.0 & 8,200 & 0.595 & 0.99 & 1.00 \\
\hline & Control-76F & 8.6 & 8,200 & 0.308 & 0.97 & 0.98 \\
\hline & Cold-44F & 0.0 & 8,200 & 0.093 & 0.88 & 0.94 \\
\hline \multirow[t]{3}{*}{$0.34 \mathrm{FAB} 50$} & Hot-97F & 2.2 & 8,200 & 0.871 & 0.99 & 0.99 \\
\hline & Control-75F & 3.0 & 8,200 & 0.452 & 0.98 & 0.99 \\
\hline & Cold-44F & 13.2 & 8,200 & 0.180 & 0.94 & 0.94 \\
\hline \multirow[t]{3}{*}{$0.34 \mathrm{FAC} 50$} & Hot-98F & 6.9 & 7,200 & 1.685 & 0.98 & 1.00 \\
\hline & Control-75F & 13.0 & 7,200 & 0.714 & 1.00 & 0.99 \\
\hline & Cold-43F & 33.9 & 7,200 & 0.238 & 0.98 & 0.95 \\
\hline
\end{tabular}

*Coefficient of Determination for Hyperbolic strength age function.

${ }^{+}$Coefficient of Determination for Strength log-age function.

TABLE 3.21

Activation Energies for High Alkali Cement Mixtures Based on Modified ASTM C1074 Approach

\begin{tabular}{|c|c|c|c|c|c|c|c|}
\hline \multirow[b]{2}{*}{ Mixture ID } & \multirow[b]{2}{*}{$\mathbf{R}^{2}$} & \multirow[b]{2}{*}{ Activation Energy (J/mol) } & \multicolumn{5}{|c|}{ Age Conversion Factor } \\
\hline & & & $41^{\circ} \mathrm{F}$ & $59^{\circ} \mathbf{F}$ & $73^{\circ} \mathrm{F}$ & $95^{\circ} \mathrm{C}$ & $113^{\circ} \mathrm{C}$ \\
\hline $0.43 \mathrm{PC}$ & 0.98 & 30,897 & 0.44 & 0.71 & 1.00 & 1.63 & 2.38 \\
\hline 0.39 FAA35 & 1.00 & 27,395 & 0.49 & 0.73 & 1.00 & 1.54 & 2.16 \\
\hline $0.39 \mathrm{FAB} 35$ & 0.93 & 35,635 & 0.39 & 0.67 & 1.00 & 1.76 & 2.72 \\
\hline $0.39 \mathrm{FAC} 35$ & 0.98 & 51,982 & 0.25 & 0.56 & 1.00 & 2.28 & 4.31 \\
\hline $0.34 \mathrm{FAA} 50$ & 1.00 & 44,695 & 0.31 & 0.60 & 1.00 & 2.03 & 3.51 \\
\hline $0.34 \mathrm{FAB} 50$ & 1.00 & 37,781 & 0.37 & 0.65 & 1.00 & 1.82 & 2.89 \\
\hline $0.34 \mathrm{FAC} 50$ & 1.00 & 45,843 & 0.30 & 0.60 & 1.00 & 2.07 & 3.63 \\
\hline
\end{tabular}


TABLE 3.22

Yield Adjusted Concrete Mixture Proportions and Test Results

\begin{tabular}{|c|c|c|c|c|}
\hline Calculated Batch Quantities & Mix1 & Mix2 & Mix3 & Mix4 \\
\hline Low Alkali cement (Cement B), lb/yd $\mathrm{yd}^{3}$ & 385 & 367 & & \\
\hline High Alkali cement (Cement C), $1 \mathrm{~b} / \mathrm{yd}^{3}$ & & & 389 & 371 \\
\hline Fly ash (FAA), lb/yd ${ }^{3}$ & 261 & & 264 & \\
\hline Fly ash (FAC), lb/yd ${ }^{3}$ & & 307 & & 310 \\
\hline Fly ash, $\%$ by Mass & 40.4 & 45.5 & 40.4 & 45.5 \\
\hline Fly ash, $\%$ by Volume & 50 & 50 & 50 & 50 \\
\hline Coarse Agg. (No.57), lb/yd ${ }^{3}$ & 2065 & 2056 & 2086 & 2077 \\
\hline Fine Aggregate, $1 \mathrm{~b} / \mathrm{yd}^{3}$ & 1248 & 1242 & 1261 & 1255 \\
\hline Mixing Water, $1 \mathrm{~b} / \mathrm{yd}^{3}$ & 220 & 229 & 222 & 231 \\
\hline$w / \mathrm{cm}$ & 0.34 & 0.34 & 0.34 & 0.34 \\
\hline ASTM C494 Type F, oz/cwt & 7.0 & 1.7 & 9.9 & 4.7 \\
\hline ASTM C494 Type C, oz/cwt & 30.0 & 30.0 & 30.0 & 30.0 \\
\hline \multicolumn{5}{|l|}{ Fresh Concrete Properties } \\
\hline ASTM C143, Slump, in. & 8.00 & 6.00 & 7.00 & 3.75 \\
\hline ASTM C231, Air, \% & 1.6 & 2.5 & 1.9 & 2.2 \\
\hline ASTM C138, Density, $1 \mathrm{~b} / \mathrm{ft}^{3}$ & 155.3 & 156.1 & 156.9 & 157.7 \\
\hline ASTM C1064, Temperature, ${ }^{\circ} \mathrm{F}$ & 72 & 72 & 75 & 75 \\
\hline \multicolumn{5}{|l|}{ Hardened Concrete Properties } \\
\hline \multicolumn{5}{|l|}{ ASTM C39, Compressive Strength, psi } \\
\hline 1 day & 2,736 & 2559 & 3542 & 161 \\
\hline 3 days & 4,136 & 5278 & 5323 & 5329 \\
\hline 7 days & 5,034 & 6758 & 6620 & 7309 \\
\hline 14 days & 5,790 & 7784 & 7773 & 8762 \\
\hline 28 days & 6,760 & 9027 & 9061 & 9897 \\
\hline
\end{tabular}

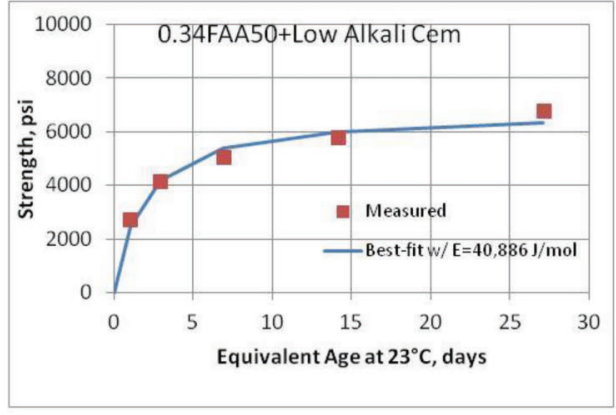

(a)

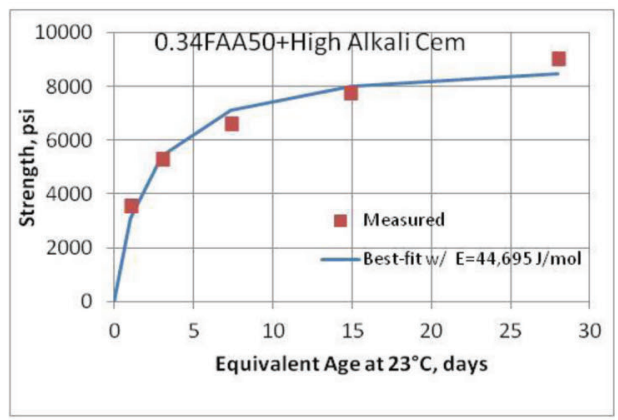

(c)

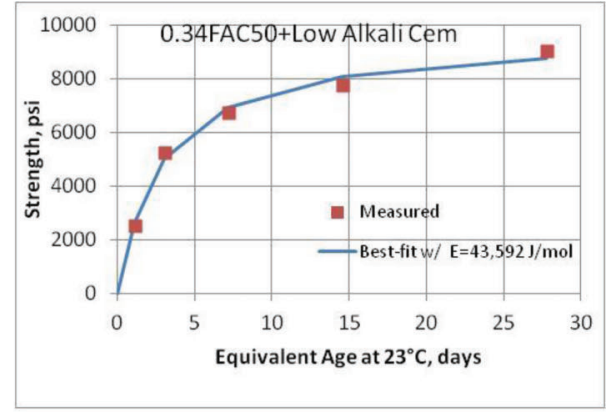

(b)

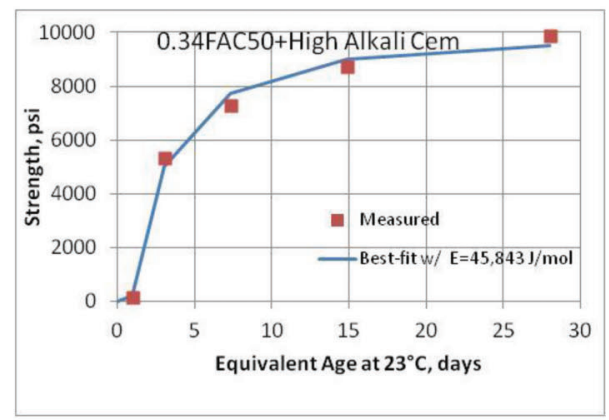

(d)

Figure 3.15 Compressive strength vs. equivalent age plots for concrete mixtures: (a) 0.34FAA50LAC, (b) 0.34FAC50LAC, (c) $0.34 \mathrm{FAA} 50 \mathrm{HAC}$, and (d) 0.34FAC50HAC. 


\section{ACKNOWLEDGMENTS}

The authors would like to extend their sincere thanks to Mr. Soliman Ben-Barka and Mr. Stuart Sherman who conducted the test program at the NRMCA Research Laboratory and Mr. Colin L. Lobo, Ph.D., P.E. for his review of this report. The authors would like to thank W.R. Grace, Boral Materials Technologies Inc., and Separation Technologies LLC for the materials provided. Funding for the study was provided by the Federal Highway Administration through a research consortium led by Purdue University.

\section{REFERENCES}

ASTM. (1993a). Annual Book of ASTM Standards, Vol. 4.01: Cement, Lime, and Gypsum. West Conshohocken, PA: ASTM International.

ASTM. (1993b). Annual Book of ASTM Standards, Vol. 4.02: Concrete and Aggregates. West Conshohocken, PA: ASTM International

Obla, K. H., Upadhyaya, S., Goulias, D., Schindler, A. K., \& Carino, N. J. (2008). New technology-based approach to advance higher volume fly ash concrete with acceptable performance. Silver Spring, MD: NRMCA Engineering Division. Retrieved from https://www.nrmca.org/research/ HVFAC_Final_Report_final.pdf 


\section{APPENDIX B. REPETITION OF SOME MORTAR MIXTURES}

A close look at Table B.1 indicates that the following mortar mixtures had Type F admixture dosage that was greater than 12 oz/cwt. (Typical manufacturer recommended dosage is $2-7 \mathrm{oz} / \mathrm{cwt}$.).

The following observations can be made:

1. Out of 42 mixtures 7 mixtures had Type $F$ admixture dosages greater than $12 \mathrm{oz} / \mathrm{cwt}$.

2. All of the 7 mixtures had fly ash FAA.

3. 5 out of these 7 mixtures had high alkali cement.

4. For similar conditions the 50\% FAA mix had much higher Type $\mathrm{F}$ dosage as compared to the 35\% FAA mix which is understandable due to the much lower water contents.

5. Higher mixing temperatures lead to slightly higher admixture dosages.

The manufacturer communicated that higher admixture dosages can be used if needed without any degradation in strength results. Nevertheless, it was decided to repeat all of above mixtures except Mixture 23 and 30. Mixture 23, 30 did not excessively high dosages and at the same time it was decided that at least two mixtures for each temperature should be repeated
(6 mixtures in all). The repetitions were done with the following objectives:

1. To confirm again whether the originally recorded admixture dosages were correct.

2. If the recorded admixture dosages were indeed correct to confirm whether the high admixture dosages led to poorer strength performance.

The repeat mixtures were conducted with the following criteria:

- Use a total of 12 oz/cwt type F admixture.

- Try to hit the lowest flow (95) that is workable to make cubes well.

- If flow is below 95 then, add slightly more water to hit the minimum flow (95).

- Test two cubes each age at 1, 2, 4, 8, 16, 28 days (equivalent ages at $73 \mathrm{~F}$ ) - as verification test the cubes will be tested during the business hours.

In Table B.2 the repeated mixtures' flow values, $w / \mathrm{cm}$, admixture dosages, and mix temperatures are provided along with those values for the original mixtures.

The strength development for each mixture and its repeat is plotted in Figure B.1. The curing temperatures for some of the mixtures and their repeats are plotted in Figure B.2.

TABLE B.1

Mortar Mixtures to Be Repeated

\begin{tabular}{lcc}
\hline \hline Mixture ID, Curing Condition & Admixture Dosage, oz/cwt & Cement Type \\
\hline Mix5 (0.34FAA50), 73F & 18 & Low alkali \\
Mix12 (0.34FAA50), 100F & 20 & Low alkali \\
Mix23 (0.39FAA35), 73F & 15 & High alkali \\
Mix26 (0.34FAA50), 73F & 32 & High alkali \\
Mix30 (0.39FAA35), 100F & 16 & High alkali \\
Mix33 (0.34FAA50), 100F & 45 & High alkali \\
Mix40 (0.34FAA50), 45F & 29 & High alkali \\
\hline
\end{tabular}

TABLE B.2

Fresh Mortar Properties of Repeated Mortar Mixtures

\begin{tabular}{|c|c|c|c|c|c|c|c|}
\hline Mix ID & Cement Type & Curing Condition & Mix \# & Flow $\%$ & $w / c m$ Ratio & F-admix. oz/cwt & Temp ${ }^{\circ} \mathbf{F}$ \\
\hline \multirow[t]{2}{*}{ FAA50 } & $\mathrm{LAC}$ & Control-75F & Mix 5 & 112 & 0.34 & 18 & 75 \\
\hline & & & $\operatorname{Mix} 5 \mathrm{R}$ & 111 & 0.36 & 12 & 73 \\
\hline \multirow[t]{2}{*}{ FAA50 } & HAC & Control-75F & Mix26 & 106 & 0.34 & 31.5 & 78 \\
\hline & & & Mix26R & 96 & 0.35 & 12 & 76 \\
\hline \multirow[t]{2}{*}{ FAA 50} & LAC & Hot-100F & Mix12 & 106 & $0.34\left(0.35^{+}\right)$ & 19.8 & Not measured \\
\hline & & & Mix 12R & 117 & 0.38 & 12 & 81 \\
\hline \multirow[t]{2}{*}{ FAA50 } & HAC & Hot-100F & Mix33 & 105 & $0.34\left(0.36^{+}\right)$ & 45.3 & 87 \\
\hline & & & Mix33R & 91 & 0.40 & 12 & 88 \\
\hline \multirow[t]{2}{*}{ FAA50 } & HAC & Cold-45F & Mix40 & 108 & $0.34\left(0.35^{+}\right)$ & 28.8 & 72 \\
\hline & & & Mix40R & 92 & 0.37 & 12 & 69 \\
\hline
\end{tabular}

LAC $=$ Low alkali cement

$\mathrm{HAC}=$ High alkali cement

${ }^{+} w / \mathrm{cm}$ ratio including water portion of the type $\mathrm{F}$ admixture. 


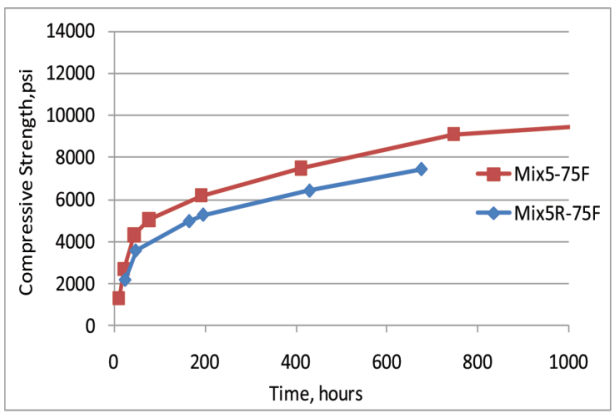

(a)

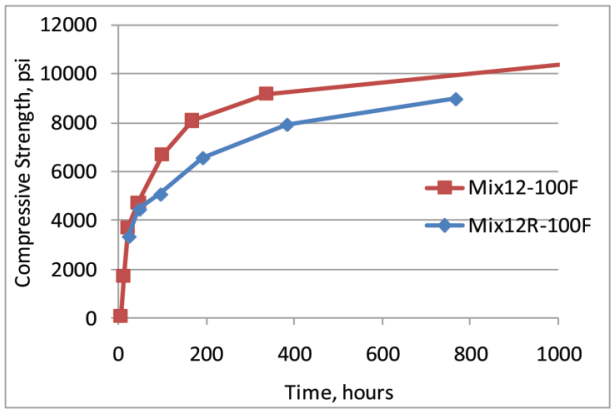

(c)

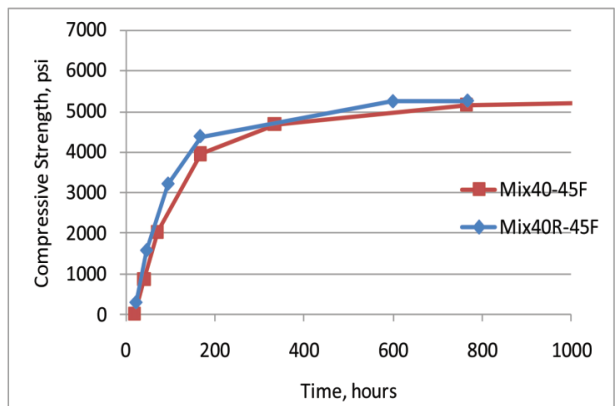

(b)

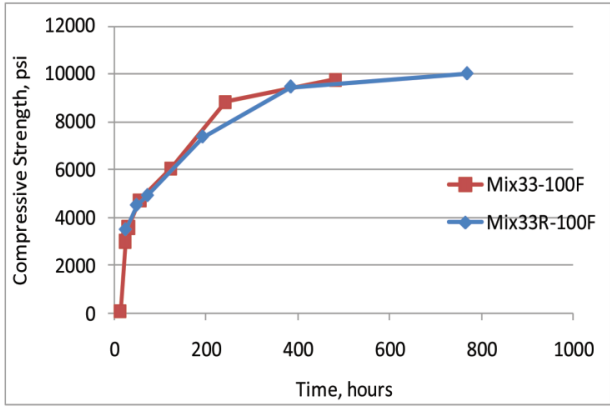

(d)

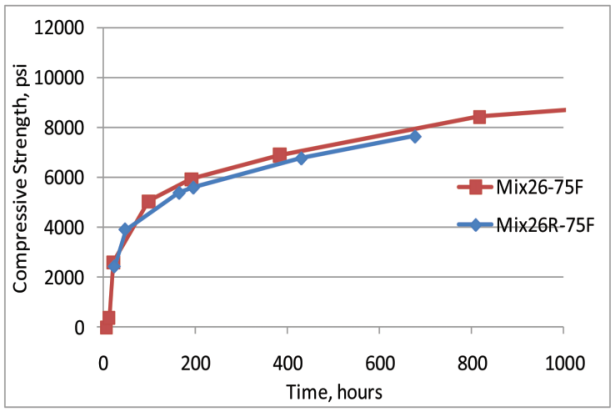

(e)

Figure B.1 Compressive strength test result of repeated mortar mixture.

From Table B.1, Figure B.1, and Figure B.2 the following observations can be made:

- When compared to Mixture 5 Mixture 5R had a slightly higher $w / \mathrm{cm}(0.36$ vs. 0.34$)$, a lower admixture dosage (12 vs. $18 \mathrm{oz} / \mathrm{cwt}$.) and had similar flow. It is clear that for Mixture 5 the originally recorded admixture dosage was correct. As illustrated in Figure B.1 (a) Mixture 5R had lower strength than Mixture 5 probably due the higher $w /$ $\mathrm{cm}$. Therefore there is no indication of strength degradation due to higher admixture dosage of Mixture 5.

- When compared to Mixture 26 Mixture 26R had a slightly higher $w / \mathrm{cm}(0.35$ vs. 0.34$)$, a significantly lower admixture dosage (12 vs. 31 oz/cwt.) and had lower flow ( 96 vs. 106). It is clear that for Mixture 26 the originally recorded admixture dosage even though significantly higher than manufacturer recommendation was correct. It is possible that with increasing admixture dosages the workability enhancement is less efficient. As illustrated in Figure B.1 (b) Mix26R had slightly lower strength than Mix26 mainly as a result of the slightly higher $w / \mathrm{cm}$. Therefore there is no indication of strength degradation due to higher admixture dosage of Mixture 26.

- When compared to Mixture 12 Mixture 12R had a higher $w / \mathrm{cm}$ (0.38 vs. 0.35 ), a lower admixture dosage (12 vs. 19.8 oz/cwt.) and had higher flow (117 vs. 106). It is clear that for Mixture 12 the originally recorded admixture dosage was correct. As illustrated in Figure B.1 (c) Mix12R had lower strength than Mix12 mainly as a result of the slightly higher $w / \mathrm{cm}$ ratio. Therefore there is no indication of strength degradation due to higher admixture dosage of Mixture 12. Figure B.2 shows that the repeat mixtures were cured at $2-3 \mathrm{~F}$ lower temperature than the original mixture but apparently that did not have a significant effect on the strength development.

- When compared to Mixture 33 Mixture 33R had a higher $w / \mathrm{cm}$ (0.40 vs. 0.36), a significantly lower admixture dosage (12 vs. 45.3 oz/cwt.) and had lower flow (91 vs. 105). It is clear that for Mixture 33 the originally recorded admixture dosage even though significantly higher than manufacturer recommendation was correct. As illustrated in Figure B.1 (d) Mix33R had the same strength as Mix 33. The higher $w / \mathrm{cm}$ of Mix 33R would 
have suggested a decrease in strength of 1000 to 1500 psi. Since this was not observed it is likely that Mixture 33 had a strength degradation of 1000 to $1500 \mathrm{psi}$ as a result of the very high admixture dosage. Figure B.2 shows that the repeat mixtures were cured at $2-3 \mathrm{~F}$ lower temperature than the original mixture. That small temperature differential is unlikely to help explain the strength anomaly.

- When compared to Mixture 40 Mixture 40R had a higher $w / \mathrm{cm}$ (0.37 vs. 0.35), a significantly lower admixture dosage (12 vs. 28.8 oz/cwt.) and had lower flow (92 vs. 108). It is clear that for Mixture 40 the originally recorded admixture dosage even though significantly higher than manufacturer recommendation was correct. As illustrated in Figure B.1 (e) Mix40R had the same strength as Mix 40. The higher $w / \mathrm{cm}$ of Mix 40R would have suggested a decrease in strength of about 800 psi.

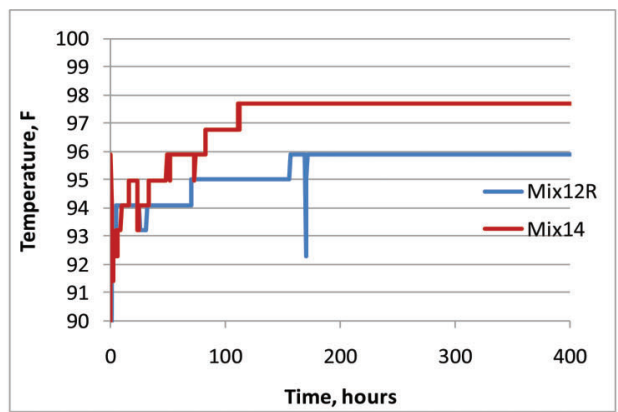

Note. Mixture 12 I-button failed to function. Mixture 14 I-button cube was in the same curing environment as Mixture 12.

(a)

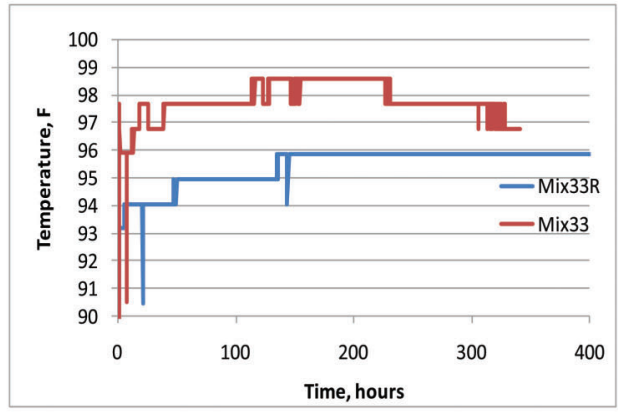

(b)

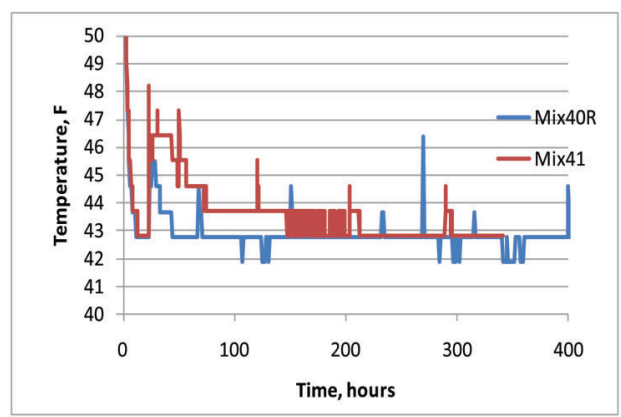

Note. Mixture 40 I-button failed to function. Mixture 41 I-button cube was in the same curing environment as Mixture 40.

(c)

Figure B.2 Curing temperature history comparisons of repeat mortar and original mortar mixtures.
Since this was not observed it is likely that Mixture 40 had a strength degradation of about 800 psi as a result of the very high admixture dosage. Figure B.2 shows that the repeat mixtures were cured at almost the same temperature as the original mixture and hence cannot explain the observed strength anomaly.

For mixtures 33 and 40 it was decided to use the originally measured strength in activation energy calculations because even though there was some strength degradation due to the higher admixture dosage it was not possible to cast mixtures at the lower $w / \mathrm{cm}$ with low HRWR dosage. The repeat mixtures had to be cast at a higher $w / \mathrm{cm}$ in spite of the HRWR dosage being at $12 \mathrm{oz} / \mathrm{cwt}$. (manufacturer recommended value of 2-7 oz/cwt). Increasing the HRWR dosage any further may result in lower $w / \mathrm{cm}$ but the potential strength increase due to the lower $w / \mathrm{cm}$ is likely to be balanced by strength degradation due to the higher HRWR dosage. 


\section{APPENDIX C. COMPRESSIVE STRENGTH TEST RESULTS OF MORTAR MIXTURES}

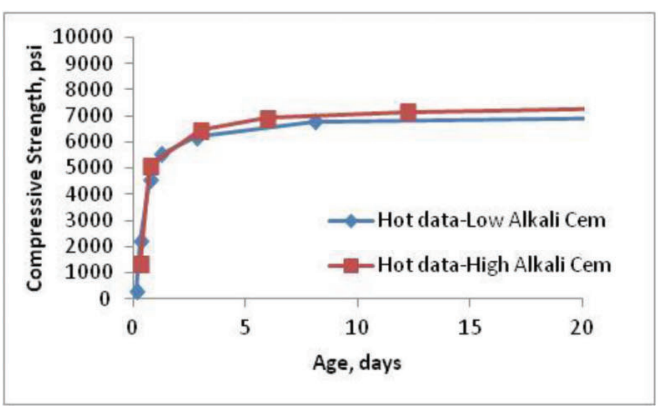

(a)

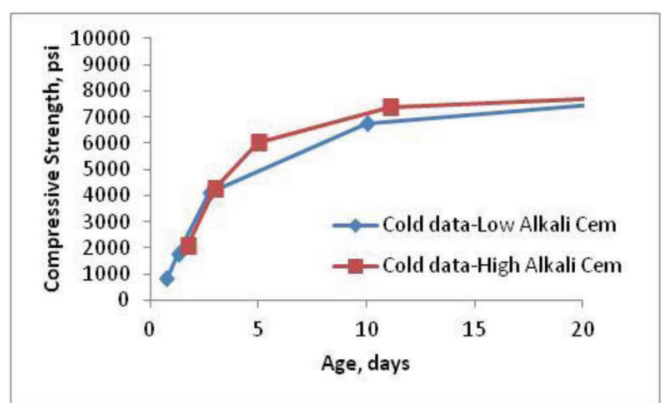

(c)

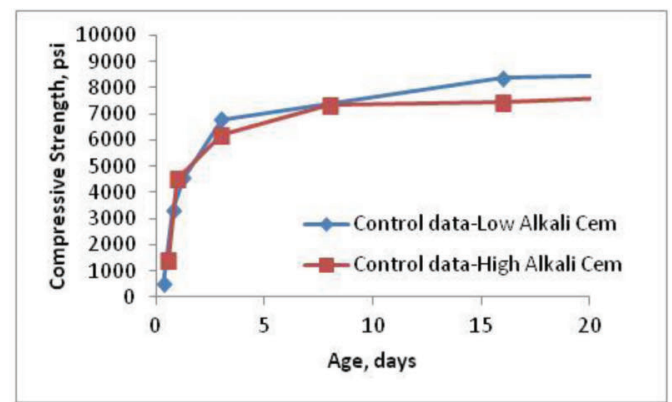

(b)

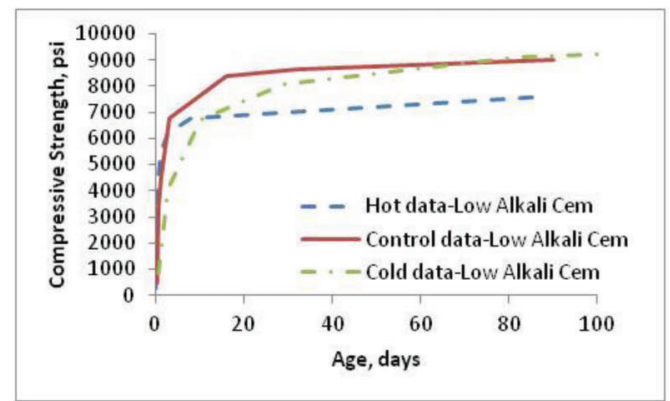

(d)

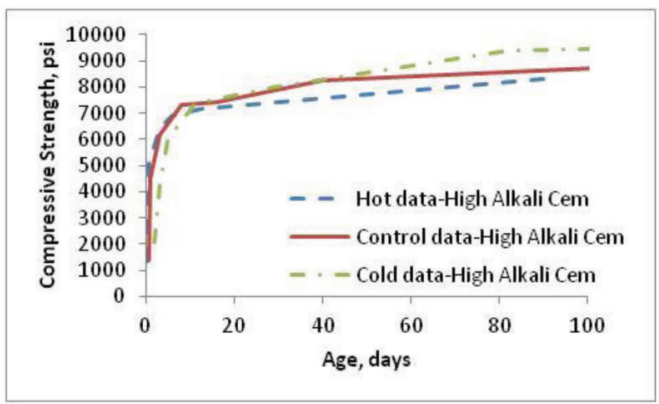

(e)

Figure C.1 Strength-age plot with low and high alkali cement at (a) Hot, (b) Control, and (c) Cold temperature; (d) strength-age plot with low alkali cement; (e) strength-age plot with high alkali cement for 0.43PC mixture. 


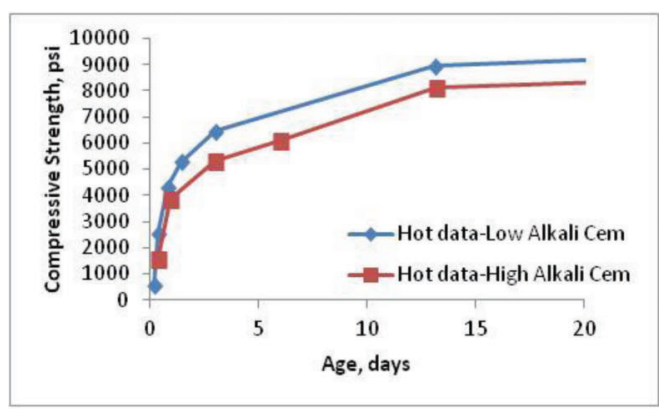

(a)

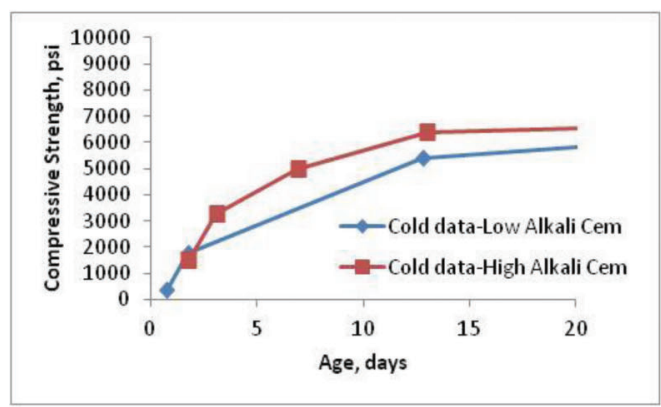

(c)

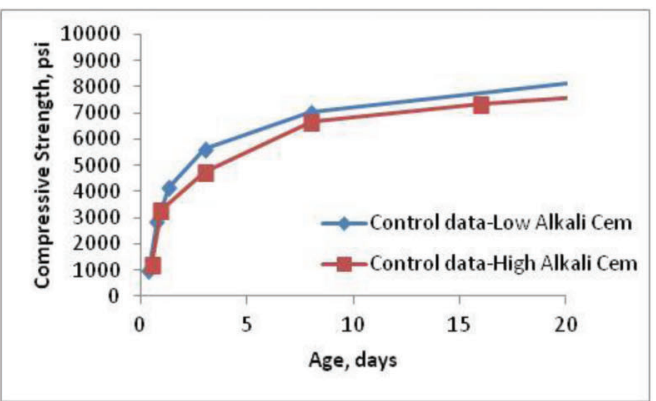

(b)

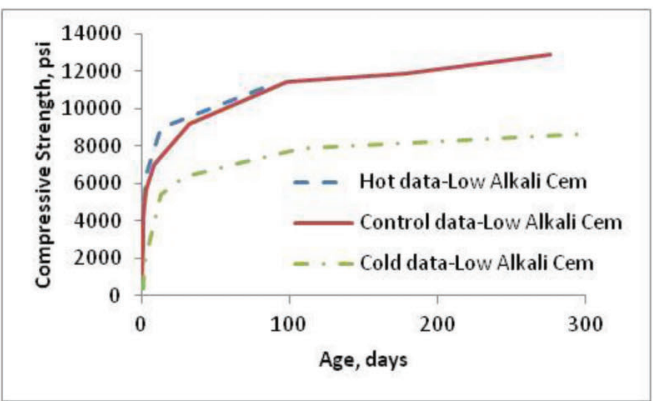

(d)

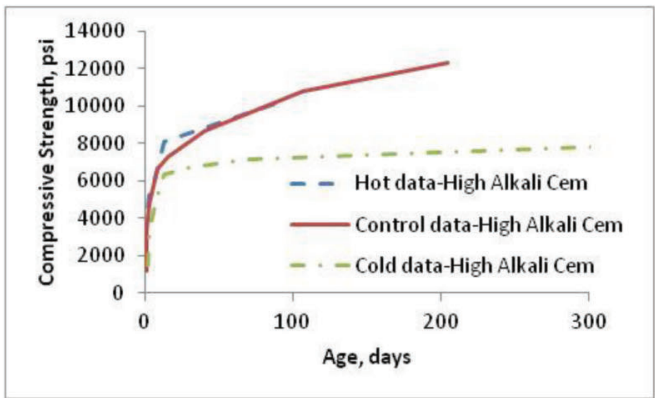

(e)

Figure C.2 Strength-age plot with low and high alkali cement at (a) Hot, (b) Control, and (c) Cold temperature; (d) strength-age plot with low alkali cement; (e) strength-age plot with high alkali cement for 0.39FAA35 mixture. 


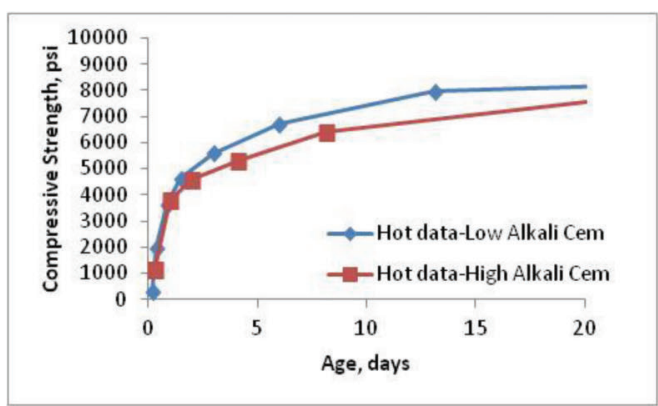

(a)

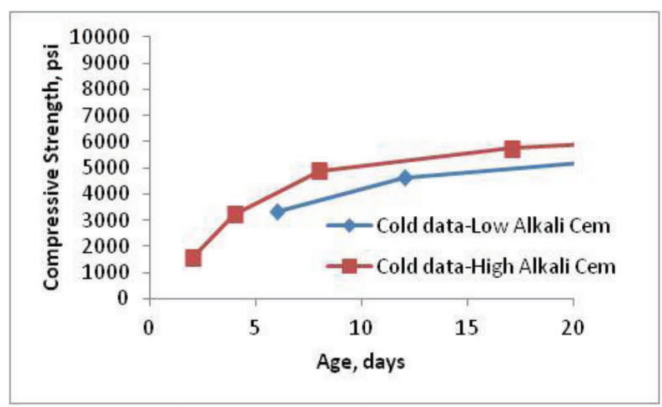

(c)

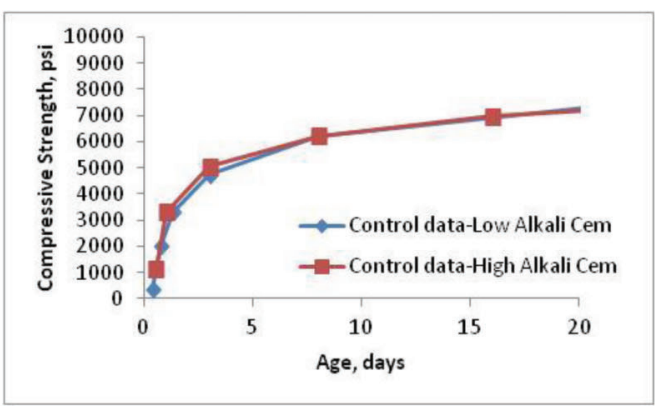

(b)

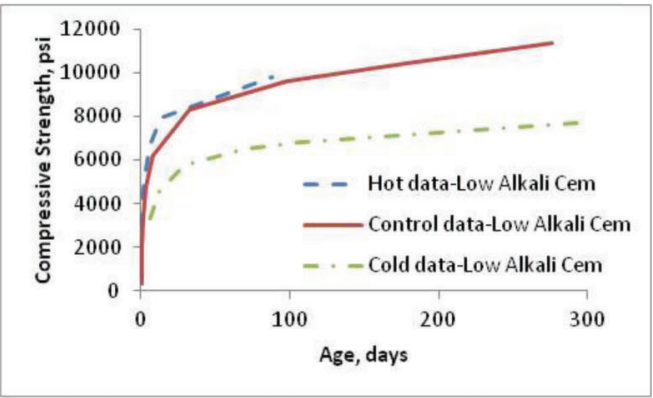

(d)

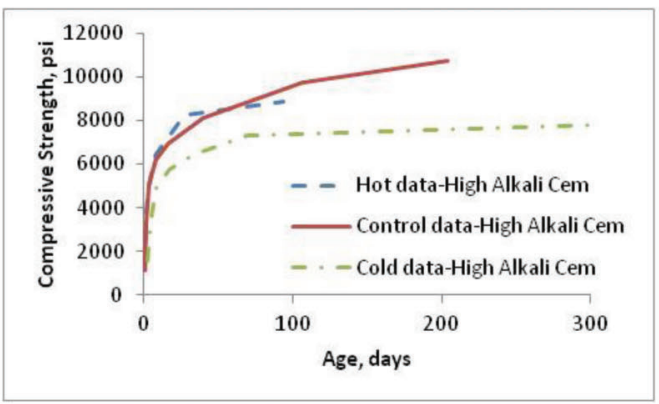

(e)

Figure C.3 Strength-age plot with low and high alkali cement at (a) Hot, (b) Control, and (c) Cold temperature; (d) strength-age plot with low alkali cement; (e) strength-age plot with high alkali cement for 0.39FAB35 mixture. 


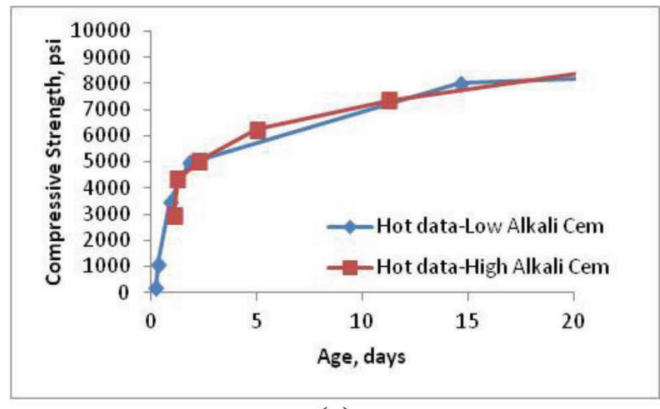

(a)

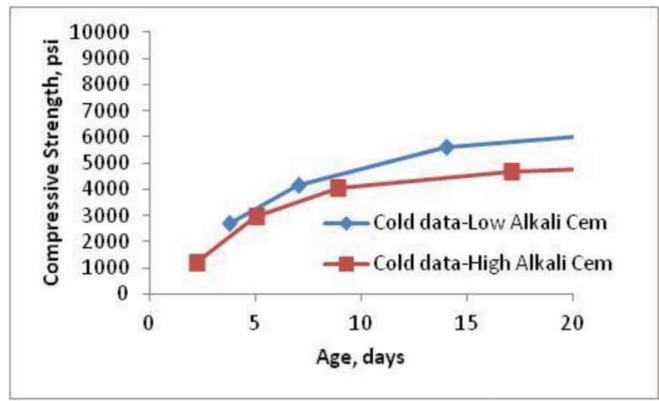

(c)

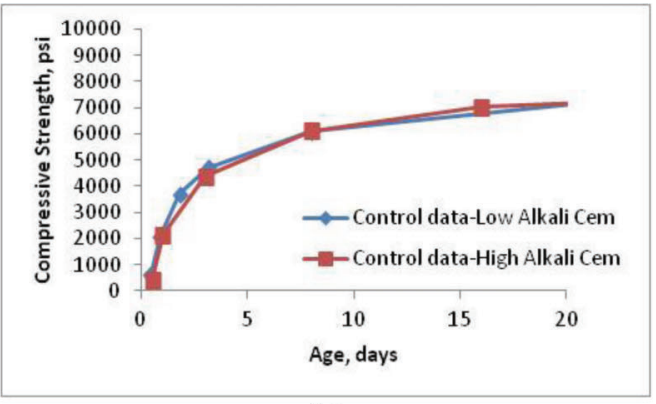

(b)

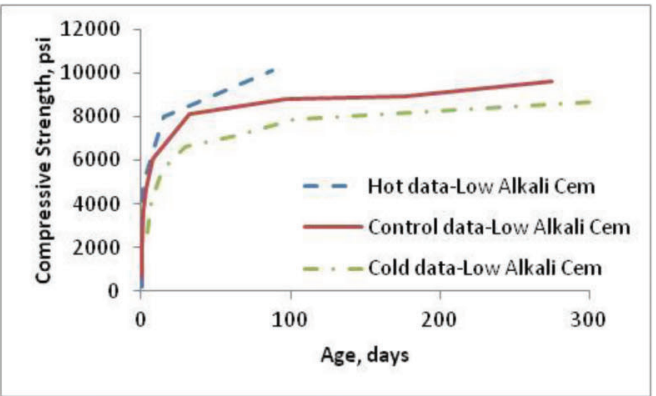

(d)

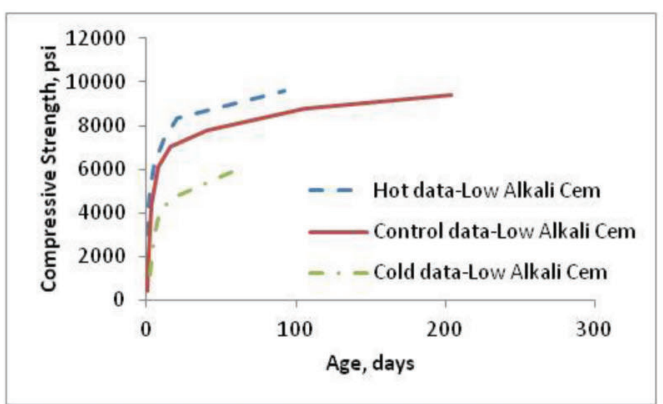

(e)

Figure C.4 Strength-age plot with low and high alkali cement at (a) Hot, (b) Control, and (c) Cold temperature; (d) strength-age plot with low alkali cement; (e) strength-age plot with high alkali cement for 0.39FAC35 mixture. 


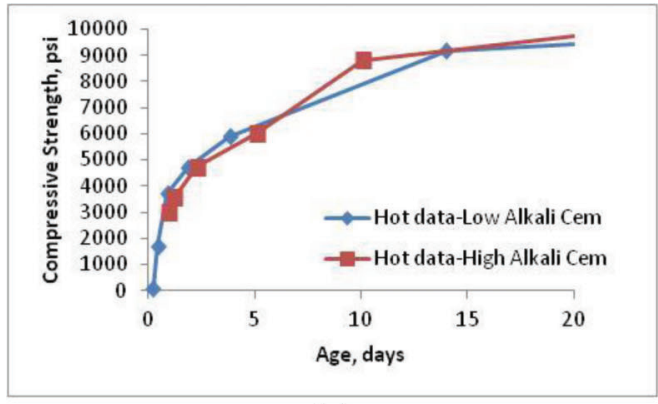

(a)

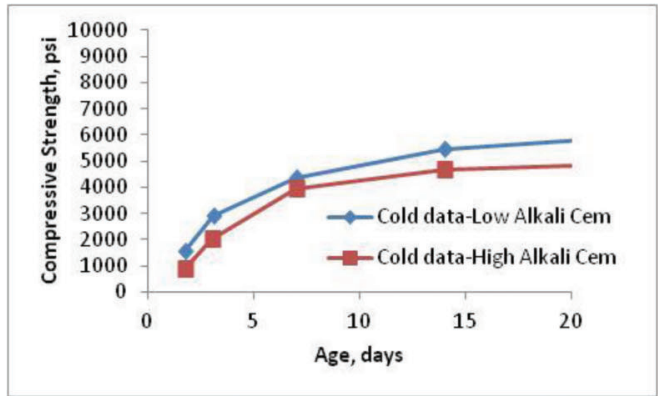

(c)

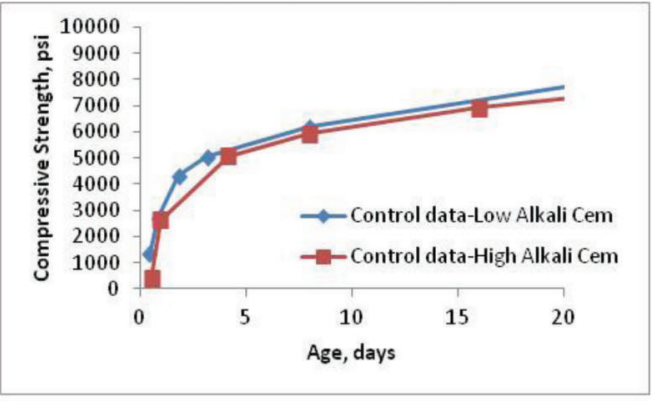

(b)

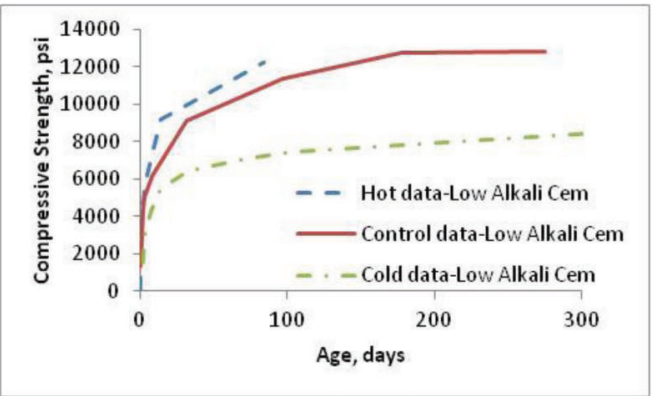

(d)

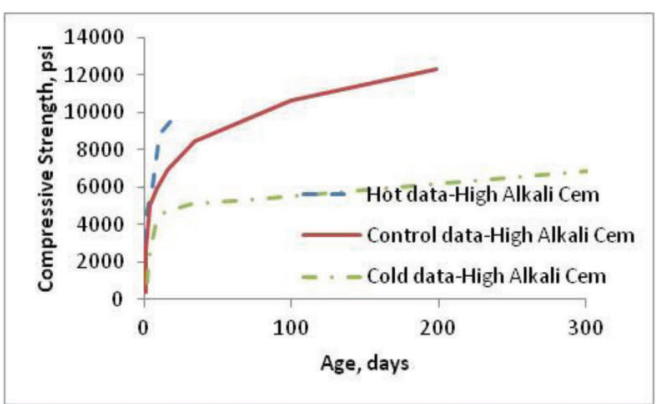

(e)

Figure C.5 Strength-age plot with low and high alkali cement at (a) Hot, (b) Control, and (c) Cold temperature; (d) strength-age plot with low alkali cement; (e) strength-age plot with high alkali cement for 0.34FAA50 mixture. 


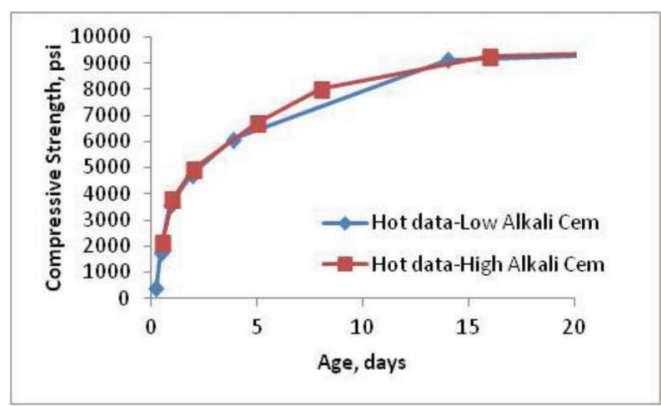

(a)

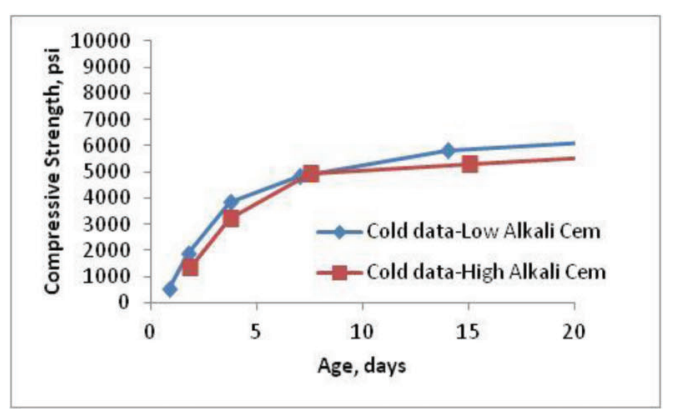

(c)

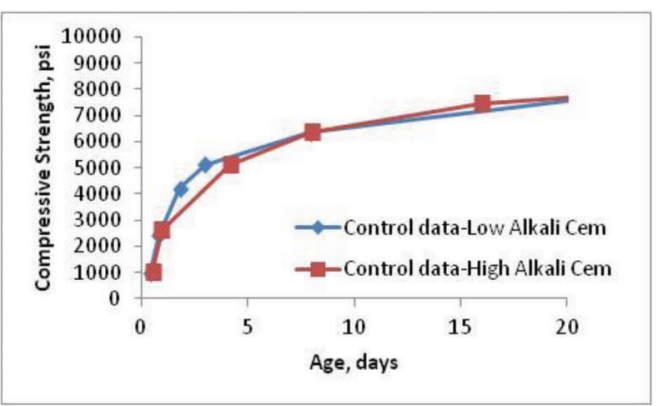

(b)

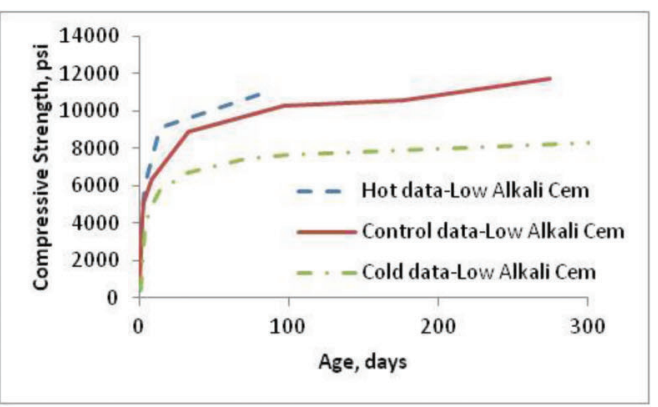

(d)

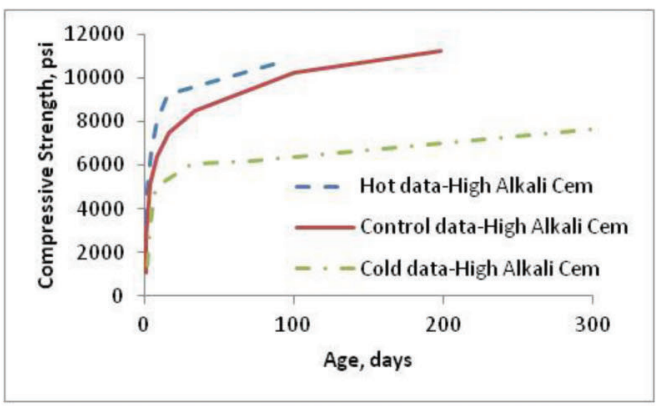

(e)

Figure C.6 Strength-age plot with low and high alkali cement at (a) Hot, (b) Control, and (c) Cold temperature; (d) strength-age plot with low alkali cement; (e) strength-age plot with high alkali cement for 0.34FAB50 mixture. 


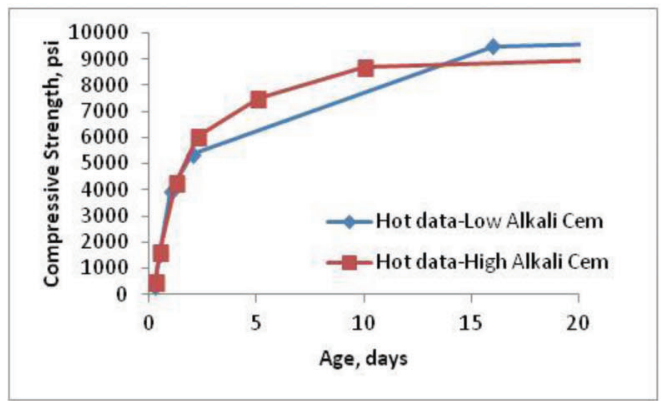

(a)

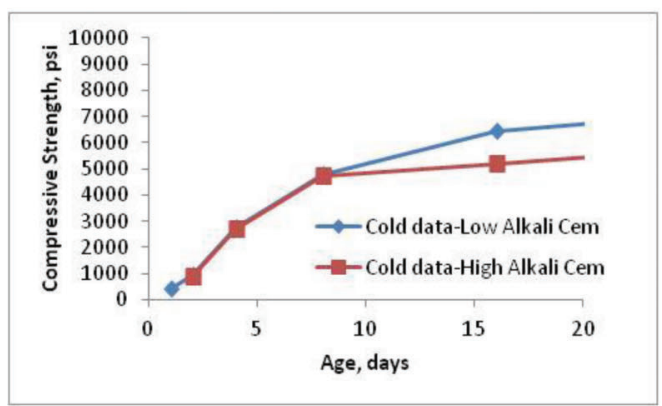

(c)

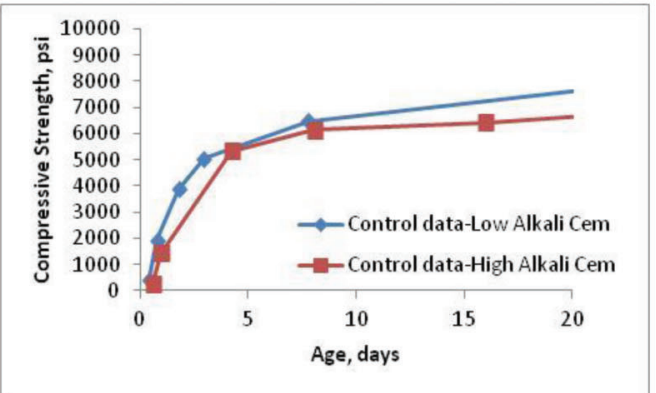

(b)

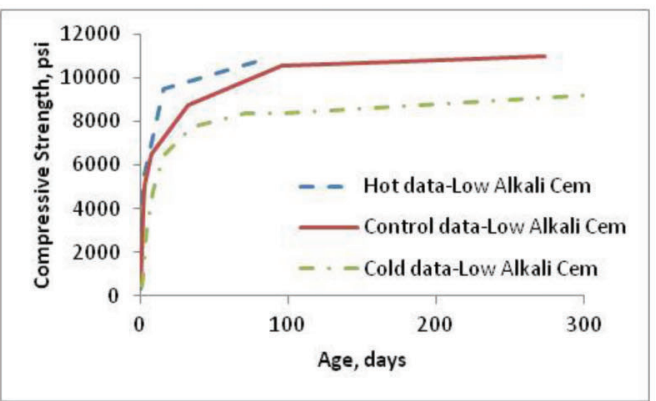

(d)

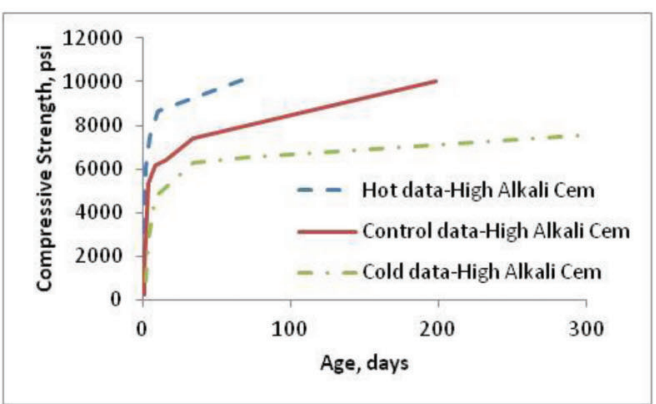

(e)

Figure C.7 Strength-age plot with low and high alkali cement at (a) Hot, (b) Control, and (c) Cold temperature; (d) strength-age plot with low alkali cement; (e) strength-age plot with high alkali cement for 0.34FAC50 mixture. 
CHAPTER 3. PHASE II, TASK 3: SETTING AND TEMPERATURE DEVELOPMENT

\section{Kevin P. Keith, Anton K. Schindler}

\section{ABSTRACT}

Fly ash is commonly used as a supplementary cementitious material (SCM) in the production of portland cement concrete. Concrete produced with high fly ash replacement levels is considered high volume fly ash (HVFA) concrete. HVFA concrete has many benefits, including reduced concrete production cost, reduced greenhouse gas emissions, and improved sustainability. Despite the advantages, there are several barriers that limit the use of HVFA concrete. One of the main limitations to the increased usage of HVFA concrete is the lack of contractor and transportation agency familiarity with the setting time and strength development of these concrete mixtures.

For this research, a laboratory-testing program was developed to examine the effect of fly ash type, fly ash dosage, cement chemical composition, and environmental conditions on the hydration development, setting times, and compressive strength development of HVFA concrete. Results from semi-adiabatic calorimetry were used to develop a hydration model for HVFA concrete. Finally, the ConcreteWorks software program was used to predict the in-place performance of selected HVFA concrete mixtures when placed in various transportation structures. It is concluded that HVFA concrete may be produced to have comparable setting times and earlyage compressive strength development to conventional portland cement concrete when used for transportation infrastructure.

\section{INTRODUCTION}

\subsection{Background}

Increases in the volume fraction of fly ash generally reduce the rate of overall hydration, which in turn leads to a decreased rate of strength development in structures. Since the hydration process is highly dependent on temperature, in-place setting times, timing of finishing operations, and rates of strength development are significantly impacted by the size of the structure built and ambient conditions experienced during placement. These effects associated with the use of HVFA concrete can be very difficult to manage during construction. Limited guidance is currently available to the concrete industry that may want to consider using HVFA concretes.

Currently, the majority of contractors have limited experience with HVFA mixtures, which places these mixtures at a disadvantage in an environment where contractor incentives are tied to rapid construction schedules using processes they know well. As a result, many contractors do not pursue the benefits of HVFA since they are concerned about many of the side effects that can occur. For example, delayed initial setting times prolong the time that the concrete finishing crew has to wait at the job site before they can do the final finishing of the concrete slab. This "idle waiting time" substantially increases concrete construction costs of primarily the slab type elements that need to be finished. Delayed initial setting times also increases the chances of plastic shrinkage cracking. The setting times of high volume Class $\mathrm{C}$ fly ash concrete can be delayed as compared to either plain portland cement or Class F fly ash concretes (Wade, Nixon, Schindler, \& Barnes, 2010). Accelerating chemical admixtures have been used to reduce these setting times. The work documented in this phase aims to evaluate the effect of fly ash dosage, type, and curing temperature on setting times of HVFA concretes.

Hydration is an exothermic process, and the total amount of heat generated may affect the in-place performance of some structures. The total heat released during hydration is a function of the composition of cementitious materials, amount of cementitious materials, and the $w / \mathrm{cm}$ of the mixture. Research is required to quantify the hydration behavior and temperature dependence of HVFA concretes that contain fly ash with different chemical compositions. This can reduce some of the concerns associated with strength gain and setting. Further, this will enable the beneficial effect of HVFA concretes to be quantified as it reduces the total temperature rise in concrete members, which may help to control durability issues related to thermal cracking (particularly for mass concrete applications) and delayed ettringite formation.

Despite the advantages associated with the use of HVFA concrete, there are several barriers which limit its use. One of the main barriers to the increased use of HVFA concrete is the concern over retarded set times, especially when high fly ash dosages are specified or cold curing conditions are encountered. Retarded set times can cause undesirable delays to concrete finishing operations. Similarly, there are concerns over the low early-age compressive strength development of HVFA concrete. Low early-age compressive strengths can require extended curing times, which can delay formwork removal and negatively impact construction sequencing. Finally, there are concerns that material incompatibilities between fly ash, chemical admixtures, and cement may arise when high-calcium Class $\mathrm{C}$ fly ashes are specified in high dosages (Spiratos, Pagé, Milvaganam, Malhotra, \& Jolicoeur, 2003). Due to these barriers, contractors and transportation agencies are often reluctant to specify HVFA concrete for transportation structures.

\subsection{Research Objectives}

The main goal of this research was to evaluate the in-place performance of HVFA concrete when used in various transportation structures. The results from this research are aimed at providing guidance to contractors, concrete producers, and transportation agencies when specifying HVFA concrete for use in transportation infrastructure. 
Auburn University, Purdue University, the National Institute of Standards and Technology (NIST), and the National Ready Mix Concrete Association (NRMCA) all worked together to address the limitations associated with HVFA concrete. One of the main limitations to the increased usage of HVFA concrete is the lack of prediction models for the in-place performance of HVFA concrete. Despite the advantages offered by HVFA concrete, there is a lack of knowledge concerning the in-place temperature development, setting times, and compressive strength development of concrete mixtures proportioned with high fly ash replacement levels. Auburn University was tasked with addressing these limitations. The primary objectives of the research conducted by Auburn University are:

- Perform laboratory testing to quantify the effect of fly ash type, fly ash dosage, cement chemical composition, and curing temperature on the hydration, setting, and compressive strength development of HVFA concrete mixtures,

- Develop a HVFA concrete hydration model, and

- Develop prediction methods to quantify the effect of fly ash type, fly ash dosage, and curing conditions on the maximum in-place concrete temperature, time to initial set, and compressive strength development of HVFA concrete placed in selected transportation structures.

\subsection{Research Methodology}

Laboratory testing was performed to by producing two control mixtures and HVFA concrete mixtures with $35 \%$ and $50 \%$ fly ash dosages. Four fly ash sources, two Class C and two Class F, were selected for laboratory testing to represent the range of fly ash chemical compositions available to the concrete industry in the United States. Two cement sources with primarily different alkali contents were evaluated in the HVFA mixtures. A total of 16 HVFA concrete mixtures were developed based on all combinations of fly ash dosage, fly ash type, and cement source. Each concrete mixture was produced at three batching temperatures $\left(40^{\circ} \mathrm{F}\right.$, $73^{\circ} \mathrm{F}$, and $105^{\circ} \mathrm{F}$ ). Semi-adiabatic calorimetry, penetration resistance testing, and compressive strength testing were conducted for all mixtures to investigate the effect of fly ash dosage, fly ash type, cement chemical composition, and curing temperature on the hydration development, setting times, and compressive strength development of HVFA concrete.

A hydration model for HVFA concrete was developed based on semi-adiabatic calorimetry testing results. The purpose of the HVFA concrete hydration model is to provide an first estimate of the hydration behavior of HVFA concrete for concrete producers and transportation agencies.

Finally, an evaluation of the in-place performance of HVFA concrete was completed by using the results obtained from the laboratory testing program and hydration model development. The ConcreteWorks software program was used to develop in-place temperature profiles for HVFA concrete placed under varying placement conditions. The in-place temperature profiles were then used to estimate the in-place temperature development, initial setting times, and compressive strength development of HVFA concretes placed in various transportation structures.

\subsection{Literature Review on the Hydration of Portland Cement and Fly Ash}

\subsubsection{Portland Cement and Fly Ash Hydration}

The hydration of portland cement is important because this process yields hydration products that possess setting and hardening characteristics. The hydration process is directly influenced by the chemical composition and fineness of the cement. Portland cement is composed of four main Bogue compounds: tricalcium silicate $\left(\mathrm{C}_{3} \mathrm{~S}\right)$, dicalcium silicate $\left(\mathrm{C}_{2} \mathrm{~S}\right)$, tricalcium aluminate $\left(\mathrm{C}_{3} \mathrm{~A}\right)$, and tetracalcium aluminoferrite $\left(\mathrm{C}_{4} \mathrm{AF}\right)$ (Mehta \& Monteiro, 2006). The cement hydration reaction is exothermic, meaning heat is released as hydration progresses. The amount of heat released as portland cement hydrates is referred to as the heat of hydration and can serve as an indication of the progress of the overall hydration process.

When fly ash is used in concrete as a supplementary cementing material (SCM), it reacts with the alkalis and calcium hydroxide $(\mathrm{CH})$ within the hydrating cement paste to form calcium silicate hydrates (C-S-H) and calcium aluminate hydrates (ACI Committee 232, 2003). Fly ash particles react with $\mathrm{CH}$ to form C-S-H, as shown by Reaction 1.1 (Mehta \& Monteiro, 2006).

$$
\text { FlyAsh }+\mathrm{CH}+\text { water } \rightarrow \mathrm{C}-\mathrm{S}-\mathrm{H}
$$

Reaction 1.1 is referred to as the pozzolanic reaction. The fly ash will continue to react with $\mathrm{CH}$ to produce $\mathrm{C}-\mathrm{S}-\mathrm{H}$ until either all of the $\mathrm{CH}$ is consumed within the pore fluid, or there is no more mixing water available (ACI Committee 232, 2003). In addition to the pozzolanic reaction, Class $\mathrm{C}$ fly ash may also react with the alkalis present in concrete to form calcium aluminate hydrates. Both of these reactions are dependent upon the breakdown of the glassy structure of the fly ash by hydroxide ions and the heat generated in early stages of hydration of the hydraulic cement (Philleo, 1991).

The pozzolanic reaction is important because it serves to convert the weak, soluble $\mathrm{CH}$ into the much stronger C-S-H hydration product, thereby increasing the durability of the hydrated cement paste. Also, the pozzolanic reaction occurs at a slow rate and releases little heat of hydration in comparison to the cementitious reactions (Mehta \& Monteiro, 2006).

The hydration of cementitious materials is temperature dependent. An increase in hydration temperature results in an accelerated hydration reaction and vice versa. Figure 1.1 shows hydration development for five mortar specimens of the same $w / c$ ratio $(w / c=0.50)$ cured at isothermal temperatures of 5, 12.5, 20, 35 and $50^{\circ} \mathrm{C}$ (Kjellsen \& Detwiler, 1992). The figure illustrates 


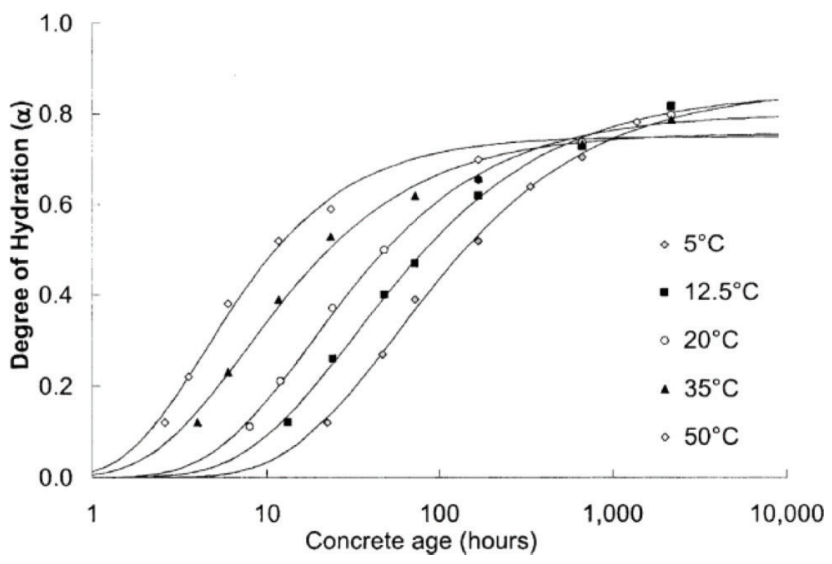

Figure 1.1 Effect of curing temperature on degree of hydration (Kjellsen \& Detwiler, 1992).

that higher curing temperatures result in an increased rate of hydration. Similarly, as the curing temperature decreases, the rate of hydration slows. The effect of curing temperature on hydration development will be discussed in more detail in subsection 1.4.4.5 when the degree of hydration formulation is introduced.

\subsubsection{Calorimetry}

The heat of hydration of a sample of paste, mortar, or concrete may be used as an indication of its degree of hydration (Powers \& Brownyard, 1948). One of the main methods used to measure heat of hydration is isothermal calorimetry. Through isothermal calorimetry, the heat of hydration of a sample is measured while it is maintained at a constant temperature (Poole, Riding, Folliard, Juenger, \& Schindler, 2007). After the first few days of testing, the hydration process begins to slow and the amount of liberated heat decreases. At this time, the error within the testing set-up may be similar in magnitude to the heat released by the sample (Poole et al., 2007). The useful duration of isothermal testing results varies from approximately 2 to 5 days depending on the sample size and testing temperature. Results from isothermal calorimetry testing are useful in determining the temperature sensitivity (activation energy) of a given concrete mixture. An example of isothermal calorimetry results obtained for mortar samples for this project (with and without fly ash replacement) is shown in Figure 1.2. It can be seen in this figure that the rate of heat evolution is dependent on the cementitious system. In this figure, the results were normalized per gram of cementitious material.

Another method commonly used to measure the heat of hydration of a concrete sample is adiabatic calorimetry. Adiabatic calorimetry is based on the principle that at any time during testing, the temperature of the sample surroundings will be equal to the temperature of the concrete (RILEM TC 119-TCE, 1997). Since it is very difficult to provide a test set-up in which there is no heat exchange to or from the testing environment, semi-adiabatic calorimetry (SAC) was developed (Cost,

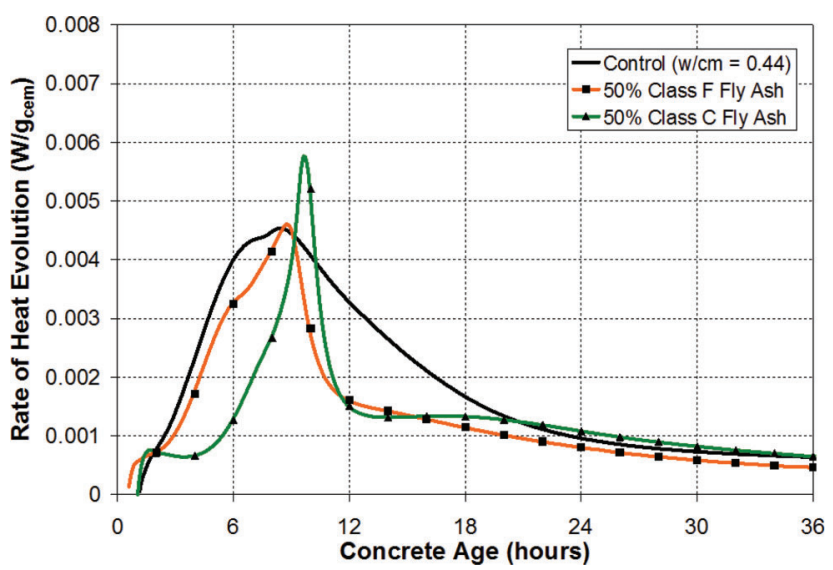

Figure 1.2 Sample isothermal calorimetry results.

2009). During semi-adiabatic calorimetry testing, a small amount of heat loss from the test set-up is expected to occur. These device specific losses are recorded and used to estimate a fully-adiabatic temperature profile. The fully-adiabatic profile must also account for the effect that the temperature difference between fully adiabatic and semi-adiabatic conditions has on the rate of hydration of the sample (Schindler \& Folliard, 2005). An example of heat of hydration data for concrete produced with $15 \%, 25 \%$ and $35 \%$ Class F fly ash collected from a semi-adiabatic calorimeter is presented in Figure 1.3.

\subsubsection{High Volume Fly Ash Concrete}

When fly ash is used as a supplementary cementitious material (SCM), the optimum replacement level is affected by the following factors: class of fly ash, chemical composition of cement, use of chemical admixtures, placement conditions, strength requirements, and curing conditions (Prusinski, Fouad, \& Donovan, 1993). Therefore, the optimum replacement level should be investigated for each concrete application.

The water-cement $(w / c)$ ratio is defined as the mass of water divided by the mass of cement. Similarly, the water-cementitious material $(w / \mathrm{cm})$ ratio is defined as the mass of water divided by the mass of cementitious materials (cement plus SCMs). It should be noted, however, that concrete produced with the same $w / \mathrm{cm}$ ratio may experience differences in strength due to differences in aggregate properties, types and sources of cementitious materials, use of chemical admixtures, entrained air contents, and curing conditions (Mindess, Young, \& Darwin, 2003).

When proportioning HVFA concrete, a lower $w / \mathrm{cm}$ ratio may necessary as compared to a portland cement only concrete. During research conducted at CANMET, Bilodeau, Malhotra, and Seabrook (2001) determined that for HVFA concrete, "the proportion of fly ash should be as high as possible and the water-cementitious materials ratio as low as possible to provide adequate earlyage strength and durability." High-range water-reducing 

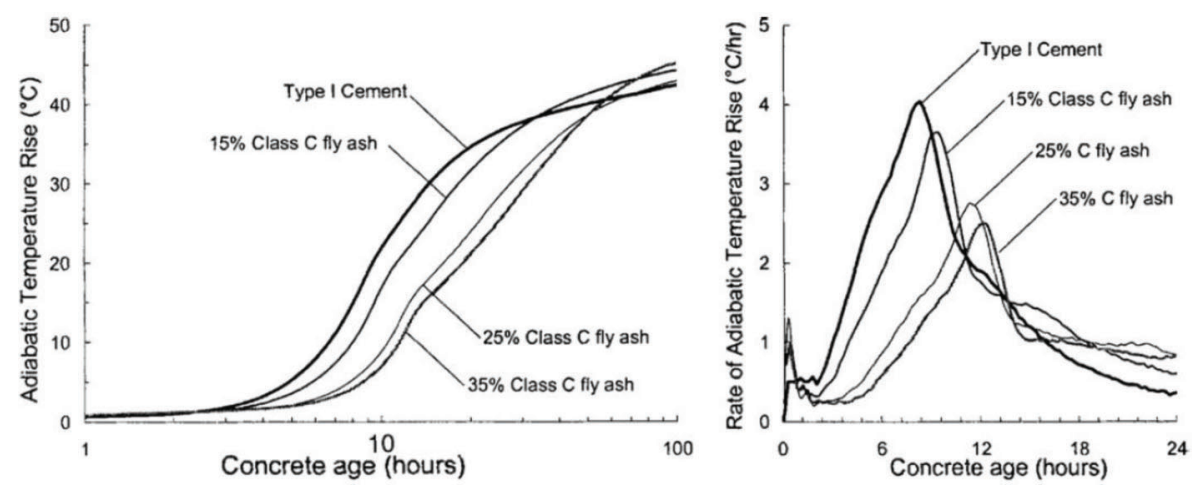

Figure 1.3 Sample semi-adiabatic calorimetry results (Schindler \& Folliard, 2005).

admixtures are typically required to maintain adequate workability at low $w / \mathrm{cm}$ ratios.

ASTM C494 (2005) Type C accelerating admixtures are defined as "an admixture that accelerates the setting and early-age strength development of concrete." Accelerators are often used during winter months to offset the effects that cold placement temperatures have on the rate of hydration and strength gain. Similarly, accelerating admixtures are commonly used in HVFA concrete to counteract the retarding effects that high fly ash contents have on the hydration process. With the correct accelerator dosage, set times and early-age strength development for HVFA concrete can be made comparable to conventional concrete.

ASTM C494 (2005) Type F and G high-range waterreducing (HRWR) admixtures are admixtures that reduce "the amount of mixing water required to produce a concrete of a given consistency by $12 \%$ or greater." HRWR admixtures are commonly used in HVFA concrete to ensure adequate workability at the low $w / \mathrm{cm}$ ratios required for adequate early-age strength. Popovics (1993) reported that the effectiveness of HRWRA in HVFA concrete is dependent on the type and dosage of the admixture and the chemical composition of the cementitious material used in the concrete.

\subsubsection{Effects of High Volume Fly Ash on Setting and} Hydration Concrete

1.4.3.1.1 Setting Time. Mehta and Monteiro (2006) define setting of concrete as "the onset of solidification in a fresh concrete mixture." Initial set defines the time at which fresh concrete can no longer be properly mixed and placed, whereas final set defines the beginning of the development of mechanical properties (Mindess et al, 2003). Initial and final set times in concrete are determined according to ASTM C403 (2006), Standard Test Method for Time of Setting of Concrete Mixtures by Penetration Resistance. Set times for HVFA concrete are influenced by the class and quantity of fly ash, type and quantity of cement, environmental conditions, concrete temperature, use of chemical admixtures, and water-to-cementitious materials ratio (Mehta \& Monteiro, 2006).
Ravina and Mehta (1986) performed a study on the fresh concrete properties of concrete produced with fly ash contents of 35,42 and $50 \%$ by weight of cementitious materials. For this study, four fly ash sources were investigated, two Class $\mathrm{F}$ and two Class $\mathrm{C}$. Ravina and Mehta (1986) reported initial set time delays of 20 minutes to 4 hours and 20 minutes and final set time delays of 1 hour to 5 hours and 15 minutes when compared to the control concrete depending on the fly ash type and dosage. ASTM Class C fly ashes resulted in longer set times when compared to equivalent concrete mixtures prepared with Class $\mathrm{F}$ fly ashes. Also, the delay in set times increased with higher fly ash replacement levels. This research, indicates that HVFA concretes exhibit delayed set times when compared to a control mixture with no fly ash replacement and a similar $w / \mathrm{cm}$.

The rate of $\mathrm{C}_{3} \mathrm{~A}$ hydration is highly dependent on the presence of sulfate ions in the hydrating cement paste. When the sulfate content is increased, the rate of $\mathrm{C}_{3} \mathrm{~A}$ hydration is slowed. Ravina and Mehta (1986) report that the delayed setting times for the concrete mixtures incorporating Class $\mathrm{C}$ fly ashes can be attributed to their higher sulfate contents.

Eren, Brooks, and Celik (1995) performed research to quantify the effect of curing temperature on setting times of HVFA concrete and slag cement concrete. They concluded that set times decrease with an increase in curing temperature and increase with a decrease in curing temperature. During cold weather, the rate of hydration is slowed, and high fly ash replacement levels can cause significant delays in initial and final set times. During hot weather, however, the retardation caused by fly ash may be beneficial to slow down the rate of hydration.

1.4.3.1.2 Heat of Hydration. When fly ash is used to replace cement in portland cement concrete, the rate of heat development and overall heat of hydration is altered (Schindler \& Folliard, 2005). In some cases, the total heat of hydration is reduced, which can be very beneficial in mass concrete construction. During mass concrete pours, the maximum temperature rise for concrete containing fly ash will depend on the chemical composition of the fly ash, replacement level, and concrete temperature at placement (Thomas, 2007). 
TABLE 1.1

Temperature Rise of HVFA Concrete Monoliths (Bisaillon et al., 1994)

\begin{tabular}{|c|c|c|c|c|c|}
\hline Mix & Cement kg/m $\left(\mathrm{lb} / \mathrm{yd}^{3}\right)$ & Class F Fly Ash kg/m $\left(\mathrm{lb} / \mathrm{yd}^{3}\right)$ & $w / c m$ & Max. Temp. ${ }^{\circ} \mathbf{C}\left({ }^{\circ} \mathbf{F}\right)$ & Time to Max Temp. (hour) \\
\hline 1 & 365 (600) Type I & - & 0.45 & $68(154)$ & 29 \\
\hline 2 & 125 (211) Type I & $155(261)$ & 0.46 & $44(111)$ & 53 \\
\hline 3 & 170 (287) Type I & $220(370)$ & 0.29 & $54(129)$ & 57 \\
\hline 4 & 330 (556) Type II & - & 0.50 & $55(131)$ & 75 \\
\hline 5 & 125 (211) Type I & $155(261)$ & 0.41 & 47 (117) & 98 \\
\hline
\end{tabular}

Langley, Carette, and Malhotra (1992) performed research to quantify the effect that high fly ash dosages have on the temperature rise for concrete. Langley et al. (1992) reported that the temperature rise caused by the hydration of HVFA concrete may be significantly reduced. When the cementitious materials content was held constant, it was found that the peak temperature of the block cast incorporating concrete with $55 \%$ Class F fly ash replacement was reduced by $29^{\circ} \mathrm{C}\left(52^{\circ} \mathrm{F}\right)$. When the total cementitious materials content was reduced and fly ash was incorporated, a reduction of $53^{\circ} \mathrm{C}\left(95^{\circ} \mathrm{F}\right)$ was achieved (Langley et al., 1992).

Similar research was conducted by Bisaillon, Rivest, and Malhotra (1994). The results from this study are shown in Table 1.1. The fly ash selected for this research had a $\mathrm{CaO}$ content of $2 \%$. Bisaillon et al. (1994) also reported decreases in peak in-place temperature when monitoring the temperature rise of large concrete monoliths $(2.5 \times 3.0 \times 4.0$ meter blocks $)$ cast with fly ash replacement levels ranging from 56 to $62 \%$.

The rate of temperature development and maximum temperature rise for HVFA concrete is dependent on the chemical composition of the fly ash (Schindler \& Folliard, 2005). Thomas, Mukherjee, Sato, and Everitt (1995) performed an isothermal calorimetry study on cement-fly ash pastes with $\mathrm{CaO}$ contents ranging from $2.6 \%$ to $27.1 \%$. The results from this study are illustrated in Figure 1.4. From this study, it was determined that as the $\mathrm{CaO}$ content of the fly ash increases, the rate of temperature development also increases.

\subsubsection{Hydration Modeling}

1.4.4.1 Degree of Hydration. Degree of hydration $(\alpha)$ is used to quantify the progress of the hydration reactions between cementitious materials and water contained in a concrete sample. The degree of hydration varies with time and at an $\alpha$ of 0 , no reactions have occurred. The maximum value of $\alpha$ is 1.0 , representing a state of complete hydration (RILEM CEA 42, 1981). It should be noted, however, that a degree of hydration of 1.0 may never be reached because not all cementitious material will hydrate (Mills, 1966).

According to RILEM CEA 42 (1981), it is "almost impossible to make a direct determination of the quantity of cement gel formed or the quantity of hydrated cement." Therefore, the degree of hydration is most often determined by indirect methods. One indirect method for determining $\alpha$ is by analysis of the chemically bound water within the hydrating cement paste.

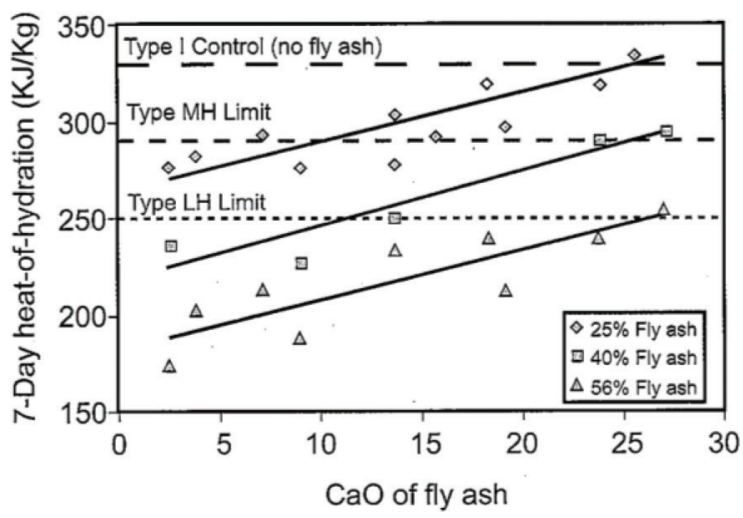

Figure 1.4 Influence of fly ash $\mathrm{CaO}$ content on heat of hydration (Thomas et al., 1995).

Another indirect method for determining the degree of hydration is based on measuring the heat of hydration of the cement paste. Powers and Brownyard (1948) found that the quantity of bound water is directly proportional to the quantity of heat developed during hydration. Therefore the degree of hydration may be determined by dividing the heat developed at a given time by the theoretical total quantity of heat available within the cementitious system. The degree of hydration formulation based on heat of hydration is as follows (Powers \& Brownyard, 1948):

$$
\alpha(t)=\frac{H(t)}{H_{T}}
$$

where:

$\alpha(t)=$ degree of hydration at time $\mathrm{t}$,

$H(t)=$ quantity of heat developed at time $t$, and

$H_{T}=$ quantity of heat developed at complete hydration.

The quantity of heat generated at a given time, $H(t)$, may be determined by the following methods: adiabatic calorimetry, isothermal calorimetry or measurement of the heat of solution. Due to unreliability of heat of solution measurements, calorimetry methods are preferred (RILEM CEA 42, 1981). The formulation given above also requires determination of $H_{T}$, the total quantity of heat available within the cementitious system. $H_{T}$ accounts for heat generated by both portland cement and SCMs at $100 \%$ hydration. The ultimate heat of hydration for a concrete mixture, $H_{T}$, can be quantified as follows (Schindler \& Folliard, 2005): 


$$
H_{T}=H_{u} \cdot C_{c}
$$

where:

$H_{T}=$ ultimate heat of hydration of the concrete $\left(\mathrm{J} / \mathrm{m}^{3}\right)$,

$H_{u}=$ total heat of hydration of cementitious materials $(\mathrm{J} / \mathrm{g})$, and

$C_{c}=$ cementitious materials content $\left(\mathrm{g} / \mathrm{m}^{3}\right)$.

The total heat of hydration of the cementitious system includes heat contribution from both portland cement and SCMs. Schindler and Folliard (2005) proposed the following approximation for the total heat of hydration of the cementitious materials:

$$
H_{u}=H_{c e m} \cdot p_{c e m}+461 \cdot p_{s l a g}+p_{F A} \cdot h_{F A}
$$

where:

$H_{u}=$ total heat of hydration of cementitious materials hydration $(\mathrm{J} / \mathrm{g})$,

$H_{c e m}=$ total heat of hydration of cement $(\mathrm{J} / \mathrm{g})$,

$p_{c e m}=$ cement weight ratio in terms of total cementitious content,

$p_{\text {slag }}=$ slag weight ratio in terms of total cementitious content,

$p_{F A}=$ fly ash weight ratio in terms of total cementitious content, and

$h_{F A}=$ heat of hydration of fly ash $(\mathrm{J} / \mathrm{g})$.

1.4.4.2 Portland Cement Heat Contribution. As seen in the formulation for $H_{u}$, the heat of hydration contribution from portland cement and fly ash must be determined. Since each portland cement compound has a unique heat of hydration, the total heat of hydration of cement at complete hydration, $H_{\text {cem }}$, may be calculated as follows (Bogue, 1947):

$$
\begin{aligned}
H_{c e m} & =500 \cdot p_{C 3 S}+260 \cdot p_{C 2 S}+866 \cdot p_{C 3 A}+420 \cdot p_{C 4 A F} \\
& +624 \cdot p_{\mathrm{SO} 3}+1186 \cdot p_{\text {Free } \mathrm{CaO}}+850 \cdot p_{\mathrm{MgO}}
\end{aligned}
$$

where:

$H_{\text {cem }}=$ total heat of hydration of cement $(\mathrm{J} / \mathrm{g})$ and

$p_{i}=$ weight ratio of $i$-th compound in terms of total cement content.

1.4.4.3 Fly Ash Heat Contribution. To calculate the total heat available within the cementitious system, the heat contribution from fly ash must be determined. Since the cementitious nature of a fly ash is correlated with its $\mathrm{CaO}$ content, fly ash heat of hydration is calculated based on $\mathrm{CaO}$ content. Kishi and Maekawa (1995) proposed a value of $209 \mathrm{~kJ} / \mathrm{kg}$ for fly ash with a $\mathrm{CaO}$ content of $8.8 \%$. Schindler and Folliard (2005) expanded the formulation for fly ash heat of hydration based on semi-adiabatic test results. The formulation for fly ash heat of hydration proposed by Schindler and Folliard (2005) is as follows:

$$
h_{F A}=1800 \cdot p_{F A-C a O}
$$

where:

$h_{\mathrm{FA}}=$ total heat of hydration of fly ash $(\mathrm{J} / \mathrm{g})$ and

$p_{F A-C a O}=$ fly ash $\mathrm{CaO}$ weight ratio in terms of total fly ash content.

In 2009, Ge and Wang modified the formulation proposed by Schindler and Folliard (2005) based on semi-adiabatic calorimetry test results obtained from concrete mixtures containing 15, 30, 45 and $60 \%$ fly ash by weight of cementitious materials. The fly ash sources investigated in this study had $\mathrm{CaO}$ contents ranging from $1.51 \%$ to $27.11 \%$. The formulation proposed by Ge and Wang (2009) is as follows:

$$
h_{\mathrm{FA}}=15.9 \cdot\left(p_{F A C a O} \times 100\right)+74.3
$$

where:

$h_{F A}=$ total heat of hydration of fly ash $(\mathrm{J} / \mathrm{g})$ and

$p_{F A C a O}=$ fly ash $\mathrm{CaO}$ weight ratio in terms of total fly ash content.

1.4.4.4 Ultimate Degree of Hydration. In an earlier subsection, it was shown that the rate of hydration is affected by curing temperature. The ultimate degree of hydration, however, is independent of curing temperature (Kjellsen \& Detwiler, 1992). The influence of curing temperature on ultimate degree of hydration is shown in Figure 1.2. The difference in ultimate degree of hydration for the samples cured at $5^{\circ} \mathrm{C}$ and $50^{\circ} \mathrm{C}$ is approximately $2 \%$.

Unless the water-cement ratio is extremely high, complete hydration will not occur $(\alpha=1.0)$. After investigation of the hydration of a wide range of cementitious materials, Mills (1966) concluded that "most, if not all, cement paste hydration stops before the cement is totally consumed." Mills determined that the ultimate degree of hydration is strongly influenced by the water-cement ratio. Based on this study, Mills developed Equation 1.8 as an approximation for the ultimate degree of hydration $\left(\alpha_{u}\right)$. The ultimate degree of hydration formulation proposed by Mills does not take into account the use of SCMs. Therefore, Schindler and Folliard (2005) expanded Mills' (1966) model as shown in Equation 1.9.

$$
\begin{gathered}
\alpha_{u}=\frac{1.031 \cdot w / c}{0.194+w / c} \\
\alpha_{u}=\frac{1.031 \cdot w / c}{0.194+w / c}+0.5 \cdot p_{F A}+0.3 \cdot p_{S L A G} \leq 1.0
\end{gathered}
$$

where:

$\alpha_{u}=$ ultimate degree of hydration,

$w / c=$ water-cement ratio by weight,

$p_{F A}=$ weight ratio of fly ash in terms of total cement content, and

$p_{S L A G}=$ weight ratio of GGBF slag in terms of total cement content. 
1.4.4.5 Hydration Model Formulation. After collecting data for the degree of hydration development of a concrete sample, the data may be represented by a bestfit mathematical model. Hansen and Pederson (1984) developed a three-parameter exponential equation to represent the heat development of a portland cement concrete sample. In 2002, Hansen and Pane modified the original equation to represent the development of degree of hydration versus time:

$$
\alpha(t)=\alpha_{u} \cdot \exp \left(-\left(\frac{\tau}{t}\right)^{\beta}\right)
$$

where:

$$
\begin{aligned}
& \alpha(t)=\text { degree of hydration at time } \mathrm{t} \\
& \alpha_{u}=\text { ultimate degree of hydration } \\
& \tau=\text { hydration time parameter } \\
& \beta=\text { hydration shape parameter, and } \\
& t=\text { concrete age or equivalent age. }
\end{aligned}
$$

1.4.4.6 Equivalent Age Maturity Method. The development of mechanical properties and hydration for a given concrete mixture is a function of its age and curing temperature history. Therefore, it is difficult to accurately estimate the in-place concrete properties based on data collected under standard laboratory conditions. The maturity method was developed to predict concrete behavior at any given time based on the measured temperature history of a concrete sample (Carino, 2004).

In 1977, Hansen and Pederson introduced the maturity function presented in Equation 1.11. This function is based on the Arrhenius equation and is commonly called the equivalent age maturity method (ASTM C1074, 2004). ASTM C1074 (2004) defines equivalent age as the amount of time at a specified temperature required to produce a maturity equal to the maturity reached by a curing period at temperatures other than the specified reference temperature. The Arrhenius method maturity function is typically evaluated using a reference temperature, $T_{R}$, of $73^{\circ} \mathrm{F}\left(23^{\circ} \mathrm{C}\right)$ in the United States or $68^{\circ} \mathrm{F}\left(20^{\circ} \mathrm{C}\right)$ in Europe.

$$
t_{e}=\sum e^{\frac{-E}{R}\left(\frac{1}{T_{c}}-\frac{1}{T_{R}}\right)} \Delta t
$$

where:

$t_{e}=$ equivalent age at the reference curing temperature (hours),

$T_{C}=$ average temperature of concrete during time interval $\Delta \mathrm{t}(\mathrm{K})$,

$T_{R}=$ reference temperature $(\mathrm{K})$,

$E=$ activation energy $(\mathrm{J} / \mathrm{mol})$, and

$R=$ universal gas constant, $8.3144 \mathrm{~J} /(\mathrm{mol} \mathrm{K})$.

The activation energy used in the Arrhenius equation defines the temperature sensitivity of the concrete mixture (Carino, 2004). When the difference between the curing and reference temperatures increases, it is important that an accurate estimate of the activation energy is used so that reliable estimates of the temperature-dependent concrete properties are achieved.
Schindler (2004) proposed the following formulation for estimating the activation energy for a given concrete mixture based on the chemical composition and fineness of the cement, as well as the presence of supplementary cementitious materials:

$$
E=22,100 \cdot f_{E} \cdot p_{C 3 A}{ }^{0.30} \cdot p_{C 4 A F}{ }^{0.25} \cdot \text { Blaine }^{0.35}
$$

where:

$f_{E}=$ activation energy factor to account for use of SCMs,

$p_{C 3 A}=$ weight ratio of $\mathrm{C}_{3} \mathrm{~A}$ in terms of total cement content,

$p_{C 4 A F}=$ weight ratio of $\mathrm{C}_{4} \mathrm{AF}$ in terms of total cement content, and

Blaine = Blaine specific surface area of cement $\left(\mathrm{m}^{2} / \mathrm{kg}\right)$.

The activation energy modification factor account for use of SCMs, $f_{E}$, is calculated as follows:

$f_{E}=11.05 \cdot p_{F A} \cdot\left(1-p_{F A C a O} / 0.40\right)+0.40 \cdot p_{S L A G}$

where:

$p_{F A}=$ weight ratio of fly ash replacement,

$p_{F A C a O}=$ weight ratio of $\mathrm{CaO}$ content for fly ash, and

$p_{S L A G}=$ weight ratio of GGBF slag replacement.

1.4.4.6.1 Application of Equivalent Age Maturity Method. After determining the activation energy for a given concrete mixture, the equivalent age for a test specimen may be calculated at any time based on the recorded temperature history using Equation 1.11. As long as the concrete has been properly placed, consolidated and cured, this equivalent age can then be used to help estimate the in-place concrete strength. When comparing concrete strength based on equivalent age, rather than real age, inaccurate correlations due to differing temperature histories may be avoided, as illustrated by Figure 1.5. Accurate prediction of the inplace concrete strength is crucial for planning construction operations such as form removal and application early-age loads (Carino, 2004).

Application of the maturity method for strength prediction requires two phases: laboratory testing and field measurement of in-place temperature history. The laboratory testing phase requires two types of testing. First, the temperature sensitivity (activation energy) of the concrete mixture must be selected or determined. Second, the strength-maturity relationship for the mixture must be determined by plotting the compressive strength of laboratory-cured concrete cylinders against the equivalent age at the time of testing (Carino, 2004).

Hansen and Pederson (1985) proposed that the strength-maturity relationship should be similar to the heat of hydration versus equivalent age relationship. This strength-maturity relationship is as follows:

$$
S(M)=S_{u} \cdot \exp \left(-\left(\frac{\tau}{M}\right)^{a}\right)
$$

where:

$S(M)=$ strength at maturity $\mathrm{M}$, 

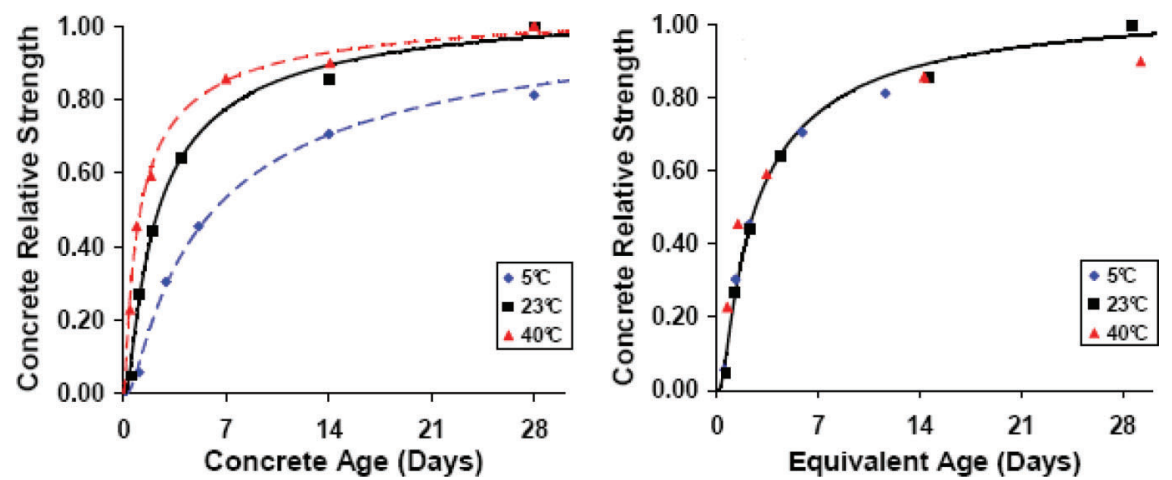

Figure 1.5 Comparison of compressive strength versus real concrete age and equivalent age for samples cured at different temperatures (Weakley, 2010).
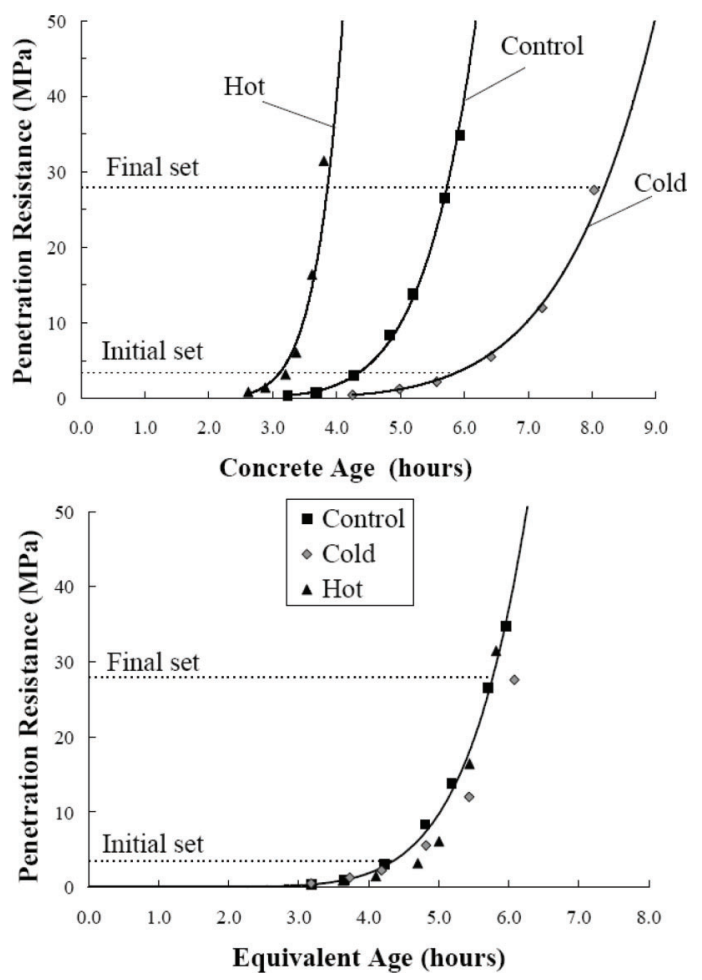

Figure 1.6 Concrete setting versus equivalent age (Wade et al., 2010).

$S_{u}=$ ultimate strength,

$\tau=$ characteristic time constant,

$M=$ maturity index, and

$A=$ shape parameter.

The ultimate strength, time constant, and shape parameter are unique for each concrete mixture. Regression analysis is used to fit Equation 1.14 to the compressive strength versus equivalent age data. After determining the strength-maturity relationship, in-place concrete strengths may be estimated based on recorded in-place concrete temperatures.

The maturity method may be used to account for the effect of temperature on concrete setting times. Initial and final set times are defined based on penetration resistance of a wet-sieved mortar sample according to
ASTM C403 (2006). Pinto and Hover (1999) performed research to determine the effect of curing temperature on setting time as determined by penetration resistance. Although the initial and final set times at each curing temperature varied significantly, the calculated equivalent ages did not, as shown in Figure 1.6. This was also confirmed by Wade et al. (2010), Weakley (2010), and Pinto and Schindler (2010). The setting times may be estimated for a given mixture at varying placement conditions if the apparent activation energy, temperature history, and equivalent ages at setting are known.

\section{LABORATORY TESTING PROGRAM}

The main objective of this research project was to provide guidance for the increased use of HVFA concrete in transportation structures. To accomplish this objective, a laboratory testing program was conducted to characterize the behavior of HVFA concrete mixtures. This section provides an overview of this testing program as well as discussion on raw material properties, concrete mixture proportions, concrete production, curing methods, and testing methods.

\subsection{Testing Program Overview}

A laboratory testing program was developed to quantify the effect that an increased volume fraction of fly ash has on the overall rate of hydration, setting times, and compressive strength development for a given concrete mixture at various temperatures.

Since the rate of hydration is dependent on the chemical composition of the cementitious system, two portland cement sources and four fly ash sources were investigated. The two portland cement sources varied in their alkali content, expressed as equivalent $\mathrm{Na}_{2} \mathrm{O}$. The "high-alkali" cement (HA) had a total alkali content of $1.04 \%$, whereas the "low-alkali" (LA) source had a total alkali content that ranged from of $0.53 \%$ to $0.57 \%$ based on the shipment. The four fly ash sources varied in their calcium oxide $(\mathrm{CaO})$ contents, ranging from approximately $1 \%$ to $28 \%$. Two Class C and two Class F fly ashes were selected to examine the effect that the fly ash chemical composition has on the hydration 
behavior and temperature sensitivity of the concrete mixture. Since the $\mathrm{CaO}$ content of a fly ash is largely a function of the coal source being burned, the range of $\mathrm{CaO}$ contents that were investigated represent the variety of fly ash sources available in the United States.

Twenty individual concrete mixtures were evaluated to examine the effect that increased volume fraction of fly ash, variation in fly ash $\mathrm{CaO}$ content, variation in cement alkali content, and changes in $w / \mathrm{cm}$ ratio have on the rate of hydration, setting behavior, compressive strength development, and temperature development of a concrete sample. Two control mixtures at $w / \mathrm{cm}$ ratios of 0.42 and 0.44 were completed for each cement source. The resulting four control mixtures were proportioned without the use of fly ash in the cementitious system to serve as a baseline for comparison purposes. These mixtures are representative of concrete that is commonly used in bridge deck applications in Alabama. In addition to the two control mixtures for each cement source, two concrete mixtures were completed for each fly ash source at $35 \%$ and $50 \%$ fly ash replacement by volume $(w / \mathrm{cm}$ ratios of 0.39 and 0.34 respectively). The fly ash mixtures were completed for each combination of cement and fly ash source leading to a total of sixteen separate HVFA concrete mixtures. In total, twenty concrete mixtures were investigated. Table 2.1 summarizes the concrete mixtures based on their $w / \mathrm{cm}$ ratio, cement source, and fly ash dosage.

The combined effect of temperature and fly ash replacement on the rate of hydration was also investigated. Therefore, each of the twenty concrete mixtures was batched at the following three temperatures: $40^{\circ} \mathrm{F}$, $73^{\circ} \mathrm{F}$, and $105^{\circ} \mathrm{F}\left(5^{\circ} \mathrm{C}, 23^{\circ} \mathrm{C}\right.$, and $\left.40^{\circ} \mathrm{C}\right)$. The hot $\left(105^{\circ} \mathrm{F}\right)$ and cold $\left(40^{\circ} \mathrm{F}\right)$ batching temperatures were selected to simulate the extreme upper and lower limits associated with concreting during the summer and winter months. By completing a mixture at all three temperatures, the temperature sensitivity of that particular mixture could be quantified.

The laboratory testing program may be separated into the following two phases:

1. Phase I: Hydration and setting time study, and

2. Phase II: Study of compressive strength development.

During Phase I, the temperature sensitivity of each mixture was determined by calculating the activation

TABLE 2.1

Concrete Mixture Summary for Each Fly Ash Source

\begin{tabular}{lcc}
\hline \hline Cement Source & w/cm & Fly Ash Dosage \\
\hline Low-Alkali Cement & 0.42 & $0 \%$ \\
& 0.44 & $0 \%$ \\
& 0.39 & $35 \%$ \\
& 0.34 & $50 \%$ \\
\hline High-Alkali Cement & 0.42 & $0 \%$ \\
& 0.44 & $0 \%$ \\
& 0.39 & $35 \%$ \\
& 0.34 & $50 \%$ \\
\hline
\end{tabular}

energy (E). This was achieved by performing isothermal calorimetry on wet-sieved mortar samples for each mixture at the three batching temperatures $\left(40^{\circ} \mathrm{F}, 73^{\circ} \mathrm{F}\right.$, and $105^{\circ} \mathrm{F}$ ). The initial and final setting times were also determined according to ASTM C403 (2008) for each batch to quantify the effect of mixing temperature on setting times. The equivalent ages for the initial and final setting times from each of the three batching temperatures were also calculated. Also during Phase I, semi-adiabatic calorimetry (SAC) testing was performed for each room temperature $\left(73^{\circ} \mathrm{F}\right)$ batch to determine the heat of hydration development of the HVFA concrete mixtures. SAC was also used to characterize the hydration development of each mixture. The results from Phase I (calculated activation energies and semiadiabatic test results) were used to develop a hydration model for HVFA concrete mixtures.

Phase II consisted of compressive strength testing for each concrete mixture batched at room temperature. For each mixture, a strength-maturity relationship was determined using the activation energy calculated in Phase I along with the measured temperature history of the specimens and their recorded compressive strengths. In addition to developing strength-maturity relationships for all mixtures, quality-control cylinders were prepared from each hot and cold batch. The qualitycontrol cylinders were tested at 28 days and compared to the room temperature results for that batch to ensure that the concrete was produced consistently at all three batching temperatures.

After developing the HVFA concrete hydration model, the results from Phases I and II were combined to evaluate the in-place performance of HVFA concrete when placed in selected transportation structures. The ConcreteWorks software program was used to estimate in-place temperature profiles for HVFA concrete mixtures placed under varying placement conditions. The placement conditions were varied by environmental conditions and concrete element type. Based on the in-place temperature profiles, the maximum in-place temperature, time to reach initial set, and compressive strength development could be determined for each HVFA concrete mixture. Using these results, the effect of fly ash dosage, fly ash type, placement conditions, and concrete element type on the in-place performance of HVFA concrete could be evaluated. The results from the ConcreteWorks analysis were designed to provide guidance on mixture proportioning and fly ash selection for HVFA concrete.

\subsection{Mixture Proportions}

Twenty different concrete mixtures (two control mixtures and eight fly ash mixtures per cement source) were used to quantify the effect of fly ash $\mathrm{CaO}$ content, fly ash dosage, and cement alkali content on the hydration and temperature development, setting times, and strength development of HVFA concrete. The nomenclature used for both the control and fly ash concrete mixtures throughout this report is shown in Figure 2.1. 


\begin{tabular}{|c|c|c|c|}
\hline \multicolumn{4}{|l|}{ Control: } \\
\hline Mix ID & w/c Ratio & Cement Source & Fresh Concrete Temperature \\
\hline$\uparrow$ & $\uparrow$ & $\uparrow$ & $\uparrow$ \\
\hline \multirow[t]{3}{*}{ CTRL } & 0.42 & Low-alkali (LA) & $73^{\circ} \mathrm{F}$ (Room) \\
\hline & 0.44 & High-alkali (HA) & $40^{\circ} \mathrm{F}$ (Cold) \\
\hline & & & $105^{\circ} \mathrm{F}(\mathrm{Hot})$ \\
\hline \multicolumn{4}{|c|}{$\begin{array}{l}\text { Example: CTRL } 44-\text { LA - 73F represents the control concrete mixture with a w/c ratio equal to } \\
0.44 \text { batched at } 73^{\circ} \mathrm{F} \text {. }\end{array}$} \\
\hline \multicolumn{4}{|l|}{ Fly Ash: } \\
\hline Cement Source & Fly Ash Dosage & $\mathrm{CaO}$ Content & Fresh Concrete Temperature \\
\hline$\uparrow$ & $\uparrow$ & $\uparrow$ & $\uparrow$ \\
\hline Low-alkali (LA) & $35 \%$ & $1 \%$ & $73^{\circ} \mathrm{F}$ \\
\hline \multirow[t]{3}{*}{ High-alkali (HA) } & $50 \%$ & $15 \%$ & $40^{\circ} \mathrm{F}$ \\
\hline & & $24 \%$ & $105^{\circ} \mathrm{F}$ \\
\hline & & $28 \%$ & \\
\hline \multicolumn{4}{|c|}{$\begin{array}{l}\text { Example: } \mathrm{LA}-35 \mathrm{FA}-15 \%-40 \mathrm{~F} \text { represents the HVFA concrete mixture with a } 35 \% \text { dosage } \\
\text { of the } 15 \% \mathrm{CaO} \text { fly ash batched at } 40^{\circ} \mathrm{F} \text {. }\end{array}$} \\
\hline
\end{tabular}

Figure 2.1 Nomenclature used for control and fly ash concrete mixtures.

The control mixture selected for this research has similar proportions to concrete routinely used for bridge deck applications. To evaluate the effect of fly ash type, fly ash dosage, and cement alkali content on hydration development, each of the HVFA concrete mixtures were proportioned by only changing the variable under consideration. Water-reducing admixture dosages were adjusted per mix to control workability. A target slump range of 2 to $7 \mathrm{in}$. was selected for all room temperature mixtures. A mid-range water-reducing admixture was used for all control mixtures. Due to low $w / \mathrm{cm}$ ratios, a high-range water-reducing (HRWR) admixture was used to provide adequate workability for all HVFA concrete mixtures.

Research conducted by Ravina and Mehta (1986) indicates that concrete proportioned with high fly ash dosages will experience delayed set times when compared to a control concrete with no fly ash replacement. Based on these results, an accelerating admixture was used for all HVFA concrete mixtures. The accelerating admixture dosages were selected with the goal of producing HVFA concrete mixtures that possess similar setting times to the control mixtures. The admixture supplier (W.R. Grace) recommended that accelerating admixture dosages of $15 \mathrm{oz} / \mathrm{cwt}$ and $30 \mathrm{oz} / \mathrm{cwt}$ be used for the $35 \%$ and $50 \%$ fly ash mixtures, respectively.

When proportioning HVFA concrete, Bilodeau et al. (2001) report that "the proportion of fly ash should be as high as possible and the water-cementitious materials ratio as low as possible to provide adequate early-age strength and durability." A preliminary mortar cube study was conducted to determine the $w / \mathrm{cm}$ ratios required to provide adequate early age strength (4 to 7 days) for the $35 \%$ and $50 \%$ fly ash mixtures. For this study, 2 in. mortar cubes were prepared with varying $w / \mathrm{cm}$ ratios for each fly ash dosage. The cubes were tested for compressive strength according to ASTM C109 (2007). Based on the results from this study, it was determined that the $w / \mathrm{cm}$ ratios should be 0.34 and 0.39 for the $50 \%$ and $35 \%$ fly ash mixtures, respectively.

All of the fly ash mixtures were proportioned to have the same paste volume as the control mixes; therefore, small adjustments were made to the fine aggregate proportions due to variations in the specific gravities of the fly ash sources. The adjustments made to the fine aggregate volumes were considered negligible. Mixture proportions for each of the twenty mixtures are given in Table 2.2. All batch weights for the aggregate are given at a saturated surface-dry (SSD) state. Also, the HRWR admixture dosages are given for both LA cement and HA cement mixtures. The LA cement dosages are given first, followed by the HA cement dosages in parentheses for each mixture.

\subsection{Raw Material Properties}

To eliminate variations during testing due to differences in material properties, a single source of each material was used for the full duration of laboratory testing. The source and properties of the aggregates and chemical admixtures are discussed in detail in Keith (2011).

\subsubsection{Portland Cement}

As was discussed previously, two cement sources with different total alkali contents were used during this research. The Type I/II "low-alkali" (LA) cement used for concrete testing was supplied by Lafarge North America from their Sugar Creek, Missouri plant. The Type I "high-alkali" (HA) cement, however, was supplied by W.R. Grace. The chemical compounds for 
TABLE 2.2

Concrete Mixture Proportions

\begin{tabular}{|c|c|c|c|c|c|c|c|c|c|c|}
\hline Item & CTRL42 & CTRL44 & $\begin{array}{c}\text { 35F } \\
\text { A-1\% }\end{array}$ & $\begin{array}{c}\text { 35F } \\
\mathbf{A}-15 \%\end{array}$ & $\begin{array}{c}\text { 35F } \\
\mathbf{A}-24 \%\end{array}$ & $\begin{array}{c}\text { 35F } \\
\mathbf{A}-\mathbf{2 8} \%\end{array}$ & $\begin{array}{c}50 F \\
\text { A-1 } \%\end{array}$ & $\begin{array}{c}\text { 50F } \\
\text { A-15\% }\end{array}$ & $\begin{array}{c}\mathbf{5 0 F} \\
\mathbf{A}-\mathbf{2 4} \%\end{array}$ & $\begin{array}{c}\text { 50F } \\
\mathbf{A - 2 8} \%\end{array}$ \\
\hline Water $\left(\mathrm{lb} / \mathrm{yd}^{3}\right)$ & 260 & 266 & 239 & 243 & 247 & 246 & 217 & 223 & 227 & 228 \\
\hline Cement $\left(\mathrm{lb} / \mathrm{yd}^{3}\right)$ & 620 & 603 & 445 & 438 & 434 & 431 & 378 & 369 & 363 & 360 \\
\hline Fly Ash (lb/yd $\left.{ }^{3}\right)$ & - & - & 166 & 185 & 195 & 200 & 260 & 289 & 303 & 310 \\
\hline Coarse Aggregate $\left(\mathrm{lb} / \mathrm{yd}^{3}\right)$ & 1920 & 1920 & 1920 & 1920 & 1920 & 1920 & 1920 & 1920 & 1920 & 1920 \\
\hline Fine Aggregate $\left(\mathrm{lb} / \mathrm{yd}^{3}\right)$ & 1210 & 1210 & 1199 & 1198 & 1198 & 1198 & 1182 & 1180 & 1179 & 1181 \\
\hline Water-Reducing Admixture (oz/cw t) & $6(9)$ & $4(9)$ & $3(9)$ & $1(2)$ & $0(1)$ & $1(2)$ & $4(6)$ & $2(5.5)$ & $4(5)$ & $1(2)$ \\
\hline Accelerating Admixture (oz/cw t) & - & - & \multicolumn{4}{|c|}{15} & \multicolumn{4}{|c|}{30} \\
\hline$w / \mathrm{cm}$ & 0.42 & 0.44 & \multicolumn{4}{|c|}{0.39} & \multicolumn{4}{|c|}{0.34} \\
\hline Fly Ash Dosage (by Volume) & - & - & \multicolumn{4}{|c|}{$35 \%$} & \multicolumn{4}{|c|}{$50 \%$} \\
\hline
\end{tabular}

Note: No mixtures are air-entrained. Water-reducing admixture dosages were adjusted to produce target slump of 2 to $7 \mathrm{in}$. Water-reducing admixture dosages presented in parentheses represent dosages used for HA cement mixtures.

TABLE 2.3

Cement Properties

\begin{tabular}{|c|c|c|c|}
\hline Parameter & HA Cement & LA Cement (1) & LA Cement (2) \\
\hline Tricalcium Silicate, $\mathrm{C}_{3} \mathrm{~S}(\%)$ & 52.51 & 56.33 & 60.40 \\
\hline Dicalcium Silicate, $\mathrm{C}_{2} \mathrm{~S}(\%)$ & 15.73 & 15.24 & 11.80 \\
\hline Tricalcium Aluminate, $\mathrm{C}_{3} \mathrm{~A}(\%)$ & 12.45 & 8.15 & 7.70 \\
\hline Tetracalcium Aluminoferrite, $\mathrm{C}_{4} \mathrm{AF}(\%)$ & 6.43 & 8.95 & 9.04 \\
\hline Free $\mathrm{CaO}(\%)$ & 0.58 & 1.35 & 1.35 \\
\hline Sulfur Trioxide, $\mathrm{SO}_{3}(\%)$ & 3.84 & 3.15 & 2.99 \\
\hline Magnesium Oxide, MgO (\%) & 2.63 & 1.62 & 2.04 \\
\hline Equivalent Alkali, $\mathrm{Na}_{2} \mathrm{O}_{\mathrm{eq}}(\%)$ & 1.04 & 0.53 & 0.57 \\
\hline Blaine Fineness $\left(\mathrm{m}^{2} / \mathrm{kg}\right)$ & 369 & 396 & 396 \\
\hline
\end{tabular}

each cement were computed according to ASTM C114 (2006) by an outside laboratory. The free lime content and Blaine fineness values were supplied by the producers. The Lafarge cement was received in two separate shipments corresponding to two separate production periods, denoted LA Cement (1) and LA Cement (2). A summary of the chemical composition for each cement as received from the independent laboratory is shown in Table 2.3.

\subsubsection{Fly Ash}

Four fly ash sources, two Class C and two Class F, were selected for testing. These fly ash sources were selected from across the United States to capture the range of fly ashes available to the concrete industry. For this project, the four sources were differentiated based on their calcium oxide $(\mathrm{CaO})$ content. Table 2.4 lists the four fly ash sources and identifies the supplier and location of the coal-fired powergeneration plant.

The four fly ash sources have varying chemical compositions based on the type of coal burned during their production. The chemical analysis results and specific gravity values for each of the fly ash sources as received from an independent laboratory are presented in Table 2.5. All testing was completed according to ASTM C311 (2006).

\subsubsection{Chemical Admixtures}

Water-reducing admixtures were used during the laboratory testing to help control the workability of both the control and fly ash mixtures. A mid-range water-reducing admixture was used for all control concrete mixtures, whereas a high-range water-reducing (HRWR) admixture was used for all HVFA concrete mixtures. Also, an accelerating admixture was used for all HVFA concrete mixtures with the goal of producing HVFA concrete with set times comparable to the control mixtures. All admixtures were supplied by W.R. Grace. The combined use of the specific HRWR admixture and accelerating admixture was recommended by W.R. Grace.

For the control mixtures, ADVA 140M was used to achieve adequate workability. The use of this admixture was required due to the selected $w / \mathrm{cm}$ ratios as well as the lack of entrained air. ADVA $140 \mathrm{M}$ is classified according to ASTM C494 (2005) as a Type A and Type $\mathrm{F}$ chemical admixture. For this research, the dosage levels used correspond to a mid-range water-reducing admixture. The ADVA 140M dosages for each control mixture are shown in Table 2.2.

For the $35 \%$ and $50 \%$ fly ash dosage mixtures, both a HRWR admixture, Advacast 575, and an accelerating admixture, Daraset HES, were used. Advacast 575 is a polycarboxylate-based Type F chemical admixture. The 
TABLE 2.4

Fly Ash Source Information

\begin{tabular}{llll}
\hline \hline Fly Ash ID & Supplier & Plant Name & Location \\
\hline FA-28 & Headwaters Resources & Burlington & Burlington, New Jersey \\
FA-24 & Lafarge North America & Muskogee & Fort Gibson, Oklahoma \\
FA-15 & Boral & Big Brown & Fairfield, Texas \\
FA-1 & ProAsh & Brandon Shores & Baltimore, Maryland \\
\hline
\end{tabular}

TABLE 2.5

Fly Ash Properties

\begin{tabular}{lcccc}
\hline \hline Parameter & FA-28 & FA-24 & FA-15 & FA-1 \\
\hline Calcium Oxide, $\mathrm{CaO}(\%)$ & 27.08 & 23.07 & 15.34 & 1.01 \\
Silicon Dioxide, $\mathrm{SiO}_{2}(\%)$ & 35.64 & 39.17 & 51.61 & 59.56 \\
Aluminum $\mathrm{Oxide}, \mathrm{Al}_{2} \mathrm{O}_{3}(\%)$ & 15.88 & 18.48 & 20.16 & 27.30 \\
Iron Oxide, $\mathrm{Fe}_{2} \mathrm{O}_{3}(\%)$ & 6.47 & 7.06 & 3.83 & 5.02 \\
Magnesium $\mathrm{Oxide}, \mathrm{MgO}(\%)$ & 6.45 & 5.04 & 0.71 & 0.06 \\
Sulfur Trioxide, $\mathrm{SO}_{3}(\%)$ & 2.14 & 1.24 & 0.88 & 0.90 \\
Sodium Oxide, $\mathrm{Na}_{2} \mathrm{O}(\%)$ & 2.19 & 1.56 & 1.47 & 2.53 \\
Potassium Oxide, $\mathrm{K}_{2} \mathrm{O}(\%)$ & 0.42 & 0.66 & 0.23 & 2.08 \\
Equivalent Alkali, $\mathrm{Na}_{2} \mathrm{O}_{\text {eq }}(\%)$ & 2.47 & 1.99 & 2.47 & 0.17 \\
Loss on Ignition $(\%)$ & 0.35 & 0.17 & 2.63 & \\
Specific Gravity & 2.71 & & \\
\hline
\end{tabular}

Advacast 575 dosages for each fly ash mixture are given in Table 2.2.

Daraset HES meets the requirements of ASTM C494 (2005) as a Type $\mathrm{C}$ chemical admixture, and was used to increase the rate of hydration for the HVFA mixtures. Daraset HES is a non-corrosive, non-chloride admixture. The accelerator dosages were kept constant at $15 \mathrm{oz} / \mathrm{cwt}$ for all $35 \%$ fly ash mixtures and $30 \mathrm{oz} / \mathrm{cwt}$ for all $50 \%$ fly ash mixtures, regardless of the batching temperature.

For this research, no air-entraining admixtures were used due to their temperature-dependent behavior and possible incompatibility with low-calcium fly ash sources.

\subsection{Concrete Production}

For each of the concrete mixtures, a $3.5 \mathrm{ft}^{3}\left(0.099 \mathrm{~m}^{3}\right)$ batch was produced at room temperature $\left(73^{\circ} \mathrm{F}\right)$. From each room temperature batch, cylinders were cast for compressive strength and semi-adiabatic calorimetry testing. Also, mortar was wet-sieved to provide setting test and isothermal calorimetry samples. In addition to the batches produced at room temperature, a $1.5 \mathrm{ft}^{3}$ $\left(0.042 \mathrm{~m}^{3}\right)$ batch was produced for each mixture at both the hot and cold batching temperatures. For each of these batches, mortar was wet-sieved to provide setting and isothermal calorimetry samples. Quality control cylinders were also cast for each hot and cold batch. The batching and mixing procedures described in the following subsections were followed for each concrete mixture that was produced to provide for consistent, reproducible results.
All materials were batched approximately one day before concrete production. At this time, the batch weights were approximated and all necessary materials were stored in 5-gallon buckets. Tight-fitting lids were placed on each bucket to prevent moisture-loss during storage. For the room temperature batches, the materials were left in the ambient conditions of an airconditioned laboratory, approximately $73^{\circ} \mathrm{F}\left(23^{\circ} \mathrm{C}\right)$ until moisture corrections were completed. For the hot and cold batches, however, the materials were stored in an insulated temperature-controlled environmental chamber. The environmental chamber was used to simulate hot and cold weather concreting conditions. To counteract the effect of mixing at ambient conditions, the materials for the hot and cold batches were over-heated and over-cooled to achieve the desired fresh concrete temperatures of 105 and $40^{\circ} \mathrm{F}$, respectively. For the hot batches, the environmental chamber was set to $120^{\circ} \mathrm{F}\left(49^{\circ} \mathrm{C}\right)$, whereas, it was set to $32^{\circ} \mathrm{F}$ $\left(0^{\circ} \mathrm{C}\right)$ for the cold batches.

Moisture corrections were completed according to ASTM C566 (1997) for all batches on the morning of mixing. Representative samples for both the fine and coarse aggregate were weighed, heated until all moisture was removed, and re-weighed to determine the moisture content to be used for determination of final batch weights. After completing the moisture corrections, the batch weights were adjusted and chemical admixtures were batched.

For each batch, the concrete mixing procedure set forth in ASTM C192 (2007) was followed. All room temperature batches were completed using a mixer with a total volume of $12 \mathrm{ft}^{3}\left(0.34 \mathrm{~m}^{3}\right)$. The hot and cold 
batches, however, were completed with a concrete mixture with a total volume of $6 \mathrm{ft}^{3}\left(0.17 \mathrm{~m}^{3}\right)$.

\subsection{Testing Methods}

Testing for this this phase of the project was broken into two phases. In the first testing phase, the activation energy was calculated for each mixture based on isothermal calorimetry results collected at each of the three batching temperatures. Also, initial and final set times were determined for each batch and the equivalent age corresponding to the initial and final set times were calculated. Finally, SAC testing was completed to characterize hydration development for each concrete mixture.

Phase II consisted of developing strength-maturity relationships for each concrete. To do this, the activation energies from Phase I were used along with the recorded temperature history of the cured compressive strength specimens.

\subsubsection{Fresh Concrete Properties Testing}

For each batch, the following fresh concrete tests were performed: slump (ASTM C143, 2008), unit weight (ASTM C138, 2008), temperature (ASTM C1064, 2008), and air content (ASTM C231, 2004). Fresh concrete testing was performed immediately following the mixing procedure described previously. For all batches, the tests were performed under the laboratory ambient conditions. After performing each of the fresh tests noted above, samples for penetration resistance testing, isothermal calorimetry, semi-adiabatic calorimetry, and compressive strength testing were collected.

\subsubsection{Penetration Resistance Testing}

Initial and final set times for each batch were determined by penetration resistance testing according to ASTM C403 (2006). According to ASTM C403, penetration resistance results are to be obtained using an upright penetrometer and a standard set of proctor needles.

Mortar was wet-sieved for the sampling can samples immediately after the fresh concrete testing was completed. The concrete was sieved through a No. 4 sieve $(4.75 \mathrm{~mm})$ with the aid of a vibrating table. When the sampling can was full, the mortar was consolidated with a vibrating table, and temperature recording was started with a maturity meter and Type $\mathrm{T}$ thermocouple wire. Although sieving for all mixture temperatures was completed in the ambient conditions of the lab, all hot and cold batch setting tests were conducted in the environmental chamber set to their respective batching temperature. A maturity meter was used to record the mortar temperatures throughout penetration resistance testing. The maturity meter recorded the mortar temperature at 30-minute increments for the duration of setting testing. With the recorded temperature history of the mortar samples, the initial and final set times could be converted to equivalent age, and the best-fit activation energy could be back-calculated.

\subsubsection{Isothermal Calorimetry}

In addition to wet-sieving mortar for the penetration resistance testing, mortar samples for each batch were also collected for isothermal calorimetry testing. Isothermal calorimetry was used to calculate the activation energy for each of the mixtures. For isothermal calorimetry testing, the mortar sample was allowed to hydrate under a constant temperature. The heat required to maintain this temperature was then recorded.

For this project, the AdiaCal TC unit manufactured by W.R. Grace and Company was used for all isothermal calorimetry. The AdiaCal TC unit has eight separate cells for testing. The isothermal calorimeter was set to the appropriate batching temperature approximately 24 hours before testing so that it could reach equilibrium. Similar to the set test samples, the mortar samples for isothermal calorimetry were wet-sieved through a No. 4 sieve $(4.75 \mathrm{~mm})$ immediately following fresh concrete testing. The mortar was placed in 2 in. (5 cm.) diameter plastic containers and weighed as quickly as possible to reduce the amount of heat lost or gained. After weighing, the sample was placed in the calorimeter and the data acquisition for that cell was started. All samples were tested for 72-94 hours, depending on mixing temperature, to ensure that all significant hydration was captured.

\subsubsection{Semi-Adiabatic Calorimetry}

Semi-adiabatic calorimetry (SAC) testing was completed for each batch to measure the heat of hydration. Based on the SAC testing results, the hydration parameters could be calculated for each mixture. The SAC used is referred to as an iQdrum device and is manufactured by Digital Site Systems, located in Pennsylvania. The iQdrum calorimeter accommodates one concrete sample at a time. For this project, 6x12 cylinders were used for all SAC testing. The sample is insulated on all sides. Each of the three iQdrum calorimeters that were used during testing was calibrated to determine correction factors to be applied during data analysis. Temperature measurements were recorded by inserting a Type $\mathrm{T}$ thermocouple directly into the concrete sample. The thermocouple was covered with a plastic sleeve before inserting it into the concrete to allow for easy removal at the end of testing and ultimately extend the life of the thermocouple sensor. A flux sensor and ambient temperature sensor are located within the unit and take recordings at the same interval as the thermocouple.

Approximately 24 hours before mixing, the calorimeter was opened and previous samples were removed to allow the device to reach equilibrium with the surrounding laboratory environment. When testing the hot and cold batches, an "equilibrium" sample that had been conditioned to the batching temperature in the environmental chamber was placed in the calorimeter approximately one hour before testing to allow the device to be as close to the fresh concrete temperature as possible. SAC testing was conducted for at least five 
days after mixing. For each batch, the cylinders for semi-adiabatic testing were cast and placed within the calorimeters as quickly as possible to minimize the amount of heat loss or gain from the specimen.

\subsubsection{Compressive Strength Testing and Curing Methods}

After measuring the fresh concrete properties and preparing samples for calorimetry and penetration resistance testing, $4 \times 8$ in. cylinders were cast for compressive strength testing according to ASTM C192 (2007). After finishing was complete, each cylinder was capped with a plastic lid to protect against moisture loss. The cylinders were left undisturbed in the laboratory for the first 24 hours. Approximately 24 hours after mixing, the cylinders were de-molded, labeled, and transported to the moist-curing room. The moist-curing room was kept at a constant temperature of $73^{\circ} \mathrm{F}$ $\left(23^{\circ} \mathrm{C}\right)$ and $100 \%$ relative humidity.

For each of the room temperature batches, twentyfive $4 \times 8$ in. cylinders were cast for compressive strength testing. Also, one cylinder was cast from each room temperature batch to collect temperature versus time data for the duration of curing. These data were collected with the use of a maturity meter with a thermocouple embedded within the center of the cylinder. The cylinders for all room temperature batches were moistcured until testing. Compressive strength testing was completed at the following concrete ages: 12 hours, 1 day, 2 days, 4 days, 7 days, 14 days, 28 days, and 56 days. At each test age, three cylinders were tested for compressive strength according to ASTM C39 (2004). The compressive strength testing results and recorded cylinder temperature histories were used to develop strength-maturity relationships for each concrete mixture.

For each of the hot and cold batches, three quality control $4 \times 8$ cylinders were cast. These cylinders were continuously moist-cured until testing at 28 days. All compressive strength testing was conducted according to ASTM C39 (2004).

\section{PRESENTATION OF RESULTS}

In this section, results from the laboratory testing program are presented and reviewed. A summary of all data collected for each concrete mixture at each of the three batching temperatures may be found in Keith (2011).

\subsection{Phase I Testing Results}

\subsubsection{Set Times from Penetration Resistance Testing}

Initial and final set times were determined for all concrete batches according to ASTM C403 (2006). As previously described, a mortar sample was wet-sieved from the fresh concrete immediately following fresh concrete testing, and penetration resistance testing was completed at the respective mixing temperatures.
A maturity meter was used to record the mortar temperature for the duration of the test. According to ASTM C403 (2006), initial set and final set are defined as the times when the penetration resistance is equal to 500 psi and 4000 psi, respectively. To determine the initial and final set times for each concrete mixture, the penetration resistance data were plotted versus concrete age. Regression analysis was then performed to fit a power function to the data, as per ASTM C403 (2006).

In the following four subsections, the effect of fly ash dosage, fly ash $\mathrm{CaO}$ content, cement alkali content, and mixing temperature on the setting times of HVFA concretes will be investigated. For all penetration resistance plots presented in this section, the solid lines represent the best-fit power curves obtained from regression analysis. Also, data markers are used to differentiate between the mixtures, and do not represent actual test points, unless otherwise indicated.

3.1.1.1 Effect of Fly Ash Dosage on Set Time. In Figures 3.1 and 3.2, penetration resistance plots are presented for all $73^{\circ} \mathrm{F}$ batches completed with the LA and HA cement sources, respectively. From these figures it can be seen that an increase in fly ash dosage resulted in delayed initial and final set times for all mixtures proportioned with both the Class $\mathrm{C}$ and Class F fly ash sources, except for the LA cement concrete mixtures proportioned with the $15 \% \mathrm{CaO}$ fly ash source. It should be noted, that the accelerating admixture dosage was doubled for the $50 \%$ fly ash mixtures (30 oz/cwt) when compared to the $35 \%$ fly ash mixture dosages (15 oz/cwt). Also, the $w / \mathrm{cm}$ ratio was not constant for all mixtures. Therefore, the effect of fly ash dosage alone on concrete setting time could not be completely isolated. The setting test results for all concrete mixtures batched at $73^{\circ} \mathrm{F}$ are summarized in Table 3.1. It is shown that the HVFA concrete proportioned with lowcalcium Class $\mathrm{F}$ ashes generally have similar, if not accelerated, set times when compared to the control mixtures for each cement source.

3.1.1.2 Effect of Fly Ash CaO Content on Set Time. As was earlier discussed that the initial and final set times for HVFA concrete will be delayed as the fly ash $\mathrm{CaO}$ content is increased. HVFA concrete mixtures proportioned with a Class $\mathrm{C}$ fly ash will exhibit longer set times when compared to a similar concrete proportioned with a Class F fly ash. In Figure 3.3, penetration resistance data for ASTM C403 (2006) are presented for all $73^{\circ} \mathrm{F}$ concrete batches.

From Figure 3.3 it may be seen that as the fly ash $\mathrm{CaO}$ content increases for a given dosage, the set times are delayed. From Table 3.1, it is shown that this trend was consistent for all HVFA concrete mixtures tested with the exception of the HA-35FA-15\% mixture. According to Ravina and Mehta (1986), the rate of $\mathrm{C}_{3} \mathrm{~A}$ hydration is highly dependent on the presence of sulfate ions in solution. The sulfate contents for the fly ashes were $0.05 \%$ for FA-1, $0.71 \%$ for FA-15, $1.24 \%$ for FA-24, and $2.14 \%$ for FA-28. Therefore, the increased 

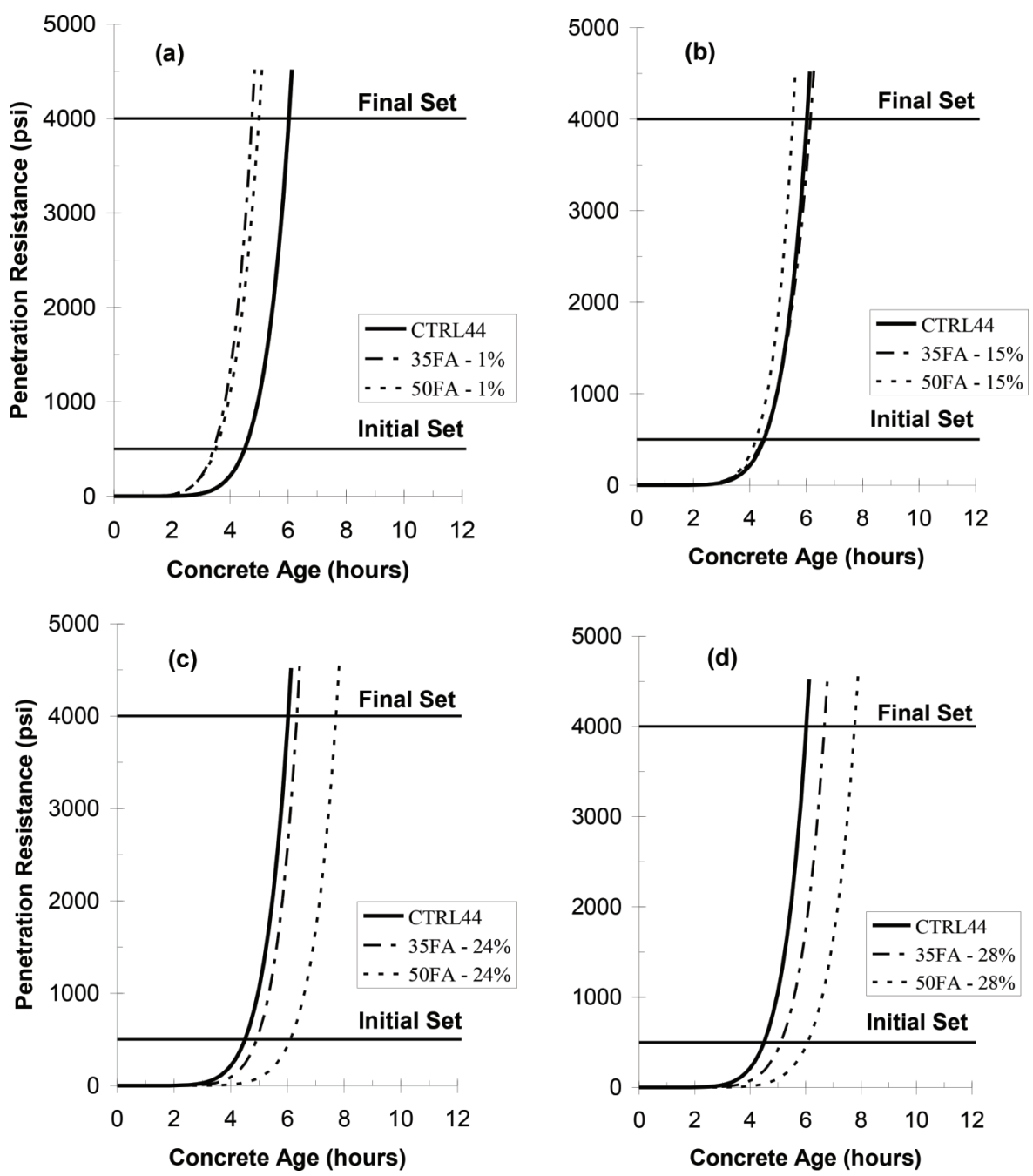

Figure 3.1 Effect of fly ash dosage on set times for (a) LA-1\%, (b) LA-15\%, (c) LA-24\%, and (d) LA-28\% mixtures (73 ${ }^{\circ}$ F).

set time delays observed for concrete proportioned with Class $\mathrm{C}$ fly ashes may be attributed to the higher sulfate contents of these ashes.

3.1.1.3 Effect of Cement Alkali Content on Set Time. The setting times of HVFA concretes are also influenced by the alkali content of the cement. For this project, two cement sources were investigated: one low alkali (LA) source and one high alkali (HA) source. The chemical composition of each cement source is presented in Table 2.3. The effect of cement alkali content on set times is shown in Figure 3.3 and Table 3.1. Set times were delayed for all HVFA concrete mixtures proportioned with the HA cement source when compared to the same mixture produced with the LA cement source. The delay in final set times ranged from 0.39 hours (35FA-15\%) to 2.39 hours (50FA-1\%).

3.1.1.4 Effect of Curing Temperature on Set Time. The effect of curing temperature on concrete setting time is illustrated in Figure 3.4 for four selected HVFA concrete mixtures. In this figure, the data markers represent penetration resistance measurements taken during testing. For this project, set times were accelerated for all hot batches $\left(105^{\circ} \mathrm{F}\right)$ and delayed for all cold batches $\left(40^{\circ} \mathrm{F}\right)$, when compared to the room temperature $\left(73^{\circ} \mathrm{F}\right)$ results. As previously discussed, the rate of hydration is temperature dependent. At high curing temperatures, the rate of hydration is increased, and initial and final setting times are accelerated. Similarly, curing at low temperatures decreases the rate of hydration and set times are delayed.

The temperature history was measured for each mortar sample during penetration resistance testing. An example of a recorded mortar temperature history is shown in Figure 3.5. The data markers shown in the mortar temperature history are used to distinguish between data series and do not represent actual data points.

Since the mortar was sieved at room temperature for all batches, all hot batch samples experienced heat loss and cold batch samples experienced heat gain during the sieving process. To account for this effect, linear interpolation was used between the measured fresh concrete temperature and the first recorded mortar 

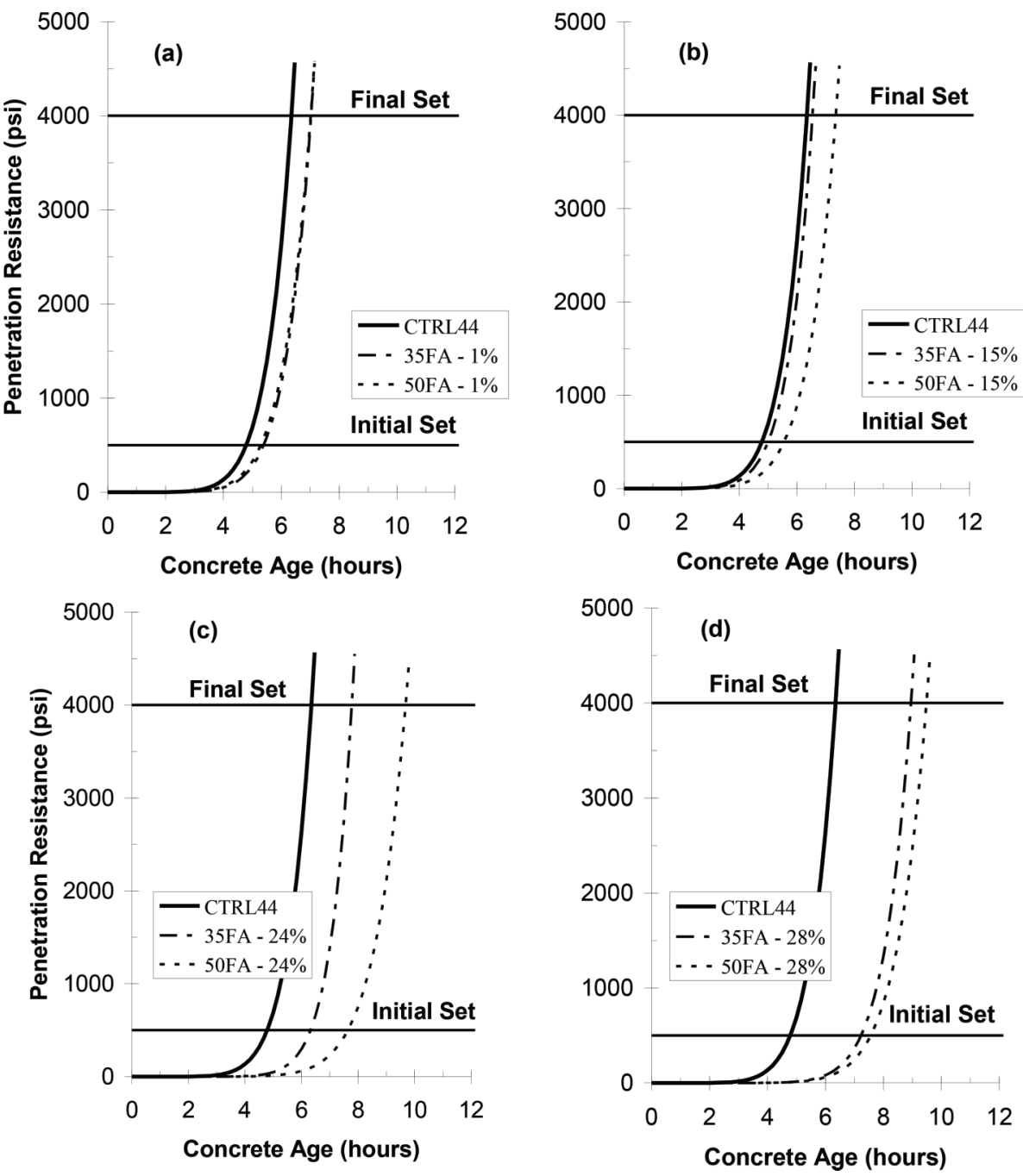

Figure 3.2 Effect of fly ash dosage on set times for (a) HA-1\%, (b) HA-15\%, (c) HA-24\%, and (d) HA-28\% mixtures $\left(73{ }^{\circ}\right.$ F).

temperature for all batches. The recorded temperature history for each mortar sample was used to calculate the equivalent age for initial and final set. Pinto and Hover (1999), Schindler (2004), and Weakley (2010) reported that although set times will vary according to curing temperature, the calculated equivalent ages for these set times will not. Therefore, initial and final set times may be estimated for various placement conditions if the activation energy of the mixture, equivalent age at setting, and concrete temperature history are known. Based on this research, best-fit activation energies were calculated for each mixture based on the setting results obtained for the hot, room, and cold batches. The best-fit activation energies from penetration resistance testing are presented later alongside the activation energies calculated from isothermal calorimetry results. Penetration resistance versus equivalent age results for the LA-35FA-15\% and HA-50FA-1\% mixtures are presented in Figure 3.6. The best-fit activation energy for the setting data is also presented for each mixture. The equivalent ages at initial set calculated using the procedure outlined above are used to estimate the in-place setting times of HVFA concretes with the ConcreteWorks temperature prediction software.

3.1.1.5 Setting Test Results Summary. As can be seen in Figures 3.1 through 3.4, fly ash dosage, fly ash $\mathrm{CaO}$ content, cement alkali content, and curing temperature all effect the time of setting for HVFA concrete mixtures. Setting times are delayed when the fly ash dosage is increased, for a similar $w / \mathrm{cm}$ ratio. Similarly, setting times are also delayed when the fly ash $\mathrm{CaO}$ content increases or the alkali content of the cement increases. Finally, setting times for HVFA concrete mixtures are temperature dependent. A decrease in curing temperature can cause significant set time delays, especially when high-calcium, Class $\mathrm{C}$ fly ashes are used at high dosages.

\subsubsection{Isothermal Calorimetry Results}

Mortar samples were wet-sieved from each concrete batch for isothermal calorimetry testing. Isothermal 

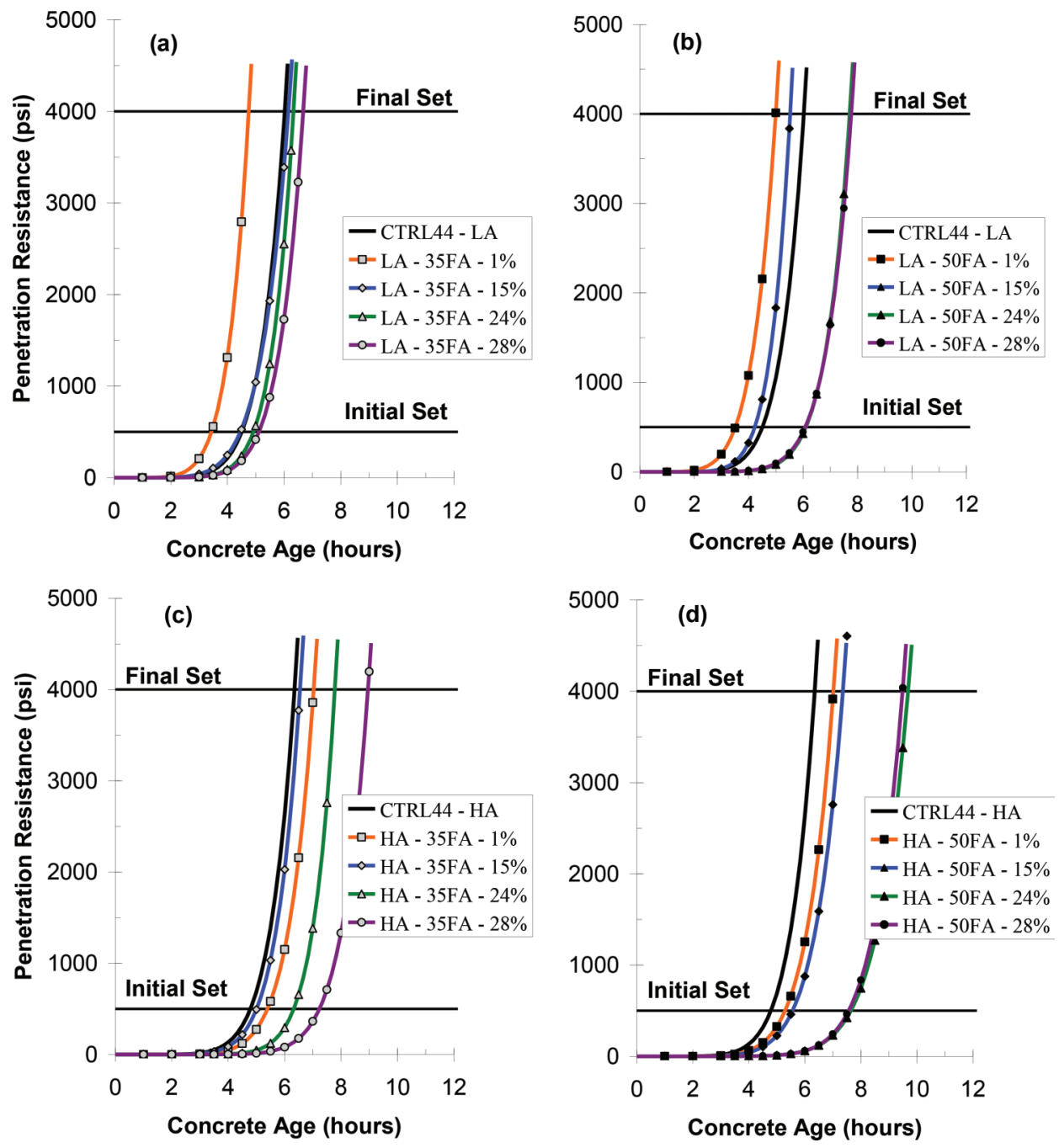

Figure 3.3 $73^{\circ} \mathrm{F}$ Setting test results for (a) LA-35FA, (b) LA-50FA, (c) HA-35FA, and (d) HA-50FA mixtures.

TABLE 3.1

Summary of Initial and Final Set Times for All $73^{\circ} \mathrm{F}$ Mixtures

\begin{tabular}{|c|c|c|c|c|}
\hline \multirow[b]{2}{*}{ Mixture } & \multicolumn{2}{|c|}{ LA Cement } & \multicolumn{2}{|c|}{ HA Cement } \\
\hline & Initial Set (hours) & Final Set (hours) & Initial Set (hours) & Final Set (hours) \\
\hline CTRL42 & 4.33 & 5.71 & 4.78 & 6.12 \\
\hline CTRL44 & 4.51 & 6.03 & 4.79 & 6.35 \\
\hline $35 \mathrm{FA}-1 \%$ & 3.44 & 4.76 & 5.40 & 7.03 \\
\hline $35 \mathrm{FA}-15 \%$ & 4.46 & 6.16 & 4.01 & 6.55 \\
\hline $35 \mathrm{FA}-24 \%$ & 4.93 & 6.34 & 6.32 & 7.78 \\
\hline $35 \mathrm{FA}-28 \%$ & 5.12 & 6.68 & 7.23 & 8.96 \\
\hline $50 \mathrm{FA}-1 \%$ & 3.51 & 5.00 & 5.30 & 7.00 \\
\hline $50 \mathrm{FA}-15 \%$ & 4.23 & 5.53 & 5.63 & 7.45 \\
\hline $50 \mathrm{FA}-24 \%$ & 6.11 & 7.72 & 7.64 & 9.68 \\
\hline $50 \mathrm{FA}-28 \%$ & 6.08 & 7.78 & 7.57 & 9.49 \\
\hline
\end{tabular}

calorimetry results are given in Figures 3.7 and 3.8 (rate of heat evolution) for all $73^{\circ} \mathrm{F}$ batches. Each isothermal calorimetry sample was tested for 72 hours to ensure that all significant hydration was captured. The results were normalized per gram of cementitious material $\left(\mathrm{g}_{\mathrm{cem}}\right)$.
Based on Figures 3.7 and 3.8, the rate of heat evolution for a mortar sample is dependent upon fly ash dosage, fly ash $\mathrm{CaO}$ content, cement chemical composition, $w / \mathrm{cm}$ ratio, and the use of chemical admixtures. In Figure 3.9, the effect of curing temperature on the rate 

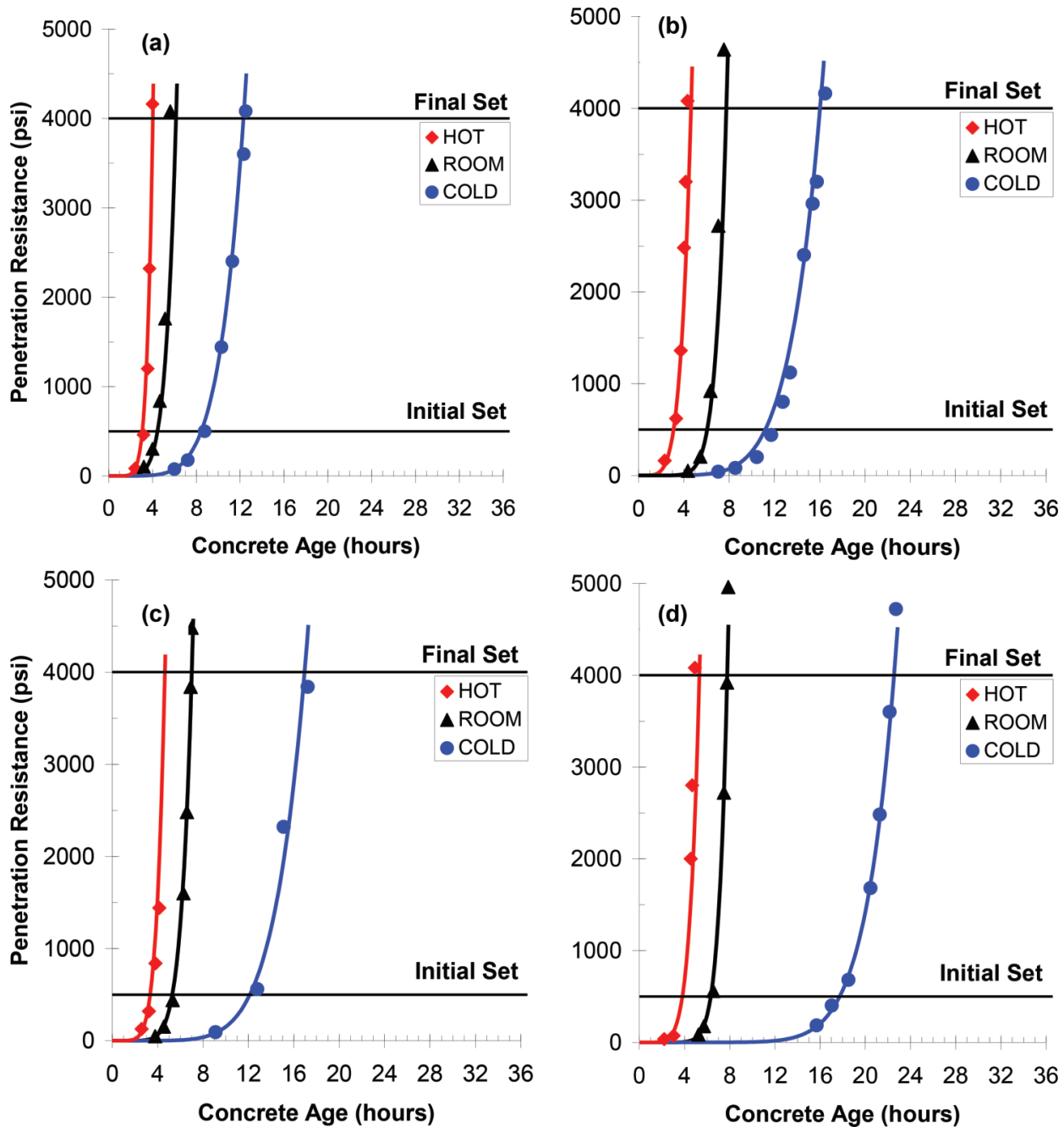

Figure 3.4 Effect of temperature on HVFA concrete set times for (a) LA-35FA-15\%, (b) LA-50FA-28\%, (c) HA-50FA-1\%, and (d) HA-35FA-24\%.

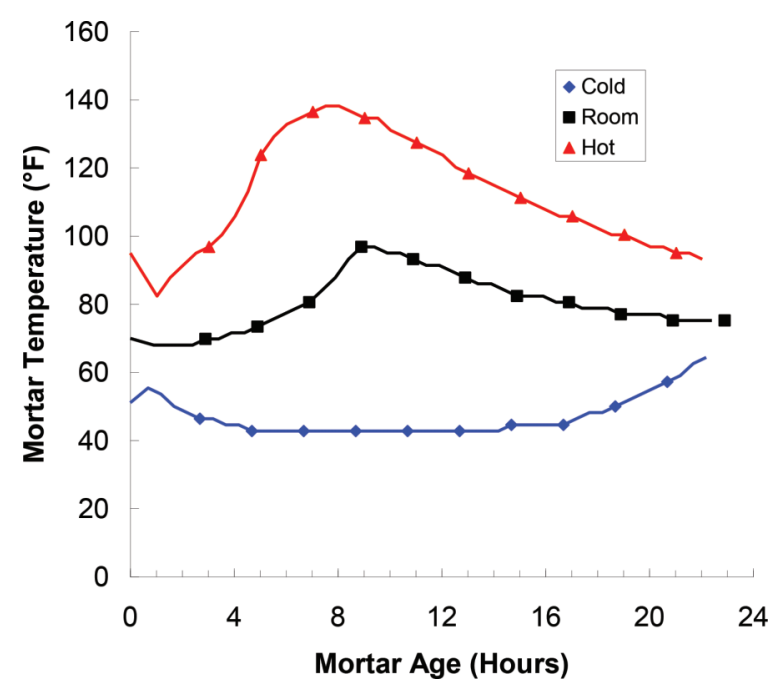

Figure 3.5 Sample mortar temperature history (LA-35F A-28\%). of heat evolution is illustrated for selected HVFA concrete mixtures. The results in Figure 3.9 indicate that the rate of heat evolution varies significantly based on the curing temperature.

The temperature sensitivity of a concrete mixture is represented by its activation energy. The activation energy is used in the equivalent age maturity method to convert concrete properties determined at a reference temperature to any other curing temperature. The activation energy was determined for each concrete mixture. To determine the activation energy for each concrete mixture, isothermal calorimetry was performed on sieved mortar samples from the hot, cold, and room temperature batches. At each mixing temperature, the degree of hydration equation (Equation 1.12) was fit to the isothermal calorimetry data. Schindler (2004) determined that the hydration shape parameter $(\beta)$ and the ultimate degree of hydration $\left(\alpha_{u}\right)$ are independent of curing temperature; therefore, they were treated as constants for each mixture. The hydration time parameter $(\tau)$ was 

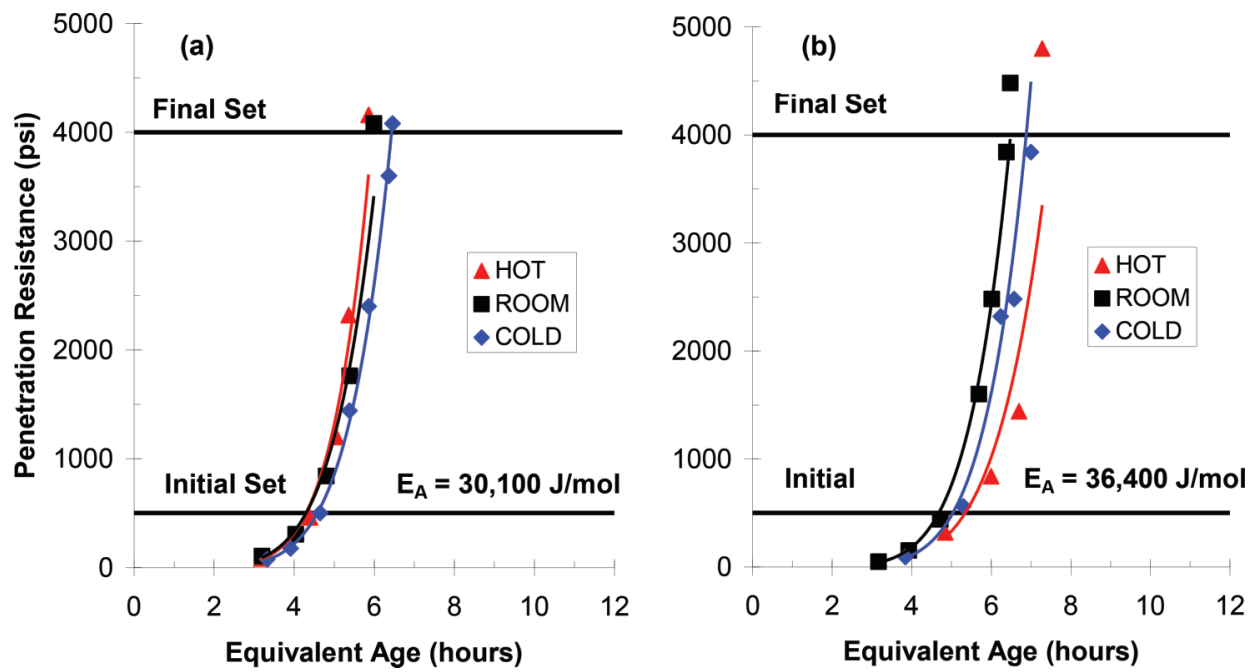

Figure 3.6 Penetration resistance versus equivalent age results for (a) LA-35FA-15\% and (b) HA-50FA-1\% concrete mixtures.
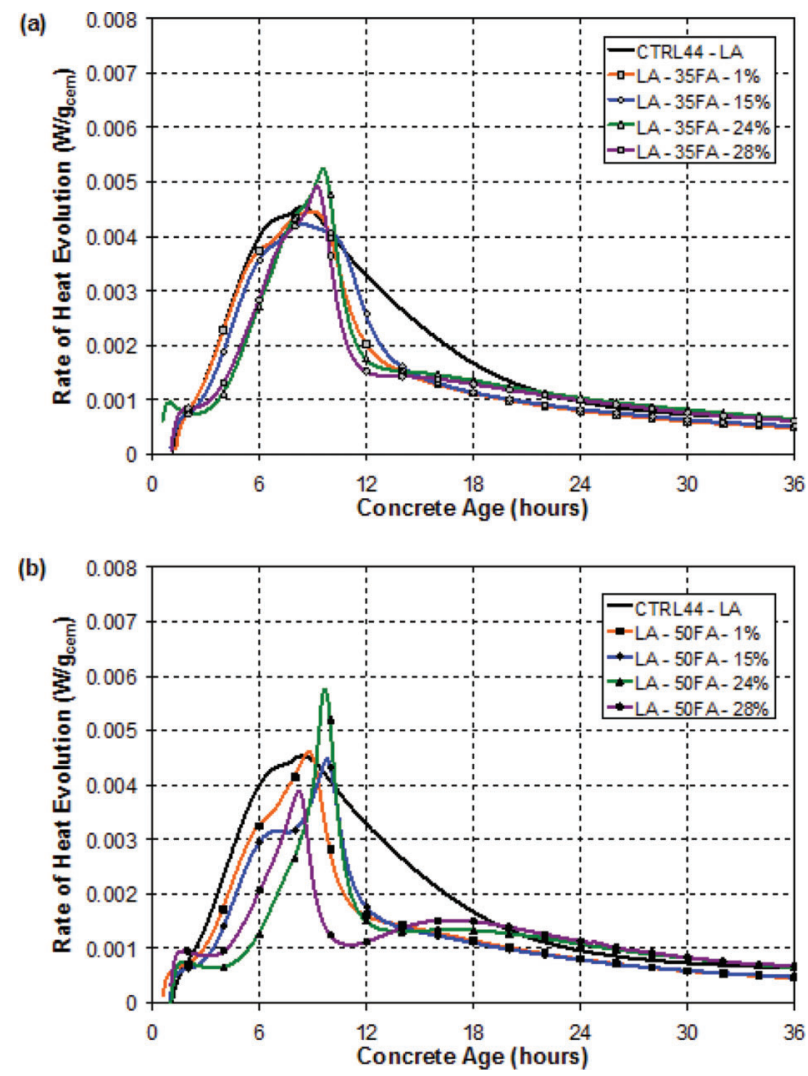

Figure 3.7 Rate of heat evolution for (a) LA-35FA and (b) LA-50FA mixtures.

the only variable that changed according to curing temperature.

After determining $\tau$ for each curing temperature, an Arrhenius plot $(\ln (\mathrm{k})$ vs. $1 / \mathrm{T})$ was developed for each mixture. Activation energies were calculated for each concrete mixture by multiplying the negative slope of the best-fit line through the Arrhenius data by the universal gas constant, R (ASTM C1074, 2004; Carino, 2004). Arrhenius rate plots and calculated activation
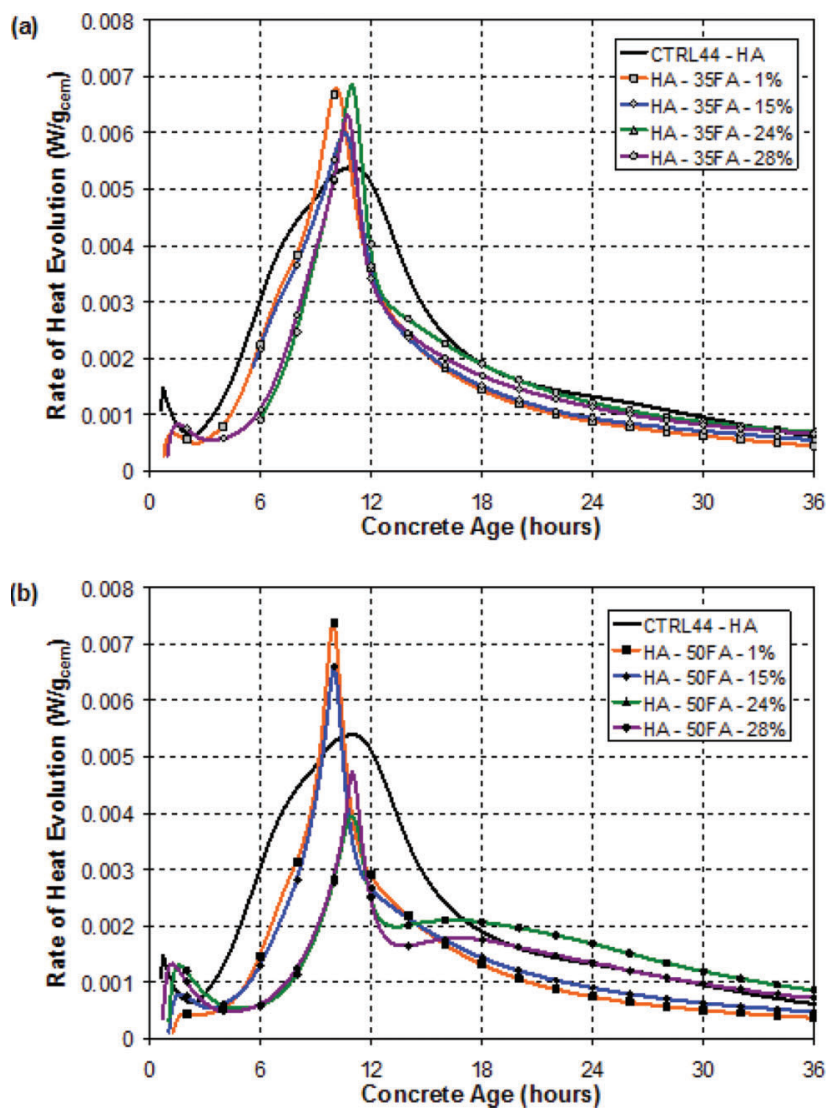

Figure 3.8 Rate of heat evolution for (a) HA-35FA and (b) HA-50FA mixtures.

energies are presented in Figure 3.10 for selected HVFA concrete mixtures.

A summary of all activation energies calculated from both isothermal calorimetry and penetration resistance testing are given in Tables 3.2 and 3.3 for the LA and HA cement sources, respectively. Also, the percent difference between the activation energy calculated from 

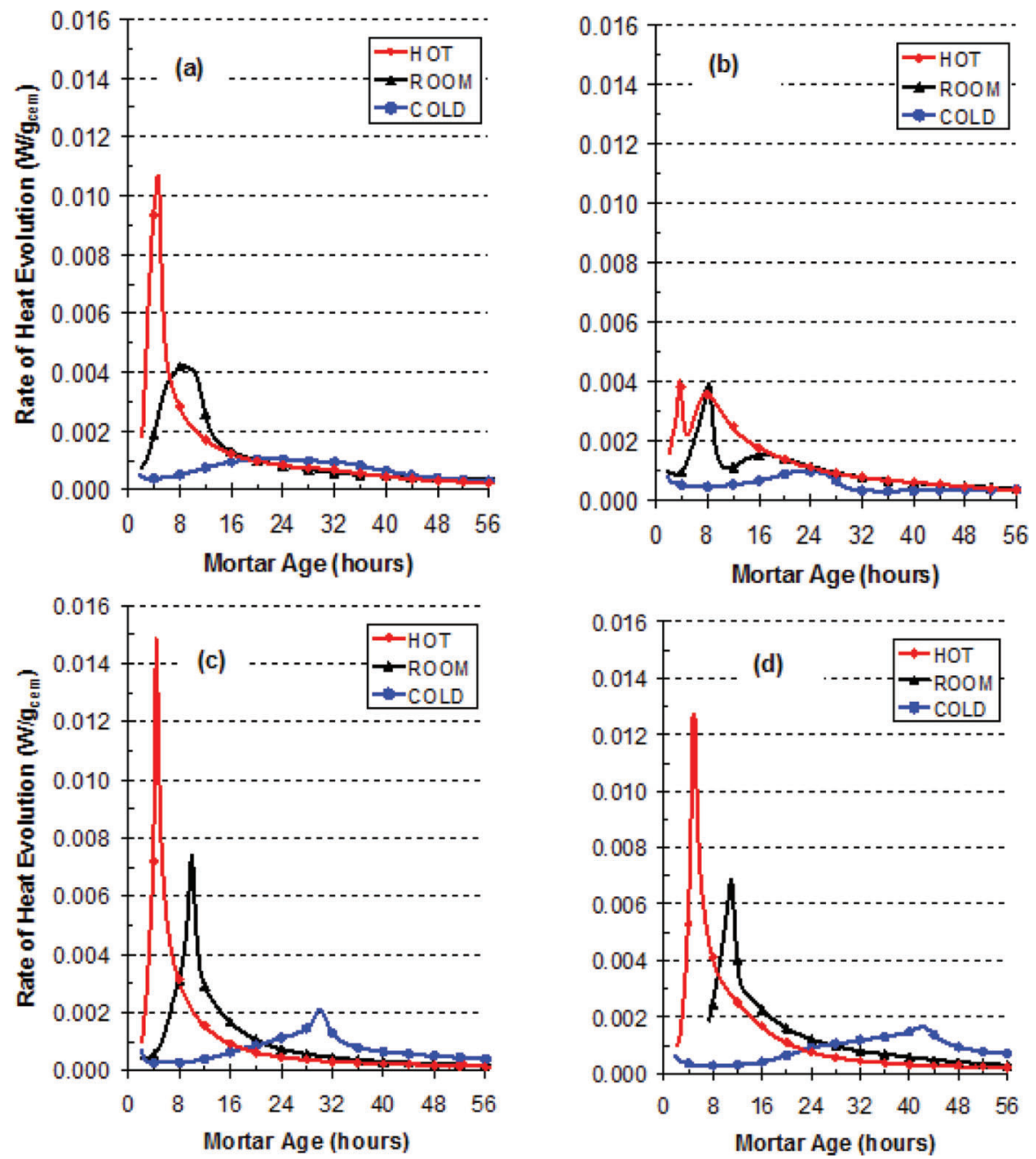

Figure 3.9 Rate of heat evolution for (a) LA-35FA-15\%, (b) LA-50FA-28\%, (c) HA-50FA-1\%, and (d) HA-35FA-24\%.

each test method is presented for each concrete mixture. The activation energy values calculated from isothermal calorimetry were used for development of the HVFA concrete hydration model. The HVFA concrete hydration model is developed, presented and discussed in section 4 . All activation energies were determined to the nearest $100 \mathrm{~J} / \mathrm{mol}$.

In Tables 3.2 and 3.3, it is shown that the activation energies calculated from penetration resistance data compare well to those obtained from isothermal calorimetry testing. Only three mixtures had a percent difference between the two activation energy values greater than $20 \%$. The activation energy values calculated from isothermal calorimetry for the LA cement mixtures ranged from $28,300 \mathrm{~J} / \mathrm{mol}$ (35FA-1\%) to $36,300 \mathrm{~J} / \mathrm{mol}$ (35FA-15\%). Similarly, the activation energy values for the HA cement mixtures ranged from 28,400 J/mol (35FA-28\%) to $34,800 \mathrm{~J} / \mathrm{mol}$ (35FA-1\%). No consistent trends in activation energies could be established based on fly ash dosage or fly ash type for the HVFA concrete mixtures.

\subsubsection{Semi-Adiabatic Calorimetry Results}

Semi-adiabatic calorimetry (SAC) testing was conducted for all mixtures batched at $73^{\circ} \mathrm{F}$ to characterize the hydration development of HVFA concrete. Testing was conducted for 120 hours to ensure that the concrete sample had hydrated sufficiently. Temperature measurements were recorded every fifteen minutes. The results from SAC testing were used to develop a database of bestfit hydration parameters to be used for generation of the HVFA concrete hydration model. A summary of the bestfit hydration parameters from SAC testing is presented in section 4 . In the following subsections, the effect of fly ash dosage and fly ash $\mathrm{CaO}$ content on the temperature development of HVFA concrete mixtures is examined.

3.1.3.1 Effect of Fly Ash Dosage on Heat of Hydration. Heat generation per volume of concrete $\left(\mathrm{MJ} / \mathrm{m}^{3}\right)$ plots for all $73^{\circ} \mathrm{F} \mathrm{LA} \mathrm{cement} \mathrm{and} \mathrm{HA} \mathrm{cement} \mathrm{concrete}$ mixtures are presented in Figures 3.11 and 3.12, respectively. The heat generation plots were calculated by multiplying the measured heat of hydration of the 

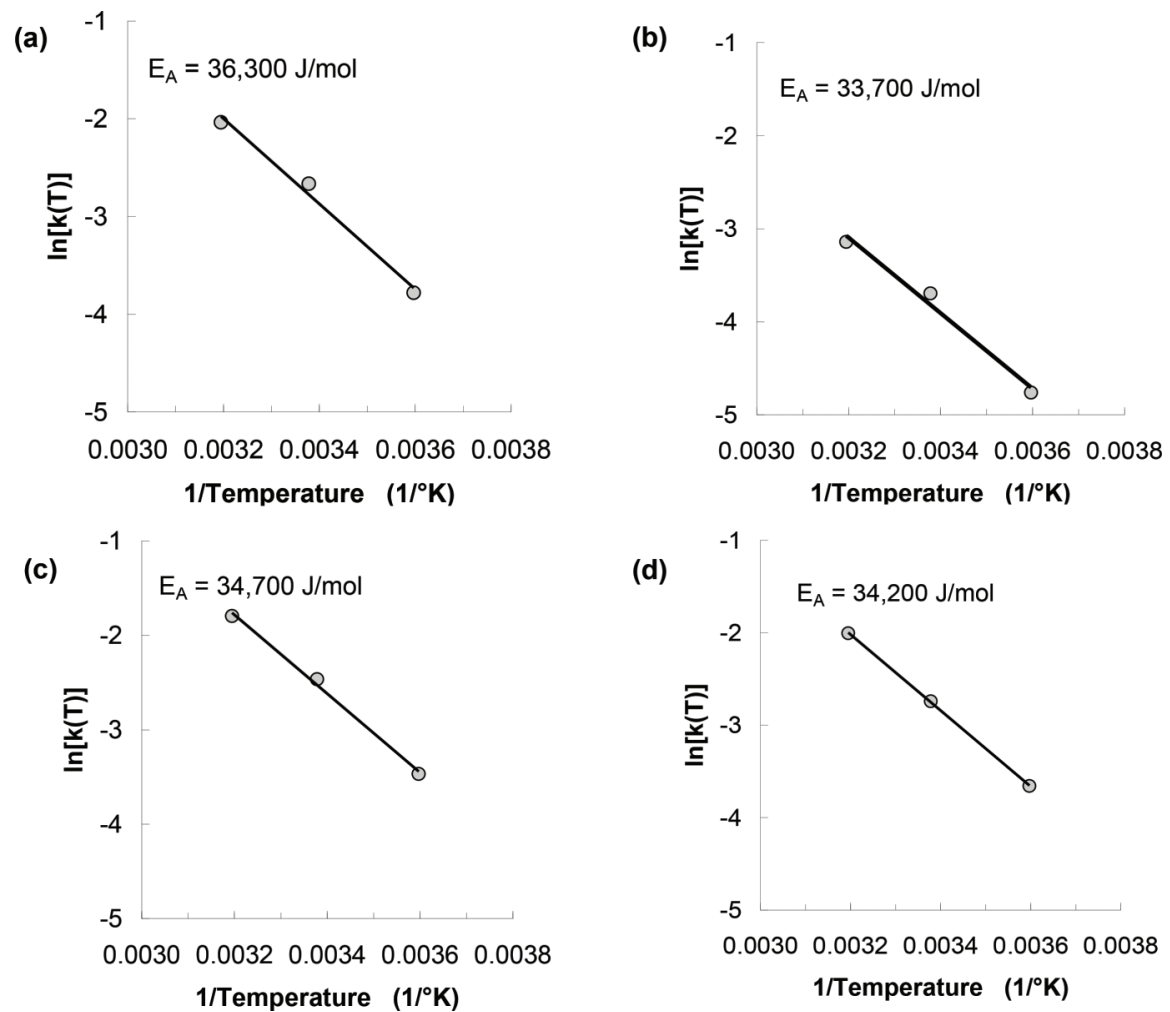

Figure 3.10 Arrhenius rate plots for (a) LA-35FA-15\%, (b) LA-50FA-28\%, (c) HA-50FA-1\%, and (d) HA-35FA-24\%.

TABLE 3.2

LA Cement Mixtures Activation Energy Summary

\begin{tabular}{lccc}
\hline \hline Mixture & $\begin{array}{c}\text { Isothermal Calorimetry } \\
\text { Activation Energy, E (J/mol) }\end{array}$ & $\begin{array}{c}\text { Penetration Resistance } \\
\text { Activation Energy, E(J/mol) }\end{array}$ & $\begin{array}{c}\text { Percent Difference } \\
(\%)\end{array}$ \\
\hline CTRL42 & 32,500 & 30,400 & 6.7 \\
CTRL44 & 33,500 & 30,100 & 10.7 \\
35FA-1\% & 28,300 & 32,600 & 14.1 \\
35FA-15\% & 36,300 & 30,100 & 18.7 \\
35FA-24\% & 32,600 & 31,200 & 4.4 \\
35FA-28\% & 35,700 & 31,200 & 13.5 \\
50FA-1\% & 29,600 & 22,800 & 3.7 \\
50FA-15\% & 30,300 & 29,200 & 0.3 \\
50FA-24\% & 29,900 & 30,000 & 1.2 \\
50FA-28\% & 33,700 & 33,300 & \\
\hline
\end{tabular}

TABLE 3.3

HA Cement Mixtures Activation Energy Summary

\begin{tabular}{lccc}
\hline \hline Mixture & $\begin{array}{c}\text { Isothermal Calorimetry } \\
\text { Activation Energy, E (J/mol) }\end{array}$ & $\begin{array}{c}\text { Penetration Resistance } \\
\text { Activation Energy, E (J/mol) }\end{array}$ & $\begin{array}{c}\text { Percent Difference } \\
\text { (\%) }\end{array}$ \\
\hline CTRL42 & 38,000 & 37,600 & 1.1 \\
CTRL44 & 35,600 & 35,400 & 0.6 \\
35FA - 1\% & 34,800 & 36,300 & 3.4 \\
35FA - 15\% & 32,000 & 38,300 & 17.9 \\
35FA - 24\% & 34,200 & 37,300 & 19.1 \\
35FA - 28\% & 28,400 & 34,400 & 4.8 \\
50FA - 1\% & 34,700 & 36,400 & 17.4 \\
50FA - 15\% & 33,500 & 39,900 & 45 \\
50FA - 24\% & 30,800 & 45,000 & 32 \\
50FA - 28\% & 28,400 & 42,500 & \\
\hline
\end{tabular}


sample by the cementitious material content of that particular concrete mixture. For both cement sources, an increase in fly ash dosage resulted in a decreased heat of hydration and less heat generated per volume of concrete, with the exception of the LA-15\% and LA-28\% concrete mixtures. For these mixtures, the heat generation was approximately equal for both fly ash dosages.

For the HA cement, Class F fly ash concrete mixtures, the $50 \%$ fly ash dosage resulted in significant reductions in heat generation $\left(10-38 \mathrm{MJ} / \mathrm{m}^{3}\right)$ when compared to the control (CTRL44-HA). The significant reduction in heat generation of the HVFA mixtures proportioned with the Class $\mathrm{F}$ fly ashes agree with past research, as described in subsection 2.5.2.5. Langley et al. (1992) reported that the peak in-place temperatures of $3.05 \times 3.05 \times 3.05$ meter concrete blocks were reduced by $52^{\circ} \mathrm{F}$ when a $55 \%$ dosage of Class $\mathrm{F}$ fly ash was used.

When compared to the control concrete results, the HVFA concrete mixtures proportioned with the Class F fly ashes (FA-1 and FA-15) generated less heat even at a high dosage and low $w / \mathrm{cm}$ ratio. The HVFA concrete mixtures proportioned with the Class $\mathrm{C}$ fly ashes (FA-24 and FA-28) were shown to have similar heat generation results to the control concrete. It should be
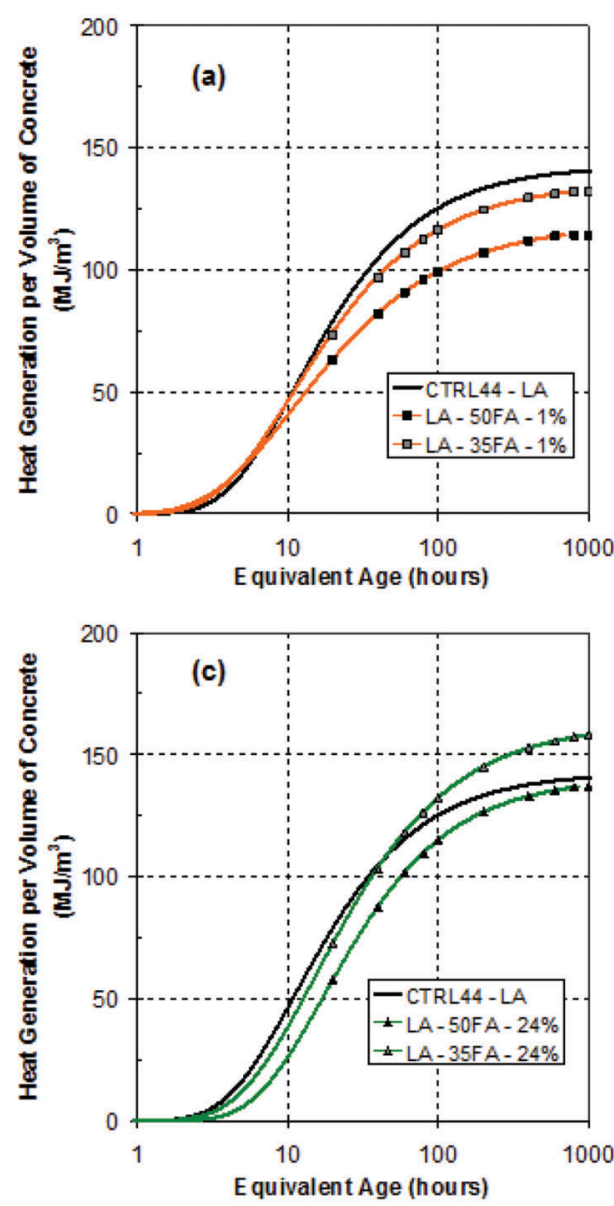

Figure 3.11 Heat generation per volume of concrete for a) LA-1\%, b) LA-15\%, c) LA-24\% and d) LA-28\% concrete mixtures $\left(1 \mathrm{MJ} / \mathrm{m}^{3}=0.725 \mathrm{kBTU} / \mathrm{yd}^{3}\right)$. noted that the $w / \mathrm{cm}$ ratio was not constant for the 35 and 50\% fly ash dosage mixtures. Generally, the heat of hydration increases according to a decrease in $w / \mathrm{cm}$ ratio. Since the $w / \mathrm{cm}$ ratio was not constant for all mixtures, the effect of fly ash dosage alone on the temperature development could not be completely isolated.

\subsubsection{Effect of Fly Ash CaO Content on Heat of} Hydration. As presented earlier, the rate of temperature development and maximum temperature rise for HVFA concrete is dependent on the chemical composition of the fly ash. The effect of fly ash $\mathrm{CaO}$ content on the heat generation of HVFA concrete is presented in Figure 3.13 for the HA cement concrete mixtures (35\% and 50\% fly ash dosages). In this figure, it is shown that as the fly ash $\mathrm{CaO}$ content is increased for a given dosage, the heat generation per volume of concrete also increases. The same trend was found for all LA cement concrete mixtures.

\subsubsection{Semi-Adiabatic Calorimetry Testing} Summary. As shown in Figures 3.11 through 3.13, fly ash dosage, fly ash $\mathrm{CaO}$ content, and cement alkali content each have an effect on the heat generation of
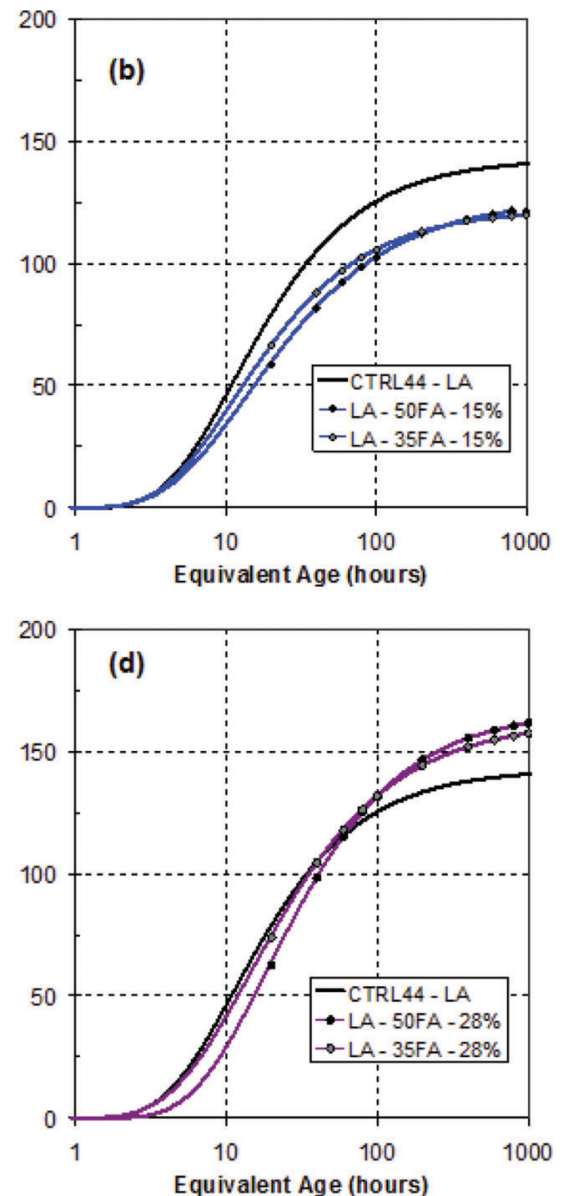

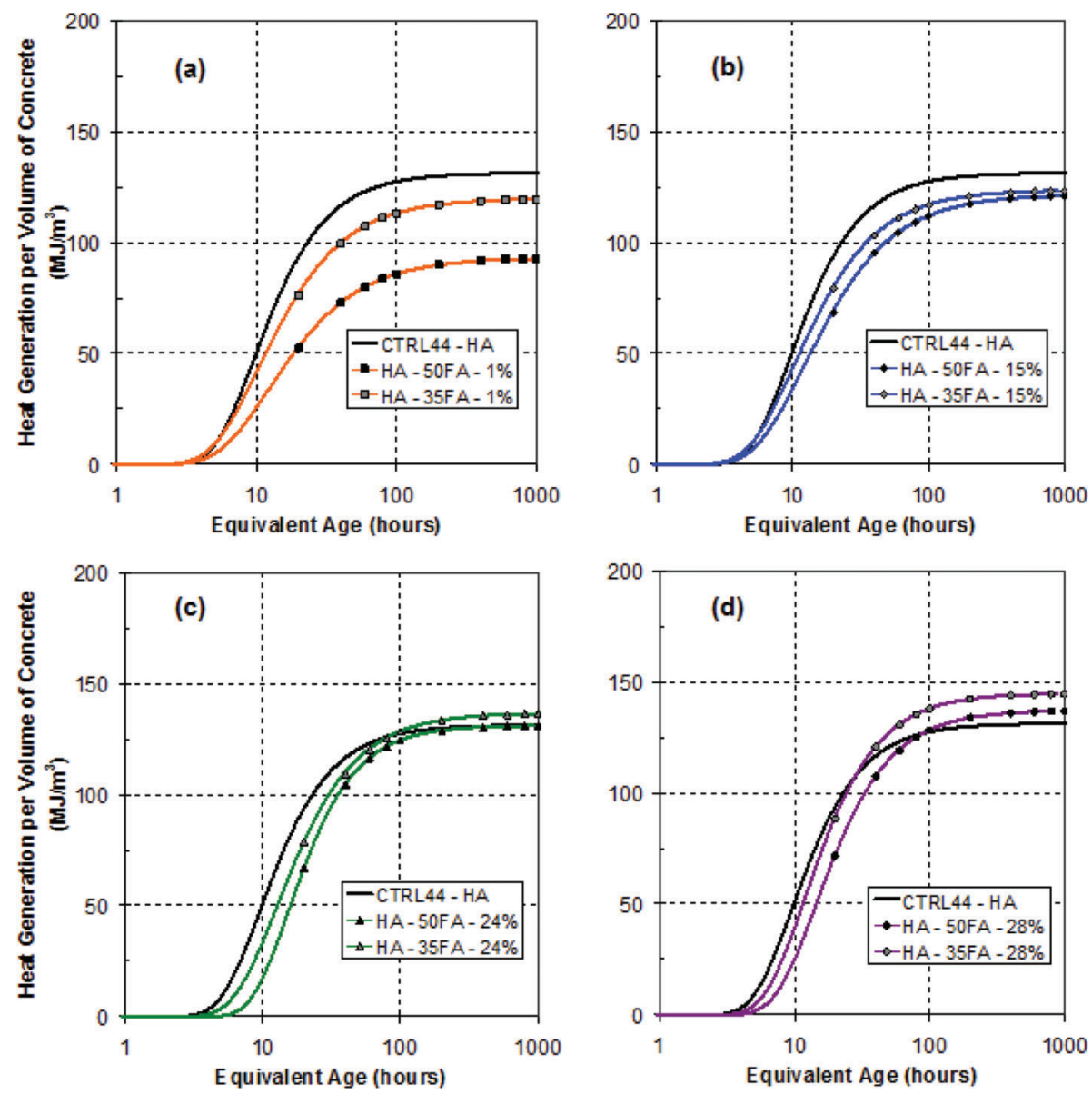

Figure 3.12 Heat generation per volume of concrete for (a) HA-1\%, (b) HA-15\%, (c) HA-24\%, and (d) HA-28\% concrete mixtures $\left(1 \mathrm{MJ} / \mathrm{m}^{3}=0.725 \mathrm{kBTU} / \mathrm{yd}^{3}\right)$.

HVFA concrete. As the fly ash dosage is increased, the heat generated by the concrete mixture will decrease. It was also shown that an increase in the fly ash $\mathrm{CaO}$ content (for a given fly ash dosage), results in more heat generated per volume of concrete. The results from SAC testing are discussed in further detail in section 4 when the HVFA concrete hydration model is developed. In that section, a review of the degree of hydration formulation is completed. and the best-fit hydration parameters $\left(\tau, \beta\right.$, and $\left.\alpha_{\mathrm{u}}\right)$ that were calculated for each mixture are presented.

\subsection{Phase II Testing Results}

\subsubsection{Compressive Strength Testing}

Immediately following fresh concrete testing, cylinders were cast from each room temperature batch for compressive strength testing. The cylinders were continuously moist-cured and tested according to ASTM C39 (2004). A maturity meter was used to record the temperature history of one cylinder from each batch. With the recorded compressive strengths and temperature history, strength-maturity relationships were developed for each mixture.
In addition to developing the strength-maturity relationship for all room temperature batches, qualitycontrol (QC) cylinders were cast from each hot and cold batch. The QC cylinders were tested after 28 days of moist-curing. A summary of strength-maturity relationship parameters and compressive strength quality-control testing results for each mixture are presented in Keith (2011). In the following subsections, the effect of fly ash dosage, fly ash $\mathrm{CaO}$ content, and cement alkali content on the compressive strength development of HVFA concrete are reviewed.

3.2.1.1 Effect of Fly Ash Dosage on Compressive Strength. One of the main limitations of HVFA concrete is low early-age strength values. Therefore, HVFA concrete is proportioned with low $w / \mathrm{cm}$ ratios to counter its reduced rate of early-age compressive strength development (Malhotra \& Mehta, 2002). For this project, HVFA concrete mixtures were proportioned with $w / \mathrm{cm}$ ratios of 0.39 and 0.34 for $50 \%$ and $35 \%$ fly ash dosages, respectively. Compressive strength testing results (moist-cured specimens) for all LA and HA cement concrete mixtures are presented in Figures 3.14 and 3.15 , respectively. In each figure, each data point represents an average of three test results for that age. 

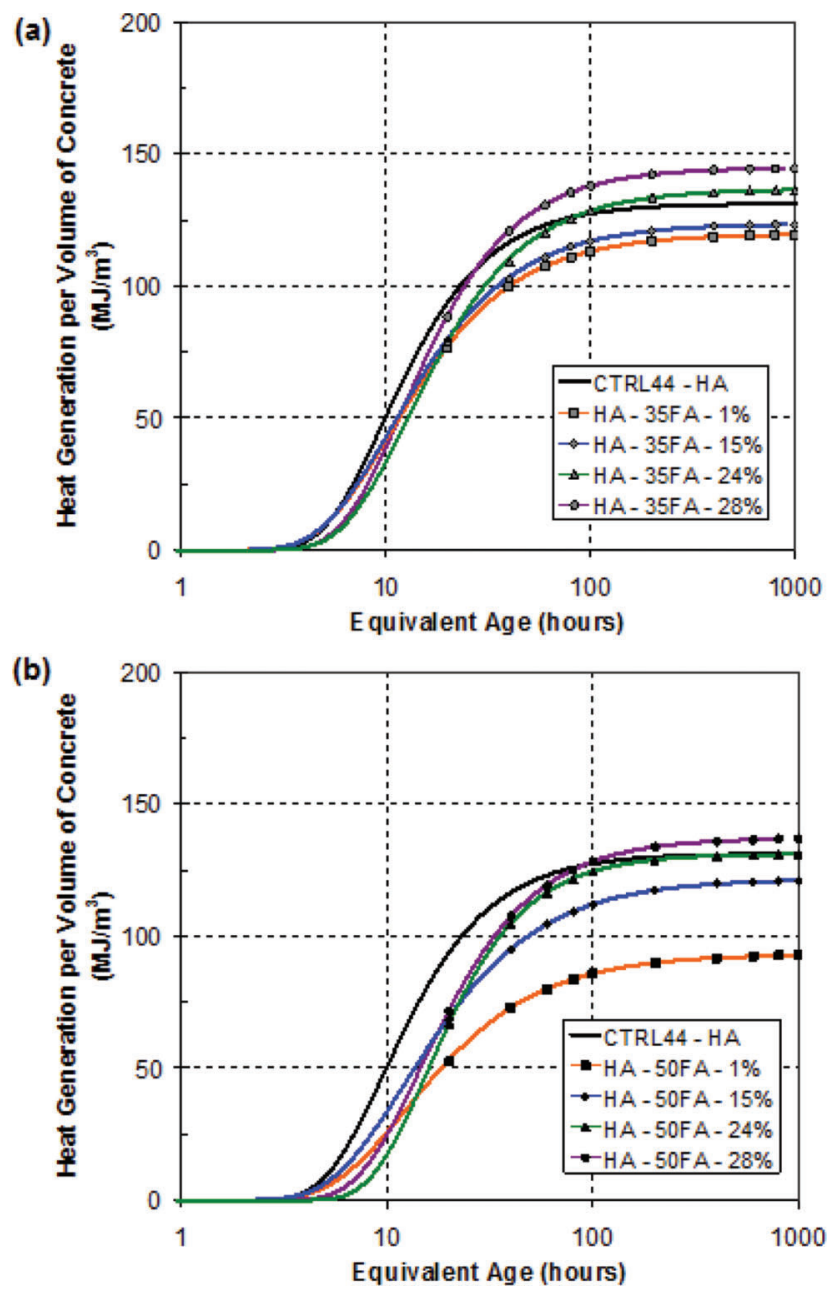

Figure 3.13 Effect of fly ash $\mathrm{CaO}$ content on heat generation for (a) HA-35FA and (b) HA-50FA concrete mixtures.

From Figures 3.14 and 3.15, it can be seen that both the $35 \%$ and $50 \%$ fly ash mixtures were proportioned to have comparable compressive strengths to the control mixtures at early ages. At four days, all mixtures (LA and HA cement) had compressive strength values within $15 \%$ of the CTRL44 strength. The results in Figures 3.14 and 3.15 also indicate that the HVFA concrete mixtures continue to gain strength at late ages due to the moist-curing conditions. In comparison, the control mixtures had minimal compressive strength gain after 28 days.

Generally, an increase in fly ash dosage for a given $w / \mathrm{cm}$ ratio will result in a decreased rate of compressive strength development at early ages. At later ages, however, the compressive strength may be increased with high fly ash dosages when compared to a control concrete with no fly ash replacement (Thomas, 2007). This improvement in late-age compressive strength for HVFA concrete will only be achieved if adequate moisture is supplied.
3.2.1.2 Effect of Fly Ash CaO Content on Compressive Strength. In Figures 3.14 and 3.15, it is shown that the HVFA concrete mixtures proportioned with the $1 \%$ $\mathrm{CaO}$ Class $\mathrm{F}$ fly ash had the lowest rate of early-age strength development for both cement sources. In Figure 3.16, the general effect of fly ash $\mathrm{CaO}$ content on the compressive strength development of HVFA concrete is illustrated. It is shown that the HVFA concrete proportioned with the Class $\mathrm{C}$ fly ash exhibits a higher rate of reaction at early ages when compared to the Class F fly ash mixture. This effect is amplified when the fly ash dosage is increased from $35 \%$ to $50 \%$. For the $50 \%$ fly ash dosage mixtures, the compressive strength of the Class F fly ash mixture surpasses the Class C fly ash mixture results at approximately 33 days. After this point, the HA-50FA-1\% mixture continues to gain strength, whereas the strength development of the HA-50FA-24\% mixture slows. The high rate of strength gain at late ages for HVFA concrete proportioned with low-calcium Class $\mathrm{F}$ fly ashes is due to the increased pozzolanic activity of these ashes. The improvement in late-age strength for HVFA is dependent on curing conditions because moisture is required for the pozzolanic reaction to convert $\mathrm{CH}$ to C-S-H (ACI Committee 232, 2003).

3.2.1.3 Effect of Cement Alkali Content on Compressive Strength. The compressive strength development of HVFA concrete is also influenced by the alkali content of the cement. In Figures 3.14 and 3.15 , it is shown that the compressive strengths were decreased at 4 days and beyond for all HVFA concrete mixtures proportioned with the HA cement source when compared to the LA cement source. The average percent reduction in the compressive strength results for the $35 \%$ and $50 \%$ fly ash dosage concrete mixtures are, $12.6 \%$ and $18.3 \%$, respectively. Jawed and Skalny (1978) reported cements with a high alkali content will generally exhibit higher strengths at early ages, but lower strengths at 28 days and beyond.

3.2.1.4 Compressive Strength Testing Summary. As shown in Figures 3.14 to 3.16, the rate of strength development for HVFA concrete is dependent on the $w / \mathrm{cm}$ ratio, fly ash dosage, fly ash chemical composition and cement chemical composition. Generally, an increase in fly ash dosage will result in lowered early-age compressive strengths. This effect may be offset; however, if HVFA concrete is proportioned with a low $w / \mathrm{cm}$ ratio and HRWR admixtures are used to control workability. The compressive strength testing results presented were used to develop a unique strength-maturity relationship for each mixture. The strength-maturity relationships for each mixture were later used to evaluate the in-place compressive strength development of HVFA concrete in transportation structures, which is described in section 5 . 

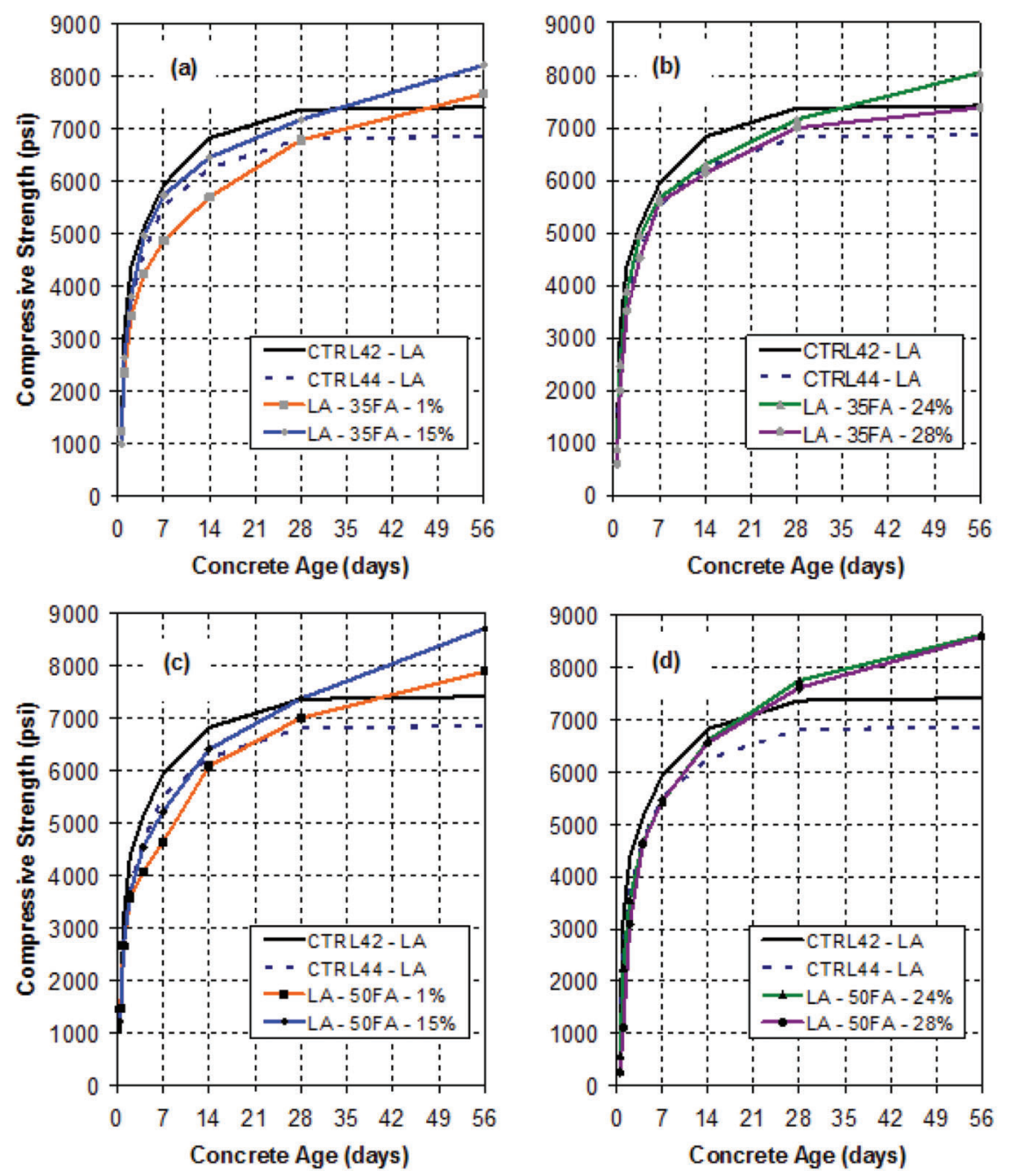

Figure 3.14 Compressive strength testing results for (a) LA-35FA-Class F, (b) LA-35FA-Class C, (c) LA-50FA-Class F, and (d) LA-50FA-Class C mixtures. 

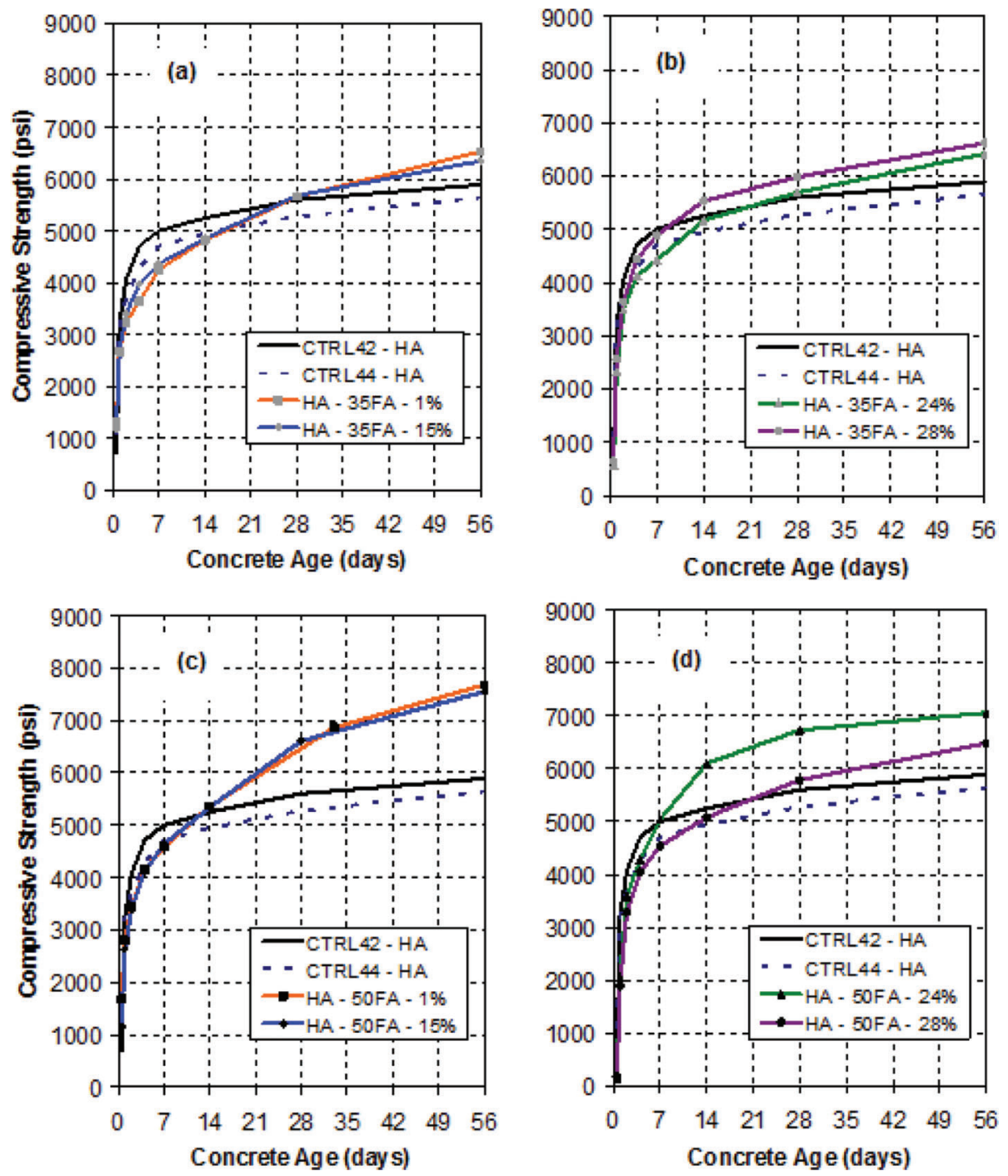

Figure 3.15 Compressive strength testing results for (a) HA-35FA-Class F, (b) HA-35FA-Class C, (c) HA-50FA-Class F, and (d) HA-50FA-Class C mixtures.
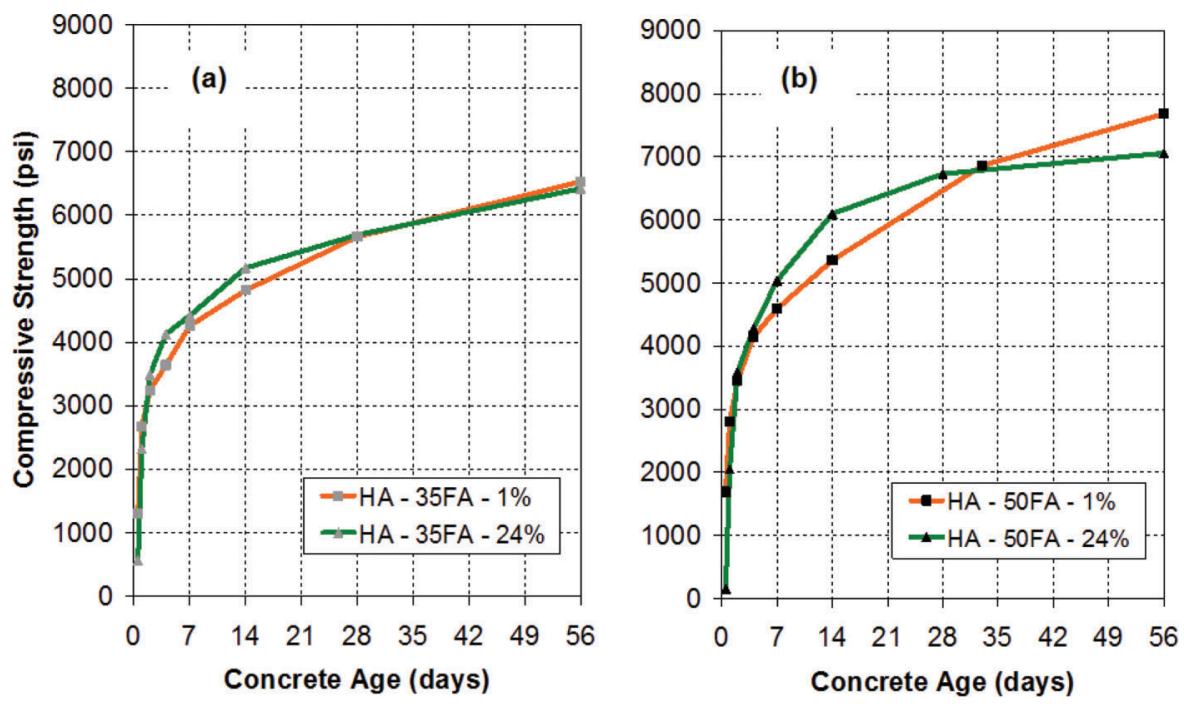

Figure 3.16 Effect of fly ash $\mathrm{CaO}$ content on compressive strength development of (a) HA-35FA and (b) HA-50FA mixtures. 


\section{DEVELOPMENT OF HVFA CONCRETE HYDRATION MODEL}

The activation energies from isothermal calorimetry testing and hydration development data from semiadiabatic calorimetry testing were used to develop a hydration model for concrete proportioned with high fly ash dosages. In this section, the modeling approach and software are introduced. Next, the calibration database used for the development of the hydration model is presented. After presenting the modeling approach and calibration database, the HVFA concrete hydration model is presented, along with an evaluation of the model. Finally, a summary is presented for significance and use of the final model.

\subsection{Modeling Approach}

As previously discussed, Hansen and Pane (2002) developed the Equation 1.10 to represent the degree of hydration development versus time for a concrete sample. This expression describes the $\mathrm{S}$-shaped degree of hydration development in terms of the hydration time parameter $(\tau)$, hydration shape parameter $(\beta)$, and ultimate degree of hydration $\left(\alpha_{u}\right)$. The hydration time parameter is expressed in terms of hours, and it represents the start of the acceleration phase of the hydration development. The hydration shape parameter $(\beta)$ is unitless, and it describes the rate (slope) of the hydration development. Finally, the ultimate degree of hydration $\left(\alpha_{u}\right)$ represents the maximum degree of hydration that may be achieved. The mixture-specific hydration parameters $\left(\tau, \beta\right.$, and $\left.\alpha_{u}\right)$ are back-calculated by fitting Equation 1.10 to the degree of hydration versus time results obtained from semi-adiabatic calorimetry testing. The effect of an increase to each of the hydration parameters to the shape of the overall hydration development are summarized in Figures 4.1a, $4.1 \mathrm{~b}$, and 4.1c. An example of the degree of hydration equation fitted to sample SAC results is presented in Figure 4.1d.

The hydration model for HVFA concrete was developed with the use of multivariate regression analysis. To complete this analysis, SAS Version 9.2 was used. The multivariate regression analysis was completed in three steps: identification of the most significant variables, development of model, and evaluation of goodness of fit.

The first step in developing the HVFA concrete hydration model was to identify the most significant variables for individually predicting both the hydration time parameter $(\tau)$ and the hydration shape parameter $(\beta)$. The chemical and physical properties of the cements, fly ash chemical compositions, and fly ash dosages were taken as explanatory variables and the $\tau$ and $\beta$ parameters were taken as the response variables for each mixture. Interactions between the variables were also considered. Since the cement and fly ash chemical properties are not independent of each other, a correlation matrix was constructed for all variables.
From the correlation matrix, variable dependence could be tested for simultaneous use in the model. In this step, the explanatory variables to be used in the final model were selected. Also, initial estimates of the coefficients for the nonlinear regression model were calculated by statistical means.

After identifying the most significant variables, the hydration model was developed. In this step, the chemical and physical properties of the cements, fly ash chemical compositions, and fly ash dosages were taken as independent variables and the degree of hydration values from semi-adiabatic testing were taken as the response variables for each mixture. Nonlinear regression analysis was used to determine the best-fit statistical values for each of the independent variables.

After developing the degree of hydration model, it was be evaluated for goodness of fit. The goodness of fit was evaluated by determining the coefficient of determination $\left(r^{2}\right)$ and the standard deviation of the absolute error $\left(S_{j}\right)$.

\subsection{Calibration Database}

The independent variables selected for the calibration database included the chemical and physical properties of the cements, the fly ash chemical compositions, the fly ash dosages, and the $w / \mathrm{cm}$ ratios for each mixture used to develop this model. This information is presented in Tables 2.2, 2.3, and 2.5, for each of the twenty concrete mixtures tested.

\subsubsection{Semi-Adiabatic Test Data}

For each concrete mixture, the hydration shape parameter $(\beta)$, hydration time parameter $(\tau)$, and ultimate degree of hydration $\left(\alpha_{u}\right)$ were selected as the response variables. The hydration parameters for each concrete mixture were obtained from semi-adiabatic calorimetry (SAC) testing. A summary of the best-fit hydration parameters for all twenty concrete mixtures (LA and HA cement sources) is presented in Table 4.1. The activation energies presented in this table were calculated from isothermal calorimetry test results obtained from three curing temperatures.

The total heat of hydration $\left(H_{u}\right)$ for each concrete mixture is also presented in Table 4.1. The total heat of hydration accounts for heat generated by portland cement and SCMs. The heat contribution of fly ash is calculated based on the fly ash $\mathrm{CaO}$ content. There are several recommendations for calculating fly ash heat contribution from past research. Kishi and Maekawa (1995) recommended a heat of hydration of $209 \mathrm{~J} / \mathrm{g}$ for a fly ash with a $\mathrm{CaO}$ content of $8.8 \%$. Schindler and Folliard (2005) proposed that the fly ash heat contribution should be calculated according to Equation 4.1. Based on SAC results, Ge and Wang (2009) proposed the relationship presented inEquations 4.2.

$$
h_{F A}=1800 \cdot p_{F A-C a O}
$$



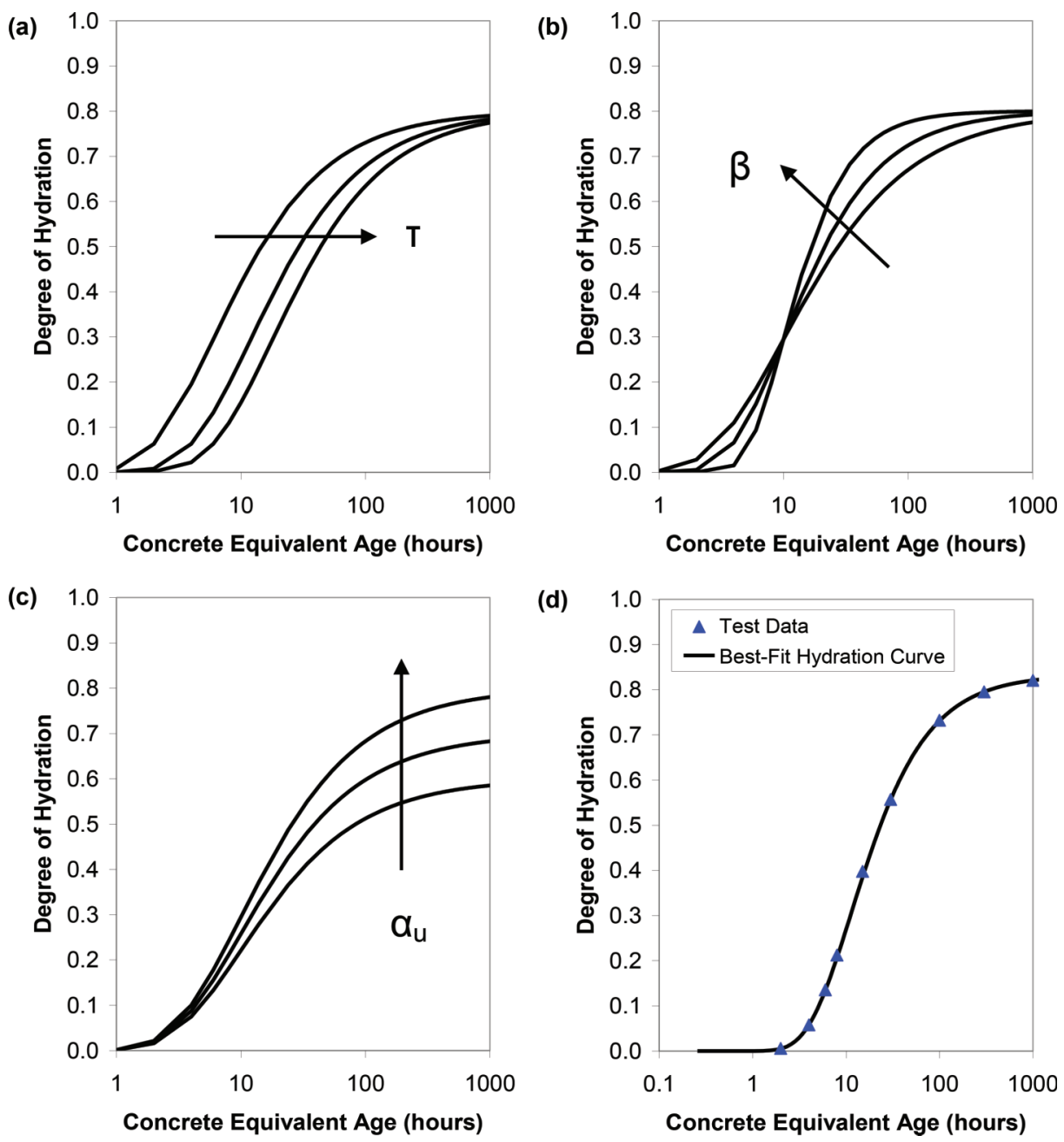

Figure 4.1 Effect of changes to: (a) hydration time parameter, (b) hydration shape parameter, and (c) ultimate degree of hydration on the overall hydration development; (d) example of hydration equation fitted to SAC testing results.

$$
h_{\mathrm{FA}}=15.9 \cdot\left(p_{F A C a O} \times 100\right)+74.3
$$

where:

$h_{\mathrm{FA}}=$ total heat of hydration of fly ash $(\mathrm{J} / \mathrm{g})$ and

$p_{F A-C a O}=$ fly ash $\mathrm{CaO}$ weight ratio in terms of total fly ash content.

A plot of fly ash heat contribution versus fly ash $\mathrm{CaO}$ content for the results presented by Kishi and Maekawa (1995), Schindler and Folliard (2005), and Ge and Wang (1999) are presented in Figure 4.2. Based on these results, a best-fit expression for fly ash heat of hydration versus fly ash $\mathrm{CaO}$ content was developed and is presented in Equation 4.3. This expression was used to calculate the heat of hydration of the fly ash used for this research.

$$
h_{\mathrm{FA}}=16.2 \cdot\left(p_{\mathrm{FACaO}} \times 100\right)+58
$$

\subsubsection{Supplemental Data from Past Research}

Schindler and Folliard (2005) created a model to predict the hydration development for concrete incor- porating fly ash and slag cement. To develop their model, two fly ash sources, one Class $\mathrm{C}$ and one Class $\mathrm{F}$, and three Type I cement sources were investigated. The fly ash dosages varied from $15 \%$ to $45 \%$ by volume of cementitious materials. SAC testing results from Schindler and Folliard's (2005) study were incorporated to expand the calibration database for this project. The best-fit hydration parameters and $w / \mathrm{cm}$ ratios for each of the mixtures are presented in Table 4.2. The fly ash dosages, indicated in Table 4.2 under mixture description, were computed on a volume basis. Finally, the activation energies presented were calculated using Equation 1.13.

\subsection{Hydration Model Development}

In the model proposed by Schindler and Folliard (2005), each of the hydration parameters $(\tau$ and $\beta)$ is composed of a "cement-only" expression and a modifier to account for the behavior of SCMs. When no SCMs are used, the exponential portions of the $\tau$ and $\beta$ expressions are equal to one, leaving the "cement-only" portion of the expression. The portland cement-only 
TABLE 4.1

Best-Fit Hydration Parameters from SAC Testing

\begin{tabular}{|c|c|c|c|c|c|}
\hline \multirow[b]{2}{*}{ Mixture Description } & \multirow[b]{2}{*}{$\mathrm{E}(\mathrm{J} / \mathrm{mol})$} & \multicolumn{3}{|c|}{ Hydration Parameters } & \multirow[b]{2}{*}{$H_{u}(J / g)$} \\
\hline & & $\beta$ & $\tau$ (hours) & $\alpha_{u}$ & \\
\hline CTRL42-LA & 32,500 & 0.966 & 10.44 & 0.812 & 466 \\
\hline CTRL44-LA & 33,500 & 0.937 & 11.41 & 0.833 & 479 \\
\hline LA-35-1 & 28,300 & 0.856 & 10.94 & 0.990 & 377 \\
\hline LA-35-15 & 36,300 & 0.895 & 11.44 & 0.762 & 433 \\
\hline LA-35-24 & 32,600 & 0.844 & 15.53 & 0.917 & 476 \\
\hline LA-35-28 & 35,700 & 0.814 & 14.60 & 0.874 & 496 \\
\hline LA-50-1 & 29,600 & 0.778 & 11.08 & 0.990 & 314 \\
\hline LA- $50-15$ & 30,300 & 0.803 & 13.96 & 0.805 & 401 \\
\hline LA-50-24 & 29,900 & 0.925 & 17.68 & 0.767 & 464 \\
\hline LA- $50-28$ & 33,700 & 0.870 & 19.00 & 0.849 & 494 \\
\hline CTRL42-HA & 38,000 & 1.468 & 9.68 & 0.728 & 462 \\
\hline CTRL44-HA & 35,600 & 1.500 & 9.66 & 0.747 & 491 \\
\hline HA-35-1 & 34,800 & 1.272 & 10.42 & 0.872 & 379 \\
\hline HA-35-15 & 32,000 & 1.281 & 10.43 & 0.769 & 435 \\
\hline HA-35-24 & 34,200 & 1.355 & 12.84 & 0.767 & 478 \\
\hline HA-35-28 & 28,400 & 1.431 & 12.05 & 0.777 & 498 \\
\hline HA-50-1 & 34,700 & 1.230 & 11.43 & 0.866 & 322 \\
\hline HA-50-15 & 33,500 & 1.193 & 12.31 & 0.764 & 408 \\
\hline HA-50-24 & 30,800 & 1.590 & 15.55 & 0.705 & 471 \\
\hline HA- $50-28$ & 28,400 & 1.403 & 14.60 & 0.689 & 501 \\
\hline
\end{tabular}

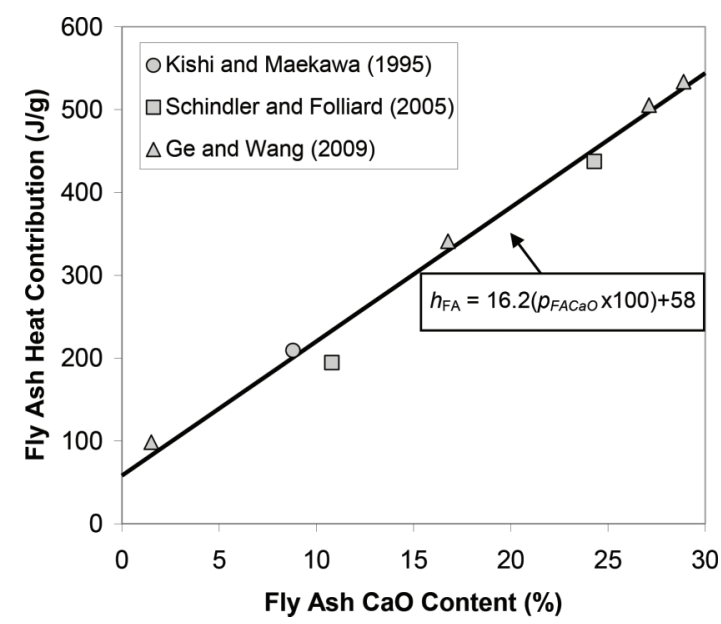

Figure 4.2 Fly ash heat contribution versus fly ash $\mathrm{CaO}$ content.

study was completed based on the four control concrete mixtures from this project and the three cement-only mixtures from Schindler and Folliard's (2005) research.

The following variables were found to provide the best statistical fit to the measured hydration parameters:

Hydration slope parameter $(\beta): 4$ parameters $=\mathrm{f}$ $\left(\mathrm{C}_{3} \mathrm{~A}, \mathrm{NaEqu}, \mathrm{Blaine}, \mathrm{p}_{\mathrm{FA}}, \mathrm{p}_{\mathrm{FA}-\mathrm{SiO} 2}\right)$

Hydration Time Parameter $(\tau): 4$ parameters $=\mathrm{f}$ $\left(\mathrm{C}_{3} \mathrm{~A}, \mathrm{C}_{2} \mathrm{~S}\right.$, Blaine, $\left.\mathrm{p}_{\mathrm{FA}}, \mathrm{p}_{\mathrm{FA}-\mathrm{CaO}}\right)$

where:

$p_{C 3 A}=$ weight ratio of $\mathrm{C}_{3} \mathrm{~A}$ in terms of total cement content,

$p_{C 2 S}=$ weight ratio of $\mathrm{C}_{2} \mathrm{~S}$ in terms of total cement content,
$N a E q u=$ cement equivalent alkali content in terms of total cement content,

Blaine = cement Blaine value $\left(\mathrm{m}^{2} / \mathrm{kg}\right)$,

$p_{F A}=$ fly ash volume ratio in terms of total cementitious content,

$p_{F A-S i O 2}=$ fly ash $\mathrm{SiO}_{2}$ weight ratio in terms of total fly ash content, and

$p_{F A-C a O}=$ fly ash $\mathrm{CaO}$ weight ratio in terms of total fly ash content.

The ultimate degree of hydration model developed by Mills (1966) was used successfully by Schindler and Folliard (2005) in the development of their hydration model for cementitious materials. Therefore, this ultimate degree of hydration model was used for development of the HVFA concrete hydration model. Similar to the approach taken by Schindler and Folliard (2005) shown in Equation 1.9, an increase in the ultimate degree of hydration due to the presence of fly ash was modeled as shown in Equation 4.4. The increase in ultimate degree of hydration, $\Delta \alpha_{\mathrm{u}}$, was found to be a function of the fly ash dosage, $p_{F A}$.

$$
\alpha_{u}=\frac{1.031 \cdot w / c m}{0.194+w / c m}+\Delta \alpha_{u}
$$

where:

$\alpha_{u}=$ ultimate degree of hydration

$w / \mathrm{cm}=$ water-cementitious material ratio by weight

$\Delta \alpha_{u}=$ increase in ultimate degree of hydration due to presence of fly ash.

The variables selected to model the hydration parameters are suitable based on engineering judgment. Cements with high $\mathrm{C}_{3} \mathrm{~A}$ and Blaine values have high early-age strength gains due to an increased rate of hydration at early ages. $\mathrm{C}_{3} \mathrm{~A}$ begins to hydrate 
TABLE 4.2

Best-Fit Hydration Parameters from SAC Testing from Schindler and Folliard (2005)

\begin{tabular}{|c|c|c|c|c|c|c|c|}
\hline \multirow[b]{2}{*}{ Cement ID } & \multirow[b]{2}{*}{ Mixture Description } & \multirow[b]{2}{*}{$w / c m$} & \multirow[b]{2}{*}{$\mathrm{E}(\mathrm{J} / \mathrm{mol})$} & \multicolumn{3}{|c|}{ Hydration Parameters } & \multirow[b]{2}{*}{$H_{u}(\mathrm{~J} / \mathrm{g})$} \\
\hline & & & & $\beta$ & $\tau$ (hrs) & $\alpha_{u}$ & \\
\hline A & Type I Cement & 0.37 & 46,000 & 0.905 & 13.69 & 0.689 & 477 \\
\hline A & 15\% Class C Fly Ash & 0.37 & 43,100 & 0.874 & 13.81 & 0.713 & 471 \\
\hline A & 25\% Class C Fly Ash & 0.38 & 41,300 & 0.772 & 23.28 & 0.793 & 468 \\
\hline A & 35\% Class C Fly Ash & 0.38 & 39,400 & 0.716 & 29.43 & 0.893 & 464 \\
\hline A & 45\% Class C Fly Ash & 0.39 & 37,500 & 0.724 & 36.66 & 0.849 & 460 \\
\hline A & 15\% Class F Fly Ash & 0.38 & 40,700 & 0.825 & 15.97 & 0.797 & 444 \\
\hline A & 25\% Class F Fly Ash & 0.39 & 37,200 & 0.786 & 18.30 & 0.831 & 421 \\
\hline A & 35\% Class F Fly Ash & 0.40 & 33,700 & 0.809 & 19.08 & 0.838 & 396 \\
\hline A & 45\% Class F Fly Ash & 0.41 & 30,100 & 0.774 & 21.73 & 0.894 & 370 \\
\hline $\mathrm{B}$ & Type I Cement & 0.50 & 42,000 & 0.719 & 16.88 & 0.887 & 513 \\
\hline $\mathrm{C}$ & Type I Cement & 0.50 & 46,300 & 0.727 & 16.32 & 0.882 & 492 \\
\hline
\end{tabular}

immediately when mixing water is added, liberating a significant amount of heat $(1350 \mathrm{~J} / \mathrm{g})$. The hydration of the calcium silicates $\left(\mathrm{C}_{3} \mathrm{~S}\right.$ and $\left.\mathrm{C}_{2} \mathrm{~S}\right)$ occurs at a slower rate than the $\mathrm{C}_{3} \mathrm{~A}$ hydration reaction, but still releases a considerable amount of heat. Schindler and Folliard (2005) used $\mathrm{C}_{3} \mathrm{~S}$ in their final hydration model. For the development of this model, $\mathrm{C}_{2} \mathrm{~S}$ replaced $\mathrm{C}_{3} \mathrm{~S}$ due to the strong correlation between $\mathrm{C}_{3} \mathrm{~S}$ and $\mathrm{C}_{3} \mathrm{~A}$ from the SAS RSQUARE selection procedure. Also, the cement sulfate content, $\mathrm{SO}_{3}$, was not used for the HVFA concrete model due to a high correlation with $\mathrm{C}_{3} \mathrm{~A}$. Finally, the cement equivalent alkali content was included in the development of the expression for $\beta$.

After selecting the independent variables for $\beta$ and $\tau$, initial values for the regression model were determined by performing multivariate linear regression analysis. The response variables were the degree of hydration values versus concrete equivalent age for each mixture. The degree of hydration values used for modeling corresponded to equivalent ages of $1,4,8,12,24,48,72$, 168,672 , and 2160 hours. A total of 299 observations were used for development of the final model. The bestfit models for $\beta, \tau$, and $\alpha_{\mathrm{u}}$ are presented in Equations 4.7 to 4.9 .

$$
\begin{gathered}
\beta=p_{C 3 A}{ }^{0.670} \cdot N a E q u^{0.080} \cdot \text { Blaine }^{2.55} \\
\exp \left(-1.37 \cdot p_{F A} \cdot p_{F A-S i O 2}-12.9\right) \\
\tau=p_{C 3 A}{ }^{-1.42} \cdot p_{C 2 S}{ }^{0.556} \cdot \text { Blaine }^{-7.32} \\
\exp \left(6.12 \cdot p_{F A} \cdot p_{F A-C a O}+43.5\right) \\
\alpha_{u}=\frac{1.031 \cdot w / c m}{0.194+w / c m}+0.428 \cdot p_{F A} \leq 1.0
\end{gathered}
$$

where:

$p_{C 3 A}=$ weight ratio of $\mathrm{C}_{3} \mathrm{~A}$ in terms of total cement content,

$p_{C 3 S}=$ weight ratio of $\mathrm{C}_{3} \mathrm{~S}$ in terms of total cement content,

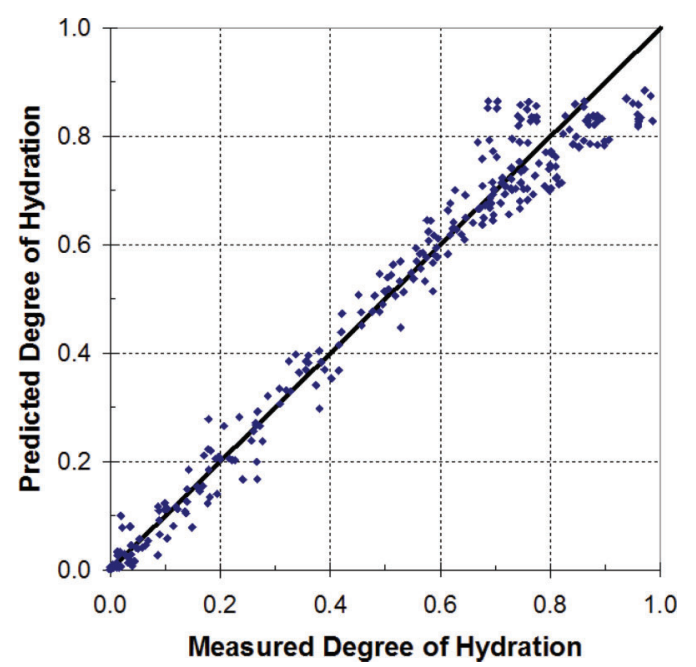

Figure 4.3 Plot of measured versus predicted degree of hydration for final model.

$N a E q u=$ cement equivalent alkali content in terms of total cement content,

Blaine $=$ cement Blaine value $\left(\mathrm{m}^{2} / \mathrm{kg}\right)$,

$p_{F A}=$ weight ratio of fly ash in terms of total cementitious content,

$p_{\text {FA-SiO2 }}=$ fly ash $\mathrm{SiO}_{2}$ weight ratio in terms of total fly ash content,

$p_{\text {FACaO }}=$ weight ratio of fly ash $\mathrm{CaO}$ in terms of total fly ash content, and

$w / \mathrm{cm}=$ water-cementitious material ratio by weight.

A scatter plot of the measured degree of hydration values versus the predicted values from the final model (Equations 4.5 to 4.7 ) is presented in Figure 4.3. This figure illustrates that the model accurately predicts the hydration development for concrete containing high fly ash dosages based on cement chemical composition, cement fineness, fly ash chemical composition, fly ash dosage, and $w / \mathrm{cm}$. The goodness of fit of the final model was evaluated by calculating the coefficient of determination $\left(\mathrm{r}^{2}\right)$ and the standard deviation of the absolute error $\left(\mathrm{S}_{\mathrm{j}}\right)$ which were 0.991 and 0.104 , respectively. This $\mathrm{r}^{2}$ indicates that $99.1 \%$ of the experimental 
variation of the response variable is explained by the final model.

\subsection{Summary}

A nonlinear regression analysis was used to develop a model to predict the hydration development of HVFA concrete mixtures. The calibration database used to develop this hydration model included SAC testing results obtained from both the laboratory testing portion of this project and Schindler and Folliard (2005). Using this model, the hydration shape parameter $(\beta)$, hydration time parameter $(\tau)$ and ultimate degree of hydration $\left(\alpha_{u}\right)$ for HVFA concrete mixtures may be computed independently based on the chemical composition of the cementitious materials and the mixture proportions of the HVFA concrete mixture. With the use of this model, the hydration parameters may be calculated quickly without the time or cost associated with SAC testing. This HVFA concrete hydration model will be used in the following section to develop design aids to predict the maximum in-place concrete temperature, time to initial set, and compressive strength development of HVFA concrete placed in selected transportation structures.

\section{EVALUATION OF THE MODELED IN-PLACE PERFORMANCE OF HVFA CONCRETE}

After completing all laboratory testing and developing the HVFA concrete hydration model, the modeled in-place performance of HVFA concrete was evaluated. The results from this analysis are intended to aid contractors and transportation agencies in estimating the maximum in-place concrete temperature, initial setting times, and compressive strength development for HVFA concrete when used in various transportation structures. In the following subsections, the analysis approach and baseline conditions that were used to complete this analysis are introduced. Also, sample in-place temperature profiles are presented. Finally, a review of the modeled in-place performance of HVFA concrete as it relates to maximum in-place concrete temperature, time to initial set, and compressive strength development is presented.

\subsection{Analysis Approach}

When HVFA concrete is used in mass concrete applications, a reduction in maximum in-place concrete temperature can reduce the susceptibility of the concrete to delayed ettringite formation (DEF). DEF is defined as the "formation of ettringite in a cementitious material by a process that begins after hardening is substantially complete and in which none of the sulfate comes from outside the cement paste" (Taylor, Famy, \& Scrivener, 2001). For DEF to occur, the concrete must be subjected to high temperatures early in the curing process. Taylor et al. (2001) reported that the critical temperature for portland cement concrete is $158^{\circ} \mathrm{F}$. Folliard et al. (2008) recommend a maximum inplace concrete temperature of $185^{\circ} \mathrm{F}$ when at least $25 \%$ Class $\mathrm{F}$ or $35 \%$ Class $\mathrm{C}$ fly ash dosages are specified. In this section, the maximum in-place concrete temperatures are estimated for HVFA concrete mixtures under varying placement conditions.

One of the main barriers to the increased use of HVFA concrete is the concern over retarded set times, especially when cold curing conditions are encountered. Retarded set times can cause delays to concrete finishing operations, causing HVFA concrete to be undesirable. Similarly, there are concerns over low early-age compressive strength development for HVFA concrete. Low early-age compressive strength development can delay formwork removal times, time to opening to traffic, and negatively impact construction sequencing. In this section, in-place setting and compressive strength development are estimated for HVFA concrete mixtures under varying placement conditions.

To evaluate the performance of HVFA concrete for each of the properties identified above, the ConcreteWorks software program was used to estimate in-place temperature profiles for HVFA concrete placed under various placement conditions. Multiple iterations were performed with the ConcreteWorks software to examine the effect of fly ash type, fly ash dosage, concrete member type, and placement temperature on the in-place temperature development, time to initial set, and compressive strength development of HVFA concrete.

The ConcreteWorks software program was developed through Texas Department of Transportation funding at the University of Texas. This software may be used to estimate the development of in-place concrete temperatures for user-defined concrete mixtures, under various placement conditions, and in various typical transportation structures. Detailed information on the heat transfer analysis method, concrete thermal properties, and concrete element models used by the ConcreteWorks software may be found in the ConcreteWorks User Manual (Riding, 2007).

For the ConcreteWorks analysis, various HVFA concrete mixtures were investigated to quantify the effect of fly ash type and dosage on the in-place performance. After selecting the database of HVFA concrete mixtures to be used for the analysis, iterations were performed to evaluate the effect of various placement conditions and element types on the development of the in-place concrete temperature for each mixture.

\subsection{Baseline Conditions for ConcreteWorks Analysis}

In the following subsections, the baseline conditions used to complete the ConcreteWorks analysis are defined. First, an overview of the HVFA concrete mixtures that were used to develop the in-place temperature profiles is presented. Next, the concrete placement temperature and ambient weather conditions are defined. Finally, each of the concrete element types and their corresponding modeling inputs are presented. 


\subsubsection{HVFA Concrete Mixtures}

Simulations were completed for each concrete mixture to develop in-place concrete temperature profiles based on varying placement conditions. The HVFA concrete mixtures were varied based on the fly ash dosage and fly ash type. Iterations were completed at fly ash dosages of $0 \%, 35 \%$, and $50 \%$ by volume of cementitious materials. For each fly ash dosage investigated, iterations were completed using two Class F and two Class $\mathrm{C}$ fly ash sources, each with varying chemical compositions. The four fly ash sources used for the ConcreteWorks analysis correspond to the four sources used for the laboratory testing phase of this research. The "low-alkali" (LA) cement source from the lab study was used for all ConcreteWorks simulations. The coarse aggregate and fine aggregate used for all simulations were a siliceous river gravel and siliceous river sand, respectively.

The mixture proportions for the $35 \%$ and $50 \%$ fly ash dosage mixtures correspond to the LA-35FA and LA-50FA concrete mixture proportions presented in Table 2.2. For each concrete mixture, the hydration parameters were required to develop the in-place concrete temperature profiles. The HVFA concrete hydration model developed in section 4 was used to estimate the required hydration parameters for each concrete mixture. The activation energy for the $35 \%$ and $50 \%$ fly ash dosage mixtures were determined from isothermal calorimetry as summarized in Table 3.2. A summary of the hydration parameter inputs for each of the concrete mixtures is presented in Table 5.1.

\subsubsection{Placement Conditions and Concrete Temperature}

The ConcreteWorks software program includes a database of environmental conditions (temperature, wind speed, percent cloud cover, solar radiation, atmospheric pressure, and relative humidity) based on thirtyyear average data for 239 U.S. cities. When defining the placement conditions for ConcreteWorks analysis, the placement time, placement date, and project location must be selected. Two project locations were investigated: Chicago, Illinois and Phoenix, Arizona. These two cities were selected to allow the full range of

TABLE 5.1

ConcreteWorks Hydration Parameter Inputs

\begin{tabular}{lccccc}
\hline \hline & \multicolumn{5}{c}{ Hydration Parameters } \\
\cline { 2 - 6 } Mix & E (J/mol) & $\boldsymbol{\beta}$ & $\tau$ (hours) & $\boldsymbol{\alpha u}$ & Hu (J/kg) \\
\hline CTRL44-LA & 33,500 & 1.199 & 9.544 & 0.716 & 478,880 \\
LA-35-1 & 28,300 & 0.901 & 9.753 & 0.838 & 369,511 \\
LA-35-15 & 36,300 & 0.936 & 13.26 & & 426,143 \\
LA-35-24 & 32,600 & 0.994 & 15.65 & & 469,017 \\
LA-35-28 & 35,700 & 1.027 & 17.37 & & 489,333 \\
LA-50-1 & 29,600 & 0.798 & 9.844 & 0.870 & 314,697 \\
LA-50-15 & 30,300 & 0.842 & 15.26 & & 401,086 \\
LA-50-24 & 29,900 & 0.917 & 19.34 & & 464,285 \\
LA-50-28 & 33,700 & 0.962 & 22.45 & & 494,019 \\
\hline
\end{tabular}

placement temperatures to be evaluated. No one city in the United States could be found that reached the average temperature extremes of 40 and $95^{\circ} \mathrm{F}$.

In addition to the placement time, date, and location, the fresh concrete temperature must also be specified. To evaluate the in-place performance of HVFA concrete, the fresh concrete temperature $\left(\mathrm{T}_{\mathrm{c} 0}\right)$ was assumed to be equal to the ambient air temperature $\left(T_{a i r}\right)$ from the ConcreteWorks historical database. Using this assumption, iterations were completed for fresh concrete temperatures equal to $40,50,60,70,80,90$ and $95^{\circ} \mathrm{F}$. The upper and lower bounds for the fresh concrete temperatures were selected to reflect the conventional limits on concrete placement temperature.

For the ConcreteWorks analysis, column elements and flatwork elements were modeled for in-place temperature development. The placement conditions for each fresh concrete temperature are defined in Tables 5.2 and 5.3 for the column elements and flatwork elements, respectively. A 12:00 p.m. time of placement was used for all column element simulations. For the flatwork elements, a 9:00 a.m. earlier placement time was selected in an effort to ensure that the peak heat of hydration of the HVFA mixtures and solar radiation peak both occur within the first 24 hours. In Tables 5.2 and 5.3, a range of placement dates is given for each fresh concrete temperature. For each range of placement dates, the ambient air temperature, $T_{a i r}$, is equal to the specified concrete temperature, $\mathrm{T}_{\text {concrete, }}$ plus/minus $2^{\circ} \mathrm{F}$.

\subsubsection{Concrete Element Types}

ConcreteWorks has built-in concrete member models and the in-place performance of HVFA concrete was evaluated by modeling the following three element types: columns, bridge decks, and pavements. In the following subsections, the inputs and modeling considerations used for each of the three element types are defined.

5.2.3.1 Mass Concrete-Square Column. To evaluate the in-place performance of HVFA concrete for mass concrete applications, three square column sizes were analyzed. ConcreteWorks models a two-dimensional horizontal cross section for column members and assumes heat transfer in the vertical direction to be zero (Riding, 2007). Therefore, the columns were defined based on their plan dimensions. The plan dimensions analyzed for the three column sizes were: $3 \times 3 \mathrm{ft}, 6 \times 6 \mathrm{ft}$, and $10 \times 10 \mathrm{ft}$. The specific ConcreteWorsk inputs used for this analysis are defined in Keith (2011).

5.2.3.2 Concrete Bridge Deck. Bridge deck in-place temperature profiles are generated by ConcreteWorks assuming one-dimensional heat transfer through a vertical cross section of the deck (Riding, 2007). For this analysis, an 8-in. thick bridge deck with metal pan formwork was used. This deck thickness is commonly used for full-depth bridge decks in the United States. 
TABLE 5.2

ConcreteWorks Placement Conditions for Column Elements

\begin{tabular}{|c|c|c|c|c|}
\hline $\mathbf{T}_{\mathrm{co}}$ & Project Location & Time of Placement & Range of Placement Dates & Placement Date \\
\hline \multirow[t]{2}{*}{$40^{\circ} \mathrm{F}$} & \multirow[t]{9}{*}{ Chicago, IL } & \multirow[t]{13}{*}{ 12:00 PM (noon) } & November $20-27$ & \multirow[t]{2}{*}{ November 24} \\
\hline & & & March 19-21 & \\
\hline \multirow[t]{2}{*}{$50^{\circ} \mathrm{F}$} & & & November 4-9 & \multirow[t]{2}{*}{ November 7} \\
\hline & & & April 6-11 & \\
\hline \multirow[t]{2}{*}{$60^{\circ} \mathrm{F}$} & & & October 7-17 & \multirow[t]{2}{*}{ October 12} \\
\hline & & & April 29-May 3 & \\
\hline \multirow[t]{2}{*}{$70^{\circ} \mathrm{F}$} & & & September $13-22$ & \multirow[t]{2}{*}{ September 17} \\
\hline & & & May $18-$ June 3 & \\
\hline $80^{\circ} \mathrm{F}$ & & & June 30-August 31 & July 15 \\
\hline \multirow[t]{2}{*}{$90^{\circ} \mathrm{F}$} & \multirow[t]{4}{*}{ Phoenix, AZ } & & May $15-$ June 2 & \multirow[t]{2}{*}{ May 22} \\
\hline & & & September 17 -October 2 & \\
\hline \multirow[t]{2}{*}{$95^{\circ} \mathrm{F}$} & & & June $7-16$ & \multirow[t]{2}{*}{ June 13} \\
\hline & & & August $11-$ September 10 & \\
\hline
\end{tabular}

TABLE 5.3

ConcreteWorks Placement Conditions for Flatwork Elements

\begin{tabular}{|c|c|c|c|c|}
\hline $\mathbf{T}_{\mathrm{c0}}$ & Project Location & Time of Placement & Range of Placement Dates & Placement Date \\
\hline \multirow[t]{2}{*}{$40^{\circ} \mathrm{F}$} & \multirow[t]{8}{*}{ Chicago, IL } & \multirow[t]{12}{*}{ 9:00 AM } & November 10-19 & \multirow[t]{2}{*}{ November 12} \\
\hline & & & March 23-28 & \\
\hline \multirow[t]{2}{*}{$50^{\circ} \mathrm{F}$} & & & October $23-30$ & \multirow[t]{2}{*}{ October 27} \\
\hline & & & April 12-17 & \\
\hline \multirow[t]{2}{*}{$60^{\circ} \mathrm{F}$} & & & September 23-October 2 & \multirow[t]{2}{*}{ September 29} \\
\hline & & & May $8-15$ & \\
\hline \multirow[t]{2}{*}{$70^{\circ} \mathrm{F}$} & & & September 4-11 & \multirow[t]{2}{*}{ September 10} \\
\hline & & & June 4-6 & \\
\hline \multirow[t]{2}{*}{$80^{\circ} \mathrm{F}$} & \multirow[t]{5}{*}{ Phoenix, AZ } & & September $17-30$ & \multirow[t]{2}{*}{ September 25} \\
\hline & & & May 16-31 & \\
\hline \multirow[t]{2}{*}{$90^{\circ} \mathrm{F}$} & & & June 22-July 8 & \multirow[t]{2}{*}{ June 24} \\
\hline & & & July 15 -August 10 & \\
\hline $95^{\circ} \mathrm{F}$ & & 10:00 AM & June 27-July 15 & July 2 \\
\hline
\end{tabular}

The specific ConcreteWorks inputs used for this analysis are defined in Keith (2011).

5.2.3.3 Concrete Pavement. The final type of concrete member considered for evaluation of the in-place performance of HVFA concrete was a concrete pavement. The specific ConcreteWorks inputs used for this analysis are defined in Keith (2011). For all concrete pavement simulations, a white curing compound was selected as the curing method. The curing compound was applied one hour after paving was completed. A concrete pavement with the following dimensions was used for all simulations:
- Pavement thickness $=14$ inches,

- Subbase 1 = asphalt concrete (3 inch thickness),

- Subbase 2 = granular (12 inch thickness), and

- Subgrade = clay.

\subsection{Sample In-Place Temperature Profiles from ConcreteWorks Analysis}

In this subsection, the effect of fly ash dosage, fly ash $\mathrm{CaO}$ content, placement temperature, and element type on the in-place temperature development of HVFA concrete is reviewed. All in-place temperature profiles presented in this section were generated using the 
baseline conditions presented in subsection 5.2. Iterations were completed for each combination of concrete element type, concrete mixture, and placement temperature, leading to a total of 315 in-place temperature profiles. Due to the large number of iterations, representative results are presented in this subsection to review the effect of each variable (fly ash dosage, fly ash type, placement temperature, and element type) on the in-place temperature development of HVFA concrete. After reviewing the effect of each variable separately, an overall evaluation of the in-place performance of HVFA concrete is described in subsection 5.4.

\subsubsection{Effect of Fly Ash Dosage on In-Place Temperature Development}

The general effect of fly ash dosage on the in-place temperature development of HVFA concrete is presented in Figure 5.1. In this figure, the temperature profiles are presented for the concrete mixtures proportioned with the $1 \% \mathrm{CaO}$ Class $\mathrm{F}$ fly ash and the $24 \% \mathrm{CaO}$ Class $\mathrm{C}$ fly ash at dosages of $35 \%$ and $50 \%$. The temperature profile for the CTRL44-LA mixture is also presented. The temperature profiles presented correspond to the $6 \times 6 \mathrm{ft}$ column element placed at $70^{\circ} \mathrm{F}$. In each plot, temperature profiles are presented for nodes located at both the center and the edge of the column elements.

In Figure 5.1, it is shown that the effect of fly ash dosage on the in-place temperature development is dependent on both the chemical composition of the fly ash and mixture proportions of the concrete. For the $1 \% \mathrm{CaO}$ Class $\mathrm{F}$ fly ash mixtures, an increase in fly ash dosage from $35 \%$ to $50 \%$ resulted in a decrease in the maximum in-place concrete temperature (measured at the center of the member) from approximately $140^{\circ} \mathrm{F}$ to $132^{\circ} \mathrm{F}$. Both of the Class $\mathrm{F}$ fly ash mixtures generated less heat than the control mixture (CTRL44-LA). For the $24 \% \mathrm{CaO}$ Class $\mathrm{C}$ fly ash mixtures, however, the same increase in dosage resulted in no change in the maximum in-place concrete temperature. Also, the Class $\mathrm{C}$ fly ash mixtures reached a higher maximum inplace concrete temperature than the control mixture. The results obtained for the Class $\mathrm{C}$ fly ash mixtures are (a)
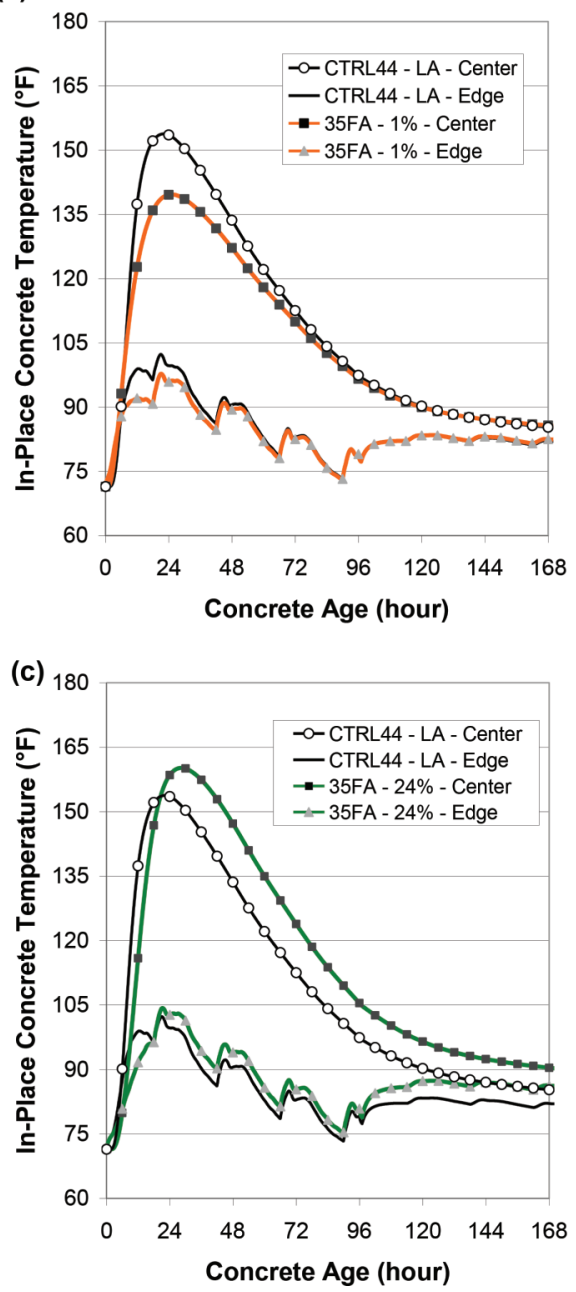

(b)

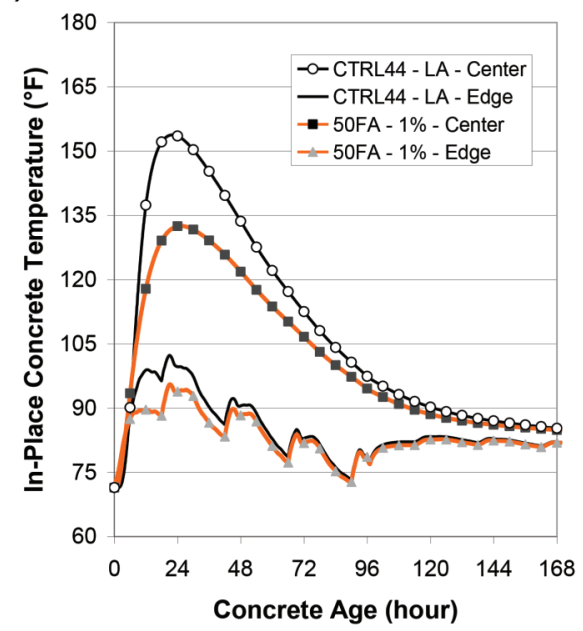

(d)

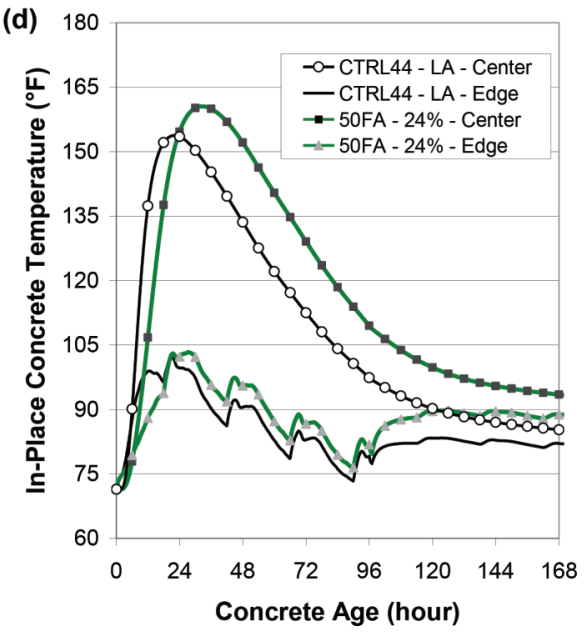

Figure 5.1 In-place temperature profiles for $6 \times 6 \mathrm{ft}$ columns placed at $70^{\circ} \mathrm{F}$ for the following mixtures: (a) $35 \mathrm{FA}-1 \%$, (b) $50 \mathrm{FA}$ $1 \%$, (c) $35 \mathrm{FA}-24 \%$, and (d) $50 \mathrm{FA}-24 \%$. 
attributed to the combined effect of the increased Class $\mathrm{C}$ fly ash heat of hydration and the low $w / \mathrm{cm}$ ratio of the mixture. The effect of fly ash $\mathrm{CaO}$ content on the inplace temperature development will be discussed in the following subsection.

\subsubsection{Effect of Fly Ash Type on In-Place Temperature Development}

The general effect of fly ash type on the maximum inplace temperature development for the $6 \times 6 \mathrm{ft}$ columns is presented in Figure 5.2. The results presented in this figure represent the estimated temperature development at the center node of the column element. The results in this figure are an example when the concrete placement temperature is $70^{\circ} \mathrm{F}$.

In Figure 5.2, it is shown that as the fly ash $\mathrm{CaO}$ content increases, for a given fly ash dosage, the maximum in-place concrete temperature also increases. In Equation 4.3, it can be seen that the heat contribution of fly ash increases based on the $\mathrm{CaO}$ content of the ash. For the $6 \times 6 \mathrm{ft}$ columns placed at $70^{\circ} \mathrm{F}$, an increase in fly ash $\mathrm{CaO}$ content from $1 \%$ to $28 \%$ resulted in an increase in the maximum in-place concrete temperature of $37^{\circ} \mathrm{F}$. Also, the HVFA concrete mixtures proportioned with a $50 \%$ dosage of the Class $\mathrm{C}$ fly ashes reached a higher in-place concrete temperature than the control mixture. The same trend was observed for the 10 x $10 \mathrm{ft}$ columns; however, the maximum in-place concrete temperature increased by $45^{\circ} \mathrm{F}$ as the fly ash $\mathrm{CaO}$ content increased from $1 \%$ to $28 \%$.

The results presented in Figure 5.2 agree with past research conducted by Langley et al. (1992), as described in subsection 1.4.3.1.2. Langley et al. (1992) reported that the temperature rise caused by the hydration of concrete proportioned with high dosages of Class F fly ashes can be significantly lower than the temperature rise of a control concrete with no fly ash replacement. For their study, the maximum temperature rise for a 10 x 10 x $10 \mathrm{ft}$ meter concrete block was reduced by $52^{\circ} \mathrm{F}$ when a $55 \%$ dosage of a Class F fly ash was used.

\subsubsection{Effect of Curing Conditions on In-Place Temperature Development}

The effect of concrete placement temperature on the in-place temperature development of HVFA concrete is illustrated in Figure 5.3. In this figure, the in-place temperature profiles at the center of the $6 \times 6 \mathrm{ft}$ column are presented when placed at $40^{\circ} \mathrm{F}$ and $95^{\circ} \mathrm{F}$. Similar temperature profiles for $6 \times 6 \mathrm{ft}$ columns placed at $70^{\circ} \mathrm{F}$ were presented in Figure 5.2.

The results presented in Figure 5.3 indicate that the in-place temperature development of HVFA concrete is highly dependent on the concrete placement temperature and curing conditions. An increase in placement temperature from $40^{\circ} \mathrm{F}$ to $95^{\circ} \mathrm{F}$ resulted in a $69^{\circ} \mathrm{F}$ increase in maximum in-place temperature from $92^{\circ} \mathrm{F}$ to $161^{\circ} \mathrm{F}$ for the $1 \% \mathrm{CaO}$ Class $\mathrm{F}$ fly ash mixture and $78^{\circ} \mathrm{F}$ increase from $114^{\circ} \mathrm{F}$ to $192^{\circ} \mathrm{F}$ for the $24 \% \mathrm{CaO}$ Class $\mathrm{C}$ fly ash mixture. The results also illustrate that the rate of temperature development is significantly affected by the concrete placement temperature. For example, when the concrete placement temperature is decreased from $95^{\circ} \mathrm{F}$ to $40^{\circ} \mathrm{F}$ for the $6 \times 6 \mathrm{ft}$ columns, the time required to reach the maximum in-place temperature for the 50FA-1\% mixture increases from approximately 22 hours to 36 hours. Similarly, the time required to reach the maximum in-place concrete temperature for the 50FA-28\% concrete mixture increases from 24 hours to 57 hours. The results presented in Figures 5.2 and 5.3 illustrate that the in-place temperature development of HVFA concrete mixtures varies according to curing conditions.

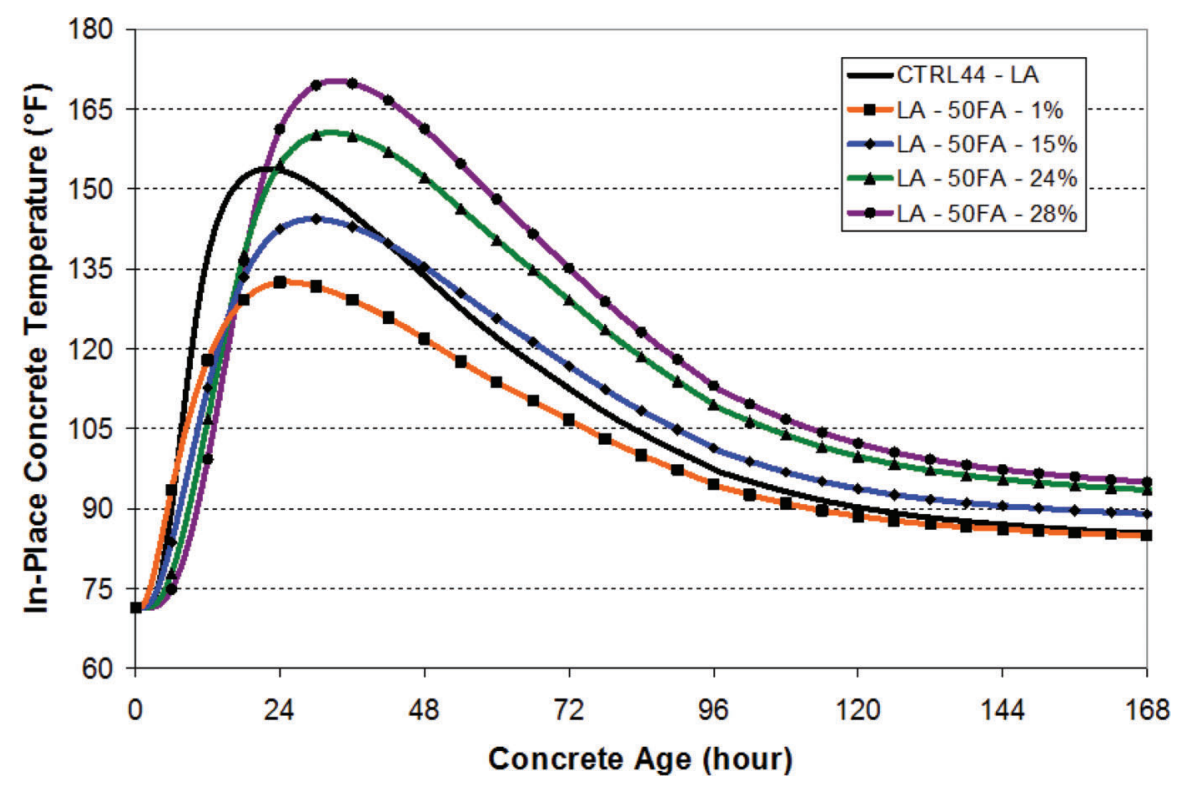

Figure 5.2 In-place temperature profiles for HVFA concrete proportioned with $50 \%$ fly ash dosage placed in $6 \times 6 \mathrm{ft}$ columns at $70^{\circ} \mathrm{F}$. 
(a)

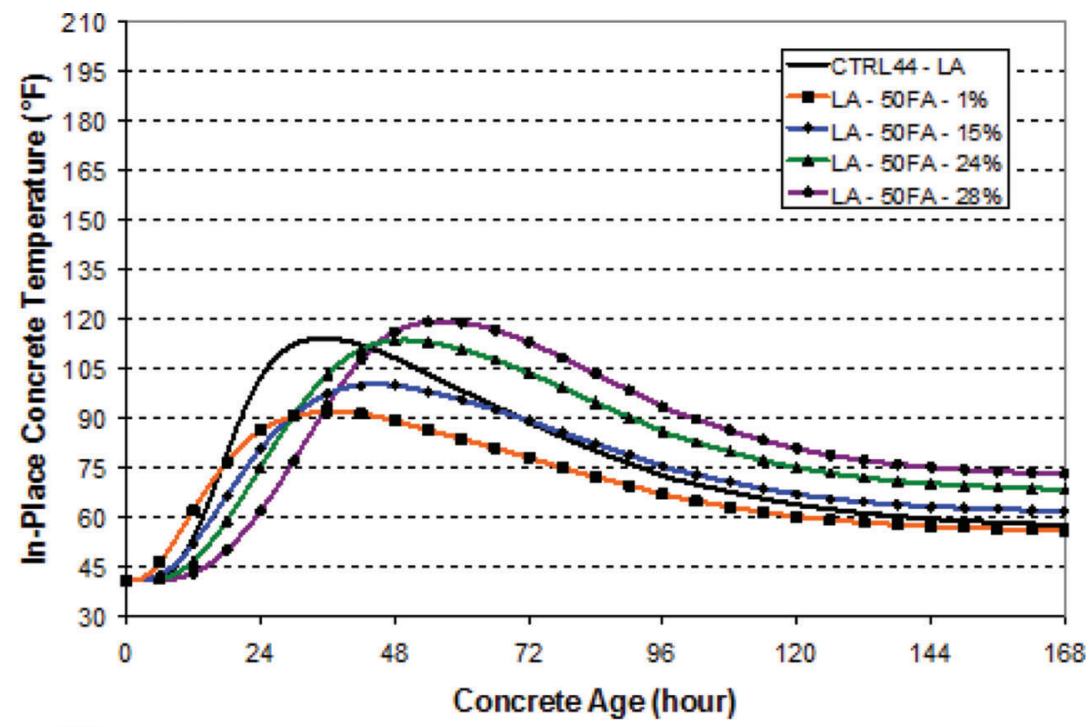

(b)

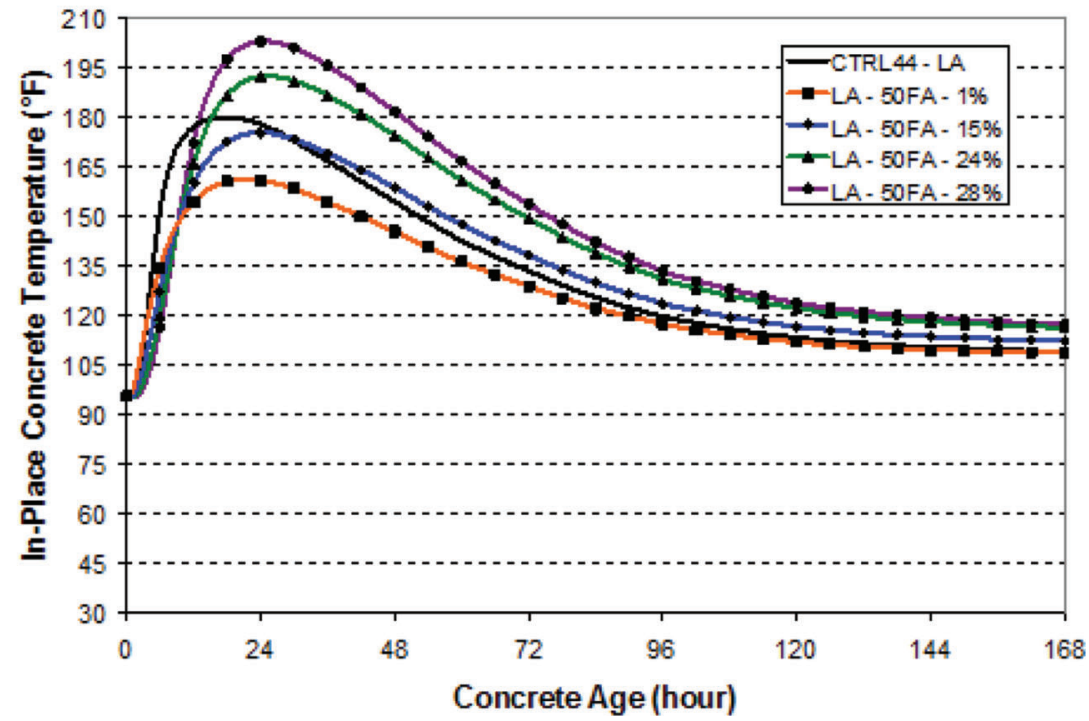

Figure 5.3 In-place temperature profiles for HVFA concrete proportioned with $50 \%$ fly ash dosage placed in $6 \times \mathrm{ft}$ columns at (a) $40^{\circ} \mathrm{F}$ and (b) $95^{\circ} \mathrm{F}$.

\subsubsection{Effect of Concrete Element Type on In-Place Temperature Development}

The general effect of element type on the in-place temperature development of HVFA concrete is presented in Figure 5.4. In this figure, in-place temperature profiles from the center of the element are presented for the $50 \%$ fly ash dosage concrete mixtures when placed in the $10 \times 10 \mathrm{ft}$ column and 8 in. bridge deck elements. The profiles presented in this figure were generated using a placement temperature of $70^{\circ} \mathrm{F}$. Results for the $6 \times 6 \mathrm{ft}$ columns placed at $70^{\circ} \mathrm{F}$ are shown previously in Figure 5.2.

From Figures 5.2 and 5.4, it is shown that as the concrete element size increases, for a given placement temperature and concrete mixture, the maximum in-place concrete temperature also increases. Also, the time required for the center of the concrete element to return to the ambient temperature conditions increases with an increase in element size. In Figure 5.4b, it is shown that the temperature development in the center of the $8 \mathrm{in}$. bridge deck element is more influenced by changes in ambient temperature conditions than the column elements. At 48 hours and beyond, the in-place concrete temperature for the bridge deck is shown to vary according to the diurnal temperature cycle. Similar results were obtained for the 14 in. pavement element. The results presented in this subsection illustrate that the in-place temperature development of HVFA concrete varies according to concrete element type.

\subsection{Evaluation of In-Place Performance of HVFA Concrete}

In the previous subsection, the general effects of fly ash dosage, fly ash type, placement temperature, and element type on the in-place temperature development 

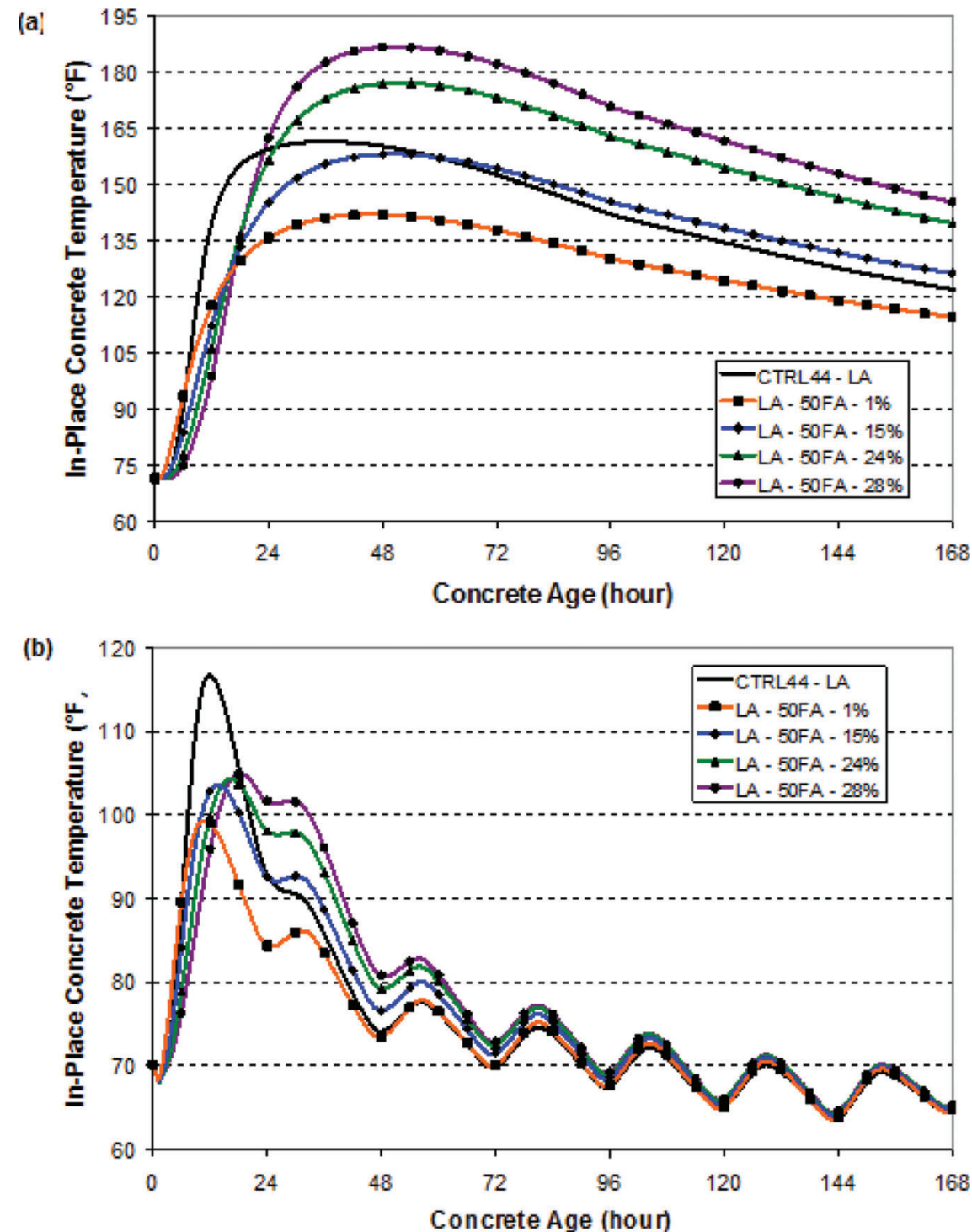

Figure 5.4 In-place temperature profiles for HVFA concrete proportioned with 50\% fly ash dosage placed in: (a) $10 \mathrm{x} 10 \mathrm{ft}$ column and (b) 8 in. bridge deck elements at $70^{\circ} \mathrm{F}$.

of HVFA concrete were each examined individually. In this subsection, the combined effect of the four variables on the in-place performance of HVFA concrete is examined. The results presented in this subsection are intended to provide guidance on the estimation of the maximum in-place concrete temperature, initial set times, and compressive strength development of HVFA concrete mixtures placed in selected transportation structures. Mixture- and material-specific results should be obtained to predict the actual behavior of the HVFA concrete developed for a project.

\subsubsection{Maximum In-Place Concrete Temperature}

In the following subsections, the ConcreteWorks analysis results for maximum in-place concrete temperature are summarized for the elements investigated. After reviewing all results, it was determined that the
HVFA concrete mixtures proportioned with the $24 \%$ and $28 \% \mathrm{CaO}$ Class $\mathrm{C}$ fly ashes had similar maximum in-place temperature development results. Therefore, only the results for the $28 \% \mathrm{CaO}$ Class $\mathrm{C}$ fly ash concrete mixtures are presented in this subsection.

5.4.1.1 Column Elements. Maximum in-place concrete temperature versus concrete placement temperature plots for the $3 \times 3 \mathrm{ft}, 6 \times 6 \mathrm{ft}$, and $10 \times 10 \mathrm{ft}$ columns are presented in Figures 5.5, 5.6, and 5.7, respectively. In these figures, the results for the control concrete mixture (CTRL44-LA) and HVFA concrete mixtures proportioned with the $1 \%, 15 \%$ and $28 \% \mathrm{CaO}$ content fly ashes are presented. All results presented in Figures 5.5 through 5.7 correspond to the maximum temperature generated at the center of the column elements. Also, the DEF temperature limits for concrete proportioned with portland cement (PC) and 
supplementary cementitious materials (SCMs) are indicated on each figure.

In Figure 5.5, it is shown that the maximum in-place concrete temperature increases linearly according to an increase in the concrete placement temperature. Also, the maximum in-place concrete temperature for the HVFA concrete mixtures proportioned with $50 \%$ fly ash dosages were consistently lower than those proportioned with the $35 \%$ dosage of the same ash.

The HVFA concrete mixtures proportioned with the Class F fly ash sources (1\% and $15 \%$ CaO content) generated less heat than the control mixture at all placement temperatures. The only HVFA mixtures with a maximum in-place concrete temperature greater than the control mixture were the 35FA-28\% and $50 \mathrm{FA}-28 \%$ mixtures placed at 90 and $95^{\circ} \mathrm{F}$. For these cases, however, the in-place concrete temperature of the HVFA mixtures only exceeded the control concrete mixture results by a maximum of $5^{\circ} \mathrm{F}$. The results presented in Figure 5.5 indicate that when HVFA concrete is used for $3 \mathrm{x} 3 \mathrm{ft}$ or smaller columns, the mixtures may be proportioned with high dosages of both Class $\mathrm{F}$ and Class $\mathrm{C}$ fly ashes without running the risk of exceeding the threshold for the formation of DEF.

The maximum in-place concrete temperature results for the $6 \times 6 \mathrm{ft}$ columns are presented in Figure 5.6. In this figure, the same linear relationship between maximum in-place concrete temperature and placement temperature is observed. For the $6 \times 6 \mathrm{ft}$ columns however, there is a larger range of maximum in-place temperature results for the HVFA concrete mixtures at each placement temperature. It is also shown that the maximum in-place concrete temperature for the Class $\mathrm{C}$ fly ash concrete mixtures exceeded the maximum inplace temperature for the control concrete mixture at all placement temperatures. Also, the results for the 35FA-15\% mixture were nearly identical to the results for the control concrete mixture at all placement temperatures.

For the HVFA concrete mixtures proportioned with the Class F fly ashes, the maximum in-place concrete temperature for the $50 \%$ dosages were lower than the results for the mixtures proportioned with $35 \%$ fly ash at all placement temperatures. These results are similar to those obtained for the $3 \times 3 \mathrm{ft}$ columns. The same trend was not observed for the Class C fly ash mixtures, however. The results presented in Figure 5.6 illustrate that an increase in fly ash dosage does not translate to reduced maximum in-place temperature development when high-calcium Class $\mathrm{C}$ fly ashes are used for large concrete elements.

Finally, the maximum in-place temperature results for the $10 \times 10 \mathrm{ft}$ columns are presented in Figure 5.7. The results obtained for the $10 \times 10 \mathrm{ft}$ columns are similar to those obtained for the $3 \times 3 \mathrm{ft}$ and $6 \times 6 \mathrm{ft}$ columns; however, the temperatures are higher. At the $10 \times 10 \mathrm{ft}$ column size, the low-calcium Class F fly ash mixtures $(\mathrm{CaO}=1 \%)$ are still shown to generate less heat than the control concrete. At all placement temperatures, the maximum in-place temperature for the $50 \mathrm{FA}-1 \%$ mixture is approximately $20^{\circ} \mathrm{F}$ less than the control mixture temperature. The results for the 50FA$28 \%$ mixture, however, range from 20 to $27^{\circ} \mathrm{F}$ higher than the control concrete results when the placement temperature is increased from $40^{\circ} \mathrm{F}$ to $95^{\circ} \mathrm{F}$.

The key findings from the results presented in Figures 5.5 through 5.7 are as follows:

- There is an approximate linear relationship between maximum in-place concrete temperature and concrete placement temperature for all HVFA concrete mixtures and column sizes that were investigated.

- For all column sizes investigated, the HVFA concrete produced with the $1 \% \mathrm{CaO}$ Class $\mathrm{F}$ fly ash generated less heat than the control concrete mixture.

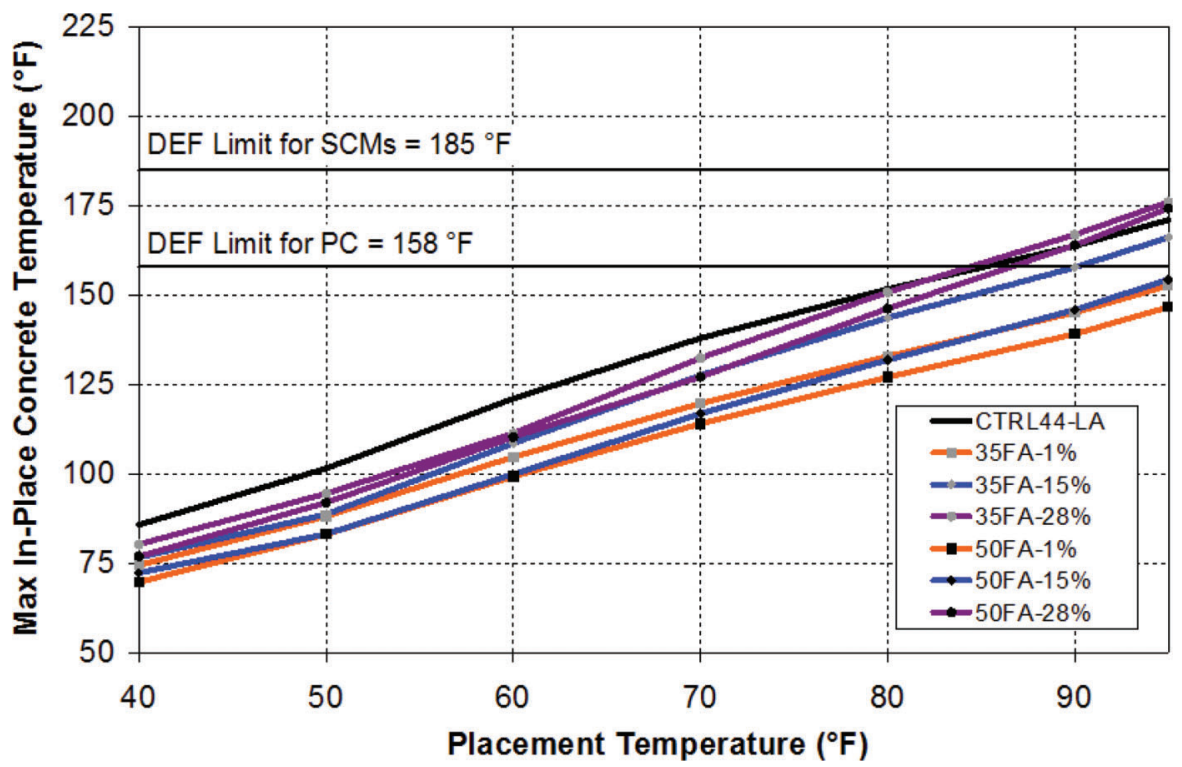

Figure 5.5 Maximum in-place concrete temperature versus concrete placement temperature for $3 \times 3 \mathrm{ft}$ columns. 


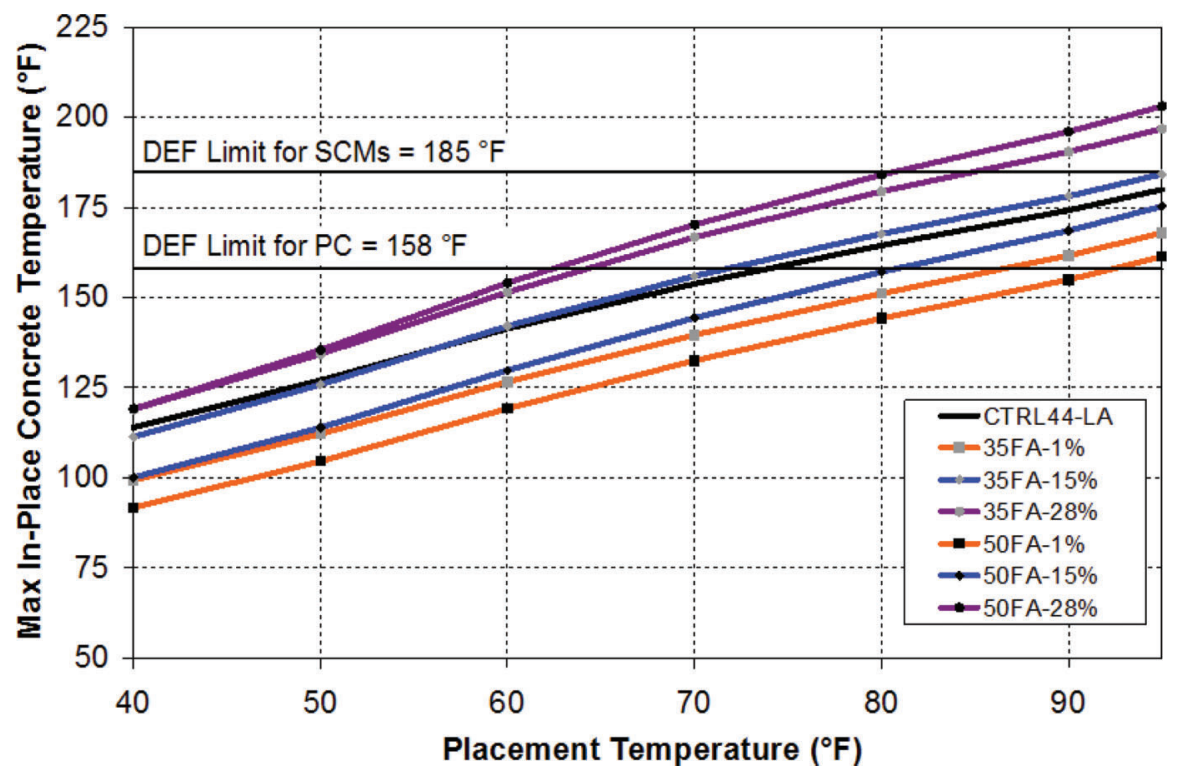

Figure 5.6 Maximum in-place concrete temperature versus concrete placement temperature for $6 \mathrm{x} 6 \mathrm{ft}$ columns.

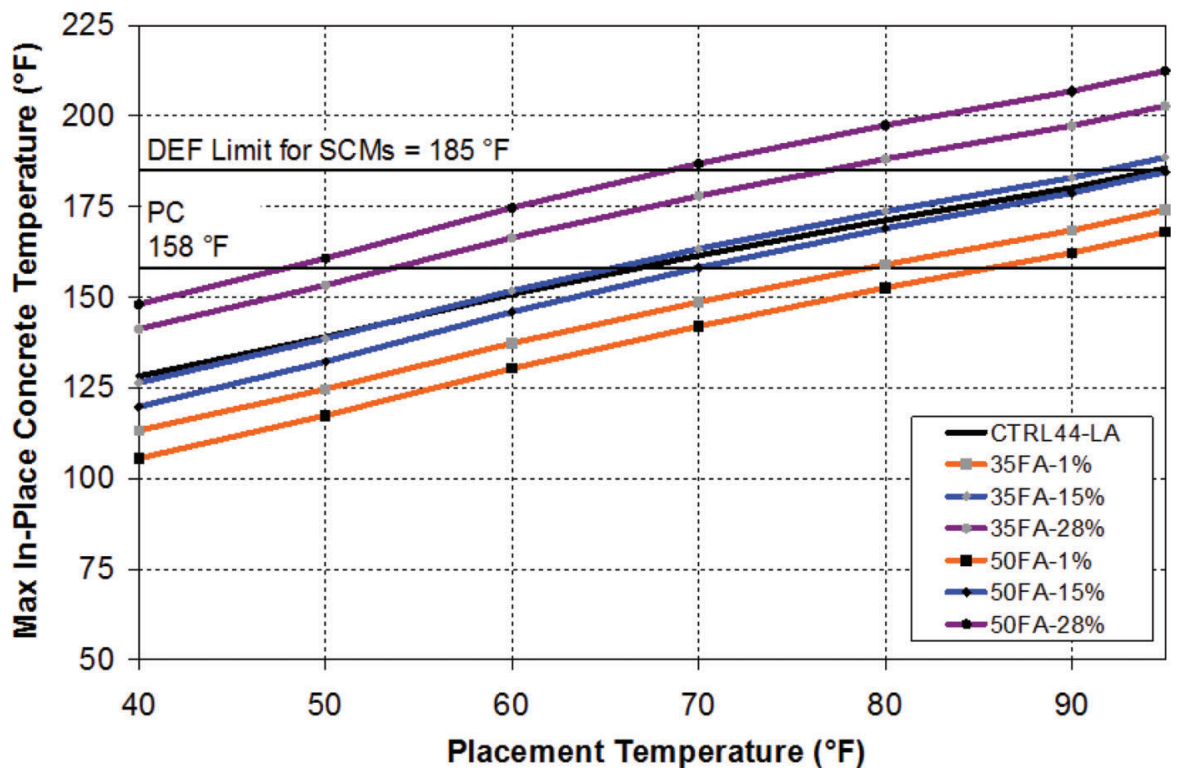

Figure 5.7 Maximum in-place concrete temperature versus concrete placement temperature for $10 \mathrm{x} 10 \mathrm{ft}$ columns.

- For both Class F fly ash mixtures, an increase in fly ash dosage from $35 \%$ to $50 \%$ resulted in a decreased maximum in-place temperature, despite the lowered $w / \mathrm{cm}$ for the $50 \%$ fly ash mixtures.

- The results from the $6 \times 6 \mathrm{ft}$ and $10 \times 10 \mathrm{ft}$ columns indicate that an increase in fly ash dosage does not translate to reduced maximum in-place temperature development when high-calcium Class $\mathrm{C}$ fly ashes are used in these types of mass concrete elements.

5.4.1.2 Flatwork. Maximum in-place concrete temperature results for the $8 \mathrm{in}$. bridge deck and $14 \mathrm{in}$. pavement elements are presented in Figures 5.8 and 5.9. The results represent the maximum temperature generated at mid-depth of the members.
The results presented in Figures 5.8 and 5.9 show that both flatwork elements had very similar in-place temperature development results. At the 40 to $70^{\circ} \mathrm{F}$ placement temperatures there is very little separation between the maximum in-place temperature results for all HVFA concrete mixtures. At the 80 to $95^{\circ} \mathrm{F}$ placement temperatures, there is increased separation between the HVFA concrete temperature results. These results indicate that the in-place temperature development for HVFA concrete placed in flatwork elements is most influenced by element size, curing method, and placement conditions rather than the fly ash dosage and fly ash $\mathrm{CaO}$ content at low placement temperatures. At all placement temperatures, the $50 \%$ fly ash dosage mixtures generated less 


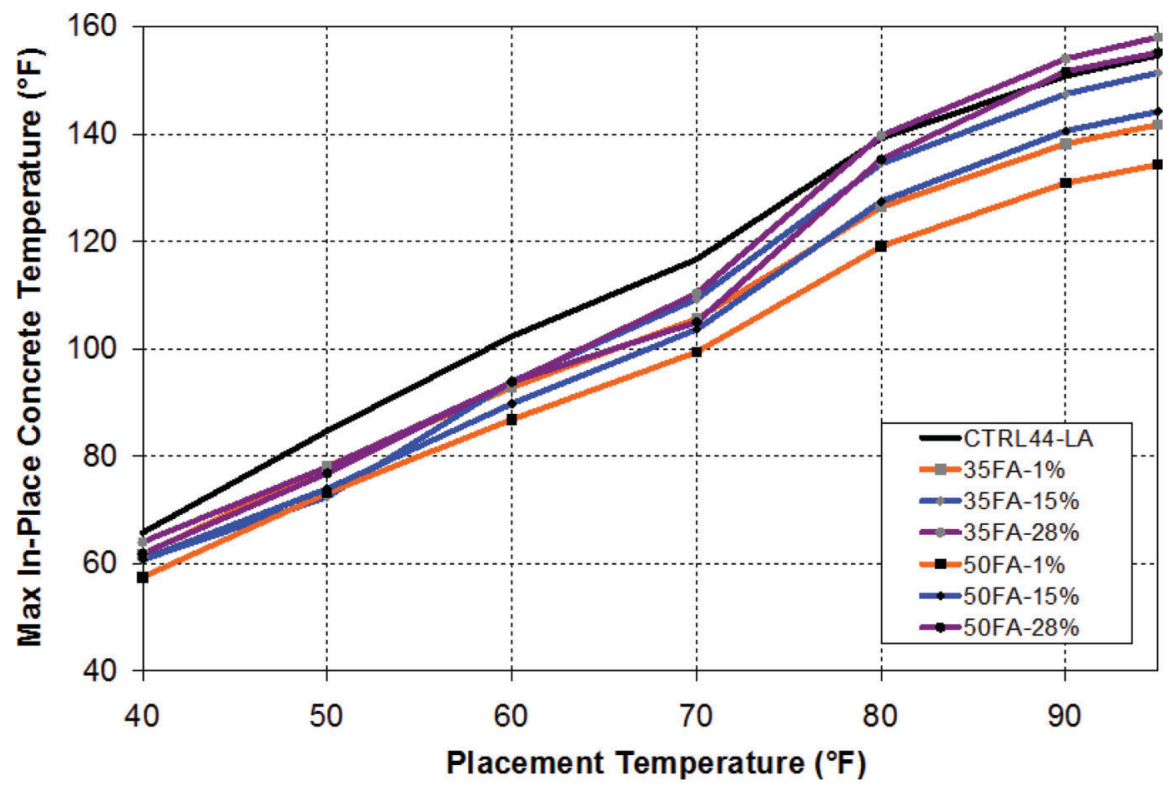

Figure 5.8 Maximum in-place concrete temperature versus concrete placement temperature for 8 in. bridge deck element.

heat than the $35 \%$ fly ash dosages for each fly ash source.

When comparing the 8 in. bridge deck and 14 in. pavement results, it is shown that the pavement element had lower maximum in-place concrete temperatures at all placement temperatures, despite having a greater thickness. The maximum difference in the in-place temperature results between the two elements was $10^{\circ} \mathrm{F}$ at the $40^{\circ} \mathrm{F}$ placement temperature and $17^{\circ} \mathrm{F}$ at the $95^{\circ} \mathrm{F}$ placement temperature. The higher in-place temperature results for the 8 in. bridge deck element can be attributed to the curing method used. The bridge deck element was cured with a blanket, whereas a white curing compound was used for the pavement element.

The results for the bridge deck and pavement elements are similar to the results presented in Figure 5.5 for the $3 \times 3 \mathrm{ft}$ column element, except that higher temperatures are reached in the larger concrete column. For all three elements, the HVFA concrete mixtures generated less heat than the control mixture at all placement temperatures, regardless of fly ash dosage and $\mathrm{CaO}$ content. The only exception to this trend were the $28 \% \mathrm{CaO}$ Class $\mathrm{C}$ fly ash mixtures at the 90 and $95^{\circ} \mathrm{F}$ placement temperatures. The key findings from the results presented in Figures 5.8 and 5.9 may be summarized as follows:

- For the flatwork elements at low placement temperatures (40 to $70^{\circ} \mathrm{F}$ ) the temperature development of HVFA concrete is most influenced by the element size, curing method, and placement conditions rather than the fly ash dosage and fly ash $\mathrm{CaO}$ content.

- For small columns (3 x $3 \mathrm{ft}$ or smaller) and flatwork elements, high-calcium Class $\mathrm{C}$ fly ash sources may be used at high dosages without high in-place concrete temperatures, even at placement temperatures exceeding $80^{\circ} \mathrm{F}$.

\subsubsection{Initial Set Time}

The in-place temperature profiles from the ConcreteWorks analysis were used to estimate the time required to reach initial set for each of the HVFA concrete mixtures. During the laboratory testing phase of this project, the equivalent age at initial and final set were determined for each room temperature batch. The equivalent ages at initial set are summarized in Table 5.4 for the HVFA concrete mixtures used for the ConcreteWorks analysis. Since the equivalent age at initial set was known for each mixture, the time required to reach initial set could be calculated based on the equivalent age maturity method and in-place concrete temperature profiles generated using ConcreteWorks.

Initial set is typically used for timing of finishing operations. Therefore, to estimate the time required to reach initial set, the temperature profile at the surface of each element was used. This location was used because the maturity of the concrete at the surface of the element will drive finishing operations.

After reviewing the ConcreteWorks results, it was found that the temperature profiles at the surface of the elements were affected by the element type, time of placement, and curing method. It was found that at the surfaces of the elements, the in-place temperature profiles for the $6 \times 6 \mathrm{ft}$ column, $10 \times 10 \mathrm{ft}$ column, and bridge deck elements do not differ significantly within the time that setting occurs. Because of the use of curing compound only, the temperature profile for the pavement element, however, varies significantly from the temperature profiles for all other elements before setting occurs. Based on these results, it can be concluded that the time required to reach initial set for the column and bridge deck elements will be similar at all placement temperatures for the same mixture. After reviewing all initial set times results, it was found 


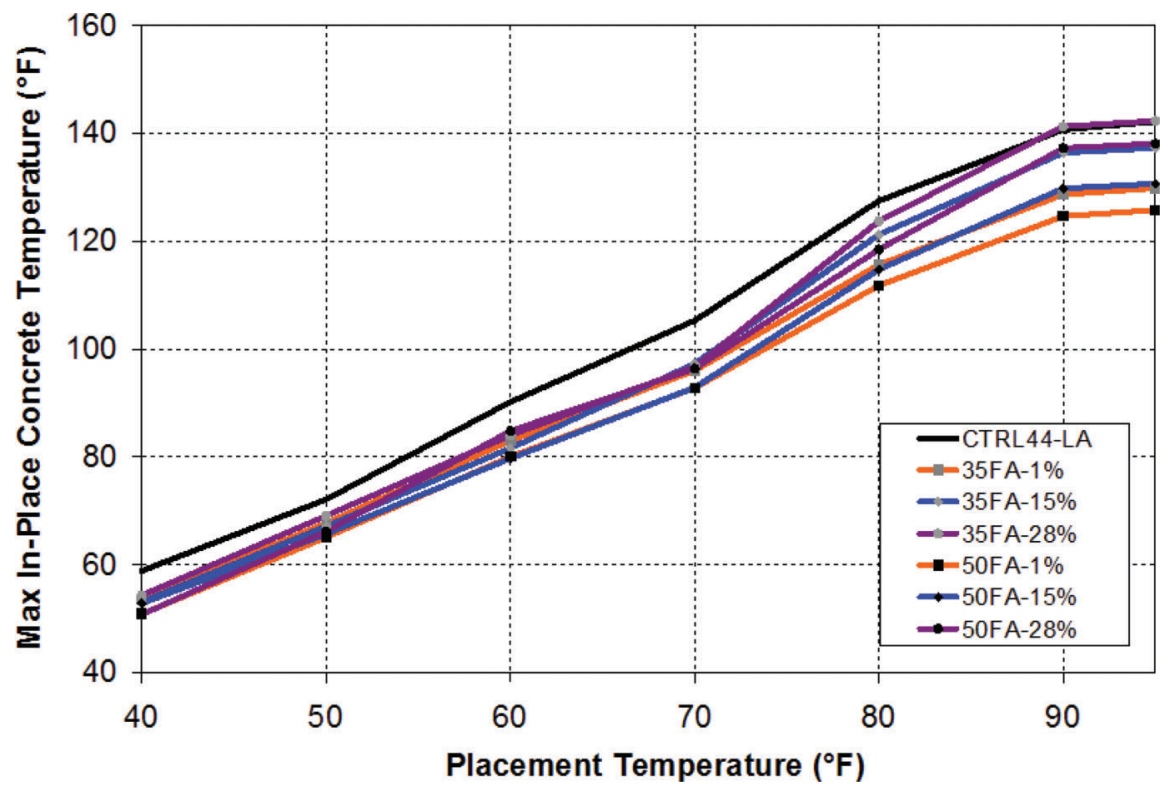

Figure 5.9 Maximum in-place concrete temperature versus concrete placement temperature for 14 in. pavement element.

TABLE 5.4

Equivalent Age at Initial Set for Mixtures Considered

\begin{tabular}{lc}
\hline \hline Mix Description & Equivalent Age at Initial Set (hours) \\
\hline CTRL44-LA & 4.38 \\
LA-35-1 & 3.54 \\
LA-35-15 & 4.57 \\
LA-35-24 & 5.15 \\
LA-35-28 & 4.73 \\
LA-50-1 & 3.61 \\
LA-50-15 & 4.12 \\
LA-50-24 & 5.78 \\
LA-50-28 & 5.73 \\
\hline
\end{tabular}

that the maximum difference in initial setting times among all 3 column sizes and the bridge deck element was 0.7 hours. The time required to reach initial set for the pavement elements, however, will be delayed, especially at low placement temperatures.

In Figure 5.10, the time required to reach initial set versus concrete placement temperature for the HVFA concrete mixtures proportioned with the 1,15 , and $28 \% \mathrm{CaO}$ fly ash sources are presented. Two plots are presented, one for the $35 \%$ fly ash dosage mixtures and one for the 50\% fly ash dosage mixtures. The results presented in this figure represent the setting times of the column and bridge deck elements. The results for the pavement element are presented in Figure 5.11.

The results presented in Figures 5.10 and 5.11 illustrate that, unlike the results obtained for maximum in-place concrete temperature, a linear relationship between initial setting time and concrete placement temperature does not exist. For all concrete elements investigated, the range in initial setting times was largest at the $40^{\circ} \mathrm{F}$ placement temperature and smallest at the $95^{\circ} \mathrm{F}$ placement temperature.
The initial setting times for the pavement elements were delayed when compared to the results for the column and bridge deck elements at all placement temperatures. The maximum difference in initial setting times for the pavement elements and column and bridge deck elements ranged from approximately 3.5 hours at the $40^{\circ} \mathrm{F}$ placement temperature to 1 hour at the $95^{\circ} \mathrm{F}$ placement temperature. These differences can be altered by the use of different curing and construction methods.

Initial set times for the HVFA concrete mixtures were delayed with increases in the fly ash $\mathrm{CaO}$ content. The HVFA concrete mixtures proportioned with the high-calcium Class $\mathrm{C}$ fly ashes exhibit the longest initial set times at all placement temperatures. At the $40^{\circ} \mathrm{F}$ placement temperature, the initial set time for the 50FA-28\% mixture placed in the pavement element was 4.8 hours greater than the control mixture set time under the same conditions. The initial set times for the $1 \% \mathrm{CaO}$ Class $\mathrm{F}$ fly ash mixtures; however, were shorter than the control mixture set times for all elements and environmental conditions.

Based on the results presented in this subsection, the following conclusions can be made concerning the in-place set time development of HVFA concrete:

- HVFA concrete may be proportioned to have similar, if not reduced, initial set times than conventional portland cement concrete when low-calcium Class F fly ashes are used with high dosages of accelerating admixtures.

- Unless very low concrete placement temperatures (40 to $50^{\circ} \mathrm{F}$ ) are expected, HVFA concrete may be used for both mass concrete elements and flatwork without encountering excessive delays in initial set times, regardless of the fly ash $\mathrm{CaO}$ content.

- An increase in fly ash dosage did not severely affect setting time results for the concrete mixtures investigated due to the use of an accelerating admixture and low $w / \mathrm{cm}$. 

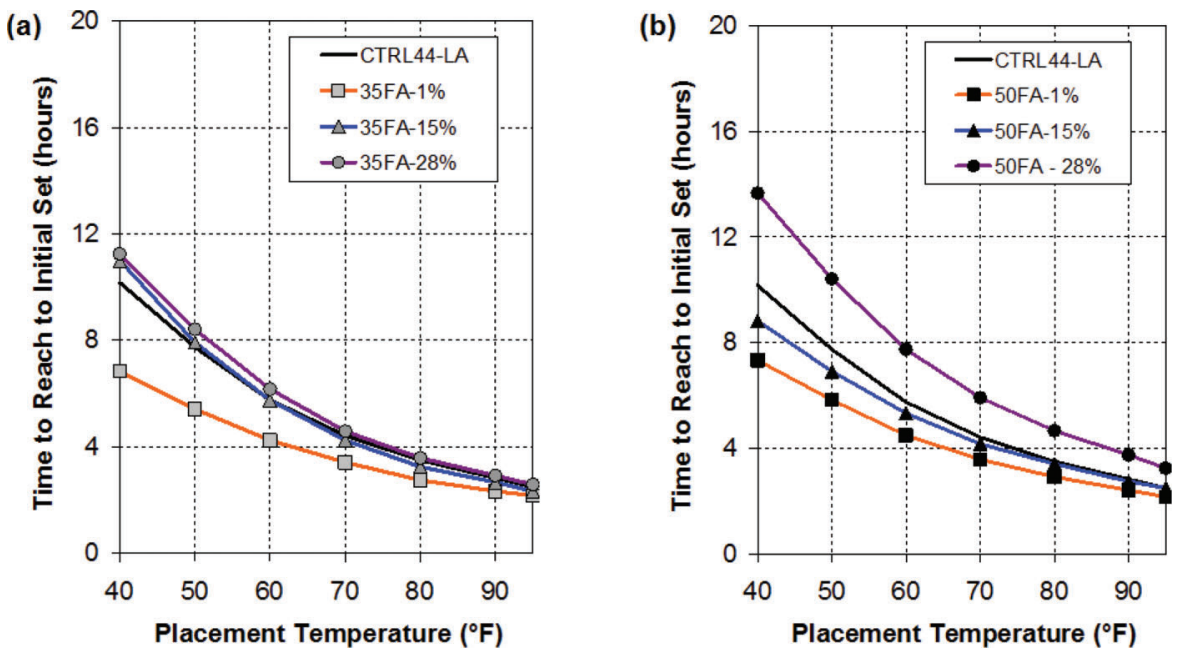

Figure 5.10 Time to reach initial for the (a) 35\% fly ash dosage and (b) 50\% fly ash dosage concrete mixtures when placed in the column or bridge deck elements.
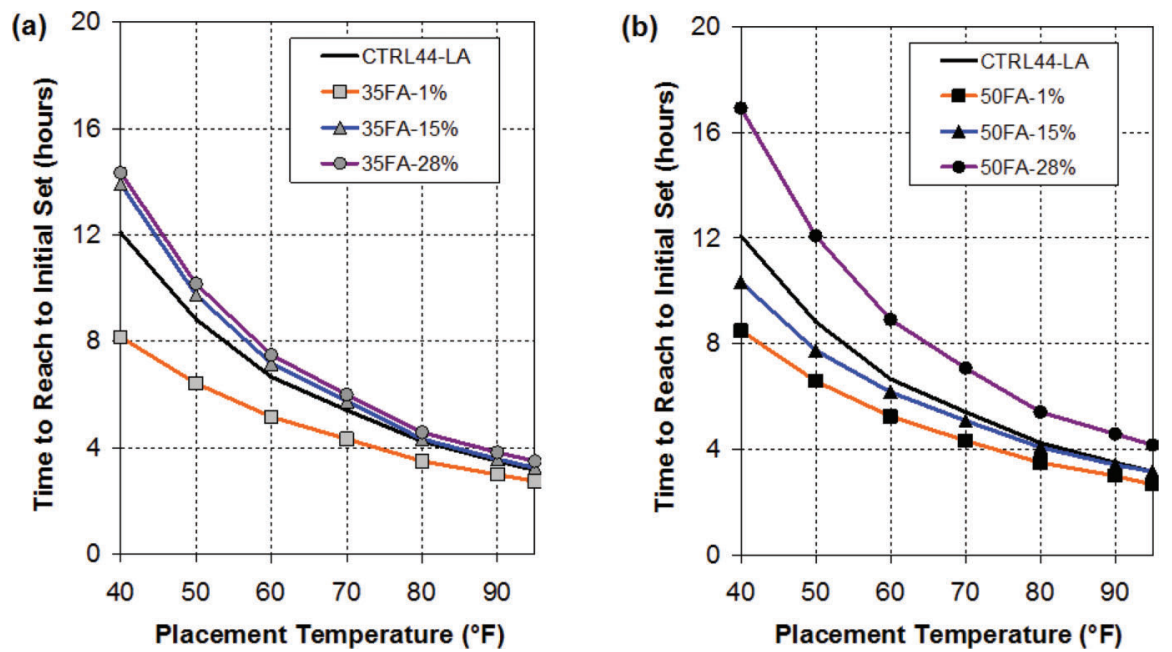

Figure 5.11 Time to reach initial for the (a) 35\% fly ash dosage and (b) 50\% fly ash dosage concrete mixtures when placed in the pavement element.

\subsubsection{Early-Age Compressive Strength Development}

In this subsection, the results from the evaluation of the in-place compressive strength development of HVFA concrete are presented. One of the main limitations to the increased use of HVFA concrete is the concern that high fly ash dosages can result in low early-age compressive strengths when compared to conventional portland cement concrete. Low early-age compressive strengths are a problem because they have the potential to negatively impact construction efficiency. The results presented in this subsection are designed to aid in the estimation of compressive strength development of HVFA concrete mixtures when placed in transportation structures under varying environmental conditions.

Formwork removal times are often based on the requirement of reaching $70 \%$ of the 28 -day design compressive strength. For this project, the control concrete mixture (CTRL44-LA) had a 28-day compressive strength of 7,100 psi. Therefore, the results in this subsection correspond to the time required to reach 5,000 psi (70\% of 7,100 psi) for each concrete mixture. For bridge decks, $70 \%$ of the specified design strength may be required for opening to traffic. Whereas for pavements, $70 \%$ of the specified design strength may be required for tie bar insertion or opening to traffic.

To complete this analysis, the temperature profile from the surface of the concrete element was used. As reported in the previous subsection, the temperature development at the concrete surface varies according to element type, placement conditions, and curing method. The flatwork elements are more susceptible to changes in environmental conditions due to their geometry and boundary conditions. The temperature development at the surface of the pavement elements is also affected by the placement time. To estimate the initial set times, the bridge deck and column elements 
were analyzed together because they share very similar temperature profiles within the first few hours after concrete placement. Because the time required to reach 5,000 psi is considerably later than the time required to reach initial set, the bridge deck and column elements must be analyzed separately when the in-place compressive strength development is considered.

The in-place compressive strength development was estimated for each HVFA concrete mixture by using the equivalent age maturity method and the temperature profiles generated with ConcreteWorks. For each HVFA concrete mixture, the time required to reach 5,000 psi was calculated using the strength-maturity parameters determined during laboratory testing. The strength-maturity parameters for all LA cement concrete mixtures are presented in Keith (2011). The activation energy values determined as per ASTM C1074 (2004) from mortar cube testing performed in Phase II-Task 2 of this project as listed in Table 5.5 were used. The strength activation energy values for all mixture are greater than those obtained from isothermal calorimetry, which is in agreement with the findings ofPinto and Schindler (2010).

The results for the time to reach $70 \%$ of the 28 -day strength versus concrete placement temperature for the $6 \times 6 \mathrm{ft}$ column, $10 \times 10 \mathrm{ft}$ column, bridge deck, and pavement elements are presented in Figures 5.12 through 5.15. All the times shown in these figures are limited to 14 days, as a time beyond 14 days will significantly delay follow-up construction activities and will thus not be typical of current practice.

The results for the compressive strength development are similar in form to the initial setting results presented in the previous subsection. As the placement temperature increases, the results converged, for all element types, which indicate the difference in the behavior of all concrete is much less during hot weather placement conditions. Also, as the fly ash dosage decreases, the time required to reach $70 \%$ of the 28 -day strength decreased for each mixture.

From the results presented in Figures 5.12 through 5.15 , it may be seen that in general an increase in fly ash $\mathrm{CaO}$ content results in less time required to reach a compressive strength that is $70 \%$ of the 28 -day strength. Typically, HVFA concretes proportioned with Class C fly ashes exhibit increased early-age compressive strengths when compared to similar Class F fly ash mixtures due to an increased rate of reaction at early ages (Smith, Raba, \& Mearing, 1982). The $28 \%$ CaO fly ash concrete mixtures required less time to reach $70 \%$ of the 28-day strength than all other HVFA mixtures at both the $35 \%$ and $50 \%$ fly ash dosages. The 35FA-15\% and $35 \mathrm{FA}-28 \%$ concrete mixtures performed similar to the control concrete regardless of the element type. Also, the 50FA-28\% concrete mixture performed similar to the control concrete when placed in the column elements. For example, the average delay in time required to reach $70 \%$ of the 28 -day strength for the 50FA$28 \%$ concrete mixture when placed in the $6 \times 6 \mathrm{ft}$ column elements was only 8 hours.

The largest delays in compressive strength development were associated with the $1 \% \mathrm{CaO}$ Class $\mathrm{F}$ fly ash concrete mixtures when placed under cold weather conditions. For example, the average delay in compressive strength development for the 50FA-1\% mixture when compared to the control ranged from 2.2 days for the $10 \times 10 \mathrm{ft}$ column elements to 6.7 days for the pavement element. These results indicate that high dosages of low-calcium Class F fly ashes should not be used under cold weather conditions when the time to achieve sufficient early-age compressive strengths are of concern.

The results presented in Figures 5.12 through 5.15 also illustrate that the compressive strength development of HVFA concrete is affected by the element type and placement temperature. The compressive strength development for the pavement element was shown to be significantly affected by cold curing conditions, especially when high dosages of Class F fly ashes were used. For the pavement element, longer curing times were required at all temperatures to reach the 5,000 psi compressive strength for the HVFA concrete mixtures and the control concrete mixture. The extended curing times required for the pavement element at cold placement temperatures may be attributed to its curing method. The pavement element was modeled with a white curing compound for all placement temperatures. The delay in compressive strength development at cold temperatures for the concrete pavement may be offset by either increasing the fresh concrete temperature prior to placement or by using cotton mats to retain the heat of the hydrating concrete in the pavement.

TABLE 5.5

LA Cement Mixtures ASTM C1074 Activation Energy Values

\begin{tabular}{|c|c|c|c|}
\hline \multicolumn{2}{|c|}{ Determined in Phase II-Task 3} & \multicolumn{2}{|c|}{ Determined in Phase II-Task 2} \\
\hline Task 3 Mixture ID & $\begin{array}{c}\text { Isothermal Calorimetry } \\
\text { Activation Energy, E (J/mol) }\end{array}$ & $\begin{array}{c}\text { ASTM C1074 Activation } \\
\text { Energy, E (J/mol) }\end{array}$ & Task 2 Mixture ID \\
\hline CTRL44 & 33,500 & 35,000 & $0.43 \mathrm{PC}$ \\
\hline $35 \mathrm{FA}-1 \%$ & 28,300 & 53,700 & 0.39 FAA 35 \\
\hline $35 \mathrm{FA}-15 \%$ & 36,300 & 52,400 & $0.39 \mathrm{FAB} 35$ \\
\hline $35 \mathrm{FA}-28 \%$ & 35,700 & 42,900 & $0.39 \mathrm{FAC} 35$ \\
\hline $50 \mathrm{FA}-1 \%$ & 29,600 & 40,900 & $0.34 \mathrm{FAA} 50$ \\
\hline $50 \mathrm{FA}-15 \%$ & 30,300 & 35,300 & $0.34 \mathrm{FAB} 50$ \\
\hline $50 \mathrm{FA}-28 \%$ & 33,700 & 43,600 & $0.34 \mathrm{FAC} 50$ \\
\hline
\end{tabular}




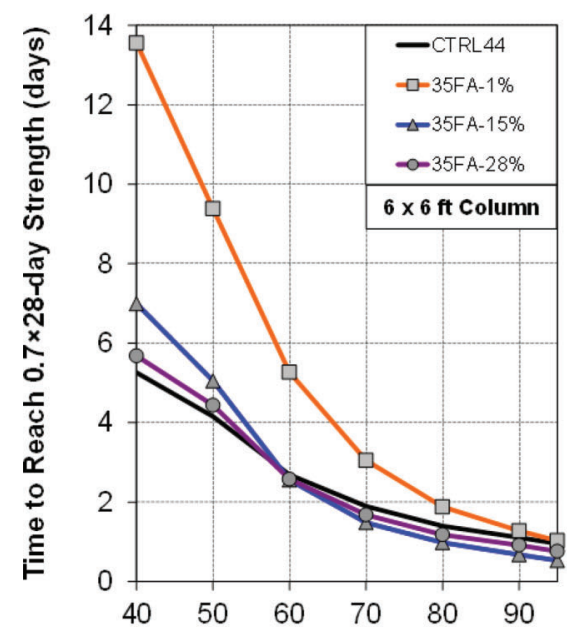

(a) Placement Temperature $\left({ }^{\circ} \mathrm{F}\right)$

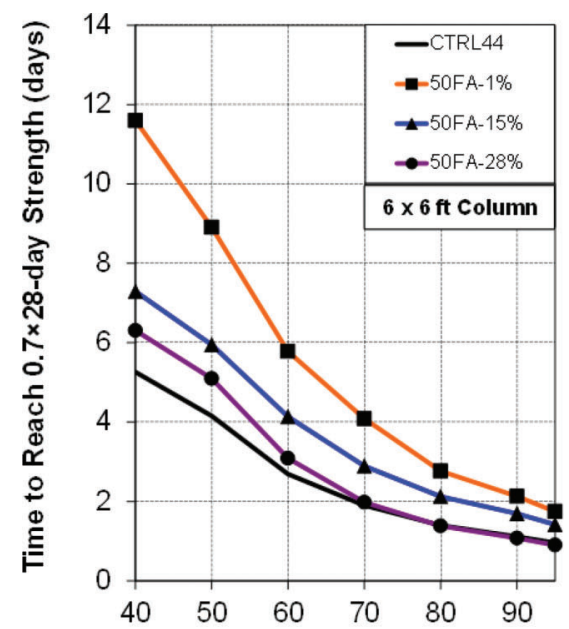

(b) Placement Temperature $\left({ }^{\circ} \mathrm{F}\right)$

Figure 5.12 Time to reach 70\% of the 28-day strength for (a) 35\% fly ash dosage and (b) 50\% fly ash dosage concrete mixtures when placed in the $6 \times 6 \mathrm{ft}$ column.

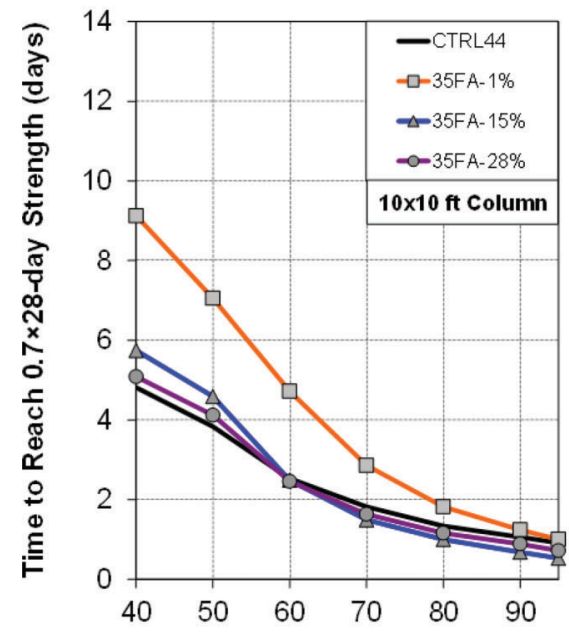

(a) Placement Temperature $\left({ }^{\circ} \mathrm{F}\right)$

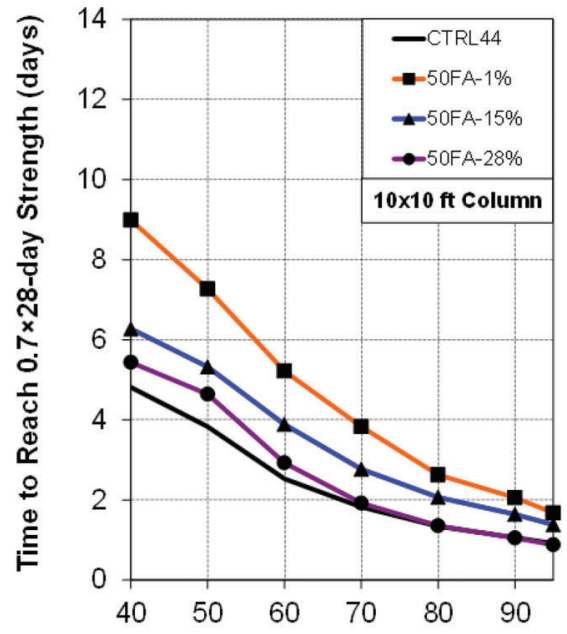

(b) Placement Temperature $\left({ }^{\circ} \mathrm{F}\right)$

Figure 5.13 Time to reach $70 \%$ of the 28-day strength for a) $35 \%$ fly ash dosage and b) $50 \%$ fly ash dosage concrete mixtures when placed in the $10 \times 10 \mathrm{ft}$ column.

Based on the results presented in this subsection, it may be concluded that HVFA concrete may be proportioned to have similar compressive strength development to that of conventional portland cement concrete. The $35 \%$ fly ash mixtures proportioned with the $15 \%$ and $28 \% \mathrm{CaO}$ fly ash sources were shown to perform comparable to the control concrete. Finally, the results indicate that HVFA concrete mixtures proportioned with high dosages of low-calcium Class F fly ashes should not be used under cold weather conditions when the time to achieve sufficient early-age compressive strengths are of concern.

\subsection{Closing Section Comments}

The results presented in this subsection are designed to provide guidance on the maximum in-place temperature development, time to reach initial set, and compressive strength development of HVFA concrete placed in transportation structures. The results presented in subsections 5.3 and 5.4 are only valid for the baseline conditions described at the beginning of the section. If the concrete mixture proportions, material properties, element geometry, or placement conditions are substantially different than what is outlined in this section, a separate analysis should be completed with ConcreteWorks. To generate in-place temperature profiles with the ConcreteWorks software, inputs for the mixture-specific activation energy, hydration parameters, and mixture proportions are required. Also, to accurately estimate the in-place setting time and compressive strength development from the ConcreteWorks temperature profiles, the equivalent age at initial set, activation energy, and strength-maturity parameters, are 


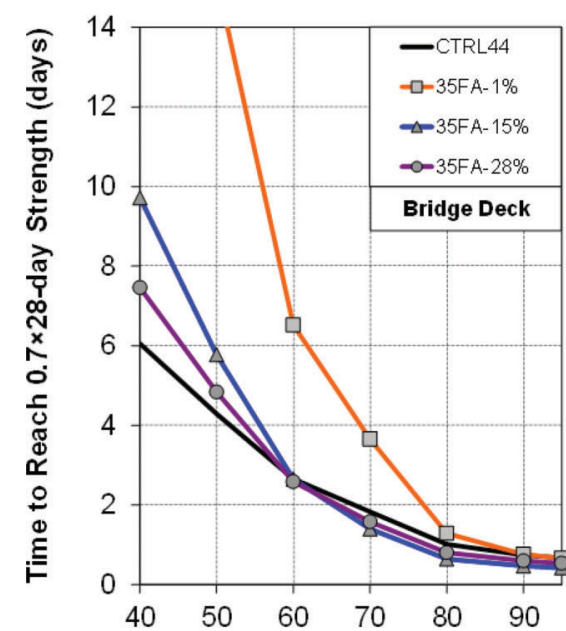

(a) Placement Temperature $\left({ }^{\circ} \mathrm{F}\right)$

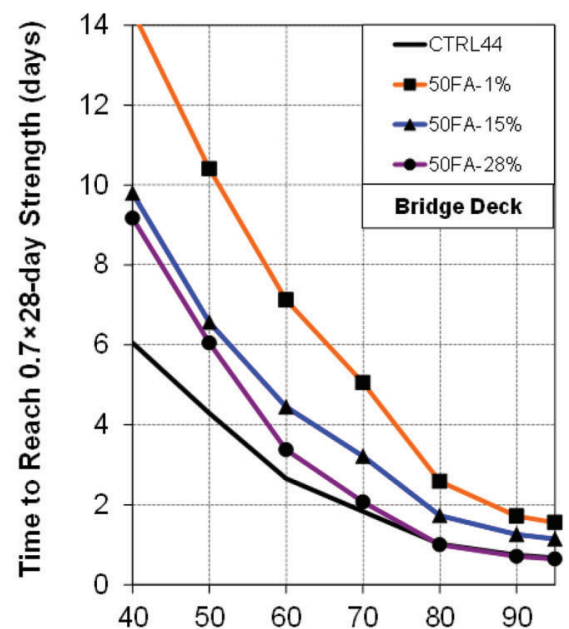

(b) Placement Temperature $\left({ }^{\circ} \mathrm{F}\right)$

Figure 5.14 Time to reach $70 \%$ of the 28-day strength for (a) 35\% fly ash dosage and (b) 50\% fly ash dosage concrete mixtures when placed in the bridge deck element.

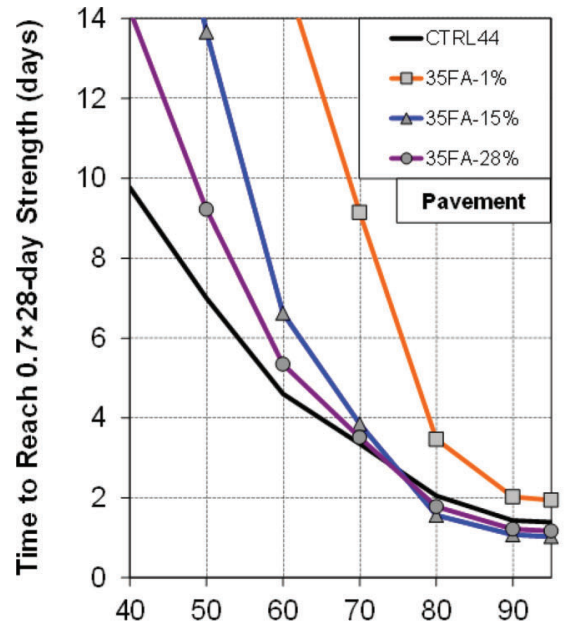

(a) Placement Temperature $\left({ }^{\circ} \mathrm{F}\right)$

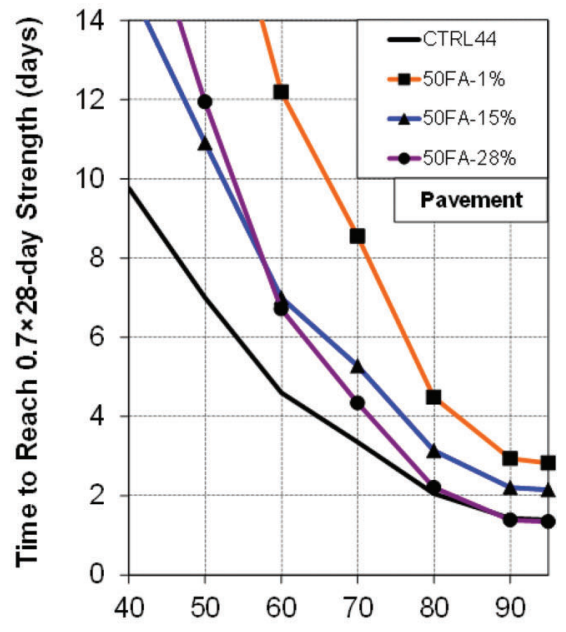

(b) Placement Temperature $\left({ }^{\circ} \mathrm{F}\right)$

Figure 5.15 Time to reach $70 \%$ of the 28-day strength for a) $35 \%$ fly ash dosage and b) $50 \%$ fly ash dosage concrete mixtures when placed in the pavement element.

required. Therefore, supplemental laboratory testing may be needed. The results presented in this section should be used as a guideline to estimate the in-place performance of HVFA concrete mixtures in various transportation structures.

\section{SUMMARY, CONCLUSIONS, AND RECOMMENDATIONS}

\subsection{Summary}

One of the main barriers to the increased use of HVFA concrete is the concern about retarded set times, especially when high fly ash dosages are specified or cold curing conditions are encountered. Retarded set times can cause delays to concrete finishing operations, causing HVFA concrete to be undesirable. Similarly, there are concerns about low early-age compressive strength development of HVFA concrete. Low earlyage compressive strengths can require extended curing times, which can negatively impact construction sequencing. Finally, there are concerns that material incompatibilities may arise when fly ash is specified in high dosages. Contractors and transportation agencies are often reluctant to use HVFA concrete due to these concerns.

A laboratory testing program was developed to investigate the effect of fly ash dosage, fly ash chemical composition, cement chemical composition, and environmental conditions on the hydration development, setting times, and compressive strength development of HVFA concrete. Concrete mixtures with $35 \%$ and $50 \%$ fly ash replacement levels were investigated. Also four fly ash sources, two Class $\mathrm{C}$ and two Class $\mathrm{F}$, were selected for testing to represent the range of fly 
ash chemical compositions available across the United States. Each concrete mixture was batched at $40^{\circ} \mathrm{F}$, $73^{\circ} \mathrm{F}$, and $105^{\circ} \mathrm{F}$. Compressive strength testing and semi-adiabatic calorimetry were conducted on all $73^{\circ} \mathrm{F}$ batches to develop strength-maturity relationships and characterize the hydration development for the HVFA concrete mixtures. Penetration resistance testing and isothermal calorimetry were conducted on all concrete batches. Results from isothermal calorimetry were used to calculate the temperature sensitivity of each HVFA concrete mixture.

The main goal of this research phase was to evaluate the in-place performance of HVFA concrete. The results from this research are aimed at providing guidance to contractors, concrete producers, and transportation agencies when specifying HVFA concrete for use in transportation infrastructure. A hydration model for HVFA concrete was developed to quantify the hydration behavior of HVFA concrete mixtures. After developing the hydration model, the ConcreteWorks software program was used to generate in-place temperature profiles for selected HVFA concrete mixtures from the laboratory testing program. Temperature profiles were generated for each concrete mixture based on varying curing conditions and concrete element types. Results from laboratory testing program (activation energies, setting data, and strength-maturity parameters) were used alongside the ConcreteWorks temperature profiles to complete an evaluation of the in-place performance of HVFA concrete. The maximum in-place concrete temperature, initial set time, and compressive strength were evaluated for various members. The final results from this analysis are intended to provide guidance to contractors, concrete producers, and transportation agencies on estimating the in-place concrete temperatures, finishing times, and form stripping times that are expected when HVFA concrete is used for transportation infrastructure.

\subsection{Conclusions}

\subsubsection{Laboratory Testing Program}

In this subsection, the conclusions corresponding to the testing and analysis conducted for each phase of the laboratory testing procedure are presented. Fly ash dosage, fly ash $\mathrm{CaO}$ content, cement chemical composition, and curing temperature were all found to affect the hydration development, time of setting, and compressive strength development for HVFA concrete mixtures. The general effect of each variable on the selected concrete properties may be summarized as follows:

- Setting times are delayed with an increase in fly ash dosage.

- Initial and final setting times are delayed when the fly ash $\mathrm{CaO}$ content is increased for a given fly ash dosage.

- Setting times for HVFA concrete are temperature dependent. As the curing temperature is decreased, set times are delayed; especially when high $\mathrm{CaO}$ Class $\mathrm{C}$ fly ashes are used at high dosages.

- Generally, as the fly ash dosage is increased, the heat generated per volume of concrete decreases.

- An increase in the fly ash $\mathrm{CaO}$ content for a given fly ash dosage, results in more heat generated per volume of concrete.

- The HVFA concrete proportioned with Class C fly ash exhibits a higher rate of early-strength development when compared to the Class F fly ash mixture.

- Generally, an increase in fly ash dosage results in lowered early-age compressive strengths. When early-age strengths are of concern, this effect can be offset by lowering the $w / \mathrm{cm}$ ratio of the HVFA mixture.

- When moist-cured, the HVFA concretes continue to gain strength at late ages, whereas the control concretes had minimal compressive strength gain after 28 days.

- Compressive strengths are decreased at most test ages for HVFA concrete mixtures proportioned with the highalkali cement source when compared to the results for the low-alkali cement source mixtures.

\subsubsection{High-Volume Fly Ash Concrete Hydration Model}

A nonlinear regression analysis was used to develop a model to predict the hydration development of HVFA concrete mixtures. The final proposed model is presented in Equations 4.5, 4.6, and 4.7. Using this model, the hydration shape parameter $(\beta)$, hydration time parameter $(\tau)$ and ultimate degree of hydration $\left(\alpha_{u}\right)$ for HVFA concrete mixtures may be computed independently based on the chemical composition of the cementitious materials and the mixture proportions of the HVFA concrete mixture. With the use of this model, the hydration parameters may be quickly estimated without the time or cost associated with SAC testing.

\subsubsection{Evaluation of the In-Place Performance of HVFA Concrete}

The conclusions relating to the in-place performance of HVFA concrete are summarized as follows:

- An increase in fly ash $\mathrm{CaO}$ content, for a given fly ash dosage, results in an increase in the maximum in-place concrete temperature, regardless of the curing conditions or element type.

- The maximum in-place concrete temperature and rate of temperature development is dependent on the concrete placement temperature and element type. At high placement temperatures, the rate of hydration is increased. This effect is amplified for large concrete elements.

- As the fly ash $\mathrm{CaO}$ content increases, for a given fly ash dosage, the maximum in-place concrete temperature increases. The use of Class F fly ash in HVFA mixtures generate less heat than the control concrete.

- For both Class F fly ash mixtures, an increase in fly ash dosage from $35 \%$ to $50 \%$ resulted in a decreased maximum in-place temperature, despite the lowered $w / \mathrm{cm}$ for the $50 \%$ fly ash mixtures. 
- An increase in fly ash dosage does not translate to reduced maximum in-place concrete temperatures when high-calcium Class C fly ashes are used for mass concrete elements. The HVFA concrete mixtures proportioned with a $50 \%$ dosage of the Class $\mathrm{C}$ fly ashes reached a higher in-place concrete temperature than the control mixture.

- When HVFA concrete is used for flatwork elements at low placement temperatures $\left(40\right.$ to $\left.60^{\circ} \mathrm{F}\right)$, the in-place temperature development is controlled by the element size and boundary conditions, rather than the fly ash dosage and $\mathrm{CaO}$ content. For small columns $(3 \times 3 \mathrm{ft}$ or smaller) and flatwork elements, high-calcium Class C fly ash sources may be used at high dosages without running the risk of exceeding the threshold for DEF formation.

- HVFA concrete may be proportioned to have similar, if not reduced, initial set times than conventional portland cement concrete when low-calcium Class F fly ashes are used with high dosages of accelerating admixtures.

- Unless very low concrete placement temperatures (40 to $50^{\circ} \mathrm{F}$ ) are expected, HVFA concrete may be used for both mass concrete elements and flatwork without encountering excessive delays in initial set times, regardless of the fly ash type.

- As an increase in fly ash $\mathrm{CaO}$ content results in less time required to reach a compressive strength that is $70 \%$ of the 28-day strength.

- Low-calcium Class F fly ashes should not be used in high dosages used under cold weather conditions - especially for flatwork and small concrete elements - when the time to achieve sufficient early-age compressive strengths are of concern.

- The in-place compressive strength development of the HVFA concrete mixtures proportioned with lower $w / \mathrm{cm}$ and the $15 \%$ and $28 \% \mathrm{CaO}$ fly ash sources are comparable to that of the control concrete mixture.

\section{REFERENCES}

ACI Committee 232. (2003). Use of fly ash in concrete (ACI 232.2R-03). Farmington Hills, MI: American Concrete Institute.

ASTM C109. (2007). Standard test method for compressive strength of hydraulic cement mortars (using 2-in. or [50$\mathrm{mm}]$ cube specimens). West Conshohocken, PA: ASTM International.

ASTM C138. (2008). Standard test method for density (unit weight), yield, and air content (gravimetric) of concrete. West Conshohocken, PA: ASTM International.

ASTM C143. (2008). Standard test method for slump of hydraulic-cement concrete. West Conshohocken, PA: ASTM International.

ASTM C192. (2007). Standard practice for making and curing concrete test specimens in the laboratory. West Conshohocken, PA: ASTM International.

ASTM C231. (2004). Standard test methods for air content of freshly mixed concrete by the pressure method. West Conshohocken, PA: ASTM International.

ASTM C232. (2007). Standard test methods for bleeding of concrete. West Conshohocken, PA: ASTM International.

ASTM C311. (2005). Standard test methods for sampling and testing fly ash or natural pozzolans for use in portland-cement concrete. West Conshohocken, PA: ASTM International.
ASTM C403. (2006). Standard test method for time of setting of concrete mixtures by penetration resistance. West Conshohocken, PA: ASTM International.

ASTM C403. (2008). Standard test method for time of setting of concrete mixtures by penetration resistance. West Conshohocken, PA: ASTM International.

ASTM C494. (2005). Standard specification for chemical admixtures for concrete. West Conshohocken, PA: ASTM International.

ASTM C566. (1997). Standard test method for total evaporable moisture content of aggregate by drying. West Conshohocken, PA: ASTM International.

ASTM C1064. (2008). Standard test method for temperature of freshly mixed hydraulic-cement concrete. West Conshohocken, PA: ASTM International.

ASTM C1074. (2004). Standard practice for estimating concrete strength by the maturity method. West Conshohocken, PA: ASTM International.

Bilodeau, A., Malhotra, V. M., \& Seabrook, P. T. (2001). Use of high-volume fly ash concrete at the Liu Centre. Ottawa, Canada: International Centre for Sustainable Development of Cement and Concrete (ICON), Materials and Research Laboratory, CANMET, Natural Resources Canada.

Bisaillon, A., Rivest, M., \& Malhotra, V. M. (1994). Performance of high volume fly ash concrete in large experimental monoliths. ACI Materials Journal, 91(2), 178-187. http://dx.doi.org/10.14359/4571

Bogue, R. H. (1947). The chemistry of Portland cement. New York, NY: Reinhold Publishing Corporation.

Carino, N. J. (2004). The maturity method. In V. M. Malhotra \& N. J. Carino (Eds.), Handbook on nondestructive testing of concrete (2nd ed., 5.1-5.47). West Conshohocken, PA: ASTM International.

Cost, T. (2009). Thermal measurements of hydrating concrete mixtures-A useful quality control tool for concrete producers (NRMCA Publication 2PE004). Silver Spring, MD: NRMCA Engineering Division.

Eren, O., Brooks, J. J., \& Celik, T. (1995). Setting times of fly ash and slag-cement concretes as affected by curing temperature. Cement, Concrete and Aggregates, 17(1), 11-17. https://doi.org/10.1520/CCA10331J

Folliard, K. J., Juenger, M. G., Schindler, A. K., Riding, K., Poole, J., Kallivokas, L. F., ... Meadows, J. L. (2008). Prediction model for concrete behavior - Final report (Research Report No. 0-4563-1). Austin, TX: Center for Transportation Research, University of Texas at Austin.

Ge, Z., \& Wang, K. (2009). Modified heat of hydration and strength models for concrete containing fly ash and slag. Computers and Concrete, 6(1), 19-40. http://dx.doi.org/10. 12989/cac.2009.6.1.019

Hansen, P. F., \& Pederson, J. (1977). Maturity computer for controlled curing and hardening of concrete strength. Nordisk Betong, 1, 19-34.

Hansen, P. F., \& Pederson, J. (1985). Curing of concrete structures. CEB Information Bulletin 166.

Keith, K. P. (2011). Characterization of the behavior of high volume fly ash concrete (Doctoral dissertation). Auburn, AL: Auburn University.

Kishi, T., \& Maekawa, K. (1995). Thermal and mechanical modeling of young concrete based on hydration process of multi-component cement minerals. In R. Springenschmid (Ed.), Proceedings of the International RILEM Symposium on Thermal Cracking in Concrete at Early Ages (pp. 11-18). London, UK: E \& FN Spon.

Kjellsen, K. O., \& Detwiler, R. J. (1992). Reaction kinetics of Portland cement mortars hydrated at different tempera- 
tures. Cement and Concrete Research, 22(1), 112-120. http:// dx.doi.org/10.1016/0008-8846(92)90141-H

Langley, W. S., Carette, G. G., \& Malhotra, V. M. (1992). Strength development and temperature rise in large concrete blocks containing high volumes of low-calcium (ASTM Class F) fly ash. ACI Materials Journal, 89(4), 362 368. http://dx.doi.org/10.14359/2514

Malhotra, V. M., \& Mehta, P. K. (2002). High-performance, high-volume fly ash concrete. Ottawa, Canada: Supplementary Cementing Materials for Sustainable Development, Inc.

Mehta, P. K., \& Monteiro, P. (2006). Concrete: Microstructure, properties and materials (3rd ed.). New York, NY: McGraw Hill.

Mills, R. H. (1966). Factors influencing cessation of hydration in water-cured cement pastes. In Symposium on the structure of portland cement paste and concrete (HRB Special Report No. 90, pp. 406-424). Washington, DC: Highway Research Board.

Mindess, S., Young, J. F., \& Darwin, D. (2003). Concrete (2nd ed.). Upper Saddle River, NJ: Pearson.

Pane, I., \& Hansen, W. (2002). Concrete hydration and mechanical properties under nonisothermal conditions. ACI Materials Journal, 99(6), 534-542.

Philleo, R. E. (1991). Concrete science and reality. In Materials Science of Concrete II (pp. 1-8). Westerville, OH: American Ceramic Society.

Pinto, R. C. A., \& Hover, K.C. (1999). Application of maturity approach to setting times. ACI Materials Journal, 96(6), 686-691. http://dx.doi.org/10.14359/795

Pinto, R. C. A., \& Schindler, A. K. (2010). Unified modeling of setting and strength development. Cement and Concrete Research, 40(1), 58-65. http://dx.doi.org/10.1016/j. cemconres.2009.08.010

Poole, J. L., Riding, K.A., Folliard, K. J., Juenger, M. C. G., \& Schindler, A. K. (2007). Methods for calculating activation energy for Portland cement. ACI Materials Journal, 104(1), 303-311. http://dx.doi.org/10.14359/18499

Popovics, S. (1993). Portland cement - fly ash - silica fume systems in concrete. Advanced Cement Based Materials, 1(2), 93-91. http://dx.doi.org/10.1016/1065-7355(93)90013-E

Powers, T. C., \& Brownyard, T. L. (1948). Studies of the physical properties of hardened Portland cement paste (Bulletin No. 22). Chicago, IL: Portland Cement Association, Research Laboratories.

Prusinski, J. R., Fouad, F. H., \& Donovan, M. J. (1993). Plant performance of high strength prestressed concrete made with Class C fly ash. In Proceedings of the 10th International Ash Use Symposium (Paper 41, Document TR-101774). Palo Alto, CA: EPRI.
Ravina, D., \& Mehta, P. K. (1986). Properties of fresh concrete containing large amounts of fly ash. Cement and Concrete Research, 16(2), 227-238. http://dx.doi.org/10. 1016/0008-8846(86)90139-0

Riding, K. A. (2007). Early age concrete thermal stress measurement and modeling (Doctoral dissertation). Austin, TX: The University of Texas at Austin.

RILEM CEA 42. (1981). Properties of set concrete at early ages-State-of-the-art report. Materials and Structures, 14(84), 400-450.

RILEM TC 119-TCE. (1997). Adiabatic and semi-adiabatic calorimetry to determine the temperature increase in concrete due to the hydration heat of the cement. In R. Springenschmid (Ed.), Prevention of thermal cracking in concrete at early ages (RILEM Report 15, pp. 315-346. London: E \& FN Spon.

Schindler, A. K. (2004). Effect of temperature on hydration of cementitious materials. ACI Materials Journal, 101(1), 72-81. http://dx.doi.org/10.14359/12990

Schindler, A. K., \& Folliard, K. J. (2005). Heat of hydration models for cementitious materials. ACI Materials Journal, 102(1), 24-33. http://dx.doi.org/10.14359/14246

Smith, R. L., Raba, C. F., \& Mearing, M. A. (1982). The utilization of Class C fly ash in concrete. In J. S. Halow \& J. N. Covey (Eds.), The Challenge of Change-Sixth International Ash Utilization Symposium proceedings, Vol. 1 (DOE/METC/82-52). Morgantown, WV: National Energy Technology Laboratory, U.S. Department of Energy.

Spiratos, N., Pagé, M., Milvaganam, N., Malhotra, V. M., \& Jolicoeur, C. (2003). Superplasticizers for concrete: Fundamentals, technology, and practice. Ottawa, Canada: Supplementary Cementing Materials for Sustainable Development.

Taylor, H. F. W., Famy, C., \& Scrivener, K. L. (2001). Delayed ettringite formation. Cement and Concrete Research, 31(5), 683-693. http://dx.doi.org/10.1016/S00088846(01)00466-5

Thomas, M. (2007). Optimizing the use of fly ash in concrete. Portland Cement Association. Skokie, IL. 24 pages.

Thomas, M. D. A, Mukherjee, P. K., Sato, J. A., \& Everitt, M. F. (1995). Effect of fly ash compsotion on the thermal cracking of concrete. ACI Special Publication, 153, 81-98. http://dx.doi.org/10.14359/1025

Wade, S. A., Nixon, J. M., Schindler, A. K., \& Barnes, R. W. (2004). Setting behavior of concrete cured at fluctuating temperatures. Transportation Research Board annual meeting compendium of papers [CD-ROM]. Washington, DC: Transportation Research Board.

Weakley, R. W. (2010). Evaluation of semi-adiabatic calorimetry to quantify concrete setting (Master's thesis). Auburn, AL: Auburn University. 
PHASE II, TASKS 4 AND 5: INTERNAL CURING AND EARLY AGE CRACKING-INTRODUCTION

Tasks 4 and 5 have been divided into four different chapters. The first chapter (Chapter 4) presents a new approach of using higher volumes of fly ash (HVFA) in concrete including internal curing (IC). It focuses on the general performance of internally cured high volume fly ash (IC-HVFA) mixtures on mechanical, shrinkage and hydration. The second chapter (Chapter 5) deals with the evaluation of the cracking potential in this type of mixtures. This is done using the Dual Ring Test. In relation to this, a large scale test is also presented (corresponding to Phase III of the Project). The third chapter (Chapter 6) includes a study on the transport properties of IC-HVFA mixtures. Finally, the fourth chapter (Chapter 7) focuses on the addition of a ternary component to high volume fly ash (HVFA) mixtures in order to reduce their delays in early age reactions and setting times. 
CHAPTER 4. PHASE II, TASKS 4 AND 5: APPLICATION OF INTERNAL CURING FOR MIXTURES CONTAINING HIGH VOLUMES OF FLY ASH

Igor De la Varga, Javier Castro, Dale P. Bentz, W. Jason Weiss

\section{ABSTRACT}

Sustainability has become an important issue in the concrete industry in recent years. One approach that has the potential to make concrete more sustainable is to replace a portion of portland cement clinker with alternative cementitious materials such as fly ash since this can reduce the carbon footprint of concrete. While fly ash is a waste material obtained from coal combustion in power plants that is broadly used in concrete applications, this work emphasizes using a greater volume of fly ash to replace cement than what is typical in current practice. This report focuses on testing performed on mixtures that would be consistent with the mortar portion of a concrete bridge deck mixture for many state departments of transportation (DOTs). In this work a relatively large percentage of cement $(40 \%, 60 \%$, or $80 \%$ by volume) is replaced with Class $\mathrm{C}$ fly ash. To overcome concerns associated with slow set and early-age strength development that are often expressed with the high volume fly ash mixtures (HVFA), the water-to-cementitious materials ratio $(w / \mathrm{cm})$ by mass has been reduced from the conventional value of 0.42 to 0.30 . To overcome potential complications that the low $w / \mathrm{cm}$ may cause in terms of self-desiccation, internal curing (IC) with prewetted lightweight aggregate was used to reduce shrinkage and increase hydration. By adopting this approach (lowering the $w / c$ and using IC), IC HVFA mixtures show additional benefits that should permit their broader application.

\section{BACKGROUND}

Fly ash has historically been used in concrete transportation structures (ACI Committee 232, 1996; Davis, Carlson, Kelly, \& Davis, 1937; Halstead, 1986; Helmuth, 1987; Malhotra \& Ramezanianpour, 1994). While one can consider fly ash as an addition or as a cement replacement, it is truly the replacement of cement with fly ash that enables the fly ash to be considered as an efficient means to reduce the clinker factor, and $\mathrm{CO}_{2}$ production in concrete (Malhotra, 1999a, 1999b; McCaffrey, 2002; Mehta, 2002; Worrell, Price, Martin, Hendirks, \& Ozawa, 2001). In addition, the use of fly ash has the potential benefits of reduced mixture cost, reduced embodied energy, and improved long-term concrete performance (Bilodeau, Sivasundaram, Painter, \& Malhotra, 1994; Malhotra, 1990; Hansen, 1990; Naik, Singh, \& Ramme, 2998; Ramlochan, Zacarias, Thomas, \& Hooton, 2003; Thomas \& Matthews, 1992). This is the reason why many producers and transportation agencies are aiming to use higher volumes of fly ash as a replacement for cement in their transportation structures. However, many hurdles exist to implementing this type of mixture. The hurdles include (1) potential incompatibilities among fly ash, admixtures, and cement; (2) strict limits on the maximum fly ash permitted and the time of the year that it can be used; (3) delays in set time (Bentz \& Ferraris, 2010) and strength development that slow construction operations; (4) concern about providing enough and proper curing; and (5) long-term durability concerning scaling and freeze/thaw issues (Krishnan, Mehta, Olek, \& Weiss, 2006; Neuwald, Krishnan, Weiss, Olek, \& Nantung, 2003). For instance, a typical class C concrete bridge in the state of Indiana requires $390 \mathrm{~kg} / \mathrm{m}^{3}$ of cementitious material, of which no more than $20 \%$ of cement by mass may be replaced by fly ash. Further, the Indiana Department of Transportation (INDOT) specification limits fly ash to be used in the concrete mixture between April 1 and October 15 of the same calendar year (INDOT, 2012).

The general approach used in this report for developing High Volume Fly Ash (HVFA) concrete is using a lower water-to-cementitious materials ratio $(w / \mathrm{cm})$ to offset the expected slower strength development. This is also in agreement with recent tendencies of developing stronger and more durable concretes through the use of lower $w / \mathrm{cm}$, corresponding to High Performance Concretes (HPC). However, these beneficial properties also come with a drawback, as HPCs have been shown to be more sensitive to early-age cracking (Shah \& Weiss, 2000; Weiss, Yang, \& Shah, 1999). This early-age cracking may be due to increased temperature rise shortly after placement due to the high cement contents that are typically used, increased plastic shrinkage cracking due to reduced bleeding rates, and increased autogenous shrinkage (Shah, Weiss, \& Yang, 1997). While plastic shrinkage and thermal volume changes have been recognized in concrete construction over the last several decades and methodologies have been developed to deal with these effects, autogenous shrinkage is a problem that has gained attention more recently (Bentur, 2003; Bentz \& Peltz, 2008; Brown, Smith, Sellers, Folliard, \& Breen, 2007; Shah, Weiss, \& Yang, 1998), because of the increased use of concrete in practice that contains water-reducing admixtures, enabling concretes to be made with lower $w / \mathrm{cm}$.

Autogenous shrinkage is a deformation not caused by external influences (i.e., moisture transfer or temperature changes). Rather, autogenous shrinkage can be thought of as an 'internal drying' caused by the hydration reactions and their accompanying chemical shrinkage. Chemical shrinkage occurs when cement reacts with water as the reacted products occupy a smaller volume than the initial constituents (L'Hermite, 1962; Le Chatelier, 1900). In fluid systems (e.g., prior to set), this chemical shrinkage does not cause much concern and the entire system collapses on itself resulting in an externally measured volume change that is equal to the chemical shrinkage. However, after the concrete begins to harden, the structure of the cementitious matrix does not enable the externally measured volume change to be 

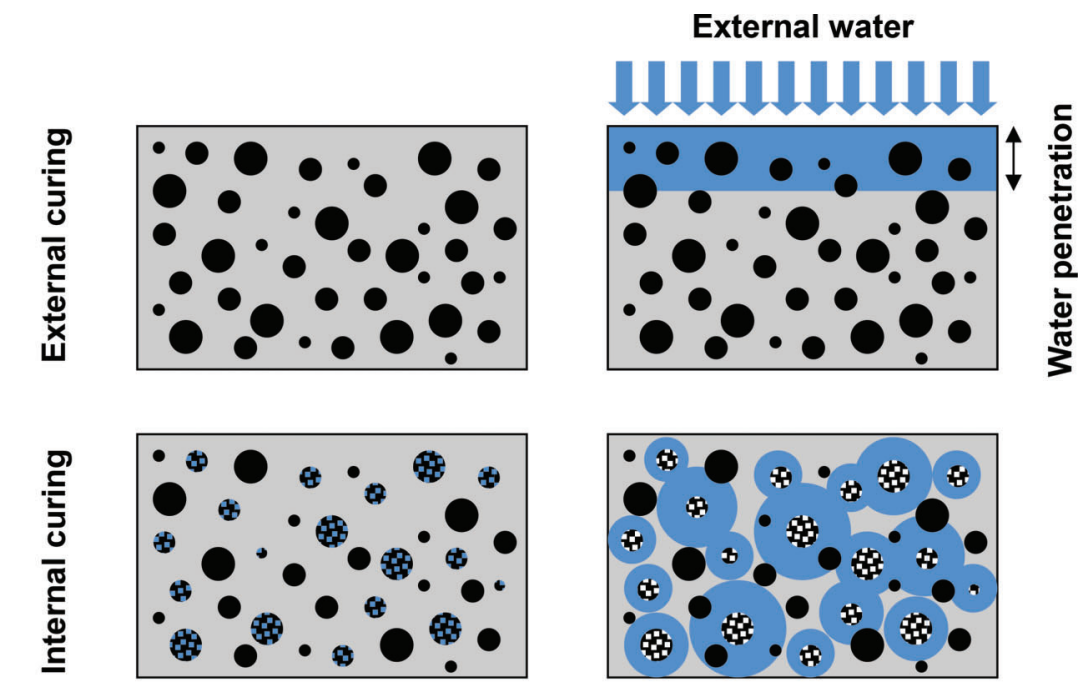

Initial specimen

After curing

\section{Normal aggregate Water filled intrusion}

Cured zone

Figure 1.1 Conceptual illustration of the differences between external and internal curing.

equal to the volume of chemical shrinkage (Sant, Lura, \& Weiss, 2006). As a result, vapor filled spaces are created within the matrix and stress begins to develop in the hardening paste (Jensen \& Hansen, 2001). This is commonly referred to as self-desiccation (i.e., internal drying). This 'internal drying' can be mitigated through the use of additional water during external curing. However, this technique has its limitations when low $\mathrm{w} / \mathrm{cm}$ concretes are used, since depercolation of the capillary pores can happen at early ages, which limits movement of water to the core of the concrete. It has been proposed by several researchers (ACI Committee 231, 2010; Bentz \& Snyder, 1999; Bentz \& Jensen, 2006; Henkensiefken, Castro, Bentz, Nantung, \& Weiss, 2009; Jensen \& Hansen, 2001; Kovler \& Jensen, 2007; Philleo, 1991) that an improved method for delivering 'curing water' to low $w / \mathrm{cm}$ concrete would be to place 'packets or inclusions' of water throughout the matrix rather than just placing the water at the surface. This is illustrated conceptually in Figure 1.1.

\section{RESEARCH APPROACH}

This study will evaluate a potential strategy for using high volumes of fly ash to replace cement. The work will seek to minimize potential reductions in early strength development that can occur with fly ash by using a lower $w / \mathrm{cm}$. While a lower $w / \mathrm{cm}$ would be beneficial in terms of improved mechanical and transport properties, it has been shown that these mixtures may be more susceptible to early-age cracking. These mixtures will be internally cured to reduce self-desiccation which will reduce autogenous shrinkage and cracking potential while enabling more of the fly ash to react.

\section{PROPORTIONING MIXTURES FOR INTERNAL CURING}

Internal curing is achieved by using a prewetted fine light-weight aggregate (LWA) to provide the internal curing water. To proportion the internally cured mixtures a methodology is used that is based on a procedure developed by Bentz and Snyder (1999), in which the amount of LWA is calculated based on the chemical shrinkage occurring in the sample. Equation 3.1 permits the calculation of the amount of LWA based on this theory. It is interesting to note that fly ash will also contribute to the chemical shrinkage; therefore the equation must be based on the binder content, chemical shrinkage of the binder, and degree of reaction of the binder, respectively (Bentz \& Weiss, 2011).

$$
M_{L W A}=\frac{C_{f} \times C S \times \alpha_{\max }}{S \times \varphi_{L W A}}
$$

where $\mathrm{M}_{\mathrm{LWA}}\left(\mathrm{kg} / \mathrm{m}^{3}\right)$ is the mass of LWA (in a dry state) that needs to be pre-wetted to provide water to fill in the voids created by chemical shrinkage, $\mathrm{C}_{f}$ $\left(\mathrm{kg} / \mathrm{m}^{3}\right)$ is the binder content of the mixture, CS ( $\mathrm{mL}$ of water per $g$ of binder) is the chemical shrinkage of the binder, $\alpha_{\max }$ (unitless) is the expected maximum degree of hydration (0 to 1), $\mathrm{S}$ (unitless) is the expected degree of saturation of the LWA and was taken to be 1 in this study when the dry LWA was soaked for $24 \mathrm{~h}$, and $\Phi_{\text {LWA }}$ ( $\mathrm{kg}$ of water $/ \mathrm{kg}$ of dry LWA) is the absorption capacity of the LWA (taken here as the $24 \mathrm{~h}$ absorption value). It has been stated that it may be more appropriate to use desorption of the LWA down to some relative humidity (e.g., $94 \% \mathrm{RH}$ ) rather than its absorption (Bentz, Lura, \& Roberts, 2005). Studies have described the measurement of this desorption and 
it is typically $90 \%-95 \%$ of the $24-\mathrm{h}$ absorption for many aggregates used in the United States, including the one used in this report (Castro, Keiser, Golias, \& Weiss, 2011; Pour-Ghaz, Castro, Kladivko, \& Weiss, 2011).

The use of HVFA concrete mixtures including IC may potentially be utilized to reduce shrinkage and shrinkage cracking Brown et al., 2007). Furthermore, the use of IC may provide a method to increase earlyage strength gain and may enable the mixture to react for a longer time since water can be supplied to the concrete over a longer time period. For example, INDOT specifications recommend that fly ash mixtures be cured for longer durations. If we consider that IC can provide water over a longer period of time, it also provides a benefit in this sense.

\section{MATERIALS AND MIXTURE PROPORTIONS}

An ordinary portland cement (OPC), ASTM C15009 Type I/II, was used in this study, with a Blaine fineness of $476 \mathrm{~m}^{2} / \mathrm{kg}$, a density of $3170 \mathrm{~kg} / \mathrm{m}^{3}$, an estimated Bogue potential phase composition of $52 \% \mathrm{C}_{3} \mathrm{~S}, 18 \%$ $\mathrm{C}_{2} \mathrm{~S}, 8 \% \mathrm{C}_{3} \mathrm{~A}$, and $9 \% \mathrm{C}_{4} \mathrm{AF}$ by mass, and a $\mathrm{Na}_{2} \mathrm{O}$ equivalent of $0.5 \%$ by mass. A Class C fly ash (ASTM C618-08a) was also used with a density of $2630 \mathrm{~kg} / \mathrm{m}^{3}$. Chemical analyses of both cement and fly ash are summarized in Table 4.1. The particle size distributions (PSDs) of the cement and fly ash are shown in Figure 4.1, each measured with laser diffraction.

The fine aggregate used was ordinary river sand with a fineness modulus of 2.71 and an apparent specific gravity of 2.58. Rotary kiln expanded shale (i.e., a lightweight fine aggregate) was used with a fineness modulus of 3.97 and a specific gravity (dry) of 1.38 . The lightweight aggregate (LWA) was measured to have a $24 \mathrm{~h}$ water absorption of $17.5 \%$ by dry mass, when this material was tested using the paper towel technique (Castro et al., 2011; NYSDOT, 2008). A polycarboxylate-based high-range water-reducing admixture (HR WRA) was added at variable dosage by mass of cement in order to maintain the same (mini) slump in all mortars (Kantro, 1980). It is interesting to note that the

TABLE 4.1

Chemical Composition of the Cement and Fly Ash Used in This Study

\begin{tabular}{|c|c|c|}
\hline & Cement & Fly Ash \\
\hline Class & $\mathrm{I} / \mathrm{II}$ & $\mathrm{C}$ \\
\hline Silicon Dioxide $\left(\mathrm{SiO}_{2}\right), \%$ & 19.97 & 38.71 \\
\hline Aluminum Oxide $\left(\mathrm{Al}_{2} \mathrm{O}_{3}\right), \%$ & 4.81 & 19.15 \\
\hline Iron Oxide $\left(\mathrm{Fe}_{2} \mathrm{O}_{3}\right), \%$ & 2.89 & 6.49 \\
\hline Sum of $\mathrm{SiO}_{2}, \mathrm{Al}_{2} \mathrm{O}_{3}, \mathrm{Fe}_{2}, \mathrm{O}_{3}, \%$ & 27.67 & 64.35 \\
\hline Calcium Oxide (CaO), \% & 63.27 & 23.51 \\
\hline Magnesium Oxide (MgO), \% & 1.54 & 5.29 \\
\hline Sulfur Trioxide $\left(\mathrm{SO}_{3}\right), \%$ & 3.27 & 1.36 \\
\hline Potassium Oxide $\left(\mathrm{K}_{2} \mathrm{O}\right), \%$ & 0.38 & 0.58 \\
\hline Sodium Oxide $\left(\mathrm{Na}_{2} \mathrm{O}\right), \%$ & 0.28 & 1.64 \\
\hline Loss on Ignition, \% & 2.85 & 0.30 \\
\hline Moisture Content, \% & - & 0.11 \\
\hline
\end{tabular}

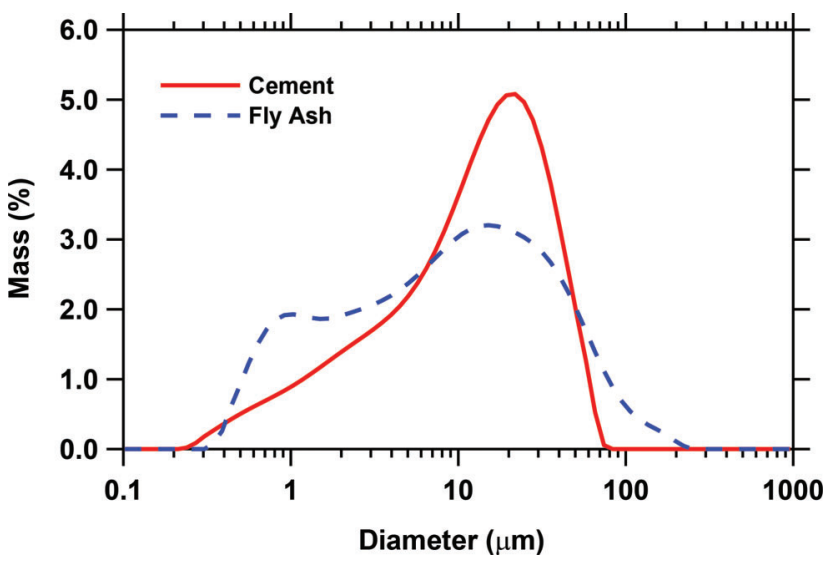

Figure 4.1 Particle size distributions (PSD) of cement and fly ash, as measured by laser diffraction.

amount of HRWRA was reduced as the fly ash content was increased (Table 4.2), likely due to the dilution in the cement content (Bentz \& Ferraris, 2010) and to the spherical shape of the fly ash particles compared to the angular shape of the cement particles. This effect may also provide a further decrease in the cost of these mixtures depending on the local availability of the materials.

A matrix of mortar mixtures was developed in which four $w / \mathrm{cm}$ levels and four fly ash replacement levels were evaluated. This matrix is shown in Table 4.2 (numbers in parentheses represent the HRWRA dosages by mass of binder for each mortar). Mortar with a $w / \mathrm{cm}$ of 0.42 and no fly ash represents a typical bridge deck concrete in the state of Indiana and is therefore taken as a reference. Not all the fly ash replacement levels were used in all mortars since fly ash enhances workability, and more prone-to-segregation specimens are thus obtained at higher $w / \mathrm{cm}$. Fly ash replacement is made on a volume basis. While many current practices replace cement with fly ash on a mass basis, due to significant differences in their specific gravities this alters the total volume of paste in the concrete, having an effect on various concrete properties (e.g., strength, shrinkage, durability, workability). By using a volume replacement, the total paste volume in the concrete is maintained as constant and this is the reason why this approach was chosen.

In two of the fly ash mortars, corresponding to $w / \mathrm{cm}$ of 0.30 and fly ash contents of $40 \%$ and $60 \%$ by volume (shaded areas), a portion of the regular fine aggregate was replaced with prewetted LWA. It is important to note that the volume of aggregate (LWA and sand) remains constant at $55 \%$, since only a portion of the sand was replaced with an equal volume of LWA. The replacement level for the LWA mixtures corresponds to the amount of LWA necessary to supply sufficient IC water to eliminate self-desiccation according to Equation 3.1, with $\mathrm{CS}=0.064 \mathrm{~mL}$ water/g binder and $\alpha_{\max }=0.83$. The volume of mortar occupied by the LWA corresponds to $14.9 \%$ and $14.5 \%$ for the $40 \%$ and $60 \%$ fly ash mortars, respectively. 
TABLE 4.2

List of Mortar Mixtures Prepared in the Study

\begin{tabular}{|c|c|c|c|c|c|}
\hline \multirow[b]{2}{*}{$w / c$ or $w / c m$} & \multicolumn{5}{|c|}{ Fly Ash Volume (\%) } \\
\hline & $0[0]$ & $20[17.2]$ & $40[35.7]$ & $60[55.5]$ & 80 [76.9] \\
\hline 0.45 & $X(0 \%)$ & - & - & - & - \\
\hline 0.42 & X $(0 \%)$ & X $(0 \%)$ & - & - & - \\
\hline 0.36 & $\mathrm{X}(0.3 \%)$ & $\mathrm{X}(0.1 \%)$ & $\mathrm{X}$ & - & - \\
\hline 0.3 & $X(0.5 \%)$ & $\mathrm{X}(0.35 \%)$ & $\mathrm{X}(0.2 \%)$ & $\mathrm{X}(0.1 \%)$ & $X(0 \%)$ \\
\hline
\end{tabular}

Note: The proportions are shown primarily as a volumetric replacement; the mass replacement has also been added in square brackets parentheses along the top row. HRWRA dosages as mass percent of binder solids shown in parentheses.

The mortars were prepared in accordance with ASTM C305-11. The LWA was oven dried, air cooled, and then submerged in water for $24 \mathrm{~h} \pm 1 \mathrm{~h}$ prior to mixing. The LWA was submerged in the total volume of water that included the mixing water needed for cement hydration and the water that would be absorbed by the LWA itself in $24 \mathrm{~h}$. The excess water (water not absorbed into the LWA during $24 \mathrm{~h}$ ) was then decanted and used as the mixing water for that particular mortar.

\section{EXPERIMENTAL RESULTS}

\subsection{Mechanical Properties}

Three $102 \mathrm{~mm}$ diameter x $204 \mathrm{~mm}$ tall [4 inch x 8 inch] cylindrical specimens were prepared in accordance with ASTM C192/C192M-07 for each mortar mixture. At each age, three specimens were used to assess compressive strength (ASTM C39-10) and two specimens were used to measure the elastic modulus (ASTM C469-02) prior to strength testing. Following demolding at 1 day, all specimens were sealed in plastic bags and stored in an environmental chamber at $23^{\circ} \mathrm{C} \pm$ $0.5^{\circ} \mathrm{C}\left[73.4^{\circ} \mathrm{F} \pm 0.9^{\circ} \mathrm{F}\right]$. The cylinders were tested at several ages: $(1,3,7,14,28,91$, and 365$) \mathrm{d}$.

The results obtained can be observed in Figure 5.1. As expected, as the $w / \mathrm{cm}$ is decreased, higher compressive strengths are achieved.

Under sealed conditions without IC and regardless of the $w / \mathrm{cm}$, their self-desiccation will ultimately produce

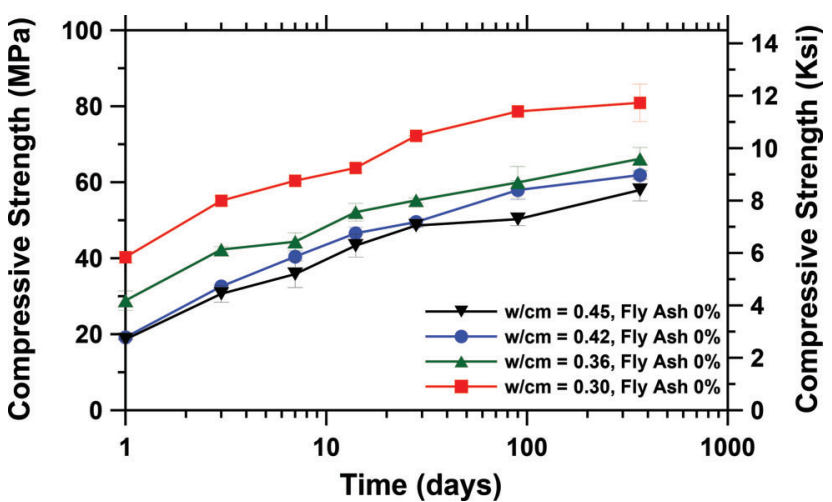

Figure 5.1 Effect of decreasing $w / \mathrm{cm}$ on the compressive strength. (Error bars represent the standard deviation from the average of three samples.) about the same volume fractions of empty porosity, although the empty pores will likely be larger in the higher $w / \mathrm{cm}$ mortar. While all of the cement in the $\mathrm{w} / \mathrm{cm}$ of 0.42 sealed system should theoretically be able to hydrate, there would be a small fraction of unhydrated cement remaining in the sealed systems with $w / \mathrm{cm}$ of 0.36 and 0.30 due to water limitations (13\% and $27 \%$, respectively, according to Powers' (1948) Model).

If fly ash is added without changing the $w / \mathrm{cm}$, a slower strength development is typically observed. Figure 5.2 shows the results. It is important to note that the reference mixture is the mortar with $w / \mathrm{cm}$ of 0.42 . Mortar containing $40 \%$ fly ash has similar $1 \mathrm{~d}$ strength, and $60 \%$ fly ash mortar has similar $14 \mathrm{~d}$ strength, with strength increasing further at later ages in both cases. At $28 \mathrm{~d}$, the concretes with $40 \%$ and $60 \%$ fly ash replacements have strengths that exceed that of the reference mixture by $52 \%$ and $17 \%$, respectively, with similar improvements observed at $91 \mathrm{~d}$ and $365 \mathrm{~d}$. Mortar containing $80 \%$ fly ash has equivalent strength at approximately $190 \mathrm{~d}$; while it exceeds the strength of the reference mortar at $365 \mathrm{~d}$ by $11 \%$.

IC through the use of prewetted LWA was also investigated using two of the mortars, and the results can be observed in Figure 5.3. Under sealed curing conditions, IC enhances the $1 \mathrm{~d}$ strength by $13 \%$ and $61 \%$ in the $40 \%$ and $60 \%$ fly ash mortars, respectively. Both internally cured $40 \%$ and $60 \%$ fly ash mortars exceed the reference strength $(w / c$ of 0.42$)$ by $54 \%$ and $32 \%$ at

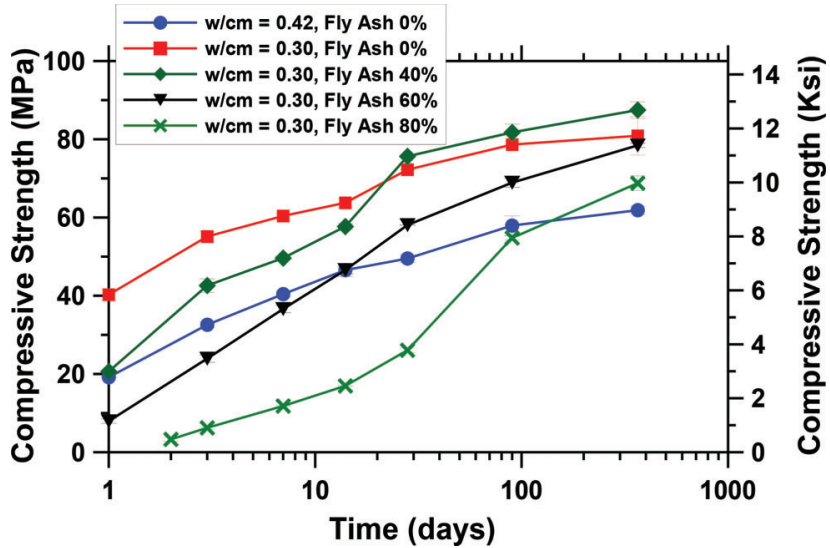

Figure 5.2 Effect of adding high volumes of fly ash on the compressive strength. (Error bars represent the standard deviation from the average of three samples.) 
$28 \mathrm{~d}$, and by $55 \%$ and $49 \%$ at $365 \mathrm{~d}$, respectively. Isothermal calorimetry will be used later in this report to indicate that these improvements in strength are most likely attributed to a higher degree of hydration reached in IC specimens. It should be noted that the HRWRA dosage was the same in the corresponding non-IC and IC mortars.

However, both IC and non-IC fly ash mortars are less stiff (i.e., lower modulus of elasticity) than plain mortars without fly ash at early ages (Figure 5.4). Considering this effect and the slower hydration occurring at early ages, more stress relaxation in restrained conditions should be expected. These property differences should produce a reduction in early-age cracking (Shin, Bucher, \& Weiss, 2011; Shin et al., 2010).

\subsection{Shrinkage Performance}

As previously mentioned, a major concern when low $w / \mathrm{cm}$ mixtures are used is autogenous deformation and cracking that can occur at early ages. Linear autogenous shrinkage measurements were performed according to ASTM C1698-09. Each mortar was encapsulated in thin, corrugated polyethylene molds, with a length-diameter ratio of approximately $420 \mathrm{~mm}$ : $25 \mathrm{~mm}$ [16.5 inches: $1 \mathrm{inch}]$. The mortar was cast into

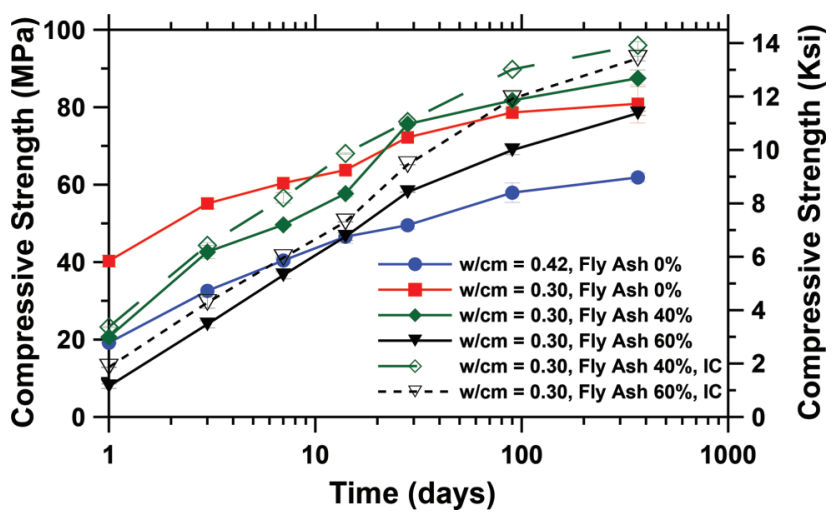

Figure 5.3 Effect of including IC in HVFA mixtures on the compressive strength. (Error bars represent the standard deviation from the average of three samples.)

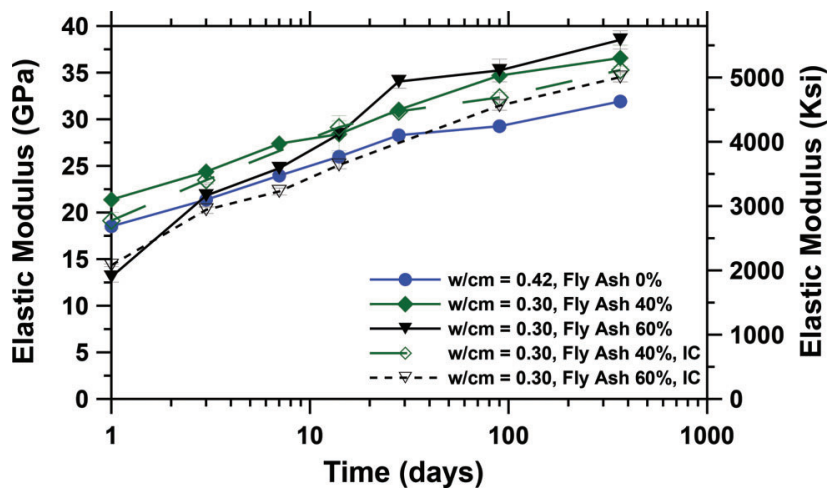

Figure 5.4 Effect of including IC in HVFA mixtures on the elastic modulus. (Error bars represent the standard deviation from the average of two samples.) the molds and vibrated. The specimens were then sealed at the ends to prevent moisture loss and placed over supports provided with spring loaded LVDT's at each end to continuously record the displacement into a data logger every 5 min during two weeks while maintained at a temperature of $23^{\circ} \mathrm{C} \pm 0.5^{\circ} \mathrm{C}\left[73.4^{\circ} \mathrm{F} \pm 0.9^{\circ} \mathrm{F}\right]$. Figure 5.5 shows the measured autogenous shrinkage in six of the mortar mixtures.

As expected, lower total autogenous shrinkage is initially observed in HVFA mortars compared with the non-fly ash mortar with $w / \mathrm{cm}$ of 0.30 , which is explained by the fact that fly ash samples contain a lower amount of cement, so that the initial reaction rate and water consumption is lower. However, at later ages, the rate of shrinkage is higher in fly ash mortars (i.e., the slope of the autogenous shrinkage response). This is important to note as this can lead to more shrinkage than a conventional concrete (e.g., between 1 week and a month) which can make this concrete more susceptible to cracking. IC further reduces autogenous shrinkage in fly ash samples, keeping them above 'zero' net shrinkage at $14 \mathrm{~d}$, indicating that the samples exhibited a slight net expansion during this period of time.

The difference in shrinkage behavior can also be explained by the size of the pores that are being emptied during hydration. The size of the emptied pores is related to the internal relative humidity $(\mathrm{RH})$ measured in the samples. Lower RH's indicate smaller pore sizes being emptied and higher autogenous deformations, since more "self-desiccation" (i.e., internal drying) is occurring in the samples.

$\mathrm{RH}$ was measured using Rotronic HygrClip2 $\mathrm{S}^{1}$ sensors $\left( \pm 0.8 \% \mathrm{RH}\right.$ at $23^{\circ} \mathrm{C} \pm 0.1^{\circ} \mathrm{C}\left[73.4^{\circ} \mathrm{F} \pm\right.$ $\left.0.2^{\circ} \mathrm{F}\right]$ ). Probes were mounted in a $75 \mathrm{~mm}$ x $68 \mathrm{~mm}[2.95$ in $x 2.7$ in] stainless steel cylinder that was placed over a water jacketed sample cup holder. The water jacket was connected with a programmable water bath; however, for this study the samples were maintained at a constant temperature $\left(23.0^{\circ} \mathrm{C} \pm 0.1^{\circ} \mathrm{C}\left[73.4^{\circ} \mathrm{F} \pm 0.2^{\circ} \mathrm{F}\right]\right)$. The $10 \mathrm{~g}$ samples were placed in $12 \mathrm{~mm}$ [0.47 in] deep cups with a $43 \mathrm{~mm}$ [1.7 in] diameter. The sensor was compared with reference salts (potassium sulfate, potassium chloride, and sodium chloride) to provide a point of calibration. Data were recorded at $5 \mathrm{~min}$ intervals. Figure 5.6 shows typical relative humidity measurements over the mortars used in this study. The lower $w / \mathrm{cm}$ mixtures have smaller size pores emptied by selfdesiccation and as such they produce a lower internal $\mathrm{RH}$. It is interesting to note that as fly ash replacement level is increased, RH also increases. This is due in part to the water demand, as there is less cement reacting and partially due to the pore size distribution. IC supplies water from the much larger pores within the LWA to fill in the water being lost from the pores so

\footnotetext{
${ }^{1}$ Certain commercial products are identified in this report to specify the materials used and procedures employed. In no case does such identification imply endorsement or recommendation by the National Institute of Standards and Technology, Purdue University or Catholic University of Chile, nor does it indicate that the products are necessarily the best available for the purpose.
} 


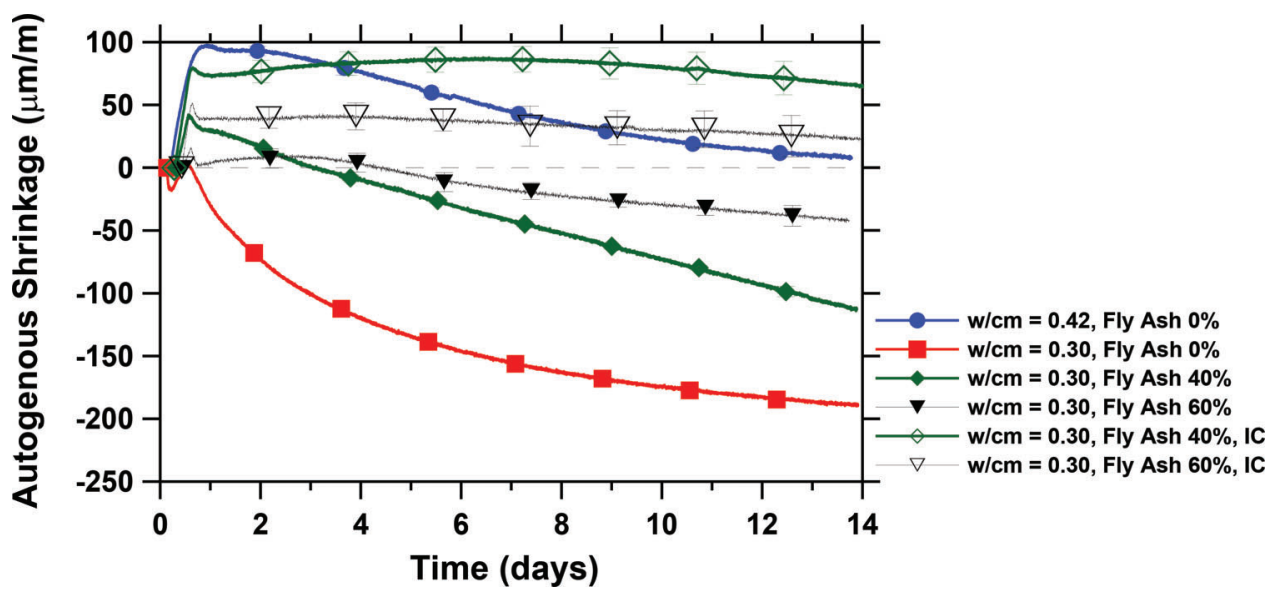

Figure 5.5 Autogenous shrinkage as a function of time. (Error bars represent the standard deviation from the average of three samples.)

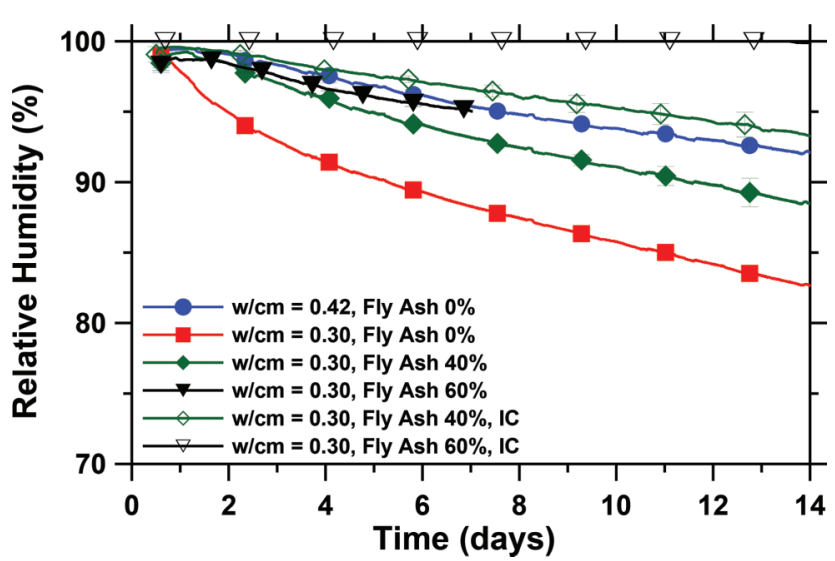

Figure 5.6 Measured internal relative humidity of an aging mortar. (Error bars represent the standard deviation from the average of two samples.)

that the smaller pores remain filled and the relative humidity is higher. Therefore, a reduction in shrinkage deformations is expected, occurring at the same time that the stresses develop in restrained samples.

As mentioned above, fly ash starts reacting at later times and consequently less autogenous shrinkage is observed at early ages. Chemical shrinkage (CS) was measured during the first $7 \mathrm{~d}$ of reaction to estimate the rate of reaction and to better understand the values for CS that should be used in Equation 3.1. Three of the mortar samples were used for assessing the chemical shrinkage according to a modified version of the ASTM C1608-07 (2007) in which the height of the water in the capillary tube was recorded continuously using a pressure sensor (Peethamparan, Weissinger, Vocaturo, Zhang, \& Scherer, 2010). Figure 5.7 shows that there is less chemical shrinkage at early ages in the samples containing fly ash when the chemical shrinkage is normalized by the mass of binder; however this will be a greater value if normalized by the mass of cement. This is important and shows why Equation 3.1 should be modified to be designed based on binder and not only portland cement.

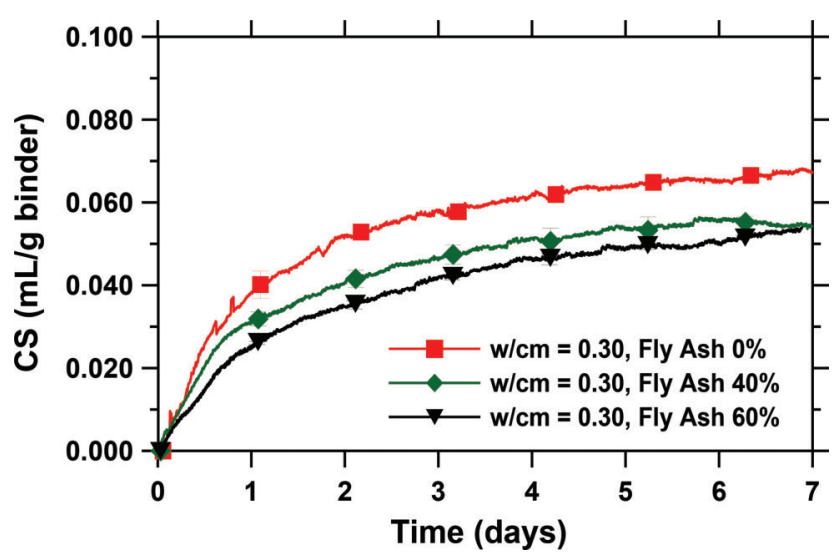

Figure 5.7 Chemical shrinkage in mortar samples. (Error bars represent plus and minus one standard deviation from the average of two samples.)

Mortar rings were prepared in accordance with ASTM C1581-04, using steel rings instrumented with 4 strain gages each. Three mortar specimens were prepared for each mixture. A data acquisition system was set up so that shrinkage deformation data was collected every $5 \mathrm{~min}$ from $30 \mathrm{~min}$ after casting the specimens. They were kept in their molds for $24 \mathrm{~h}$ in an environmental chamber at $23^{\circ} \mathrm{C} \pm 0.5^{\circ} \mathrm{C}\left[73.4^{\circ} \mathrm{F} \pm 0.9^{\circ} \mathrm{F}\right]$. After $24 \mathrm{~h}$, the specimens were demolded, sealed with aluminium foil to minimize evaporation, and placed again back in the environmental chamber. By doing this, the effect of internal curing water in the sample could be better observed. Further shrinkage measurements were taken up to $28 \mathrm{~d}$.

Again, a lower shrinkage rate is observed in HVFA mortars at early ages, if compared to a plain mortar with $w / c$ of 0.30 and no fly ash. This is translated into lower stresses developed in the samples during the first days of curing (Figure 5.8). However, as chemical reaction and chemical shrinkage develop more slowly and to a higher extent, specimens with fly ash are more likely to crack at later ages. For example, in this study, the $w / \mathrm{cm}=0.30,40 \%$ fly ash mortar without IC cracked after $18 \mathrm{~d}$, while the $w / \mathrm{cm}=0.30$ no fly ash mor- 


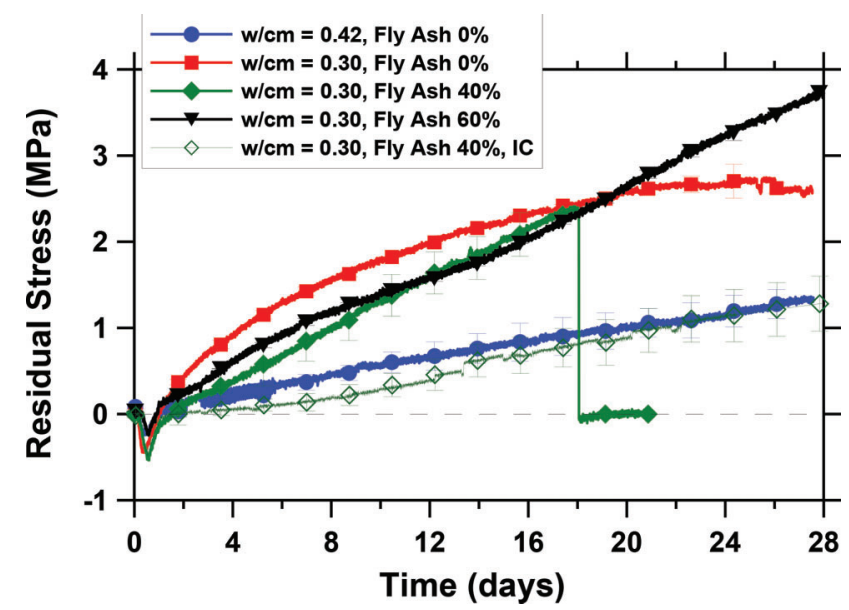

Figure 5.8 Stress developed in ring specimens as a function of time. (Error bars represent the deviation from the average of two samples.)

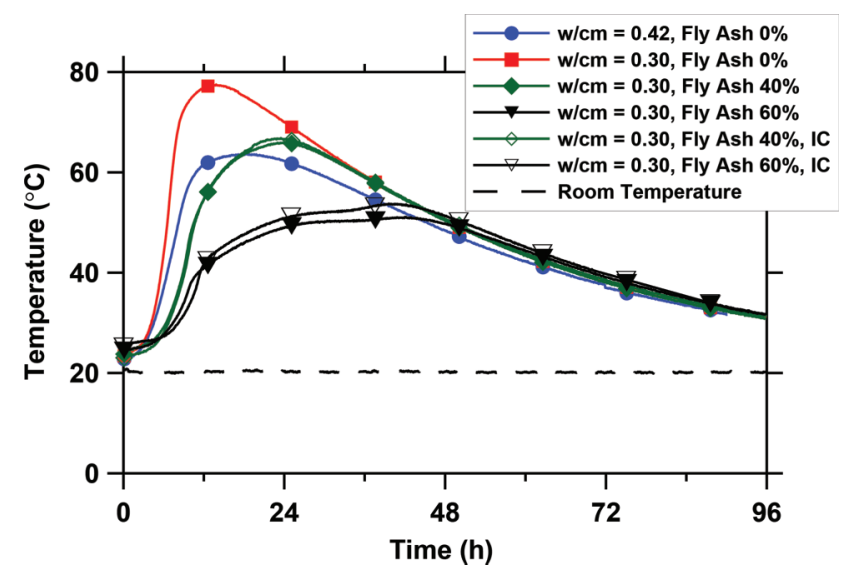

Figure 5.9 Temperature rise as a function of time.

tar did not crack during the $28 \mathrm{~d}$ evaluation period. The $40 \%$ fly ash IC mortar exhibited a stress development quite similar to that of the original $w / \mathrm{cm}=0.42$ mortar without fly ash throughout the $28 \mathrm{~d}$ measurement.

\subsection{Semi-Adiabatic Temperature Rise}

Early-age cracking due to the thermal effects can be common in high performance concrete due to its low $w / \mathrm{cm}$ and high paste volume. To demonstrate another potential benefit of using higher volumes of fly ash in concrete, a simple test was developed. This test consisted of placing a mortar cylinder $(15.25 \mathrm{~cm} \times 17.8 \mathrm{~cm}$ [6 in $x \quad 7$ in]) inside an insulated chamber made from a high refractory ceramic insulation material (Zircar Microsil ${ }^{1}$ ), creating a quasi(semi)-adiabatic chamber with a very low thermal coefficient. The temperature history of the mortars was measured over $4 \mathrm{~d}$ in the insulated chamber as shown in Figure 5.9.

The maximum heat developed by the $40 \%$ fly ash mortar produces a temperature that is approximately the same as the plain mortar with a $w / \mathrm{cm}$ of 0.42 (being only $3.4 \%$ higher). A lower temperature rise was obser- ved in the $60 \%$ fly ash mortar (20\% lower compared to the reference mortar). While these observations have been made by others using supplementary cementitious materials, this shows a clear indication that the lower $w / \mathrm{cm}$ does not result in substantially greater heat rise when fly ash is used. IC does not appreciably change the temperature profile for either the $40 \%$ or the $60 \%$ fly ash mortars.

\subsection{Influence of Fly Ash On the Hydration Rate and Strength Development}

HVFA mixtures are often characterized by their slower rate of early-age reaction and strength development. This can be examined using isothermal calorimetry. Approximately $15 \mathrm{~g}$ of an externally mixed mortar were weighed and placed in a glass ampoule, which was then capped and placed into the isothermal calorimeter about $10 \mathrm{~min}$ after cement was mixed with water. Figure 5.10 shows the results obtained in the mortars being studied in this research over the course of $14 \mathrm{~d}$.

The results were normalized by $\mathrm{mL}$ of initial water (i.e., initial porosity). By doing this, and assuming that the volume of created hydration products is proportional to the heat generated, one can potentially relate the space-filling capabilities of the hydration products with strength (Bentz, Barrett, De la Varga, \& Weiss, 2012). A slower heat development is observed with increasing fly ash replacement level as the fly ash is less reactive than the cement, though the fly ash can provide additional space between the cement particles and can act as a nucleating agent which both increase the hydration rate of the cement. After $14 \mathrm{~d}$, the rate of heat evolved in the $40 \%$ fly ash IC mixture is higher than that in the plain mortars, indicating more reaction taking place in the fly ash systems. This increased rate of reaction can help explain the increased autogenous shrinkage rate, increased chemical shrinkage rate, and increased strength at intermediate ages.

It is interesting that if one plots the cumulative heat release versus strength development (Figure 5.11), all of the mixtures prepared in this study follow a single line $\left(\mathrm{R}^{2}=0.972\right)$. This correlation between early age cumula-

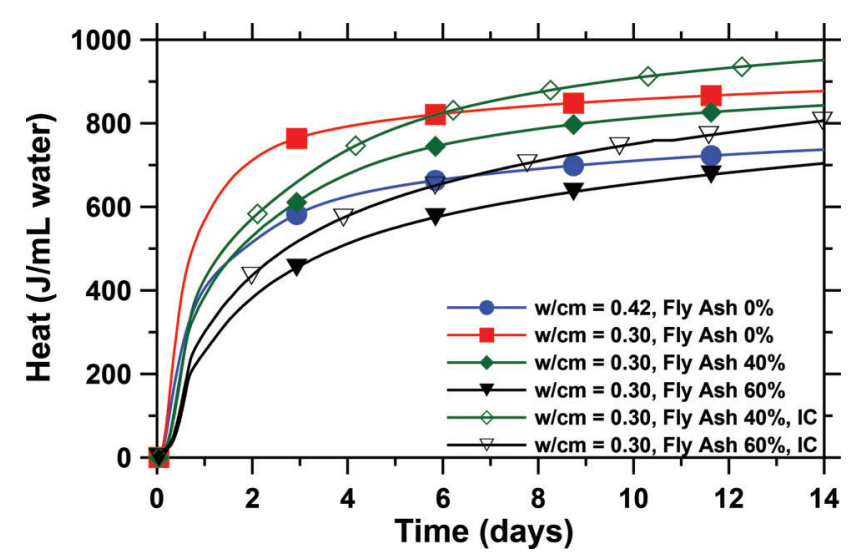

Figure 5.10 Cumulative heat released (Isothermal) curves vs time on a per milliliter of water basis. 


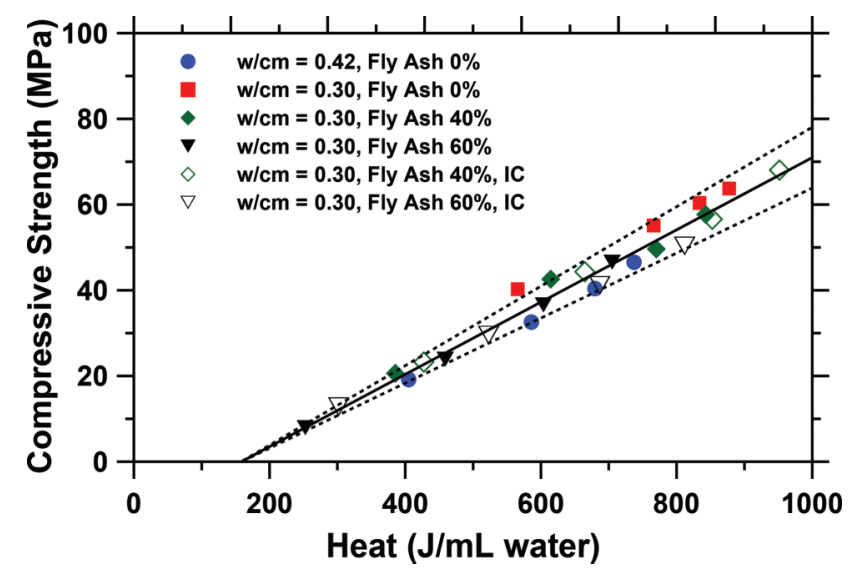

Figure 5.11 Measured compressive strengths vs. measured cumulative heat on a per milliliter initial water basis for mortars at the ages of $1,3,7$, and $14 \mathrm{~d}$. Solid line shows the best fit while the two dashed lines indicate $\pm 10 \%$ from the best fit.

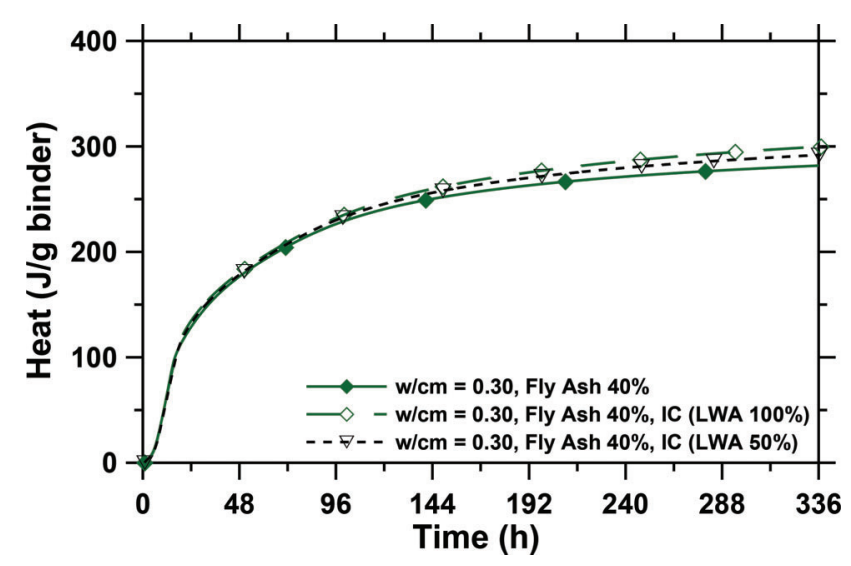

Figure 5.12 Effect of changing IC replacement level.

tive heat release and strength has been observed in other systems as well (Bentz, 2010a; Bentz, Durán-Herrera, \& Galvez-Moreno, 2012; Bentz, Sato, De la Varga, \& Weiss, 2012). Figure 5.11 can be a powerful tool for using isothermal calorimetry to estimate strength at early ages in these mortar mixtures.

The use of IC results in a slight increase in the heat developed at early ages $(24 \mathrm{~h})$ due to increased cement reaction (Castro, De la Varga, \& Weiss, 2012; Geiker, 1983; Lura, Winnefeld, \& Klemm, 2010) however it does not appreciably alter the rate of reaction as shown in Figure 5.12. Though not used in this work, powder additions such as rapid set cement, calcium hydroxide, fine limestone, or aluminates or the addition of a conventional chemical admixture (e.g., non-chloride accelerator) could be strategies to mitigate these early-age deficiencies, if they exist, (retardation and the accompanying set time delays) in HVFA mixtures (Bentz, 2010b; Bentz, Sato, et al., 2012; Gurney, Bentz, Sato, \& Weiss, 2012).

\section{CONCLUSIONS}

A matrix of several HVFA mortars was prepared for use in this study. The mixtures all had a similar fresh workability performance as defined in terms of slump. Numerous tests were performed to evaluate the properties of these mortars including: compressive strength, modulus of elasticity, free and restrained shrinkage, measurement of internal relative humidity, isothermal calorimetry, and semi-adiabatic temperature rise. The $w / \mathrm{cm}$ was reduced to compensate for slow strength development with high volumes of fly ash. Internal curing was used to counteract the drawbacks of using low $w / \mathrm{cm}$ concretes in terms of shrinkage cracking, while providing additional water to support the fly ash reactions. Several conclusions can be drawn from this study:

1. HVFA mixtures with $w / \mathrm{cm}$ of 0.30 have higher strength at later ages ( $28 \mathrm{~d}, 91 \mathrm{~d}$, and $365 \mathrm{~d})$ compared to the reference mortar. In particular, the $40 \%$ fly ash mortar had an equivalent $1 \mathrm{~d}$ strength if compared to a mortar with $w / \mathrm{cm}$ of 0.42 (reference mixture in this study), whereas the $60 \%$ fly ash mortar had equivalent strength at $14 \mathrm{~d}$.

2. IC enhances the $1 \mathrm{~d}$ strength by $13 \%$ and $61 \%$ in the $40 \%$ and $60 \%$ fly ash mortars, cured under sealed conditions, respectively. The effect of IC at later ages is more evident; it results in a higher strength achieved if compared to the reference $w / c=0.42$ mortar $(55 \%$ and $49 \%$ higher at $365 \mathrm{~d}$ in internally cured $40 \%$ and $60 \%$ fly ash mortars, respectively).

3. A lower modulus of elasticity is obtained when either fly ash, IC, or a combination of both are included in the mixture. IC addition reduces the elastic modulus by an average of $7 \%$ and $11 \%$ in $40 \%$ and $60 \%$ fly ash mortars, respectively.

4. The slower hydration reaction in the fly ash mixtures results in less initial autogenous shrinkage deformations at early ages. However, the rate of autogenous shrinkage remains high in the fly ash mixtures at later ages. This increases their potential for cracking at later ages. The use of IC can be a beneficial method to reduce stress development caused by restrained shrinkage. The reduction in shrinkage observed is mainly due to a higher internal RH maintained in these systems.

5. IC does not significantly offset the retardation or slow strength observed when high fly ash volumes are used. Other means to accelerate the hydration process may be available (Bentz, 2010b; Bentz, Sato, et al., 2012; Gurney et al., 2012).

6. Lowering the $w / \mathrm{cm}$ increases the potential of heat generation and thermal cracking; however the addition of high volumes of fly ash has shown benefits in this regard.

7. Although IC does not alter the initial rate of the hydration reaction during the first hours (where other means of mitigating the retardation effect observed in HVFA mixtures should be used), the importance of properly calculating the amount of LWA needed in internallycured concrete mixtures is exemplified in Figure 14, where different amounts of LWA were used producing a difference in the degree of hydration achieved in each system at later ages (i.e., $7 \mathrm{~d}$ ).

A detailed economic analysis was not performed, since economics are strongly influenced by local costs and availability of materials. However, replacement of cement with fly ash should produce a cost reduction that, in the vast majority of cases, will outweigh the increased cost of using LWA. When a life cycle analysis 
is considered and/or if carbon trading is considered, the HVFA mixtures should have even more benefits.

\section{ACKNOWLEDGMENTS}

The authors of this work acknowledge the Federal Highway Administration for partially supporting this project together with Purdue University and NIST (Project BAA No. DTFH61-08-R-00034). The contents of this report reflect the views of the authors, who are responsible for the facts and the accuracy of the data presented herein, and do not necessarily reflect the official views or policies of the Federal Highway Administration, nor do the contents constitute a standard, specification, or regulation. The experiments reported in this paper were conducted in the Pankow Materials Laboratories at Purdue University. The authors acknowledge the support that has made this laboratory and its operation possible.

\section{REFERENCES}

ACI Committee 231. (2010). Report on early-age cracking: Causes, measurement and mitigation (ACI 231R-10). Farmington Hills, MI: American Concrete Institute.

ACI Committee 232. (1996). Use of fly ash in concrete (ACI 232.2R-96). Farmington Hills, MI: American Concrete Institute.

ASTM C1608-07. (2007). Standard test method for chemical shrinkage of hydraulic cement paste. West Conshohocken, PA: ASTM International.

Bentur, A. (Ed.). (2003). Early age cracking in cementitious systems-Report of RILEM Technical Committee 181EAS - Early age shrinkage induced stresses and cracking in cementitious systems (RILEM Report 25). Paris, France: RILEM Publications SARL.

Bentz, D. P. (2010a). Blending different fineness cements to engineer the properties of cement-based materials. Magazine of Concrete Research, 62(5), 327-338. http://dx.doi.org/ 10.1680/macr.2008.62.5.327

Bentz, D. P. (2010b). Powder additions to mitigate retardation in high-volume fly ash mixtures. ACI Materials Journal, 107(5), 508-514. http://dx.doi.org/10.14359/51663971

Bentz, D. P., Barrett, T., De la Varga, I., \& Weiss, W. J. (2012). Relating compressive strength to heat release in mortars. Advances in Civil Engineering Materials, 1(1), 1-14. https://doi.org/10.1520/ACEM20120002

Bentz, D. P., Durán-Herrera, A., \& Galvez-Moreno, D. (2012). Comparison of ASTM C311 strength activity index testing vs. testing based on constant volumetric proportions. Journal of ASTM International, 9(1), 1-7. https://doi. org/10.1520/JAI104138

Bentz, D. P., \& Ferraris, C. F. (2010). Rheology and setting of high volume fly ash mixtures. Cement and Concrete Composites, 32(4), 265-270. http://dx.doi.org/10.1016/j.cemcon comp.2010.01.008

Bentz, D. P., \& Jensen, O. M. (2006). Mitigation strategies for autogenous shrinkage cracking. Cement and Concrete Composites, 26(6), 677-685. http://dx.doi.org/10.1016/ S0958-9465(03)00045-3

Bentz, D. P., Lura, P., \& Roberts, J. W. (2005). Mixture proportioning for internal curing. ACI Concrete International, $27(2), 35-40$.
Bentz, D. P., \& Peltz, M. A. (2008). Reducing thermal and autogenous shrinkage contributions to early-age cracking. ACI Materials Journal, 105(4), 414 420. http://dx.doi.org/ $10.14359 / 19904$

Bentz, D. P., Sato, T., De la Varga, I., \& Weiss, W. J. (2012). Fine limestone additions to regulate setting in high volume fly ash mixtures. Cement and Concrete Composites, 34(1), 11-17. http://dx.doi.org/10.1016/j.cemconcomp.2011.09.004

Bilodeau, A., Sivasundaram, V., Painter, K., \& Malhotra, V. (1994). Durability of concrete incorporating high volumes of fly ash from sources in the USA. ACI Materials Journal, 91(1), 3-12. http://dx.doi.org/10.14359/4411

Bentz, D. P., \& Snyder, K. A. (1999). Protected paste volume in concrete: Extension to internal curing using saturated lightweight fine aggregate. Cement and Concrete Research, 29(11), 1863-1867. http://dx.doi.org/10.1016/S0008-8846 (99)00178-7

Bentz, D. P., \& Weiss, W. J. (2011). Internal curing: A 2010 state-of-the-art review. Washington, DC: U.S. Department of Commerce, National Institute of Standards and Technology. Retrieved from http://ws680.nist.gov/publication/ get_pdf.cfm?pub_id=907729

Brown, M., Smith, C., Sellers, G., Folliard, K., \& Breen, J. (2007). Use of alternative materials to reduce shrinkage cracking in bridge decks. ACI Materials Journal, 104(6), 629-637. http://dx.doi.org/10.14359/18967

Castro, J., De la Varga, I., \& Weiss, J. (2012). Using isothermal calorimetry to assess water absorption of fine LWA in mortars. Journal of Materials in Civil Engineering, 24(8), 996-1005. http://dx.doi.org/10.1061/(ASCE)MT.1943-5533. 0000496

Castro, J., Keiser, L., Golias, M., \& Weiss, J. (2011). Absorption and desorption properties of fine lightweight aggregate for application to internally cured concrete mixtures. Cement and Concrete Composites, 33(10), 1001-1008. http:// dx.doi.org/10.1016/j.cemconcomp.2011.07.006

Davis, R. E., Carlson, R. W., Kelly, J. W., \& Davis, A. G. (1937). Properties of cements and concretes containing fly ash. ACI Journal Proceedings, 33(5), 577-612. http://dx.doi. org/10.14359/8435

Geiker, M. (1983). Studies of Portland cement hydration by measuring of chemical shrinkage and a systematic evaluation of hydration curves by means of the dispersion model (Doctoral dissertation). Kongens Lyngby: Technical University of Denmark.

Gurney, L., Bentz, D. P., Sato, T., \& Weiss, W. J. (2012). Reducing set retardation in high volume fly ash mixtures with the use of limestone: Improving constructability for sustainability. Transportation Research Record, 2290, 139146. http://dx.doi.org/10.3141/2290-18

Halstead, W. J. (1986). Use of fly ash in concrete (NCHRP Synthesis 127). Washington, DC: Transportation Research Board, National Research Council. Retrieved from http:// onlinepubs.trb.org/Onlinepubs/nchrp/nchrp_syn_127.pdf

Hansen, T. C. (1990). Long-term strength of high fly ash concretes. Cement and Concrete Research, 20(2), 193-196. http://dx.doi.org/10.1016/0008-8846(90)90071-5

Helmuth, R. (1987). Fly ash in cement and concrete. Skokie, IL: Portland Cement Association.

Henkensiefken, R., Castro, J., Bentz, D., Nantung, T., \& Weiss, J. (2009). Water absorption in internally cured mortar made with water-filled lightweight aggregate. Cement and Concrete Research, 39(10), 883-892. http://dx.doi.org/ 10.1016/j.cemconres.2009.06.009

INDOT. (2012). Concrete pavements. Section 500 in 2012 standard specifications. Indianapolis, IN: Indiana Depart- 
ment of Transportation. Retrieved from http://www.in.gov/ dot/div/contracts/standards/book/sep11/5-2012.pdf

Jensen, O. M., \& Hansen, P. F. (2001). Water-entrained cementbased materials: I. Principles and theoretical background. Cement and Concrete Research, 31(4), 647-654. http://dx. doi.org/10.1016/S0008-8846(01)00463-X

Kantro, D. L. (1980). Influence of water-reducing admixtures on properties of cement paste-a miniature slump test. Cement, Concrete and Aggregates, 2(2), 95-102. https://doi. org/10.1520/CCA10190J

Kovler, K., \& Jensen, O. M. (Eds.). (2007). Internal curing of concrete-State of the art report of RILEM Technical Committee 196-ICC (RILEM Report 41). Paris, France: RILEM Publications SARL.

Krishnan, A., Mehta, J. K., Olek, J., \& Weiss, W. J. (2006). Technical issues related to the use of fly ash and slag during late-fall (low temperature) construction season (Joint Transportation Research Program Publication No. FHWA/IN/ JTRP-2005/05). West Lafayette, IN: Purdue University. http://dx.doi.org/10.5703/1288284313382

L'Hermite, R. G. (1962). Volume changes of concrete. In Chemistry of cement: Proceedings of the Fourth International Symposium, Vol. 1 (pp. 659-694). Washington, DC: U.S. Department of Commerce.

Le Chatelier, H. (1900). Sur les Changements de Volume qui Accompagnent le durcissement des Ciments. Bulletin Societe de l'Encouragement pour l'Industrie Nationale, Seme serie, tome 5, Paris.

Lura, P., Winnefeld, F., \& Klemm, S. (2010). Simultaneous measurements of heat of hydration and chemical shrinkage on hardening cement pastes. Journal of Thermal Analysis and Calorimetry, 101(3), 925-932. http://dx.doi.org/10.1007/ s10973-009-0586-2

Malhotra, V. M. (1990). Durability of concrete incorporating high-volume of low calcium (ASTM Class F) fly ash. Cement and Concrete Composites, 12(4), 271-277. http://dx. doi.org/10.1016/0958-9465(90)90006-J

Malhotra, V. M. (1999a). Making concrete "greener" with fly ash. ACI Concrete International, 21(5), 61-66.

Malhotra, V. M. (1999b). Role of supplementary cementing materials in reducing greenhouse gas emissions. In O. E. Gjorv \& K. Sakai (Eds.), Concrete technology for a sustainable development in the 21 st century. London, UK: E \& FN Spon Press.

Malhotra, V. M., \& Ramezanianpour, A. (1994). Fly ash in concrete (2nd ed.). Ottawa, Canada: CANMET, Canada Centre for Mineral and Energy Technology.

McCaffrey, R. (2002). Climate change and the cement industry. Global Cement and Lime Magazine (Environmental Special Issue), 15-19.

Mehta, P. K. (2002). Greening of the concrete industry for sustainable development. ACI Concrete International, 24(7), 23-28.

Naik, T., Singh, S., \& Ramme, B. (1998). Mechanical properties and durability of concrete made with blended fly ash. ACI Materials Journal, 95(4), 454 462. http://dx.doi. org $/ 10.14359 / 388$

Neuwald, A., Krishnan, A., Weiss, W. J., Olek, J., \& Nantung, T. E. (2003). Concrete curing and its relationship to measured scaling in concrete containing fly ash. In Transportation Research Board annual compendium of papers [CD-ROM]. Washington, DC: Transportation Research Board.

NYSDOT. (2008). Moisture content of lightweight fine aggregate (Test Method No. NY 703-19 E). Albany, NY: New York State Department of Transportation, Materials Bureau.
Peethamparan, S., Weissinger, E., Vocaturo, J., Zhang, J., \& Scherer, G. (2010). Monitoring chemical shrinkage using pressure sensors. ACI Special Publication, 270, 77-88. http://dx.doi.org/10.14359/51663740

Philleo, R. E. (1991). Concrete science and reality. In Materials Science of Concrete II (pp. 1-8). Westerville, OH: American Ceramic Society.

Pour-Ghaz, M., Castro, J., Kladivko, E. J., \& Weiss, J. (2011). Characterizing lightweight aggregate desorption at high relative humidities using a pressure plate apparatus. Journal of Materials in Civil Engineering, 24(8), 961-969. http://dx. doi.org/10.1061/(ASCE)MT.1943-5533.0000422

Powers, T. C. (1948). A discussion of cement hydration in relation to the curing of concrete (Bulletin No. 25). Chicago, IL: Portland Cement Association, Research Laboratories.

Ramlochan, T., Zacarias, P., Thomas, M. D. A., \& Hooton, R. D. (2003). The effect of pozzolans and slag on the expansion of mortars cured at elevated temperature: Part I: Expansive behaviour. Cement and Concrete Research, 33(6), 807-814. http://dx.doi.org/10.1016/S0008-8846(02)01066-9

Sant, G., Lura, P., \& Weiss, J. (2006). Measurement of volume change in cementitious materials at early ages: Review of testing protocols and interpretation of results. Transport Research Record, 1979, 21-29. http://dx.doi.org/10.3141/ 1979-05

Shah, S. P., \& Weiss, W. J. (2000). High performance concrete: Strength, permeability, and shrinkage cracking. In Symposium proceedings: PCI/FHWA/FIB International Symposium on High Performance Concrete, September 2527, 2000, Orlando, Florida: The economical solution for durable bridges and transportation structures (pp. 331-339). Chicago, IL: Precast/Prestressed Concrete Institute.

Shah, S., Weiss, W. J., \& Yang, W. (1997). Shrinkage cracking in high performance concrete. In L. S. Paul (Ed.), Symposium proceedings: PCI/FHWA International Symposium on High Performance Concrete, October 20-22, New Orleans, Louisiana: Advanced Concrete Solutions for Bridges and Transportation Structures. Chicago, IL: Precast/Prestressed Concrete Institute.

Shah, S., Weiss, W. J., \& Yang, W. (1998). Shrinkage crackingCan it be prevented? ACI Concrete International, 20(4), $51-55$.

Shin, K.-J., Castro, J., Schlitter, J., Golias, M., Pour-Ghaz, M., Henkensiefken, R., Weiss, J. (2010). The role of internal curing as a method to improve durability. In S.-H. Kim \& K. Y. Ann (Eds.), Handbook on concrete durability (pp. 379-428). Republic of Korea: Middleton Publishing.

Shin, K.-J., Bucher, B. E., \& Weiss, W. J. (2011). The role of lightweight synthetic particles on the restrained shrinkage cracking behavior of mortar. Journal of Civil Engineering Materials, 23(5), 597-605. http://dx.doi.org/10.1061/ (ASCE)MT.1943-5533.0000213

Thomas, M. D. A., \& Matthews, J. D. (1992). Carbonation of fly ash concrete. Magazine of Concrete Research, 44(160), 217-228. http://dx.doi.org/10.1680/macr.1992.44. 160.217

Weiss, W. J., Yang, W., \& Shah, S. P. (1999). Factors influencing durability and early-age cracking in high strength concrete structures. ACI Special Report, 189, 387-409. http://dx.doi.org/10.14359/5863

Worrell, E., Price, L., Martin, N., Hendriks, C., \& Ozawa, L. (2001). Carbon dioxide emissions from the global cement industry. Annual Review of Energy and the Environment, 26, 303-329. http://dx.doi.org/10.1146/annurev.energy.26.1.303 
CHAPTER 5. PHASE II, TASKS 4 AND 5: INFLUENCE OF INTERNAL CURING ON THE ISOTHERMAL AND SEMI-ADIABATIC CRACKING BEHAVIOR OF HIGH VOLUME FLY ASH MIXTURES

\section{Igor De la Varga, Timothy J. Barrett, Dale P. Bentz, W. Jason Weiss}

\section{ABSTRACT}

Fly ash is increasingly being used to replace cement in concrete due to economic and sustainability benefits. While mixtures with high volumes of fly ash (HVFA) have reduced heat generation during hydration, concerns about the rate of early-age strength development, scaling, and requirement for longer curing times may limit their use. The authors have developed an approach of using higher volumes of fly ash in conjunction with low water-to-cementitious materials ratios $(w / \mathrm{cm})$ and internal curing. Reducing the $w / \mathrm{cm}$ increases the rate of early-age strength development. To counteract the autogenous shrinkage, internal curing is used to supply the necessary curing water. This report uses a recently developed dual ring test method to quantify the earlyage tensile stress development due to autogenous deformation (autogenous shrinkage) when the concrete is prevented from shrinking freely. This is done under isothermal and semi-adiabatic temperature conditions. This was done using six mortar mixtures. Two plain mixtures were tested with $w / c$ of 0.42 (similar to a conventional bridge deck) and a $w / c$ of 0.30 , respectively. Two mixtures were prepared with $w / \mathrm{cm}=0.30$ where $40 \%$ and $60 \%$ of the cement was replaced with fly ash (volumetric replacement). Finally, internal curing was used for the mixtures with $w / \mathrm{cm}$ of 0.30 and $40 \%$ and $60 \%$ of the cement replaced with fly ash. The results of the isothermal tests show that autogenous shrinkage and the resulting tensile stress are considerably reduced in high volume fly ash mixtures with internal curing. Additionally, thermal shrinkage was also evaluated in three of these mixtures using semi-adiabatic tests. This test consists of superimposing autogenous and thermal shrinkage. The replacement of cement with large amounts of fly ash reduces the stress due to temperature changes, thus increasing the cracking capacity. Internally cured mixtures have a high potential to further reduce the internal stresses. As a result, HVFA mixtures with a substantially lower carbon footprint can be used while also providing a lower potential for cracking.

\section{INTRODUCTION}

In 2008, the American Concrete Institute (ACI) and the Portland Cement Association (PCA) announced a joint concrete sustainability initiative. This initiative was developed since the sustainability of the concrete is a critical issue for the industry. While the goal of that initiative is broad sweeping, as it set out to "outline broad goals and strategies to which all segments of the concrete industry can align their own corporate activities to assure a common concrete industry messageensuring that concrete is designed, specified, produced, installed, and applied, enabling an environmentally and socially responsible, healthy, and prosperous environment that improves the quality of life" (ACI, 2008), it can be argued that an important component of this work must be the reduction of the emission of greenhouse gases per cubic yard of concrete that is produced. As the production of cement clinker is known to produce $\mathrm{CO}_{2}$ through the combustion of fossil fuel and through the conversion of limestone to calcium oxide (decalcination) in the kiln, it appears that one tangible method to reduce $\mathrm{CO}_{2}$ emission for the built infrastructure is to reduce the amount of cement clinker employed per cubic yard of concrete.

The authors of this report have previously developed an approach to suggest how a typical concrete bridge deck mixture with a water-to-cement ratio $(w / \mathrm{cm})$ of 0.42 can be modified to replace a substantial portion of the cement with fly ash (De la Varga, Castro, Bentz, \& Weiss, 2012). Due to concerns that have been raised with extended set time and reduce rates of strength gain, this approach consists of reducing the $w / \mathrm{cm}$ in the HFVA mixtures to provide similar set and strength development to the conventional mixture. Results from this approach can be seen in Table 1.1 for a few selected mixtures (details of which are provided later in the report).

It can be noticed that the mortar portion of the conventional bridge deck mixture had a compressive strength of 19.2 MPa, 32.6 MPa, and 49.5 MPa at $1 \mathrm{~d}, 3$ d, and $28 \mathrm{~d}$, respectively. However, when $40 \%$ of the cement was replaced with fly ash and the $w / \mathrm{cm}$ was reduced to 0.30 , the strength was $7 \%, 31 \%$, and $53 \%$ higher than that of the reference mixture at $1 \mathrm{~d}, 3 \mathrm{~d}$, and $28 \mathrm{~d}$, respectively. On the other hand, when $60 \%$ of the cement was replaced with the same fly ash and the $w / \mathrm{cm}$ was reduced to 0.30 , the strength was $59 \%$ lower, $27 \%$ lower, and $17 \%$ higher than that of the reference mixture at $1 \mathrm{~d}, 3 \mathrm{~d}$, and $28 \mathrm{~d}$, respectively. Similarly, the set time of the conventional mixture was $5 \mathrm{~h}$, while the set times of the mixtures with $40 \%$ and $60 \%$ cement replacements were $7.7 \mathrm{~h}$ and $\mathrm{t} 11 \mathrm{~h}$, respectively (an approach for how to mitigate delays in set times is described in Chapter 4 of this report).

While the results in the previous paragraph appear quite positive, it can be noticed that by reducing the water to cement ratio, the autogenous shrinkage of the mixture increased (negative sign in Table 1.1 indicates shrinkage). Also, it is commonly reported that higher performance mixtures (generally obtained through increased cement contents, finer cements, and silica fume additions) may have a higher heat of hydration and can be therefore more susceptible to thermal cracking. As a result of this concern, the research team has taken additional steps to try to reduce the potential for cracking due to autogenous and thermal cracking in the HFVA mixtures. It is believed that the reduction in cement content should reduce the heat generated due to 
TABLE 1.1

Performance Comparison between Typical Concrete Bridge Deck Mix Design and Low w/cm HVFA Mixtures

\begin{tabular}{|c|c|c|c|c|c|}
\hline \multirow{2}{*}{\multicolumn{2}{|c|}{ Property }} & \multirow{5}{*}{$\begin{array}{c}\text { Test Method } \\
\text { ASTM C 39-12 }\end{array}$} & \multicolumn{3}{|c|}{ Mixtures" } \\
\hline & & & \multirow{2}{*}{$\frac{\mathbf{0 . 4 2 - 0} \%}{19.2}$} & \multirow{2}{*}{$\frac{\mathbf{0 . 3 0 - 4 0} \%}{20.6}$} & \multirow{2}{*}{$\frac{0.30-60 \%}{8}$} \\
\hline Compressive Strength (MPa) & $1 \mathrm{~d}$ & & & & \\
\hline & $3 \mathrm{~d}$ & & 32.6 & 42.6 & 23.9 \\
\hline & $28 \mathrm{~d}$ & & 49.5 & 75.6 & 58 \\
\hline Time of Set (h) & & ASTM C 403-08 & 5 & 7.7 & 11 \\
\hline \multirow[t]{3}{*}{ Autogenous Shrinkage (in/in) } & $1 \mathrm{~d}$ & \multirow[t]{3}{*}{ ASTM C 1698-09 } & 97 & 29 & 3 \\
\hline & $7 \mathrm{~d}$ & & 44 & -42 & -17 \\
\hline & $14 \mathrm{~d}$ & & 8.1 & -113 & -41 \\
\hline
\end{tabular}

${ }^{*}$ Typical values for mortar mixtures.

TABLE 1.2

Performance Comparison between Typical Concrete Bridge Deck Mix Design and Low w/cm IC-HVFA Mixtures

\begin{tabular}{|c|c|c|c|c|c|c|c|}
\hline \multirow{2}{*}{\multicolumn{2}{|c|}{ Property }} & \multirow[b]{2}{*}{ Test Method } & \multicolumn{5}{|c|}{ Mixtures* $^{*}$} \\
\hline & & & $0.42-0 \%$ & $0.30-40 \%$ & $0.30-40 \%-\mathrm{IC}$ & $0.30-60 \%$ & $0.30-60 \%-\mathrm{IC}$ \\
\hline \multirow{2}{*}{$\begin{array}{l}\text { Compressive } \\
\text { Strength (MPa) }\end{array}$} & $1 \mathrm{~d}$ & \multirow[t]{2}{*}{ ASTM C 39-12 } & 19.2 & 20.6 & 23.2 & 8 & 12.9 \\
\hline & $28 \mathrm{~d}$ & & 49.5 & 75.6 & 76.3 & 58 & 65.1 \\
\hline Time of Set (h) & & ASTM C 403-08 & 5 & 7.7 & 6.7 & 11 & 10.3 \\
\hline \multirow{3}{*}{$\begin{array}{l}\text { Autogenous } \\
\text { Shrinkage (in/in) }\end{array}$} & $1 \mathrm{~d}$ & \multirow[t]{3}{*}{ ASTM C 1698-09 } & 97 & 29 & 74 & 3 & 37 \\
\hline & $7 \mathrm{~d}$ & & 44 & -42 & 86 & -17 & 34 \\
\hline & $14 \mathrm{~d}$ & & 8.1 & -113 & 65 & -41 & 23 \\
\hline
\end{tabular}

*Typical values for mortar mixtures.

hydration and therefore reduce the potential for thermal cracking. To reduce the autogenous shrinkage, the research team proposes the use of internal curing (IC). While several strategies exist to internally cure concrete, the work in this report uses pre-wetted lightweight aggregate. Table 1.2 compares the results obtained in internally cured HVFA with the mixtures shown in Table 1.1.

As it can be observed, the autogenous shrinkage of the IC-HVFA mixtures is considerably reduced compared to the reference $(65 \mu \mathrm{in} / \mathrm{in}$ and $23 \mu \mathrm{in} / \mathrm{in}$ for the $40 \%$ and $60 \%$ internally cured fly ash mixtures at $14 \mathrm{~d}$, compared to $8.1 \mu \mathrm{in} / \mathrm{in}$ for the reference). The reasons for this are further explained in De la Varga et al. (2012).

\section{OBJECTIVES}

Two series of dual ring tests (Schlitter, Senter, Bentz, Nantung, \& Weiss, 2010) will be performed. The first series (isothermal) holds the temperature constant for $2 \mathrm{~d}, 4 \mathrm{~d}$, and $7 \mathrm{~d}$, at which time the temperature is subsequently reduced at a rate of $2^{\circ} \mathrm{C} / \mathrm{h}$ until a temperature of $-9^{\circ} \mathrm{C}$ is reached. By reducing the temperature of the rings, the temperature required to cause cracking can be quantified. This approach enables one to quantify the stress material development due to autogenous shrinkage and assess how close the material is to cracking at early ages.

The second series (semi-adiabatic) is conducted where the dual ring specimen is exposed to controlled semi-adiabatic temperature profiles. The simultaneous effects of autogenous and thermal shrinkage can be considered in this case.

\section{MATERIALS AND EXPERIMENTAL PROCEDURES}

\subsection{Materials}

An ordinary portland cement (OPC) (ASTM C15009 Type I/II) was used in this study, with a Blaine fineness of $476 \mathrm{~m}^{2} / \mathrm{kg}$, a specific gravity of 3.17 , an estimated Bogue composition of $52 \% \quad \mathrm{C}_{3} \mathrm{~S}, 18 \% \quad \mathrm{C}_{2} \mathrm{~S}$, $8 \% \quad \mathrm{C}_{3} \mathrm{~A}, 9 \% \quad \mathrm{C}_{4} \mathrm{AF}$, and a $\mathrm{Na}_{2} \mathrm{O}$ equivalent of 0.5 . A class C fly ash (ASTM C618-08a) was also used at $40 \%$ and $60 \%$ volume replacements of cement. The fly ash had a specific gravity of 2.63. Chemical analysis of the cement and fly ash is summarized in Table 3.1. 
The fine aggregate consisted of regular river sand with a fineness modulus of 2.71 and an apparent specific gravity of 2.58. Rotary kiln expanded shale (i.e., a fine lightweight aggregate) was used with a fineness modulus of 3.97 and a specific gravity (dry) of 1.38 . The LWA was measured to have a $24 \mathrm{~h}$ water absorption of $15.9 \%$ by mass, when this material was tested using the paper towel technique (Castro, Kesier, Golias, \& Weiss, 2011; NYSDOT, 2008). A high-range water-reducing admixture (HRWRA) was added at variable dosage by mass of binder in order to maintain the same slump in all mortars. Thus, the amount of HRWRA was reduced as the fly ash content was increased (see Table 3.2).

\subsection{Mixture Proportioning}

Six different mixtures were utilized in this study. Each of the mixtures was designed to have a similar workability as determined using a mini-slump cone test (Kantro, 1980). Four of the mixtures were mortars with a conventional fine aggregate (55\% by volume), different $w / \mathrm{cm}$, and different amount of fly ash replacing a volume of cement (designated in Table 3.2 as $0.42-0 \%$, $0.30-0 \%, 0.30-40 \%$, and $0.30-60 \%$ - with the number on the left representing $w / \mathrm{cm}$, and the number on the right

TABLE 3.1

Chemical Composition of the Cement and Fly Ash Used in This Study

\begin{tabular}{|c|c|c|}
\hline & Cement & Fly Ash \\
\hline Class & $\mathrm{I} / \mathrm{II}$ & $\mathrm{C}$ \\
\hline Silicon Dioxide $\left(\mathrm{SiO}_{2}\right), \%$ & 19.97 & 38.71 \\
\hline Aluminum Oxide $\left(\mathrm{Al}_{2} \mathrm{O}_{3}\right), \%$ & 4.81 & 19.15 \\
\hline Iron Oxide $\left(\mathrm{Fe}_{2} \mathrm{O}_{3}\right), \%$ & 2.89 & 6.49 \\
\hline Sum of $\mathrm{SiO}_{2}, \mathrm{Al}_{2} \mathrm{O}_{3}, \mathrm{Fe}_{2}, \mathrm{O}_{3}, \%$ & 27.67 & 64.35 \\
\hline Calcium Oxide (CaO), \% & 63.27 & 23.51 \\
\hline Magnesium Oxide (MgO), \% & 1.54 & 5.29 \\
\hline Sulfur Trioxide $\left(\mathrm{SO}_{3}\right), \%$ & 3.27 & 1.36 \\
\hline Potassium Oxide $\left(\mathrm{K}_{2} \mathrm{O}\right), \%$ & 0.38 & 0.58 \\
\hline Sodium Oxide $\left(\mathrm{Na}_{2} \mathrm{O}\right), \%$ & 0.28 & 1.64 \\
\hline Loss on Ignition, \% & 2.85 & 0.30 \\
\hline Moisture Content, \% & - & 0.11 \\
\hline
\end{tabular}

representing the volume fraction of fly ash replacing the same volume of cement). The $0.42-0 \%$ mixture would represent the mortar fraction of a typical concrete mix design used in a bridge deck in the State of Indiana, and it is taken as the reference mixture in this study. Both fly ash mortar mixtures were also prepared with a portion of the fine aggregate replaced by pre-wetted LWA. These mixtures are designated in Table 3.2 as $0.30-40 \%-\mathrm{L}$ and $0.30-60 \%-\mathrm{L}$. The volume of mortar occupied by the LWA corresponds to $14.9 \%$ and $14.5 \%$ for the $40 \%$ and $60 \%$ fly ash mortars, respectively. It is important to note that though these designations are based on a total volume basis, the volume of aggregate (LWA and sand) remained constant at 55\% since only the sand was replaced with LWA. The amount of LWA was that necessary to eliminate self-desiccation, according to a published approach for determining the LWA replacement volume (Bentz \& Snyder, 1999; Bentz \& Weiss, 2011), where the cement factor and chemical shrinkage employed are account for both of the cement and fly ash.

\subsection{Mixing Procedure}

The mixing procedure was performed in accordance with ASTM C305-06 (2006). The normal weight sand was oven-dried and air-cooled for $24 \mathrm{~h}$ before mixing. The LWA was oven dried, air cooled, and then submerged in water for $24 \mathrm{~h} \pm 1 \mathrm{~h}$ prior to mixing. The volume of water used to submerge the LWA included both mixing water and the water the LWA would absorb in $24 \mathrm{~h}$. The excess water (water not absorbed in $24 \mathrm{~h}$ ) was then decanted and used as the mixing water. The fly ash and cement were conditioned for $24 \mathrm{~h}$ at room temperature.

The fine aggregate was added to a "buttered" mixer, along with a small amount of mixture water, and then mixed for a short amount of time to minimize dust. If LWA was used, it was added right after the normal fine aggregate and mixed with it for a short amount of time. The cement and fly ash (if any) were then added to the mixer. Water was then added, noting the time of water-to-cement contact, followed by the HRWRA.

TABLE 3.2

Mixture Proportions

\begin{tabular}{|c|c|c|c|c|c|c|}
\hline \multirow[b]{2}{*}{ Mortar Mixture } & \multicolumn{2}{|c|}{ Plain } & \multicolumn{2}{|c|}{ Fly Ash } & \multicolumn{2}{|c|}{ Internal Curing } \\
\hline & $0.42-0 \%$ & $0.30-0 \%$ & $0.30-40 \%$ & $0.30-60 \%$ & $0.30-40 \%-\mathrm{L}$ & $0.30-60 \%-\mathrm{L}$ \\
\hline Volume Fraction of Aggregate, $\%$ & 55 & 55 & 55 & 55 & 55 & 55 \\
\hline Water/Cementitious Material (w/cm) & 0.42 & 0.30 & 0.30 & 0.30 & 0.30 & 0.30 \\
\hline Cement, $\mathrm{kg} / \mathrm{m}^{3}$ & 612 & 731 & 453 & 307 & 453 & 307 \\
\hline Fly Ash, kg/m ${ }^{3}$ & 0 & 0 & 252 & 384 & 252 & 384 \\
\hline Fly Ash, \% & 0 & 0 & 40 & 60 & 40 & 60 \\
\hline Water, $\mathrm{kg} / \mathrm{m}^{3}$ & 257 & 219 & 211 & 207 & 211 & 207 \\
\hline Water for $\mathrm{IC}, \mathrm{kg} / \mathrm{m}^{3}$ & - & - & - & - & 38 & 37 \\
\hline Fine Aggregate, $\mathrm{kg} / \mathrm{m}^{3}$ & 1418 & 1418 & 1418 & 1418 & 998 & 1006 \\
\hline LWA, $\mathrm{kg} / \mathrm{m}^{3}$ & 0 & 0 & 0 & 0 & 236 & 232 \\
\hline HRWRA, g/100g Cementitious Material & 0.0 & 0.5 & 0.2 & 0.1 & 0.2 & 0.1 \\
\hline
\end{tabular}

$1 \mathrm{~kg} / \mathrm{m}^{3}=1.69 \mathrm{lb} / \mathrm{yd}^{3}$. 
The materials were mixed for three minutes, rested for three minutes while the bottom of the bowl was scraped with a spoon, then mixed for an additional two minutes.

\subsection{Semi-Adiabatic Chamber}

A semi-adiabatic chamber with a thermal conductivity of $0.019 \mathrm{w} / \mathrm{m}^{\circ} \mathrm{K}$ at $20^{\circ} \mathrm{C}$ was used to monitor the early-age temperature evolution (semi-adiabatic temperature profiles) of the mortar mixtures shown in Table 3.2. However, just three of the mixtures were selected for accomplishing the second objective of the research (evaluation of thermal shrinkage). A $150 \mathrm{~mm}$ diameter x $150 \mathrm{~mm}$ tall sample was cast and placed inside the semi-adiabatic chamber. The temperature of the sample was measured with a Type T thermocouple which was placed inside the sample at a depth of $75 \mathrm{~mm}$. The temperature was monitored for $4 \mathrm{~d}$ at 5 min intervals.

\subsection{Dual Ring Test (DRT)}

The dual ring test (DRT) operates by casting an annulus of mortar between two restraining rings in two lifts, being vibrated with a handheld vibrator after each lift. The temperature of the test is controlled by placing a copper tubing coil that is connected to an ethyl glycol system on top of the rings and sample. Due to the low coefficient of thermal expansion of the rings (i.e., they are made of Invar), the dual ring test has the ability to remain volumetrically stable over varying temperatures. The rings, sample, and temperature control coil are sealed in a highly insulated chamber with a thermal conductivity of $0.019 \mathrm{~W} / \mathrm{m} \cdot \mathrm{K} 189$ at $20^{\circ} \mathrm{C}$ (approximately half that of conventional glass fiber insulation). The temperature of the ethyl-glycol is controlled through an external programmable $28 \mathrm{~L}$ water bath system that enables the bath temperature to be lowered below freezing to 196 approximately $-20^{\circ} \mathrm{C}$. The rings are instrumented with four equally spaced CEA-00 strain gages that measure the strain developed in the inner and outer restraining rings. The gages have a grid resistance equal to $120 \% \pm 1460.3 \% \Omega$, Gage Factor $2.05 \% \pm 0.5 \%$ at $24{ }^{\circ} \mathrm{C}$, temperature correction of Gage Factor $+1.2 \pm$ 1470.2 , and an operating temperature range of $-75^{\circ} \mathrm{C}$ to $+175^{\circ} \mathrm{C}$. A data acquisition system was set up so that the strain and temperature of the rings were recorded every $5 \mathrm{~min}$. The recorded strains are used to calculate the residual stress accumulation in the sample. The induced stresses from temperature changes can be used to show the reserve capacity and determine how near the specimen is to cracking (Schlitter et al., 2010).

\section{RESULTS AND DISCUSSION}

\subsection{Isothermal Conditions}

The mortar mixtures shown in Table 3.2 were used in this part of the study. The mortar specimens were placed in the dual ring and held at a constant temperature of $23^{\circ} \mathrm{C} \pm 0.2^{\circ} \mathrm{C}$ for $2 \mathrm{~d}, 4 \mathrm{~d}$, or $7 \mathrm{~d}$, at which point the temperature was decreased at a constant rate of $2^{\circ} \mathrm{C} / \mathrm{h}$. As the temperature of the specimens was decreased, a sharp increase was observed in their residual stress. In some samples, this increase in stress reached the tensile strength of the sample which resulted in cracking, shown by an instantaneous decrease in stress in the figures. Figures 4.1, 4.2 and 4.3 show a comparison of the $2 \mathrm{~d}, 4 \mathrm{~d}$, and 7-d stress development, respectively. From these data, the remaining stress capacity of the material can be utilized to assess the cracking capacity of the mixtures. The remaining stress capacity indicates how near the sample is from cracking (magnitude shown with an arrow in Figure 1 left). The larger the remaining stress capacity, the better the performance, in terms of preventing cracking.

Using fly ash (with or without internal curing) as a replacement for cement reduces the stress development in the specimens. For instance, at $7 \mathrm{~d}$ (Figure 3), the $0.30-60 \%$ mixture exhibited a $57 \%$ average reduction in residual tensile stress compared to the $0.30-0 \%$ mortar mixture, and a similar stress development to that of the $0.42-0 \%$ mortar mixture. This reduction can be attributed directly to the high volume of fly ash replacement in the system. The fly ash reacts at a slower rate than the cement it replaced, leading to a slower rate and quantity of shrinkage, thus reducing the residual stress accumulation. However, this yields in lower initial strength development, as reported by (De la Varga et al., 2012).

Including IC further reduces the stress development in the fly ash specimens. For instance, the $0.30-60 \%-\mathrm{L}$ mixture resulted in relatively low residual stress accumulation when held at a constant temperature, an $86 \%$ reduction in residual stress over the $0.30-0 \%$ mixture and a $68 \%$ improvement upon the $0.30-60 \%$ mixture. This can be attributed to the reduced autogenous shrinkage and a low modulus of elasticity as a result of IC with fine LWA (Raoufi, Schlitter, Bentz, \& Weiss, 2011). The result is a material that has the benefits of a low $w / c m$ (e.g. reduced transport properties, high strength), while not incurring the detrimental effects of restrained autogenous shrinkage.

The increase in stress due to thermal volume change, induced by reducing the temperature, can be used to quantify how close the specimen was to cracking. This excess stress is a measure of remaining tensile stress capacity that the specimen has. A comparison of the remaining stress capacity of each mixture can be seen in Figure 4.4. In some instances, the sample was unable to be cracked, signified by the arrow symbolizing the potential for higher capacities than measured. At early ages, the mortars have a relatively low modulus of elasticity and have yet to undergo significant residual stress development, which results in larger quantities of remaining stress capacity. As shrinkage occurs in the system, the residual stress developed begins to reduce the net effective tensile capacity of the sample, thereby reducing the remaining stress capacity. The use of HVFA replacement alleviates some of the residual stress accumulation at early ages, but the slower reaction reduces the overall tensile capacity of the material. The resulting 

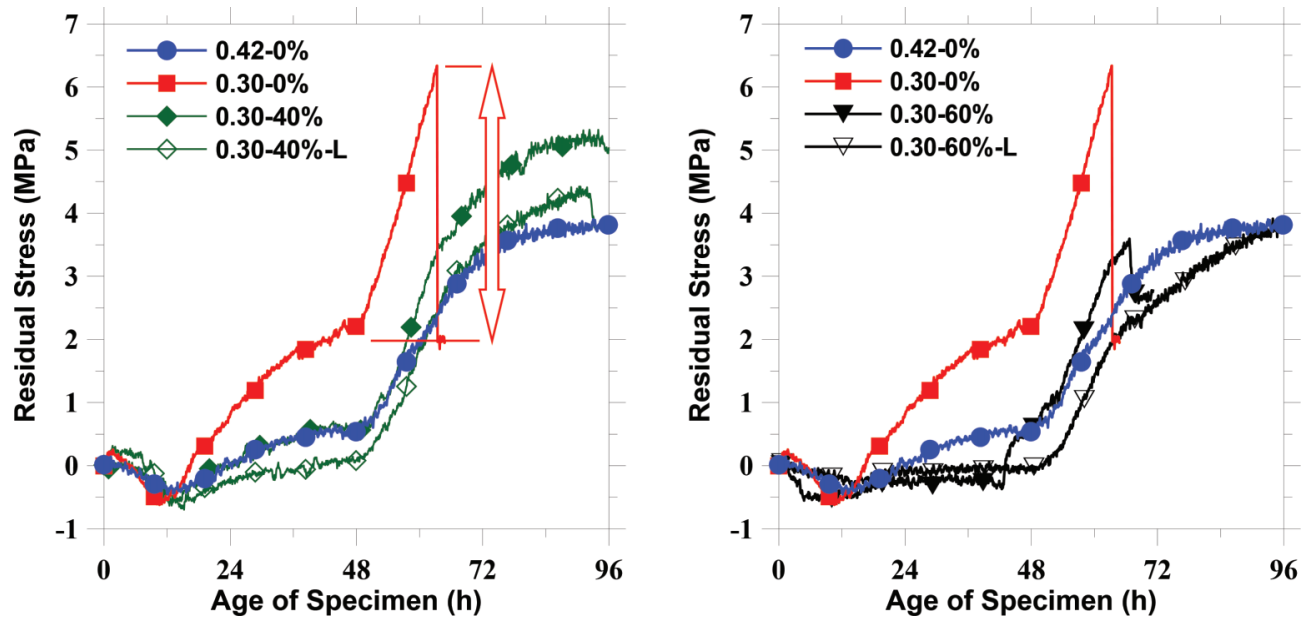

Figure 4.1 2-day residual stress comparison.
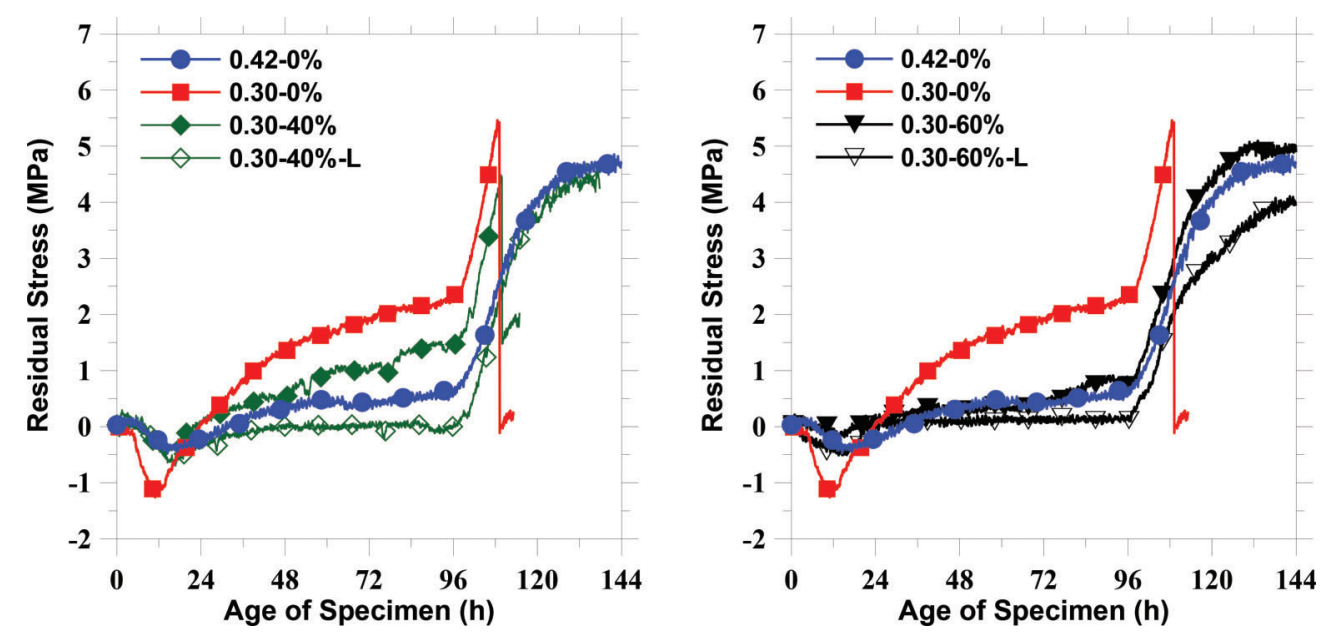

Figure 4.2 4-day residual stress comparison. Three of the mixes did not crack at any time: $0.42-0 \%, 0.30-40 \%-\mathrm{L}$, and $0.30-60 \%-\mathrm{L}$.
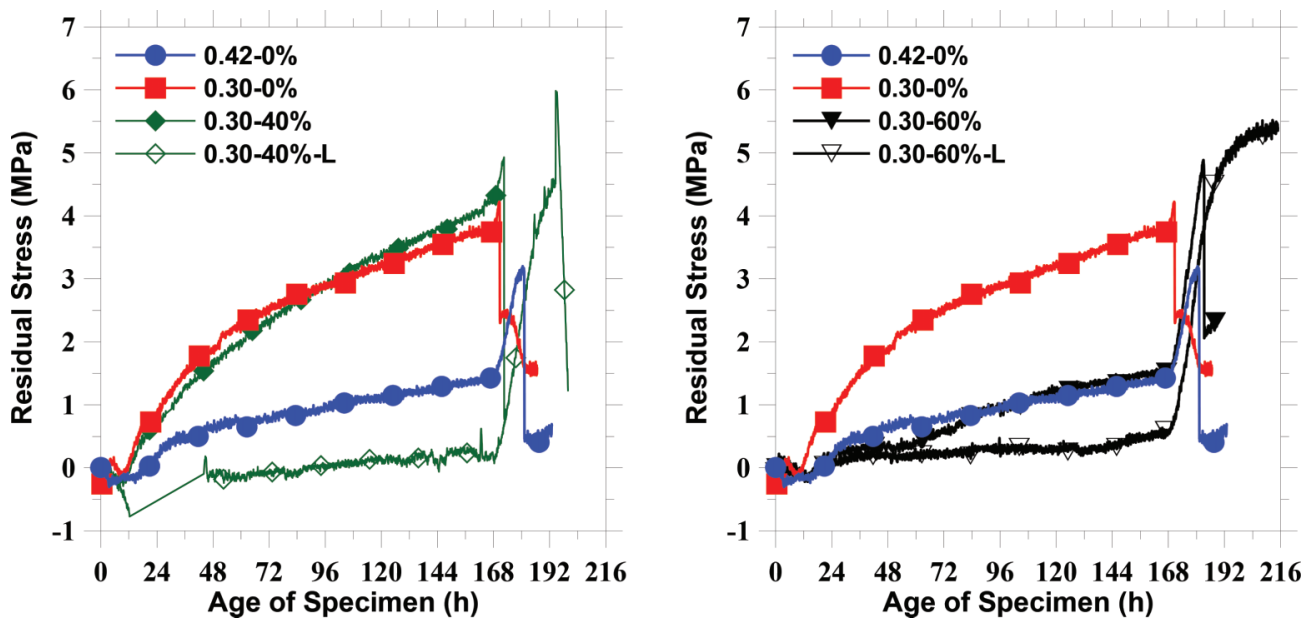

Figure 4.3 7-day residual stress comparison.

effects are roughly equivalent cracking capacities between the HVFA mixtures and the $0.30-0 \%$ mixture at early ages (i.e., $2 \mathrm{~d}$ ), and higher than the reference mixture ( 0.42 with no fly ash). At later ages (i.e., $7 \mathrm{~d}$ ), the reduction in shrinkage due to the hydration of cement in the $0.30-60 \%$ mixture results in 6.6 times and 1.9 times the remaining stress capacity over the $0.30-0 \%$ and $0.42-0 \%$ mixtures, respectively. However, the $0.30-40 \%$ mixture does not 


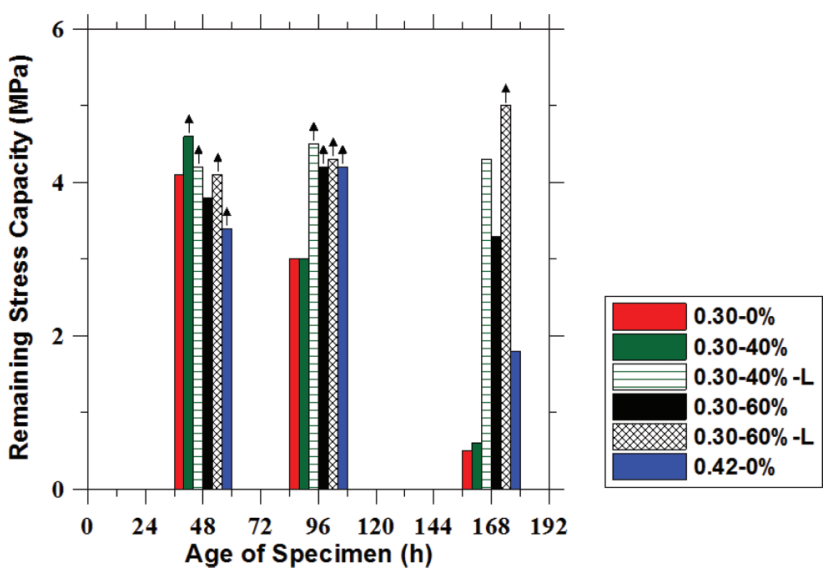

Figure 4.4 Remaining stress capacity comparison at $2 \mathrm{~d}, 4 \mathrm{~d}$, and $7 \mathrm{~d}$ of age.

show any increase in remaining stress capacity compared to the $0.30-0 \%$.

When IC is utilized, the residual stresses are significantly reduced, resulting in a material that has not yet experienced a reduction in its net effective tensile capacity. Since damage from shrinkage is not incurred, the material is much more resilient toward cracking, and most importantly, the samples do not experience a reduction in remaining stress capacity over time, but rather an increase due to the development of strength at later ages. At $7 \mathrm{~d}$ of age, the $0.30-60 \%-\mathrm{L}$ mixture showed a reserve capacity of 2.8 times that of the reference $0.42-0 \%$ and up to 10 times that of the 0.30 $0 \%$. The $0.30-40 \%-\mathrm{L}$ mixture also shows a higher remaining stress capacity compared to those of the $0.42-0 \%$ and $0.30-0 \%$ (2.4 and 8.6 times, respectively). These results demonstrate that HVFA replacement with the inclusion of IC can be effectively used to improve the cracking resistance of concretes.

\subsection{Semi-Adiabatic Conditions}

In structures, a concrete mixture will typically experience a temperature rise at early ages due to the heat of hydration, followed by a gradual reduction in temperature as the mixture equilibrates with its surroundings. In mass concrete, the temperature of the concrete in the interior decreases slowly over long periods of time. In many structures, the concrete temperature rises then decreases over the next few days to reach equilibrium with its surroundings. This could be represented with semi-adiabatic temperature profiles.

Semi-adiabatic temperature profiles of the six mortar mixtures presented were measured as shown in Figure 4.5. As a result, it can be observed that all of the HVFA mortar mixtures have a slower rate of temperature rise and a lower maximum temperature than that of the 0.30-0\% mortar. The maximum heat that was developed by the $0.30-40 \%$ mortar is approximately the same as the reference $0.42-0 \%$ mortar; however, there was a delay of $9 \mathrm{~h}$ in the time that the peak temperature was observed for the fly ash mixture. A lower temperature

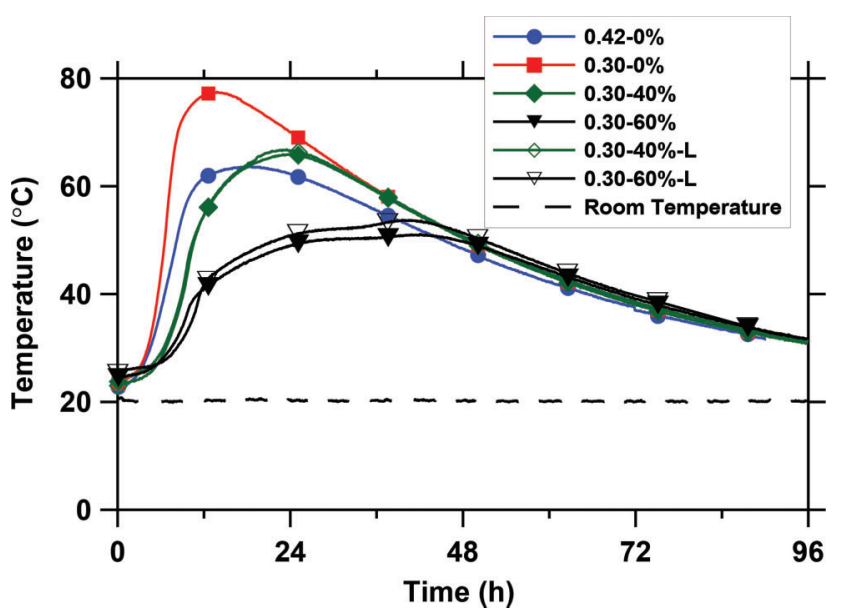

Figure 4.5 Semi-adiabatic temperature profiles.

rise was also observed in the $0.30-60 \%$ mortar. It can be noticed that IC does not significantly change the temperature profiles.

The temperature profiles shown in Figure 5 can be generated in the dual ring in order to see the benefits that HVFA mixtures have not only due to the slower rate of reaction, but also due to the lower change (i.e., decrease) in temperature that would induce less thermal stresses in practice, and thus make these concretes less likely to experience thermal cracking.

The three mortar mixtures shown in Table 3.2 that were used for the semi-adiabatic tests were: $0.30-0 \%$, $0.30-40 \%$, and $0.30-40 \%-\mathrm{L}$. The temperature of the dual ring was controlled to match the temperature profile of the $0.30-0 \%$ mixture shown in Figure 4.5. This would be the worst case scenario as the temperature rise is the highest in both magnitude and rate.

The stresses developed in the dual ring for the three mixtures can be seen in Figure 4.6. Figure 4.6 shows that the $0.30-0 \%$ and $0.30-40 \%$ mixtures have similar behavior, with the mixture containing $40 \%$ fly ash cracking at a slightly earlier age and lower stress level. When IC was used with the fly ash, the stress due to autogenous shrinkage is essentially eliminated. As such, the response for the $0.30-40 \%$ - $\mathrm{L}$ mixture is mainly that of the thermal component of the shrinkage. This mixture was able to withstand a total temperature reduction of $-67.5^{\circ} \mathrm{C}$ and still exhibited no cracking. It seems that the compressive stresses at early ages (i.e., expansion) were beneficial to the mixture, since it provided a slight compression in the sample prior to any shrinkage. As the temperature was reduced at later ages, this compressive stress was reduced while no significant shrinkage stresses developed. This observed behavior may also be attributed to the more compliant nature of the material due to the lower stiffness obtained with lightweight aggregate (Raoufi et al., 2011). At the end of the test, when the temperature was reduced at a faster rate in attempts to induce cracking, the shrinkage stress was also lower in the internally cured mixture. 


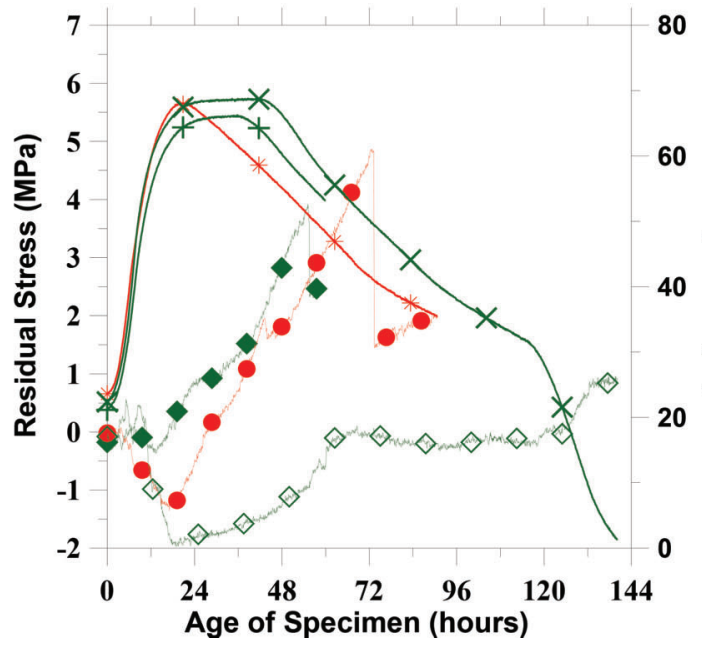

80

60

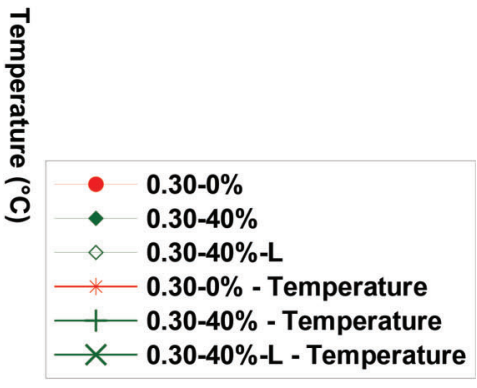

Figure 4.6 Semi-adiabatic residual stress development.

Considering that the fly ash specimens would be exposed to a different temperature profile (Figure 4.5) than that of the $0.30-0 \%$, this would theoretically be an additional thermal benefit in terms of cracking potential, as the temperature profiles of the fly ash specimens show lower temperature rise and rate.

\section{LARGE SCALE APPLICATION}

This work corresponds to the Phase III 'Large Scale Testing and Evaluation', but it is presented in this chapter as the approach followed in the dual ring work is similar to that of the large scale testing. The objective of Phase III of the research is to develop a clean set of temperature development and strength development data for a beam section exposed to different thermal conditions. This data is valuable for evaluating the temperature development and strength development profiles from Tasks 2 and 3, as well as the effects of curing and crack resistance described in Tasks 4 and 5. This data will also be made available to the research community for future researchers to use in validating models.

A large concrete beam ( $8 \mathrm{ft}$. $\mathrm{x} 1 \mathrm{ft} . \mathrm{x} 4$ in) $[2.44 \mathrm{~m} \mathrm{x}$ $0.30 \mathrm{~m} \times 0.10 \mathrm{~m}]$ with $w / c$ of 0.30 was cast. The beam was restrained at both ends, thus simulating a conventional concrete bridge deck (see Figure 4.7). The objective of this study is to assess the cracking potential due to thermal and autogenous shrinkage and evaluate the benefits of using fly ash and internal curing in a large scale concrete specimen. Thermal shrinkage was induced through a metallic panel that is in direct contact with the concrete beam. Copper tubes are attached to the metallic panel and fed with a glycol solution capable of producing a large range of temperatures. These copper tubes are protected with an insulation material. A final wooden panel will be added to the set up to make the system more rigid and durable. The panel is used to transfer a semi-adiabatic temperature profile characteristic of that mix design in the same way as was done in the DRT. This temperature profile was

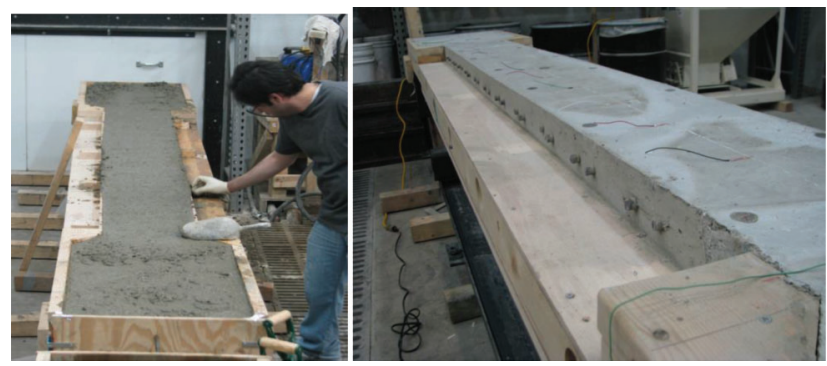

Figure 4.7 Concrete beam preparation.

TABLE 3.3

Measured Heat Capacities of the Raw Materials

\begin{tabular}{lc}
\hline \hline Material & $\begin{array}{c}\text { Heat Capacity } \\
{[\mathbf{J} /(\mathrm{g} \cdot \mathbf{K})]( \pm \text { std. dev. })}\end{array}$ \\
\hline Cement A & $0.743 \pm 0.001$ \\
Fly ash A & $0.720 \pm 0.001$ \\
Sand & $0.736 \pm 0.001$ \\
Lightweight Aggregate & $0.628 \pm 0.003$ \\
\hline
\end{tabular}

generated using real data from a weather station that, together with other concrete properties (e.g., thermal properties), was inserted as an input value in the FEM MASSE model.

\subsection{Thermal Property Measurements}

Five sets of mortar cylinders, along with their component raw materials, were received from Purdue at NIST for the measurement of their thermal properties (specifically thermal conductivity, heat capacity, and emissivity). These thermal properties were measured to provide critical inputs for thermal and mechanical analysis software packages (such as FEMMASSE, ConcreteWorks, and HIPERPAV). Thermal conductivity and heat capacity were assessed using a Hot Disk Thermal Constants Analyzer (Bentz, 2007; Bentz, Peltz, Durán-Herrera, Valdez, \& Juárez, 2011), while 
TABLE 3.4

Measured Thermal Properties of the Five Mortar Mixtures

\begin{tabular}{lcccc}
\hline \hline Mixture & Specific Gravity & Thermal Conductivity [W/(m/K)] & Heat Capacity [J/(kg·K)] & Emissivity (cut surface) \\
\hline $0.30-0 \%$ & $2.29 \pm 0.03$ & $1.949 \pm 0.026$ & $0.85 \pm 0.13$ & $0.903 \pm 0.004$ \\
$0.42-0 \%$ & $2.20 \pm 0.01$ & $1.880 \pm 0.062$ & $0.91 \pm 0.06$ & $0.902 \pm 0.003$ \\
$0.30-40 \%$ & $2.26 \pm 0.01$ & $1.847 \pm 0.035$ & $0.81 \pm 0.01$ & $0.901 \pm 0.003$ \\
$0.30-40 \%-\mathrm{L}$ & $2.06 \pm 0.01$ & $1.456 \pm 0.089$ & $0.89 \pm 0.15$ & $0.908 \pm 0.005$ \\
$0.42-0 \%-\mathrm{L}$ & $2.23 \pm 0.02$ & $1.826 \pm 0.062$ & $0.93 \pm 0.02$ & $0.900 \pm 0.002$ \\
\hline
\end{tabular}

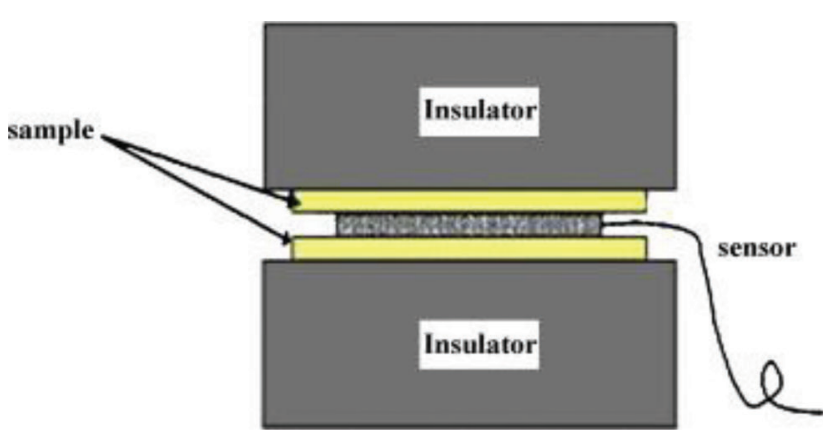

Figure 4.8 Basic configuration for measuring thermal properties of mortar samples using the Hot Disk Thermal Constants Analyzer.

emissivity (reflectivity) was evaluated using a GierDunkle reflectometer (ASTM E408-71, 2008; Bevans, Luedke, \& Nelson, 1966). First, the heat capacities of the raw materials were measured using a gold pan cell on the Hot Disk, with a typical specimen mass of $1 \mathrm{~g}$ of powder ( $0.5 \mathrm{~g}$ for the lightweight aggregate (LWA) sand). The measured heat capacities are provided in Table 3.3, along with the standard deviation obtained from 3 replicate measurements on the same sample.

The received $100 \mathrm{~mm}$ diameter x $200 \mathrm{~mm}$ height mortar cylinders were cut into four sections (nominally $50 \mathrm{~mm}$ in height) and pre-conditioned in a $25^{\circ} \mathrm{C}, 50 \%$ $\mathrm{RH}$ environment for a minimum of $7 \mathrm{~d}$, before being evaluated using the Hot Disk equipment. The two middle sections of the cylinders were used to create a matched pair of specimens for evaluation using the Hot Disk as shown in Figure 4.8. The mass of each preconditioned cylinder section, along with its dimensions, was measured and used to calculate its specific gravity. The measured thermophysical properties (average values \pm one standard deviation) are summarized in Table 3.4. As would be expected, under this pre-conditioning regimen, the mortars with internal curing exhibit a lower thermal conductivity, due to the presence of the less dense LWA, particularly for the lower water-tocementitious material ratio $(w / \mathrm{cm})$ of 0.3 .

The emissivities reported in Table 3.4 are the average value measured on the six cut faces from the four cylinder sections. Measured values were slightly higher for the cast and finished surfaces of the cylinders (both for pre-conditioned and rewetted surfaces), and it is suggested that for modeling, an emissivity value of 0.92 be employed for dry surfaces and a value of 0.91 for wet surfaces.

\section{CONCLUSIONS}

High volume fly ash concretes are used in conjunction with internal curing (i.e., through pre-wetted lightweight aggregate). Reducing the $w / \mathrm{cm}$ of the HVFA mixtures guarantees sufficient early-age strength in comparison to a typical mixture used in Department of Transportation applications (De la Varga et al., 2012). When IC is used in conjunction with low $w / \mathrm{cm}$ HVFA mixtures, a reduced potential for early-age cracking is observed. Results of the semi-adiabatic tests show that while plain concrete may be susceptible to cracking, the use of fly ash as a cement replacement combined with internal curing provides sufficient resistance to shrinkage cracking, thereby improving the sustainability and durability of structures. Future research has been started to correlate the small geometry of the dual ring test to a large scale beam. The results of this study show that HVFA replacement with the inclusion of IC is a feasible method of improving the sustainability and durability of concrete.

\section{REFERENCES}

American Concrete Institute (ACI). (2008). [Press release]. Retrieved from http://www.concrete.org/general/Press Releases/sdc\%20pca\%20meeting.htm

ASTM C305-06. (2006). Standard practice for mechanical mixing of hydraulic cement pastes and mortars of plastic consistency. West Conshohocken, PA: ASTM International.

ASTM E408-71. (2008). Standard test methods for total normal emittance of surfaces using inspection-meter techniques. West Conshohocken, PA: ASTM International.

Bentz, D. P. (2007). Transient plane source measurements of the thermal properties of hydrating cement pastes. Materials and Structures, 40(10), 1073-1080. http://dx.doi.org/ 10.1617/s11527-006-9206-9

Bentz, D. P., Peltz, M. A., Durán-Herrera, A., Valdez, P., \& Juárez, C. A. (2011). Thermal properties of high-volume fly ash mortars and concretes. Journal of Building Physics, 34(3), 263-275. http://dx.doi.org/10.1680/macr.2008.62.5.327

Bentz, D. P., \& Snyder, K. A. (1999). Protected paste volume in concrete: Extension to internal curing using saturated lightweight fine aggregate. Cement and Concrete Research, 29(11), 1863-1867. http://dx.doi.org/10.1016/S0008-8846 (99)00178-7

Bentz, D. P., \& Weiss, W. J. (2011). Internal curing: A 2010 state-of-the-art review. Washington, DC: U.S. Department of Commerce, National Institute of Standards and Technology. Retrieved from http://ws680.nist.gov/publication/ get_pdf.cfm?pub_id $=907729$ 
Bevans, J. T., Luedke, E. E., \& Nelson, K. E. (1966). A device for the rapid measurement of total emittance. Journal of Spacecraft and Rockets, 3(5), 758-760. http://dx.doi.org/10. 2514/3.25051

Castro, J., Keiser, L., Golias, M., \& Weiss, J. (2011). Absorption and desorption properties of fine lightweight aggregate for application to internally cured concrete mixtures. Cement and Concrete Composites, 33(10), 1001-1008. http:// dx.doi.org/10.1016/j.cemconcomp.2011.07.006

De la Varga, I., Castro, J., Bentz, D., \& Weiss, J. (2012). Application of internal curing for mixtures containing high volumes of fly ash. Cement and Concrete Composites, 34(9), 1001-1008. http://dx.doi.org/10.1016/j.cemconcomp.2012. 06.008

Kantro, D. L. (1980). Influence of water-reducing admixtures on properties of cement paste-a miniature slump test.
Cement, Concrete and Aggregates, 2(2), 95-102. https://doi. org/10.1520/CCA10190J

NYSDOT. (2008). Moisture content of lightweight fine aggregate (Test Method No. NY 703-19 E). Albany, NY: New York State Department of Transportation, Materials Bureau.

Raoufi, K., Schlitter, J., Bentz, D., \& Weiss, J. (2011). Parametric assessment of stress development and cracking in internally cured restrained mortars experiencing autogenous deformations and thermal loading. Advances in Civil Engineering, 2011. http://dx.doi.org/10.1155/2011/870128

Schlitter, J. L., Senter, A. H., Bentz, D. P., Nantung, T., \& Weiss, W. J. (2010). A dual concentric ring test for evaluating residual stress development due to restrained volume change. Journal of ASTM International, 7(9), 1-13. https:// doi.org/10.1520/JAI103118 
CHAPTER 6. PHASE II, TASKS 4 AND 5: FLUID TRANSPORT IN HIGH-VOLUME FLY ASH MIXTURES WITH AND WITHOUT INTERNAL CURING

Igor De la Varga, Robert P. Spragg, Carmelo Di Bella, Javier Castro, Dale P. Bentz, W. Jason Weiss

\section{ABSTRACT}

The transport of fluid and ions in concrete mixtures is central to many aspects of concrete deterioration. As a result, transport properties are frequently measured as an indication of the durability that a concrete mixture may be expected to have. This paper is the second in a series investigating the performance of High Volume Fly Ash (HVFA) mixtures with low water-tocementitious ratios $(w / \mathrm{cm})$ that are internally cured. While the first paper focused on strength and shrinkage, this paper presents the evaluation of the transport properties of these mixtures. Specifically, the paper presents results from: Rapid Chloride Migration (RCM), Rapid Chloride Penetration Test (RCPT), apparent chloride diffusion coefficient, surface electrical resistivity, and water absorption. The test matrix consisted of mortar samples with two levels of fly ash replacement ( $40 \%$ and $60 \%$ by volume) with and without internal curing provided with pre-wetted lightweight fine aggregates (LWA). These mixtures are compared to plain ordinary portland cement (OPC) mortars. The results indicate that HVFA mixtures with and without internal curing provide benefits in terms of reduced transport coefficients compared to the OPC mixtures.

\section{INTRODUCTION}

Fly ash is a by-product of coal combustion in power plants that can be used in concrete as a cement replacement (ACI Committee 232, 2003). The replacement of cement with fly ash can contribute positively in improving the sustainability of the concrete construction industry in a number of ways: (1) using less cement so that the clinker factor per cubic yard of concrete is reduced, and (2) using a waste product (fly ash) that no longer needs to be landfilled. The amount of fly ash replacement for cement that is typically used in concrete pavements and transportations structures is limited by specifications to approximately $20 \%-25 \%$ by mass, due to strength and de-icer scaling concerns (Obla, Lobo, \& Kim, 2012). High Volume Fly Ash (HVFA) concrete mixtures where cement replacement is increased to be on the order of $50 \%$ or more have demonstrated the potential to perform very well or achieve specific characteristics in a series of studies (Malhotra \& Mehta, 2002). For example, the use of HVFA in mass concrete applications can reduce the heat of hydration and resulting thermal effects (Malhotra, 1990), thereby minimizing early-age cracking.

Concerns with the use of HVFA are related to slow rate of hydration which can result in slow strength gain and extended curing times. To overcome these limitations, the research team has taken an approach that can be used to offset the slow strength development of HVFA mixtures (De la Varga, Castro, Bentz, \& Weiss, 2012). This approach consists of reducing the water-tocementitious materials mass ratio $(w / \mathrm{cm})$. However, as the $w / \mathrm{cm}$ is reduced, increased autogenous shrinkage is typically observed (Pease, Hossain, \& Weiss, 2004). In addition, the low $w / \mathrm{cm}$ concrete mixtures may become more difficult to water cure, as the curing water can only penetrate a few millimeters due to the much denser microstructure that forms (Bentz \& Weiss, 2011). This issue of self-desiccation in high performance concrete has been discussed by Philleo (1991; Bentz \& Weiss, 2011). Internal curing (IC) has been developed as one potential approach to improve curing and to distribute curing water throughout the concrete's cross section. IC consists of mixing in pre-wetted porous lightweight aggregates (LWA) that act as water reservoirs within the concrete, and subsequently exploiting the mechanism of chemical shrinkage to draw water from the LWA to the paste as needed during hydration, thereby curing the concrete from within (Bentz \& Weiss, 2011).

The use of HVFA mixtures with a low $w / \mathrm{cm}$ may be expected to improve performance in terms of reduced transport properties (i.e., water and ion penetrability). Transport properties depend on total porosity, pore size distribution, pore connectivity, and pore tortuosity (EuroLightCon, 1998). Internal curing elevates the rate of hydration at later ages, which refines the pore structure and reduces the transport of water and ions through the cement matrix at a given age (Castro, 2011). Bentz (2009) suggested that the quality of the interfacial transition zone (ITZ) is another factor to consider in transport performance when comparing mixtures with and without IC, but to a lesser degree than the pore tortuosity of the bulk matrix (Delagrave, Bigas, Ollivier, Marchand, \& Pigeon, 1997). It has been shown that IC improves the quality of the ITZ (Bentz \& Stutzman, 2008; Peled, Castro, \& Weiss, 2010), which also reduces transport.

This paper presents a series of tests that highlight the transport properties of HVFA mixtures with IC, following up on an initial study that focused on mechanical properties, shrinkage, and early-age cracking (De la Varga et al., 2012).

\section{MATERIALS AND MIXTURE PROPORTIONS}

As previously mentioned, this paper considers an approach to use a HVFA mixture as a potential substitute for a typical concrete mixture design. For example, a conventional pavement mixture design in the state of Indiana would consist of a water-to-cement ratio, $w / c=0.42$ to achieve specific performance requirements, including early-age strength. To use higher volumes of fly ash, the $w / c(w / c m)$ has to be reduced to compensate for the otherwise low early age strength. As a result, a $w / \mathrm{cm}=0.30$ was used with $40 \%$ and $60 \%$ replacement by volume of cement with fly ash. The 
testing matrix consisted of six mixtures. Two plain mortar mixtures were prepared using ordinary portland cement $(w / c=0.30 ; w / c=0.42)$. Two HVFA mortars were prepared with $w / \mathrm{cm}$ of 0.30 with $40 \%$ and $60 \%$ of the cement replaced with fly ash (by volume). These two HVFA mortar mixtures were also prepared with internal curing. The mixture proportions of the mortars are summarized in Table 2.1. The mixture with a $w / c$ of 0.42 would correspond to a typical mixture design used in transportation structures (e.g., bridge deck). In Table 2.1, "L" stands for LWA and identifies the internally-cured mortars.

An ordinary portland cement (OPC), ASTM C15009 Type I/II, was used in this study, with a Blaine fineness of $476 \mathrm{~m}^{2} / \mathrm{kg}$, a density of $3170 \mathrm{~kg} / \mathrm{m}^{3} \pm 10 \mathrm{~kg} / \mathrm{m}^{3}$, an estimated Bogue potential phase composition of $52 \% \mathrm{C}_{3} \mathrm{~S}, 18 \% \mathrm{C}_{2} \mathrm{~S}, 8 \% \mathrm{C}_{3} \mathrm{~A}$, and $9 \% \mathrm{C}_{4} \mathrm{AF}$ by mass, and a $\mathrm{Na}_{2} \mathrm{O}$ equivalent of $0.5 \%$ by mass. A Class $\mathrm{C}$ fly ash (ASTM C618-08a) was also used with a density of $2630 \mathrm{~kg} / \mathrm{m}^{3} \pm 10 \mathrm{~kg} / \mathrm{m}^{3}$. The fine aggregate used was ordinary river sand with a fineness modulus of 2.71 and an apparent specific gravity of 2.58. Rotary kiln expanded shale (i.e., a lightweight fine aggregate) was used with a fineness modulus of 3.97 and an oven dry specific gravity of 1.38. The lightweight aggregate (LWA) was measured to have a $24 \mathrm{~h}$ water absorption of $17.5 \%$ by dry mass, when this material was tested using the paper towel technique (Castro, Keiser, Golias, \& Weiss, 2011; NYSDOT, 2008). A polycarboxylate-based high-range water-reducing admixture (HRWRA) was added at variable dosage by mass of cement in order to maintain the same (mini) slump in all mortars (Kantro, 1980). While the fly ash replacement for cement was performed on a volumetric basis, the $w / \mathrm{cm}$ of the last five mortars was maintained constant on a mass basis (following current industry practice), implying that these mixtures have variable initial capillary porosities.

In this study, sealed curing conditions were used for many of the mortar specimens, both those with and without IC. These curing conditions were selected as providing the most representative match for the curing that would be experienced by the interior of a concrete member in the field (Golias, Weiss, \& Bentz, 2013). To proportion the internally cured mixtures a methodology is used that is based on a procedure developed by Bentz, and reported in Bentz and Weiss (2011), in which the amount of LWA is calculated based on the chemical shrinkage occurring in the sample. In this study, a chemical shrinkage value of $6.4 \%$ was assumed for all the internally cured mixtures.

\section{TEST METHODS}

A series of tests were performed to assess the transport properties of the plain, HVFA, and internally cured HVFA mixtures. A total of five different tests methods were used. The tests are divided into three main groups. The first set of tests (3.1 to 3.3) is related with the movement (i.e., diffusion) of chloride ions in concrete. The second test (3.4) describes the electrical properties (e.g., surface resistivity) of the mixtures. Finally, the third test (3.5) describes water absorption (ASTM C1585).

\subsection{Rapid Chloride Migration-NT Build 492}

The rapid chloride migration test ( $\mathrm{RCM}$ ) was conducted to determine the non-steady state chloride migration coefficients following the NT Build 492 procedure (Hooton, Nagi, \& Ozyildirim, 2000; Nordtest, 1999). The RCM test accelerates the chloride transport by applying an electrical potential across the specimen for a specified period of time. The test samples were prepared from cylinders with a diameter of $102 \mathrm{~mm}$ [4 in.] and length of $204 \mathrm{~mm}$ [8 in.]. The day after casting, the samples were demolded, sealed in double plastic bags and stored at $(23 \pm 0.5)^{\circ} \mathrm{C}\left[(73.4 \pm 0.8)^{\circ} \mathrm{F}\right]$ until the age of testing was reached. The reason for this is that the IC effects are better observed in sealed-cured samples rather than in moist-cured samples. Additionally, as mentioned above, sealed-curing provides the most representative match for the curing that would be experienced by the interior of a concrete member in the field (Golias et al., 2013). The cylinders were cut into disks having a length of $(51 \pm 2) \mathrm{mm}[(2 \pm 0.08)$ in.] from the central

TABLE 2.1

Mixture Proportions of the Mortars Used in This Study

\begin{tabular}{|c|c|c|c|c|c|c|}
\hline Mortar Mixture & $0.42-0 \%$ & $0.30-0 \%$ & $0.30-40 \%$ & $0.30-60 \%$ & $0.30-40 \%-\mathrm{L}$ & $0.30-60 \%-\mathrm{L}$ \\
\hline Cement, $\mathrm{kg} / \mathrm{m}^{3}$ & 612 & 731 & 453 & 307 & 453 & 307 \\
\hline Fly Ash, $\mathrm{kg} / \mathrm{m}^{3}$ & - & - & 252 & 384 & 252 & 384 \\
\hline Water, $\mathrm{kg} / \mathrm{m}^{3}$ & 257 & 219 & 211 & 207 & 211 & 207 \\
\hline Water for $\mathrm{IC}, \mathrm{kg} / \mathrm{m}^{3}$ & - & - & - & - & 38 & 37 \\
\hline Fine Aggregate (SSD), $\mathrm{kg} / \mathrm{m}^{3}$ & 1418 & 1418 & 1418 & 1418 & 998 & 1006 \\
\hline Oven Dry LWA, $\mathrm{kg} / \mathrm{m}^{3}$ & - & - & - & - & 236 & 232 \\
\hline HRWRA, g/100g cementitious material & - & 0.5 & 0.2 & 0.1 & 0.2 & 0.1 \\
\hline Fly Ash, \% (by volume) & - & - & 40 & 60 & 40 & 60 \\
\hline Water/cementitious material (w/cm) & 0.42 & 0.30 & 0.30 & 0.30 & 0.30 & 0.30 \\
\hline Equivalent $w / c$ & 0.42 & 0.30 & 0.47 & 0.67 & 0.47 & 0.67 \\
\hline Volume Fraction of Aggregate, $\%$ & 55 & 55 & 55 & 55 & 55 & 55 \\
\hline
\end{tabular}

$1 \mathrm{~kg} / \mathrm{m}^{3}=1.69 \mathrm{lb} / \mathrm{yd}^{3}$. 
part of the original cylinder. The samples were vacuumsaturated and placed in a rubber sleeve. The top portion of the sleeve is used to create a reservoir where $0.3 \mathrm{M}$ $\mathrm{NaOH}$ is placed in contact with the upper surface of the sample. The bottom of the sample is placed in a solution of $10 \% \mathrm{NaCl}$. The test is illustrated in Figure 3.1. An initial electrical potential of $30 \mathrm{~V}$ is applied to the specimen. This potential is adjusted according to the current response (as outlined in the NT Build standard; Nordtest, 1999) and the applied potential is maintained for a $24 \mathrm{~h}$ period.

At the end of the $24 \mathrm{~h}$ time period in which voltage was applied, the sample was rinsed with distilled water and the surface was wiped with a cloth. The sample was then split into two pieces as shown in Figure 3.2. A 0.1 $\mathrm{mol} / \mathrm{L}$ silver nitrate solution is sprayed on the fractured surface of the sample. Where sufficient chloride is present, the silver nitrate causes white silver chloride to precipitate as shown in Figure 3.2. The chloride penetration depth is measured at 10 locations across the section and used to determine an average depth of penetration.

The chloride non-steady state migration coefficient $\left(\mathrm{D}_{\mathrm{nssm}}\right)$ was calculated using Equation 3.1 (Luping, 1997).

$$
\begin{gathered}
D_{n s s m}=\frac{0.0239(273+T) \cdot L}{(U-2) \cdot t} \\
\cdot\left(x_{d}-0.0238 \sqrt{\frac{\left(273+T \cdot L \cdot x_{d}\right.}{U-2}}\right.
\end{gathered}
$$

where $D_{n s s m}$ is the non-steady state migration coefficient $\left(x 10^{-12} \mathrm{~m}^{2} / \mathrm{s}\right), U$ is the absolute value of the applied voltage (V), $T$ is the average value of the initial and final temperatures in the solution (C), $L$ is the thickness of the specimen $(\mathrm{mm}), x_{d}$ is average value of the average chloride penetration depth $(\mathrm{mm})$ and $t$ is the test duration (h). A single specimen was tested for each mortar mixture at each testing age, with a coefficient of variation of $9 \%$ according to NT Build 492.

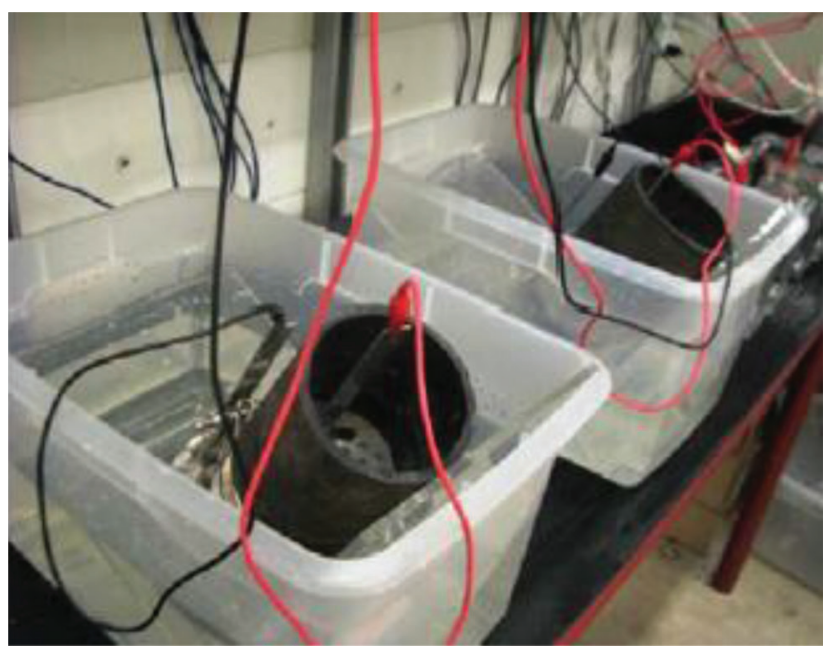

Figure 3.1 Rapid Chloride Migration (RCM) experimental set-up.

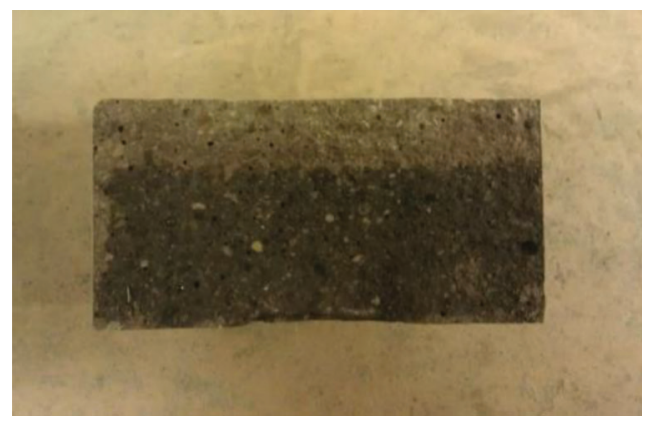

Figure 3.2 A sample from the Rapid Chloride Migration (RCM) test that was split and sprayed with Silver Nitrate $\left(\mathrm{AgNO}_{3}\right)$.

\subsection{Rapid Chloride Penetration Test (RCPT)}

ASTM C 1202 (or AASHTO T277) was performed on cylindrical samples with diameter of $102 \mathrm{~mm}$ [4 in.] and length of $204 \mathrm{~mm}$ [8 in.]. The day after casting, the samples were demolded, sealed in double plastic bags and stored at $(23 \pm 0.5)^{\circ} \mathrm{C}\left[(73.4 \pm 0.8)^{\circ} \mathrm{F}\right]$ until their testing age was reached.

At the time of testing, a disk was cut from the center of the cylinder that was $102 \pm 2 \mathrm{~mm}[4 \pm 0.08 \mathrm{in}$.$] in$ diameter and $51 \pm 2 \mathrm{~mm}[2 \pm 0.08$ in.] long. The disk was taken from the same cylinder that was prepared for the RCM test described above. A single specimen was tested for each mortar mixture at each testing age, with a coefficient of variation of $12.3 \%$ according to ASTM C1202. Prior to testing, each sample was vacuum saturated. During the test, one surface of the sample was exposed to sodium chloride solution $(3 \% \mathrm{NaCl})$ and the other surface was exposed to sodium hydroxide solution $(0.3 \mathrm{~mol} / \mathrm{L} \mathrm{NaOH})$. A $60 \mathrm{~V}$ external potential was applied and the current at 5 min intervals was recorded for a $6 \mathrm{~h}$ period. Figure 3.3 shows the experimental set up with four samples being tested simultaneously.

Alternative interpretations of this test have been proposed, namely that the resistance after a short duration test (usually $1 \mathrm{~min}$ or $5 \mathrm{~min}$ ) be used to calculate the resistivity (ASTM C1760-12, 2012; Riding, Poole, Schindler, Juenger, \& Folliard, 2008; Snyder, Ferraris, Martys, \& Garboczi, 2000).

\subsection{Apparent Chloride Diffusion Coefficient}

Based on ASTM C1543, $102 \mathrm{~mm}$ [4 in.] by $102 \mathrm{~mm}$ [4 in.] cylinders were prepared from four of the mortar mixtures shown in Table 1: $0.42-0 \%, 0.30-0 \%, 0.30$ $60 \%$, and $0.30-60 \%-\mathrm{L}$. Two specimens were tested for each mortar mixture at each testing age. The specimens were sealed-cured for $28 \mathrm{~d}$, and then dried at $50 \% \mathrm{RH}$ and $(23 \pm 0.5)^{\circ} \mathrm{C}\left[(73.4 \pm 0.8)^{\circ} \mathrm{F}\right]$ for an additional $28 \mathrm{~d}$. After this time period an epoxy was used to seal the sides of the cylinders to prevent evaporation during the test and to impose uni-directional control of the chloride penetration. The chlorides came from a salt solution $(3 \% \mathrm{NaCl})$ poured on top of the cylinders and held in place by a dike attached to the cylinder. A cover 


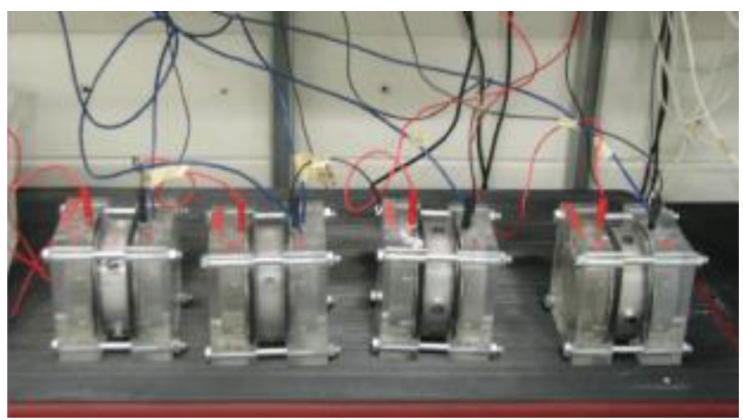

Figure 3.3 Rapid Chloride Penetration Test (RCPT) cells.

was placed on the top to avoid evaporation of the salt solution during the following ponding periods:

- 3,6 months (after specified curing period).

- 3,6 months after 1 year sealed-curing.

Although this test allows for uni-directional chloride penetration, the method of conditioning the samples may have induced some initial absorption, so it is not a pure diffusion test. In addition, the exposure of the bottom face to a $50 \% \mathrm{RH}$ environment during the test induces evaporation at that surface, meaning that chlorides can also migrate by wicking action.

After each ponding period was over, the samples were ground in layers of $1 \mathrm{~mm}$ so that the powder could be analyzed in terms of its total chloride content by titration. Chloride penetration profiles were assessed and apparent chloride diffusion coefficients were estimated based on Fick's Second Law, shown in Equation 3.2.

$$
\frac{C_{x}-C_{0}}{C_{s}-C_{0}}=1-\operatorname{erf}\left(\frac{x}{\sqrt{4 \cdot D_{a} \cdot t}}\right)
$$

where $C_{x}(\%)$ is the chloride concentration at any point $x(\mathrm{~m})$ at any time $t(\mathrm{~s}), C_{o}$ is the background chloride concentration $(\%), C_{s}$ is the surface chloride concentration $(\%), D_{a}$ is the apparent chloride diffusion coefficient $\left(10^{-12} \mathrm{~m}^{2} / \mathrm{sec}\right)$, and erf is the error function.

\subsection{Surface Resistivity}

The electrical resistivity was also measured using cylindrical samples that were $102 \mathrm{~mm}$ [4 in.] in diameter and $204 \mathrm{~mm}$ [8 in.] long and cured in lime water, using the four-point Wenner probe surface testing device (i.e., Wenner; see Figure 3.4), as described in AASHTO TP 95-11. Three specimens were tested for each mortar mixture at each testing age.

In this test, current is applied at the outer probes and the potential difference is measured between the two inner probes. Assuming that the sample has homogeneous semi-infinite geometry, the mortar resistivity is described by Equation 3.3 (Spragg et al., 2013):

$$
\rho=R \cdot K
$$

where $R$ is the measured resistance $(\Omega)$, and $k$ is the geometry correction shown in Equation 3.4 based upon

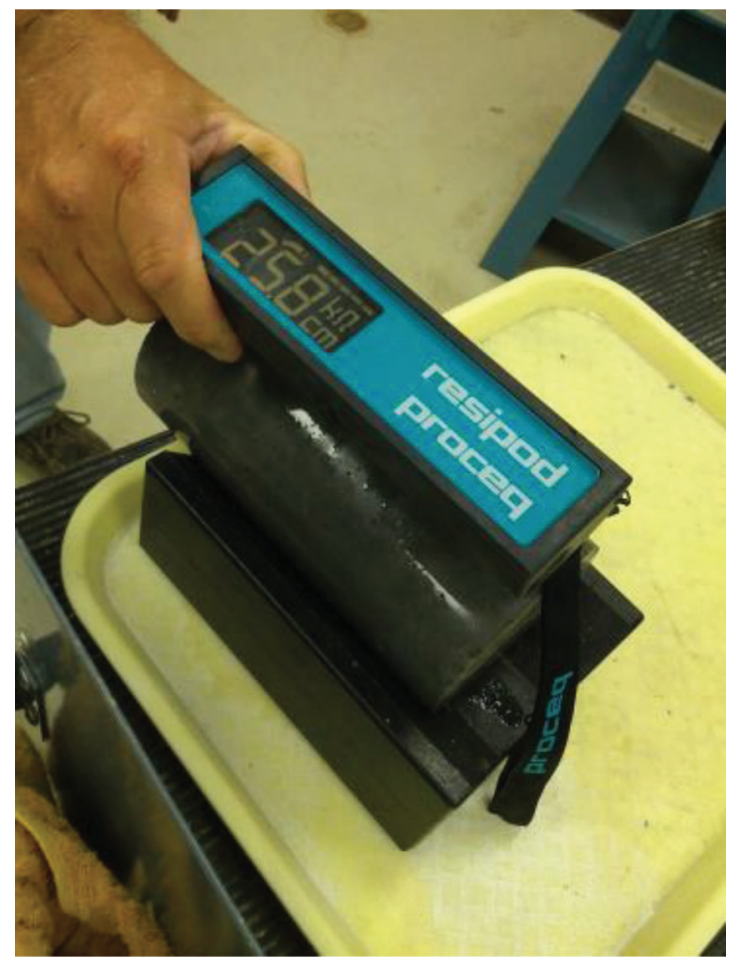

Figure 3.4 Wenner probe surface testing device ${ }^{2}$.

a fit of the data presented by Morris, Moreno, and Sagüés (1996).

$$
k=\frac{2 \pi a}{1.10-\frac{0.730}{\frac{d}{a}}+\frac{7.82}{\frac{\left(\frac{d}{a}\right)^{2}}{a}}}
$$

This approximation is only valid for cylindrical specimens with $d / a \leq 4.0$ and $L / a \geq 5.0$, where $d$ is the diameter of the cylinder, $a$ is the interprobe distance of the Wenner apparatus, and $L$ is the length of the cylinder.

Pore solution extraction was also conducted at different ages: $10 \mathrm{~min}$ and every one hour while still in the fresh state by a Millipore pressure filtering system, using nitrogen gas at pressures up to $200 \mathrm{kPa}$. The procedure has been previously used and described by Penko (1983). Extractions performed on hardened samples were conducted at ages of $1 \mathrm{~d}$ and $3 \mathrm{~d}$ using a high pressure die at pressures up to $380 \mathrm{MPa}$, as described byBarneyback and Diamond (1981).

After extraction, pore solution resistance was measured at different ages. This was done through the use of a conductivity cell, with stainless steel electrodes at the ends of a short polycarbonate tube. The geometry factor of $0.212 \mathrm{~cm}$ of the cell was determined using the

\footnotetext{
${ }^{2}$ Certain commercial products are identified in this paper to specify the materials used and procedures employed. In no case does such identification imply endorsement or recommendation by the National Institute of Standards and Technology, EMPA, Purdue University or Universidad del Desarrollo, nor does it indicate that the products are necessarily the best available for the purpose.
} 
ratio of area to length and was confirmed using a solution of known conductivity.

Using a Solatron 1260 Impedance Gain-Phase analyzer with a frequency range of $1 \mathrm{MHz}$ to $10 \mathrm{~Hz}$, and a $500 \mathrm{mV}$ AC Signal, the measured resistance was obtained and corrected by the geometry factor according to Equation 3.3 to obtain the electrical resistivity.

\subsection{Water Absorption}

The amount of water absorbed and the rate at which water is absorbed (referred to as sorptivity) can provide useful information concerning pore connectivity and pore volume, both of which are important in evaluating the durability of cementitious materials.

Replicate 102 mm x 204 mm [4 x 8 in.] cylinders were prepared from each mortar mixture in Table 2.1. Each cylinder was cast, demolded at $24 \mathrm{~h}$, and sealed in a double layer of plastic bags. Two cylinders were cast for each testing age of $28 \mathrm{~d}$ or $90 \mathrm{~d}$. After each curing age, one of the two cylinders was demolded and three $51 \pm$ $2 \mathrm{~mm}[2 \pm 0.08$ in.] thick specimens were cut from the central portion of the cylinder. The specimens were then placed in an isothermal $(23 \pm 0.5)^{\circ} \mathrm{C}\left[(73.4 \pm 0.8)^{\circ} \mathrm{F}\right]$ environment at $65 \pm 1 \% \mathrm{RH}$ until they reached mass equilibrium, defined as less than a $0.02 \%$ in mass over a period of $15 \mathrm{~d}$. This conditioning method lasted approximately 1 year and was chosen to provide consistent moisture content in all of the samples (Castro, Bentz, \& Weiss, 2011; Golias, Castro, \& Weiss, 2012).

Prior to absorption testing, the side surface of the samples was sealed with aluminum tape and the top surface was covered with plastic wrap. This was to prevent drying of the sample from these surfaces. Other researchers (Castro et al., 2011) have used epoxy rather than aluminum tape as the test was carried out for longer ages (i.e., 1 year). The remainder of the procedure was conducted in accordance with ASTM C1585-11.

The amount of absorbed water is normalized by the cross-section area of the specimen exposed to the fluid using Equation 3.5:

$$
i=\frac{m_{t}}{a \cdot p}
$$

where $i$ is the normalized absorbed fluid volume $\left(\mathrm{mm}^{3} /\right.$ $\left.\mathrm{mm}^{2}\right), m_{t}$ is the specimen mass at time $\mathrm{t}(\mathrm{g}) ; a$ is the area of the specimen exposed to the fluid (i.e., that of the bottom face) $\left(\mathrm{mm}^{2}\right)$, and $\rho$ is the density of the absorbed fluid (taken to be $0.001 \mathrm{~g} / \mathrm{mm}^{3}$ at $23^{\circ} \mathrm{C}$ for water).

The absorbed fluid volumes are then plotted as a function of the square root of time. The initial sorptivity is determined from the slope of the curve during the first $6 \mathrm{~h}$ according to ASTM C1585-11, while secondary sorptivity is determined using the slope of the same measurements between $1 \mathrm{~d}$ and $8 \mathrm{~d}$, as outlined in ASTM C1585. The initial and secondary sorptivities can be used to evaluate the connectivity of the pore network (Castro et al., 2011).

\section{RESULTS AND DISCUSSION}

\subsection{Chloride Transport Tests}

The measured non-steady state migration chloride coefficients from four of the mortar mixtures are provided in Table 4.1. The 0.42-0\% mortar had the greatest values of diffusion coefficients at all ages. While at early ages, the diffusion coefficients for the $0.30-0 \%, 0.30$ $60 \%$, and $0.30-60 \%-\mathrm{L}$ mortars are comparable, it is evident that at later ages (after 1 year) the diffusion coefficient decreases significantly in both fly ash mortars. The reduction in the diffusion coefficient of the HVFA mixtures over time suggests that there is plenty of curing water available in these systems (just provided from the mix water). In other words, the equivalent $w / c$ of these mixtures would correspond to a $w / c=0.67$, allowing the achievement of higher degrees of hydration, thereby reducing the diffusion coefficient.

It should be noted that the bulk diffusion coefficients of actual in-place concrete may actually be lower than the values shown for the internally-cured mortar. First, the internally cured mortar is cut to perform the test. As such, the cutting of the concrete exposes porous lightweight aggregate surfaces. When these cut aggregates are exposed to the solution, the chloride can easily diffuse/absorb quickly into the exposed LWA portions of the mortar which may not represent what happens in field concretes where the LWA are not exposed. As such, these results represent conservative estimates for the diffusion coefficient. In addition, since the sample was vacuum-saturated, the conductivity of the lightweight aggregate may alter the electrical response of the concrete (Weiss, Bullard, Snyder, Bentz, \& Castro, 2013).

Similarly, the results from the RCPT for the same four mortar mixtures are shown in Table 4.2. The charge passed values shown are much higher than what

TABLE 4.1

Chloride Diffusion Coefficients Obtained from the Rapid Chloride Migration Test

\begin{tabular}{lcccc}
\hline \hline & \multicolumn{4}{c}{ Diffusion Coefficients $\left(\mathbf{1 0}^{-\mathbf{1 2}} \mathbf{~ m}^{\mathbf{2}} / \mathbf{s}\right)$} \\
\hline Time [d] & $0.42-0 \%$ & $0.30-0 \%$ & $0.30-60 \%$ & $0.30-60 \%-\mathrm{L}$ \\
$\mathbf{2 8}$ & 13.8 & 3.68 & 5.54 & 3.49 \\
$\mathbf{5 6}$ & 18.2 & 4.58 & 2.46 & 3.97 \\
$\mathbf{9 1}$ & 13.9 & 4.40 & 1.41 & 2.03 \\
$\mathbf{3 6 5}$ & 7.94 & 2.25 & 0.29 & 0.48 \\
\hline
\end{tabular}

TABLE 4.2

Rapid Chloride Penetration Test Results for the Mortars Samples

\begin{tabular}{lcccc}
\hline \hline \multicolumn{4}{c}{ Charge Passed [C] } \\
\hline Time [d] & $\mathbf{0 . 4 2 - 0} \%$ & $\mathbf{0 . 3 0 - 0} \%$ & $\mathbf{0 . 3 0 - 6 0} \%$ & $\mathbf{0 . 3 0 - 6 0 \%}-\mathbf{L}$ \\
$\mathbf{2 8}$ & $*$ & 14026 & 3137 & 4398 \\
$\mathbf{5 6}$ & 15263 & 8894 & 2167 & $*$ \\
$\mathbf{3 6 5}$ & 5083 & 1061 & 129 & 114 \\
\hline
\end{tabular}

*Test not performed. 
is typically reported (in concretes) since these results were obtained in mortars with only 55\% aggregates volume. As it can be observed, the charge passed decreases over time in all mortar mixtures, which is attributed to increased hydration. In other words, more hydration products are formed, thus decreasing the number of interconnected pores of the samples. When comparing the two plain mortars $(0.42-0 \%$ and $0.30-0 \%)$, it is evident that less charge will pass through the lower $w / c$ mortar $(42 \%$ and $79 \%$ less at $56 \mathrm{~d}$ and $365 \mathrm{~d}$ respectively). Both HVFA mortars also show lower charge passed values throughout the testing ages compared to the control $0.42-0 \%$ and $0.30-0 \%$ mixtures.

The use of the RCPT method can be thought of essentially as a measure of concrete resistivity. However, it should be noted that RCPT was performed with high voltage and the samples heated during testing, which increases the charge passes due to the Joule effect. As such, there is no need to continue the test for $6 \mathrm{~h}$, and changes in the current during this time are most likely due to increases in temperature, not chloride penetration (Synder, 2001; Snyder et al., 2000). It is also important to emphasize that even though it is well known that the use of supplementary cementitious materials will improve the pore structure reducing the penetrability, on the other hand, they are also able to modify the chemistry of the pore solution. As a consequence, any change in the alkalinity of the system due to the mineral admixtures may result in misleading values.

The lower chloride diffusion coefficients obtained in the HVFA mixtures (both IC and non-IC) are translated into less chloride penetration from the concrete surface compared to the control $0.42-0 \%$ mixture at all different curing/ponding time periods used in this study. This is observed in Figure 4.1. However, the internally cured HVFA mixture shows larger amounts of chloride within the first $3 \mathrm{~mm}$ to $4 \mathrm{~mm}$ (this makes sense since the LWA used consisted of fine particles with a nominal maximum size of $4.76 \mathrm{~mm}$ ). Other studies have attributed this effect to the fact that the top surface of the samples are cut, thereby exposing the pores of the LWA at the surface of the sample. The exposed LWA pores are much larger than the paste
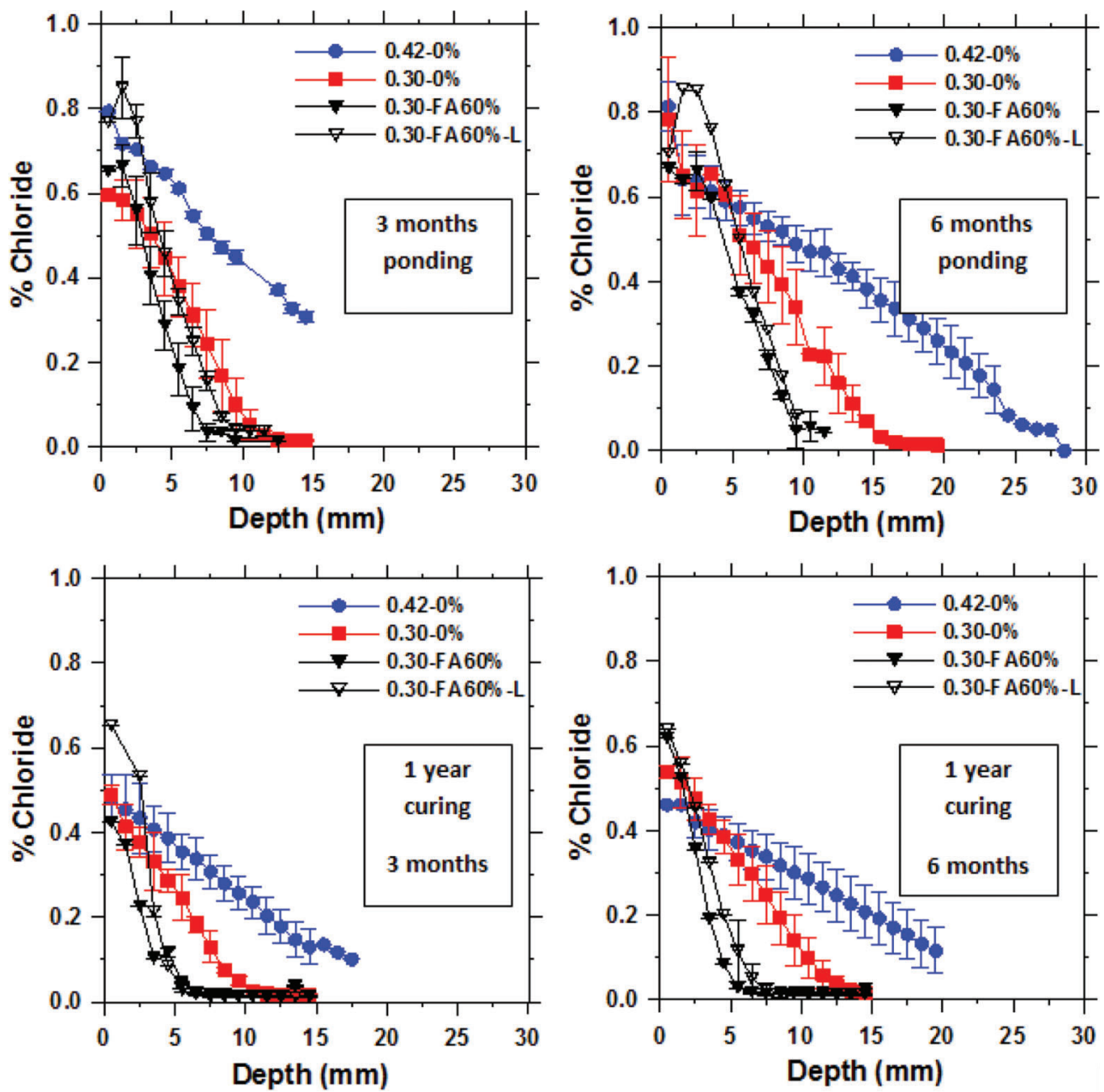

Figure 4.1 Chloride penetration profiles at different curing/ponding time periods. (Error bars represent the standard deviation from the average of three samples.) 
pores. As a consequence, LWA pores can work as reservoirs for the chloride solution which can be easily absorbed and rapidly diffuse into the LWA pores.

The apparent chloride diffusion coefficients can be calculated using these profiles and Equation 3.2. They are provided in Table 4.3. It can be observed how the apparent chloride diffusion coefficient decreases when the samples were cured for an additional year, increasing their degree of hydration and, therefore, decreasing the chloride penetration. This is observed in all four mixtures. While the $0.42-0 \%$ mortar shows higher values of apparent diffusion coefficients compared to the other mixtures, both HVFA mixtures show similar results between them, but still lower values than the $0.30-0 \%$ mortar. Therefore, an improvement in transport properties is observed in the HVFA mixtures if compared to either the $0.42-0 \%$ or $0.30-0 \%$ mortars.

\subsection{Electrical Tests}

Figure 4.2 shows the "normalized" surface resistivity measurements for four of the mortars described in Table 2.1. The mortars were stored in lime water so they can be considered to be close to saturation. The normalization is performed by dividing the bulk surface resistivity by the pore solution resistivity at the different ages shown in Figure $4.2(1 \mathrm{~d}, 3 \mathrm{~d}, 7 \mathrm{~d}, 14 \mathrm{~d}, 28 \mathrm{~d}, 56 \mathrm{~d}$, $91 \mathrm{~d}$, and $365 \mathrm{~d})$. This corresponds to plotting the formation factor, as explained in Equation 4.1. The formation factor characterizes the solid microstructure and it is directly related to the transport properties of a porous medium (NIST, n.d.).

$$
F=\frac{\rho_{t}}{\rho_{o}}
$$

where $F$ is the formation factor, $\rho_{t}$ is the mortar resistivity $(\Omega . \mathrm{m})$, and $\rho_{o}$ is the pore solution resistivity $(\Omega . \mathrm{m})$.

However, after $7 \mathrm{~d}$, pore solutions extractions are difficult to perform since the amount of liquid in the samples is low. Instead, an extrapolation from the pore solution resistivity vs. degree of hydration (DOH) curve was performed. These two parameters are linearly related after a certain degree of hydration (Spragg et al., 2013). Estimating the DOH at the different ages mentioned above, it was then possible to calculate the pore solution resistivity, and thus, normalize the results in
Figure 4.2. However, the resistivity values obtained from sealed-cured specimens do not correspond to values from samples that have been saturated. Thus, an additional correction for this must be performed. The approach taken was to calculate the sealed-to-saturated pore solution resistivity ratio using a model developed at NIST (n.d.), that can estimate the pore solution resistivity in both conditions (i.e., sealed and saturated). The tested (sealed) pore solution resistivity was then corrected to saturated conditions with that factor for each mortar mixture.

As observed in Figure 4.2, the HVFA specimens have similar resistivity values to that of the $0.42-0 \%$ mortar at $91 \mathrm{~d}$, and keep increasing over time, even surpassing the $0.30-0 \%$ mortar at about $300 \mathrm{~d}$ of hydration. After 1 year, both HVFA mixtures reach resistivity values up to 3 times larger than those obtained by the $0.42-0 \%$ mortar.

\subsection{Water Absorption Test}

Figure 4.3 shows the absorbed water throughout the $8 \mathrm{~d}$ of testing as a function of the square-root of time. It is observed that there is a decrease in the amount of absorbed water when the samples were cured during

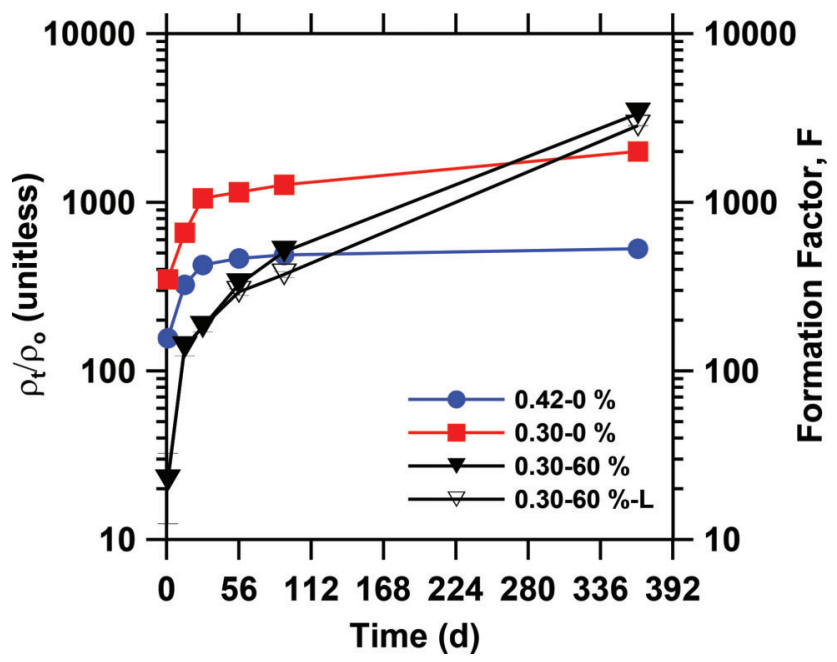

Figure 4.2 Formation factor as a function of time of mortars samples stored in lime water up to $365 \mathrm{~d}$. (Error bars represent the standard deviation from the average of three samples.)

TABLE 4.3

Apparent Chloride Diffusion Coefficients Approximated Using Fick's 2nd Law

\begin{tabular}{|c|c|c|c|c|}
\hline \multirow[b]{2}{*}{ Time [d] } & \multicolumn{4}{|c|}{ Apparent Diffusion Coefficients $\left(10^{-12} \mathrm{~m}^{2} / \mathrm{s}\right)$} \\
\hline & $0.42-0 \%$ & $0.30-0 \%$ & $0.30-60 \%$ & $0.30-60 \%-\mathrm{L}$ \\
\hline 91 (ponding) & $17.6(0.3)$ & $3.47(0.5)$ & $1.12(0.1)$ & $1.75(0.1)$ \\
\hline 180 (ponding) & $11.6(0.7)$ & $3.19(0.5)$ & $1.10(0.1)$ & $0.94(0.1)$ \\
\hline $\begin{array}{l}365 \text { (curing) + } \\
91 \text { (ponding) }\end{array}$ & $12.1(0.8)$ & $2.65(0.4)$ & $0.72(0.1)$ & $0.89(0.1)$ \\
\hline $\begin{array}{l}365 \text { (curing) + } \\
180 \text { (ponding) }\end{array}$ & $10.6(0.9)$ & $2.10(0.4)$ & $0.32(0.1)$ & $0.61(0.1)$ \\
\hline
\end{tabular}

Note: Numbers in parenthesis represent the standard deviation of two samples. 
$28 \mathrm{~d}$ and $90 \mathrm{~d}$ before drying. This happens in all the samples prepared and it is attributed to the higher degree of hydration reached, thus decreasing the capillary porosity and the amount of absorbed water. Figure 4.3(a) and (b) show the comparison of the $0.42-0 \%$ mortar with the HVFA mortars. It can be noticed that, after $8 \mathrm{~d}$ of testing, the $0.42-0 \%$ mortar absorbs approximately three times more water than the low $w / \mathrm{cm}$ HVFA mortars, which is in agreement with the fact that a higher volume of larger pores would be present in the high $w / c$ mortar.

When analyzing the effect that IC has on the HVFA mortars (Figure 4.3(c) and (d)), it can be observed that, while the IC mortars absorbed between $25 \%$ and $50 \%$ more water than the non-IC HVFA mortars at $28 \mathrm{~d}$, there is a decrease of $25 \%$ in the amount of water absorbed in the IC samples cured for $90 \mathrm{~d}$. It is important to remember at this point that the IC-HVFA mortars are intended to replace the $0.42-0 \%$ (reference mixture corresponding to a typical bridge deck concrete mix). The IC-HVFA mortars cured for $90 \mathrm{~d}$ have a similar performance in terms of water absorption as a plain 0.30 mixture. This would positively influence the durability of the material.

Figure 4.4(a) and (b) show the initial sorptivities calculated as the slope of the absorption vs. the square root of time during the first $6 \mathrm{~h}$ of testing. Initial sorptivity of the $0.42-0 \%$ mortar is approximately 4 times higher than the $0.30-0 \%$ mortar. The inclusion of fly ash in the low $w / \mathrm{cm}$ mortar does decrease the rate of water absorption in comparison with the $0.42-0 \%$ mortar as well. The inclusion of IC does not reduce the amount of water absorbed at $28 \mathrm{~d}$, being the same for the $40 \%$ fly ash mortar, and higher for the $60 \%$ fly ash mortar. However, IC slightly decreases the absorption rate at later ages (e.g., 90 d) in the HVFA mortars, which is attributed to a further hydration.

Figure 4.4(c) and (d) show the secondary calculated as the slope of the absorption vs. the square root of time between $1 \mathrm{~d}$ and $8 \mathrm{~d}$ of testing. Trends are similar

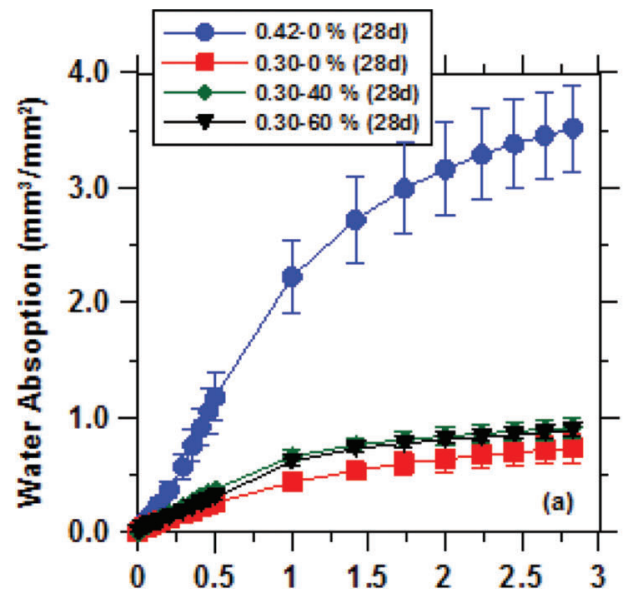

Time After Exposure ( $\left.\mathbf{d}^{1 / 2}\right)$

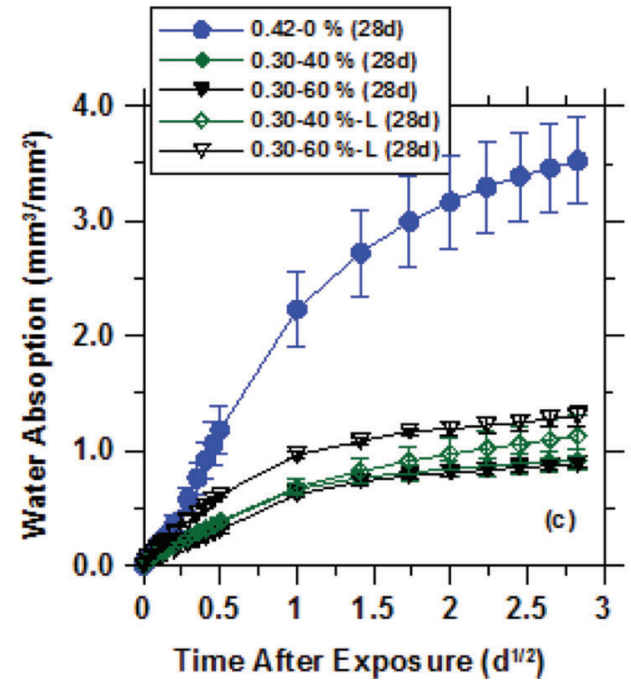

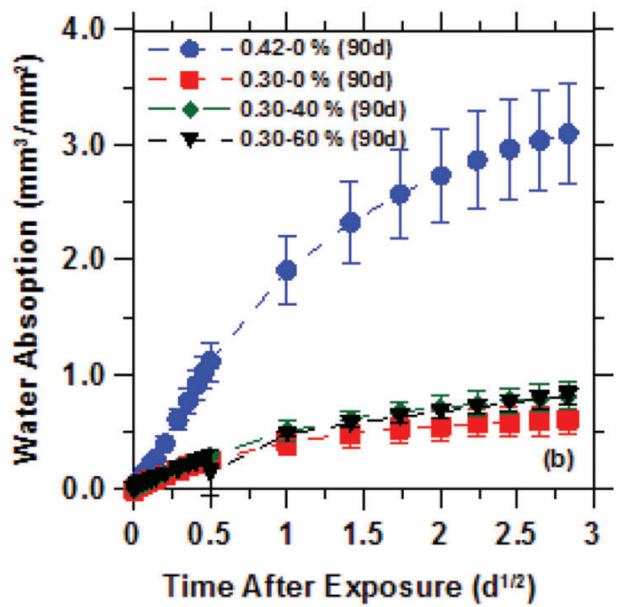

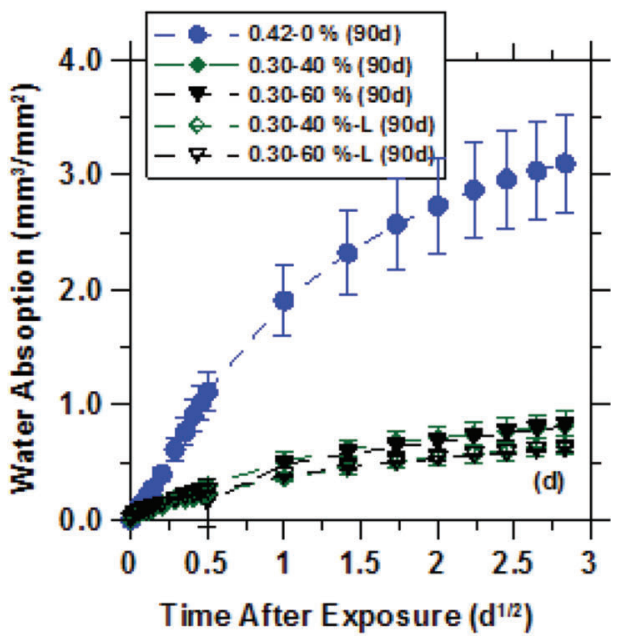

Figure 4.3 Absorbed water of sealed-cured mortars samples (a) as a function of fly ash content curing during $28 \mathrm{~d}$, (b) as a function of fly ash content curing during $90 \mathrm{~d}$, (c) including IC and curing $28 \mathrm{~d}$, and (d) including IC and curing $90 \mathrm{~d}$. (Error bars represent the standard deviation from the average of three samples.) 


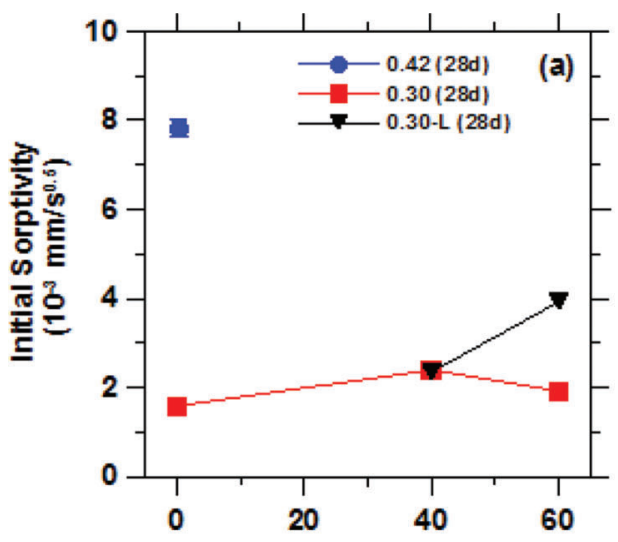

Fly Ash Volume Fraction (\%)

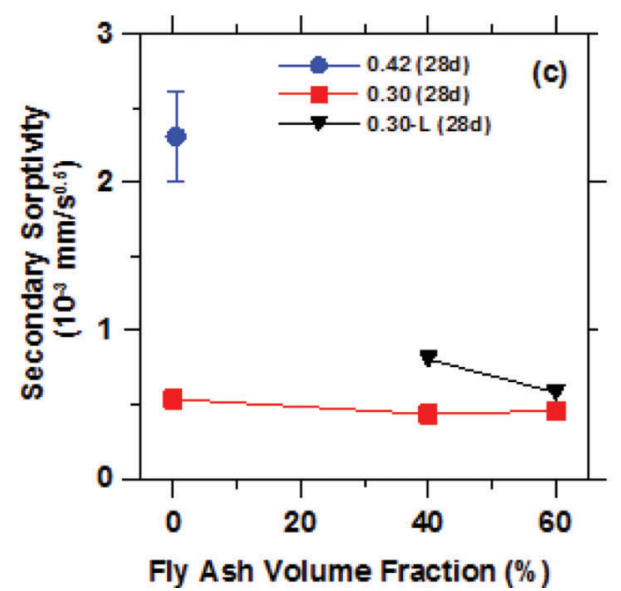

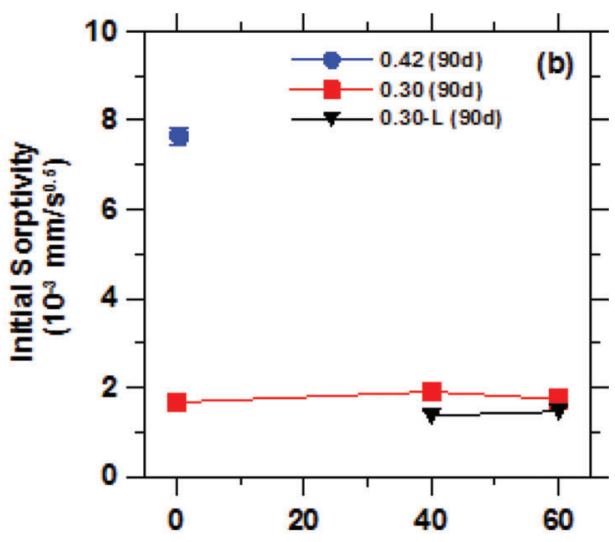

Fly Ash Volume Fraction (\%)

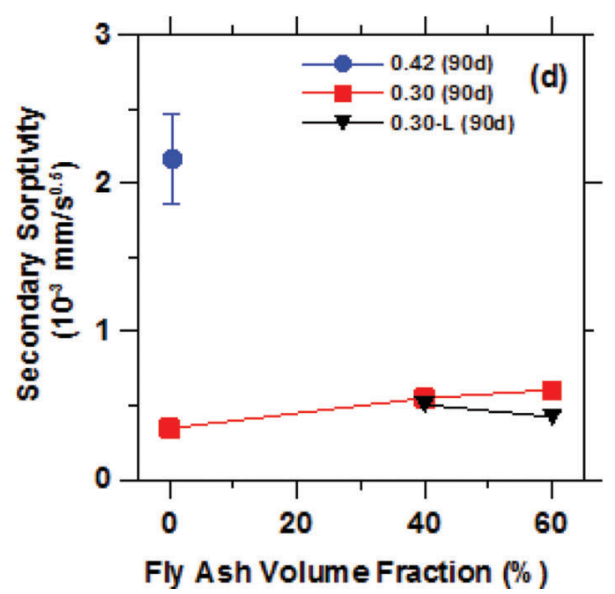

Figure 4.4 (a) and (b) Initial sorptivity of the sealed-cured mortars samples; (c) and (d) Secondary sorptivity of the sealed-cured mortars samples.

to those obtained for the initial sorptivity. Internally cured HVFA mixtures absorb a much lower amount of water and at a slower rate than a mortar with a $w / c$ of 0.42 .

The water absorption results must be carefully interpreted when LWA are included in the mixtures, as they can act as empty water reservoirs. This might be more relevant at early ages, especially when samples have been exposed to a drying environment (i.e., $65 \pm 1 \%$ $\mathrm{RH}$ ), as indicated in the conditioning method used for the water absorption test (Castro et al., 2011). At 28 d, it may be possible that the water contained in the LWA particles in the high volume fly ash mixtures is not completely consumed by the cement reaction; thereby, the degree of hydration is not high enough to achieve capillary pore depercolation within the cement paste matrix. As such, when samples are dried, water in LWA may evaporate, but leaving behind a path for water to re-ingress during a subsequent absorption test. At later ages (e.g., $90 \mathrm{~d}$ ), a higher degree of hydration is achieved, densifying the cement paste matrix and, therefore, isolating the LWA particles within the cement paste matrix. In this case, the LWA particles will not be easily accessible for water to re-ingress during the absorption test, even after the sample has been dried.

\section{SUMMARY AND CONCLUSIONS}

This paper is the second paper in a series investigating the performance of HVFA mixtures with low $w / c$ that are internally cured. The low $w / \mathrm{cm}$ HVFA mixtures with and without IC have been compared to a typical $w / c$ (i.e., 0.42) used in concrete transportation structures (e.g., bridge deck). The study has demonstrated the benefits of using IC in low $w / \mathrm{cm}$ HVFA mixtures in reducing fluid transport by performing various transport tests on mortar mixtures. The following conclusions can be drawn:

- Chloride diffusion is considerably reduced in low $w / \mathrm{cm}$ HVFA mixtures (with or without IC) compared to a control $w / c=0.42$ mixture. However, the chloride penetration within the first $3 \mathrm{~mm}$ to $4 \mathrm{~mm}$ from the top surface of a specimen cut from a cast cylinder is larger in the IC HVFA mortars due to the surface exposure of porous LWA. This effect has been reported elsewhere (Weiss et al., 2013) and must be considered when evaluating transport properties in internally cured mixtures. 
- Electrical measurements (i.e., surface resistivity) indicate that the low $w / \mathrm{cm}$ HVFA mixtures have similar resistivity values as the control $0.42-0 \%$ mortar at $91 \mathrm{~d}$, but improve over time. After 1 year, both HVFA mixtures reach resistivity values up to 3 times larger than those obtained by the $0.42-0 \%$ mortar. This is attributed to denser microstructure achieved in the HVFA specimens (both IC and non-IC).

- The amount and rate of water absorbed in HVFA samples with and without IC is considerably reduced compared to a control $w / c=0.42$ mortar.

In conclusion, while the transport properties of low $w / \mathrm{cm}$ HVFA mixtures (with or without IC) are considerably reduced compared to a control $w / c=0.42$ mixture, IC does not really provide a further reduction of the fluid and ion transport in concrete with similar cementitious content. However, IC provides benefits in increased strength and reduced cracking as indicated in a previous study (De la Varga et al., 2012), without detrimentally impacting transport properties, which are likely controlled first and foremost by the high level of replacement of cement with fly ash and the pore refinement/increased alkali sorption that such replacement provides. Nevertheless, and from a practical perspective, if low $w / \mathrm{cm}$ HVFA with IC mixtures substitute a typical $w / c=0.42$ bridge deck concrete, the reduction in fluid transport would be considerable, thus improving the durability of the material.

\section{REFERENCES}

ACI Committee 232. (2003). Use of fly ash in concrete (ACI 232.2R-03). Farmington Hills, MI: American Concrete Institute.

ASTM C1760-12. (2012). Standard test method for bulk electrical conductivity of hardened concrete. West Conshohocken, PA: ASTM International.

Barneyback, R. S., \& Diamond, S. (1981). Expression analysis of pore fluids from hardened cement pastes and mortars. Cement and Concrete Research, 11(2), 279-285. http://dx. doi.org/10.1016/0008-8846(81)90069-7

Bentz, D. P. (2009). Influence of internal curing using lightweight aggregates on interfacial transition zone percolation and chloride ingress in mortars. Cement and Concrete Composites, 31(5), 285-289. http://dx.doi.org/10.1016/j.cemconcomp.2009.03.001

Bentz, D. P., \& Stutzman, P. E. (2008). Internal curing and microstructure of high performance mortars. In D. P. Bentz \& B. J. Mohr (Eds.), ACI SP-256: Internal curing of high performance concretes-Laboratory and field experiences (pp. 81-90). Farmington Hills, MI: American Concrete Institute.

Bentz, D. P., \& Weiss, W. J. (2011). Internal curing: A 2010 state-of-the-art review. Washington, DC: U.S. Department of Commerce, National Institute of Standards and Technology. Retrieved from http://ws680.nist.gov/publication/ get_pdf.cfm?pub_id $=907729$

Castro, J. (2011). Moisture transport in cement based materials: Applications to transport test and internal curing (Doctoral dissertation). West Lafayette, IN: Purdue University.

Castro, J., Bentz, D., \& Weiss, J. (2011). Effect of sample conditioning on the water absorption of concrete. Cement and Concrete Composites, 33(8), 805-813. http://dx.doi.org/ 10.1016/j.cemconcomp.2011.05.007

Castro, J., Keiser, L., Golias, M., \& Weiss, J. (2011). Absorption and desorption properties of fine lightweight aggregate for application to internally cured concrete mixtures. Cement and Concrete Composites, 33(10), 1001-1008. http:// dx.doi.org/10.1016/j.cemconcomp.2011.07.006

De la Varga, I., Castro, J., Bentz, D., \& Weiss, J. (2012). Application of internal curing for mixtures containing high volumes of fly ash. Cement and Concrete Composites, 34(9), 1001-1008. http://dx.doi.org/10.1016/j.cemconcomp.2012. 06.00

Delagrave, A., Bigas, J. P., Ollivier, J. P., Marchand, J., \& Pigeon, M. (1997). Influence of the interfacial zone on the chloride diffusivity of mortars. Advanced Cement Based Materials, 5(3-4), 86-92. http://dx.doi.org/10.1016/S10657355(96)00008-9

EuroLightCon. (1998). LWAC material properties state-ofthe-art. In Economic design and construction with light weight aggregate concrete. Brite-EuRam III, 47-8.

Golias, M., Castro J., \& Weiss J. (2012). The influence of the initial moisture content in lightweight aggregate on internal curing. Construction and Building Materials, 35, 52-62. http://dx.doi.org/10.1016/j.conbuildmat.2012.02.074

Golias, M., Weiss, J., \& Bentz, D. (2013). Influence of exposure conditions on the efficiency of internal curing in concrete. Advances in Civil Engineering Materials, 2(1), 522533. https://doi.org/10.1520/ACEM20120023

Hooton, D., Nagi, M. A., \& Ozyildirim, H. C. (2000). The rapid chloride permeability test. HPC Bridge Views, 12, 2-4.

Julio-Betancourt, G. A., \& Hooton, R. D. (2004). Study of the Joule effect on rapid chloride permeability values and evaluation of related electrical properties of concretes. Cement and Concrete Research, 34(6), 1007-1015. http://dx. doi.org/10.1016/j.cemconres.2003.11.012

Kantro, D. L. (1980). Influence of water-reducing admixtures on properties of cement paste-a miniature slump test Cement, Concrete and Aggregates, 2(2), 95-102. https://doi. org/10.1520/CCA10190J.

Luping, T. (1997). Chloride diffusion coefficient of concrete and relevant test methods: the state-of-the art and suggestions for future work (NORDTEST Project No. 1351-97). Stockholm, Sweden: RISE Research Institutes of Sweden.

Malhotra, V. M. (1990). Durability of concrete incorporating high-volume of low calcium (ASTM Class F) fly ash. Cement and Concrete Composites, 12(4), 271-277. http://dx. doi.org/10.1016/0958-9465(90)90006-J

Malhotra, V. M., \& Mehta, P. K. (2002). High-performance, high-volume fly ash concrete. Ottawa, Canada: Supplementary Cementing Materials for Sustainable Development, Inc.

Morris, W., Moreno, E. I., \& Sagüés, A. A. (1996). Practical evaluation of resistivity of concrete in test cylinders using a Wenner array probe. Cement and Concrete Research, 26(12), 1779-1787. http://dx.doi.org/10.1016/S0008-8846(96)00175-5

NIST. (n.d.). Estimation of pore solution conductivity. Retrieved April 2013, from http://concrete.nist.gov/poresolncalc. html

Nordtest. (1999). Concrete, mortar and cement-based repair materials: Chloride migration coefficient from non-steadystate migration experiments (NT Build 492). Espoo, Finland: Nordtest. Retrieved from http://210.42.35.80/ G2S/eWebEditor/uploadfile/20110819235419966.pdf

NYSDOT. (2008). Moisture content of lightweight fine aggregate (Test Method No. NY 703-19 E). Albany, NY: New York State Department of Transportation, Materials Bureau. 
Obla, K., Lobo, C., \& Kim, H. (2012). Greatly increased use of fly ash in hydraulic cement concrete (HCC) for pavement layers and transportation structures: Volume I. Silver Spring, MD: NRMCA Engineering Division. Retrieved from https://www.nrmca.org/research_engineering/Documents/ Lab_fly_ashFinal_report.pdf

Pease, B., Hossain, A. B., \& Weiss, J. (2004). Quantifying volume change, stress development, and cracking due to early-age autogenous shrinkage. ACI Special Publication, 220, 23-39. http://dx.doi.org/10.14359/13147

Peled, A., Castro, J., \& Weiss, J. (2010). Atomic force microscopy examinations of mortar made by using water-filled lightweight aggregate. Transportation Research Record, 2141, 92-101. http://dx.doi.org/10.3141/2141-16

Penko, M. (1983). Some early hydration processes in cement paste as monitored by liquid phase composition measurements (Doctoral dissertation). West Lafayette, IN: Purdue University. Retrieved from http://docs.lib.purdue.edu/dissertations/AAI8324046/

Philleo, R. E. (1991). Concrete science and reality. In Materials science of concrete II (pp. 1-8). Westerville, OH: American Ceramic Society.
Riding, K. A., Poole, J. L., Schindler, A. K., Juenger, M. C. G., \& Folliard, K. J. (2008). Simplified concrete resistivity and rapid chloride permeability test method. ACI Materials Journal, 105, 390-394. http://dx.doi.org/10.14359/19901

Snyder, K. A. (2001). The relationship between the formation factor and the diffusion coefficient of porous materials saturated with concentrated electrolytes: Theoretical and experimental considerations. Concrete Science and Engineering, 3(12), 216-224.

Snyder, K. A., Ferraris, C., Martys, N. S., \& Garboczi, E. J. (2000). Using impedance spectroscopy to assess the viability of the rapid chloride test for determining concrete conductivity. Journal of Research-National Institute of Standards and Technology, 105(4), 497-510.

Spragg, R., Villani, C., Snyder, K., Bentz, D., Bullard, J. W., \& Weiss, J. (2013). Factors that influence electrical resistivity measurements in cementitious systems. Transportation Research Record, 2342, 90-98. http://dx.doi.org/10.3141/2342-11

Weiss, J., Bullard, J., Snyder, K., Bentz, D., \& Castro, J. (2013). Electrical properties of concrete with conductive aggregate: Implications for internal curing. Unpublished manuscript, Lyles School of Civil Engineering, Purdue University. 
CHAPTER 7. PHASE II, TASKS 4 AND 5: ADDITION OF A TERNARY COMPONENT TO HIGH VOLUME FLY ASH MIXTURES TO REGULATE SETTING

\section{Dale P. Bentz, Taijiro Sato, Igor De la Varga,} W. Jason Weiss

\section{INTRODUCTION}

Isothermal and semi-adiabatic calorimetry results obtained in this study, along with direct measurements of setting times, have consistently indicated a significant retardation produced by the incorporation of high contents of the (high calcium) Class $\mathrm{C}$ fly ash into the blended cement. This is exemplified by the isothermal calorimetry curves shown in Figure 1.1 for $w / \mathrm{cm}=0.36$ pastes prepared with and without a $40 \%$ volumetric replacement of cement by the Class $\mathrm{C}$ fly ash (both pastes prepared without any HRWRA). In this case, a retardation on the order of $3 \mathrm{~h}$ is observed. For $w / \mathrm{cm}=$ 0.3 pastes prepared with a HRWRA, the retardation is increased to about $4 \mathrm{~h}$. Previous studies (Bentz, 2010b; Bentz \& Ferraris, 2010) using this same Class C fly ash with a different cement have indicated that this retardation can be mitigated by the addition of either calcium hydroxide or a rapid set cement, after first adding $2 \%$ gypsum to that system to restore normal hydration peaks (see Figure 1.2). The fact that the behavior of cement/fly ash blends depends on both components is exemplified by the results in Figure 1.3, where a $2 \%$ gypsum addition to the current Type I/II cement/C fly ash blended paste actually significantly increased the retardation. In Figure 1.3, it can also be observed that for a paste prepared with just the Class $\mathrm{C}$ fly ash, there was a significant heat release within the first hour, with the fly ash paste exhibiting early stiffening (initial set) and a noticeable increase of temperature within its storage container.
Recognizing that a gypsum addition is not beneficial for this particular cement/fly ash combination, isothermal calorimetry studies were next conducted to examine the influence of the addition of either a rapid set cement or an additional source of aluminate, via a calcium aluminate cement or an aluminum hydrate, on this retardation. While calcium hydroxide additions have also been observed to eliminate this retardation for a different (Type II/V) cement blended with this same Class C fly ash (Bentz, 2010b), they were not investigated in the current experiments due to their tendency to reduce compressive strengths (Bentz, Ferraris, De la Varga, Peltz, \& Winpigler, 2010). The isothermal calorimetry results in Figure 1.4 indicate that the rapid set cement, particularly at a $10 \%$ addition level, can provide sufficient early age reactions to offset the retardation of the portland cement by the Class $\mathrm{C}$ fly ash. In this case, the rapid set cement does not reduce the retardation produced by the fly ash, but instead provides the development of mechanical properties (setting) via its own hydration reactions that are not substantially retarded by the fly ash. These heat release measurements have been corroborated with rheological and conventional Vicat needle measurements of setting in a previous study (Bentz \& Ferraris, 2010).

As shown in Figure 1.5, the rapid set cement additions (Figure 1.4) were more effective than providing an additional source of aluminate. Based on the curves in Figure 1.5 (a), the aluminum hydrate did not contribute to the hydration reactions, serving mainly as an inert filler. Conversely, the paste prepared with an addition of $10 \%$ of the calcium aluminate cement exhibited a particularly abnormal hydration behavior with enhanced reactions within the first few hours followed by a substantially reduced reaction rate at later ages. At this point, the rapid set cement appears as the most viable and reliable powder addition option for mitigating excessive retardation in the systems

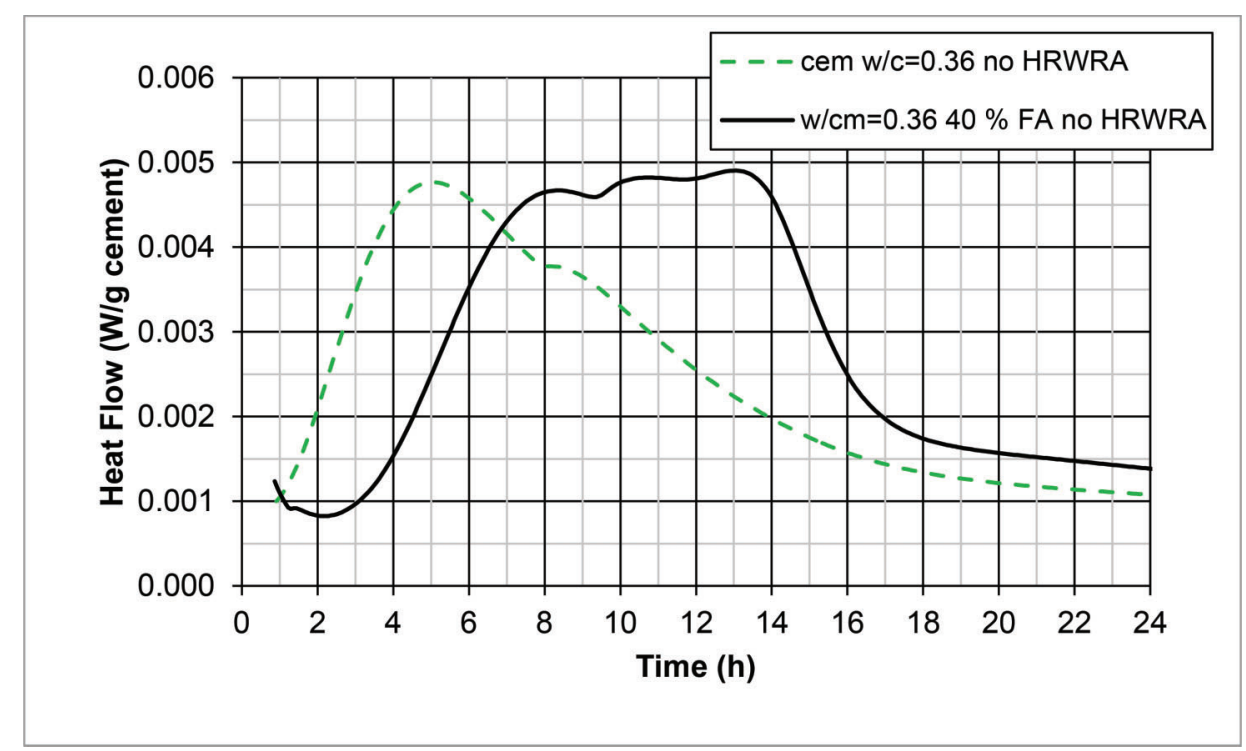

Figure 1.1 Isothermal calorimetry for $w / c m=0.36$ pastes, indicating about $3 \mathrm{~h}$ of retardation due to the Class $\mathrm{C}$ fly ash. 


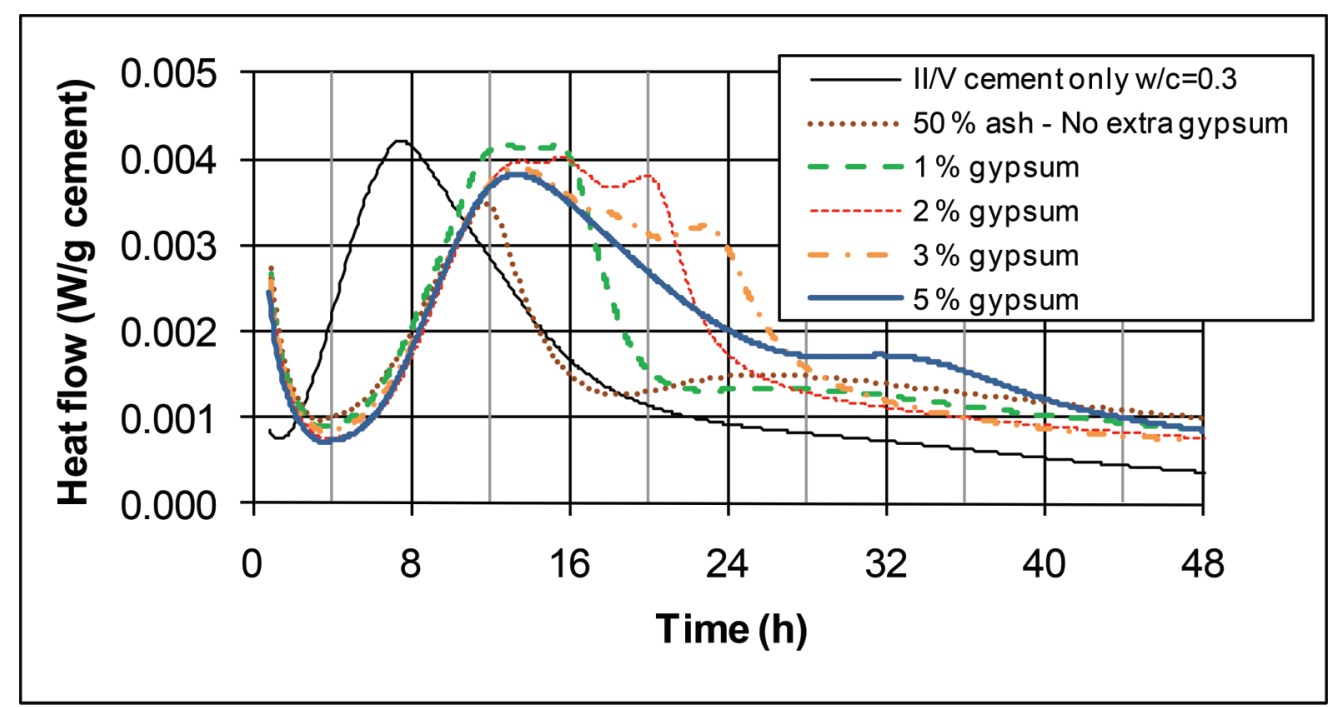

Figure 1.2 Isothermal calorimetry for $w / \mathrm{cm}=0.3$ pastes prepared with various additions of gypsum to a $50 \% \mathrm{Class} \mathrm{C}$ fly ash blended cement (Bentz, 2010b).

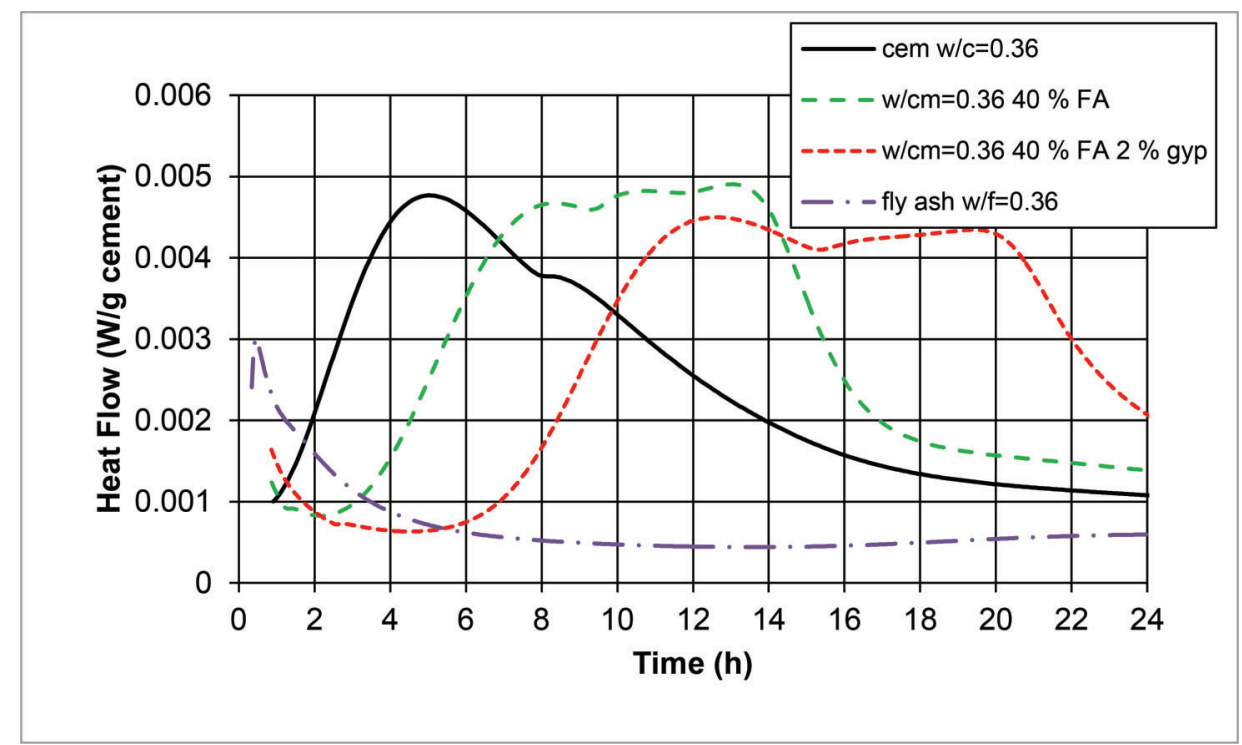

Figure 1.3 Isothermal calorimetry curves for $w / \mathrm{cm}=0.36$ pastes prepared with the Type I/II cement and the high calcium Class $\mathrm{C}$ fly ash.

containing high contents of the Class $\mathrm{C}$ fly ash. As illustrated by the concrete mixtures presented elsewhere in this report, switching to a finer Type III cement or using chemical admixtures can also offer some reduction in the extended setting times of these HVFA blends, when the admixtures are used at fairly high dosages.

These initial efforts with the rapid set cement were supplemented by evaluation of the use of (fine) lime- stone fillers to reduce the extended setting times of the HVFA mixtures, based on some recent results of Canadian and European research groups (De Weerdt, Kjellsen, Sellevold, \& Justnes, 2011; Sato \& Beaudoin, 2011). This part of the research program has been summarized in a manuscript that has been published in Cement and Concrete Composites (Bentz, Sato, De la Varga, \& Weiss, 2012). The following summary has been extracted from that manuscript. 


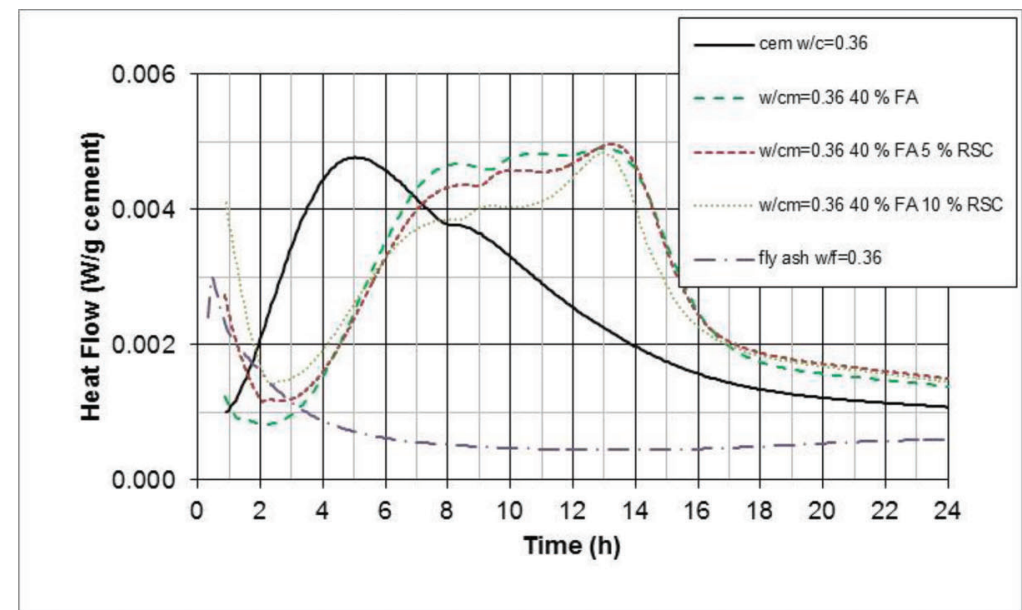

(a)

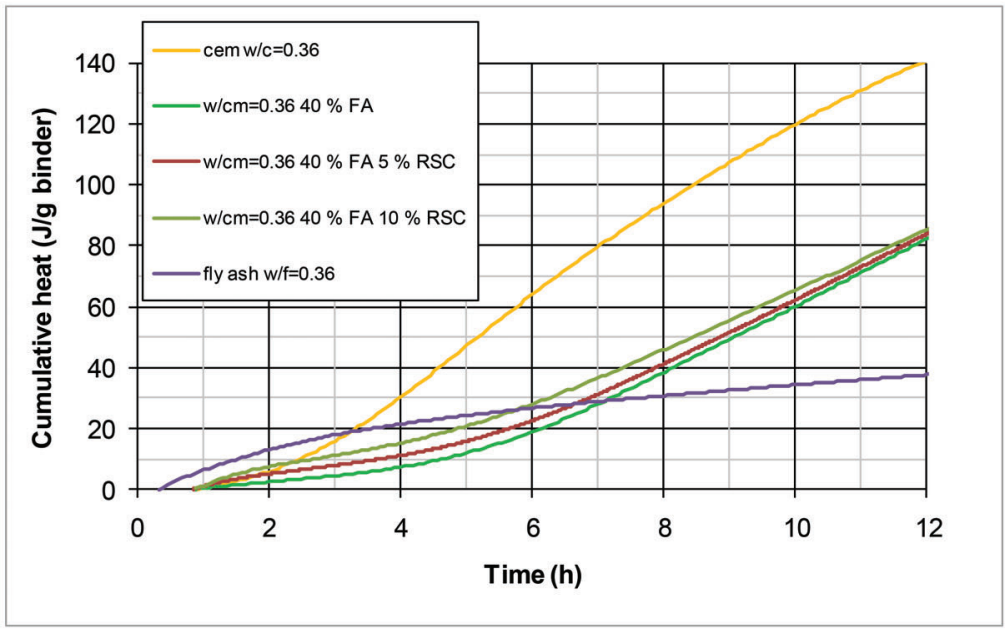

(b)

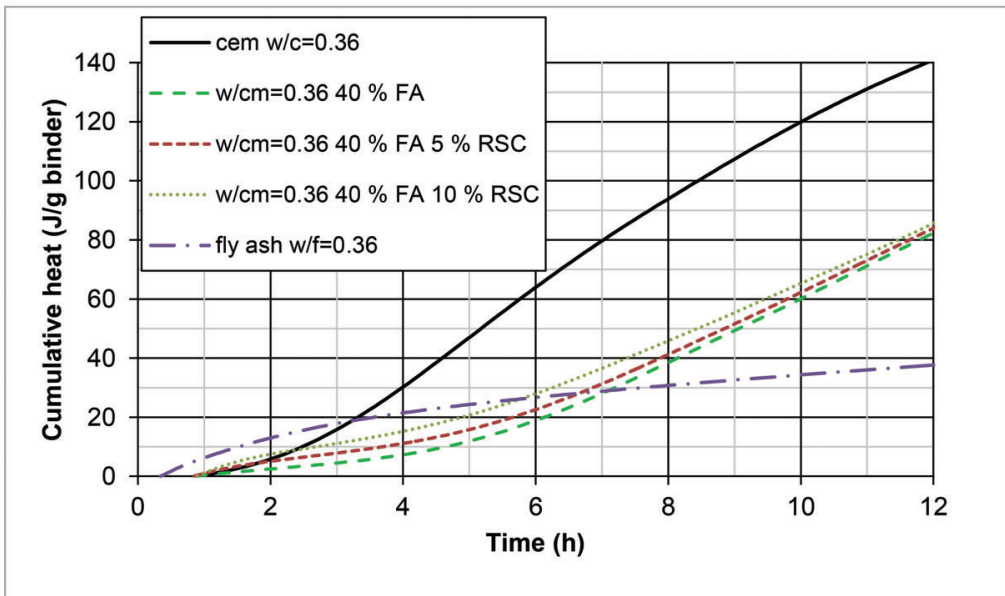

(c)

Figure 1.4 Isothermal calorimetry heat flow (a) and cumulative heat release (b and c) curves for $w / \mathrm{cm}=0.36 \mathrm{blended}$ cement pastes prepared with two levels of addition of a rapid set cement. RSC $=$ rapid set cement. 


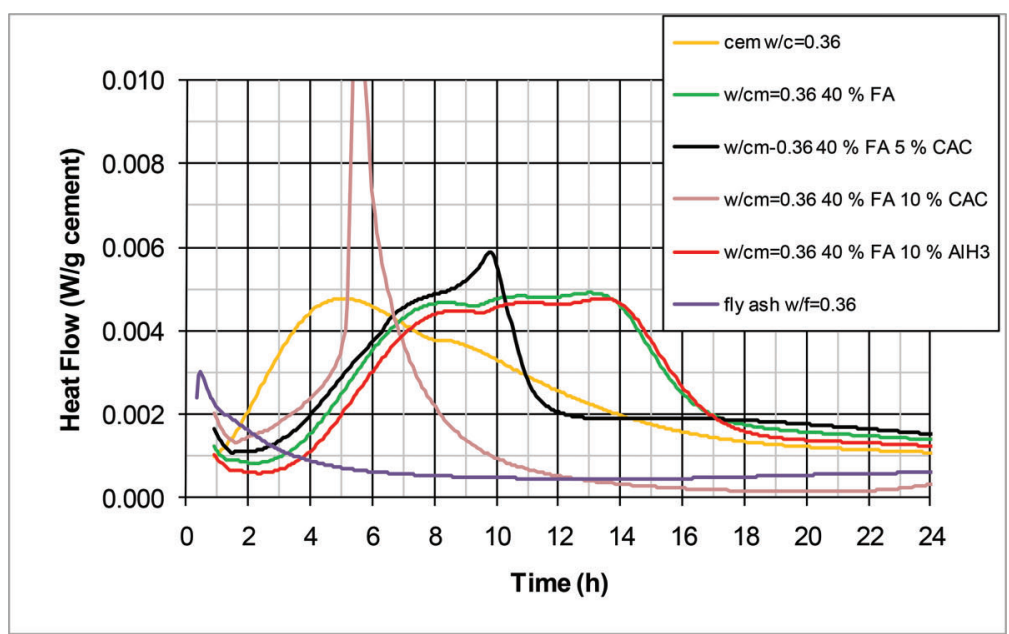

(a)

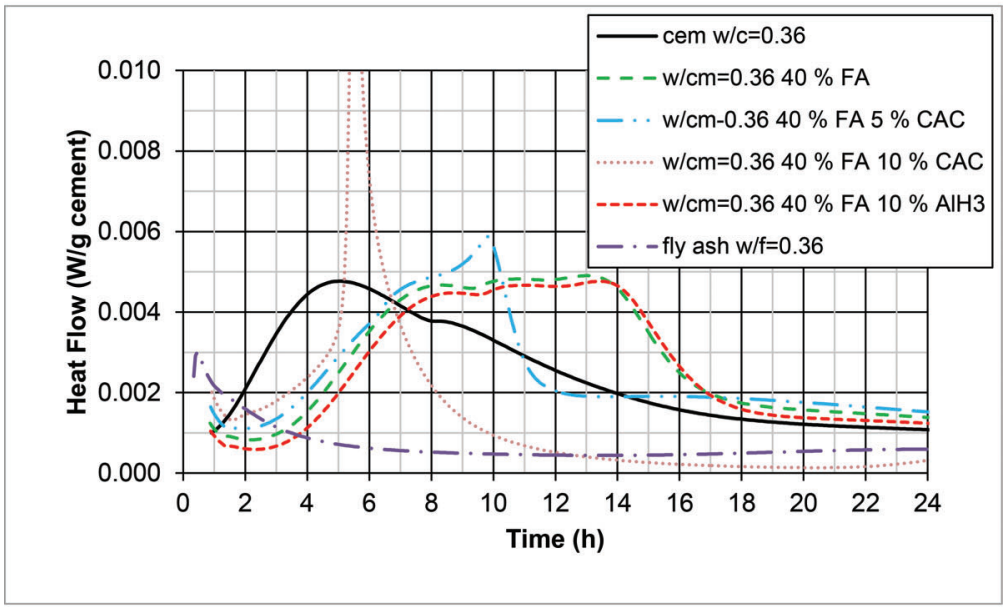

(b)

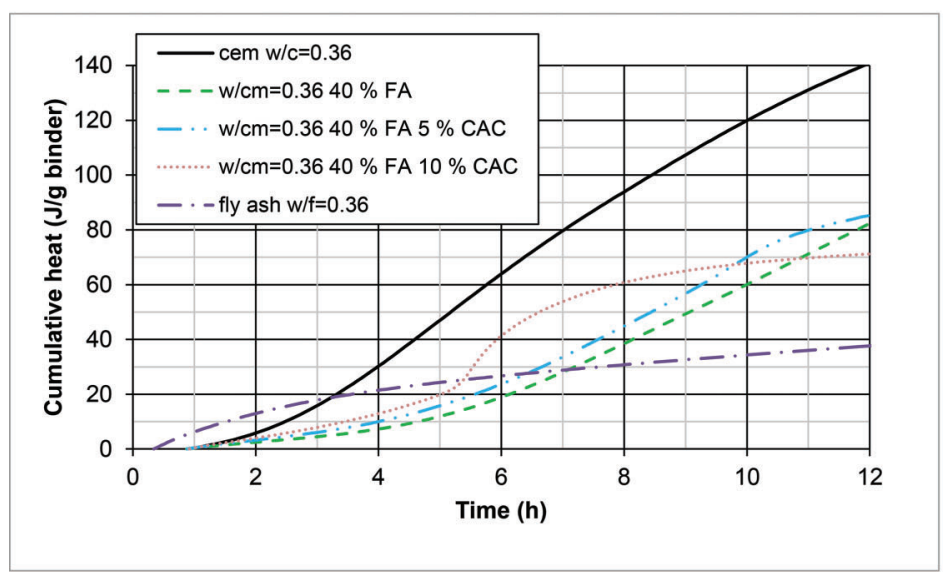

(c)

Figure 1.5 Isothermal calorimetry heat flow (a) and cumulative heat release (b and c) curves for $w / \mathrm{cm}=0.36 \mathrm{blended}$ cement pastes prepared with various additions of aluminate. $\mathrm{CAC}=$ calcium aluminate cement. 


\section{MATERIALS AND EXPERIMENTAL METHODS}

An ordinary portland cement (ASTM C150 Type I/II) was used in this study, with a Blaine fineness of $476 \mathrm{~m}^{2} / \mathrm{kg}$, a specific gravity of 3.18 , an estimated Bogue potential phase composition of $52 \% \mathrm{C}_{3} \mathrm{~S}, 18 \%$ $\mathrm{C}_{2} \mathrm{~S}, 8 \% \mathrm{C}_{3} \mathrm{~A}$, and $9 \% \mathrm{C}_{4} \mathrm{AF}$ by mass, and a $\mathrm{Na}_{2} \mathrm{O}$ equivalent of 0.5 . A Class $\mathrm{C}$ fly ash (ASTM C618-08a) was employed, with a specific gravity of 2.65. Its detailed chemical composition is provided elsewhere (De la Varga, Castro, Bentz, \& Weiss, 2012), but it contained major oxides of $38.7 \% \mathrm{SiO}_{2}, 19.2 \% \mathrm{Al}_{2} \mathrm{O}_{3}, 6.5 \% \mathrm{Fe}_{2} \mathrm{O}_{3}$, and $23.5 \% \mathrm{CaO}$ by mass. The particle size distributions (PSDs) of the cement and fly ash are provided in Figure 2.1. Three limestone powders, one a nano-limestone (designated by $\mathrm{n}$ ) and the other two having median particle diameters of $4.4 \mu \mathrm{m}$ (c) and $16.4 \mu \mathrm{m}$ (o), respectively, were obtained from their manufacturers. The specific gravity of each limestone powder is 2.7 . In addition, a finely ground silica with a specific gravity of 2.65 and a median particle diameter of $2.7 \mu \mathrm{m}$ (designated by s) was also investigated as a fine particle activator. The measured PSDs of these four materials, as determined by laser diffraction, are provided in Figure 2.2. It is recognized that the measurement of the PSD of the nano-limestone powder in this manner is severely limited by the agglomeration of the nano-sized particles and that the actual particles are likely much smaller than the sizes indicated by the curve in Figure 2.2. Previously, microscopy techniques have been employed to determine that the "true" particle sizes of this nanolimestone are in the range of $50 \mathrm{~nm}$ to $120 \mathrm{~nm}$ (Sato \& Beaudoin, 2011). Following a dispersion technique developed by Sato, Daillo, and Trischuk (2011), laser diffraction has yielded a somewhat finer PSD than shown in Figure 2.2, with a median particle diameter of $180 \mathrm{~nm}$. With the exception of the coarsest of the limestone powders, the fillers are generally significantly finer than either the cement or the fly ash.

For the fairest comparison of setting times and compressive strengths, cement pastes (and the paste fraction of the mortars) were prepared with constant volume

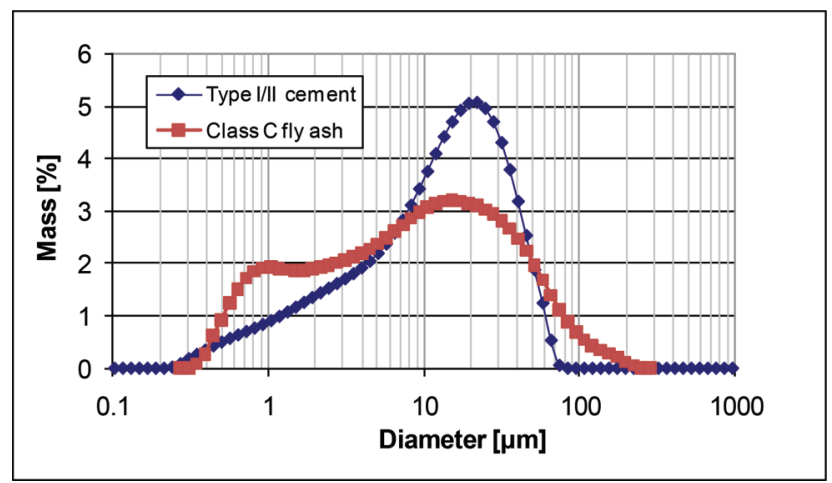

Figure 2.1 Measured particle size distributions for the cement and fly ash investigated in the study. Results are the average of six individual measurements and error bars (one standard deviation) would fall within the size of the symbols.

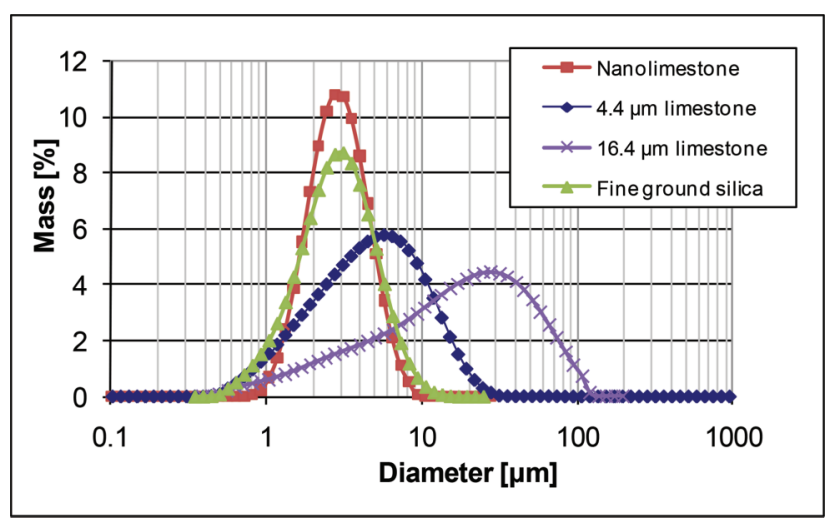

Figure 2.2 Particle size distributions for the limestone and silica powders investigated in the study.

fractions of water and powders, based on a control mixture with a water-to-cement ratio by mass $(w / c)$ of 0.30 . This low $w / c$ (or $w / c m$ for the mixtures with fly ash) was chosen to provide sufficient early age strength in the HVFA mixtures (De la Varga et al., 2012). For comparison purposes, a plain cement paste with $w / c=$ 0.42 by mass was also prepared as being more representative of typical transportation concrete designs (De la Varga et al., 2012). Mixture designs for the nine pastes examined in the present study are provided in Table 2.1. The mixture names indicate the volume percentages of the three components, cement: fly ash: filler, with the letter following the filler content indicating the specific filler employed (n, c, o, or s). A polycarboxylate-based high range water reducing agent (HRWRA) was used at various dosages in the paste mixtures, with the chosen dosage being based on that required in mortars to provide adequate workability for casting cubes. No HRWRA was necessary in the $w / c=0.42,100 \%$ cement mixture. Mixing was performed in a high shear blender following the procedure developed by the Portland Cement Association, currently under consideration as an ASTM standard. After mixing, truncated conical specimens were prepared for evaluation of setting times using the Vicat needle and small glass vials were loaded with paste for measurement of heat release using isothermal calorimetry.

The Vicat needle penetration tests were conducted according to the ASTM C191 standard, but with the following modification to minimize evaporation from the specimen surface during the test. A moist sponge was held in place in the bottom of a polystyrene foam cup using toothpicks, and the inverted cup placed on top of the truncated conical cement paste specimen, in an effort to maintain a near $100 \%$ relative humidity environment surrounding the hardening cement paste. The cup was removed prior to each measurement and returned immediately after recording the needle penetration. All set time measurements were conducted inside a walk-in environmental chamber maintained at $25.0 \pm 1.0^{\circ} \mathrm{C}$. In the ASTM C191 standard, the single laboratory precisions are listed as $12 \mathrm{~min}$ and $20 \mathrm{~min}$ for initial and final times of setting, respectively. 
TABLE 2.1

Mixture Proportions for Cement Pastes

\begin{tabular}{|c|c|c|c|c|c|c|c|c|c|}
\hline Ingredient/Mixture & $100 w 30$ & $100 w 42$ & 60:40:0 & $55: 40: 5 n$ & $55: 40: 5 c$ & $60: 35: 5 c$ & 60:30:10c & 60:30:10o & $60: 35: 5 s$ \\
\hline Cement & $400 \mathrm{~g}$ & $400 \mathrm{~g}$ & $257.1 \mathrm{~g}$ & $237.6 \mathrm{~g}$ & $237.6 \mathrm{~g}$ & $256.9 \mathrm{~g}$ & $256.7 \mathrm{~g}$ & $256.7 \mathrm{~g}$ & $257.1 \mathrm{~g}$ \\
\hline Fly ash & - & - & $142.9 \mathrm{~g}$ & $144 \mathrm{~g}$ & $144 \mathrm{~g}$ & $124.9 \mathrm{~g}$ & $107 \mathrm{~g}$ & $107 \mathrm{~g}$ & $125 \mathrm{~g}$ \\
\hline Nano-limestone & - & - & - & $18.3 \mathrm{~g}$ & - & - & - & - & - \\
\hline $4.4 \mu \mathrm{m}$ limestone & - & - & - & - & $18.3 \mathrm{~g}$ & $18.2 \mathrm{~g}$ & $36.3 \mathrm{~g}$ & - & - \\
\hline $16.4 \mu \mathrm{m}$ limestone & - & - & - & - & - & - & - & $36.3 \mathrm{~g}$ & - \\
\hline $2.7 \mu \mathrm{m}$ silica & - & - & - & - & - & - & - & - & $17.9 \mathrm{~g}$ \\
\hline Water & $120 \mathrm{~g}$ & $168 \mathrm{~g}$ & $128.6 \mathrm{~g}$ & $127.8 \mathrm{~g}$ & $127.8 \mathrm{~g}$ & $128.5 \mathrm{~g}$ & $128.4 \mathrm{~g}$ & $128.4 \mathrm{~g}$ & $128.6 \mathrm{~g}$ \\
\hline HRWRA & $3.2 \mathrm{~g}$ & - & $0.68 \mathrm{~g}$ & $0.22 \mathrm{~g}$ & $0.22 \mathrm{~g}$ & $0.24 \mathrm{~g}$ & $0.34 \mathrm{~g}$ & $0.34 \mathrm{~g}$ & $0.24 \mathrm{~g}$ \\
\hline$w / \mathrm{cm}$ & 0.30 & 0.42 & 0.321 & 0.324 & 0.324 & 0.321 & 0.321 & 0.321 & 0.321 \\
\hline
\end{tabular}

Following the general guidelines provided in the ASTM C1702 standard, isothermal calorimetry was conducted for a period of at least $1 \mathrm{~d}$ using single or replicate paste specimens having a mass of between $5.4 \mathrm{~g}$ and $5.6 \mathrm{~g}$. The prepared paste was first placed in the glass calorimeter specimen vials, the vials were sealed, and the sealed vials were then loaded into the calorimeter along with a reference vial containing only dry cement powder. Using this procedure, the initial "mixing" peak that occurs when water contacts cement was not examined in this study. For this technique, the average absolute difference between replicate specimens of cement paste was previously measured to be $2.4 \times 10^{-5} \mathrm{~W} / \mathrm{g}$ (cement), with a maximum absolute difference of $0.00011 \mathrm{~W} / \mathrm{g}$ (cement), for measurements conducted between $1 \mathrm{~h}$ and $7 \mathrm{~d}$ after mixing.

Data for setting times and heat release of the pastes were supplemented by measurements of isothermal calorimetry (for $7 \mathrm{~d}$ ) and compressive strength ( $1 \mathrm{~d}, 7 \mathrm{~d}$, $28 \mathrm{~d}$, and $56 \mathrm{~d}$ ) on companion mortar mixtures for a subset of the pastes listed in Table 3.2 in Chapter 5, also prepared with a constant volume fraction of water and powders. For preparation of the mortars, a blend of four silica sands (specific gravity of 2.61) was used, with a constant sand volume fraction of 0.55 . Mortars were mixed in a planetary mixer according to ASTM C30506 procedures; cubes were prepared, stored in double sealed plastic bags for $24 \mathrm{~h}$, demolded, and stored in saturated limewater until the age of testing.

\section{RESULTS AND DISCUSSION}

The investigated cement and fly ash were chosen based on previous results that had indicated a substantial retardation of early hydration and setting for an HVFA mixture (De la Varga et al., 2012). These results are confirmed by the setting and heat flow and cumulative heat release isothermal calorimetry curves shown in Figure 3.1, Figure 3.2, and Figure 3.3 respectively. In Figure 3.2, the results are normalized per gram of cement powder, while in Figure 3.3, they are normalized per $\mathrm{ml}$ of (initial) water. The former focuses attention on the reactivity of the cement, assuming the other powders to be basically inert during the first hours of hydration. The latter examines the heat released per unit volume of capillary porosity; assuming that the volume of created hydration products is proportional to the heat generated, it could potentially relate to the space-filling capabilities of the ongoing reactions (e.g., set and strength development). The initial and final setting times of the various paste mixtures are also summarized in Table 3.1. The blended paste with $40 \%$ fly ash by volume has an initial setting time that is delayed by over $2 \mathrm{~h}$ with respect to the $100 \%$ portland cement control, while its final setting time is likewise delayed by more than $2.5 \mathrm{~h}$. Similarly, in Figure 3.2, the isothermal calorimetry curve for the $40 \%$ fly ash mixture is shifted to the right by approximately $2.5 \mathrm{~h}$ relative to the curve for the $100 \%$ cement, $w / c=0.30$ control, along with the shape of the main hydration peak being somewhat altered.

The two $100 \%$ cement pastes exhibit similar setting times. While one would normally expect a lower $w / c$ paste to exhibit faster setting, in this case, the lower $w / c$ is confounded with the addition of the HRWRA. As can be seen in Figure 3.2, the isothermal calorimetry curve for the $w / c=0.30$ cement paste with HRWRA is shifted to later times by about $1.5 \mathrm{~h}$ with respect to that produced by the $w / c=0.42$ cement paste with no HRWRA addition. From Figure 3.2 and Figure 3.3, it is clear that less hydration is required for the setting of the $w / c=0.30$ paste than for the $w / c=0.42$ paste, as would be expected. Thus, the retardation produced by the addition of the HRWRA is offset by the reduction in needed hydration to produce setting for a lower $w / c$, and in this particular case, the setting times of the two different $w / c$ pastes are basically equivalent.

The results for the nano-limestone powder indicate that a $5 \%$ replacement by volume of cement with the nano-limestone restores the setting times to those of the $100 \%$ cement, $w / c=0.30$ control paste. As has been observed previously (Sato \& Beaudoin, 2011), the ability of nano-limestone to accelerate the reactions at early ages is clearly indicated in the isothermal calorimetry curves in Figure 3.6, where the curve with the nano-limestone is shifted more than $3 \mathrm{~h}$ to the left with respect to the $40 \%$ fly ash (no limestone) curve. This acceleration in time is also accompanied by an increase in height of the main hydration peak, particularly of the second hump of the peak corresponding to renewed reaction of the aluminate phases. The net effect of these differences, as illustrated by the cumulative heat release 


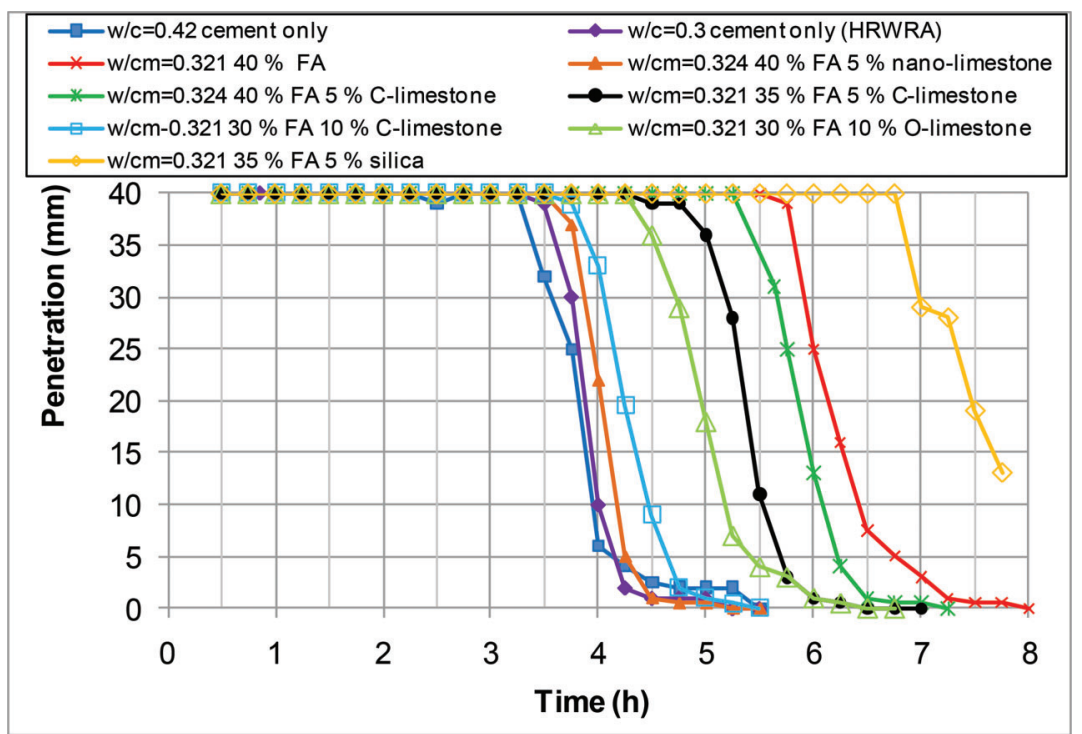

Figure 3.1 Measured Vicat needle penetrations vs. time for the nine cement pastes.

TABLE 3.1

Measured Setting Times and Concurrent Heat Releases for Cement Pastes

\begin{tabular}{|c|c|c|c|c|c|c|c|c|c|}
\hline Property & $100 w 30$ & $100 w 42$ & 60:40:0 & $55: 40: 5 n$ & $55: 40: 5 c$ & $60: 35: 5 c$ & 60:30:10c & $60: 30: 100$ & $60: 35: 5 s$ \\
\hline Initial set time & $3.81 \mathrm{~h}$ & $3.75 \mathrm{~h}$ & $6 \mathrm{~h}$ & $3.95 \mathrm{~h}$ & $5.75 \mathrm{~h}$ & $5.29 \mathrm{~h}$ & $4.15 \mathrm{~h}$ & $4.84 \mathrm{~h}$ & $7.33 \mathrm{~h}$ \\
\hline Final set time & $5.5 \mathrm{~h}$ & $5.5 \mathrm{~h}$ & $8.25 \mathrm{~h}$ & $5.5 \mathrm{~h}$ & $7.5 \mathrm{~h}$ & $7 \mathrm{~h}$ & $5.5 \mathrm{~h}$ & $7 \mathrm{~h}$ & $>7.8 \mathrm{~h}$ \\
\hline $\begin{array}{l}\text { Cumulative heat at initial } \\
\text { set }(\mathrm{J} / \mathrm{ml} \text { water })\end{array}$ & 36.4 & 56.4 & 40.2 & 44.7 & 58.5 & 51.3 & 44.3 & 48.7 & 48.6 \\
\hline $\begin{array}{l}\text { Cumulative heat at final } \\
\text { set }(\mathrm{J} / \mathrm{ml} \text { water })\end{array}$ & 105 & 124 & 85 & 95 & 111 & 106 & 88 & 118.4 & - \\
\hline
\end{tabular}

curves in Figure 3.3, is that at an age of $12 \mathrm{~h}$, the heat release in the $5 \%$ nano-limestone paste exceeds that of the $40 \%$ fly ash blend by more than $50 \%$. As shown in Figure 3.4, this also resulted in a $12 \%$ increase in the $1 \mathrm{~d}$ mortar cube compressive strength, while strengths at later ages were similar in the two systems.

For the particular cement and fly ash combination selected for this study, there is a measurable influence of limestone fineness on early-age performance, as has been observed previously (Sato \& Beaudoin, 2011). The first demonstration of this effect can be found in comparing results for the 55:40:5 blends prepared with either the nano-limestone or the $4.4 \mu \mathrm{m}$ limestone powder, with equivalent HRWRA dosages. In this case, the much finer nano-limestone produces a significant increase in early age hydration rates and a concurrent decrease in setting times of about $2 \mathrm{~h}$. A similar result is found in comparing the performance of the 60:30:10 blends with either the $4.4 \mu \mathrm{m}$ or $16.4 \mu \mathrm{m}$ limestone powders at equivalent HRWRA dosages. Here, the 4.4 $\mu \mathrm{m}$ limestone reduced the setting times back to within the vicinity of the values observed for the control paste, while the setting times exhibited by the paste with the $16.4 \mu \mathrm{m}$ limestone still exceeded those of the control specimens by an hour or more.

There are at least two mechanisms by which the limestone powder can be influencing the early age behavior of these blended cement pastes. First, the limestone powder can be functioning as a fine filler, providing additional surfaces for the nucleation and growth of hydration products (Bentz, 2006; Gutteridge \& Dalziel, 1990). In this case, the greatly increased surface area of the nano-limestone relative to the other two limestones would be expected to accelerate the early age reactions, in agreement with the present results. Second, the limestone powder could potentially provide an additional source of calcium ions to the pore solution, even though $\mathrm{CaCO}_{3}$ has a relatively low solubility at the elevated $\mathrm{pH}$ levels that will quickly be encountered in typical cement paste pore solutions. But, it must be recognized that for these specimens, the initial contact always occurs between the pre-blended powders and a circumneutral distilled water ( $\mathrm{pH}$ near 7 ), in which the solubility of calcium carbonate will be much higher than in a typical $\mathrm{pH} \sim 13$ pore solution.

Some support for this latter mechanism is provided by comparing the results for the 60:35:5 systems prepared with either the $4.4 \mu \mathrm{m}$ limestone or the $2.7 \mu \mathrm{m}$ silica, particularly focusing on the isothermal calorimetry curves in Figure 3.2 and Figure 3.3. For the silica substitution, the dormant period is actually lengthened relative to that observed for the $40 \%$ fly ash blended cement with no fillers. However, once hydration starts in earnest, the system with the 5\% silica exhibits a 


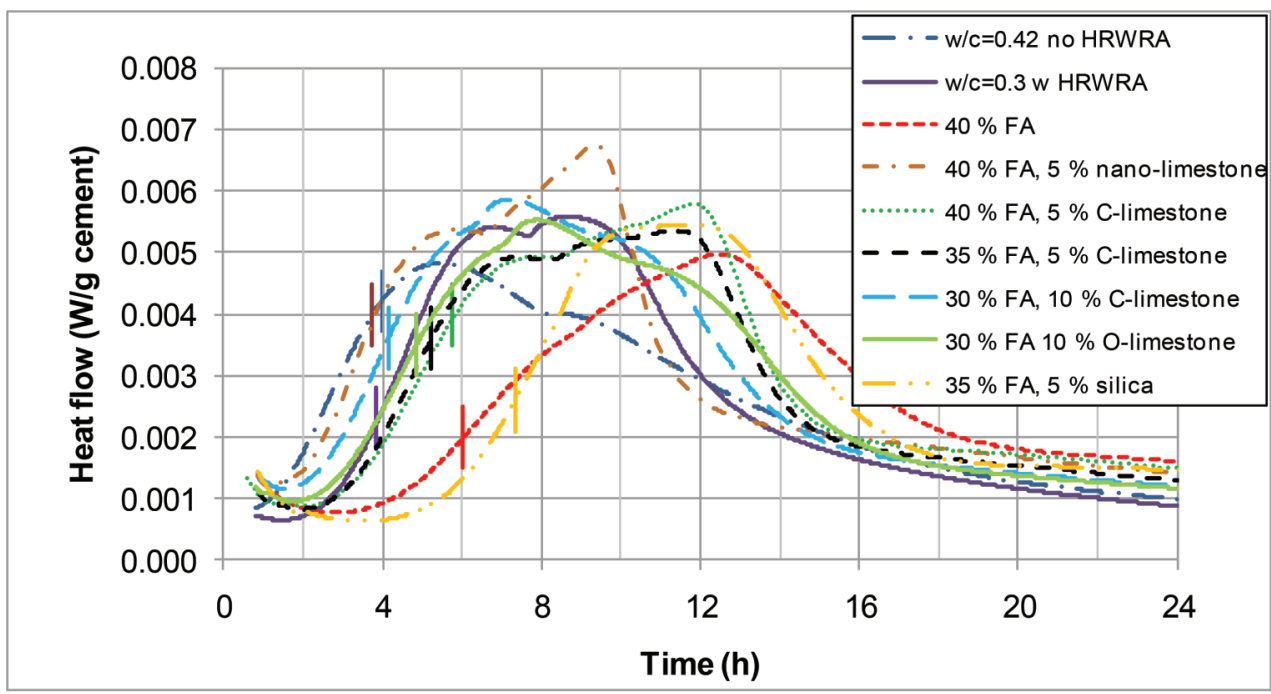

Figure 3.2 Isothermal calorimetry curves vs. time for the nine cement pastes. For each curve, the short vertical line indicates the initial setting time.

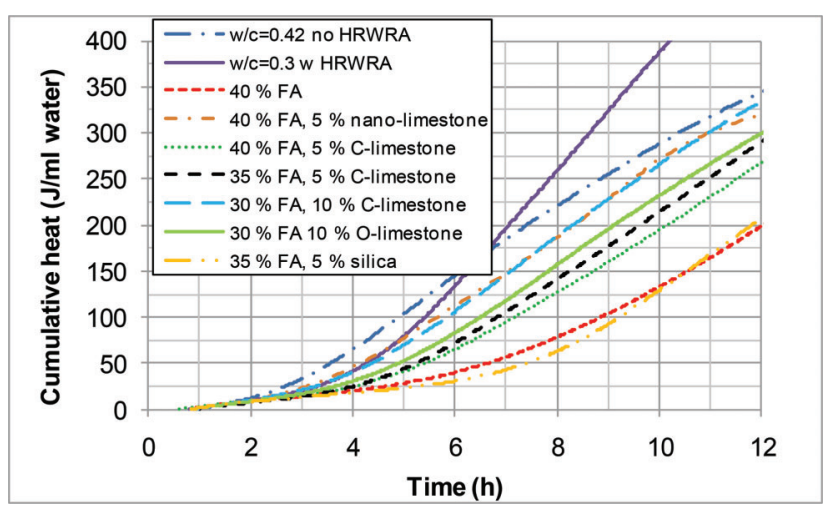

Figure 3.3 Cumulative heat release curves vs. time for the nine cement pastes on a per $\mathrm{ml}$ of water basis.

higher hydration rate. The lengthening of the dormant period suggests that the silica (certainly not supplying calcium ions to solution) may in fact be decreasing available calcium, as perhaps some of the fine silica is amorphous, as opposed to crystalline, and therefore more reactive in a high $\mathrm{pH}$ solution. Conversely, with the limestone powder perhaps supplying calcium ions to the pore solution and certainly providing ample additional surface area for the nucleation and growth of hydration products, the dormant period is decreased and the subsequent hydration is accelerated. Clearly, additional research is needed to isolate and clarify the contributions of these two mechanisms.

These same effects might not be observed at all in a system that is not on the edge of calcium deficiency, as exemplified by the results obtained with limestone and quartz powders (each about $5 \mu \mathrm{m}$ in diameter) in a Class F fly ash blended cement that were recently presented in reference (De Weerdt et al., 2011). As another example of the influence of the silica powder on early age reactions, isothermal calorimetry results for mortars with various volumetric substitutions for cement by a silica powder (slightly coarser than the one employed in the current study) are provided in Figure 3.5 (De la Varga, 2013). In this case, the three mortars with silica substitutions were prepared with the same dosage of HRWRA, while the HRWRA dosage in the $100 \%$ cement mortar was double this value to provide adequate flow (De la Varga et al., 2012). Focusing on the various silica substitution levels, one finds that in going from $40 \%$ to $60 \%$ silica, the early age hydration is accelerated, while in going from $60 \%$ to $80 \%$, an increase in the dormant period is observed. However, once hydration begins in earnest, the hydration rates in the $80 \%$ system are similar to those observed for the other two substitution levels. At this highest substitution level, it appears that the silica powder may be finally consuming a sufficient quantity of calcium ions to delay the onset of the primary hydration peak. This hypothesis must be tempered by the facts that the $w / c$ of the $80 \%$ silica mixture is higher than that in any of the other mixtures (which could also provide some contribution to a dilution of pore solution concentrations) and that the HRWRA to cement ratio is also highest in the $80 \%$ silica mixture.

Mortar cube compressive strengths are provided in Figure 3.4 for a subset of the mixtures, with their corresponding isothermal calorimetry curves being provided in Figure 3.6. In general, the trends observed in the mortar calorimetry curves are the same as those obtained for their equivalent pastes in Figure 3.2. These mortars were prepared to verify that the limestone powder replacements were not having any serious deleterious influences on compressive strengths. In general, the $1 \mathrm{~d}$ compressive strengths of the mixtures with limestone exceed that of the $40 \%$ fly ash blended cement paste, with later age strengths being generally comparable, in agreement with the general observations from a previous blended cement study (Sandberg \& Roberts, 2005). If the goal of an HVFA blend is to match the compressive strength of the w/c $=0.42,100 \%$ cement mortar, 


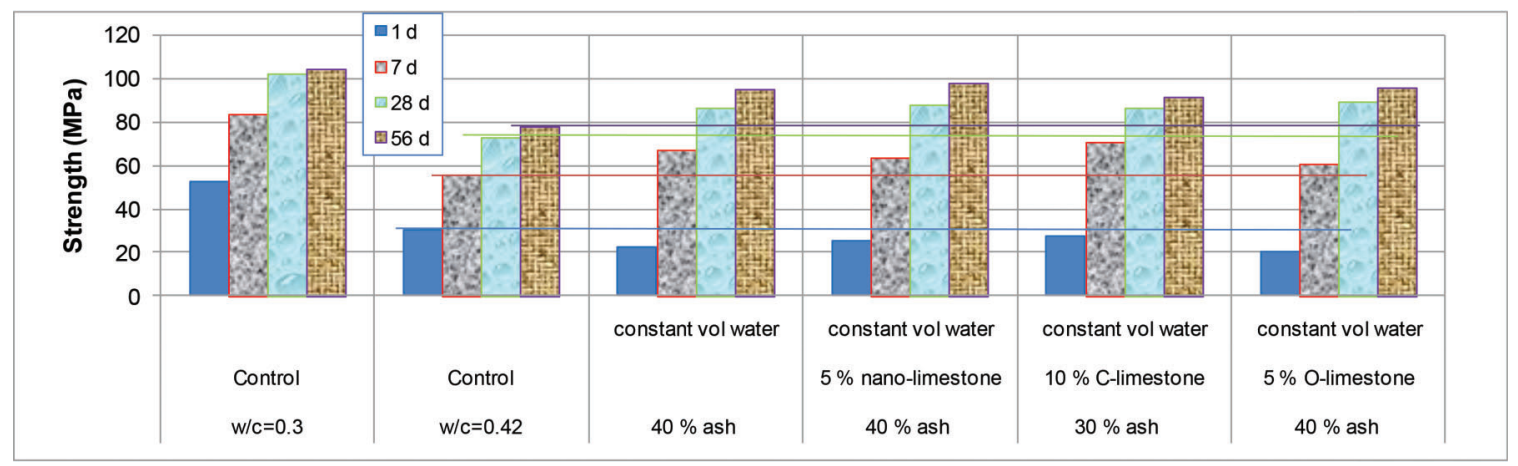

Figure 3.4 Measured compressive strengths for mortar cubes at four ages.

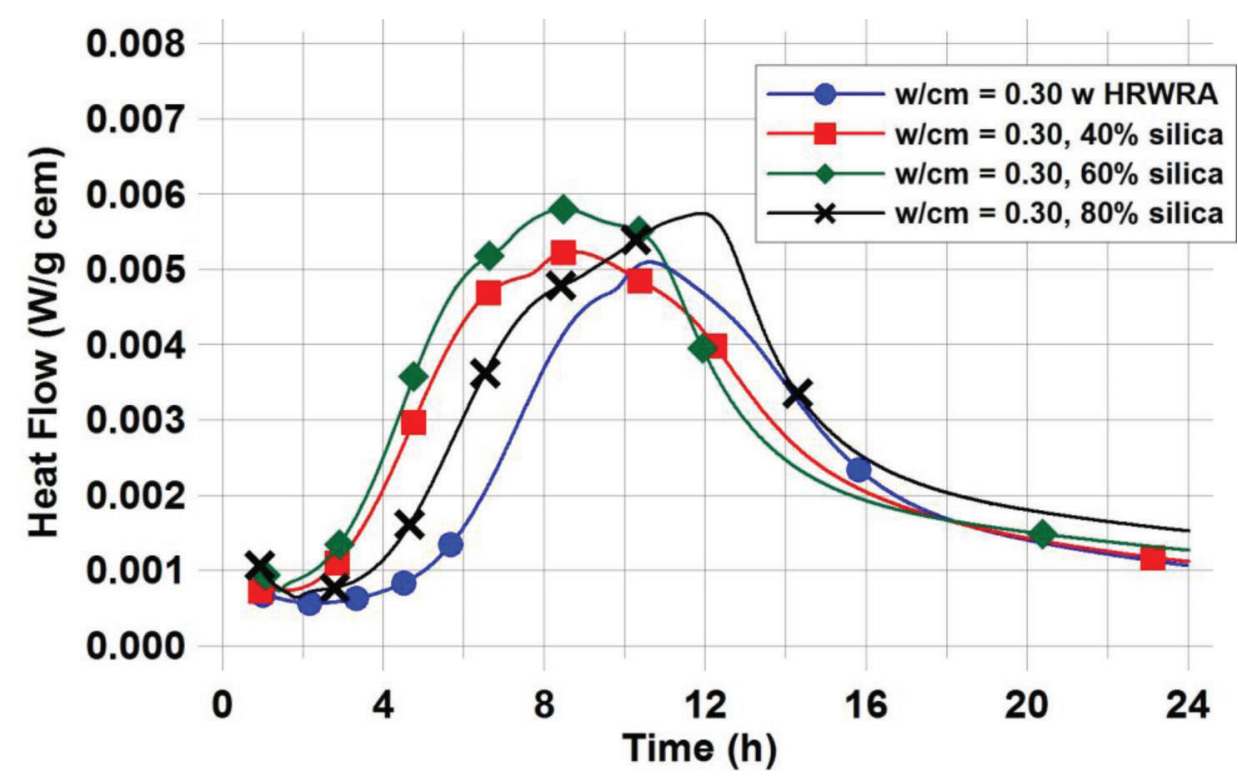

Figure 3.5 Isothermal calorimetry results for mortars with various volumetric substitutions of silica powder for cement (De la Varga, 2013).

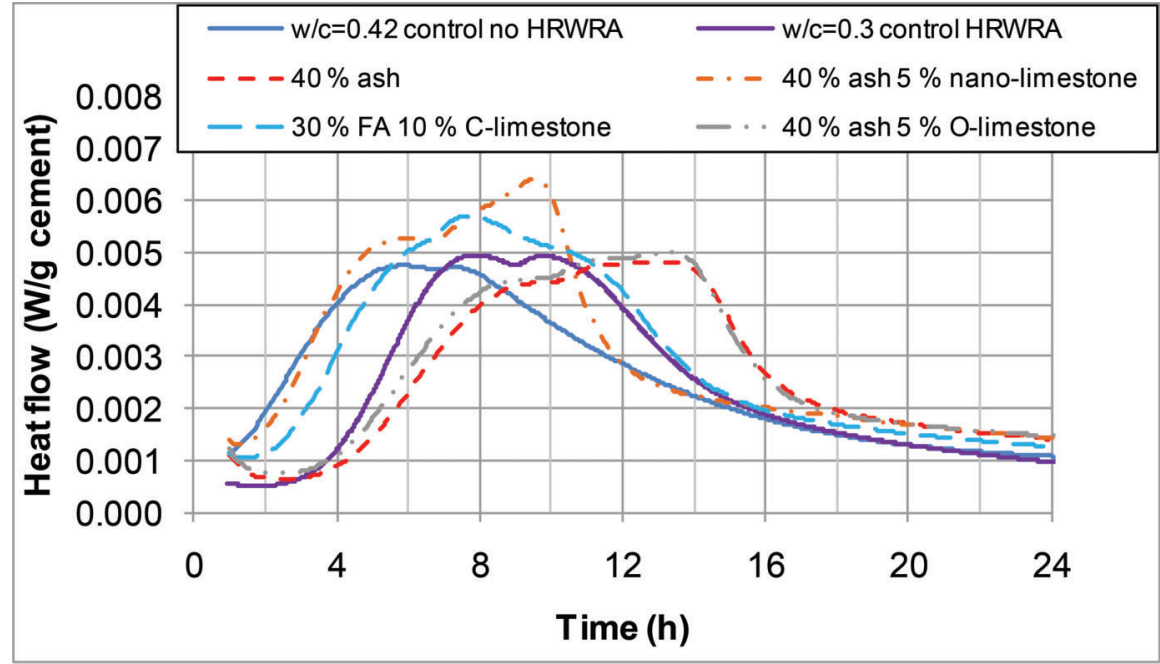

Figure 3.6 Isothermal calorimetry curves vs. time for the six mortar mixtures. 

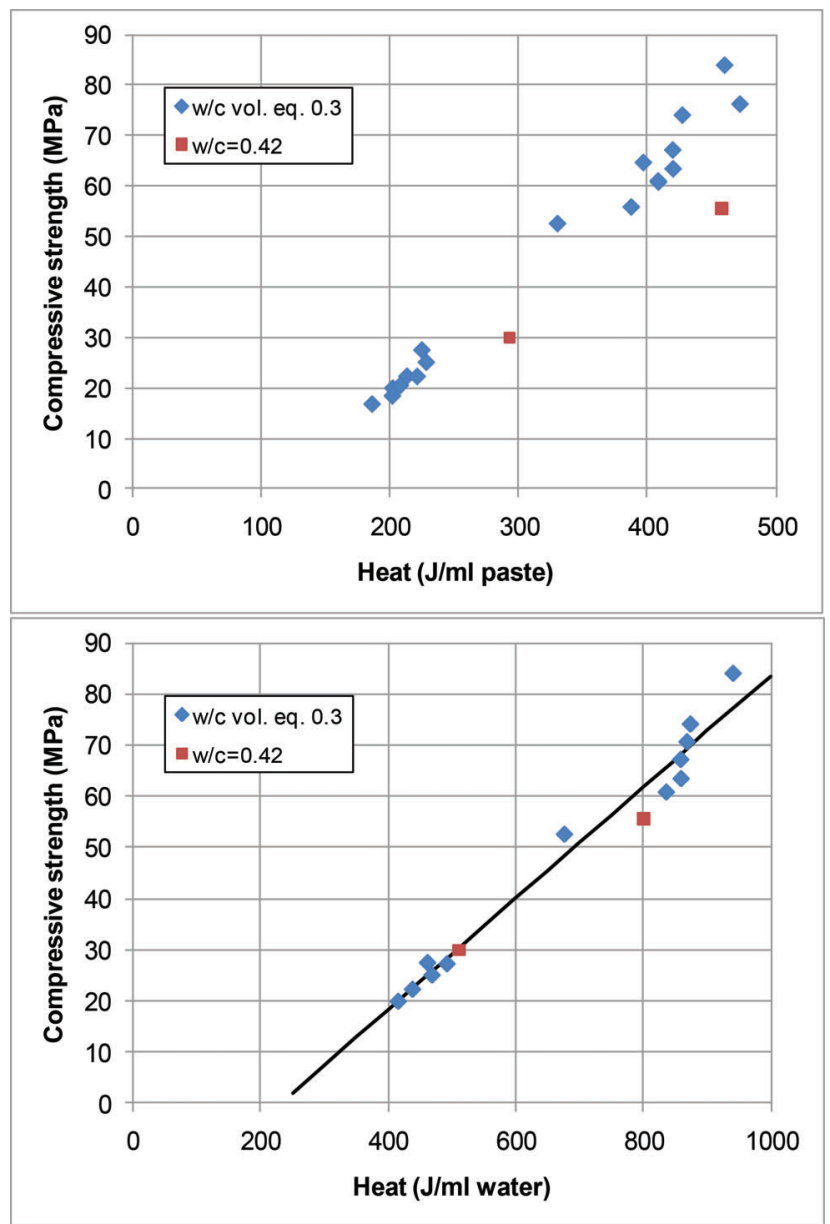

Figure 3.7 Measured compressive strengths vs. measured cumulative heat release on either a per $\mathrm{ml}$ paste basis (top) or a per $\mathrm{ml}$ initial water basis (bottom) for mortars at ages of $1 \mathrm{~d}$ and $7 \mathrm{~d}$.

the various mixtures with fly ash or fly ash and limestone exceed this goal at the ages of $7 d, 28 d$, and $56 d$, but fall somewhat short at an age of $1 \mathrm{~d}$. For the $1 \mathrm{~d}$ results, of the subset of mortars that were evaluated, the 60:30:10c system comes the closest to matching the strength of the $100 \%$ cement control. In this case, benefits are obtained from both the 10\% limestone replacement for fly ash and the accompanying reduction of the fly ash content from $40 \%$ to $30 \%$, as the latter is chiefly responsible for the retardation present in these binary and ternary mixtures. It should be pointed out, though, that this 60:30:10c mixture still obtains a $40 \%$ reduction in cement content relative to a $100 \%$ cement, $\mathrm{w} / \mathrm{c}=0.30$ mixture, and a $33 \%$ reduction in cement content (volume) relative to

its $\mathrm{w} / \mathrm{c}=0.42$ counterpart, illustrating the potential sustainability benefits of these ternary blends.

A further topic to be addressed within the scope of the present experiments is the relationship between isothermal calorimetry and the development of mechanical resistance (setting and strength) for a cement paste or mortar. As mentioned previously, a higher $w / c$ mixture will generally require a greater degree of hydration to achieve setting, as observed in the present study
(Table 3.1). However, for a constant volume fraction of water, as employed for eight of the paste mixtures examined in this study, one might expect a reasonable correlation between heat release and setting or strength development. With this in mind, the cumulative heat release values up to the times of initial and final set have been included in Table 3.1. These cumulative heat release values do not include the contributions from the initial mixing and dissolution of cement phases, as data acquisition typically began $1 \mathrm{~h}$ after the initial contact between cement and water. Assuming that most of the heat generation during this first hour is due to the input of mixing energy and dissolution processes, neglecting this contribution when considering a potential relationship between heat release and the formation (precipitation) of hydration products would seem to be a reasonable approach. For the eight paste mixtures with a constant volume fraction of water (and powders), for the cumulative heat release at the times of initial set, an average value of $46.6 \mathrm{~J} / \mathrm{ml}$ water with a coefficient of variation of $15 \%$ is determined. The equivalent values for the times of final set are $101.2 \mathrm{~J} / \mathrm{ml}$ water and $12 \%$, with one less data point due to the lack of a final setting time determination for the ternary blend with the fine silica powder. These coefficients of variation for cumulative heat release at initial and final set are similar to those found in a previous study for fly ash/cement blends with various powder additions (gypsum, rapid set cement, and calcium hydroxide; Bentz \& Ferraris, 2010).

The relationship between cumulative heat release and strength development is explored in Figure 3.7 that plots the $1 \mathrm{~d}$ and the $7 \mathrm{~d}$ compressive strengths for the mortar mixtures vs. their cumulative heat release (at the same age) on both a per $\mathrm{ml}$ of paste and a per $\mathrm{ml}$ of (initial) water basis. When plotted against the cumulative heat release on a per $\mathrm{ml}$ paste basis, the results for the $w / c=0.42$ mortar are separated from the others that all contain the same initial volume fraction of water. However, when plotted against the cumulative heat release on a per $\mathrm{ml}$ of (initial) water basis, all of the results for these mortars are seen to basically lie on a single straight line with some scatter $\left(\mathrm{R}^{2}=0.972\right)$. This reasonable correlation between early age cumulative heat releases and strengths has been observed previously for portland cements of different finenesses (Bentz, 2010a), and has also recently been demonstrated for blended cements prepared with a variety of fly ashes and natural pozzolans, when a constant volumetric proportioning paradigm was employed (Bentz, Durán-Herrera, \& Galvez-Moreno, 2012).

\section{REFERENCES}

Bentz, D. P. (2006). Modeling the influence of limestone filler on cement hydration using CEMHYD3D. Cement and Concrete Composites, 28(2), 124-129. http://dx.doi.org/10.1016/ j.cemconcomp.2005.10.006

Bentz, D. P. (2010a). Blending different fineness cements to engineer the properties of cement-based materials. Magazine 
of Concrete Research, 62(5), 327-338. http://dx.doi.org/10. 1680/macr.2008.62.5.327

Bentz, D. P. (2010b). Powder additions to mitigate retardation in high-volume fly ash mixtures. ACI Materials Journal, 107(5), 508-514. http://dx.doi.org/10.14359/51663971

Bentz, D. P., Durán-Herrera, A., \& Galvez-Moreno, D. (2012). Comparison of ASTM C311 strength activity index testing vs. testing based on constant volumetric proportions. Journal of ASTM International, 9(1), 1-7. https://doi.org/10. 1520/JAI104138

Bentz, D. P., \& Ferraris, C. F. (2010). Rheology and setting of high volume fly ash mixtures. Cement and Concrete Composites, 32(4), 265-270. http://dx.doi.org/10.1016/j.cemcon comp.2010.01.008

Bentz, D. P., Ferraris, C. F., De la Varga, I., Peltz, M. A., \& Winpigler, J. (2010). Mixture proportioning options for improving high volume fly ash concretes. International Journal of Pavement Research and Technology, 3(5), 234-240.

Bentz, D. P., Sato, T., De la Varga, I., \& Weiss, W. J. (2012). Fine limestone additions to regulate setting in high volume fly ash mixtures. Cement and Concrete Composites, 34(1), 11-17. http://dx.doi.org/10.1016/j.cemconcomp.2011.09.004

De la Varga, I. (2013). Increased fly ash volume and internal curing in concrete structures and pavements (Doctoral dissertation). West Lafayette, IN: Purdue University. Retrieved from http://docs.lib.purdue.edu/dissertations/AAI3605112/

De la Varga, I., Castro, J., Bentz, D., \& Weiss, J. (2012). Application of internal curing for mixtures containing high volumes of fly ash. Cement and Concrete Composites, 34(9), 1001-1008. http://dx.doi.org/10.1016/j.cemconcomp.2012. 06.008

De Weerdt, K., Haha, M. B., Le Saout, G., Kjellsen, K. O., Justnes, H., \& Lothenbach, B. (2011). Hydration mechanisms of ternary Portland cements containing limestone powder and fly ash. Cement and Concrete Research, 41(3), 279-291. http://dx.doi.org/10.1016/j.cemconres.2010.11.014

De Weerdt, K., Kjellsen, K. O., Sellevold, E., \& Justnes, H. (2011). Synergy between fly ash and limestone powder in ternary cements. Cement and Concrete Composites, 33(1), 30-38. http://dx.doi.org/10.1016/j.cemconcomp.2010.09.006

Gutteridge, W. A., \& Dalziel, J. A. (1990). Filler cement: The effect of the secondary component on the hydration of Portland cement: Part I. A fine non-hydraulic filler. Cement and Concrete Research, 20(5), 778-782. http://dx.doi.org/10. 1016/0008-8846(90)90011-L

Sandberg, P. J., \& Roberts, L. R. (2005). Cement-admixture interactions related to aluminate control. Journal of ASTM International, 2(6), 1-14.

Sato, T., \& Beaudoin, J. (2011). Effect of nano- $\mathrm{CaCO}_{3}$ on hydration of cement containing supplementary cementitious materials. Advances in Cement Research, 23(1), 1-11. http://dx.doi.org/10.1680/adcr.9.00016

Sato, T., Daillo, F., \& Trischuk, K. (2011). Dispersion of nano$\mathrm{CaCO}_{3}$ coated with various surfactants for accelerating hydration of cement. Unpublished report. 


\section{Standard Method of Test for}

\section{Evaluating Stress Development and Cracking Potential due to Restrained Volume Change Using a Dual Ring Test}

\section{AASHTO Designation: T XXX-12}

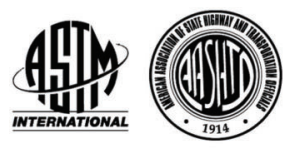

1. SCOPE

1.1. This test method covers the evaluation of stress development and cracking potential in concrete when volume changes caused by shrinkage and temperature changes are restrained. The procedure is comparative for the degree of restraint of the ring and is not intended to determine the time of initial cracking of a concrete cast in any specific type of structure.

1.2. The values stated in SI units are to be regarded as the standard.

1.3. This standard does not purport to address all of the safety problems, if any, associated with its use. It is the responsibility of the user of this standard to establish appropriate safety and health practices and determine the applicability of regulatory limitations prior to use.

2. REFERENCED DOCUMENTS

2.1. AASHTO Standards:

R 39, Making and Curing Concrete Test Specimens in the Laboratory

2.2. ASTM Standards:

C 305, Practice for Mechanical Mixing of Hydraulic Cement Pastes and Mortars of Plastic Consistency

C 403/C403M, Test Method for Time of Setting of Concrete Mixtures by Penetration Resistance

C 1581, Standard Test Method for Determining Age at Cracking and Induced Tensile Stress Characteristics of Mortar and Concrete under Restrained Shrinkage

C 1698, Standard Test Method for Autogenous Strain of Cement Paste and Mortar

F 1684, Standard Specification for Iron-Nickel and Iron-Nickel-Cobalt Alloys for Low Thermal Expansion Applications

3. SUMMARY OF TEST METHOD 
3.1. This test method measures the strains developed in two concentric metal rings providing restraint to a mortar specimen undergoing volume change. The measured strains in the restraining rings are used to calculate the stress development in the concrete specimen. This enables the stress that develops due to restraint to be quantified along with an assessment of the cracking potential of the concrete mixture.

3.2. This test method utilizes restraining rings made of a low thermal expansion iron-nickel alloy (Invar( $\left.{ }^{\circledR}\right)$, allowing for the temperature of the test to be varied, while the rings remain nominally volumetrically stable. The test equipment is enclosed in an insulated chamber, with the temperature being controlled throughout the entirety of the test. The methodology set forth in this standard is to utilize the thermal capabilities of the dual ring test to assess the potential for cracking at 2, 4, and 7 days of age. The temperature of the test specimen is held at a constant $23+/-2^{\circ} \mathrm{C}$ until the appropriate age, upon which time the temperature is reduced at a rate of $2^{\circ} \mathrm{C} /$ hour until cracking is induced. This temperature drop approach yields the remaining stress capacity of the concrete specimens at early ages, quantifying the potential for cracking in the specimen at the given ages.

4.

SIGNIFICANCE AND USE

4.1. This test method is suitable for evaluating the effects of concrete variations on the cracking potential of restrained concrete elements. These variations may include aggregate source, aggregate gradation, aggregate absorption, aggregate-paste bond, cement type, cement content, water content, supplementary cementitious materials, fiber reinforcement, or chemical admixtures.

4.2. Actual cracking during service or construction is dependent upon many variables including degree of restraint, hydration effects of the cement, curing methods, and environmental factors. This method is useful for comparing the relative likelihood of early age cracking and for aiding in the selection of appropriate mixture designs. This method may also be utilized to evaluate the effect of temperature change on the thermal stress development and cracking tendency.

4.3. The details of the test method apply to nominal dimensions of specimens as set forth in this standard. Other specimen geometries may be used with appropriate changes being made to ensure an equivalent degree of restraint in the specimens. The pertinent calculations can be found in Reference 12.2.

4.4 The numerical results from this test method must be interpreted with caution, especially in applications such as quality control and acceptance testing. 
Other concrete tests that may be related to cracking tendency are the compressive strength, tensile strength, elastic modulus, Poisson's ratio, unrestrained linear autogenous strain, the single restrained ring test, and creep/relaxation.

5.

5.1 .

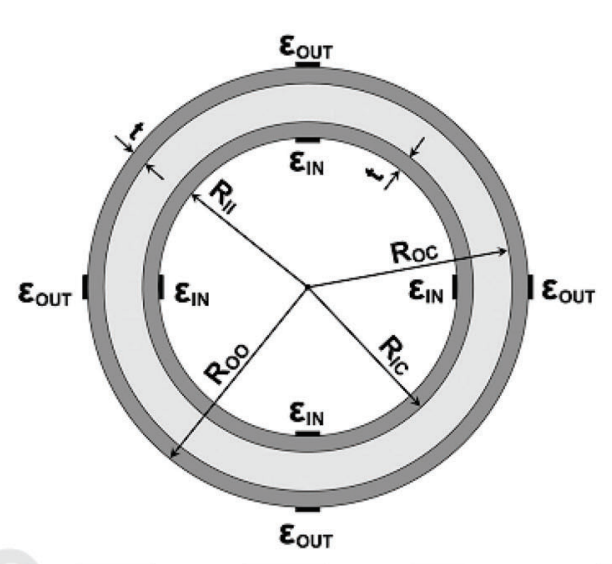

(a)

Restraining Rings - The standard restraining rings shall be made of a low thermal expansion iron-nickel alloy (Invar(R) as specified in ASTM F1684 or equivalent. The inner and outer rings shall have wall thicknesses of $19 \mathrm{~mm} \pm 3 \mathrm{~mm}(0.75 \mathrm{in} \pm 0.12 \mathrm{in})$, as provided by the specified dimensions in Table 1. The height of the rings shall be $76.2 \mathrm{~mm} \pm 3 \mathrm{~mm}$ ( $3 \mathrm{in} \pm 0.12$ in). The inner and outer faces shall be round, true, seamless, and polished smooth.

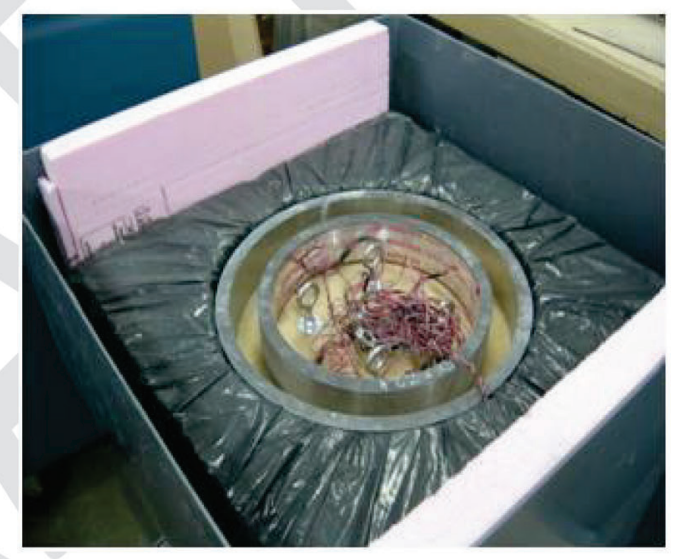

(b)

Figure 1 - Geometry of dual ring test (a) and dual ring test setup in insulating chamber (b) (Reference 12.2)

Table 1 - Dimensions of dual ring test

\begin{tabular}{cc}
\hline Ring Face & Radius (mm) \\
\hline $\mathrm{R}_{\mathrm{II}}$ & $146 \pm 3$ \\
$\mathrm{R}_{\mathrm{IC}}$ & $165 \pm 3$ \\
$\mathrm{R}_{\mathrm{OC}}$ & $203 \pm 3$ \\
$\mathrm{R}_{\mathrm{OO}}$ & $222 \pm 3$ \\
\hline
\end{tabular}

Note 1 - Bond low coefficient of thermal expansion type strain gages at four equidistant, midheight locations on both the interior of the inner ring and the exterior of the outer ring. 
Data Acquisition - The data acquisition unit shall be compatible with the strain instrumentation and automatically record each strain gauge independently at 5 minute intervals. It is recommended that the system be capable of correction for thermal changes.

5.3. Base Form - The base form shall be nonabsorbent. Fabricate the base form of resin-coated or polyethylene-coated plywood to minimize frictional restraint of the concrete.

Insulating Chamber - The dual ring test setup shall be enclosed in an insulating chamber throughout the entirety of testing. The insulating chamber shall be constructed such that it achieves an overall heat transfer coefficient of $0.04 \mathrm{~W} / \mathrm{m}^{2} / \mathrm{K}$ or lower. Details of this calculation are can be found in Reference 12.2.

Temperature Control System - A temperature control system shall be used to regulate the temperature of the testing apparatus. The temperature control system shall have a minimum operating range of $30^{\circ} \mathrm{C}$ to $-10^{\circ} \mathrm{C}$ with an operating rate of at least $2.0^{\circ} \mathrm{C} / \mathrm{h}$.
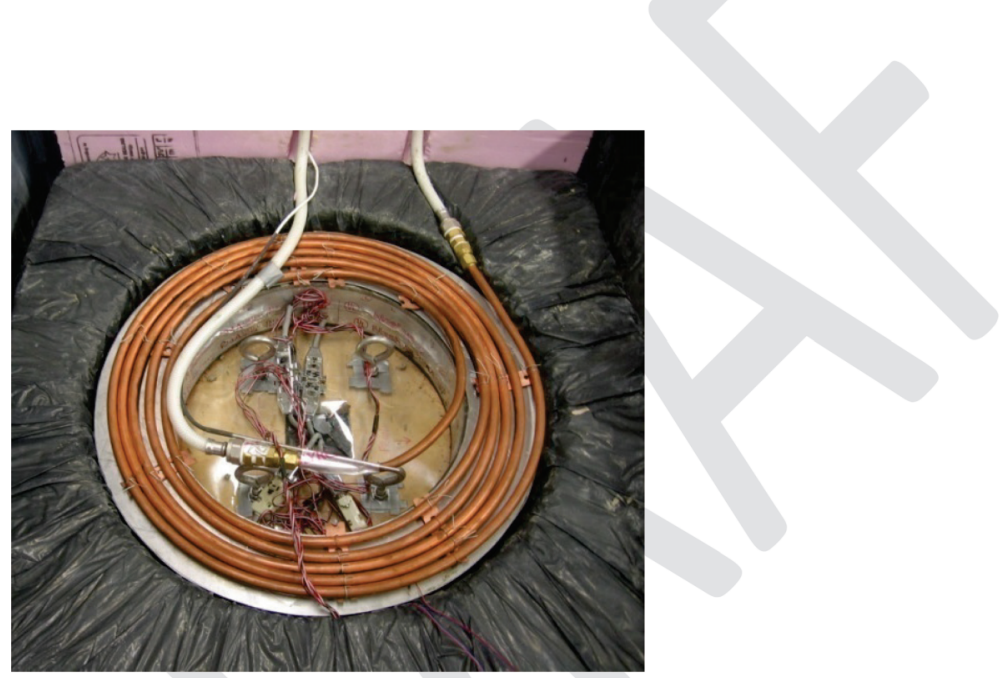

Figure 2 - Temperature control system for dual ring test (Reference 12.2)

Note 2 - An electrical heating and cooling panel is recommended in order to minimize additional moisture from being introduced into the testing chamber.

Temperature Monitoring - Thermocouples or equivalent shall be utilized to automatically record the ambient temperature and the temperature of the restraining rings and specimen. Attach the thermocouples to the non-testing face of the restraining rings near the strain gage sites using an appropriate conductive tape or equivalent method.

6. 
6.1. Coat the base form with a suitable form release agent. The test faces of the rings (outer face of inner ring and inner face of outer ring) shall be covered with a suitable adhesion resistant material such as acetate sheets or a form release agent.

6.2. Center the invar rings on the base form within the insulating chamber.

Note 3 - It is recommended to use a set of fabricated spacers to ensure the rings are concentric.

6.3. Mix and cure the test ring specimen in accordance with the applicable requirements of R 39 and ASTM C 305.

6.4. Cast the annulus of concrete between the two restraining rings in two equal lifts. The concrete shall be vibrated in situ after each lift using a suitable hand vibrator. Vibrate the concrete equidistantly 30 times per layer, ensuring that the vibrator head slightly penetrates into the previous layer with each insertion.

6.5 After consolidation, strike off the concrete surface. Clean any excess concrete from the top and sides of the form. Finish the specimen with the minimum manipulation necessary to achieve a finished, flat, and even surface.

7. TESTING PROCEDURE

7.1. Place the temperature control system on the finished specimen and testing apparatus. Program the appropriate temperature profile for the given experiment.

Note 4 - The temperature profile for the approach implemented in this standard is to set isothermal testing conditions at $23^{\circ} \mathrm{C} \pm 1{ }^{\circ} \mathrm{C}$ until 2 , 4, or 7 days, whereafter the temperature is reduced at a rate of $2{ }^{\circ} \mathrm{C} / \mathrm{h}$ to induce thermal cracking or until the lower temperature limit of the equipment is achieved.

Note 5 - At very early ages, a thermal increase (beyond the $23{ }^{\circ} \mathrm{C}+1{ }^{\circ} \mathrm{C}$ limit) may be seen due to the heat of hydration. This is an artifact of the testing limitations and should be considered normal, as the material is highly compliant at early ages and thus little effect on the results of the testing procedure outlined in Note 4 are seen. Generally, the temperature of the specimen should return to $23{ }^{\circ} \mathrm{C} \pm 1{ }^{\circ} \mathrm{C}$ within 24 hours of casting.

7.2. Temperature compensation for the invar ring strain gauge measurements shall be performed by monitoring the temperature change on the ring surface at gauge sites.

Note 6 - Prior to testing, a temperature compensation curve for each strain gauge shall be created by cycling the invar rings (without any specimen) through any applicable temperature ranges. For the procedure described in Note 4, the temperature cycle should be from $30{ }^{\circ} \mathrm{C}$ to $15{ }^{\circ} \mathrm{C}$ and back, at a rate of $2{ }^{\circ} \mathrm{C} / \mathrm{h}$. Plot the strain readings as a function of temperature to 
create the temperature compensation curve. It is recommended that a data acquisition system with built in temperature compensation capabilities be utilized, otherwise the temperature compensation shall be performed manually during the calculations.

7.3. Record the individual strain gauge readings on the inner and outer invar rings at 5 minute intervals from the finish of casting until the end of testing.

Note 7 - An instantaneous strain decrease of 20 microstrain in one or more gauges usually indicates cracking. When using fiber-reinforced concrete mixtures, a distinct decrease in strain may not be apparent.

7.4. Place any additional insulating panels on top of the testing apparatus then seal the insulating chamber for the remainder of the testing period.

8.

\section{CALCULATION AND INTERPRETATION OF RESULTS}

8.1. The circumferential residual stress in the specimen at the inner face of the specimen, $\sigma_{\theta}\left(\mathrm{R}_{\mathrm{IC}}\right)$, is provided by Equation 1a:

$\sigma_{\theta}\left(R_{I C}\right)=-\varepsilon_{I N} \cdot E_{\mathrm{I}} \cdot\left(\frac{R_{I C}^{2}-R_{I I}^{2}}{2 \cdot R_{I C}^{2}}\right) \cdot\left[\frac{R_{O C}^{2}+R_{I C}^{2}}{\left(R_{O C}^{2}-R_{I C}^{2}\right)}\right]-\varepsilon_{O U T} \cdot E_{\mathrm{I}} \cdot\left(\frac{R_{O O}^{2}-R_{O C}^{2}}{2 \cdot R_{O C}^{2}}\right) \cdot\left[\frac{2 \cdot R_{O C}^{2}}{\left(R_{O C}^{2}-R_{I C}^{2}\right)}\right]$

where:

$\varepsilon_{\mathrm{IN}}=$ average of the four strain gages on the inner ring less their temperature compensation $(\mu \mathrm{m} / \mathrm{m})$

$\varepsilon_{\mathrm{OUT}}=$ average of the four strain gages on the outer ring less their temperature compensation $(\mu \mathrm{m} / \mathrm{m})$

$\mathrm{E}_{\mathrm{I}} \quad=$ Young's elastic modulus of restraining rings, taken to be $141,000 \mathrm{MPa}(20,450 \mathrm{ksi})$ for Invar ${ }^{\circledR}$

$\mathrm{R}_{\mathrm{II}} \quad=$ Radius to the inner face of the inner ring $(\mathrm{mm})$

$\mathrm{R}_{\mathrm{IC}}=$ Radius to the inner face of the concrete specimen (mm)

$\mathrm{R}_{\mathrm{OC}}=$ Radius to the outer face of the concrete specimen $(\mathrm{mm})$

$\mathrm{R}_{\mathrm{OO}}=$ Radius to the outer face of the outer ring $(\mathrm{mm})$

Using the standard dimensions as specified in section 5.1, Equation la can be simplified to the following:

$\sigma_{\theta}\left(R_{I C}\right)=-0.53 \varepsilon_{I N} E_{\mathrm{I}}-0.58 \varepsilon_{\text {OUT }} E_{\mathrm{I}}$

8.2. To calculate the remaining stress capacity, $\sigma_{\text {Remaining }}\left(R_{I C}\right)$, of the specimen when using the testing approach described in Note 4, subtract the residual stress in the specimen prior to

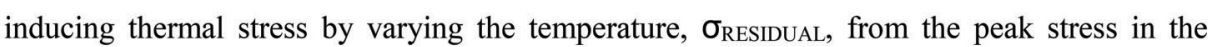
specimen after thermal cracking occurs, $\sigma_{\mathrm{CRACK}}$. In the event that cracking cannot be induced, $\sigma_{\text {CRACK }}$ is taken as the maximum stress withstood by the specimen. The remaining stress capacity represents the additional stress necessary to cause cracking in the specimen at that given age. This value yields insight as to how close the specimen is to cracking under the 
applied conditions. Report the temperature differential that the specimen endured during the testing for remaining stress capacity.

$\sigma_{\text {Remaining }}\left(R_{I C}\right)=\sigma_{C R A C K}-\sigma_{R E S I D U A L}$

Note 8 - This procedure shall be repeated at 2,4 , and 7 days of age, so as to generate a remaining stress capacity curve for the given mixture.

9.

\section{REPORT}

9.1 .

Report the following, if known:

9.1.1. Properties of the concrete mixture - batch materials and proportions, air content, consistency, unit weight, and temperature of fresh concrete;

9.1.2. Variations in forming, casting, or curing;

9.1.3. Restraining ring thicknesses and specimen dimensions;

9.1.4. Temperature of testing chamber, restraining rings, and concrete mixture at time of casting;

9.1.5. Plot of residual tensile stress versus time with specimen temperature profile versus time superimposed on the secondary y-axis;

9.1.7. Remaining stress capacity in the specimen at specified ages with the corresponding temperature differential required to generate said thermal stresses; and

9.1.8. If cracking occurs before the minimum temperature is reached, report the time-to-cracking to one-tenth of a day.

\section{PRECISION AND BIAS}

10.1. Precision:

10.1.1. Single-Operator Precision: The single operator coefficient of variation of a single test result has been found to be x.x \%. Therefore, the results of two properly conducted tests by the same operator on the same concrete material at the same age are not expected to differ by more than $\mathrm{xx} \%$ of their average.

10.1.2. Multi-Laboratory Precision - No data is currently available on experimental variation between multiple laboratories.

11.

\section{KEYWORDS}


11.1. Concrete, restrained ring test, dual ring test, shrinkage, thermal cracking

12.

REFERENCES

12.1. A. Radlinska, et al., "The Ring Test: A Review of Recent Developments," in International RILEM Conference on Volume Changes of Hardening Concrete: Testing and Mitigation, Lyngby, Denmark, 2006, pp. 205-214

12.2

Schlitter, J.L., Senter, A.H., Bentz, D.P., Nantung, T., and Weiss, W.J., “A Dual Concentric Ring Test for Evaluating Residual Stress Development due to Restrained Volume Change", Journal of ASTM International, Vol. 7, No. 9, 2010. 


\section{Project Partners}

This work was partially supported by the Federal Highway Administration through the Cooperative Agreement No. DTFH61-09-H-00005 in support of the Exploratory Advanced Research (EAR) Program. The work was led and performed by Purdue University, NIST, NRMCA, and Auburn University. The authors wish to thank the many agencies and vendors we have collaborated with during the development of this report. The contents of this work reflect the views of the authors, who are responsible for the facts and the accuracy of the data presented herein, and do not necessarily reflect the official views or policies of the sponsoring organizations. These contents do not constitute a standard, specification, or regulation.

\section{Publication}

This report was published in collaboration with the Joint Transportation Research Program and Purdue University. The full content of this technical report is available for download at https://doi.org/10.5703/1288284316554.

\section{Open Access and Collaboration with Purdue University}

The Indiana legislature established the Joint Highway Research Project in 1937. In 1997, this collaborative venture between the Indiana Department of Transportation and Purdue University was renamed as the Joint Transportation Research Program (JTRP) to reflect state and national efforts to integrate the management and operation of various transportation modes. Since 1937, the JTRP program has published over 1600 technical reports. In 2010, the JTRP partnered with the Purdue University Libraries to incorporate these technical reports in the University's open access digital repository and to develop production processes for rapidly disseminating new research reports via this repository. Affiliated publications are also part of the collection. As of 2017, the JTRP collection has over 1.5 million downloads, with some particularly popular reports having over 20,000 downloads. 